\title{
II Tolomeo
}

e-ISSN 2499-5975

Vol. 22

Dicembre | December | Décembre 2020

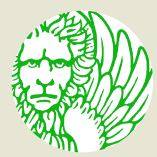

Edizioni Ca'Foscari

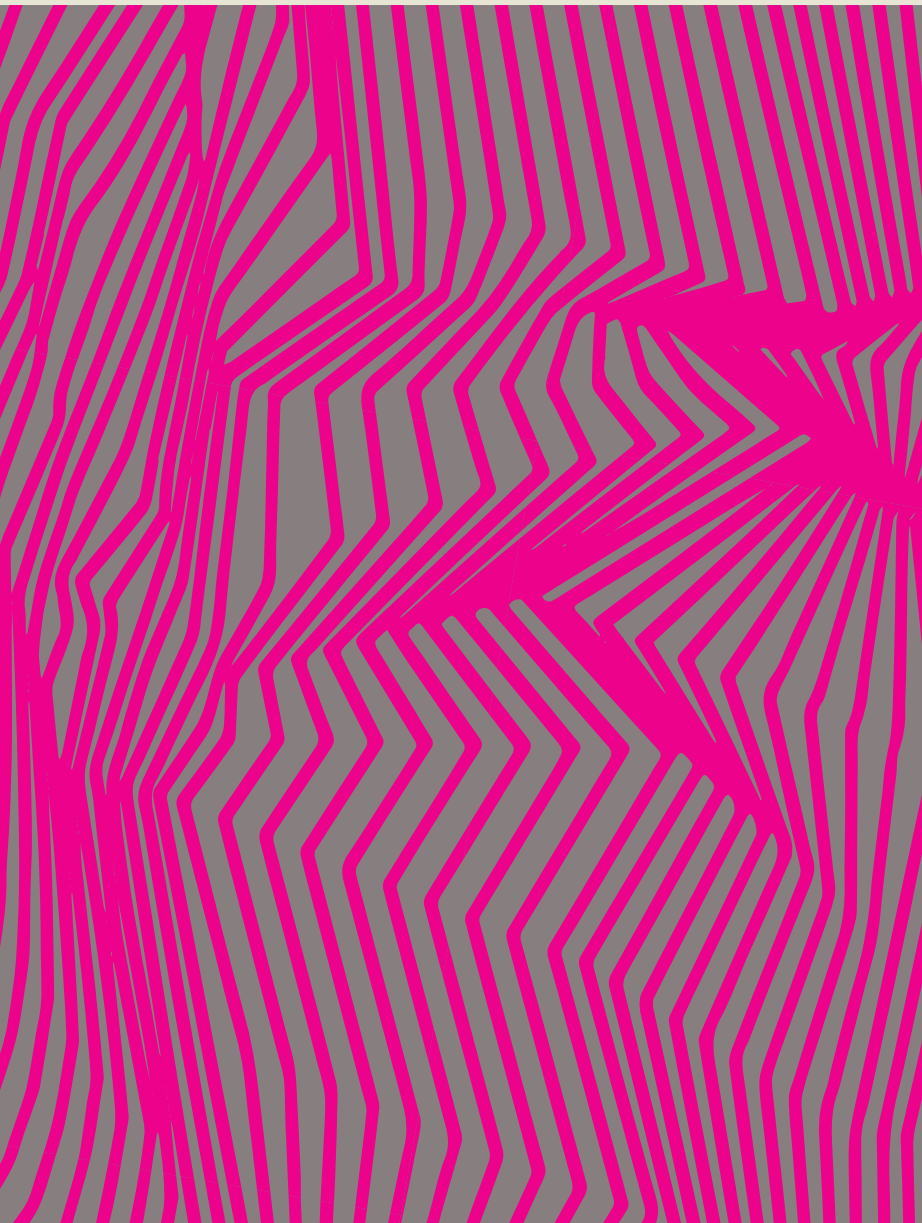




\section{Il Tolomeo}

Rivista di studi postcoloniali

A Postcolonial Studies Journal

Journal d'études postcoloniales

Direttore| Director|Directeur

Alessandro Costantini

Edizioni Ca' Foscari - Digital Publishing

Fondazione Università Ca' Foscari Venezia

Dorsoduro 3246, 30123 Venezia

URL http://edizionicafoscari.unive.it/it/edizioni/riviste/il-tolomeo/ 


\section{Il Tolomeo}

\section{Rivista di studi postcoloniali | A Postcolonial Studies Journal | Journal d'études postcoloniales}

Rivista annuale |Annual journal | Revue annuelle

Direttore scientifico | General Editor | Directeur Alessandro Costantini (Università Ca' Foscari Venezia, Italia)

Consiglio di direzione|Board of Directors |Comité de direction Shaul Bassi (Università Ca'Foscari Venezia, Italia) Alessandro Costantini (Università Ca'Foscari Venezia, Italia) Marco Fazzini (Università Ca'FoscariVenezia, Italia)

Comitato scientifico editoriale | Editorial Scientific Board | Comité scientifique Shaul Bassi (Università Ca'FoscariVenezia, Italia) Carmen Concilio (Università degli Studi di Torino, Italia) Alessandro Costantini (Università Ca'Foscari Venezia, Italia) Marco Fazzini (Università Ca'FoscariVenezia, Italia) Serenella lovino (University of North Carolina, USA) Lila Lamrous (Université Clermont Auvergne, France) Pablo Mukherjee (University of Warwick, UK) Michal Obszynski (University of Gdańsk, Poland) Yolaine Parisot (Université Paris Val de Marne, France)

Comitato scientifico internazionale|InternationalAdvisory Board/Comité scientifique international Giulio Marra (fondatore | founder | fondateur I/ Tolomeo, Università Ca' Foscari Venezia, Italia) Francesca Romana Paci (cofondatore | co-founder | cofondateur // Tolomeo, Università del Piemonte Orientale, Italia) Elleke Boehmer (University of Oxford, UK) Andrea Cali (Università del Salento, Italia) Yves Chemla (Université Paris V, France) Kathleen Gyssels (University of Antwerpen, Belgium) Graham Huggan (University of Leeds, UK) Jean Jonassaint (Syracuse University, USA) Józef Kwaterko (UniwersytetWarszawski, Polska) Chris Mann (Rhodes University, South Africa) Marco Modenesi (Università di Milano, Italia) Radhika Mohanran (Cardiff University, UK) Armando Pajalich (Università Ca' Foscari Venezia, Italia) Sandra Ponzanesi (University College Utrecht, Nederland) Itala Vivan (Università degli Studi di Milano, Italia) Tony Voss (University of Kwa-Zulu Natal, Southafrica)

Comitato di redazione | Editorial Board | Comité de rédaction Gerardo Acerenza (Università di Trento, Italia) Esterino Adami (Università degli Studi di Torino, Italia) Michela A. Calderaro (Università degli Studi di Trieste, Italia) Marta Cariello (Università degli Studi della Campania «Luigi Vanvitelli», Italia) Carmen Concilio (Università degli Studi di Torino, Italia) Alessandro Costantini (Università Ca' Foscari Venezia, Italia) Irene De Angelis (Università degli Studi di Torino, Italia) Lucio De Capitani (Università Ca' Foscari Venezia, Italia) Sara Del Rossi (UniwersytetWarszawki, Polska) Adriano Elia (Università degli Studi Roma Tre, Italia) Marco Fazzini (Università Ca FoscariVenezia, Italia) Alice Girotto (Università Ca'Foscari Venezia, Italia) Maria Paola Guarducci (Università degl Studi Roma Tre, Italia) Yannick Hamon (Università Ca' Foscari Venezia, Italia) Marie-Christine Jamet (Università Ca' Foscari Venezia, Italia) David Newbold (Università Ca' Foscari Venezia, Italia) Silvia Riva (Università degli Studi di Milano, Italia) Biancamaria Rizzardi (Università di Pisa, Italia) Giuseppe Serpillo (Università degli Stud di Sassari, Italia) Eliana Vicari (Université Paris Nanterre, Nanterre Cedex) Alessia Vignoli (Instytut Romanistyki, UniwersytetWarszawski, Polska) Ilaria Vitali (Università degli Studi di Macerata, Italia) Anna Zoppellari (Università degli Studi di Trieste, Italia)

Segreteria di redazione | Editorial assistants | Secrétaires de rédaction Silvia Boraso (Università Ca' Foscari Venezia, Italia) Lucio De Capitani (Università Ca’ Foscari Venezia, Italia)

Direzione e redazione | Head office | Siège Dipartimento di Studi Linguistici e Culturali Comparati, Università Ca' Foscari Venezia | Dorsoduro 1405, 30123 Venezia| tolomeo@unive.it

Editore|Publisher|Éditeur Edizioni Ca' Foscari - Digital Publishing | Dorsoduro 3246, 30123 Venezia, Italia|ecf@unive.it

C 2020 Università Ca' Foscari Venezia

(C) 2020 Edizioni Ca’ Foscari - Digital Publishing per la presente edizione

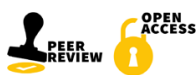

\section{(ㄷ)(1)}

Quest'opera è distribuita con Licenza Creative Commons Attribuzione 4.0 Internazionale

This work is licensed under a Creative Commons Attribution 4.0 International License

Certificazione scientifica delle Opere pubblicate da Edizioni Ca'Foscari - Digital Publishing: tutti i saggi pubblicati hanno ottenuto il parere favorevole da parte di valutatori esperti della materia, attraverso un processo di revisione anonima sotto la responsabilità del Comitato scientifico della rivista. La valutazione è stata condotta in aderenza ai criteri scientifici ed editoriali di Edizioni Ca' Foscari.

Scientific certification of the works published by Edizioni Ca' Foscari - Digital Publishing: all essays published in this volume have received a favourable opinion by subject-matter experts, through an anonymous peer review process under the responsibility of the Scientific Committee of the journal. The evaluations were conducted in adherence to the scientific and editorial criteria established by Edizioni Ca' Foscari. 
Vol. 22 - Dicembre | December | Décembre 2020

\title{
Sommario | Table of contents | Résumé
}

\author{
TESTI CREATIVI | CREATIVE WORKS | CRÉATIONS
}

\section{Beyrouth}

Maha Badr

\section{Beirut}

Maha Badr, translation by Gregory Dowling

\section{Beirut}

Maha Badr, traduzione di Alessandro Costantini

Injury

At the Berlin Rathaus Shoneberg Flea Market

Ingrid de Kok

\section{Ferita}

Al mercato delle pulci di Rathaus Shoneberg, Berlino Ingrid de Kok;

a cura di Maria Paola Guarducci e Francesca Terrenato

\section{Still}

Grosse Hamburger Strasse, Berlin

Ingrid de Kok

\section{Fermo immagine}

Grosse Hamburger Strasse, Berlino

Ingrid de Kok;

a cura di Maria Paola Guarducci e Francesca Terrenato

\section{Border Travel}

Ingrid de Kok 


\section{Oltre il confine}

Ingrid de Kok;

a cura di Maria Paola Guarducci e Francesca Terrenato

\section{Allowances}

Ingrid de Kok

\section{Si può}

Ingrid de Kok;

a cura di Maria Paola Guarducci e Francesca Terrenato

\section{Lament}

Ingrid de Kok

\section{Lamento}

Ingrid de Kok;

a cura di Maria Paola Guarducci e Francesca Terrenato

Playing

Ingrid de Kok

\section{Suono}

Ingrid de Kok;

a cura di Maria Paola Guarducci e Francesca Terrenato

Ingrid de Kok. Parole, immagini, suoni

Maria Paola Guarducci, Francesca Terrenato

Waiting for the Virus

With Apologies to C.P. Cavafy and E. Keeley

Douglas Reid Skinner

Aspettando il virus

Porgendo le scuse a C.P. Cavafy e E. Keeley

Douglas Reid Skinner; traduzione di Marco Fazzini

Douglas Reid Skinner

Marco Fazzini 
DOSSIER. Postcolonial Approaches to the Environmental Humanities and Ecocriticism | Approches postcoloniales des humanités environnementales et de l'écocritique |Approcci postcoloniali alle scienze umane ambientali e all'ecocritica

Colonizzazione ambientale, defuturing capitalocenico e pratiche eco-artistiche decoloniali a sud Il 'caso Taranto'

Luigi Carmine Cazzato, Antonella D’Autilia

"The Road to Awesomeness": The Environment, Language and Rhetoric in Chetan Bhagat's Postcolonial India

Esterino Adami

Decolonizing the Anthropocene: 'Slow Violence' and Indigenous Resistance in Cherie Dimaline's The Marrow Thieves

Chiara Xausa

Ha Jin's Ecocritical Irony in the Stylistic Analysis of the Personal Pronouns in "A Tiger Fighter is Hard to Find" Isabella Marinaro

Dwelling, Dispossession, and "Slow Violence" in the Time of Climate Change

The Representation of Refugees in Amitav Ghosh's

The Hungry Tide

Nicola Pilia

Maïssa Bey: romancière de l'agentivité de femmes

Entre réécriture de l'Histoire et invention de soi

Lila Lamrous

Les catastrophes naturelles dans les romans caribéens d'expression française

Alessia Vignoli

La critique environnementale dans les récits animaliers de René Maran

Lia Milanesio 
La Peste d'Albert Camus : une analyse de la société coloniale algérienne à travers le prisme de l'épidémie

Ruy Duarte de Carvalho's Neo-Animist Project:

An African Approach to Posthumanism

Alice Girotto

Note della Redazione

VARIA

Mélancolie de Janvier

Yves Chemla

Sur les pas de Louis-Philippe Dalembert. Un hommage à la carrière du " gavroche caraïbe »

Silvia Boraso

Le transfert culturel chez Ousmane Sembène : du péritexte auctorial au péritexte traduit

Littérature des Français du Maghreb et métissage linguistique

Les cas de Jean Pélégri et Jean-Pierre Koffel

Anna Zoppellari

Language Colonization and English Hybridization: The Use of Irish English Lexis in Twentieth Century Irish Drama Fabio Luppi

INTERVISTE | INTERVIEWS | INTERVIEWS - DOCUMENTI | DOCUMENTS | DOCUMENTS

Jacques Stephen Alexis au Moulin d'Andé : L'espace d'un scintillement

Kathleen Gyssels

An Ecocritical Journey Through the Narrative of the Canadian Novelist Alissa York: How Literature Explores Environmental Problems 
La vérité sort de la bouche du cheval, un roman

pour découvrir l'altérité

Quelques mots avec Meryem Alaoui

Martina Gennari

RECENSIONI | REVIEWS | COMPTES RENDUS

Sandra Federici

L'entrance des auteurs africains dans le champ de la bande dessinée européenne de la langue française (1978-2016)

Alessandro Scarsella

Luigi Gaffuri

Racconto del territorio africano

Silvia Boraso

Langue française et arts du spectacle à Montréal : aperçu de deux parcours biographiques

Cristina Brancaglion

Alessandra Ferraro

« Représentations littéraires de la différence culturelle »

Fabiana Fianco

Francis-Joachim Roy

\section{Les chiens}

Silvia Boraso

Daniel-Henri Pageaux

Lectures Indiaocéanes. Essais sur les francophonies

de l'Océan Indien

Sara Pagone

"Univers de la radio ». Ponts, 19, 2019

Anna Michieletto

Fawzia Zouari

Molière et Shéhérazade

Sara Alouani

Agnès Schaffauser

Salim Bachi

Sara Alouani 
Michela A. Calderaro

Sheer Bliss: A Creole Journey

Amanda T. Perry

Sara Florian

Caribbean Counterpoint: The Aesthetics of Salt

in Lasana Sekou

Lucio De Capitani

Listen, the Landscape: The Nature Narratives in Jacob Ross' Short Story Collection Tell No-One About This

Michelle Ramos-Rodriguez

Carla Tempestoso

Il teatro di Caryl Phillips. Transnazionalità, identità e appartenenze

Giuseppe Capalbo

Inua Ellams

Three Sisters

Ilaria Oddenino

NECROLOGI | OBITUARIES | NÉCROLOGIES

In memory of Jacques Coursil, 1938-2020

Paola Irene Galli Mastrodonato

BIO-BIBLIOGRAFIE | BIO-BIBLIOGRAPHIES |

BIO-BIBLIOGRAPHIES

Bio-bibliographies 
Testi Creativi | Creative Works | Créations 



\section{Beyrouth}

\section{Maha Badr}

Beyrouth,

Que veux-tu de cette flambée dans l'azur

Brûles-tu tes pierres dans le délice du feu

Exploses-tu tes larmes blanchies de sommeil

Éclates-tu rouge le rêve doux des glaces

Beyrouth,

Tu râles

As-tu honte de ton corps défait

As-tu peur du silence des ombres

Ressens-tu le remords d'une furie fiévreuse

Beyrouth,

Au loin

Entends-tu le murmure des débris

Vois-tu la fumée nébuleuse du chagrin

Sens-tu le deuil dans l'élan des flots

Beyrouth,

Beyrouth,

Beyrouth,

Au nom de la rose

Soulage le cri d'une poussière

Maha Badr, le 12 août 2020 


\section{Note}

Maha Badr, chercheuse, professeure et auteure littéraire libanaise, habitant Beyrouth, remet dans les mains des lecteurs du Tolomeo un petit poème et un grand don. Elle nous offre en partage son émotion à elle encore toute vivante et souffrante, jaillie du sol couvert de décombres et du ciel embrasé par les flammes de l'explosion.

Ce petit (c'est là un terme d'affection) poème est beau. Et douloureux. On dit que les chants désespérés sont les chants les plus beaux; je préfère penser que le sont les chants douloureux, mais qui gardent au fond un espoir: car son texte se clôt sur une nuance, sur une touche qui a couleur d'Espoir. Car c'est une forme d'auto-pietas que l'amour pour soi-même, mais avec et au milieu des autres.

Alessandro Costantini 


\section{Beirut}

Maha Badr, translation by Gregory Dowling

Beirut,

What do you seek from this blaze in the blue sky

Are you burning your stones in the delight of fire

Are you exploding your white tears of somnolence

Are you shattering in red the sweet dream of mirrors

Beirut,

You gasp

Are you ashamed of your undone body

Are you afraid of the silence of shadows

Do you feel the remorse of a feverish fury

Beirut,

In the distance

Do you hear the murmur of the debris

Do you see the cloudy smoke of chagrin

Do you feel grief in the dash of the waves

Beirut,

Beirut,

Beirut,

In the name of the rose

Lighten the cry of the dust 



\section{Beirut}

Maha Badr, traduzione di Alessandro Costantini

Beirut,

Cosa cerchi in questa fiammata nel cielo azzurro?

Bruci forse le tue pietre nella delizia del fuoco?

Fai scoppiare le tue lacrime bianche di sonno?

Mandi in mille pezzi rossi il sogno dolce degli specchi?

Beirut,

tu rantoli

Ti vergogni del tuo corpo sfatto?

Hai paura del silenzio delle ombre?

Avverti il rimorso di una furia febbrile?

Beirut,

In lontananza

Lo senti il mormorio delle macerie?

Vedi il dolore nelle nuvole di fumo?

Indovini il lutto nello slancio delle onde?

Beirut,

Beirut,

Beirut,

Nel nome della rosa

Rendi lieve il grido della polvere 



\section{Injury At the Berlin Rathaus Shoneberg Flea Market}

Ingrid de Kok

All the dolls are bald.

Some heads are porcelain

some straw-stuffed cotton

others rubbery, cold.

A few are blind, several stare across embroidered thread.

Some marble eyes, cobalt blue, cannot close or cry.

If they had souls or skulls

there might be something heraldic

coiled inside, perhaps wings,

the cauls of fallen seraphim.

Some arms are broken off, stacked in a pile

for locking back if we can, into torso sockets.

Necks are limp, held by string, almost new-born floppy

but more like rabbits

hanging on a poacher's peg.

Most are naked, sexless, but one wears a grey nightdress and that makes it worse. 
I watch them tipped from a hessian sack onto a long table

in the corner of the market

next to a stack of lightbulbs, plugs, extension cords, screws and spanners, other small rusted tools.

The trader smiles, wants to make a sale.

But I hesitate, wonder to myself

why he buys and sells

spanners, plugs and dolls.

Perhaps he's not a specialist.

He may be what he seems:

a poor guy in a threadbare belt, collecting this and that.

If I'm half delirious

blame it on the weather.

40 degrees, slowing my breath

softening my brain.

I should stop stuttering say clearly that I'll buy a doll, the one with pockmarked cheeks, take her home on the train.

But I won't be able to mend her, make her well again.

I know that. Not here.

I would have to hide her away. 


\section{Ferita}

\section{Al mercato delle pulci di Rathaus Shoneberg, Berlino}

Ingrid de Kok; a cura di Maria Paola Guarducci e Francesca Terrenato

Tutte le bambole sono calve.

Testa di porcellana alcune

altre di pezza e paglia

oppure di gomma, fredda.

Ce ne sono di cieche, certe ti guardano da filo cucito. Altre da biglie blu cobalto, senza palpebre o pianto.

Se avessero anima, o teschio lì dentro potrebbe annidarsi un emblema, forse due ali, placente di serafini caduti.

Ci sono braccia spezzate, ammucchiate per chi voglia tentare l'incastro, nelle orbite cave dei torsi.

Colli flosci, tenuti da spago, quasi da tenero neonato o piuttosto da coniglio penzolante dal carniere.

Le più sono nude, asessuate, ma una indossa una vestina grigia ed è peggio ancora. 
Le guardo cadere

da un sacco di iuta

su un tavolaccio

in fondo al mercato

accanto a lampadine, prese, prolunghe,

viti e chiavi inglesi,

altra ferraglia rugginosa.

L'uomo sorride, spera di vendere.

lo invece esito, mi chiedo

perché compra e vende

chiavi, prese e bambole.

Forse non è uno specialista.

0 è proprio quel che sembra:

un poveruomo con cinghia lisa

che raccoglie alla rinfusa.

Sarà colpa del tempo

se quasi vaneggio.

Quaranta gradi, respiro lento

il cervello galleggia.

Basta balbettare, dovrei

solo dire che ne compro una,

la bambola col viso butterato,

per portarla a casa in treno.

Ma non la saprei guarire, metterla a posto.

Lo so. Non qui.

E dovrei tenerla nascosta. 


\section{Still Grosse Hamburger Strasse, Berlin}

Ingrid de Kok

On Grosse Hamburger Strasse

There is silence

It is always silent

On Grosse Hamburger Strasse

Even four boys drinking pilsener

Opposite the frozen families waiting

For the transport

Are mute

The missing house

Hushed

And a man

Who enters the gate

Of the evacuated cemetery

His head covered

Makes no print

No sound

Not even a shuffle.

How grief saturates

The air

Rain is coming

How grief sediments

The stones

Rain is coming.

How no sign

Of guilt

Or remembrance

Brings back voices

To this suspended place

The old inhabitants

Forever stilled 
The new owners

Arrested in history's gaze.

Nobody moves

On Grosse Hamburger Strasse

Except shadows gathering

Then dispersing

And this afternoon also

As gentle rain falls on the stones

And upon the earth below

Without a splatter of sound. 


\title{
Fermo immagine Grosse Hamburger Strasse, Berlino
}

\author{
Ingrid de Kok; a cura di Maria Paola Guarducci e Francesca Terrenato
}

C'è silenzio

Sulla Grosse Hamburger Strasse

Sempre silenzio

Sulla Grosse Hamburger Strasse

Pure i quattro ragazzi con birra

Davanti alle famiglie che impietrite

Attendono il convoglio

Sono muti

La casa scomparsa

Zittita

E un uomo

A capo coperto

Passando il cancello

Del cimitero evacuato

Non lascia impronta

Né fa rumore

Neanche un fruscio.

Come satura l'aria

Il dolore

Fra poco piove

Come addensa la pietra

Il dolore

Fra poco piove.

Perché nessun segno

Di colpa

Di memoria

Riporta le voci

In questo luogo sospeso

Gli antichi abitanti

Per sempre fermati 
I nuovi padroni

Fissi nell'occhio della storia.

Nessuno si muove

Sulla Grosse Hamburger Strasse

A parte le ombre riunite

E poi disperse

Anche in un pomeriggio

Di pioggia lieve che cade

Sulle pietre e giù sulla terra

Senza suono di gocce. 


\section{Border Travel}

\section{Ingrid de Kok}

All that can be said

today

is this.

A small boy

left his bear

behind

right ear muddy

left leg squashed

trodden in the rush

when someone

in a uniform

his own children

at home perhaps

put the boy and his mother

onto a bus

back

across the border

to start again

this time

without

the bear 



\section{Oltre il confine}

\section{Ingrid de Kok; a cura di Maria Paola Guarducci e Francesca Terrenato}

Solo questo

oggi

va detto.

Un bimbo

ha perso

l'orsetto

orecchio infangato

zampa schiacciata

calpestato nella furia

mentre un uomo

in uniforme

con figli suoi

a casa sua forse

metteva bimbo e mamma

su un pullman

respinti

oltre il confine

per ricominciare

stavolta

senza

l'orsetto 



\section{Allowances}

\section{Ingrid de Kok}

Allow a favourite child Favourite poem or ballet Favoured lie to yourself Favoured time of day

Allow a favourite place Perhaps the Alps, perhaps Karoo Vote for weather that thunders Or temperate blue

Favourite painting? Mine, Las Meninas of course Who cares how predictable If the perfect choice

Most prized plant?

Brunfelsia at the gate Decanting its purple bouquet Onto grey morning slate

Though other beloveds Are always lying in wait Say cornflowers, Giotto The Milky Way, day break

But you, you really are my chosen one Duty free, no alternative, no sequel Though they keep telling us Favourites are disallowed, all things being equal. 



\section{Si può}

Ingrid de Kok; a cura di Maria Paola Guarducci e Francesca Terrenato

Si può avere un figlio preferito Poesia preferita o balletto

Bugia preferita da dirsi

$\mathrm{O}$ un preferito momento.

Si può avere una meta preferita Magari le Alpi, forse il Karoo Votare per tuoni e tempeste O per il sereno del cielo blu

Un quadro preferito?

Il mio, Las Meninas, di certo

È scontato? Fa niente

lo lo trovo perfetto

La pianta più amata?

Il bouquet della brunfelsia

Viola stillante sopra lapidi

Mattinali di grigia ardesia

Pure altri amori restano

In attesa del loro turno

I fiordalisi, sciami di stelle

Giotto e il fare del giorno

Ma tu, dentro di me sei tu l'eletto

Senza costi, alternative, conseguenze

Anche se lo sappiamo bene

Non è giusto accordare preferenze. 



\section{Lament}

\section{Ingrid de Kok}

The great grey eagle owl

Throws her weightless shawl

Over evening

Fashioning earth's second ceiling

Under which small sounds

Scuttle and die

While above the owl's feathers

In open neutral sky

An unnameable smaller bird

Keens, grieves for its children

Or even for us, calls to itself

There being no heaven to call to. 



\section{Lamento}

Ingrid de Kok; a cura di Maria Paola Guarducci e Francesca Terrenato

Il grande gufo grigio

Lancia il lievissimo manto

Sopra la sera

E veste il controsoffitto della terra

Sotto cui minimi suoni

Silurati muoiono

Mentre sopra le piume del gufo

Nel cielo aperto neutrale

Un uccellino senza nome

Grida, piange per i suoi figli

O per noi, e invoca se stesso

Perché non c'è un dio lassù. 



\section{Playing}

Ingrid de Kok

Every morning I get up

and play my violin

I know you know

I don't have a violin

but I play it.

I watch

a silhouette

on the path

beckon another

illumination or tracing.

Everything hums

not only fridges,

children, bees,

women thinking of other things

as they work.

Watch, draw, sing,

play the violin. 

Vol. 22 - Dicembre | December | Décembre 2020

\section{Suono}

Ingrid de Kok; a cura di Maria Paola Guarducci e Francesca Terrenato

Ogni giorno mi alzo

e suono il violino

lo so che lo sai

un violino non ce l'ho

ma lo suono.

Osservo

un'ombra

sul sentiero

disegnare un'altra

traccia o rivelazione.

Non solo il frigo

canta sommesso,

anche un bambino, un'ape,

una donna che pensa ad altro

mentre lavora.

Osserva, disegna, canta, suona il violino. 



\section{Ingrid de Kok. Parole, immagini, suoni}

Maria Paola Guarducci

Università degli Studi Roma Tre, Italia

Francesca Terrenato

Sapienza Università di Roma, Italia

Già nota a lettori e lettrici de Il Tolomeo e al pubblico italiano per le sue frequentazioni del nostro paese, in cui ha vinto premi importanti ed è stata più volte tradotta, Ingrid de Kok (Stilfontein, Sudafrica, 1951) partecipa a questo numero della rivista con sei poesie inedite, diverse tra loro ma ugualmente rappresentative della sua composita poetica. Autrice di sei raccolte di versi (Familiar Ground, 1988; Transfer, 1997; Terrestrial Things, 2002; Seasonal Fires, 2006; Other Signs, 2011, Mappe del Corpo a cura di Paola Splendore, 2008), accademica, intellettuale engagée e infaticabile e curiosa viaggiatrice, de Kok appartiene alla generazione dei bianchi dissidenti che ha vissuto e combattuto l'apartheid. Sostenitrice della democrazia guidata da Nelson Mandela, questa generazione, che annovera artisti e gente comune, non ha mai cessato di esercitare il diritto alla critica nei confronti del potere anche quando, archiviato l'entusiasmo della liberazione, i primi nodi sono arrivati al pettine. Si ritrova dunque, anche nei versi delle poesie qui presentate, l'interesse di Ingrid de Kok per quei soggetti sempre estromessi dalle decisioni e piuttosto oggetti su cui il potere si esercita e di cui ha bisogno. Le minoranze, i bambini, le donne: gruppi e individui non intrinsecamente 'fragili' o espressione di alterità, bensì resi tali affinché l'autorità possa legittimarsi e fare il suo corso. Prima di tutto Ingrid de Kok è però poeta: i versi che qui presentiamo portano i segni della sua ricerca formale per dare corpo a senso e suono, memoria e racconto in un percorso rigoroso rivelatore di spazi vuoti ma eloquenti, posture insolite e innaturali dell'umano in cui a volte si celano le verità più scomode. Ricordano, infatti, certi ritratti della sua connazionale Marlene Dumas le bambole monche e calve di Injury/Ferita, rovesciate su un banco del mercato delle pulci di Berlino accanto a fili elettrici e 
prese che ben oltre la loro banalità di asettici oggetti del quotidiano evocano sottilmente e in modo macabro certe pratiche naziste messe in atto non troppo lontano di lì, né nello spazio né nel tempo, persistenti quindi in una sorta di reminiscenza costante che contamina tutto. La memoria è anche al centro dell'altra poesia berlinese, Still/ Fermo immagine, complesso componimento che lega la Storia rappresentata dal memoriale ebraico su Grosse Hamburger Strasse alla contemporaneità: un fermo immagine che al tempo stesso blocca e rende eterna - still nell'intraducibile doppia accezione di aggettivo, 'immobile', e avverbio, 'ancora' - la memoria della Shoah. Ovunque la natura parla in de Kok, sudafricana avvezza a panorami ruvidi, estremi, struggenti e crudeli ma anche miti e confortevoli, attraverso immagini di fiori e piante, acqua, pioggia, pietre, fango, albe, cieli stellati, sole, freddo e caldo. In questa varietà di luoghi e di cose, si muovono a pieno diritto gli animali, altro grande tema dell'universo poetico di Ingrid de Kok: anche loro, però, sono infestati dal contatto con le persone, quando non condannati dalla naturale legge del più forte - conigli braccati, uccellini predati - che li rende vittime e dunque metafore di tanta umanità che soffre. Risuonano tuttavia anche echi di momenti più intimi - Allowances/Si può - legati a un privato femminile, un mondo di affetti e consuetudini a tratti segreto - Playing/Suono - sempre rivendicato e difeso, ascrivibile alla tradizione poetica che fa capo a Emily Dickinson e ai suoi versi lapidari e felici, svincolati dal canone, eppure così classici. C'è anche il grande tema del materno, l'attenzione tutta femminile ai più piccoli, siano neonati inermi, bambole da adottare o bambini migranti; questi ultimi costretti a ricominciare di continuo, rimpallati tra un confine e l'altro, in un corto circuito tra coordinate geografiche in cui gli adulti smarriscono senso e umanità. Dire il silenzio: sembra questa la sfida più alta che i versi di Ingrid de Kok si prefiggono e nelle note del violino che non c'è eppure suona - Playing/Suono - questo silenzio prende effettivamente forma.

Nella traduzione si è cercato quanto più possibile di mantenere l'aspetto ellittico dell'inglese, ma la resa in italiano ci ha spesso costrette a radicali cambiamenti di ordine nelle parole, così come il tentativo di mantenere assonanze, rime e soprattutto tempi del verso ci ha in alcuni casi imposto dei piccoli tagli. Confidiamo che il senso si sia mantenuto, assieme all'immediatezza delle immagini di de Kok e che, seppure nella perdita di qualche frammento, i suoi versi abbiano trovato il loro equilibrio anche nella nostra lingua. 


\section{Waiting for the Virus With Apologies to C.P. Cavafy and E. Keeley}

Douglas Reid Skinner

What are we waiting for, assembled in our houses?

The virus is due here soon.

Why isn't anything going on in Parliament?

Why aren't the members sitting there and legislating?

Because the virus is on its way.

What's the point of members making laws now?

Once the virus is in our houses, it will do the legislating.

Why did our Leader get up so early, and why is he sitting at the entrance to Parliament, flanked by experts?

Because the virus is arriving and he's intent on returning it whence it came.

He's even got a scroll to wave, loaded with titles and imposing names.

Why have our ministers of state come out today wearing their best Armani suits and shirts? Why have they put on Bulgari watches pearl earrings, rings sparkling with sapphires and emeralds? Why is the Speaker carrying the royal mace, beautifully worked in silver and gold?

Because the virus is coming today and things like that might impress a virus. 
Why don't our distinguished orators turn up as usual to make their speeches, say what they have to say?

Because the virus is coming today and it pays no heed to rhetoric and public speaking.

Why all the sudden puzzlement, all the anxiety? (People's faces have become so serious.)

Why are the streets and squares emptying so rapidly, why is everyone going home lost in thought?

Because night has fallen and the virus is here.

Some of our men just in from the border say there are more viruses on the way.

Now what's going to happen to us with this virus? Some say it's a kind of solution. 


\section{Aspettando il virus Porgendo le scuse a C.P. Cavafy e E. Keeley}

Douglas Reid Skinner; traduzione di Marco Fazzini

Ma cosa aspettiamo, assembrati dentro casa?

Il virus arriverà presto.

Perché non succede nulla in Parlamento?

Perché i deputati non ci vanno e non legiferano?

Perché il virus ci sta già arrivando.

Che senso ha per i deputati fare leggi proprio ora?

Quando il virus arriverà a casa nostra, sarà lui a legiferare.

Perché il nostro Capo s'alza così presto, e se ne sta seduto all'entrata del Parlamento, fiancheggiato dagli esperti?

Perché il virus sta arrivando e lui è pronto a restituirlo al luogo dell'origine. $\mathrm{Ha}$ addirittura un documento da sventolare, carico di titoli, di nomi imponenti.

Perché i nostri ministri sono usciti oggi indossando camicie e i più bei vestiti d'Armani? Perché si sono messi braccialetti e orologi di Bulgari, anelli scintillanti di zaffiri e smeraldi?

Perché il Presidente dei Comuni fa oggi da mazziere, col quel bastone regale in argento e oro?

Perché il virus arriverà oggi

e cose del genere potrebbero magari spaventarlo. 
Perché i nostri esimi oratori non si palesano com'è loro solito a rilasciare discorsi, a dire quello che sempre dicono?

Perché il virus arriverà oggi

e non si cura della retorica e dei pubblici discorsi.

Perché tutta questa improvvisa confusione, questa ansia?

(ma come sono diventate serie le facce della gente.)

Perché strade e piazze si svuotano di fretta,

e tutti se ne vanno a casa persi tra pensieri?

Perché la notte è sopraggiunta e il virus è arrivato.

E alcuni dei nostri uomini proprio al confine dicono

che altri virus si stanno mettendo in movimento.

Ma cosa ci succederà adesso con questo virus?

Qualcuno dice che magari è una bella soluzione. 


\section{Douglas Reid Skinner}

\section{Marco Fazzini}

È nato a Upington, in Sudafrica, nel 1949. Ha vissuto a Cape Town, Johannesburg, New York, San Francisco e Londra, dove vive attualmente lavorando come curatore e scrittore. A Città del Capo, negli anni Ottanta e nei primi anni Novanta, ha curato le riviste letterarie Upstream e New Contrast, e creato e diretto la casa editrice The Carrefour Press, pubblicando alcuni dei maggiori poeti di lingua inglese del Sudafrica. Tra questi ricordiamo: Stephen Watson, Don Maclennan e Douglas Livingstone.

Attualmente dirige la rivista sudafricana di poesia Stanzas, è il curatore e il traduttore per la competizione annuale Avbob Poetry Competition, e si occupa di una piccola casa editrice dal nome Crane River. Le sue sillogi principali sono: Reassembling World (1981), The House in Pella District (1985), The Unspoken (1988), The Middle Years (1993), Blue Rivers (2011), Heaven: New \& Selected Poems (2014) e Liminal (2017). Ha tradotto (da solo o in collaborazione con altri poeti) da varie lingue, tra cui: afrikaans, francese, ebraico, italiano, oltre ai volumi The Secret Ambition (una scelta di poesie di Valerio Magrelli). 



\section{Dossier}

Postcolonial Approaches to the Environmental Humanities and Ecocriticism | Approches postcoloniales des humanités environnementales et de l'écocritique | Approcci postcoloniali alle scienze umane ambientali e all'ecocritica 



\title{
Colonizzazione ambientale, defuturing capitalocenico e pratiche eco-artistiche decoloniali a sud Il 'caso Taranto'
}

\author{
Luigi Carmine Cazzato \\ Università di Bari "Aldo Moro", Italia
}

\section{Antonella D'Autilia}

Università di Bari "Aldo Moro", Italia

\begin{abstract}
The present essay aims at offering some interpretative keys for the ecological disaster of one of the most beautiful sites in the Mediterranean: Taranto. It does so through the ecocritical tools of postcolonial and decolonial thinking and the analysis of an intense iconography of eco-artistic urbanist engagements, which have dramatically exemplified what it has meant living in the midst of the largest of Europe's steelworks. These engagements, having "one foot in literature and the other on land" (Glotfelty, Fromm 1996, XIX), attempt to decolonize the imaginary related to the deeply discordant relationship between the city of Taranto and 'its' steelworks, which is more than twice the size of the same city. We have chosen, after Jason Moore (2016), the adjective 'capitalocenic' rather than the more voguish 'anthropocenic', since the environmental changes are not the end result of an abstract human action, but rather of the tangible centurylong rule of capital: the earthless (Vázquez 2017) mode of production of modernity that à la Descartes denies the earth into which it gets its claws and, as a consequence, it denies the deep knitting of "humanity-in-nature/nature-in-humanity" (Moore 2015).
\end{abstract}

Keywords Capitolocene. Coloniality. Defuturing. South.

Sommario 1 Cartesio, Gissing e il donkey's method. - 2 Il Mezzogiorno capitalocenico nello spazio della differenza imperiale. - 3 Taranto: fra colonial mimicry e defuturing. - 4 Ferite 'coloniali' e tentativi decoloniali di guarigione estesica.

$\begin{array}{lll} & \text { Peer review } & \\ & \text { Submitted } & 2020-08-25 \\ \text { Edizioni } & \text { Accepted } & 2020-10-24 \\ \text { Ca'Foscari } & \text { Published } & 2020-12-22\end{array}$

\section{Open access}

(C) 2020 | ()( Creative Commons Attribution 4.0 International Public License

Citation Cazzato, L.C.; D’Autilia, A. (2020). "Colonizzazione 


\section{Cartesio, Gissing e il donkey's method}

George Gissing, grande estimatore tardo-vittoriano della Magna Grecia e del sud, arrivò a Taranto nel 1897 quando il nuovo borgo umbertino aveva già occupato, con le sue grandi e brutte costruzioni giallastre, come scrisse nel suo By the Ionian Sea (1901), il terreno dove si stanziarono anticamente Fenici, Greci e Romani. Proseguendo il suo viaggio lungo le rive dello Jonio, poco fuori la città, Gissing si imbatté nella scena di un contadino che arava la terra con un aratro che avrebbe potuto avere mille anni e, grazie a un asinello, tracciava un solco che pareva «the merest scratch of a furrow» (Gissing 1901, 33). Non solo l'aratro tracciava appena un graffio sul terreno, ma l'umile e gentile contadino, ci dice Gissing, aveva una pazienza incondizionata col suo asino, il quale dettava il ritmo del lavoro.

The donkey's method of ploughing was to pull for one minute, and then rest for two; it excited in the ploughman not the least surprise or resentment. Though he held a long stick in his hand, he never made use of it; at each stoppage he contemplated the ass, and then gave utterance to a long 'Ah-h-h!' in a note of the most affectionate remonstrance. They were not driver and beast, but comrades in labour. It reposed the mind to look upon them. (Gissing 1901, 33; corsivi degli Autori)

La domanda è: perché la scena in cui l'aratro guidato dal duo uomoasino che dissoda la terra accarezzandola fu un ristoro per la mente di Gissing? Probabilmente per l'autore di The Nether World, in fuga dalla mostruosa civiltà industriale inglese, fu una sorta di ritorno reale e mentale a quando non vi era contrasto fra contadino e bestia, fra uomo e animale, insomma, fra Cultura e Natura. Cultura e Natura erano, per dirla con le sue parole, compagni di lavoro e non sostanze in conflitto fra di loro, come è stato da Cartesio in poi. Come risaputo, con la separazione da parte del filosofo francese fra corpo e mente si impose la logica dell'aut/aut: o Natura o Cultura. Soprattutto, si decretò il controllo della Cultura sulla Natura e l'utilizzo dei suoi corpi, facendo diventare gli esseri umani «padroni e possessori della natura» (Descartes 2003, 162-3).

Nell'esplicitare il costrutto di world-ecology, tramite il quale considerare il mondo come co-prodotto di Cultura e Natura, Moore (2015, 5) si oppone al dualismo cartesiano radicato nei secoli: «An alternative begins neither with 'humans' nor with 'nature' but with the relations that co-produce manifold configurations of [...] humanity-in-nature/nature-in-humanity». Il problema non è solo di natura ontologica ma anche epistemologica poiché, a causa della matrice della colonialità (Quijano 2010), la differenza viene trasformata in gerarchia, come denunciano da un quarto di secolo i pensatori decoloniali. Questa ma- 
trice, nata al tempo della conquista dell'America, non solo gerarchizza le differenze 'razziali', ma sopprime forme di conoscenza e mondi che non si conformano con l'idea di euromodernità. Infine, la colonialità «cemented the dichotomy between the human/civilized (European) world [...] and the nonhuman/uncivilized (the nonmodern, racialized dark peoples of the world)» (Escobar 2018, 94). Quindi, da una parte la cultura europea dall'altra la natura non europea. ${ }^{1}$ Pertanto, il progetto della modernità/colonialità ha previsto sia zone di sfruttamento da parte dell'uomo sull'uomo (sul proletariato bianco maschile retribuito) sia zone di appropriazione da parte dell'uomo di altri esseri umani (le donne dedite alla riproduzione e gli schiavi destinati alla produzione, al pari non retribuiti) e dell'extra-umano, ovvero la 'cheap nature' di cui parla Moore (2015), anch'essa, per così dire, non retribuita. Insomma, oltre l'energia pagata male, questo progetto aveva bisogno di energia pagata affatto, sia di origine umana (schiavi e donne) $)^{2}$ che extra-umana (colonie).

\section{Il Mezzogiorno capitalocenico nello spazio della differenza imperiale}

Ci si starà chiedendo: cosa c'entra tutto ciò con il caso dell'acciaieria tarantina? Ci entra almeno in due modi. Il primo è che il sistema delle gerarchie prodotte dalla matrice coloniale del potere è divenuto, nel tempo, il cavallo di Troia per poter attuare progetti eurocentrici di 'civilizzazione', 'modernizzazione' e 'sviluppo' su tutto il pianeta. Il secondo modo è che la colonialità non ha operato solo fuori dall'Europa - sul crinale della differenza coloniale - ma anche dentro di essa, ai suoi 'margini', sul crinale della differenza imperiale (Mignolo 2007; Tlostanova 2018). Approfondiamo.

Nonostante la logica dell'epistemologia occidentale consista in un metadiscorso che costantemente approva se stesso attraverso la disapprovazione dell'altro, questa logica opera anche all'interno dell'identità occidentale. Madina Tlostanova lo spiega efficacemente a questo modo:

To put it simply, starting from about the sixteenth century a global imperial hierarchy appeared in the emerging world system. With-

Per quanto il saggio sia stato concepito congiuntamente, i paragrafi 1-2-3 sono da attribuire a Luigi Carmine Cazzato e i paragrafi 4-5 a Antonella D’Autilia.

1 In questo senso, il noto mappamondo di Visscher del 1652, contenente ai quattro angoli l'allegoria dei quattro continenti, tutti in compagnia di animali tranne l'Europa, è più che eloquente.

2 A tal proposito, si può vedere lo studio di Federici 2004. 
in this hierarchy several imperial leagues were formed and transformed in the course of time. In the post-Enlightenment modernity Spain, Italy, and Portugal moved to the position of the South of Europe and hence to the internal imperial difference that never collapsed into absolute or insurmountable forms. The Ottoman sultanate and Russia, on the contrary, became the zones of the external imperial difference, as they were rooted in different (from the core European norm) religions, languages, economic models, and ethnic-racial classifications. Both internal and external imperial others were never allowed to join the first league and become equal to Great Britain, France, or the United States today. (Tlostanova 2018, 2; corsivi degli Autori)

Va da sé che all'Italia è stato attribuito un certo grado di inadeguatezza a causa del ritardo nel tempo (la sua, appunto, tarda storia nazionale e imperiale) e della sua marginalità nello spazio (la collocazione geografica a sud). Questa inadeguatezza o ritardo nella corsa verso il progresso la situa automaticamente in un non alto grado nella scala della colonialità, che prevede la discriminazione meridionista (Cazzato 2017) fra avanzato nord-ovest e arretrato sud-est europeo. Se poi consideriamo la stessa discriminazione all'interno del territorio italiano fra sviluppato e ricco nord e sottosviluppato e povero sud, il posizionamento su questa scala peggiora di parecchio, collocando il Mezzogiorno in uno spazio di subalternità analogo a quello occupato dai popoli colonizzati, nonostante l'assenza di un processo coloniale formale. L'autore del Pensiero meridiano ci ricorda che non si può non rivendicare «la connessione tra un sud, quello italiano, e il sud del mondo» (Cassano 2005, IX). Ė quello che si proverà a fare in questo contributo. E forse il ritorno alla città di Taranto, al tempo dell'arrivo del progresso dal nord, che con la sua lucida armatura in acciaio è andato in soccorso all'arretratezza del sud nei suoi consunti e antiquati panni, potrebbe chiarire meglio quanto detto fin qui.

Questo processo è stato una lunga corsa che, partita dopo l'Unità d'Italia, ha subito una prepotente accelerata nel dopoguerra. Al tempo di Gissing, Taranto non era la città industriale che sarebbe diventata durante gli anni del boom economico con la nascita dell'Italsider (poi ILVA, e adesso ArcelorMittal). Tuttavia, già si intravvedevano le prime avvisaglie, se l'autore inglese scrive che il posto dove si lavorava l'antica e famosa porpora di Taranto, seconda solo a quella di Tiro e descritto qualche decennio prima dal grande archeologo Lenormant, «had vanished, swallowed up, with all remnants of antiquity, by the graceless Arsenal» (Gissing 1901, 34). Lo sgraziato arse- 
nale era, per Gissing, come per Cesare Brandi mezzo secolo dopo, ${ }^{3}$ il simbolo del «mechanic triumph of to-day» (34) e per questo antipatico, nonostante fosse «the pride of Taranto, and the source of its prosperity» (34). Tuttavia, il vero orgoglio e la vera prosperità della città arrivarono più tardi, quando nel 1960 ci fu la posa della prima pietra di quello che sarebbe diventato il centro siderurgico più grande d'Europa. Un anno prima Pier Paolo Pasolini, un altro nemico del progresso o dello «sviluppo senza progresso» (Pasolini 2009, 455-8), trova che la città «brilla sui due mari come un gigantesco diamante in frantumi... Taranto, città perfetta. Viverci è come vivere all'interno di una conchiglia, di un'ostrica aperta» (Pasolini 2017, 162).

Purtroppo, Taranto era la città perfetta anche in un altro senso, quello pensato dalla classe dirigente italiana di allora, che decise di prendere seriamente coscienza della realtà meridionale per mutarla, come disse il Presidente Giuseppe Saragat. Pasolini fece appena a tempo, dunque, a vedere la Taranto perfetta: mentre lui la contemplava come fosse un gioiello, erano già all'opera i tentacoli delle magnifiche sorti e progressive del grande design modernizzante del Capitalocene. Preferiamo chiamare così questo progetto poiché, per quanto sorretto da intenzioni e azioni antropogeniche, non è un progetto semplicemente antropocenico, come viene oramai comunemente chiamato. È un design capitalocenico perché, come sostiene Moore, siamo appunto nell'era del capitalismo «as a world-ecology of power, capital, and nature» (Moore 2016, 6). Fu Dino Buzzati a prestare le sue parole alle incredibili, oggi, immagini del documentario Il pianeta acciaio di E. Marsili del 1962. Egli colse il senso storico dell'evento in tutta la sua violenza ontologica e retorica, senso consegnato alla voce di Armando Foa. È doloroso, ma leggiamo:

Guardate, un paesaggio classico: il mare, la riva deserta, gli ulivi, il sole, le cicale, la pace, la sonnolenza, tutto rimasto immobile e intatto dai tempi della Magna Grecia. Ma ora state attenti, lo metteranno a ferro e fuoco, letteralmente. Perché? Quando vivevano Platone ed Archimede questo ulivo era già nato. A morte! [qui il brutale primo piano di un bulldozer che scaraventa a terra in un lampo un imponente ulivo dal tronco maestoso] Dopo duemila anni, divelto da una forza infernale. Schiantato giù nella polvere come fosse uno stecco. Si aggirano gli orribili dinosauri di metallo. Via gli ulivi, via le vecchie casupole, via le cicale e l'antico incanto mediterraneo. Via! Le bestiali macchine vogliono fare il deser-

3 La città dei due mari «potrebbe essere stupenda: e invece è squallida» (Brandi 2004, 129), ben servita com'è «dai militari, dai preti, dagli architetti» e dalla Cassa del Mezzogiorno, espressione necessaria e inadeguata del Mezzogiorno «scivolato progressivamente verso una situazione coloniale» $(91,129)$. 
to: una landa piatta senza un filo d'erba. L'hanno già fatta. Poche ore sono bastate per cancellare i millenni. E adesso bestioni preistorici mugolanti di soddisfazione fra il sole e il polverone insistono a spianare, testare, livellare. Ma perché hanno devastato così? Perché? Perché gli ulivi, il sole, le cicale significavano sonno, abbandono, rassegnazione e miseria, e ora qui invece gli uomini hanno costruito una cattedrale immensa di metallo e di vetro per scatenarvi dentro il mostro infuocato che si chiama acciaio e che significa vita. (Marsili 1962)

Qui sentiamo tutta la violenta hybris cartesiana, lockiana e faustiana dell'Occidente illuminista che, inebriato dalla possanza dei suoi muscoli artificiali, in poche ore veramente cancella millenni: furiose ruspe neofuturiste sradicano ventimila ulivi secolari per far posto a un deserto, esteso 1500 ettari e pari al doppio dell'intera città, su cui dovevano essere conficcati i pilastri della cattedrale della nuova vita, del nuovo futuro. Appare in tutta la sua cruda evidenza il volto della modernità, che nega il suo legame con la terra e la Terra. Ė la earthlessness di cui parla Rolando Vázquez, che descrive bene la natura di questo progetto eurocentrico:

On the one hand, modernity's anthropocentrism, built on the separation between the 'human' and 'nature,' requires the negation of earth. Modernity's notion of humanity and civilization is produced as earthlessness. This negation is implemented through forms of classification, appropriation, extraction, consumption and pollution. On the other hand, modernity's Eurocentrism required the negation of other worlds and was built on the separation between civilization and barbarism, between the human and the savage, the developed and the underdeveloped, the consumer and the subaltern. (Vázquez 2017, 2)

Concludendo, il progetto egemone della civiltà dell'acciaio è stato un progetto capitalocenico di doppia negazione distruttiva: la negazione cartesiana della natura attraverso uno sradicamento 'terrestre', nel doppio senso cui abbiamo accennato prima: gli ulivi secolari strappati dalla terra, come inutili stecchi, rappresentavano anche lo sradicamento dal pianeta; la negazione (di tipo coloniale) della cultura dell'altro subalterno: i tarantini come i primitivi d'Europa, ancora legati a sonnolenti e superati stili produttivi, che significavano non già vita vera ma vita vegetativa. 


\section{Taranto: fra colonial mimicry e defuturing}

Oggi la parola 'vita' pronunciata davanti alle immagini di un quartiere senza futuro (il Tamburi), è impronunciabile, oscena. Eppure, per il bellunese Buzzati allora non era così. Era esattamente il contrario, poiché migliaia di contadini trovarono lavoro e quindi fiducia nei propri mezzi:

Venivano dai campi, dai pascoli, dalla rassegnazione. Oggi si sentono già uomini diversi. Si sentono vivi e moderni, non hanno più un senso di vergogna e di invidia quando vedono passare le macchine e i camion prelati Torino, Genova, Milano, con al volante quei tipi del nord, con le facce così industriali. Adesso si sentono uguali, altrettanto forti, altrettanto bravi. (Marsili 1962)

Walter Tobagi nel 1979 chiamerà questi «uomini diversi» metalmezzadri: metà contadini e metà metallurgici, non più contadini, ma nemmeno pienamente operai. ${ }^{4}$ Insomma, erano una strana e bizzarra copia degli uomini del nord: una sorta di mimic men, se volessimo dirla con Naipaul (1967). Certo, lo spazio della differenza imperiale non è quello della differenza coloniale e tuttavia, se anglicizzarsi per gli indiani voleva dire «emphatically not to be English» (Bhabha 2004, 87; corsivo nell'originale), in maniera analoga per i meridionali industrializzarsi come i settentrionali voleva dire, altrettanto chiaramente, non essere settentrionali. Voleva dire solo indossare la loro maschera, la loro faccia industriale, senza averne l'anima. Oggi, a oltre mezzo secolo di distanza, gli effetti della colonial mimicry non possono essere più chiari. Così come è chiaro che, dopo un secolo e mezzo di questione meridionale, i meridionali sono rimasti 'meridionali' ma con un problema nuovo: se prima dovevano scegliere fra povertà ed emigrazione, adesso devono scegliere fra lavoro e salute, quindi vita. Quella vita sfavillante che doveva arrivare sui nastri trasportatori dell'acciaio infuocato. Insomma, per dirla con una massima in voga fra i decolonialisti, Taranto deve affrontare «modern problems for which there are no longer modern solutions» (Escobar 2018, 67). Che vuol dire: passata la sbornia dell'arrivo del progresso e del futuro, a Taranto rimangono i problemi dell'hangover, ovvero il costo ambientale di questa sbornia che lascia senza futuro. A quel tempo nessuno, tranne rare eccezioni, ${ }^{5}$ volle vedere o prevedere. Solo le pagine private di

4 Così Leogrande: «quella categoria serve ancora a spiegare parte del fallimento dell'industrializzazione dall'alto. Inquinamento a parte, la fabbrica ha portato salari e occupazione, ma non ha creato né tanto meno qualcosa che assomigli alla 'classe operaia' di Torino o Milano» («Tobagi e i 'metalmezzadri'», Corriere del Mezzogiorno, 3 giugno 2010).

5 Una di queste eccezioni fu Antonio Cederna che nel 1972 sul Corriere della Sera scrisse che quello tarantino gli sembrava «un processo barbarico d'industrializzazio- 
un ufficiale sanitario, disseppellite da Alessandro Leogrande, esprimevano dubbi e paure sul disastro in fieri nel 1965, non certo quelle del Direttore del Centro Siderurgico, per il quale, secondo l'ufficiale,

non conta la tutela della città da un grave danno ecologico, contano la difesa del prestigio personale e gli interessi di alcuni esponenti politici, che ritengono di poter disporre a loro piacimento delle sorti del nostro territorio, come si trattasse di una colonia africana da sfruttare. ${ }^{6}$ (cit. in Leogrande 2014)

A quel tempo, che Taranto fosse come una sorta di colonia da sfruttare non lo diceva pubblicamente quasi nessuno. Oggi questa tesi è entrata nell'agone del dibattito culturale e politico (si veda l'ultimo paragrafo). Allo stesso modo, è entrato nel senso comune che il progresso viaggia sempre insieme al regresso, e che quindi aggiunge e sottrae futuro al contempo. Fry (2009) chiama questo fenomeno «defuturing». Paradossalmente, la modernità costituitasi programmaticamente sul culto del nuovo, del progresso senza fine, insomma sul culto del futuro, ha portato alla forclusione del futuro. Tim Parks, espatriato ed esperto osservatore inglese delle cose italiane (nonché traduttore di Moravia e Calvino), nel suo viaggio in treno da Milano a Palermo del 2012, anno in cui scoppiò il caso Taranto grazie al coraggio dei giudici, ${ }^{7}$ scommette che l'acciaieria, causa di 11.500 morti per malattie respiratorie nei soli precedenti sette anni, non chiuderà. Ma aggiunge: «in any event, it's likely that in the near future the trains will be taking more and more men and less and less steel northwards» (Parks 2014, 229). Se a fine '800 la scelta era fra morire da brigante o emigrante, adesso è fra morire (e far morire) da metalmezzadro o emigrare di nuovo: un ritorno al non-futuro.

Pertanto, le proposte decoloniali sul sud del mondo possono valere anche per Taranto che è un sud che fa parte del nord del mondo, ma un nord imperfetto, quello della differenza imperiale interna. Come il sud del mondo, anche Taranto ha subito la prospettiva progettuale arrivata da nord, che doveva risvegliarla dall'antica sonnolenza. Anche Taranto, sotto l'impatto della modernità, ha visto andare in rovina il suo oikos (Moore 2015). ${ }^{8}$ Anche la vita dei tarantini è pri-

ne [che] non ha ancora pensato alle elementari opere di difesa contro l'inquinamento e non ha nemmeno piantato un albero a difesa dei poveri abitanti dei quartieri popolari sotto vento» («Taranto strangolata dal 'boom'», 18 aprile 1972).

6 Sulla metafora africana si può vedere Cazzato 2019.

7 Cf. «Il gip: 'Ilva mossa da logica del profitto sequestro per tutelare la vita umana'», La Repubblica, 26 luglio 2012; https://bari.repubblica.it/cronaca/2012/07/27/ news/ilva_il_gip-39795626/.

8 Oikeios è quel luogo, se non ameno, almeno favorevole di cui parlava il filosofo e botanico greco Teofrasto, dimensione che per Moore $(2015,35)$ diventa «the creative, hi- 
vata di futuro, se è vero che viene negata la vita a quelli appena nati (+ 54\% di tumori nei bambini rispetto alla media regionale) e a quelli che nasceranno. La colonialità capitalocenica distrugge l'alterità umana ed extraumana, impoverendo le strade che portano al futuro in Amazzonia, o in India, come a Taranto.

Il focus dei prossimi paragrafi è su alcune forme di impegno artistico visuale che irrompono sulla scena locale e nazionale come contro-narrazione decoloniale.

\section{$4 \quad$ Ferite 'coloniali' e tentativi decoloniali di guarigione estesica ${ }^{9}$}

Una panoplia di idee e una densa iconografia, hanno caratterizzato il ricco corpus di esperienze estetiche dell'impegno diretto dei vari eco-artisti che hanno ricostruito la storia della convivenza tarantina con l'industria siderurgica. Esse emergono come realtà immanenti agli esercizi ecocritici di resilienza costruttiva attivati dai numerosi movimenti sociali cittadini, sorti in opposizione alla riproduzione di relazioni di potere, atte a tenere in piedi una devastante gestione del territorio prevalentemente votata alla monocoltura dell'acciaio.

Moore ha posto l'accento sull'evidenza che «il mondo soggettivo del valore è stato forgiato attraverso la soggettività dell'immaginazione del capitale» (Moore 2017, 98). Nello stesso frangente teorico, tuttavia, ci ha messo in guardia sul potenziale decostruttivo generabile dall'introduzione di determinate istanze simbolico-culturali all'interno dei rapporti di valore riletti in un'ottica capitalocenica su scala planetaria. In base a quanto detto, un importante contributo alla causa delle eco-mobilitazioni tarantine è dato da un'intensa attività di contro-immaginazione riscontrabile in alcuni esempi emblematici di «imaginative literature» (Huggan, Tiffin 2010, 12), intesa come strumento di resistenza attiva ai processi di accumulazione di «natura sociale astratta» (Moore 2017, 90) innescatisi a Taranto a partire dall'insediamento del polo siderurgico.

Ilvarum Yaga: cento disegnatori contro la Strega Rossa [figg. 1-4] ne è un esempio. ${ }^{10}$ Si tratta di una mostra collettiva, in cui cento fumettisti italiani su invito del curatore dell'iniziativa Piero Angelini, hanno

storical, and dialectical relation between, and also always within, human and extrahuman place».

9 Il riferimento qui è all'articolo di Mignolo, Vázquez 2013, in cui si contrappone l'estesica all'estetica e alle ferite coloniali le cure estesiche decoloniali. Sulla questione Mignolo ritorna nel 2019 con «Reconstitución epistémica/estética: la aesthesis decolonial una década después», tradotto in italiano in Mignolo 2019.

10 Ilvarum Yaga: cento disegnatori contro la Strega Rossa = Mostra collettiva (Taranto, 8-30 novembre 2019). Crac Puglia (Centro di Ricerca Arte Contemporanea). http:// 
Luigi Carmine Cazzato, Antonella D'Autilia

Colonizzazione ambientale, defuturing capitalocenico e pratiche eco-artistiche decoloniali a sud
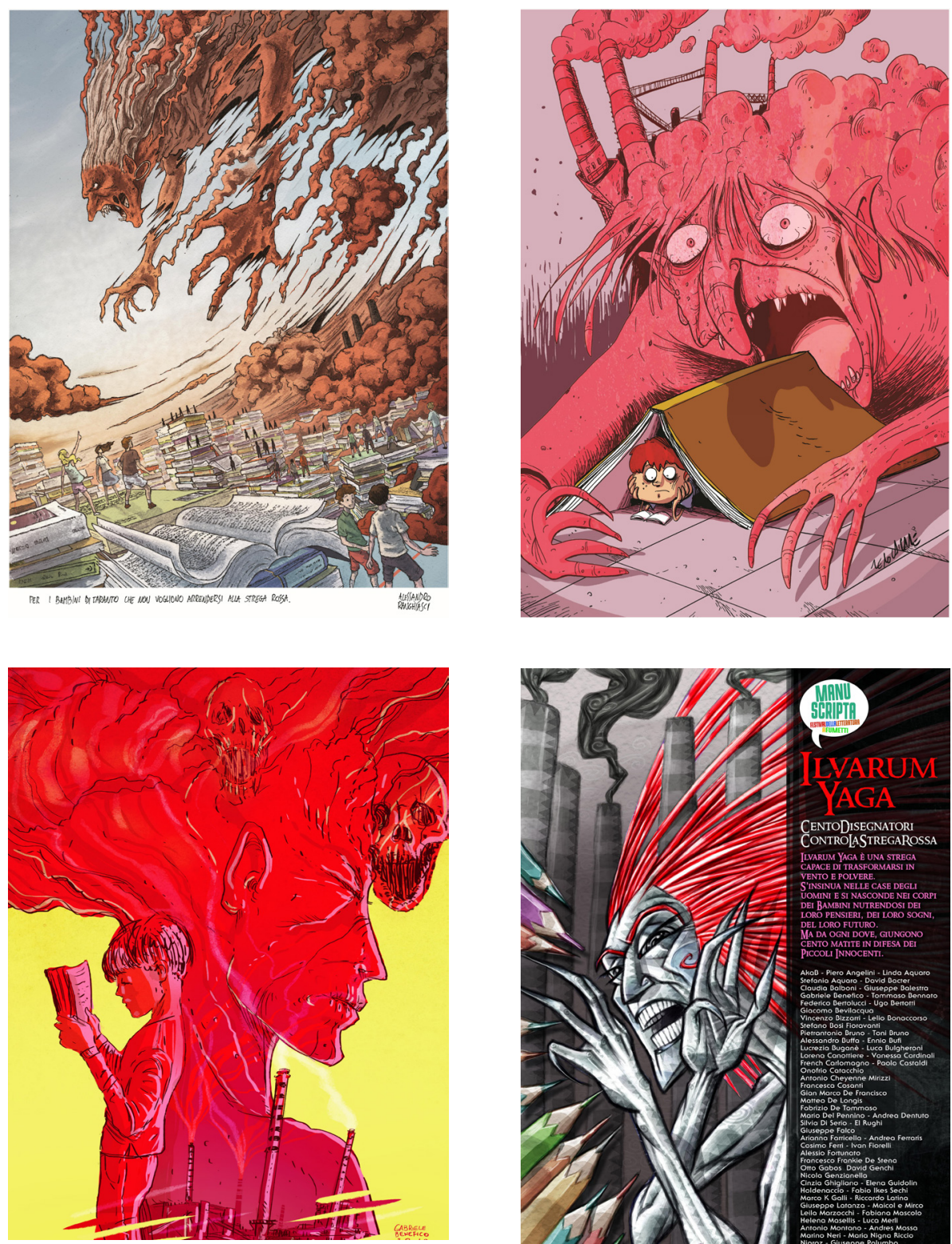

Figura 1 Ranghiaschi 2019, Ilvarum Yaga

Figura 2 Zerocalcare 2019, Ilvarum Yaga

Figura 3 Benefico 2019, Ilvarum Yaga

Figura 4 Ilvarum Yaga: cento disegnatori contro la Strega Rossa

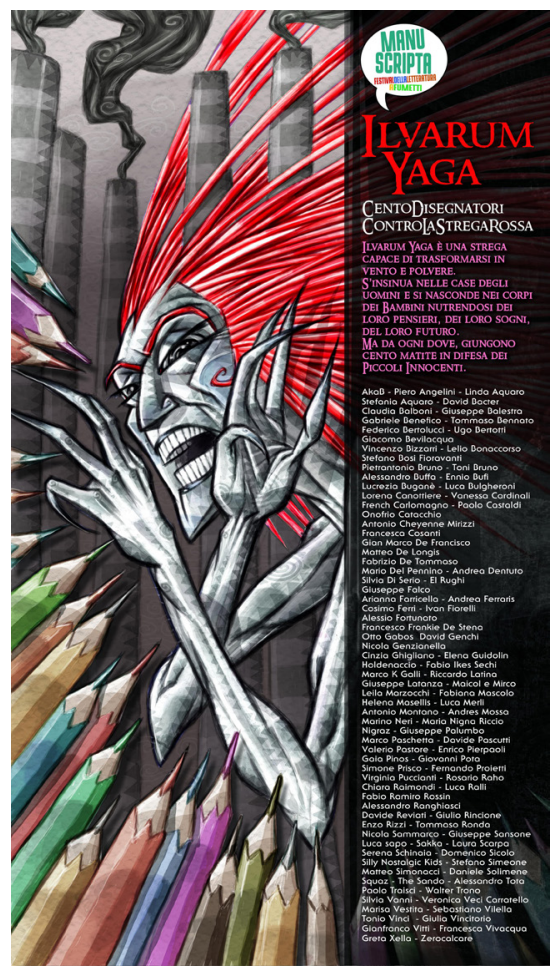


dato vita nelle loro tavole a una dialettica emotivo-rappresentazionale - con la strega/ILVA - di straordinaria ricchezza euristica, inscenando la sottrazione capitalistica di cui è stata oggetto la città, sullo sfondo di panorami industriali su cui incombe il potente respiro di varie personificazioni della fabbrica, dipinta con le sembianze di una strega, di una madre-matrigna, e altre mitologiche identità mostruose nella loro azione di devastazione culturale e fisica, cui si oppongono i bambini di Taranto armati di libri e dei loro pensieri [figg. 1-4].

La forma d'arte, dunque, intrecciata a doppio filo con questioni internazionali di giustizia ecologica e che qui si vuole analizzare, è il fumetto. Il fumetto, nelle sue ormai mutevoli e innumerevoli forme, sembra sia stato un medium, o linguaggio/ambiente (Barbieri 1991), assai adatto per la contro-narrazione. Probabilmente, lo è stato per la sua doppia dimensione semiotica, iconica e verbale al contempo, che, per quanto scritta, è molto vicina al linguaggio del parlato e quindi riduce la distanza comunicativa, facilitando la divulgazione critica. Quindi, non è un caso che Taranto sia stata protagonista di una corposa lista di volumetti, e-comics, graphic novel, che hanno ritratto - direttamente e indirettamente - la storia dello sfruttamento ecologico e le forme di 'indisciplinamento culturale' figlie delle lotte cittadine anti-ILVA. Nel dibattito scientifico internazionale, molti autori condividono l'ipotesi che il fumetto sia un medium piuttosto trascurato: sia come focus d'analisi, sia come mezzo letterario dal potenziale decostruttivo (Schmitt 1992; Berger 1971). Schmitt e Berger in effetti, soffermandosi, tra le altre, su opere come Mutt \& Jeff, Katzenjammer Kids il primo, Peanuts e Li'l Abner il secondo, intervengono sulle peculiarità e le virtù di questo particolare linguaggio artistico, portando a galla l'evidente inconsistenza di tesi che ipotizzano la subordinazione del fumetto alle altre forme letterarie. ${ }^{11}$

Analogamente, Rosie Ibbotson si è occupata dell'analisi del rapporto tra rappresentazione visuale e violenza ambientale, operando una rilettura critica dell'attività artistica e del pensiero politico del movimento tardo ottocentesco Arts and Craft definendolo «as one of

manuscripta.terraterra.eu/2019/11/05/a-taranto-arriva-ilvarum-yaga-centodisegnatori-contro-la-strega-rossa/.

11 Data la vastità delle produzioni riconducibili a tali forme espressive, non è possibile in questa sede tracciarne una genealogia parziale, tuttavia è possibile addurre alcuni esempi di come tale medium attraverso le sue proprietà semiotiche, oltre ad essere un mezzo di alfabetizzazione visiva, assolva alla funzione di abbattimento di alcune barriere cognitive e porti alla luce mondi subalterni e sotterranei. Pensando al contesto italiano un caso studio in tal senso, ci viene offerto da Monica Boria, la quale analizzando il legame fra il fumetto e il movimento del '77, ci fa comprendere come autori quali Pazienza, Tamburini, Liberatore, Mattioli e altri, portando avanti il progetto avanguardistico del movimento: «sperimentano sia con il linguaggio visivo che con quello linguistico, lasciano entrare la realtà dell'epoca e sviluppano una estetica del tutto personale» (Boria 2007, 120). 
many forms of visual culture implicated in the imperial Anthropocene» (Ibbotson 2020, 32). Sulla base di tale rilettura, sono rilevabili numerose analogie con le forme di impegno estetico-immaginativo tarantino. In effetti condividendo col movimento in questione la critica olistica all'industrialismo, hanno anch'esse messo in risalto l'agentività delle immagini, questa volta all'interno di un regime visuale che, articolandosi su ordini mutevoli di intertestualità e mediante processi di risignificazione, ha messo in risalto il rimosso processo di colonialità e centralità della questione ecologica nello spazio diegetico.

\section{$5 \quad$ Fumetti postcoloniali}

In tale direzione appaiono rilevanti il saggio a fumetti ILVA. Comizi d'acciaio, nato dalla matita di Kanjano e dalla penna di Carlo Gubitosa, e il graphic novel L'ora $X$ realizzato a sei mani da Erri De Luca, Cosimo Damiano Damato e Paolo Castaldi. Queste opere si caratterizzano per un incipit che, con varie affinità tonali e uno stile iperrealistico, narrano come una terra baciata dalla natura e dalla storia venga ferita dalle ruspe, primi agenti del progresso-regresso industriale. Entrambi i lavori si aprono, dunque, mettendo in mostra l'enorme impatto di un'attività eteronormativa di colonizzazione ambientale, che si staglia sullo sfondo della Taranto degli anni '60. La profondità della dimensione verbo-visuale essenzialmente critica di questi lavori emerge attraverso il connubio di immagini e testi politicamente carichi che, parafrasando i documentari dell'epoca, ritraggono «la trasformazione profonda che giungerà a mutare sostanzialmente il volto e la vita del Mezzogiorno, del Mezzogiorno agricolo, del Mezzogiorno povero, del Mezzogiorno fermo da troppi secoli all'avara civiltà dell'ulivo» (Gubitosa, Cangiano 2013, 35).

La mimesi dell'azione distruttiva capitalocenica che accompagna l'abbattimento degli ulivi secolari per far posto all'Italsider, nell'Ora $X$ è rappresentata soprattutto in una delle tavole iniziali, in cui è visibile il dettaglio di un tronco d'ulivo con fattezze antropomorfe che viene sradicato dal terreno [fig. 5]. La parte terminale del tronco e le radici, nel momento in cui vengono strappate dalla terra, assumono la fisionomia di un profilo quasi umano. I contorni di questa figura in tale atto divengono più indefiniti e sembrano assumere la forma di fiotti di sangue, come a voler simboleggiare la ferita provocata dalla separazione violenta della città dalla sua natura millenaria.

Allo stesso modo, la narranalisi esordiale di ILVA. Comizi d'acciaio si concentra prevalentemente nella manifestazione esteriore dell'attività di biocolonizzazione che caratterizzò l'industrializzazione barbarica di Taranto, in un processo fisico, quasi corporeo. In una delle tavole, il piano ravvicinato di un albero abbattuto, appena stramazzato al suolo, affiora dal campo ed il tronco è cinto da una corda: effetto 
Luigi Carmine Cazzato, Antonella D’Autilia

Colonizzazione ambientale, defuturing capitalocenico e pratiche eco-artistiche decoloniali a sud
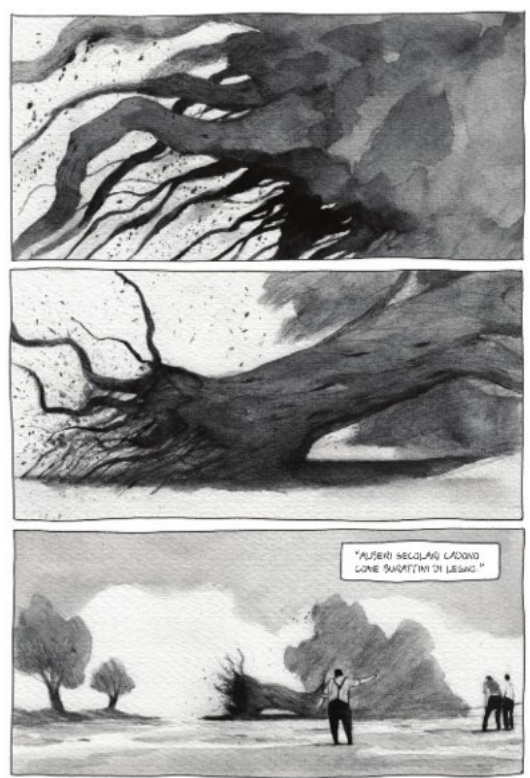

Figura 5

Erri De Luca et al., L'ora X

Una storia di Lotta Continua, 2019
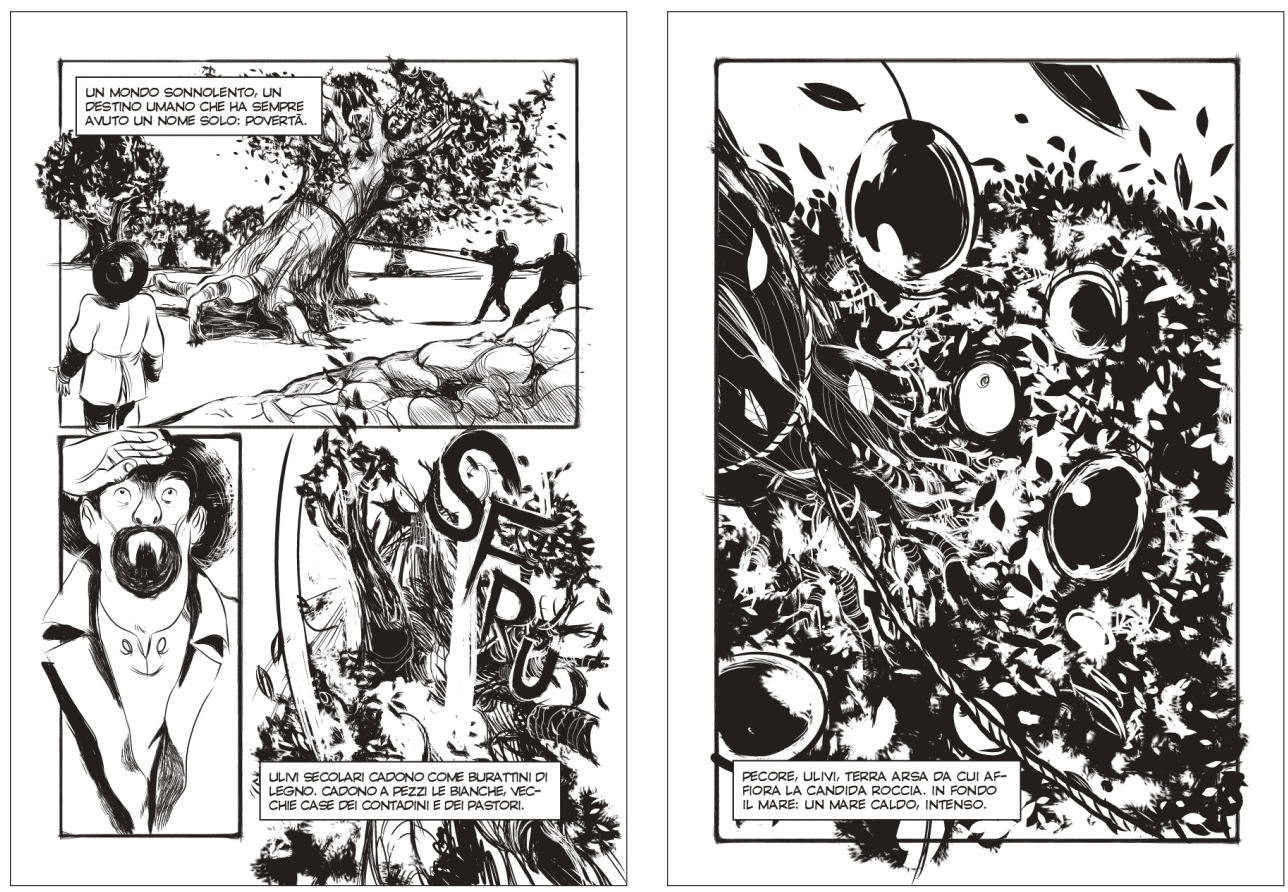

Figure 6-7 Gubitosa \& Kanjano, ILVA. Comizi d'acciaio, 201 
che, unitamente alla scelta di utilizzare la visione dall'alto, conferisce al soggetto dell'immagine un senso di sottomissione e debolezza [figg. 6-7]. Guardando alcuni dettagli delle foglie e delle olive sembra quasi che l'albero sia la personificazione di un'entità che sta per morire.

Ai margini dell'espressione di una tale polisemia esegetico-emozionale, si colloca la validazione di una prassi conflittuale consonante con la linea argomentativa di Gennaro Ascione, che propone una revisione dei canoni interpretativi della nozione di emancipazione su vari livelli, tra cui il riesame del ruolo proattivo della differenza colonial/imperiale come primum movens nella formulazione di tale concetto e delle sue manifestazioni nella realtà: «the colonial difference is systematically excluded from the realm of possible angles from which the narrative dimension of emancipation can be observed and reconstructed» (Ascione 2016, 130). Ecco allora che la didascalia della figura 6 («Un mondo sonnolento, un destino umano che ha sempre avuto un nome solo: povertà») ha un innegabile valore euristico. Tale affermazione è volta a storicizzare le radici della subalternità tarantina cristallizzate nella costruzione artificiale diadica di una dilatazione temporale, caratterizzante il Sud Italia, da sempre considerato deficitario rispetto al nord, che è suo moderno ed efficiente avamposto.

Tra l'altro il carattere performativo, insito nella riedificazione iconografico-testuale dell'ambiente urbano in questi paesaggi, risiede nella definizione di connessioni cognitive che espandono lo spettro della relazione tra arte e natura, che diviene oggetto di ridefinizione. Curtin (2005), infatti, ci fa notare che i filtri interpretativi dettati dalla storia coloniale hanno una casualità diretta nei nostri giudizi percettivi in rapporto al nostro modo di pensare ai luoghi e alle persone. Propone, quindi, il superamento del dualismo tra ecocentrismo radicale e antropocentrismo e di quel paradosso di matrice eurocentrica che ha spinto la natura umana fuori dal naturale. Nella loro disamina sul rapporto fra studi postcoloniali e letteratura ecocritica Cilano, DeLoughrey (2007) avviano una proficua discussione partendo dalle riflessioni dello storico indiano Ramachandra Guha, il quale segnala come la tendenza dell'ambientalismo, per lo più di matrice occidentale, al sovradimensionamento della visione biocentrica rispetto a quella antropocentrica ingeneri un'interpretazione astorica di natura non umana. Tali constatazioni acquistano maggiore rilevanza laddove, proseguendo nella lettura dei fumetti su Taranto, si intravvede la convergenza di questioni di giustizia sociale e problemi di giustizia ambientale su di un'unica cintura critica in cui, secondo la lezione di Moore (2015), affiora l'esigenza di un'analisi che proceda dall'unità fra umanità-nella-natura e natura-nella-umanità.

Continuando a scorrere le pagine di ILVA. Comizi d'acciaio, L'ora $X$ e volgendo lo sguardo verso le altre narrazioni grafiche sul tema ILVA degli artisti locali e non, è rintracciabile quella tendenza che Davis ravvisa nei fumetti urbani a restituire al dibattito scientifico 
elementi di analisi per un effettivo ripensamento del concetto di città socialmente e spazialmente giuste: «especially those dealing with 'urban' spaces located in the emerging 'global cities' of the South, repeatedly depict various states of discriminatory infrastructural development, failure and violence» (Davies 2019, 3).

Nel capitolo del lavoro di Gubitosa e Kanjano intitolato «Il treno», gli autori si impegnano in un'operazione paradigmatica di collegamento fra i conflitti eco-urbani di Taranto e le mobilitazioni delle popolazioni indigene in Amazzonia, ove il drenaggio di risorse minerarie è strettamente interconnesso con l'approvvigionamento di minerale di ferro dell'ILVA. La trattazione di tali aspetti comparatistici procede mettendo in scena storie locali, codificabili mediante il concetto di earthlessness, che oltrepassano i confini europei: l'alterità espressiva del fumetto si muove verso un recupero di «nonanthropocentric ways of relating to earth and of worlding the world» (Vázquez, 2017, 10). Il volume contiene tra l'altro un'eloquente postfazione scritta da Alessandro Marescotti, presidente del network ecopacifista PeaceLink, che sottolinea come: «la polvere metallica che finisce nei polmoni dei tarantini è la stessa che viene maledetta dagli indigeni dell'Amazzonia dove viene estratta e a volte fusa anche in loco» (Gubitosa, Cangiano 2013, 184) e ricollega le lotte di Taranto e del Brasile alle rivolte contadine che oppongono la nonviolenza gandhiana alla costruzione della maxi-acciaieria Posco. In effetti, Marescotti prefigura una sorta di futuro postcoloniale, quando dichiara: «Come le colonie che si liberarono dall'oppressione e dall'interesse economico superiore, noi ci libereremo dal colonialismo che ci ha distrutto, derubato e ucciso, sottraendoci persino la dignità». ${ }^{12}$

La storia narrata nel fumetto è ambientata a Carajàs in Brasile, una zona che ospita il più ricco giacimento di ferro al mondo, dove alcuni bambini delle comunità rurali indigene giocano fra i paesaggi spettinati dalle scorie del siderurgico. La vertente testuale si snoda proprio attorno a tale circostanza e l'esito drammatico della vicenda prende piede quando uno di questi ragazzi indigeni imbattutosi coi suoi compagni in un enorme ammasso di carbone ardente ci cade dentro ustionandosi le gambe. Tale contingenza conduce verso il climax della vicenda narrata e proietta l'andamento della storia verso i principali assi semantici attorno a cui gravita la storicità intrinseca allo schema compositivo dell'episodio. Quando il dato linguistico sposa la trasposizione grafica delle mobilitazioni contadine di Carajàs porta alla luce l'identità delle formule discorsive presenti nei conte-

12 Marescotti, A. (2013). «Ilva di Taranto: il diritto di resistenza per la resistenza del diritto». Il fatto quotidiano.it, 23 settembre 2013; https://www.ilfattoquotidiano. it/2013/09/28/ilva-di-taranto-il-diritto-di-resistenza-per-la-resistenzadel-diritto/726403/. 
Luigi Carmine Cazzato, Antonella D'Autilia

Colonizzazione ambientale, defuturing capitalocenico e pratiche eco-artistiche decoloniali a sud
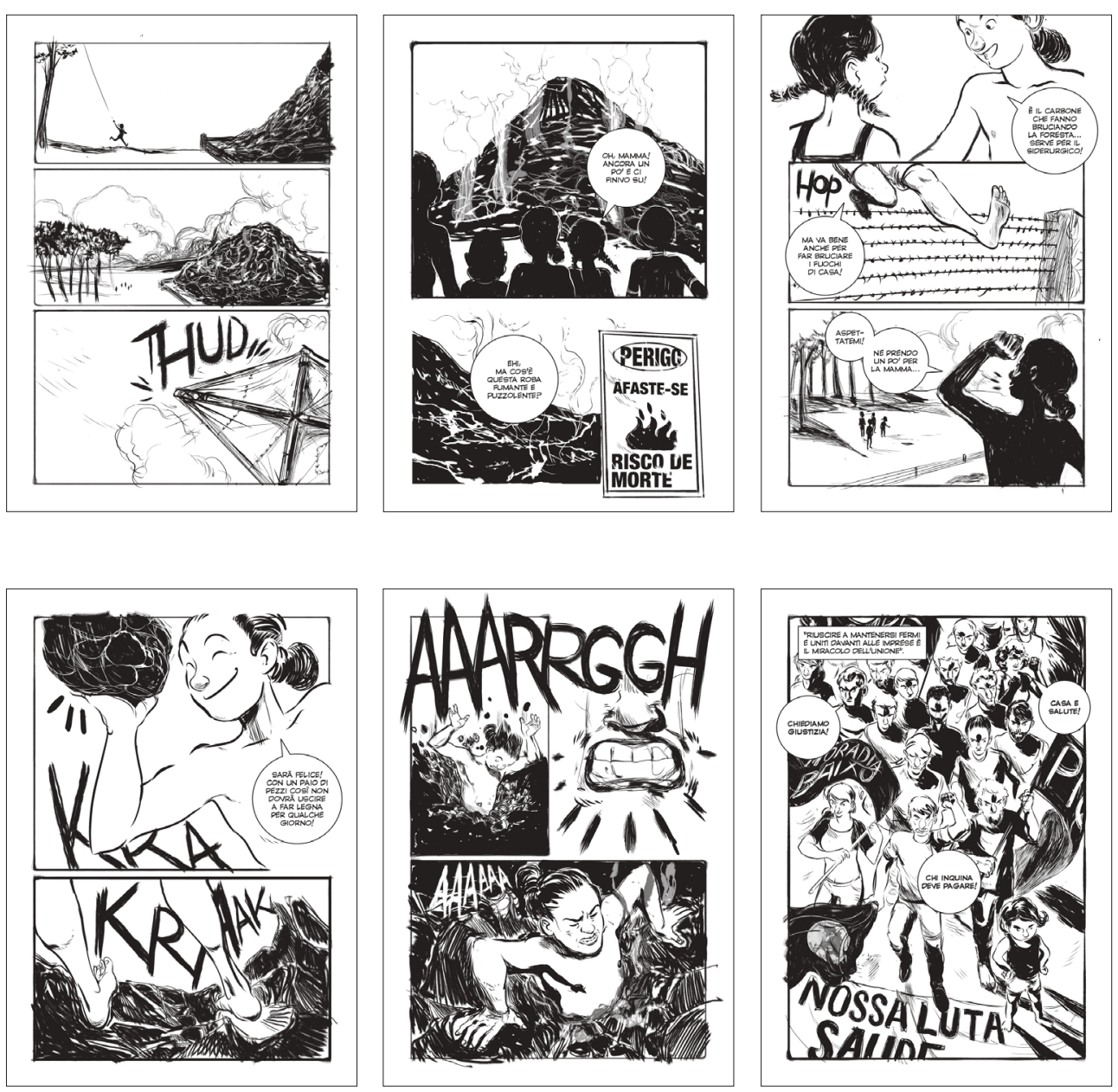

Figure 8-13 Gubitosa \& Kanjano, ILVA. Comizi d'acciaio, 2013 
nuti delle medesime rivendicazioni in Brasile come a Taranto (emblematiche le espressioni: «Casa e salute!», «Chi inquina deve pagare!»). Inoltre, viene messo in rilievo come: «identities are constantly negotiated across the boundaries of East and West and hybridization challenges notions of identities and cultures as fixed stable, or bounded entities that exist in isolation» (Mehta, Mukherji 2015, 62).

In virtù di quanto detto fin qui è rilevabile quanto tali fumetti da un punto di vista complessivo assolvano alla stessa funzione del fumetto sudafricano Bitterkomix, quando Dony riferendosi ad esso afferma che «the subversive character of the magazine may thus well suit the postcolonial label as traditionally understood in terms of mimickry and resistance to past hegemonic discourses and practices» (Dony 2014, 12). Ebbene, come la striscia a fumetti The World of Lily Wong ha avuto un ruolo di primo piano nell'esteriorizzazione delle mitologie politico-culturali che caratterizzarono la natura della transizione di Hong Kong da un governo coloniale alla sovranità cinese (Kluver 2000) - e Goorgoorlou è divenuto l'archetipo dell'economia informale senegalese a forte impronta neoliberista sulle pagine di Le Cafard Libéré (Seck 2018) - allo stesso modo sfogliando ILVA. Comizi d'acciaio ci si imbatte in biografie individuali e soggettività storiche in grado di rendere il senso di una rappresentazione collettiva che esula dalle cronache mainstream sull'ILVA. Se l'identificazione del visualizzatore e l'astrazione iconica incarnano l'abilità dei fumettisti (Seck 2018), il fumetto postcoloniale in questo senso ha il merito di cogliere quell'anello mancante fra semiosi e mondo fisico, che porta a un doppio risultato. Da una parte, vi è una veduta prospettica dei limiti intrinseci del concetto di modernità come realtà storica con pretese universali, dall'altra un'agentività messa in opera dalla creazione di relazioni logico-semantiche tali da rievocare quelli che sono stati definiti da Vázquez «relational worlds», in opposizione a quel processo in cui «modernity has relegated nature, bodies and other worlds of meaning to a negated alterity, to forms of nonexistence, to worldlessness» (Vázquez 2017, 2).

Sicché quasi a metà del fumetto di Gubitosa e Kanjano è presente un capitolo che mette in evidenza l'effetto giroscopico dell'operazione di ribaltamento degli esercizi di defuturing capitalocenico, messa in moto da forme di relazionalità orientate al recupero di una Taranto come «historical site of experience» (Vázquez 2017, 8) e da una svolta genetica dell'eco-attivismo locale in direzione di una fuoriuscita da una cultura monodimensionale delle mobilitazioni. In effetti, il capitolo «Il pastore», può essere letto come dimostrazione figurale e ontologica dell'insurrezione ideologico-trasformativa operata da alcune eco-sentinelle del capoluogo ionico, che - dovendo aggirare l'architettura normativa che legittimava il perpetrarsi di quel disastro ecologico che poco tempo più tardi sarebbe salito agli onori della cronaca - trasformarono «il frutto del lavoro contadino nel- 

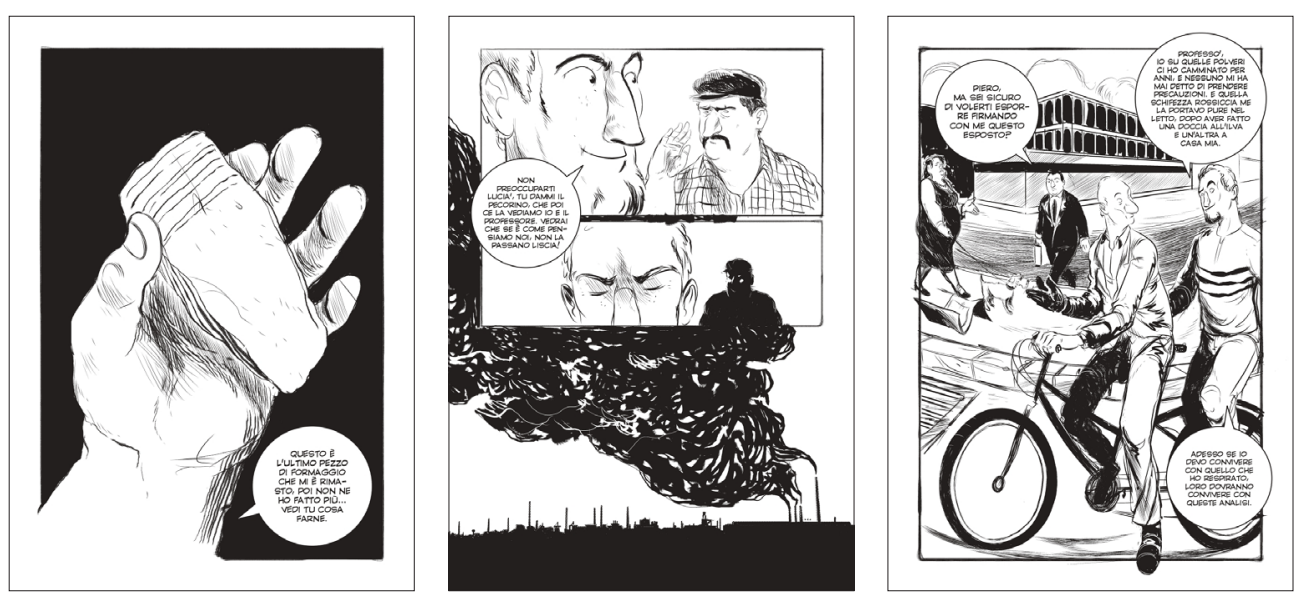

Figure 14-16 Gubitosa \& Kanjano, ILVA. Comizi d'acciaio, 2013

la prova materiale di una tremenda verità negata per anni dal potere politico, mediatico e industriale» (Gubitosa, Cangiano 2013, 122). Parte di quella «verità negata» fu accertata dai giudici: era l'avvelenamento da diossina causato dalle emissioni di fumi e polveri del siderurgico. Sul finire dell'episodio, in una tavola che narra un pezzo rimarchevole della storia di Taranto, i co-protagonisti (il professore eco-pacifista e il coraggioso operaio) gettano le basi per quello che sarebbe divenuto un vero e proprio manifesto metodologico per la fioritura dell'associazionismo tarantino: una costellazione di associazioni, che, oltre a muoversi nei tradizionali luoghi del conflitto sociale, considerano «nature not just as the stage upon which the human story is acted out, but as an actor in the drama» (Glotfelty, Fromm 1996, XXI).

È il dramma capitalocenico della ferita 'coloniale' inferta sul corpo unico della natura, umana e extra-umana. Taranto in particolare e il sud in generale sono stati vittime sacrificali sull'altare del dio progresso nella modernità/colonialità, sia nello spazio della differenza coloniale (il sud globale) sia nello spazio della differenza imperiale (il sud europeo). Il fumetto, uno degli ultimi arrivati sulla scena del crimine artistico, ha messo generosamente a disposizione il suo spazio estesico e semiotico sincretico per lenire il dolore delle ferite procurate e indicare un'altra epistemologia, un altro modo possibile di stare al mondo: quella del sud (de Sousa Santos 2014). In conclusione, e per dirla con Larkosh-Lenotti (2006), ciò che viene indicata è una «sud-alternity»: la subalternità (gramsciana) che si trasforma in una alterità meridiana, in cui gli asini dovrebbero tornare a dettare il ritmo del lavoro e della vita. 


\section{Bibliografia}

Ascione, G. (2016). Science and the Decolonization of Social Theory: Unthinking Modernity. London: Palgrave Macmillan.

Barbieri, D. (1991). I linguaggi del fumetto. Milano: Bompiani.

Bhabha, H. (2004). The location of Culture. London; New York: Routledge.

Berger, A. (1971). «Comics and Culture». The Journal of Popular Culture, 5, 164-77.

Boria, M. (2007). Il movimento del Settantasette e il fumetto. Leinen, F.; Rings, G. (eds), Bilderwelten, Textwelten - Comicwelten: Romanistische Begegnungen mit der "neunten" Kunst. München: Meidenbauer, 105-23.

Brandi, C. (2004). Pellegrino di Puglia. Roma: Editori Riuniti.

Cassano, F. (2005). Il Pensiero meridiano. Roma; Bari: Laterza.

Cazzato, L. (2017). Sguardo inglese e Mediterraneo italiano. Alle radici del meridionismo. Milano: Mimesis.

Cazzato, L. (2019). «Italia come Africa e Africa come Italia: movimenti migratori, confini reali, espansioni immaginarie da S.T. Coleridge a Erri De Luca». California Italian Studies, 9(1), 1-17.

Cilano, C.; DeLoughrey, E. (2007). «Against Authenticity: Global Knowledges and Postcolonial Ecocriticism». Interdisciplinary Studies in Literature and Environment, 14(1), 71-86.

Curtin, D. (2005). Environmental Ethics for a Postcolonial World. Lanham (Maryland): Rowman \& Littlefield.

Davies, D. (2019). Urban Comics, Infrastructure and the Global City in Contemporary Graphic Narratives. Abingdon: Routledge.

De Luca, E. et al. (2019). L'ora X. Una storia di lotta continua. Milano: Feltrinelli.

Descartes, R. (2003). Discorso sul metodo. Torino: UTET.

de Sousa Santos, B. (2014). Epistemologies of the South: Justice Against Epistemicide. Boulder (Colorado): Paradigm Publishers.

Dony, C. (2014). «What is a Postcolonial Comic?» Chronique de Littérature Internationale, 7 November, 12-13. https://orbi.uliege.be/bitstream/2268/173763/1/What\%20is\%20a\%20postcolonial\%20 comic_cdony_mixedzone.pdf.

Escobar, A. (2018). Designs for the Pluriverse: Radical Interdependence, Autonomy, and the Making of Worlds Single. Durham; London: Duke University Press.

Federici, S. (2004). Caliban And the Witch: Women, the Body, and Primitive Accumulation. New York: Autonomedia.

Fry, T. (2009). Design Futuring: Sustainability, Ethics and New Practice. Oxford; New York: Berg Pub Ltd.

Gissing, G. (1901). By the Ionian Sea. Notes of a Ramble in Southern Italy. London: Chapman and Hall.

Glotfelty, C.; Fromm, H. (1996). The Ecocriticism Reader: Landmarks in Literary Ecology. Athens: University of Georgia Press.

Gubitosa, C.; Cangiano, G. (2013). Ilva, comizi d'acciaio. Padova: Becco Giallo.

Huggan, G.; Tiffin, H. (2010). Postcolonial Ecocriticism: Literature, Animals, Environment. New York: Routledge.

Ibbotson, R. (2020). «Crafting 'Nature'. Ecocriticism, Environmental Violence and the Transnational Arts and Crafts Movement». Coughlin, M.; Gephart, E. (eds), Ecocriticism and the Anthropocene in Nineteenth-Century Art and Visual Culture. New York: Routledge, 32-48. 
Kluver, R. (2000). «Comic Effects: Postcolonial Political Mythologies in the World of Lily Wong». Journal of Communication Inquiry, 24(2), 195-215.

Larkosh-Lenotti, C. (2006). "On Gramsci, "Epistemic Interference" and the Possibilities of Sud-Alternity». Annali d'Italianistica, 24, 311-26.

Leogrande, A. (2014). «Lo stato d'eccezione tarantino». Alfabeta2, 34, gennaio-febbraio.

Marsili, E. (1962). Il pianeta acciaio. https://www.youtube.com/ watch?v=PJcFrWx76IE.

Mehta, B.; Mukherji, P. (2015). Postcolonial Comics: Texts, Events, Identities. Abingdon: Routledge.

Mignolo, W.D. (2007). «Delinking: The Rhetoric of Modernity, the Logic of Coloniality and the Grammar of Decoloniality». Cultural Studies, 21(2-3), 449-514.

Mignolo, W.D. (2019). «Ricostituzione epistemico-estetica: l'aesthesis decoloniale un decennio dopo». ECHO, 1, 229-42. https://doi.org/10.15162/2612$6583 / 1141$.

Mignolo, W.D.; Vázquez, R. (2013). «Decolonial AestheSis: Colonial Wounds/Decolonial Healings». Social Text Online. https://www.socialtextjournal.org/periscope_article/decolonial-aesthesis-colonialwoundsdecolonial-healings/.

Moore, J.W. (2015). Capitalism in the Web of Life. London: Verso.

Moore, J.W. (2016). Anthropocene or Capitalocene: Nature, History, and the Crisis of Capitalism. Oakland: PM Press.

Moore, J.W. (2017). Antropocene o Capitalocene? Scenari di ecologia-mondo nella crisi planetaria. Verona: Ombre Corte.

Naipaul, V.S. (1967). The Mimic Men. London: Andre Deutsch.

Parks, T. (2014). Italian Ways: On and Off the Rails from Milan to Palermo. London: Vintage.

Pasolini, P.P. (2009). Saggi sulla politica e sulla società. Milano: Mondadori. Collana I meridiani.

Pasolini, P.P. (2017). La lunga strada di sabbia. Milano: Guanda.

Quijano, A. (2010). «Coloniality and Modernity/Rationality». Mignolo, D.W.; Escobar A. (eds), Globalization and the Decolonial Option. London; New York: Routledge, 22-32.

Schmitt, R. (1992). «Deconstructive Comics». The Journal of Popular Culture, 25(4), 153-62.

Seck, F. (2018). «Goorgoorlou, the Neoliberal 'homo senegalensis': Comics and Economics in Postcolonial Senegal». Journal of African Cultural Studies, 30(3), 263-78.

Tlostanova, M. (2018). What Does It Mean to Be Post-Soviet? Decolonial Art from the Ruins of the Soviet Empire. Durham; London: Duke University Press.

Vázquez, R. (2017). «Precedence, Earth and the Anthropocene: Decolonizing design». Design, Philosophy papers, 15(38), 77-91. 


\title{
"The Road to Awesomeness": The Environment, Language and Rhetoric in Chetan Bhagat's Postcolonial India
}

\author{
Esterino Adami \\ Università degli Studi di Torino, Italia
}

\begin{abstract}
This paper aims to discuss how environment, language and rhetoric interplay in the postcolonial context, in particular by focusing on Making India Awesome (2015), a recent collection of essays in which journalist and novelist Chetan Bhagat offers suggestions to handle many of the challenges of India, ideally positioning the country 'on the road to awesomeness'. Although ecology is not specifically treated, it obviously constitutes the backdrop of the themes of the book as it intertwines with broad social and cultural domains. I will look at the postcolonial environmental intertext, and its ideological implications, which the author builds up via specific frames, metaphors and devices from an interdisciplinary perspective informed by postcolonial critique, environmental humanities and ecolinguistics. The purpose of the analysis thus is to provide a critical reflection on how language shapes, creates and hides values at the interface between the postcolonial and the environmental.
\end{abstract}

Keywords Chetan Bhagat. Postcolonial Environment. India. Ideology. Rhetoric.

Summary 1 Introduction: The Environment in the Postcolony .- 2 Theoretical Background: Environmental Studies, Postcolonial Critique and Rhetoric . - 3 The Author, the Text and the Context. - 4 Bhagat's Writing: Changing India and Persuading People. - 5 Behind Ecocriticism: Ideology and the Nation?. - 6 Conclusion.

$\begin{array}{lll} & \text { Peer review } \\ & \text { Submitted } & 2020-07-10 \\ \text { Edizioni } & \text { Accepted } & 2020-09-07 \\ \text { Ca'Foscari } & \text { Published } & 2020-12-22 \\ & \text { Open access } & \end{array}$

(C) 2020 | ()( Creative Commons Attribution 4.0 International Public License 


\section{Introduction: The Environment in the Postcolony}

Over the last years, general attention to the environment and environmental awareness has experienced a remarkable growth in the attempt to spotlight crucial questions of ecology, power imbalance and society. It is now almost a truism to affirm that the environment is part and parcel of human life, but much recent scholarship has underlined how its very conceptualisation needs to be revised to extend to and include a vast system of forces, elements and notions, in which human beings constitute only one of the actors of a wide and dynamic scenario (Heise 2008). The emergence of a new environmental philosophy, or ecosophy, emphasises the living aspect of human experience and reflects on holistic dimensions such as wellbeing, the continuum between present and future, and the importance of resilience.

Such considerations inform and influence the field of environmental humanities, whose tools, frames and theories are used to address a number of issues, from cultural suppression of autochthonous communities, destructive processes of development and the rethinking of social practices. All these themes frequently appear in the postcolonial agenda too, thus endorsing the connection between the two fields, as abundantly demonstrated by both creative and academic works. Hence stems an interdisciplinary critical work that, according to DeLoughrey, Didur, Carrigan $(2015,5)$, "advocates for the power of the imagination as expressed collectively across the full range of cultural practices". Since language constitutes the building blocks of discourse, it is imperative to focus on the key role it performs in the construction, circulation and naturalisation of ideas, or ideologies, for instance by investigating those texts that deal with environmental preoccupations and postcolonial contingencies. Thanks to specific linguistic strategies, in fact, values and meanings can be foregrounded in order to gain attention, or on contrary can be relegated to peripheral positions, or even totally silenced.

In this paper, I set out to discuss how the environment, language and rhetoric interplay in the postcolonial context, in particular by focusing on Making India Awesome (Bhagat 2015), a recent collection of essays in which journalist and novelist Chetan Bhagat offers suggestions to handle many of the challenges of contemporary India, ideally positioning the country "on the road to awesomeness" (175). Although ecology is not specifically treated, it constitutes the backdrop of many themes of the book as it intertwines with broad social and cultural areas to form "the entire network of human and nonhuman material existence" (Mukherjee 2010, 15). In fact, questions of exploitation, poverty and marginalisation are inextricably linked to the contexts depicted by Bhagat, and as such may be rendered as powerful, provoking or ideological narratives. My research purpose here is twofold. Firstly, I propose to scrutinise this text from an 
interdisciplinary perspective that combines the methods and tools of postcolonial critique, environmental humanities and ecolinguistics (e.g. DeLoughrey, Didur, Carrigan 2015; Stibbe 2015). Secondly, since no text is ideology-free, I argue that even Bhagat's writing does not seem to entirely detach itself from a form of unconscious pride and nationalistic rhetoric in spite of the author's progressive views and ecological hopes, hence the need for a critical reflection on how language shapes, intensifies and hides values (Cockcroft, Cockcroft 2005; Jeffries 2010).

\section{Theoretical Background: Environmental Studies, Postcolonial Critique and Rhetoric}

I start with the outline of a theoretical background whose critical scaffolding delineates the connections between environmental studies and postcolonial critique, two coterminous fields that share much work and commitment. Scholars working in both areas in fact have repeatedly highlighted how the ruthless exploitation of natural resources, the progressive destruction of biodiversity and the risks of industrialisation processes are common research objects. The rise of ecocriticism and environmental humanities, following a kind of environmental turn of the last two decades, has brought to light other shared pathways too, spanning topical issues as diverse as the annihilation of aboriginal populations in Australia, the contradictions of Canadian 'ecological' projects and the dystopian power of climate change in South Asian literatures. As Huggan, Tiffin (2015, 6) hold, "postcolonial studies has come to understand environmental issues not only as central to the projects of European conquest and global domination, but also as inherent in the ideologies of imperialism and racism on which those projects historically - and persistently - depend". As a matter of fact, even with the achievement of political independence, some of these territories have perpetuated colonial policies, often under the rubrics of progress and development, and therefore ecological questions turn out to be crucial in the postcolonial world. Novelists and theorists like Ken Saro-Wiwa, Arundhati Roy and Amitav Ghosh, in different times, contexts and genres, have given global visibility to many environmental concerns, interrogating the pernicious entanglements of environmental and societal questions, or reimagining the relation between human life and other forms of life in a holistic manner.

If we turn an ecocritical lens on the postcolonial world, thus, we can observe an extensive map showing different relations between local communities, committed writers, political fractions at play with centripetal and centrifugal forces, often negotiating, or fighting, neoliberal policies that are the by-product of formerly imperial conditions. 
In this light, the material aspect of life goes hand in hand with the natural and the cultural ones because the production of commodities not only determines the size of the labour market but also obviously generates consequences in terms of exploitation, pollution and suitability. As Mukherjee $(2010,73)$ notes, "eco- and postcolonial criticism have been discovering how to cross-fertilize each other through an ongoing dialogue, and a stronger materialist re-articulation of their positions should make this exchange about culture and society even more fruitful". In other words, this type of interdisciplinary approach takes, adapts and elaborates tools and theories from cultural criticism to deconstruct the (in)visible representation and treatment of the environment in postcolonial texts, also considering the role of historical capital and the obfuscating borders of the globalised world.

The metaphors and discourses that celebrate development, progress and welfare can be analysed in this light too and Chetan Bhagat seems to begin his reflection on contemporary India from such a position because he uses factual cases not only to discuss, but also to provide suggestions about how to solve social and economic problems that are implicitly related to the environment. The articulation of Bhagat's text reflects his double role (as a novelist and a motivational speaker), takes a narrative form and is driven by the principles of rhetoric, such as the Aristotelian ethos (the character of the speaker), pathos (the arousing of emotions in the audience) and logos (the linguistic realisations of the speech), in the construction of discourse (see Burke 2014). Specifically, the author applies techniques and tropes "to structure and elaborate an argument, and to move the emotions" (Wales 1995, 406), activating forms of functional persuasion, which indicates "all kinds of persuasive discourse (spoken or written) concerned with everyday life, here real people are being persuaded to a real purpose" (Cockcroft, Cockcroft 2005, 5). Thus, the writer tries to convince readers to discard unwise social practices, which often have a very negative impact on nature, and adopt responsible behaviours, which are expected to contribute to national wellbeing and life in general. However, a closer look at the book in reality can reveal how, to a certain extent, it ambiguously situates itself close to some forms of populism, whose environmental awareness is not always transparent. In what follows, I concentrate on the linguistic strategies through which the author proposes his solutions to some of the challenges of contemporary India. From this angle, Bhagat's stylistic choices are viewed as signs of discourse and ideology that cumulatively galvanise current narratives of both denunciation and celebration, thus offering a sketched portrait of the attitudes, contradictions and sentiments that characterise India's social, cultural and natural arena. 


\section{The Author, the Text and the Context}

This section briefly presents and contextualises the author, his work and style. In the Indian subcontinent, Chetan Bhagat is extremely famous, not only thanks to his literary career, but also because of his role as correspondent, Bollywood screenwriter and social media influencer, thus demonstrating how new media platforms now are parallel to, or replace, traditional medias in building, circulating and amplifying discourses and news stories (Chaudhuri 2010), including of course debates on the environment, sustainability and public perception (Nambiar 2014). Bhagat's production spans both fiction and non-fiction, with novels such as Five Point Someone (2004), One Night @ the Call Center (2005), and The Girl in Room 105 (2018). All these stories share a number of motifs and features that outline the new Indian scene as they speak about young generations, the rise of opulence in the main urban centres and the economic and post-industrial transformation undergoing in the country. The novels hybridise various genres, from the coming-of-age tradition to the realms of crime and romance, and in spite of their mass-market connotations they mirror social and cultural traits of present-day India, in particular the English-speaking metropolitan contexts. Thus, Bhagat's books have enriched the formation of a new canon for Indian English literature (Varughese 2013, 13-17) with questions of identity, globalisation and power, and have even inaugurated a sort of subgenre, namely the so-called "call centre fiction".

However, Bhagat is also a very productive columnist, contributing to both English-language and Hindi-language newspapers like The Times of India and Dainik Bhaskar, and has authored three non-fictional volumes, i.e. What Young India Wants (2012), Making India Awesome (2015) and the recent India Positive (2019). These texts almost become a sort of how-to-do manual in their attempt to resolve difficulties of India today, thus revealing their kinship to forms of neoliberalism (Simpson, Mayir 2010, 39), but at the same time they openly seek to raise awareness, construct community and promote values. Frequently they deal with macro issues such as politics, economics and human rights and they encourage the adoption of new or different manners, with the ultimate purpose to reach high levels of prosperity and wealth for all. However, a discourse of general reform and democratic enhancement should not avoid a serious reflection on the environment and its deep connection with the world's human component to trigger a rethinking of the binary polarity of nature and culture. Bhagat sincerely commits himself to a debate on the improvements of various portions of the Indian population, but he does not specifically include the question of ecology in his writing, and somehow blends auspicious objectives, wise suggestions and nationalist pride, proposing what Daftuar (2015) terms "easy advice". 
Endowed with a title that almost seems to anticipate Trumpian populism, Making India Awesome (2015) collects a series of pieces and columns about the main social, economic and political challenges of India, originally written for newspapers and magazines. From the very title, the buzzword 'awesome' is emphatically utilised to portray an improved, ideal and perhaps even utopian general canvas for the country. The book is organised in four parts: "Awesome Governance: Politics and Economy", "Awesome Society: Who We are as a People and What We Need to Change", "Awesome Equality: Women's Rights, Gay Rights and Minority Rights", and "Awesome Resourc-

es: The Youth". Overall, the writer unravels the textual rendition of 'change', either desirable or already achieved, for instance with examples or situations, behaviours and conditions seen before and after the colonial period, the development of certain territories, or the promulgation of specific laws. Many of these narratives hint at implicit environmental topics, from the provision of services to slums and districts to the preparation of food, and the related themes of developing agriculture and alimentary industry. In my analysis, I consider how the rhetorical and narrative format chosen by Bhagat activates the types of ideological patterning that are ecolinguistically salient (Stibbe 2015, 35).

\section{Bhagat's Writing: Changing India and Persuading People}

One of the pieces in which the environmental intertext particularly stands out is entitled "Cleanliness Begins at Home", and is in line with a general discourse about the conditions of hygiene and sanitation structures in the country. Often associated with stereotyped images of dirt, insalubrity and pollution, this is a motif that dominated the colonial era (Schülting 2016), but also resonates across postcolonial texts, from the novels by Mulk Raj Anand such as Untouchable (1935) to the 2017 film Toilet: a Love Story, directed by Shree Narayan Singh. In his unsophisticated prose, the author starts by triggering a comparison: "Indians who travel abroad are often awestruck by cleanliness levels in the developed world" (Bhagat 2015, 99) and then develops his argument with examples, metaphors and other linguistic devices. He also openly mentions the Swachh Bharat project, a nationwide initiative that took place between 2014 and 2019 to eradicate problems derived from open defection and improve solid waste management. However, while praising the initiative as a whole, Bhagat stresses how such issues should not be viewed merely in terms of campaigns showcasing the establishment or celebrities, because they concern everyday routines adopted by millions of citizens, with a great impact on society and the environment. In fact, the real question at stake here is about identity and not merely a lack of cleanliness 
in the Indian population, since for the author "we are not dirty people. Indians keep their homes scrupulously clean" (99). In this way, a collective identity is built and foregrounded and serves as a starting point for the promotion of behavioural change.

Rather than linking the problem to the authorities' role, the essay brings in the sense of individual responsibility by suggesting public involvement and effort: "if we truly want to be a clean country, we need to take steps to ensure we minimize filth in the first place, rather than hope someone will pick up a broom and clean it" (100). The sense of the citation is marked by the manner adverb 'truly' whilst the writer's personal commitment emerges from inclusive pronouns ('we'). To construct and validate a pragmatic persuasive discourse, i.e. to convince people to change or modify quotidian habits, Bhagat utilises various rhetorical techniques. A micro-level linguistic analysis here can unveil how modality in particular is instrumental for the assemblage of the text and the promotion of a specific viewpoint. For Griffiths $(2006,110)$, "modality is a term for a cluster of meanings centred on the notions of necessity and possibility", although in reality its conceptual scope is larger and typically refers to the speaker's (or writer's) attitude toward an object or topic, therefore determining how the language used conveys specific meanings and feelings. Modality can be labelled as deontic, boulomaic and epistemic, respectively referring to sense of duty, idea of desire and knowledge system, and operates by various means such as verbs, evaluative terms and expressions of perception. Bhagat's essay, and in general the entire book, displays a positive modal shading (Gibbons, Whiteley 2018, 113), which profiles an opinionated narrating voice expressing volition, obligation and judgement.

A recurrent textual pattern that strengthens the author's view lies in the use of conditional constructions, a modal strategy that allows a speculative process, in which we imagine the consequence or effect of a certain action. The quotation above has an example of hypothetical form ("if we truly"), but more can be found in following excerpt, along with other modalised items and stylistic devices:

The only way it can, and will, become clean is if we minimize and prevent the creation of filth in the first place, and the only way that will happen is when all of us together think, 'What is outside my home is also mine'.

This sense of community recognition of a greater good and collective ownership is the only way for the situation to change. Else, we risk this cleanliness drive becoming another social fad that will be forgotten when the novelty wears off.

Of course, infrastructural improvements, such as new treatment plants for solid, sewage, industrial and agricultural waste, are required. New sets of indices, whether they be measures of 
cleanliness or density of dustbin distribution, too are needed. Laws and fines have their place as well. All that is indeed the government's job and it will be judged on it.

However, all this will come to naught if we Indians don't change our mentality about what is my space and what isn't. The country is ours. You obviously can't clean all of it, but you can be aware of at least a little bit of area around you. If every Indian has a concept of 'my 10 metres', or a sense of ownership about a 10-metre radius around him or her, magic can happen. [...]

Whenever there is a collective sense of ownership, we have higher cleanliness levels. It is for this reason that most college campuses are cleaner than the city outside, despite housing thousands of youngsters inside.

So get out there, scan your 10 metres. Can you improve anything? A swachh Bharat is indeed possible. The first step is 'swachh manasikta' or clean mindsets. Are you game? (100-1)

As Jeffries (2010,123-4) argues, "modality also draws on the issues of hypothetical worlds and speaker preferences/certainties, and the focus here is on persuasion by evaluative opinion, as well as persuasion by imagining different possibilities". The rhetorical dimension of the extract is intensified by the use of conditional forms, or hypothetical syllogism, triggered by what Cockcroft, Cockcroft $(2005,128$, emphasis in the original) define as "the big IF", in this case the speculation about the possibility for Indians to modify their lifestyles to improve the living condition of the country. We should also notice how the conjecture proposed by the writer is activated by a rather emphatic phrase ('the only way'), a sort of slogan or 'sound bite' that generates unilateral hypothetical thinking and speculation. In this light, Bhagat's text uses various modal means, in particular of boulomaic kind, to conceptualise an alternative, but somehow feasible, reality, in which India is imagined as a clean and unpolluted country. Modal items include conditional structures ('if/whether'), modal auxiliary verbs (for possibility like 'can', and volition like 'will') and adjectives/ adverbs ('obviously, possible'), which deliver an ideological message because they sustain the promotion of hygiene, here seen as a component not only for individual life but also for collective wellbeing.

A remarkable aspect of the text regards the representation of the sense of community, with the creation of complicity and solidarity, and this is marked by the use of inclusive pronoun 'we' (thus identifying the writer as part of a national community) and especially by the use of evaluative language. For Stibbe (2015, 83), "appraisal patterns are of key interest in ecolinguistics because of their power to influence whether people think of an area of life positively or negatively". In the passage, Bhagat's vocabulary in fact comprises a range of appraising words, for example adjectives, both explicit, 
in particular through comparatives like 'higher/cleaner', and implicit, e.g. terms with positive connotations such as 'new' or 'improvement'. But evaluative language can also be realised via other linguistic categories, for instance with positively-oriented lexis ('minimize and prevent the creation of filth'), or on the contrary inherently negative lexis ('naught'). To reinforce the power of appraising items, the author also employs apostrophic address (Gibbons, Whiteley 2018, 168 ) by asking his readers direct questions ('Can you improve anything? Are you game?') to further motivate and engage with them to take action, and therefore raise ecological sensitivity, foregrounded by symbolic and loaded terms such as 'treatment plants'.

I now tackle the theme of food, namely another topic that can be fruitfully investigated from an ecostylistic viewpoint, given its correlation with significant mechanisms such as exploitation of territories (e.g. intensive agriculture), production (e.g. the use of chemicals or unhealthy ingredients), and distribution, (e.g. the transport systems necessary for carrying products across a country). Historically and culturally, food in India constitutes a domain that deeply ties up with the environmental sphere at different levels, and as illustrations I can mention widespread alimentary practices such as vegetarianism (followed by many religious communities throughout the country) or the Ayurvedic diet, whose guidelines follow principles of physical and spiritual wellbeing, acknowledging the role of humans within a multifaceted scenario. Bhagat specifically treats the idea of food in two essays, "Our fatal attraction to food" and "Junk food's siren appeal", which essentially deal with the sophistication of common foods. From the former, I extract the following parts:

This reminds me of a study released by the Centre for Science and Environment (CSE) in 2012, referring to harmful substances in some of the yummiest snacks brought into India by our caring MNCs. A huge reaction ensued. Over tea and bhujias, cold drinks and samosas, butter chicken and naan, Indian held discussions on how what they considered the love of their life - delicious, yummy food - could be harming them. The CSE study hit where it hurt most - instant noodles, potato chips and cold drinks are all middle-class indulgences. In scientific mumbo-jumbo, like trans-fat content and percentage daily intake, terms few understand, it said something like 'This stuff is bad for you'.

The MNCs jumped, engaging public-relations firms to clarify that they had been misunderstood. After all, anybody advertising their products with cute baby voices or other emotional tugs like grandparent-hugging, could hardly be making anything harmful. If you believe the ads, chips and colas make you a more loving, endearing person and burgers and burgers and fried chicken help you make better friends. [...] 
The CSE and FDA report aside, one doesn't need a laboratory to figure out that some of the things we eat are bad for us. Here are some simple facts. A juice brand sells mango nectar that can have eight spoons of sugar per glass. A pack of instant noodles is nothing but refined processed starch (plus MSG and lead, it seems). The malt-based so-called nutritional milk additives for children are mostly sugar. Expensive breakfast cereals can't beat the health value inherent in a few simple rotis. Fried potato chips and burgers with patties that were frozen months ago are quite obviously not healthy.

It isn't just the MNCs. The mithais and namkeens that are part of our traditional heritage, the thick gravies served in Indian restaurants and some homes, and the samosas and pakodas we regularly see being sold at railway stations are equally bad for us. Simple, healthy meals with low oil and sugar are the best. And yet, no one - the government, the MNCs or people like us - seems to care. We shall pay the price in the next ten years. Obesity levels will increase, fitness will decline and healthcare costs will rise. The affluence we feel so proud of will actually come back to us. (Bhagat 2015, 97-8)

The linguistic choices that characterise this passage confirms the author's persuasive aim and his interest in environmental issues, in particular by calibrating different registers, varieties and usages. Indeed, on the one hand the text exhibits specialised terminology with acronyms (e.g. FDA: Food Drug Administration; MSG: monosodium glutamate; MNCs: multinational companies), as well as exact dates and technical details from authoritative sources, often described as 'facts', and therefore true things that can be accessed and verified by anyone. But on the other it incorporates forms of jargon ('mumbo-jumbo') and diatopic items of Indian English (Sailaja 2009), in particular food terms such as bhujia (a crispy snack), mithai (a dry sweet), and namkeen (a savoury snack). The effect that derives from this heteroglossic style is to gain trust from the readers, who are glad to see precise and reliable scientific references, as well as to establish proximity that leads to an inclusive sense of community and denounces problems stemming from the use of processed and unhealthy foods.

The entire essay seems to be grounded upon a particular type of frame (Stibbe 2015, 46-8), namely a cognitive structure through which a story and certain aspects of reality are defined and constructed, which can be spelled out as 'food is life', thus establishing links with society, tradition and identity. However, the author develops such a frame to produce a message of warning by means of opposition and negation (Jeffries 2010, 108-9), i.e. signalling a threat to healthy food, which in turn embeds a threat to the environment and life in general. The good properties of traditional food are here re- 
placed by the characteristics of heavy and unhealthy products (sweet drinks, frozen meat), which appear to be connected with the activity of multinational corporations, often seen as prototypes of global exploitation in both natural and human terms. The premodifying adjective 'caring', moreover, carries a judgement of irony for the work of these companies.

Bhagat also highlights the importance of participants in a frame, here his readers and people in general, who are asked to actively react to the text by changing their food and shopping habits. In fact, the writer insists on the agency of individuals, that is, the power of people to decide and pursue the goal of collective wellbeing. Although the passage implicitly celebrates the healthy nature of Indian food as a response to sophisticated (and clearly unsustainable) food production, the last paragraph condemns a host of popular foods too, which are bad for the body and whose regular consumption leads to serious problems. The argumentation is strategically built thanks to an adversative connective ('yet'), which introduces a negative statement ('no-one') orchestrated as a form of meta-criticism since it is organised in an inclusive form ('people like us', implying the writer and the readers), which aims to shake people's minds and generate awareness. Two other devices are worth noticing: the deontic modal auxiliary verb 'shall', by which a certain bleak future scenario is mentally evoked in a categorical way, a sort of future obligation looming onto society, through an economic metaphor ('to pay the price') and then the key word 'affluence', whose positive semantic load is here nullified and turned into a negative element of suffering. The very harmful aspects of certain food habits are then rendered in a three-part list, a textual pattern that for Jeffries $(2010,73)$ "seems to imply completeness, without being comprehensive, and often appears to supplant real content". In this way, linguistically and symbolically, the essay enhances its persuasive force and it also triggers a wide debate about ecological and social matters in postcolonial India.

\section{$5 \quad$ Behind Ecocriticism: Ideology and the Nation?}

Generally, Bhagat's book represents an attempt to change and solve several pernicious situations that are at the heart of social and environmental injustice in India, spanning from uneven access to education to the management of airports, or the recognition of civil rights for the so-called minor communities. Taken together, these pieces corroborate ecological discourses of denunciation as they foster beneficial principles of ecosophy that sustain a rethinking of practices, attitudes and behaviours, with the general objective of improving life conditions and its related dimensions. However, as Stibbe (2015, 35) argues, discourses may also overarch ambivalent positions, and 
embed elements that somehow depart from a consistently ecological orientation. Some sections of the book, in fact, seem to accord to other intentions, in particular a kind of rhetorical celebration of the nation that promotes the emergence of the country in all sectors. From this angle, the author's slightly ambiguous attitude can be read against the broad social and cultural fabric of contemporary India, in which voices of nationalism are spreading and appropriating all social and cultural domains, in an effort to impose a certain view of the country, specifically crystallised as a Hindu-only nation. It is true that the writer dedicates some pages of his work to other religious groups (e.g. the Muslim community) and stigmatises communal violence, gender discrimination and other forms of intolerance, but nonetheless Bhagat's writing does not seem to entirely detach itself from a form of unconscious pride and nationalistic rhetoric in spite of the writer's progressive ideas and ecological hopes. Therefore, it is vital to detect whether language can generate rhetorical manipulations or camouflages.

The ideology of growth here does not specifically consider the environmental dimension, but simply foregrounds mythicized images of power and nation, as we can observe at the very beginning of the book, whose introductory section is arranged in an epistolary way to attract the reader's attention and build consensus by highlighting ideas of cultural belonging. Let us look at the following extracts:

Dear Reader,

Thank you for picking up this book. This is not a story. There is no romance in here, nor are there page-turning, thrilling moments. Rather, this book is about a dream both you and I share - to make India a better place. (Bhagat 2015, 1)

By God's grace and thanks to my readers' love, my books have reached almost all corners of the country. Each book is a unique Indian story, about people from a particular place of India. The stories have worked all over India. Doesn't this mean that, at some level, we are homogenous? We can and do emphasize with Krish Malhotra's attempts at getting married to a girl outside his community (2 States). A reader in Rajasthan can relate to Madhav Jha's struggle with spoken English (Half Girlfriend). As a motivational speaker, I have travelled across India; I have visited over a hundred cities in the last three years. While there are geographical differences, I find that ultimately, as Indians, we are the same. The average Indian anywhere in the country is looking for a better quality of life, a certain amount of hope and security and the freedom to make personal choices. The issues that really matter to us are the same. (11-13) 
The tone of the writing is similar to a form of captatio benevolentiae, realised by a series of techniques such as the apostrophic address to the real/implied reader ('Dear Reader') and the balance with person deixis, namely pronouns that here give the impression of a sort of face-to-face, even intimate conversation ('you and I') in order to sanction empathetic bonds and closeness. But the extracts also piece together echoes from both non-fictional and fictional domains, the complex reality of the country and the literary worlds imagined by the author. In this regard, it is important to bear in mind the function of the persuasive text in which the author's personality and stance are translated in terms of public image, political charisma and corporate identity. As a result, "the contemporary cult of celebrity commodifies and celebrates everyone in the public eye, from popular heroes such as footballers, musicians and film stars to bishops, politicians and even certain kinds of criminals" (Cockcroft, Cockcroft $2005,30)$. Not only is Bhagat a very popular novelist in India, but he is also a journalist, a commentator and a public figure, whose opinions, views and beliefs are widely spread through a number of media. By mentioning some of his literary works (Viswamohan 2011), he authenticates the positive message of his stories, juxtaposing the real and the fictional, and therefore seems to augment the reliability of his position for the wide readership.

The writer repeatedly communicates a sense of belonging as a form of collective identity for the nation ('we are the same'), for example with the invocation to God, astutely defined via generic label, and therefore with an inclusive value for numerous religious groups, as well as the use of hyperbolic expressions ('all over India'), which reinforce other positively connoted phrases ('a better place', 'a better quality of life'). However, there are hedges, i.e. expressions used to mitigate the force of an utterance (Wales 1995, 15), in this case the sense of national rhetoric, for example when he confesses "I am not perfect, nor are all my thoughts". The main effect of this type of wording is to exhibit a collaborative attitude as readers are advised to do certain things (through the mechanics of persuasive language), but at the same time they are attributed an active role in the process of change, which is ultimately targeted at reaching wellbeing and welfare, metaphorically viewed as awesomeness.

Bhagat's project appears to adhere to a host of different cultural, social and economic initiatives that celebrate the country's efforts to become a new world superpower and that seem to hide the complexities (and flaws) of a country like India. An example of such tendency can be seen in the tourism campaign known as 'India Shining', which is meant on the one hand to attract international visitors, and therefore to engender positive results in commercial terms, and on the other to play a part in a broader process of development and amelioration for the country. The author does not speak about this 
programme, but the 'dream' of awesomeness that he unceasingly presents as an umbrella term for welfare and progress to some extent shares the same principles. In so doing, the author builds up a hypothetical demarcation line, before and after the 'real' change, with the realisations of "the spectacular embodiment of the postcolony's transition to capitalism in the twenty-first century that potentially not only enhance the national reputation but also lay bare the conflicts and anxieties under the full global gaze" (Kaur 2016, 622). In a nutshell, the writer's intention to provide readers with suggestions and reflections about a new way to understand society, nature and the world with the goal to improve prosperity for all takes the form of denouncing narratives, but is not devoid of partial evaluations and prejudices that pivot around national pride, mythicize the image of the country, and ultimately seem to endorse consumerist (and therefore markedly damaging) perspectives.

\section{Conclusion}

In this paper, I have endeavoured to integrate different analytical approaches, from postcolonial criticism to environmental studies and critical stylistics, to offer a reading of Chetan Bhagat's Making India Awesome, a non-fictional text that thanks to its persuasive style encourages readers to change old habits to improve welfare and wellbeing. The author's intention is to assess challenging or unhealthy situations, and pragmatically generate a reaction in people, thus fulfilling a beneficial purpose in terms of awareness to the environment, both natural and human. Yet, a closer reading of some extracts of the volume permits to see how the writer's project is still marked by a certain dose of ambiguity, whereby the aim of improving society, and the territory, is not accompanied by a full reflection on how certain measures need to be implemented, bearing in mind that 'development' can be a dense word not necessarily meaning the amelioration of the entire society and the country. The term indeed complicates its semantic (and ideological) weight because it may weave in layers of meaning by hiding "a predatory socio-economic system - global capitalism - that effectively spreads inequality at the same time as it champions its own adherence to freedom, democracy and human rights" (Huggan, Tiffin 2015, 32).

The often monolithic rhetoric of the nation, moreover, seems to illuminate only the presence of upper classes and the rising bourgeoisie, thus further marginalising those peripheral subjects that constitute specific micro-contexts. From this perspective, the celebratory and populist notion of 'awesomeness', similarly to what happens with the expanding power of news media (Nambiar 2014), appropriates echoes of ecological discourse too, but it finally emerges as a driving 
(often acritical) force that may run across unwise practices, whose impact is frequently destructive and affect both material and immaterial life, for example with the disfigurement of the land, the annihilation of autochthonous populations or the increment of pollution with the excuse of more opportunities for people. The risk here is to lose the interconnectedness of fields and domains, or, to put it in Mukherjee's $(2010,15)$ words, "precisely this network of politics, culture, ecology, physical space and non-human matter that we should understand as 'environment'".

In his essays, Bhagat has employed the reader-oriented narrative format to encode possible worlds by setting up particular frames, i.e. attention-getting stories of pride, appeal and redemption that shake off the postcolonial frustration of the global margins to embrace and foreground ambitious targets. Ultimately, the author's persuasive discourse can be interpreted as an idea of social, cultural and environmental renovation, supporting a form of ecosophy, albeit a linguistic and stylistic investigation discloses its limits and partial criticalities.

\section{Bibliography}

Bhagat, C. (2015). Making India Awesome. New Delhi: Rupa.

Burke, M. (2014). "Rhetoric and Poetics. The Classical Heritage of Stylistics". The Routledge Book of Stylistics. Oxon: Routledge, 11-30

Chaudhuri, M. (2010). "Indian Media and its Transformed Public". Contributions to Indian Sociology, 44(1-2), 57-78.

Cockcroft, R.; Cockcroft, S. (2005). Persuading People. An Introduction to Rhetoric. Houndmills: Palgrave Macmillan.

Daftuar, S. (2015). "Easy advice. Review of Make India Awesome". The Hindu. https://www.thehindu.com/books/literary-review/swatidaftuar-reviews-making-india-awesome/article7641323.ece.

DeLoughrey, E.; Didur, J.; Carrigan, A. (2015). “Introduction. A Postcolonial Environmental Humanities". Global Ecologies and the Environmental Humanities. Postcolonial Approaches. Oxon: Routledge, 1-32.

Dhar, S. (2013). "The Fiction of Chetan Bhagat and the Discourse of Motivation". Sen, K.; Rituparna, R. (eds), Writing India Anew: Indian-English Fiction 2000 2010. Benjamin: Amsterdam University Press, 161-9.

Gibbons, A.; Whiteley, S. (2018). Contemporary Stylistics. Language, Cognition, Interpretation. Edinburgh: Edinburgh University Press.

Griffiths, P. (2006). An Introduction to English Semantics and Pragmatics. Edinburgh: Edinburgh University Press.

Heise, U.K. (2008). Sense of Place and Sense of Planet. Oxford: Oxford University Press.

Huggan, G.; Tiffin, H. (2015). Postcolonial Ecocriticism. Literature, Animals, Environment. Oxon: Routledge.

Jeffries, L. (2010). Critical Stylistics. The Power of English. Houndmills: Palgrave Macmillan. 
Kaur, R. (2016). "I Am India Shining': The Investor-Citizen and the Indelible Icon of Good Times". The Journal of South Asian Studies, 75(3), 621-48.

Mukherjee, U.P. (2010). Postcolonial Environments. Nature, Culture and the Contemporary Indian Novel in English. Basingstoke: Palgrave Macmillan.

Nambiar, P. (2014). Media Construction of Environment and Sustainability in India. New Delhi: Sage Publications Pvt. Ltd.

Sailaja, P. (2009). Indian English. Edinburgh: Edinburgh University Press.

Schülting, S. (2016). Dirt in Victorian Literature and Culture. Writing Materiality. Abingdon: Routledge.

Simpson, P.; Mayir, A. (2010). Language and Power. Oxon: Routledge.

Stibbe, A. (2015). Ecolinguistics. Language, Ecology and the Stories we Live by. Abingdon: Routledge.

Varughese, E.D. (2013). Reading New India. Post-Millennial Indian Fiction in English. London: Bloomsbury.

Viswamohan, A.I. (2011). "Marketing Lad Lit, Creating Bestsellers: the Importance of Being Chetan Bhagat". Postliberalization Indian Novels in English: Politics of Global Reception and Awards. London: Anthem Press, 19-30.

Wales, K. (1995). A Dictionary of Stylistics. London: Longman. 


\title{
Decolonizing the Anthropocene: 'Slow Violence' and Indigenous Resistance in Cherie Dimaline's The Marrow Thieves
}

Chiara Xausa

Alma Mater Studiorum, Università di Bologna, Italia

\begin{abstract}
Through a reading of Cherie Dimaline's 2017 young adult novel The Marrow Thieves, a survival story set in a futuristic Canada destroyed by global warming, this article explores the conceptualization and reimagination of the Anthropocene in contemporary postcolonial and Indigenous theory and fiction. Firstly, I will argue that literary representations of climate change can be complicit in producing hegemonic strands of Anthropocene discourse that consider human destructiveness and vulnerability at undifferentiated species level. Secondly, I will suggest that the novel's apocalypse reveals the processes of colonial violence and dispossession that have culminated in the eruptive event of environmental catastrophe, rather than portraying a story of universal and disembodied human threat that conceals oppression against Indigenous people.
\end{abstract}

Keywords Cherie Dimaline. Climate fiction. Indigenous knowledge. Slow violence. Apocalypse.

Summary 1 Decolonizing the 'Anthropos'. - 2A Silencing Apocalypse. - 3 Cherie Dimaline's The Marrow Thieves.

$\begin{array}{lll} & \text { Peer review } \\ & \text { Submitted } & 2020-07-15 \\ \text { Edizioni } & \text { Accepted } & 2020-08-24 \\ \text { Ca'Foscari } & \text { Published } & 2020-12-22 \\ & \text { Open access } & \end{array}$

(ㄷ) 2020 | @(i) Creative Commons Attribution 4.0 International Public License 


\section{Decolonizing the 'Anthropos'}

In the past two decades, climate change and its effects have been articulated in a range of literary works and have especially become major trends in anglophone fiction. Since then, a lot of literary criticism has explored the cultural challenges of writing climate fiction, and the ecopolitical value of environmental literature. Environmental humanities scholars such as Ursula K. Heise, Adam Trexler and Adeline Johns-Putra have been increasingly investigating the main challenges faced by authors of climate fiction, such as the more-thanhuman complexity of climate change, the novel's anthropocentric tendencies, the planetary scale and the slowly unfolding pace of human environmental impact.

This article aims to investigate the extent to which literary imagining has been complicit in determining what counts as crisis, nature, human and planetary, and in producing hegemonic strands of Anthropocene discourse that consider human destructiveness and vulnerability at undifferentiated species level, de-emphasizing moral responsibilities. Through a reading of Cherie Dimaline's Young Adult novel The Marrow Thieves (2017), I will explore how the notion of the Anthropocene is challenged and reimagined in contemporary postcolonial and Indigenous theory ${ }^{1}$ and fiction. I argue that besides casting a new light on the relationship between climate change, colonial and neo-colonial violence and structural inequalities generated by intersecting systems of oppression, Dimaline amplifies the marginalized and forgotten experiences of those who are already experiencing the climate apocalypse.

Before approaching the novel, it will be necessary to introduce the current debate about the Anthropocene and its literary representations. The term Anthropocene, denoting the current geological age characterized by a significant human impact on global ecosystems, was first introduced in 1980 by the American biologist Eugene F. Stoermer, and subsequently popularized in 2000 by atmospheric chemist Paul J. Crutzen, together with Stoermer. The 35 members of the Anthropocene Working Group (AWG), an interdisciplinary research group established in 2009 to investigate, evaluate and critically analyze the still informal geological time unit, have identified July 16 th 1945, the time of the world's first nuclear bomb explosion at Alamogordo, New Mexico, as the possible beginning of the Anthropocene (Zalasiewicz et al, 2014).

1 As argued by Colorado-born citizen of the Cherokee Nation Daniel Heath Justice (Justice 2018, 8), the capital 'I' "affirms a distinctive political status of peoplehood, rather than describing an exploitable commodity, like an "indigenous plant" or a "native mammal". The proper noun affirms the status of a subject with agency, not an object with a particular quality". 
Drawing upon multiple Indigenous scholars who posit that the Anthropocene is a continuation of practices of extraction, dispossession and environmental transformation that started five hundred years ago and are still ongoing, rather than a new event, Heather Davis and Zoe Todd argue that the question of when the Anthropocene began is of the utmost importance and opens up diverse political implications. To begin the project of decolonizing the Anthropocene, they advance a dating of the Anthropocene that goes beyond its current universalizing (read: Eurocentric) framing, and places the 'golden spike' that might mark the advent of the epoch at 1610. Before them, geographers Simon L. Lewis and Mark A. Maslin had suggested that among the various proposed dates two might be described as 'golden spikes' (more precisely, Global Stratographic Section and Points, or GSSPs): 1610 and 1964. While the authors refer to the latter approach as the 'bomb peak', what is more important for the present discussion is that the former is named the 'Orbis' hypothesis, from the Latin for 'world', to indicate the beginning of the modern world system and the globalization of trade that followed the intensive European colonization of the Americas. This period of large decline in human numbers (stirred up by diseases carried by Europeans, war, enslavement and famine) led to the near cessation of farming and reduction in fire use which vastly increased carbon sequestration: such decline of atmospheric carbon dioxide reached its minimum in 1610. According to the authors, what makes 1610 a suitable marker for the Anthropocene is also the "mixing of previously separate biotas, known as the Colombian Exchange" (Simon, Maslin 2015, 174) and the subsequent "geologically unprecedented homogenization of Earth's biota" (Simon, Maslin 2015, 174-5). ${ }^{2}$

One of the most striking contributions of Lewis and Maslin's approach is that it introduces the violent legacies of colonialism into the Anthropocene debate. Similarly, Davis and Todd underscore that proposing a 1610 start date, or placing the 'golden spike' at the beginning of the colonial period, allows us to trace the origins of the current ecological crises in a "proto-capitalist logics based on extraction and accumulation through dispossession" (Davis, Todd 2017, 764), that continue to shape our current epoch. Not only colonialism, particularly settler colonialism, was always about processes of terraforming, but it can also be read as an anticipation of the conditions of the Anthropocene:

2 It should be stressed, though, that members of the AWG have questioned this proposed dating of the Anthropocene: they claim that "1610 is not an ideal stratigraphic marker for an epoch-scale boundary. It is one small dip of several in the Holocene epoch" and that "by the time of the authors' other suggested date of 1964, the 'great acceleration' in human activity was well under way" (Zalasiewicz et al., 2015, 436). 
The forced displacement that many tribal communities suffered involved adaptation to entirely new environments, to new climates, new ecosystems, new plants and animals. These processes of environmental transformation and forced displacement can be understood as climate change, or more broadly, a preview of what it is like to live under the conditions of the Anthropocene. (Davis, Todd 2017, 771)

Further, by casting a new light on the continuities between colonial exploitation and climate change, the authors question and challenge the depoliticizing universalism intrinsic in the term Anthropocene. The 'Anthropos' after which geologists have named the current epoch does not seem to have a class, a race, a gender and, by inviting us to think at undifferentiated species level, runs the risk of erasing power hierarchies. As such, this universalizing logic has led many scholars to prefer more revealing terms (such as Capitalocene [Haraway 2015; Moore 2015; Malm 2016], Eurocene [Grove 2016] or White Supremacy Scene [Mirzoeff 2016]) ${ }^{3}$ over Anthropocene. As postcolonial ecocritic Rob Nixon (2011) suggests, both human responsibility for climate change and vulnerability to environmental harm are 'unevenly universal'. Postcolonial, decolonial and critical race studies join hands with ecofeminism(s) to posit that the dichotomy between humans and nonhumans is developed alongside racial and gendered hierarchies of difference (Gergan et al. 2018) and to challenge the 'racial blindness' (Yusoff 2018) and gender blindness of the universal human subject (read: white maleness) implied in the concept of the Anthropocene.

\section{A Silencing Apocalypse}

As noted by Susan Watkins, contemporary white male-authored postapocalyptic fiction "tends towards conservatism" (Watkins 2020,1) and a desire and longing for the confirmation of the status quo. Ideas of human civilization rely on traditional patriarchal and imperialist values and gesture towards a future that is either a "restoration of what has been lost during the apocalypse" or a "nostalgic mourning for the past" (Watkins 2020, 1). Conventional post-apocalyptic imagination cannot seem to move beyond traditional gender narratives (namely: the protection of the heteronormative nuclear family unit and the obsession with the father-son bond). Similarly, post-apocalyptic climate fiction tends to revolve around men: the protagonists with decision mak-

3 "Given that the Anthropos in Anthropocene turns out to be our old friend the (imperialist) white male, my mantra has become, it's not the Anthropocene, it's the white supremacy scene" (Mirzoeff 2016, 123). 
ing authorities are often white male heroes, mainly research scientists and government officials. Power and agency are divided along racial, ethnic and gender lines, and women are a silent backdrop and a site of innate vulnerability (Gaard, 2017; Mcgreavy, Lindenfeld, 2014). The most prominent examples are Cormac McCarthy's The Road (2006) and Roland Emmerich's The Day After Tomorrow (2004). ${ }^{4}$

If the "paradigmatic figure of the Anthropocene is the European or Western white male scientist", as Andrew Baldwin (2017) suggests quoting Yusoff $(2018,218)$, then climate fiction becomes a useful entry point for conceptualizing the meaning of the Anthropocene. The theme of climate justice is almost entirely absent from mainstream cultural representations of climate change; those whose agency is most constrained, however, are also the most vulnerable to the violence of climate shift, as well as the main victims.

Hsu and Yazell (2019) term 'structural appropriation' the process in which mainstream post-apocalyptic climate fiction projects into white American characters and readers the structural violence of climate catastrophe that has already been experienced by colonized, postcolonial and Indigenous populations. These future scenarios are often inhabited by small - and sometimes elitist - groups of survivors struggling to regenerate US culture and society, both endangered by a "third-worlding the West as a result of apocalyptic social collapse" (Hsu, Yazell 350). If, as Lawrence Buell has suggested, the "[a]pocalypse is the single most powerful master metaphor that the environmental imagination has at its disposal" (Buell 1995 285), analyzing what these narratives conceal and obscure instead of revealing is an urgent task. Indeed,

rather than exploring environmental apocalypses that have already happened to populations outside the US (or to sovereign Indigenous nations putatively located "within" the US), post-apocalyptic fiction re-inscribes colonial and racial logics in imagined futures that, in many cases, have been unmoored from histories of race and empire. (Hsu, Yazell 2019, 349)

4 Conversely, many contemporary YA dystopian novels feature strong young women as central characters and attempt to deconstruct the idea of women as victims. When it comes to racial tensions, however, these novels are ambivalent at best and tend to perpetuate the hegemonic status quo, as highlighted by Mary J. Couzelis in The Future is Pale. Race in Contemporary Young Adult Dystopian Novels. Couzelis analyzes three contemporary dystopian novels recommended by the American Library Association's list for the best young adult books (Lois Lowry's The Giver [1993], Scott Westerfeld's Uglies [2005], and Suzanne Collins's The Hunger Games [2008]), and observes that they "ignore race or present a monochromatic future implying that other ethnicities do not survive in the future or that their participation in the future is not important" (Couzelis 2013, 131). 
In these narratives of apocalypse climate change is portrayed as a universal human threat that is experienced by a universal human subject (read: white Western man), silencing the manifold vulnerabilities to environmental catastrophes that have been conceptualized by global climate justice movements. The authors linger over a significant episode staged in Cormac McCarthy's The Road (2006), when the father and son protagonists of the novel come across an old plantation house that has become a cannibal shelter. At first glance, this allusion seems to reveal that the novel is attempting to shed light on the structural violence of plantation slavery and thus explore the continuities between past, present and future apocalypse; however, Hsu and Yazell contend that this scene only serves to create a negative model for humanity that contrasts with the two protagonists. As noted by Eddie Yuen, what renders this language of catastrophe problematic is its being

'apocalyptic' only in the Hollywood sense: [...] devoid of ethical content. It says nothing of who we are and where we are going. (Yuen 2012, 678)

Besides being written from a universal and un-embodied position, stories about apocalyptic environmental disasters can be very escapist: they give the reader the illusion that a techno-science approach will solve the problems of climate change without addressing social injustices and without a radical change in the way we live and we consume. There is an urgent need for utopian visions of the future that do not focus on the advances of Western technology and economics but rather recognize the disparities accentuated by climate change and imagine hopeful futures where no one (human and non-human) is left behind.

With regard to narratives of ultimate destruction, fear caused by implausible stories struggles to galvanize action in response to climate change. As Stephanie LeMenager (2017) suggests, much of the climate fiction of Europe, white America, Britain and Scandinavia is indeed concerned with what Roy Scranton has termed 'learning to die' in the Anthropocene: according to Scranton one of the main challenges of the Anthropocene is learning to die not as individuals, but as a civilization, because it is too late to imagine effective responses to the challenge of living in the Anthropocene.

But who is learning to die as a civilization? As the Potawatomi philosopher Kyle Whyte makes clear in Indigenous Science (Fiction) for the Anthropocene: Ancestral Dystopias and Fantasies of Climate Change Crises, narratives of apocalypse that project climate crisis in "horrific science fiction scenarios" (Whyte 2018, 225) obscure ongoing oppression against Indigenous people, and conceal their perspectives on the continuities between colonial violence and climate change. "Having endured one or many more apocalypses" (236) and having suffered the most severe hardship arising from environmental transformation 
due to different forms of colonialism, most Indigenous people live in a present which is already dystopian. Among such dreadful transformations Whyte mentions "ecosystem collapse, species loss, economic crash, drastic relocation, and cultural disintegration" (226) and a disrupted relationship with the nonhuman. A key feature of what the philosopher has defined "living Indigenous science fiction" (230) is the contrast between a spiraling time of constant change and ongoing crisis - which sheds light on the role of colonial dispossession in environmental transformation - on the one hand, and linear narratives of upcoming crisis and dire futures of climate change on the other.

\section{Cherie Dimaline's The Marrow Thieves}

The Marrow Thieves, a Young Adult novel published in 2017 by Métis writer, activist and member of the Georgian Bay Métis Community in Ontario Cherie Dimaline, is a survival story set in a futuristic Canada destroyed by global warming. In this age of "rising waters, tectonic shifts, and constant rains" (Dimaline 2017a, 26) white people have lost the ability to dream as a result of environmental trauma. The novel makes clear that at the foundation of this ecological crisis does not lie a hostile nature that is allegedly retaliating against human beings, but rather a neocolonialist and capitalist logic, its consumptive force and its turning of habitats, environments and bodies into resources to be subjugated, transformed and exploited. To save themselves from the water they have poisoned and from the air they have polluted so much that "the earth shook and melted and crumbled" (47), settlers turn to Indigenous people for salvation. North America's Indigenous People are being hunted for their bone marrow, that could restore the dreams of the rest of the world:

Dreams get caught in the webs woven in your bones. That's where they live, in that marrow there. (18)

The Marrow Thieves' protagonist and first-person narrator is Frenchie, a fifteen-year-old fictional character from Cherie Dimaline's community, as the writer confesses in an interview (Dimaline 2017b). Early in the novel, he escapes capture while his brother falls into the hands of government's Recruiters, hunting Indigenous people to bring them to marrow-stealing 'factories'. As he flees the Recruiters, he is rescued from Miig, a middle-aged Anishinaabe men, who invites him to join an intergenerational group of Indigenous people also seeking safety. Together, they struggle for survival, attempt to reunite with their loved ones, and take refuge form the recruiters.

Miig is the keeper of the so-called 'Story', the account of the events that led to the post-apocalyptic world. As much of the importance of 
the novel lies in the articulation of stories that keep culture intact, I quote this 'Story' at length:

Anishnaabe people, us, lived on these lands for a thousand years. [...] We welcomed visitors, who renamed the land Canada. [...] We lost a lot. Mostly because we got sick with new germs. And then when we were on our knees with fever and pukes, they decided they liked us there, on our knees. And that's when they opened the first schools. We almost lost our languages. [...]

Then, the wars for the water came. America reached up and started sipping on our lakes. And where were the freshest lakes and the cleanest rivers? On our lands, of course. Anishnaabe were always the canary in the mine for the rest of them. Too bad the country was busy worrying about how we didn't pay an extra tax on Levi's jeans and Kit Kat bars to listen to what we were shouting. [...] The water wars raged on, moving north seeking our rivers and bays, and eventually, once our homelands were decimated and the water leeched and the people scattered, they moved on to the towns. [...] The water Wars lasted ten years before a new set of treaties and agreements were shook on between world leaders in echoing assembly halls. The Anishnaabe were scattered, lonely, and scared. On our knees again, only this time there was no home to regroup at. (Dimaline 2017a, 26)

What this passage implies is a strong continuity between the legacies of residential schools and past exploitation on the one hand, and a postapocalyptic and post-climate change future on the other. ${ }^{5}$ Far from being implausible and disconnected from the material reality, Dimaline's dystopian world sheds light on the ongoing repetition of past and current traumas. By putting the story of residential schools through the lens of future, and through the lens of climate change, the novel's apocalypse reveals the processes of colonial violence and dispossession that have culminated in the eruptive event of environmental catastrophe, rather than portraying a story of universal and dis-embodied human threat that conceals oppression against Indigenous people. Further, Frenchie and the other members of the group do not respond to the dangers posed by climate change by 'learning to die' in the Anthropocene: they have already experienced such threat of loss - loss of

5 Residential schools were government-sponsored boarding schools established to assimilate Indigenous children into Euro-Canadian culture by adopting Christianity and speaking English or French, eradicating all aspects of Indigenous cultural and spiritual identity. The residential school system operated in Canada from 1876 to late 1990s, although the origins can be traced to as early as the 1830s. It is estimated (Miller 2012) that 150,000 First Nation, Inuit and Métis children attended residential schools, while the number of school-related deaths remains unknown (as many as 6,000 children may have died). 
culture, loss of language, loss of lands. The story moves perhaps more slowly than other post-apocalyptic narratives on climate change, particularly in the YA field, but I read this slowness as a strategy to enlighten what Rob Nixon has termed the 'slow violence' of climate change, which is "neither spectacular nor instantaneous, but rather incremental" and "occurs gradually and out of sight" (Nixon 2011, 2). The main casualties of slow violence are the unseen 'poor' (hence the title Slow Violence and the Environmentalism of the Poor) lacking resources. Nixon brings together postcolonial studies, ecocriticism and literary studies to address the representation of climate crises in an age when the media often chooses the instant sensational event over the long-term effects of disasters that are "anonymous and star nobody" (3).

Besides underscoring the links between colonization and the Anthropocene, the novel voices a more contemporary threat to land, language, culture and identity that might be increased by current vulnerabilities to climate change. Once again, this mirrors the history of settler colonialism in the Americas. In a future devastated by extreme weather events a huge part of the population would be displaced, and the so-called uninhabited traditional territory would be the first space to be reclaimed by the dominant society (Dimaline 2017b). As Miig recounts, indeed, after the advent of climate crises the Indigenous people of North America were removed from lands that "were deemed 'necessary' to the government, same way they took reserve land during wartime" (Dimaline 2017a, 88). Like land, Indigenous culture is under serious threat: when settlers find out that Indigenous marrow holds the cure for the rest of the world, their initial openness and real interest towards Indigenous forms of knowledge soon turns into appropriation and commodification:

At first, people turned to Indigenous people the way the New Agers had, all reverence and curiosity, looking for ways we could help guide them. They asked to come to ceremony. [...] And then they changed on us, [...] looking for ways they could take what we had and administer it themselves. How could they best appropriate the uncanny ability we kept to dream? (88)

After having asked for volunteers, they turned to history and built new residential schools where the marrow was forcibly extracted. I propose to read Indigenous ability to dream in spite of environmental threat as an allegory of Indigenous knowledge on climate change: a potential resource to be exploited and decontextualized instead of allowing Indigenous communities to make meaningful and contributions.

Kinship relations too are an explicit target of attack. As Coloradoborn citizen of the Cherokee Nation Daniel Heath Justice underscores in Why Indigenous Literatures Matter, one of the fundamental purposes of residential schools was to "dismantle Indigenous resistance 
through a direct, sustained attack on families and the full network of relations and practices that enabled health and self-determination" (Justice 2018, 85). Marrow-stealing schools function in similar ways: every member of the group's connections to family members has been broken by government's Recruiters, with devastating results for all of them. Frenchie has first lost his father, then his mother, last of all his brother; Miig has lost his husband Isaac; Minerva, the elder of the group, was feeding her new grandson when the Recruiters "busted into her home, took the baby, and raped her" (Dimaline 2017a, 98). Bodies too, indeed, are sites of struggle, especially women's bodies. The chronological order of the novel is sometimes interrupted by a number of flashbacks or 'coming-to-stories', in which some characters of the group share the circumstances that led to their separation from their communities. Besides Minerva, we get to know the background story of Wab, an eighteen-year-old girl who is also a rape survivor. The rape profoundly debilitates her body, to the point that she gives up running, having previously depended on her strong legs to survive and earn a living as a messenger. Through these female characters, referred as "the dissenting voice to the way things are" (32), the novel stresses that bodies can carry indelible marks of a violence caused by intersecting forms of oppressions: racial, colonial and gender violence, but also the violence of climate shift.

It would be tempting to read The Marrow Thieves as a bleak story of dispossession and climate catastrophe; what needs to be stressed, however, is that Dimaline's authorial focus lies in subverting toxic colonial stories about Indigenous people to voice persistence and survival. In an interview with Publishing Perspectives, talking about the Métis Nation on the Georgian Bay, forcibly removed from Drummond Island, she explains that

removals and relocations of a culture are specific to my community, although experienced in different ways by all Indigenous people. It's part of our stories. And it's a huge piece of why we share stories and keep that history intact, just as we've kept our culture intact. [...] My community has struggled and survived, and I'm enormously proud to be able to carry our voices forward. (Dimaline 2017d)

Similarly, Justice (2018) stresses the importance of stories of "that which continues, that which remains" (56), stories about the "now" that subvert dominant colonial narratives seeing Indigenous people as disappearing historical artifacts. North American Indigenous people are more that descendants of those who survived the apocalypse: they are "survivors, too" (5) of the apocalypse of colonization and environmental transformation that continues today. Furthermore, I argue that Dimaline's choice to propose a story about the 'now' of climate change helps readers to deconstruct dominant apocalyptic 
narratives concealing, obscuring and appropriating the structural violence of environmental crises that has already been experienced by colonized, postcolonial and Indigenous populations.

The Marrow Thieves is a healing story of survival where Indigenous youth are able to see themselves in the future (Dimaline 2017b). By the end of the novel, Frenchie and the other members of the group are still trying to escape from the Recruiters and learning to coexist with climate change and a drastically altered environment. As such, the novel does not propose a "naïve story of hard effort overcoming all struggles" (Justice 2018, 137); instead, it was

hard, desperate work. We had to be careful we weren't making things up, half remembered, half dreamed. We felt inadequate. We felt hollow in places and at certain hours we didn't have names for in our languages. (Dimaline 2017a, 214)

Yet they keep resisting because they have each other ("we were still hopeful. Because we had each other. New communities to form" 88), because they establish new forms of kinship and relationality that rebuild what settler colonialism has mutilated. As Justice suggests, it is telling that near the novel's end Frenchie uses the word 'family' to describe his small group of fellow Indigenous runaways. In this context, the term serves to contrast colonial notions of 'Indian blood' - figured here as bone marrow - used by settler governments to define who is 'Indian', control access to Indigenous land, create standards of Indigenous authenticity and claim belonging without Indigenous kinship, as established by the Indian Act, first introduced in 1876. It is through resistant kinship practices which include inter-generational dialogue that the protagonists step towards a more hopeful future. As opposed to more mainstream YA narratives about climate change that focus on inter-generational conflicts and raise ethical questions about inter-generational responsibility (see The Carbon Diaries 2015 by Saci Lloyd), in The Marrow Thieves the relationship between ancestor and future generations strengthens the struggles of the present while providing "secret sources of agency" that empower protagonists and help them "survive the dystopia" (Whyte 2017, 231). We could think of relationship - with the human community, with ancestors, but also with the land and the more-than-human world - as the driving force of the novel. As Miig states near the novel's conclusion,

we can start healing the land. We have the knowledge, kept through the first round of these blasted schools, from before that, when these visitors first make their way over here like angry children throwing tantrums. When we heal our land, we are healed also. (Dimaline 2017a, 193) 


\section{Bibliography}

Baldwin, A. (2017). "Rearranging Desire: On Whiteness and Heteronormativity". Baldwin, A.; Bettini, G. (eds), Life Adrift. Climate Change, Migration, Critique. London: Rowman \& Littlefield International, Ltd.

Buell, L. (1955). The Environmental Imagination: Thoreau, Nature Writing, and the Formation of American Culture. Cambridge: Harvard University Press.

Chakrabarty, D. (2009). "The Climate of History. Four Theses". Critical Inquiry, 35(2), 197-222.

Clark, T. (2015). Ecocriticism on the Edge: The Anthropocene as a Threshold Concept. New York: Bloosmbury Academic.

Couzelis, M.J. (2013). "The Future Is Pale: Race in Contemporary Young Adult Dystopian Novels". Basu, B. et al. (eds), Contemporary Dystopian Fiction for Young Adults: Brave New Teenagers. Abingdon: Routledge, 131-44.

Davis, H.; Todd, Z. (2017). "On the Importance of a Date, or, Decolonizing the Anthropocene". ACME: An International Journal for Critical Geographies, 16(4), 761-80.

Dillon, G. (ed.) (2012). Walking the Clouds: An Anthology of Indigenous Science Fiction. Tucson: University of Arizona Press.

Dimaline, C. (2017a). The Marrow Thieves. Toronto: Cormorant Books Inc.

Dimaline, C. (2017b). "How Cherie Dimaline Found Hope in a Dystopian Future". CBC Radio, October 2. https://bit.ly/39J64IN.

Dimaline, C. (2017c). "Cherie Dimaline: Hopes and Dreams in the Apocalypse". Interviewed by Deborah Dundas. The Star. November 6. https://www. thestar.com/entertainment/books/2017/11/06/cherie-dimaline-hopes-and-dreams-in-the-apocalypse.html.

Dimaline, C. (2017d). "Indigenous Writers in Canada: Interview with Author Cherie Dimaline”. Interviewed by Carla Douglas. Publishing Perspectives. November 3. https://publishingperspectives.com/2017/11/indigenous-writers-canada-interview-author-cherie-dimaline/.

Gaard, G. (2017). Critical Ecofeminism. London: Lexington Books.

Gergan, M. et al. (2018). "Earth beyond Repair: Race and Apocalypse in Collective Imagination". Environment and Planning D: Society and Space, 38(1), 91-110.

Goodbody, A.; Johns-Putra, A. (eds) (2018). Cli-fi: A Companion. Oxford; New York: Peter Lang.

Grove, J. (2016). "The New Nature". Boston Review, January 1. http://bostonreview.net/forum/new-nature/jairus-grove-jairus-groveresponse-jedediah-purdy.

Haraway, D. (2015). "Anthropocene, Capitalocene, Plantationocene, Chthulucene: Making Kin”. Environmental Humanities, 6(1), 159-65.

Huggan, G.; Tiffin, H. (eds) (2010). Postcolonial Ecocriticism: Literature, Animals, Environment. New York: Routledge.

Hsu, H.L.; Yazell, B. (2019). "Post-Apocalyptic Geographies and Structural Appropriation". Morgan, N. et al. (eds), Routledge Companion to Transnational American Studies. New York: Routledge.

Justice, D.H. (2018). Why Indigenous Literatures Matter. Waterloo: Wilfrid Laurier University Press. Kindle Edition.

LeMenager, S. (2017). "Climate Change and the Struggle for Genre". Menely, T.; Taylor, J.O. (eds), Anthropocene Reading: Literary History in Geologic Times. University Park (PA): The Pennsylvania State University Press, 220-38. 
Lewis, S.L.; Maslin, M.A. (2015). "Defining the Anthropocene". Nature, 519, 17180. https://doi.org/10.1038/nature14258.

Malm, A. (2016). Fossil Capital. London: Verso.

McGrevy, B.; Lindenfeld, L. (2014). "Entertaining Our Way to Engagement? Climate Change Films and Sustainable Development Values". International Journal of Sustainable Development, 17(2), 123-36.

Miller J.R. (2012). "Residential Schools in Canada". The Canadian Encyclopedia, October 10. https://www.thecanadianencyclopedia.ca/en/article/residential-schools.

Mirzoeff, N. (2016). "It's Not the Anthropocene, It's the White Supremacy Scene, Or, The Geological Color Line". Grusin, R. (ed.), After Extinction. Minneapolis: University of Minnesota Press, 123-50.

Moore, J. (2015). Capitalism in the Web of Life: Ecology and the Accumulation of Capital. New York: Verso.

Nixon, R. (2011). Slow Violence and the Environmentalism of the Poor. Boston: Harvard University Press.

Streeby, S. (2018). Imagining the Future of Climate Change: World Making through Science Fiction and Activism. Oakland: University of California Press.

Trexler, A. (2015). Anthropocene Fictions: The Novel in a Time of Climate Change. Charlottesville: University of Virginia Press.

Watkins S. (2020). "Introduction: Rewriting and Transforming Traditions". Contemporary Women's Post-Apocalyptic Fiction. Palgrave Studies in Contemporary Women's Writing. London: Palgrave Macmillan, 1-40. https://doi. org/10.1057/978-1-137-48650-9_1.

Whyte, K.P. (2017). "Our Ancestors' Dystopia Now: Indigenous Conservation and the Anthropocene". Heise, U. et al. (eds), The Routledge Companion to the Environmental Humanities. New York: Routledge, 208-15.

Whyte, K.P. (2018). "Indigenous Science (Fiction) for the Anthropocene: Ancestral Dystopias and Fantasies of Climate Change Crises". Environment and Planning E: Nature and Space, 1(1-2), 224-42.

Yuen, E. (2012). "The Politics of Failure Have Failed: The Environmental Movement and Catastrophism". Lilley, S. et al. (eds), Catastrophism: The Apocalyptic Politics of Collapse and Rebirth. PM Press. Kindle Edition.

Yusoff, K. (2018). A Billion Black Anthropocenes or None. Minneapolis: University of Minnesota Press.

Zalasiewicz, J. et al. (2014). "When Did the Anthropocene Begin? A Mid-Twentieth Century Boundary Level Is Stratigraphically Optimal”. Quaternary International, 383, 1-8.

Zalasiewicz, J. et al. (2015). "Disputed Start Dates for the Anthropocene". Nature, 520, 436 . 



\section{Ha Jin's Ecocritical Irony in the Stylistic Analysis of the Personal Pronouns in "A Tiger Fighter is Hard to Find"}

Isabella Marinaro

Sapienza Università di Roma, Italia

Abstract This paper aims at showing how stylistics and ecocriticism, both being strongly 'centripetal' and 'interdisciplinary' fields, can cooperate in the exploration of a literary text. This is the case of a short story written by the Chinese-American writer $\mathrm{Ha}$ Jin. Also thanks to the help of the digital analysis, the paper mainly focuses on the stylistic analysis of the personal pronouns and of the narrator's point of view to show the stinging and subversive irony which pervades the narration of the vicissitudes of a Chinese TV film crew who is asked to shoot a scene where a muscular hero has to fight bare-handed against a real Siberian tiger. The analysis also aims to show how the story becomes the opportunity for the writer to depict the narrow frame of mind of the blind dictatorship of his homeland and its deplorable consequences on both humans and animals. Thus, the whole exploration, in the end, reveals the ecocritical lens through which Ha Jin narrates the story.

Keywords Ha Jin. Personal pronouns. Ecocriticism. Irony. Digital stylistics.

Summary 1 Introduction. -2 Brief presentation of the Author. -3 Summary of the Short Story. - 4 Analysis: Introductory Considerations. - 4.1 Stylistic Analysis of the Personal Pronouns and the Narrator's Point of View. -5 Conclusion.

$\begin{array}{lll} & \text { Peer review } \\ & \text { Submitted } & 2020-06-30 \\ \text { Edizioni } & \text { Accepted } & 2020-09-19 \\ \text { Ca'Foscari } & \text { Published } & 2020-12-22 \\ & \text { Open access } & \end{array}$

(ㄷ) 2020 | @(i) Creative Commons Attribution 4.0 International Public License 


\section{Introduction}

In his attempt to describe ecocriticism, ${ }^{1}$ Warren argues that this discipline is particularly strong when it becomes 'interdisciplinary': "interdisciplinary focus suggests that new ways of knowing can emerge from disciplinary crossing" (Warren 2010, 771-2). In point of fact, the very nature of ecocriticism suggests that this discipline has to be interdisciplinary, especially when considering its definition: "the study of the relationship between literature and the physical environment" (Glotfelty, Fromm, cit. in Garrard 2016, 61). Warren further reinforces his argument by adding that "the ecocritical impulse reaches into a host of areas, each of them vibrant with new knowledge, new ideas" (Warren 2010, 772).

Such "interdisciplinary focus" seems to be in agreement with contemporary stylistics, as the predominant characteristic of stylistics is that it is a "bridge discipline" (Leech [2008] 2013, 2), that is to say a discipline whose nature is strongly interdisciplinary in its foundations and in its applications (Montini 2020, 9). ${ }^{2}$ Given that "stylistics is the study of style" (Montini 2020,17) and style is "the way in which language is used in a given context, by a given person, for a given purpose and so on" (Leech, Short [1981] 2007, 1), the interdisciplinarity of contemporary stylistics is an epistemological strong point, especially when taking into account that one of its main objectives is "to define the meaning of a text and its interpretation" (Montini 2020, 11). Therefore, the multiplicity of approaches will provide a more accurate interpretation of a text which a singular perspective cannot ensure (Montini 2020, 11). ${ }^{3}$ Thus, what ecocriticism and stylistics have in common is their openness to other disciplines, approaches and knowledge.

In this article, an attempt has been made to carry out a stylistic analysis of a short story titled "A Tiger Fighter is Hard to Find", ${ }^{4}$ written by the Chinese American writer Ha Jin, in order to provide an ecocritical interpretation of this text. The objective of this paper is to re-

1 See the following articles and books for a more complete outline of ecocriticism: Garrard 2004; Gifford 2008; Corti 2013; D’Arcy Wood 2013; Oppemann 2014. Suggested reading on stylistics: Stockwell 2005; Burke 2014; Stockwell, Whiteley 2014; Sotirova 2016; Montini 2017, 2020.

2 "La stilistica inglese contemporanea si presenta innanzitutto secondo un'impostazione fortemente interdisciplinare nel suo statuto e nelle sue applicazioni" (Montini 2020, 9).

3 “Questo aspetto, però, anziché essere visto come una debolezza epistemologica, ne è un significativo punto di forza dal momento che, se uno dei principali obiettivi è definire il significato di un testo e la sua interpretazione, la molteplicità degli approcci servirà a comporre una 'verità' sul testo che una prospettiva unica non potrà garantire" (Montini 2020, 11).

4 Ha Jin 2001. 
veal what linguistic tools are employed by the writer in order to voice his sharp criticism of Chinese political narrow-mindedness. Special attention will be paid to the stylistic analysis of the most recurrent personal pronouns used in the short story. Moreover, apart from a political criticism against a specific regime which, as is shown in the story, is the very origin of all evils, this article aims at showing how certain aspects of Ha Jin's language contribute to an ecocritical critique. Ha Jin's political denunciation is also voiced through his ironic descriptions of clumsy attempts of a Chinese TV crew asked to shoot a muscular actor in the heinous act of fighting bare-handed a tiger.

Before entering the core of the analysis, a short presentation of the author and a summary of the short story with some introductory considerations about the symbolical importance of the tiger in Chinese traditional culture will be provided. Then, a stylistic analysis of the personal pronouns employed by Ha Jin, in particular those referring to the TV crew and the tiger will be proposed, as well as the narrator's point of view will be analysed. The stylistic analysis will be carried out with the aid of AntConc, a free text analysis application, particularly useful in data-driven analysis of text and key words. In the final part of this article, attention will be drawn to $\mathrm{Ha}$ Jin's use of irony in his short story when he presents the unfortunate consequences of dictatorship, specifically the objectification and victimisation of animals and humans. The acts of torturing and killing the tiger to fulfil the order from the local political institution in the name of the Chinese Communist Propaganda is seen as an ecocritical reading which reveals the distorted mechanisms at the basis of a solely anthropocentric view. Such reading is reinforced by the contribution of Derrida's philosophy which criticises the Western anthropocentric view according to which animals are objectified by humans.

\section{Brief presentation of the Author}

Ha Jin is the pen name of Jin Xuĕfēi, meaning 'Snow Flying'. He is a Chinese writer in English whose language has often been labelled as ironic, mostly for the effects produced by the literal translation of Chinese expressions into English with the consequent effect of plunging readers into the reality of some aspects of Chinese culture which he criticizes. It is typical of Ha Jin's style to translate Chinese metaphors, idiomatic expressions and toponyms to create an idiosyncratic narration which allows readers to actively perceive the feelings of his characters and understand their habits (Ibáñez 2016). Not always has his peculiar English been received in a positive way, since some critics define it as "crippled", or "shaky", (Messud 2000, Garner 2000, Tsai 2005) just for the same reasons for which he has been applauded as a brilliant example of bilingual creativity, or translation literature, 
or, in other words, as one of the many expressions of literature currently appearing in global English (Ibáñez 2016; Haomin Gong 2014).

\section{Summary of the Short Story}

This story is part of a collection of twelve short stories titled The Bridegroom, all narrating, as most of Ha Jin's work, private happenings of common Chinese people. All the stories of the collection take place short before the end of the twentieth century, thus in a postCultural Revolution China. This book came out after his winning of the 1999 National Book Award for the novel Waiting (Vintage, 1999).

The story takes place in 1981 in a province of Northeast China and narrates the vicissitudes of a Chinese TV film crew who are explicitly asked to shoot a film where a renowned muscular actor, called Wang Huping and nicknamed Prince for his uncontested allure, has to fight bare-handed against a tiger. The real reason for this request, according to the political authorities, is to provide the audience with the "role model" "for the revolutionary masses to follow" (Ha Jin 2001,54 ) by recalling Wu Song's legendary deeds. ${ }^{5}$ Wang Huping, in particular, soon becomes obsessed with the task of showing his virile strength by killing a tiger just like the ancient hero did (Liangyan Ge 2006, 40).

Everything starts when the director receives this command in a letter sent by the provincial governor's office. He is immediately puzzled as to how to accomplish the task of "strengthen[ing] people's hearts and instill[ing] into them the spirit that fears neither heaven nor earth" (Ha Jin 2001, 54).

The whole story is pervaded by the writer's irony. In a fictional context, irony comes "from the contrast in values associated with two different points of view" (Leech, Short [1981] 2007, 223); in particular, it may take place when the contrast is between an implied point of view stated in the narration and the one of the writer. The initial situation revolves around a sense of duty felt by the director and crew which prompts them to obey that order and the actual impossibility of complying with it given the evident unequal balance of strengths between a tiger and a man.

Despite the fact that the tiger is a protected endangered species, the crew succeed in catching one enormous specimen thanks to a grant from the local administration office. The actor is scared, but,

$5 \mathrm{Wu}$ Song is the hero of a sixteenth century Chinese novel titled Shuihu zhuan recounting the adventures of one hundred and eight bandit heroes in the twelfth century. In the initial part of the novel, Wu Song succeeds in killing a tiger with his bare hands thus showing his virility and heroism (Liangyan Ge, 2006, 39-43). 
nonetheless, the director convinces him to face the wild beast. The scene of the combat has to be necessarily shot in one take, and it requires two men with tranquillizer guns and a jar of liquor for Wang Huping, who is also given a cudgel. The tiger and Prince are soon involved in a ridiculous fight, revolving around cudgel blows and the tiger springing around. The actor manages to hit the animal only when it collapses under the influence of the tranquillizer.

Of course, the director is not satisfied with the shooting, but Wang Huping is nonetheless exulting convinced that he has killed it with his bare hands: "I killed him! I killed him!" (Ha Jin 2001, 58).

He is so hot-headed that he continues babbling about killing the tiger as the crew doctor gives him a sedative before clutching him, dragging him away and sending him to hospital for his frenzied comments. Despite the actor's mental state, a second shooting is done, but unsuccessfully.

The crew come to the conclusion that what is actually wrong is not the animal, but the actor and the only possible solution is to shoot the scene with a fake tiger, that is to say, someone of the crew wearing the skin of the tiger. The problem at this point is to kill a protected animal, but the director finds a way to do it after talking to a friend of his in the local administration. A soldier is called to kill the tiger without damaging its skin. Pieces of the animal are sold on the black market which supplies Chinese traditional medicine.

Meanwhile, Wang Huping is excited at the idea of fighting against a fake animal, without realising that there is no bravery in doing that. On the way to the set, Prince grins at the crew, gnashes his teeth and makes hissing sounds through his nose, while his eyes show ferocity. Seeing him in such state, nobody in the crew is available to shoot the combat scene against him.

Hoping to find a volunteer, the director offers an opulent eightcourse dinner as a reward in a renowned restaurant. The crew truck driver, called Little Dou, accepts and wears the tiger's skin while Prince is invited to attack, but, of course, without the cudgel. Prince punches the truck driver-tiger incessantly while pitiful requests for mercy are coming from underneath the tiger's skin. Even though the fake tiger is motionless, Wang Huping goes on kicking its flanks, neck, head: "Kill this paper tiger! I'm going to finish him off!" (67). When the fight is over, he shouts out: "I killed another tiger! I'm a real tiger-fighter!" (67). Little Dou under the tiger's skin is taken to hospital wounded and unconscious.

The film production is completed at last, broadcast by some TV stations in the Northeast and the crew are promised a financial bonus in case the film receives an award.

The story ends with both the truck driver and Prince still hospitalized, but the one who actually seems to have suffered irrecoverable damage is Prince, who is sent to a psychiatric hospital for good. 
While paying a visit to him, the narrator finds Prince's hands full of scars caused by punching sandbags. He tells Prince that there are over three hundred messages for him, but he does not specify that most of them are written by girls who are still in love with him and that he has been sent chocolates, raisins, fountain pens, and "even photos of themselves" (70). The narrator is puzzled by the whole story and wonders: "How come when a man becomes a poor wretch he's all the more splendid to the public?" (70). Prince will end his life deeply convinced, "like an imbecile" (70), that he is a real tiger-fighter.

\section{Analysis: Introductory Considerations}

The plot shows scenes of comicality, due to the evident irony of the writer when depicting the clumsy attempts of the crew to fulfil the absurd order from the local political institution issued in the name of a chauvinistic political attitude. Beyond a political attack against the Chinese Communist narrow-mindedness, there is also a clear ecocritical approach which shows the ill-fated consequences of violation against nature, embodied in the heinous act of killing the tiger to make the filming of the scene easier for the muscular actor.

In fact, the end of the story is bitter because none of the directly involved characters ends up happy. This is inevitable as the tiger is killed, and thus nature is violated. With a slight hint of comicality, Ha Jin shows that there is a sort of nemesis for this act of violation: the muscular actor, Wang Huping-Prince, is permanently hospitalized in a psychiatric ward. He is ridiculed by the narrator, who depicts him as an imbecile convinced of being a hero, a tiger-fighter. The truck driver, Little Dou, who has accepted to play the tiger only in exchange for an opulent lunch, needs a long hospitalization. Moreover, readers are not even told if the TV film wins an award or not.

The real victim is the tiger, a majestic wild male Siberian specimen, the real prince of nature, who is first encaged, then tortured, and killed in the end. The crew, a synecdochical referent for humans, ends up as proper nature exploiters: not only do they capture, torture and kill the tiger, but, appallingly enough, trade its body parts on the black market. All this in the name of an absurd order framed in the Communist propaganda of those years, whose aim is to convince people of the rightness of a narrow-minded political conviction.

Wang Huping ends up in a psychiatric ward, where he pathetically goes on practising kung-fu. The film is probably not going to win any award. This is an inevitable ending of this story, especially if we contextualise the story inside a Chinese frame with all the symbolisms the tiger bears in ancient Chinese traditional culture. As a matter of fact, in China the tiger is considered the king of all wild animals, representing a powerful and untamed Yang masculine energy. Its fierce 
appearance is associated with the elements of sun and fire, of rage and, very importantly, it is considered the symbol of universal balance and justice, and capable of driving off demons (Eberhard 1986, 3624). According to ancient Chinese popular tradition, the tiger was so sacred and feared by people that its very name was considered as a taboo (Eberhard 1986, 362-4): harming a tiger implies chaos and the collapse of the universe. By extension, being traditionally the king of wild animals, the tiger in this story embodies nature which is violated by Chinese totalitarianism that has overthrown the ancient harmony of Chinese cultural tradition and has objectified nature.

\subsection{Stylistic Analysis of the Personal Pronouns and the Narrator's Point of View}

From a stylistic point of view, a further dose of comic effect is provided by the use of the personal pronouns in the story, which contribute to focussing on the narrator's point of view and explain the real frame of mind of Wang Huping, Prince, or the fake "tiger-fighter".

A fruitful starting point when exploring a text from a stylistic point of view is to analyse what it foregrounds, that is to say, what in that text 'deviates' from the linguistic norm. Moreover, a stylistic analysis has to be necessarily "rigourous", "retrievable" and "replicable" (Simpson 2004, 3-4), that is to say transparent in its procedures, based on objective data and textually grounded (Montini 2017, 12-13). For a stylistician, any other way of analysing a text runs the risk of being bizarre and biased. Since the process of interpretation is always a gap-filling process (Eco 1995, 49), we may assert that stylistics provides an objective kind of analysis.

At this point we are going to concentrate on the use of personal pronouns in the text, with particular reference to the first persons 'we'/'I' and the third person masculine object pronoun 'him', which are the most frequently occurring pronouns in the short story employed in the short story to narrate the vicissitudes of the TV crew.

Starting any text, thus not necessarily a literary text, with a personal pronoun is a device used by writers to plunge readers into the plot immediately, 'in medias res', thus avoiding any kind of introduction. In this way, readers' attention is promptly activated (Wales [1990] 2016, 260). Ha Jin's short story starts with the personal pronoun 'we', referred to the film crew and to the narrator, who, in this way, is understood to be a member of the crew directly involved in the story. When we process the text with a digital tool, such as 
Isabella Marinaro Ha Jin's Ecocritical Irony in "A Tiger Fighter is Hard to Find"



Figure 1 Word list resulting from the word processing of the story

AntConc, ${ }^{6}$ we notice that this pronoun is more frequent than ' $\mathrm{I}$ ', despite the fact that the story is told by a single narrator: he is the narrator and, at the same time, the member of the TV crew, as well as the accomplice in their wrongdoings. As a matter of fact, in the text 'we' is present 72 times, while ' $I$ ' is present 52 times [fig. 1]. ${ }^{7}$ It must be added that 'I' not only refers to the narrator - who speaks only 16 times in the first person singular -, but to any other character who speaks in the first person singular throughout the text, that is to say also Wang Huping/Prince, Secretary Feng (the prop master), Director $\mathrm{Yu}$, Old Min (the man who refuses to play the role of the tiger), and Little Dou (the truck driver who accepts to play the part of the tiger). Therefore, the dominant personal subject pronoun is 'we': this detail conveys that the narrator feels personally engaged in what is happening to the film crew. The narration is subjective, told through the eyes of one narrator who feels deeply involved in the vicissitudes and in the wrongdoings of the TV crew.

6 AntConc is a free text analysis application which can be used for the analysis of single or multiple text files, especially concerning a data-driven analysis of text and keywords (https://www.laurenceanthony. net/software/antconc/).

7 The text is 5018 words long. 
The fact that the most frequent personal pronoun in the story is 'he' is not very meaningful as regards the interpretation of the text, since all the characters - even the tiger - are male. This fact explains the high frequency of this pronoun, which appears exactly 89 times.

Nevertheless, even though the narrator feels involved in the vicissitudes of the crew, during the narration he gradually becomes definitely aware of the foolishness of the whole matter, despite the fact that since the very beginning he has been a little sceptical, even though curious, about the shooting of a real combat between a tiger and a man: "I stood behind a large elm, hugging my ribs" (Ha Jin $2001,58)$, he says at the first shot, and "I had my doubts" (63) at the proposal of shooting the film with a fake tiger. He is doubtful about the realistic effect of shooting a film with a man wearing a tiger skin:

I thought that we couldn't possibly get a skin identical to the real tiger's. After I expressed my misgivings, people fell silent for a long time. (64)

He also becomes gradually detached from the tragi-comical development of the story when he states "I avoided looking at him" (65) referring to Wang Huping's bizarre behaviour before facing the fake tiger. Nevertheless, he does not desist from being critical about the foolish expedient of a fight between Little Dou/tiger and Wang Huping - who is overexcited at this point:

I said, 'If he cripples Little Dou, it'll cost us lots.' - 'Don't put such jinx on us!' the director snapped at me. I held my tongue. (67)

However, he stops making objections and, adopting a passive attitude, quickly falls silent. What is left for him to do, at the end of the story, is paying visits to the presumed tiger-fighter and his victim, just to report on the bitter ending of this crazy enterprise.

Perhaps the most interesting piece of data emerging from the Word List as a result of the digital processing of the short story is that the most frequently used noun is the noun 'tiger'. As a matter of fact, this noun recurs 86 times, that is to say it is the most meaningful noun in the story [fig. 2]. It is worth noticing the personal pronoun attributed to the wild animal throughout the narration: everybody refers to the feline with the personal pronoun 'it', apart from Huping, who always uses the objective personal pronoun 'him': this feature is a further indication which reveals that the tiger is objectified throughout the short story. According to English grammar rules, the pronoun 'it' is preferable for animals in general, especially when nobody is involved with them on an emotional level. The personal pronouns 'he'/'him' and 'she'/'her' are used to address animals when somebody is emotionally involved with them, as if they were equated 


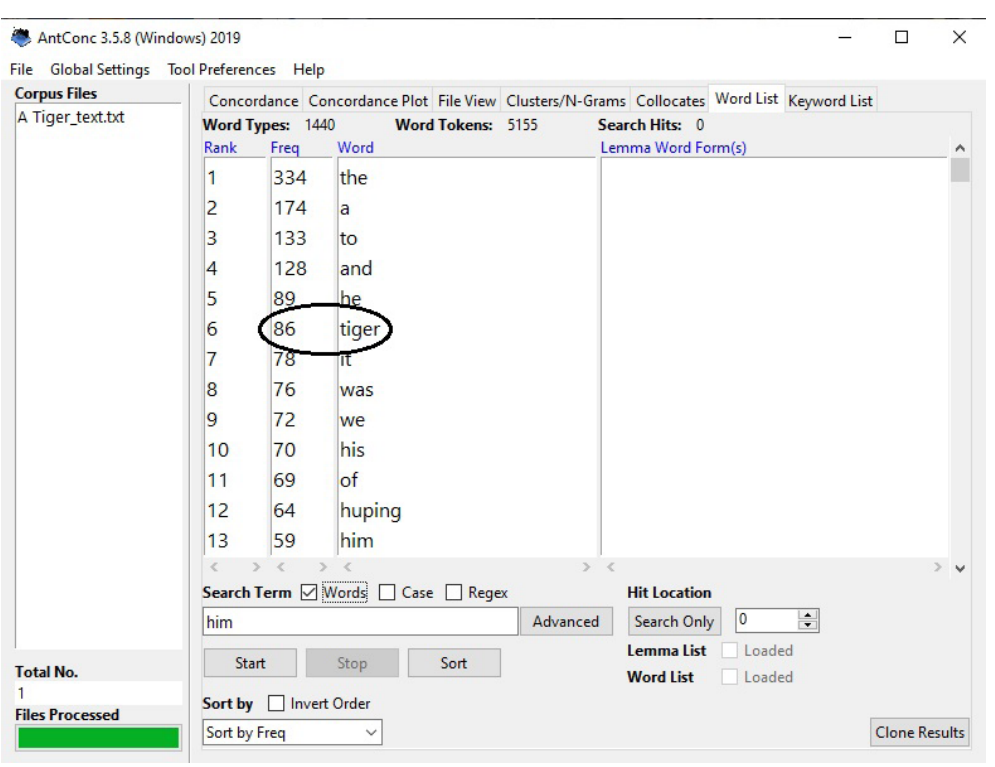

Figure 2 Occurrence of the word 'tiger' resulting from the digital text processing

with humans for this reason. Wang Huping always refers to the tiger with a pronoun which is proper for a person. This can be considered a deviation from the norm, thus it foregrounds the text and, for that reason, it deserves further analysis. For the crew the tiger is always 'it', which means that the animal is being objectified, while for its opponent only the animal is always 'him', thus 'he'. This could be explained if contextualised in Prince's delirium, which makes him feel that he and his opponent are at the same level, as if the animal were a man, hopefully as muscular as he is. It is clear that the more powerful the rival is, the more powerful the one who defeats him is, especially when, for Wang Huping, the real issue at stake is Wu Song's emulation and, consequently, a proof of virility. However, just for this reason, and in this context, the distorted representation of Wang Huping's rival creates a comical effect, created by the narrator particularly through accurate juxtapositions of images of Prince's exultation for being convinced of having killed the tiger, and of the pitiful reaction of the people around him [tab.1].

Prince is exulting for having being chosen to fight against a tiger for his masculine poise. However, the narrator does not spare pitiful details on the uneven struggle between the feline and the actor from the very first shot by ridiculing Wang Huping's immediate reaction 
Table 1 Juxtapositions regarding Wang Huping' behaviour and the reactions to it

\begin{tabular}{ll}
\hline Wang Huping's behaviour & $\begin{array}{l}\text { Other people's reactions to Wang } \\
\text { Huping's behaviour }\end{array}$ \\
\hline $\begin{array}{l}\text { "I killed him! I'm the number one tiger- } \\
\text { fighter!" Huping shouted. (58) }\end{array}$ & $\begin{array}{l}\text { People ran up to him and tried to calm } \\
\text { him down. (...) The medic poured some } \\
\text { water into the bowl and took out a } \\
\text { sedative tablet. He made Huping take } \\
\text { the medicine. (58) }\end{array}$ \\
\hline $\begin{array}{l}\text { (...) to our astonishment, he burst out } \\
\text { singing like a hero in a revolutionary }\end{array}$ & $\begin{array}{l}\text { Two men clutched Huping's arms } \\
\text { and dragged him away while he was } \\
\text { opera (...). (59) }\end{array}$ \\
$\begin{array}{ll}\text { babbling about plucking out the tiger's } \\
\text { heart, liver, and lungs. (...) "He's punch- } \\
\text { drunk." (59) }\end{array}$ \\
\hline $\begin{array}{l}\text { Whenever someone came into sight } \\
\text { he'd shout "Hey, I killed the tiger"! (59) }\end{array}$ & $\begin{array}{l}\text { We worried about him, so we called in a } \\
\text { pedicab and sent him to the hospital for } \\
\text { a checkup. (...) the diagnosis was mild } \\
\text { schizophrenia. (59) }\end{array}$ \\
\hline
\end{tabular}

at the tiger's attack. Prince identifies himself with the Chinese legendary hero, Wu Song, still the outcome of the clash is indecorous:

With a crack the front half of the cudgel flew away. Hupping dropped the remaining half, just as Wu Song does in the story. The beast rushed forward, reached for Huping's leg, and ripped his pants, then jumped up, snapping at his throat. (58)

The descriptions of the fights between Wang Huping and the tiger enhance a perception of absurdity regarding the whole adventure, which consequently, casts an ironic light on the events as seen by the narrator.

Addressing the tiger using the pronoun 'him' paves the way for the last feature of the story which deserves special attention, namely the way Wang Huping refers to the fake tiger.

Once it has been ascertained that no man is capable of facing a tiger, let alone bare-handed and blindly driven by a sense of obedience and vanity, the only solution which seems to be left is killing the tiger and using its skin for a human actor to play a fake tiger. Despite his mental disorder, Wang Huping is called to fight against a pitiful tiger puppet and his behaviour leaves the readers amused:

On the way, Huping grinned at us, gnashed his teeth, and made hisses through his nose. His eyes radiated a hard light. That spooked me, and I avoided looking at him. (65) 
Convinced of being on the cusp of definitely conquering the longed title of tiger-fighter, Prince states, thinking of what is expecting him: "I'll whip him!" (65) and "Kill this paper tiger! I'm going to finish him off!" (67).

The narrator's ironic tone is further enhanced by the use of the personal pronoun 'him', even though Prince refers to his rival as a 'paper tiger' being unaware that this time he is not going to fight a real animal. It is clear that his mental state prevents him from being able to actually distinguish between the real wild beast and the unlucky man playing that role.

There is more to it. Under the skin of the wretched tiger, as readers are perfectly aware, there is a truck driver, Little Dou, and the very adjective 'little' conveys a lot about the pitiful mission with which Prince is entrusted.

When analysing the use of the personal pronouns, we notice that the narrator addresses Little Dou/tiger by using the pronoun 'it', and not 'him'. Only Prince insists on 'him', but readers are well aware of his madness and of the fact that he does not know that his opponent is a fake tiger. This is, perhaps, one of the pivotal points which reveals Ha Jin's critical lens, through his narrator. This deviating use of personal pronouns, especially the use of 'it' for a human, foregrounds the text and unveils the bitter irony of the Chinese writer. An irony which, playing with the distorted vision of humans and animals, turns into ecocriticism whose ultimate target is both the stubbornness of Chinese Communism and the ill-fated consequences of violation against nature.

As a matter of fact, among the twelve stories which form the collection titled The Bridegroom, this is the only story which provides an evident vein of harsh ecocriticism. Ha Jin's stinging irony is subversive and clearly reveals the writer's political view of contemporary China.

The act of killing a tiger, above all for all that this animal represents in Chinese traditional culture (Eberhard 1986, 362-4) is vile and appalling and it is accomplished only to obey a political order and satisfy the director's thirst for success. The victims of such blind obedience are first of all the tiger, but also all the crew in their mad attempt to carry out the order issued by the authority. Wang Huping and Little Dou are the characters who are affected the most. The only possible consequence, human failure, is suggested at the end of the story when the two characters are hospitalised.

Applying the principles of Derrida's philosophy, further ecocritical interpretation of this text can be achieved. Even though the French author's analysis, in particular the one carried out in The Animal that Therefore I Am (Derrida 2006), regards Western philosophy, we can attempt to extend his thought and employ it as a key to interpret $\mathrm{Ha}$ Jin's short story. What equates the Western philosophy to the Chi- 
nese Communist attitude described in the story is the relationship between humans and animals based on strength and subjugation which characterizes the anthropocentric view of life and the Communist propaganda action. According to Derrida, all Western philosophy is to be read as strongly anthropocentric. Going back to one of Kant's statements, Derrida reflects on how deeply "human", and exciting for humans, is the impulse of fighting and, consequently, for taming what humans consider 'inferior', subjectable, such as animals can be. According to the French philosopher, men always fight an inner clash against "non-human animals", which are reduced to play sparring partners. Such a vision is inevitably catastrophic because it is based on an unbalanced distribution of power between humans and the rest of the living beings. This causes a total chaos since the subjugation and objectification of "non-human animals" inevitably harms humans too.

Derrida claims that "non-human animals" are prisoners of anthropocentric views: such interpretation well explains why Little Dou, wearing the tiger's skin, becomes "it" to everybody, as the stylistic analysis has proven. Little Dou, in the role of the fake tiger, is 'reduced' to the state of a "non-human animal", consequently he is addressed with the pronoun of "it" by the TV crew, and only Wang Huping, with his mental disorder, refers to him by employing the pronouns "he/him. It can be argued that the crew admire the tiger for its majestic aspect. However, adopting Derrida's framework, they are convinced that it is at their disposal, no matter if that implies killing the feline, just because of the fact that it is an animal. Applying a Derridean lens, the superiority to animals that humans feel is the real reason why Wang Huping keeps on beating the fake tiger with unwonted fury, as the following examples from the text show: "Huping struck its head with his cudgel [...] striking its back and head" (Ha Jin 2001, 57) and "I'll whip him" (65) are some of the expressions referred to the fight against Prince and the real tiger and, later, the narrator describes the actor's fury against the fake animal:

"Huping leaped on its back and began riding it around, shouting, "Kill!" Gripping its forelock with his left hand, he hit the tiger hard on the head with his right fist. “Oh, Mama!” the tiger squealed. "He's killing me!" (66).

And also:

“Huping was slapping the tiger's face and spat on it as well."(67)

Such cruelty towards both the real tiger and the fake one, goes beyond Prince's madness, as it represents the expression of repressed rage of humans against "non-human animals". 
Ha Jin's story tackles the specific situation of a dictatorship and its absurd expressions of stubbornness and violence. As a matter of fact, this short story depicts the vicious mechanisms which inevitably emerge in contexts of dehumanisation, or, better, objectification. We can notice Wang Huping's instinctive rage, under the false appearance of gentle manners (thanks to which he has been given the nickname Prince), as the extension of the brutality of the dictatorship which he obeys and which is blindly violent, no matter if against people or nature.

In the end, this blind stubbornness is embodied in the absurd order written in the letter, in the servile obedience of the crew director, in the wilfulness of most members of the crew to continue the shooting, in Wang Huping's arrogance, as well as in the cruelty against the tiger and against Little Dou. The victims are all the characters of the story: not only the tiger, or Prince and Little Dou, but the whole crew who hope for an award which will never arrive. Even the narrator is a victim as he ends up simply going to pay visits to the two broken fighters. The linguistic strategy employed by Ha Jin of making the narrator use the pronoun 'we', rather than ' $\mathrm{I}$ ', makes it clear that the narrator is as a member of the TV crew who is an accomplice in the crime against nature. The narrator, therefore, takes active parts in the objectification of nature. In this way, Ha Jin depicts the narrator and the crew as perpetrators of Chinese Communist totalitarianism which, given its nature, exploits the other as a means to reach an objective without worrying about him/her. In this story, Ha Jin has adopted an ecocritical lens to point his finger against the chains of the Cultural Revolution and that is evidently shown by the writer's irony employed to describe the TV crew's clumsiness which pervades the narration and that culminates in the final part where the narrator ends up by visiting the two 'fighters' in the hospital. The narrator, thus, is portrayed as a tormentor for joining the TV crew through the use of personal pronoun 'we'. Yet, he is also a victim when he is depicted as incompetent for not having been able to openly rebel against the crime.

\section{Conclusion}

This paper has attempted to employ a stylistic exploration as a method of analysis to emphasise an ecocritical perspective and the writer's ironic vein in the commentary of a political story which points an accusing finger at the unfortunate consequences of blind dictatorship on both humans and animals and which reduces them both to the position of victims. The analysis started with the exploration of the use of the pronouns employed by Ha Jin to refer to the tiger and to the narrator to show both the focus of the narration and the 
victimization of humans and animals. The synergy between the stylistic analysis and the ecocritical reading has unveiled the distorted and unlimited mechanisms which are at basis of an unfettered anthropocentric view. Such condition inevitably has an impact on human beings and nature, which is violated by humans. The tiger is always reduced to 'it', notwithstanding the fact that its part is performed by a human. The only person who refers to the animal with the pronouns 'he/him' is the schizophrenic Prince. The tiger is exploited to the advantage of the Chinese Communist propaganda: the ancient harmony which saw the tiger as a supreme symbol of energy is broken and none of the members of the film crew is safe. The two fighters, Prince and Little Dou, end up pitifully due to the fact that they have presumed to tame and kill the tiger, that is to say nature. Not even the narrator ends up happy: his passive involvement in the vicissitudes of the film crew, his being definitely 'we' with the crew, bestows on him the minor role of assisting the two fighters, who are both hospitalised.

\section{Bibliography}

Burke, M. (ed.) (2014). The Routledge Handbook of Stylistics. London; New York: Routledge.

Corti, E. (2013). "Natura, ecologismo e studi letterari: una ricognizione introduttiva", in "Green Studies: natura, letteratura e ambiente", monogr. no., Ácoma, 5, 5-22. http://acoma.it/sites/default/files/pdfarticoli/44corti.pdf.

D’Arcy Wood, G. (2013). "Che cosa sono gli studi sulla sostenibilità", in "Green Studies: natura, letteratura e ambiente", monogr. no., Ácoma, 5, 23-35. http:// www.acoma.it/sites/default/files/pdf-articoli/44wood.pdf.

Derrida, J. (2006). L'animale che dunque sono. Italian trans. by Massimo Zannini. Milano: Jaca Book.

Eberhard, W. (1986). Dictionary of Chinese Symbols. London; New York: Routledge.

Eco, U. (1995). Six Walks in the Fictional Woods. Cambridge (MA): Harvard University Press.

Garner, D. (2000). "Ha Jin Cultural Revolution”. The New York Times Magazine,

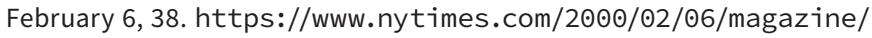
ha-jin-s-cultural-revolution.html.

Garrard, G. (2004). Ecocriticism. London; New York: Routledge.

Garrard, G. (2016). "Ecocriticism". Adamson, T.; Gleason, W.A.; Pellow, D.N. (eds), Keywords for Environmental Studies. New York: NY Press, 61-4. http://www.jstor.org/stable/j.ctt15zc5kw.24.

Gifford, T. (2008). "Recent Critiques of Ecocriticism", New Formations, 64, 1524. https://www.questia.com/library/journal/1G1-178452219/ recent-critiques-of-ecocriticism.

Ha Jin (1999). Waiting. London: Vintage.

Ha Jin (2001). "A Tiger-Fighter is Hard to Find". The Bridegroom. London: Vintage. 
Haomin Gong (2014). “Language, Migrancy, and the Literal: Ha Jin's Translation Literature". Concentric and Cultural Studies, 40(1), 147-67. https://

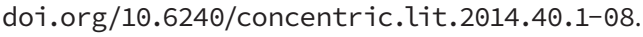

Ibáñez, J.R. (2016). “'All the Guns Must Have the Same Caliber': A Kachruvian Study of Ha Jin's Chineseness in 'Wind and Clouds over a Funeral'". Concentric: Literary and Cultural Studies, September, 195-220. https://doi. org/10.6240/concentric.lit.2016.42.2.11.

lovino, S.; Oppemann, S. (2014). Material Ecocriticism. Bloomington; Indianapolis: Indiana University Press.

Leech, G. [2008] (2013). Language in Literature. London; New York: Routledge. Leech, G.; Short, M. [1981] (2007). Style in Fiction. Harlow: Pearson Longman.

Liangyan Ge (2006). "The Tiger-Killing Hero and the Hero-Killing Tiger". Comparative Literature Studies, 43(1-2), 39-56.

Messud, C. (2000). "Tiger-Fighter Meets Cowboy Chicken”. The New York Times on the web, Books. https://archive.nytimes.com/www.nytimes. com/books/00/10/22/reviews/001022.22messudt.html.

Montini, D. (2017). "Preface. Stylistics Reloaded: Tradition and Prospects of an Impure Discipline". Fictions. Studi sulla narratività. Pisa; Roma: Fabrizio Serra Editore, 9-20.

Montini, D. (2020). La stilistica inglese contemporanea. Roma: Carocci Editore.

Simpson, P. (2004). Stylistics. A Resource Book for Students. London; New York: Routledge.

Sotirova, V. (2016). The Bloomsbury Companion of Stylistics. London; New York: Bloomsbury.

Stockwell, P. (2005). "Language and Literature: Stylistics". Aarts, B.; McMahon, A. (eds), The Handbook of English Linguistics. Oxford: Blackwell, 742-58.

Stockwell, P.; Whiteley, S. (eds) (2014). The Cambridge Handbook of Stylistics. Cambridge: Cambridge University Press.

Tsai, N. (2005). "Waiting for a Better Translation", Translation Review, 70, 58-67.

Wales, K. [1990] (2016). A Dictionary of Stylistics. London; New York: Routledge.

Warren, J. (2010). "Placing Ecocriticism". Interdisciplinary Studies in Literature and Environment, 17(4), 770-2. http://www.jstor.org/stable/44087672. 


\title{
Dwelling, Dispossession, and "Slow Violence" in the Time of Climate Change The Representation of Refugees in Amitav Ghosh's The Hungry Tide
}

Nicola Pilia

Università degli Studi di Torino, Italia

\begin{abstract}
In this essay, I will analyse the crucial issues of dwelling and dispossession concerning refugees in the novel The Hungry Tide by Amitav Ghosh. Political and environmental displacement is addressed within the framework of 'slow violence' as proposed by the landmark work of Rob Nixon, Slow Violence and the Environmentalism of the Poor (2011). With the intention to define the Morichjhãpi refugees as a foreshadowing of the climate migrations involving the lives of the subalterns in South Asia, as argued by Brandon Jones (2018), the essay provides a historical background of the Morichjhãpi Massacre and studies the forced eviction narrated in the novel through the pages of Nirmal's diary. Together with Kusum, the Marxist professor experiences the tragedy of the subalterns in the ever-changing ecosystem of the Sundarbans, bridging the gap between environmental and postcolonial categories while providing fruitful insights within the notions of human history and ecological deep time.
\end{abstract}

Keywords The Hungry Tide. Ecocriticism. Displacement. Migrations. Slow Violence.

Summary 1 Introduction. - 2The Morichjhãpi Massacre between History and Fiction. - 3 Nirmal, Kusum, and the Representation of Refugees: Dwelling and Dispossession. 4 "Slow Violence", Deep Time and the Shadow of Climate Change in The Hungry Tide.

$\begin{array}{lll} & \text { Peer review } \\ & \text { Submitted } & 2020-06-30 \\ \text { Edizioni } & \text { Accepted } & 2020-10-19 \\ \text { Ca'Foscari } & \text { Published } & 2020-12-22\end{array}$

Open access

() 2020 | ()( Creative Commons Attribution 4.0 International Public License 


\section{Introduction}

With The Hungry Tide (2004), Amitav Ghosh analyses the significance of place to the formation and expression of personal and social identities in postcolonial India, foregrounding narratives that involve the unfolding of events across time in the dynamic ecosystem of the Sundarbans. Through this paper, my aim is to address the vulnerability of marginalised subjects before the power of nature, especially investigating the community of refugees portrayed in Nirmal's journal, and propose a study of the novel's environmental themes in the wake of Rob Nixon's definition of "slow violence", useful to account for, eventually, the geological dimension of the novel. My reading concentrates on the figure of Nirmal and the representation of the dispossessed: adopting a postcolonial-ecocritical point of view, I will briefly examine the historical background that led to the 1979 Morichjhãpi Massacre, reclaimed in the novel as one of its central plot lines. The episode will allow me to devote attention to issues such as centralised control imposed by the adoption of Project Tiger's developmental rhetoric, neocolonial corporate power disguised as conservation narratives, and abuse towards the poor, already burdened in the past by the forced relocation in apparently hostile 'empty' lands. Besides, with this paper, I wish to propose a reading of the novel which is alert to the discomforting presence of climate change: considering Nirmal the cornerstone of political and geological discourses, I will address the deep-time temporal dimension of The Hungry Tide and study how the refugees in the novel may be said to have anticipated the demands of today's climatic migrations. In order to bridge the gap between social and environmental history, the importance of slow violence will be underscored as a way to describe the problems of the subalterns and human responsibility to today's environmental devastation.

In the following section, the historical dimension of the struggle is presented and, while outlining facts and circumstances, I will seek to follow the blurring of fiction and history that revolves around the theme of dispossession and violence against the Dalit community in the novel. In the third section, I will mostly focus on the characters of Nirmal and Kusum, both sufferers, on different levels, of the outburst of violence towards the community of refugees who had settled in the Sundarbans in the 70s. Seeking to investigate the political discourse that permeates Ghosh's novel, I will refer to the contradictory notions of 'development' and 'belonging' described by Huggan and Tiffin (2015) as imposed narratives of power affecting the downtrodden. In the last part, the novel will be discussed through the lenses of Rob Nixon's notion of slow violence, with the intention of establishing connections between state-mandated violence, the presence of climate change and their repercussions towards the lives of those at the margins in the Global South. 


\section{The Morichjhãpi Massacre between History and Fiction}

Following a narratological approach already adopted in most of his works, Amitav Ghosh intertwines different narratives in the novel across distant temporal lines. Nirmal's account of the facts discloses as his nephew Kanai, a middle-class Indian entrepreneur from New Delhi proceeds in reading his journal, projecting the plot into the past. Besides providing a detailed historical account of colonial and postcolonial settlement in the Sundarbans, Nirmal's journal also offers the perspective of an outsider among outsiders, whose point of view is surely bound to be overshadowed: "I am writing these words in a place that you will probably never have heard of" (Ghosh 2005, 67), he writes in order to introduce the island of Morichjhãpi, theatre of a massacre conveniently marginalised in Indian history (Das 2015, 40). Nirmal, a retired Marxist teacher who had been arrested in the past for his ideals and for the same reason forced to leave the city of Calcutta, appears naively enthusiastic about the villagers: at the beginning of his diary he casually discovers the newly established community and proposes himself to help the refugees, as he romantically believes he is witnessing the outset of a utopian society in the tidal ecosystem. The newcomers have suffered the plight of displacement, as the reader soon gets to know, and are now trying to establish a fixed abode in an ever-changing land that opposes such processes discursively and materially.

These postcolonial migrants embody a narrative of multiple evictions and dispossession. ${ }^{1}$ Following post-partition communal conflicts, many Hindu East Bengalis started migrating to escape persecution in their homeland. After being initially forced to settle in hastily made resettlement camps, in 1978 a group of refugees originally from the Khulna district of East Bengal started marching to Morichjhãpi, an uninhabited island in the Sundarbans, with the hope that the new Communist government would fulfil its promises of granting them a place to live, having supported their cause earlier: the political ascendency of the Left is reported to have encouraged the refugees to seek shelter within Bengal as Morichjhãpi, an island in the forests of the Sundarbans, had indeed been cleared in 1975, its mangrove vegetation replaced by a governmental programme to increase state revenue (Jalais 2005). However, as Rajorshi Das explains, the government refused to entertain their demands, initially imposing an economic blockade, and subsequently sinking the boats of the islanders. ${ }^{2}$ Many of the refugees consequently died of starva-

1 Historical account of the Hindu East Bengalis' dispossession follows Das 2015 and Jalais 2005.

2 Jalais 2005 offers a full-fledged analysis of the facts and their political implications. 
tion and cholera while others were killed in police firing during acts of state-mandated brutality.

The refugees' eviction was justified on environmental grounds: seeking to make room for a conservation enterprise called Project Tiger, Jyoti Basu, at that time Chief Minister of West Bengal, described the settlement of Morichjhãpi as illegal trespassing on a State Forest Reserve and on land designated for Tiger preservation (Das 2015, 41). Ross Mallick estimates as many as 17,000 deaths among the refugees and, despite the scarcity of proofs, Annu Jalais claims that only one in four refugees survived. The refugees, having fled to India from Bangladesh in waves after Partition in 1947 and then, in increasing numbers, after the 1971 Bangladesh War of Independence, were mainly Dalits who had tried to resettle from situations of cultural and physical displacement. According to Ramachandra Guha, Project Tiger was "a network of parks [...] managed primarily for the benefits of rich tourists", whose designation "was made possible only by the physical displacement of existing villages and their inhabitants" (Guha 1989, 75): it was originally intended to create artificial portions of 'cleared' land available to the touristic few, hence undermining long-term residents' livelihood conditions. Despite recognising the successful outcome of the World Wildlife Fund-sponsored conservation project, Weik admits that the Bengali Government has to be blamed for killing and manslaughter while pretending to act on behalf of animals. Thus, conservation rhetoric justified the takeover of land and natural resources, as Jaising aptly remarks. Morichjhãpi settlers were not encroaching upon the resources of the human natives but conversely those of the non-human ones, therefore violating the Forest Acts by their unauthorised occupation of a part of the Sundarban Reserve Forest, according to the government.

The Morichjhãpi Massacre, despite being covered in the Calcutta press at the time, had long since lapsed into oblivion: at the time of Ghosh's writing The Hungry Tide, only Ross Mallick's 1999 scholarly account was available in English (Fletcher 2011, 6). For this reason, we can say that the author gives an important contribution in advocacy of a socio-political context overlooked by the political debate, thus confirming what is well stated by Huggan and Tiffin about imaginative literature and its power to mediate functions of social and environmental advocacy while standing as a catalyst for action and engaged cultural critique (Huggan, Tiffin 2015, 14). The detailed account of the historical facts of Morichjhãpi in The Hungry Tide is a major example of a text that preserves its aesthetic function while drawing attention to its social and political usefulness, following the narration of a group of people in perpetual struggle with the natural and political environment; this offers the possibility to reconcile the interplay of postcolonial criticism and ecological questions in favour of a renovated unitary notion of environmental and social justice. 


\section{Nirmal, Kusum, and the Representation of Refugees: Dwelling and Dispossession}

Nirmal, the character who best exemplifies the fluidity of historical and environmental categories in the novel, is often read by commentators as in opposition to his wife Nilima, whose life is "entirely rooted in her sociocultural milieu" (Das 2006, 180). According to S. Das, Nirmal intrinsically appears as a violator of the law established by Nilima's commitment to Lusibari: following the poetic abstractions of Rilke, he chases "a romantic ideal across the margin of history" eventually collapsing "in the illusory space between what the Bangladeshi refugees claim as home and what renders them homeless" (1801). Poetry and unsettlement characterize his social consciousness, which gradually develops into active commitment throughout the novel. Forced to flee to the tide country in the middle of his life, where one's existence can be lived "on the margins of greater event", he discovers that "no place was so remote as to escape the flood of history" (Ghosh 2005, 77). Initially his engagement with the tide country is a forced construction in the wake of post-war conflicts and the post-partition hideous political climate, where opposition against revolutionary thinkers permeates Indian culture. At the beginning, he admits, in the Sundarbans "nothing was familiar [...]. How was it possible that these islands were a mere ninety-seven kilometres from home and yet so little was known about them?" (79). Years later, after retiring from working as headmaster of Lusibari school in 1979, he feels the need to support the cause of the Morichjhãpi refugees, an issue that would deeply change his erratic spirit and cause his mental breakdown, according to Nilima's words (117). Despite his initial scepticism towards local mythology and religion, he gradually develops a peculiar connection to the land through his commitment to the Morichjhãpi refugees, not limiting himself to a static perception of his personality but acknowledging that, in the tide country, "transformation is the rule of life" (224). A first account made by his wife precisely describes to her nephew Kanai the historical circumstances which led to the refugees' forced relocation in the island of Morichjhãpi:

Morichjhãpi, said Nilima, was a tide country island a couple of hours from Lusibari by boat. It fell within a part of the Sundarbans reserved for tiger conservation, but unlike many such islands it was relatively easily accessible from the mainland. In 1978 a great number of people suddenly appeared on Morichjhãpi. In this place where there had been no inhabitants before there were now thousands, almost overnight [...].

"Most of them were Dalits, as we say now," said Nilima. "Harijans, as we used to say then." 
But it was not from Bangladesh that these refugees were fleeing when they came to Morichjhãpi; it was from a government resettlement camp in central India. In the years after Partition the authorities had removed the refugees to a place called Dandakaranya, deep in the forests of Madhya Pradesh, hundreds of miles from Bengal.

"They called it resettlement," said Nilima, "but people say it was more like a concentration camp or a prison. The refugees were surrounded by security forces and forbidden to leave. Those who tried to get away were hunted down". (118)

The author provides the reader with all the necessary details to delve into the context of the episode. History is the main source for Nilima's reconstruction: she adequately sets her account in a post partition narrative that involves acts of forceful eviction and internment of the peasant population. The word resettlement is a key word here, a linguistic euphemism which masquerades the acts of violence perpetrated by the government. As she explains, the refugees had decided to settle in the Sundarbans assuming that they would not face opposition from the State Government. Nilima's attitude seems dismissive towards the refugees, she shows no eagerness to take part in the clash between the refugees and the government, her description of the acts of violence perpetrated in the Sundarbans are elusive, outcome of a "miscalculation" (119), and her husband's obsession with this oppressed group of people is nothing more than a form of idealism that will turn the couple into two strangers in the same house. She cannot feel an intimate shared experience with those people that had partially betrayed her sense of a fixed community committing an act of transgression by transcending physical and mental borders with the threat of upsetting the intimate stability of her newly-established community-oriented programme.

No sooner does Kanai start to read Nirmal's diary than a more nuanced point of view of the history of Morichjhãpi discloses to the reader. His account begins with the admittance of a defeat: "the truth was that I had not written a single word in all my time in Lusibari; not just that, I had even abandoned my other great pleasure - reading" (144). Partly for "superannuation", partly for the desire for following higher ideals of knowledge he decides, after retirement, to visit some other schools of the region. During one of his trips with the boatsman Horen across the archipelago, he finds himself "picked up and shaken by huge waves" (160) while travelling, so the pair decides to take shelter on a nearby island. The two men approach a small shack - "of the usual kind, made with bamboo and palm-leaf thatch" (161). Inside this small dwelling, the casual encounter with Kusum is narrated in a naïve quasi-romantic style: "I looked down and saw a young woman kneeling in front of me, touching my feet. That I could not identify 
her was no more a surprise that she should know me [...]. As a schoolteacher, then this happens with almost everyone you meet. [...] new faces do not match the old" (162). While the rain is still pouring outside, the two men can learn about Kusum's story, which constitutes one of the essential nodes of the novel.

Kusum's words come to Nirmal like a flooding torrent: Shakti Jaising defines her character as a "network narrative" that "features the experiences of two generations of metropolitan and rural characters whose lives intersect in the Sundarbans" (Jaising 2015, 67). Hers is a deep account of grief and loss: she recounts her travel to a mining town called Dhanbad in search of her mother, where she is saved by a food retailer named Rajen ${ }^{3}$ and, thanks to him, discovers that her mother, previously betrayed by a snake oil salesman, now works "in a place where truck-drivers came [...] and buy women for the night" (163). Soon after Kusum's marriage with Rajen and her mother passing away, her connection to the tide country becomes evident: she starts to fathom that her personal idea of belonging cannot match with the iron and rails of her temporary sojourn, dreaming of "stormtossed islands straining at their anchors, and of the rivers that bound them in golden fetters, [...] high tide [...] islands submerged like underwater clouds" (164). After a railway incident that kills her husband, leaving her alone with their son Fokir, she gets to know about the "great march to the East". The story of the refugees displaced from Bangladesh in search of a fixed abode shakes her unquiet spirit, as they appear "like ghosts, covered in dust, strung out in a line, shuffling behind the rail tracks", with children on their shoulders and bundles on their backs. Most importantly, this group of dispossessed people speak Bangla, the very same language as Kusum and the other dwellers of the tide country. Thus, the refugees are first portrayed as a colony of ghosts who, however, can rely on the use of words to describe their situation and their stateless condition deprived of the basic rights. This is how a spokesperson from this group replies to Kusum, once questioned about their destination:

"Once we lived in Bangladesh, in Khulna jila: we're tide country people, from the Sundarbans' edge. When the war broke out, our village was burned to ash; we crossed the border, there was nowhere else to go. We were met by the police and taken away; in buses they drove us to a settlement camp. We'd never seen such a place, such a dry emptiness; the earth was so red it seemed to be stained with blood. For those who lived there, that dust was as good as gold; they loved it just as we love our tide country mud. But no matter how we tried, we couldn't settle there: rivers ran 
in our heads, the tides were in our blood. [...] We sent some people ahead, and they found the right place; it's a large empty island called Morichjhãpi. For months we prepared, we sold everything we owned. But the police fell on us the moment we moved. They swarmed on the trains, they put blocks on the road - but we still would not go back; we began to walk". (164-5)

This reading of the postcolonial dispossessed does not spare the postcolonial state from harsh criticism: The Hungry Tide depicts the invisibility of those "living in the aftermath of emancipatory moments/projects such as Independence and postcoloniality" (Nayar 2010, 105); they come after such movements that, among their outcomes, saw the creation of welfare states and social mechanisms of charity and development but, at the same time, overlooked the lives of those at the margins. The Indian State appears inadequate to recognize and welcome those who are stateless, denying its land to those who need it with the presumption of keeping it inviolable. On addressing the inadequacy of the postcolonial state, Pramod Nayar implies that India has created two opposed narrations, portraying men antithetical to the environment they inhabit: people are doubly dispossessed by state actions, first through war and then through evictions and forced relocations; the characters find their own counternarratives to the institutional "pedagogy of national identity" (113), where violence emerges from silence and voice is given to what has been suppressed in ghostly forms.

The circumstances around the refugees precipitate quite rapidly: Nirmal is taken aback when he notes that a wooden watchtower has been erected in Morichjhãpi for better controlling the land and several men are required to patrol the island's shore; however, inducements to leave and, later, threats prove ineffective as the settlers remain adamant in their decision not to leave their land. The strong measures taken by the government start with banning all movements in and out of the island under the provision of the Forest Preservation Act, then forbidding people from gathering, treated as a criminal offence. In Lusibari it is rumoured that dozens of police boats were encircling Morichjhãpi and, by employing gas and conventional warfare, prevented anyone from bringing rice or water to the islanders.

During one of their final journeys to Morichjhãpi, Nirmal and Horen witness a scene of police brutality against some settlers that were hoping to enter the island with some provisions, after probably slipping out in search of external aid. A group of settlers is encircled by policemen in their motorboat; Nirmal finds himself in the middle of the tragedy and cannot avoid sharing his emotional commitment to the cause. The refugees assert here their status of dispossessed, a cry that mirrors Nirmal's own feelings as a displaced urban subject living in the Sundarbans, who is now able to understand and identi- 
fy with the settlers' anxiety "because of his lifelong struggle to find an effective political and artistic voice" (Jaising 2015, 71-2):

Surely the settlers would turn back now? In our hearts we prayed they would. But what happened instead was something unforeseen: the people in the boat began to shout in unison, "Amra kara? Bastuhara." Who are we? We are the dispossessed!

How strange it was to hear this plaintive cry wafting across the water. It seemed at that moment not to be a shout of defiance but rather a question being addressed to the very heavens, not just for themselves but on behalf of a bewildered humankind. Who, indeed, are we? Where do we belong? And as I listened to the sound of those syllables, it was as if I were hearing the deepest uncertainties of my heart being spoken to the rivers and the tides. Who was I? Where did I belong? In Calcutta or in the tide country? In India or across the border? In prose or in poetry?

Then we heard the settlers shouting a refrain, answering the questions they had themselves posed: "Morichjhãpi chharbona." We'll not leave Morichjhãpi, do what you may.

Standing on the deck of the bhotbhoti, I was struck by the beauty of this. Where else could you belong, except in the place you refused to leave.

I joined my feeble voice to theirs: "Morichjhãpi chharbona!"

[...]

At first it seemed the policemen might have decided to look the other way and let the boat pass.

That their intention was utterly otherwise became clear when the motorboat wheeled around in the water. Picking up speed, it came shooting toward the wobbling nouko with its boatload of passengers and provisions. It rammed the boat square in the middle: in front of our eyes the timbers flew apart. Suddenly the water was full of struggling men, women and children. (Ghosh 2005, 254)

Ghosh seems to suggest that the traditional idea of belonging cannot account for the social complexity of the Sundarbans estuarine ecosystem. Huggan and Tiffin have investigated upon the fights over land between European and indigenous settlers in the colonies and the anxiety resulting from the overshadowed awareness of land expropriation and the suppression of local knowledge by the normalising act of legal narratives of possession. ${ }^{4}$ According to them, possession does not imply belonging: the evicted refugees live in a perpetual state of home and not-at-home which is a paradoxical consequence of the possibility of dwelling without any perception of feeling at home. Entitle- 
ment to one's land encompasses the tensions between emotional possession and laws governing ownership which, in a postcolonial state, create a conundrum that violently puts the citizens against the political forces. Subaltern people are "residual presences from a precapitalist era" (Nixon 2011, 164), whose rights to the soil are overwritten by the logic of private property; the land does not belong to them and to "dwell in movement" constitutes an unacceptable contradiction since it does not directly improve the "civilizational spectacle of the nation" (164). Drawing from nature's observation, Nirmal realises how transformation is a dominant force also for the society of the tide country, challenging the idea of static boundaries or fixed identities: the developmental agenda of the nation-state is short-sighted because it ignores the ever-changing relationship between humans and their surroundings, as Jaising remarks too (Jaising 2015, 67, 75).

Such a misleading developmental narration, while advocating for tiger protection, mirrors a Western approach to ecological problems like tiger extinction: it calamitously considers endangered ecosystems and animals as disconnected from the livelihood of local peoples who are simultaneously put at risk. The non-human world is seen as a priority over the life of subaltern groups targeted by their own governments with the cooperation of international organisms. The West Bengal Government perpetrates a strong operation of mass eviction over the group of refugees, who demonstrated their cohesion eventually reclaiming their stateless exceptionalism by asserting their "dispossessed" status. This drastic removal campaign is conducted employing off-duty policemen and criminal gangs: murders and rapes on a massive scale are among the consequence of state-mandated brutality against the dispossessed. The tiger, hunted to near-extinction especially during colonial times, stands in the common narration as antithetical to men's appeals, following neocolonial presumptions which envision environmental justice and conservation as separated from social justice and in conflict with the concerns of indigenous people.

The siege goes on for many days, even after being declared illegal by the High Court. On arriving once again in Morichjhãpi, Nirmal realises how the blockade has affected the lives of the settlers. Starvation has shaped Kusum and Fokir's body, while many others are dead due to contaminated water. It is a moment of apparent calm before the final clash and Kusum, in her last reported words, affirms all her lack of faith in such conservation measures that affect the life of the poor:

[They say that] "This island has to be saved for its trees, it has to be saved for its animals, it is a part of a reserve forest, it belongs to a project to save tigers, which is paid for by people from all around the world". Every day, sitting here with hunger gnawing at our bellies, we would listen to these words over and over again. Who are these people, I wondered, who love animals so 
much that they are willing to kill us for them? Do they know what is being done in their name? Where do they live, these people? Do they have children, do they have mothers, fathers? As I thought of these things, it seemed to me that this whole world had become a place of animals, and our fault, our crime, was that we were just human beings, trying to live as human beings always have, from the water and the soil. No one could think this a crime unless they have forgotten that this is how humans have always lived - by fishing, by clearing land and by planting the soil. (Ghosh 2005, 261-2)

She seems aware of the ecological reach of the struggle, as her figure in Nirmal's journal combines fable and moral drama. The fiction of 'national progress' is once again revealed as a mere abstraction: development, as Huggan and Tiffin maintain, is a rhetorical issue which aims at the preservation of the middle-class society requiring the sacrifice of subaltern people. The episode shows how "environmental issues cannot be separated from questions of social justice and human rights" (Huggan, Tiffin 2015, 54) and, on a wider perspective, "ecological disruption is co-extensive with damage to the social fabric" (54), as development implies "top-down forms of economic management" (54) reflecting the neocolonialist imperatives of global corporate commerce. In the novel, the postcolonial state perpetrates coercive allocation and management of natural resources that are versions of 'ecological imperialism' ${ }^{5}$ with its disastrous effects, provoking a cultural-and-ecological crisis which erodes the social structures that make pluralism possible and hits marginalised societies already excluded by the centralised machinery of the state.

\section{4 "Slow Violence", Deep Time and the Shadow of Climate Change in The Hungry Tide}

I will now try to read the blending of environmental and postcolonial issues regarding the notion of "slow violence" as presented by Rob Nixon. With this section, I wish to offer a reading of The Hungry Tide which brings the ecocritical and political dimensions we have previously outlined on a deeper level, well rooted in discussions on climate change and contemporary environmental threats. Nirmal, whose trajectory in the novel has been traced above, encompasses along his pages both historical dimension and geological perspectives, evoking the all-transformative agency of the tide country and anticipating, in some lyrical and foreboding passages of the novel, the devastating effects that natural phenomena might bring about in the tide coun- 
try. I would like to suggest that the history of the Morichjhãpi Massacre could be framed as a comparable narrative as those analysed by Nixon in his exhaustive 2011 work of literary criticism, Slow Violence and the Environmentalism of the Poor. By doing so, The reality of climate change will be employed to address the effects of slow violence in a novel, The Hungry Tide, which does not tackle the climatic crisis openly but dedicates much attention to the history of those refugees who are caught in a volatile and environment and may be presented as a foreshadowing of today's climatic migrations.

Slow violence, Nixon explains, is a form of violence that eludes eye-catching definitions in an age dominated by the spectacularisation of the facts in the economy of media communication. It unfolds over years, decades, even centuries sometimes, occurring gradually and out of sight in a vortex of delayed destruction dispersed across time and space. It is by definition neither spectacular nor instantaneous, but rather incremental and accretive (Nixon 2011, 2), and its calamitous repercussions can be detected across a range of temporal scales. It has an exponential character and it commonly affects "those people lacking resources" (4), whose unseen poverty confers them the status of "disposable people" and makes them the perfect target of slow violence. The poor are way more likely to experience the proximity of environmental threat, which endangers their lives in different ways. Among the most common forms of environmental violence against the poor, Nixon talks about toxicity, resources depletion, petro-imperialism, megadam construction, neocolonial tourism, corporate deregulation, and antihuman conservation practices; these forces, he says, "disproportionately jeopardize the livelihoods, prospects, and memory banks of the global poor" (5).

Slow forms of environmental catastrophes operate by displacements, as places are rendered inhabitable since the effects of the "violent geographies of fast capitalism" (7) imply temporal, geographical, symbolic, and technological displacement:

For if the past of slow violence is never past, so too the post is never fully post: industrial particulates and effluents live on in the environmental elements we inhabit and in our very bodies, which epidemiologically and ecologically are never our simple contemporaries. (Nixon 2011, 8)

Slow violence poses a number of representational challenges for writers and activists who face the difficult task of giving shape to formless threats, whose "catastrophic acts [...] are low in instant spectacle but high in long-term effects" (10). Casualties of environmental violence are normally part of a system which does not consider them as such; indeed, they are disposable and expendable for a broader project that usually involves processes of forced industrialization. 
On explaining the dynamics of displacement, Nixon talks about the superimposition of an 'official landscape' upon a 'vernacular one' (17): by doing that, the socioenvironmental struggles of a community are severed from the accumulated cultural meaning, surrendering to a construction of an uninhabited landscape. As stated previously, in The Hungry Tide different narratives of place from below try to oppose to the fallout brought about by developmental agendas whose primary beneficiaries live elsewhere. This is the case of the refugees who become conservation refugees as soon as the West Bengal Government forces them to find a new land while seeking to provide finite resources for the touristic few. ${ }^{6}$ Nixon is categorical with his lashing against neoliberal ways of consumption that internationalize profits and externalize risks: although the barriers of free trade have been pushed down, neoliberal policymakers have erected even higher barriers which segregate "inordinate wealth from inordinate poverty" (20).

Given the apparent lack of representational resources of a group of people who struggle for survival, Nirmal's role in The Hungry Tide is essential to make the threat accessible, helping "counter the layered invisibility" (16) to the history recesses. It is a strong act of redefinition capable of opening up "connective avenues" (23) between environmental and social justice and new forms of globalization "from below". A fictional engaged writer, like Nirmal, opposes the silencing history of land theft, forced removal and coercive labour. Through his journal, he undertakes a work that counters the distancing network of free market resource development, "a rhetoric that displaces onto future generations [...] the human and ecological costs of such development" (26). Interestingly, Nixon explains that physically-displaced people hold, on the one hand, "an official, centripetal logic of national development" and on the other "a terrifying, centrifugal narrative of displacement, dispossession and exodus", an evacuation from land and from public awareness that reflects the tensions between culturally-constructed and geological threats (152).

The consequences of environmental politics of devastation resonating along time and space are evident in the legacy of the refugees of Morichjhãpi. Ghosh's work offers a dramatization of occluded temporal relationship on a transnational space, opposing the particular/ local element to hegemonic forces of conservation that create private profit by blaming local populations for the way they manage natural resources. Corporate colonialism employs distancing strategies

6 Idea taken from Nixon 2011, 18: "Too often in the global South, conservation, driven by powerful transnational nature NGOs, combines an antidevelopmental rhetoric with the development of finite resources for the touristic few, thereby depleting vital resources for long-term residents". 
to impose the 'foreign burden' upon local forms of resistance, taking advantage of "neoliberal lawlessness in cahoots with corrupt, legally immune local politicians" (56). The ebbing and flowing of transnational capital impact on the rural subsistence communities on a suspended, concealed time which only exists "in the penumbral realms of the longue durée" (62): the recourse to deep time, a rhetorical element which is present along Nirmal's diary, is a necessary strategy to reclaim the settlers from the state of invisibility and amnesia constituting, according to Nixon, "the dialectic of ordinary disaster" (65).

Reading The Hungry Tide in the time of climate change puts forward interesting challenges regarding the notion of deep time. Concerns over Western environmentalism and the threatened subaltern lives of the poor widen the discourse of displacement in a longer temporal perspective "capable not only of understanding the history of colonialism, environmentalism and globalization that conditioned events like the Morichjhãpi Massacre, but also of anticipating the increasing agential challenges climate and geology will pose in cases of forced migration in South Asia" (Jones 2018, 642). Owing to the menace of rising sea level in the Sundarbans region, it should be noted that the dynamics of climate change could have been present on Ghosh's mind while drafting the novel. Deep time and climate change can be read here with the same lens we have used above to describe slow violence: its actions far exceed any historical notion, a longer temporal perspective should be adopted to address issues of conservation and claims over nature. A broader perspective is employed by Nirmal to depict Lusibari to the eyes of a young Fokir, Kusum's son: he narrates episodes from the colonial past of the region, a journey into the past back to the "worst storm of all" (Ghosh 2005, 203), that of 1737. On such occasion, "a wall of water twelve metre in height" (204) shocked the tide country at the same time when an earthquake destroyed thousands of dwellings in Kolkata. Immediately after, Nirmal overcomes the historical dimension of the environmental disaster in the Sundarbans sinisterly revealing that "a storm will come, the water will rise, and the bãdh [i.e. the embankment] will succumb, in part or in whole. It is only a matter of time" (205). The fragility of the land is put in direct contact with a perspective that goes beyond history, representing humankind at the mercy of and active participant in the long enduring geological and climatological forces; Nirmal seems to aspire to "consciously reconcile modern colonialist responsibility for human violence and environmental exploitation in South Asia with the accidental consequences of stratigraphic encroachment and global climate change" (Jones 2018, 645).

Brandon Jones, reading the novel as anticipatory of the climatic fiction genre, describes The Hungry Tide as dominated by the tension between a transcultural conflict involving Western environmentalism and subaltern agency, and at the same time the presence of a 
planetary crisis developing on a much longer period of time under the looming presence of climate change, both of them embodied in the Morichjhãpi Massacre. Nirmal's first person journal encompasses "biographical experience, colonial history, geological shifts, biodiversity loss and tidal ecology in a mode of perception that looks both far into the past and into the future" (645). Nirmal's writing mediates our knowledge of phenomena that exceed the scale of immediate experience, an important role that fiction writing must assume in the time of the Anthropocene. Given the high level of complexity of the issue of climate change, with its economic, ethical and of course scientific implications, fictional writing can offer a critical tool for rendering the unimaginable scale of time that is nevertheless an exceedingly felt presence for the human and nonhuman residents in the Sundarbans. Through Nirmal's journal, the reader learns about the volatility of the region: in the last few years, as Jones claims, "the number and severity of cyclones have increased, tidal surges have become more extreme and the erosion of island embankments has noticeably intensified due to sea levels rising faster than anywhere on earth" (645), also stating that the entire ecosystem will face severe consequences of these rapid changes, since the changes will affect the inhabitants of the region including dense vegetations, Bengali tigers, all the aquatic life and 4.3 million people (645). Nirmal's ecological interest is evident in acknowledging the rapid changes that natural phenomenon are undergoing in the region:

I remembered how, when I first came to Lusibari, the sky would be darkened by birds at sunset. Many years had passed since I'd seen such flights of birds. When I first noticed their absence, I thought they would soon come back but they had not. I remembered a time when at low tide the mudbanks would turn scarlet with millions of swarming crabs. That colour began to fade long ago and now it is never seen anymore. Where had they gone, I wondered, those millions of swarming crabs, those birds?

[...] It was as if I could see those signs everywhere, not just in myself but in this place that I had lived in for almost thirty years. The birds were vanishing, the fish were dwindling and from day to day the land was being reclaimed by the sea. What would it take to submerge the tide country? Not much - a miniscule change in the level of the sea would be enough. (Ghosh 2005, 215)

His words offer a description of nature in the ecological perspective of deep time. Ecological awareness, here inscribed within the backdrop of postcolonial history, does not create a totalizing narrative that suppresses the history of oppression represented in the Massacre. Nirmal's diaristic work integrates short-sighted politics of dis- 
possession with the geological rhythm of the environment so that the "trans-historical mutability"7 of nature evoked in the novel trivialises the political struggles for controlling the watery ecosystem of the Sundarbans. A different viewpoint is advocated while moving beyond "narrow nationalistic, ethnic and racial binaries to embrace an ecological perspective that is compelled by the understanding that we live [...] in one world" (Kaur 2007, 127).

The Morichjhãpi refugees are displaced both on political and environmental grounds. Their newly established utopian community look at the past, along their history of political dispossession and at the same time advocate for the need to find new paradigm of resistance in a deep temporal perspective, predicting the forthcoming threats suffered by climate refugees in South Asia. In a land that accelerates the process of slow violence, climate-induced refugees ask for a new definition of society which opposes to the forced relocation processes "whose vigorously unimagined condition becomes indispensable to maintaining a highly selective discourse of national development" (Nixon 2011, 150). Narratives of unitary national ascent impose physical and imaginative displacement: it is an imaginative work of expulsion that realizes itself through police brutality, lorries, and bulldozers but finds its synthesis on an indirect bureaucratic level that "creates and sustains the condition for administered invisibility"8 (151). 'Surplus people' and 'ghosted communities ${ }^{9}$ are forcibly removed and relocated out of sight, often resettled in overcrowded conditions or in scarcely productive 'empty' lands. They are developmental refugees and their trajectory, reflecting processes of slow violence perpetrated by the combination of political and environmental agents, anticipates climate-induced migratory phenomena; by representing their plight, The Hungry Tide bridges the gap between postcolonial and ecocritical approaches to the climatic crisis offering new resources for critical thinking in the Anthropocene.

7 Idea taken from Kaur 2007.

8 See Nixon 2011, 151: “Assaults on a nation's environmental resources frequently entail not just the physical displacement of local communities, but their imaginative displacement as well, indeed on the prior rhetorical and visual evacuation of those communities from the idea of the developing nation-state. This imaginative work of expulsion typically predates the arrival of the police, the dogs, the lorries, the bulldozers, and the engineers. Thus the direct violence of physical eviction becomes coupled to an indirect bureaucratic and media violence that creates and sustains the conditions for administered invisibility. The result is what I have called spatial amnesia, as communities, under the banner of development, are physically unsettled and imaginatively removed, evacuated from place and time and thus uncoupled from the idea of both a national future and a national memory".

9 Both definitions are widely used by Nixon 2011. 


\section{Bibliography}

Crosby, A.W. (1986). Ecological Imperialism: The Biological Expansion of Europe, 900-1900. Cambridge: Cambridge University Press.

Das, R. (2015). "Reading Amitav Ghosh's The Hungry Tide through the History and Legacy of Morichjhapi". The Golden Line, 1(2), 40-3. http://goldenline.bhattercollege.ac.in/V1/V1N2_Goldenline.pdf.

Das, S.S. (2006). "Home and Homelessness in The Hungry Tide: A Discourse Unmade". Indian Literature, 50(5), 179-85. https://www.jstor.org/stable/23340744.

Fletcher, L. (2011). "Reading the Postcolonial Island in Amitav Ghosh's The Hungry Tide". Island Studies Journal, 6(1), 3-16. https://www. researchgate.net/publication/266369339_Reading_the_Postcolonial_ Island_in_Amitav_Ghosh\%27s_The_Hungry_Tide.

Ghosh, A. (2005). The Hungry Tide. London: HarperCollins.

Guha, R. (1989). "Radical American Environmentalism and Wilderness Preservation: A Third World Critique". Environmental Ethics, 11(1) 71-83.

Huggan, G.; Tiffin, H. (2015). Postcolonial Ecocriticism: Literature, Animals, Environment. London: Routledge.

Jaising, S. (2015). "Fixity Amid Flux: Aesthetics and Environmentalism in Amitav Ghosh's The Hungry Tide". ARIEL: A Review of International English Literature, 46(4), 63-88. www.muse.jhu.edu/article/602108.

Jalais, A. (2005). "Dwelling on Morichjhapi: When Tigers Became 'Citizens', Refugees 'Tiger-Food'” Economic and Political Weekly, 40(7), 1757-62.

Jones, B. (2018). "A Postcolonial Utopia for the Anthropocene: Amitav Ghosh's The Hungry Tide and Climate-Induced Migration". Modern Fiction Studies, 64(4), 639-58. www.muse.jhu.edu/article/711930/pdf.

Kaur, R. (2007). "'Home Is Where the Orcaella Are': Toward a New Paradigm of Transcultural Ecocritical Engagement in Amitav Ghosh's The Hungry Tide". ISLE: Interdisciplinary Studies in Literature and Environment, 14(1), 125-41.

Mallick, R. (1999). "Refugee Resettlement in Forest Reserves: West Bengal Policy Reversal and the Marichjhapi Massacre". The Journal of Asian Studies, 50(1), 104-25.

Nayar, P.K. (2010). "The Postcolonial Uncanny: The Politics of Dispossession in Amitav Ghosh's The Hungry Tide". College Literature, 37(4), 88-119. https://www.jstor.org/stable/27917766.

Nixon, R. (2011). Slow Violence and the Environmentalism of the Poor. Cambridge (MA): Harvard University Press.

Nixon, R. (2005). "Environmentalism and Postcolonialism". Loomba, A. et al. (eds), Postcolonial Studies and Beyond. Durham (NC): Duke University Press: 233-51.

Weik, A. (2007). "The Home, the Tide and the World: Eco-cosmopolitan Encounters in Amitav Ghosh's The Hungry Tide". Journal of Commonwealth and Postcolonial Studies, 13(2), 14(1), 120-41. 



\title{
Maïssa Bey: romancière de l'agentivité de femmes Entre réécriture de l'Histoire et invention de soi
}

\section{Lila Lamrous}

Université Clermont Auvergne (CELIS), France

\begin{abstract}
The study of Maïssa Bey's novel Surtout ne te retourne pas allows to examine how the Francophone novel represents an earthquake as a poetic, metaphorical and political shockwave. The novel is part of a literary tradition but also shows the singularity of the writing and the engagement of the Algerian novelist Maïssa Bey. It allows to examine the feminine agentivity in the context of the disaster camps in Algeria: from the ravaged space/country emerge the voices of women who enter into resistance to improvise, invent their lives and their identities. The earthquake allows them to free themselves, to take a subversive point of view at society and their status as women in an oppressive patriarchal society. The staged female characters arrogate to themselves the right to reread history and take their destiny back.
\end{abstract}

Keywords Maghreb Francophone Literature. Women's Literature. Agency. Poetics and engagement. Postcolonial theory.

Sommaire 1 Introduction. - 2 Le séisme : une « onde de choc ». - 3 Expérience collective de femme et « fin d'un monde ». 4 Un être « hypogé ».

$\begin{array}{lll} & \text { Peer review } \\ & \text { Submitted } & 2020-04-11 \\ \text { Edizioni } & \text { Accepted } & 2020-06-19 \\ \text { Ca'Foscari } & \text { Published } & 2020-12-22 \\ & \text { Open access } & \end{array}$

(ㄷ) 2020 | () Creative Commons Attribution 4.0 International Public License 


\section{Introduction}

Maïssa Bey est venue à l'écriture pendant la guerre civile algérienne, la décennie noire particulièrement néfaste pour les intellectuels et pour les femmes. Son souci, d'abord à travers une écriture de l'urgence puis dans des romans plus autofictionnels mais toujours porteurs d'un engagement fort, a été constamment de mettre en scène ceux que la société algérienne marginalise, exclut et condamne au silence. Ces mises en scène rendent visibles notamment les femmes, légitiment leur vision de la réalité et de l'Histoire et font résonner la force émancipatrice et subversive de leur voix. En mettant en mots le désir d'être de ces femmes algériennes, M. Bey propose une écriture « née du désir de redevenir sujet, de remettre en cause, frontalement, toutes les visions d'un monde fait par et pour les hommes, de découvrir et d'éclairer autrement ce que l'on croyait connaître " (Bey, rapporté par Kaouah 2004, 68). De Cette Fille-là (2001) à Nulle autre Voix (2018), Maïssa Bey déconstruit en particulier les rôles sociaux et l'être de surface que la société impose aux femmes et met en scène des personnages féminins capables de se reconstruire en tant que sujets, conscients autant d'une vérité en eux que des potentialités de leur être, puisque « Je est un autre » ainsi que le signale l'exergue ${ }^{1}$ de Surtout ne te retourne pas (2005).

C'est bien dans ce projet récurrent de l'écrivaine que s'inscrit Surtout ne te retourne pas, roman qui donne à voir de façon moins référentielle que métaphorique la destruction d'une ville et les répercussions sur les survivants qui, placés en situation de vulnérabilité, questionnent le sens du séisme. Ou plutôt le roman questionne la vulnérabilité des femmes construite par la société et que le tremblement de terre révèle, déplace comme pour mieux rendre possible la redéfinition de l'identité féminine. Nous montrerons donc comment le personnage féminin révélé par le tremblement de terre prospecte, par la parole conquise, aussi bien dans la ville (pays) ravagée que dans l'espace délabré de la mémoire et de l'imaginaire, de l'inconscient et de l'onirisme jusqu'à atteindre les confins de son être pour le faire advenir. C'est au terme de ce trajet erratique, ce « voyage » (tant horizontal, sur un champ de ruines et dans la rencontre collective, que vertical dans les confins de l'être), qu'il devient alors sujet de l'Histoire et de son destin. Maïssa Bey propose ainsi une possible agentivité de femmes. ${ }^{2}$

1 Exergue emprunté à Arthur Rimbaud (1871), Lettre du Voyant, 15 mai 1871. Paris : La Pléiade, 343-4.

2 Le concept d'agentivité, qui provient de la philosophie analytique de l'action, a été exploré dans les études féministes et de genres de même que dans les études littéraires. En nous appuyant sur les travaux de Judith Kegan Gardiner et surtout de Susan Hekman (1995), nous pouvons examiner, dans un texte littéraire, la construction et le parcours 


\section{Le séisme : une « onde de choc »}

Surtout ne te retourne pas est d'abord un roman de séisme qui s'inscrit dans une tradition littéraire, du Maghreb entre autres, et pose des questions d'écriture que l'auteure algérienne va résoudre en investissant le registre métaphorique de la description.

La première indication paratextuelle, un « avertissement » de l'auteure à forte fonction phatique, met en relation le projet scripturaire avec un fait réel : le tremblement de terre de Boumerdès (Algérie) survenu en 2003. Le roman constitue une réponse à un phénomène naturel « dont l'onde de choc nous a ébranlés » (5) autant que le tsunami de 2004 qui est immédiatement rapproché. Il prend sa source dans cette actualité mais sans se prétendre témoignage, documentaire ou reconstitution plus ou moins fidèle de l'événement, il s'exhibe comme fiction romanesque : « Ceci est un roman » (5), la formule pose d'emblée, et de façon catégorique, le cadre générique. Le séisme constitue alors une matière factuelle qui propose un soubassement diégétique et autorise une lecture non pas compassionnelle mais participative en ce sens où il émeut, interpelle l'humain par sa force destructrice et interrogative. Le lecteur retrouve les «ingrédients » attendus d'un récit de séisme : mouvement et rumeur de la terre ébranlée, déroute des populations, fracas des destructions, sidération et peur à nouveau des secousses, puis la vie dans des camps de sinistrés, ouvrant une vie précaire et provisoire où il faut apprendre à se reconstruire... Ces éléments diégétiques structurent le récit, le jalonnent de repères tout en produisant un effet de réel, mais ils restent surtout un canevas sur lequel l'écriture peut tisser une toile métaphorique. Cette écriture de la représentation du séisme est du reste questionnée pour son pouvoir évocateur et sa visée poétique ; en effet, l'auteure, dès les premières pages, met en opposition deux façons d'appréhender ce réel dramatique : « Langage scientifique » et « langage humain » sont confrontés (65-6) pour rappeler que, du point de vue de la victime, qu'est la narratrice, le séisme ouvre le temps de l'incompréhension, met en danger (voire en faillite) la raison et les habitudes. La narratrice souligne le caractère construit, autoritaire du discours scientifique dont la visée explicative, rendue possible parce que celui-ci intervient après l'événement, est en réalité aveugle à la peur et au ressenti des populations. Les mots du domaine des sciences sont efficaces sans doute pour catégoriser les types de catastrophes, décrire, définir avec précision les causes, mesurer en durée, fréquence et gravité ce mouvement de la terre :

d'un personnage féminin qui agit sur son devenir à partir d'une prise de conscience qui lui permet de repenser le monde dans lequel il inscrit sa quête d'être et d'émancipation. Ce concept doit naturellement être utilisé en prenant en compte les spécificités du contexte historique, politique et religieux du patriarcat algérien. 
J'ai appris, quand j'étais au lycée et au hasard de mes lectures très diverses, que, dans le langage scientifique, il y a souvent beaucoup de mots pour dire la même chose. Pour rester dans les expressions courantes, connues de tous, le premier mouvement sera de dire : tremblement de terre. Certains diront peut-être : secousse sismique. / Plus savamment, on parlera de secousse tellurique, du latin Tellus, terre. Ou bien encore, pour ne pas faillir à la traditionnelle rivalité scientifique entre racines latines et grecques, séisme, du grec, seismos, « secousse ».

Le tout développé, expliqué, exposé par des gens graves, imposants, entourés d'appareils, d'écrans, de tracés, ceux qu'on voit à la télévision après chaque catastrophe. Toujours les mêmes. Et qui parlent avec des mots graves, pesants, imposants, comme Tectonique des plaques, Extension, Coulissage, Compression, Magnitude, Épicentre, Échelle, Cicatrices, Failles, Fractures, sans oublier les Répliques. Inévitables, prévient-on. Des secousses, des milliers de secousses d'intensité variable, mais suffisamment nombreuses, suffisamment perceptibles, suffisamment virulentes pour précipiter les populations déjà traumatisées et paniquées hors de leurs maisons et entretenir la peur. (65-6)

Mais la narratrice remet en question la possibilité pour ce discours de cerner l'onde de choc sur l'homme puis de la commenter ; car il s'agit pour elle, prioritairement, d'explorer le chaos selon la perspective du sujet, dans ce qu'il traduit du dérèglement d'un monde et de l'humain :

En langage humain, simplement, essentiellement humain, ce sont de tout autres mots. Des mots plus directs, plus abrupts, des mots délétères qui ne laissent aucun espace, aucun interstice par où pourraient s'infiltrer les Lumières de la Science : effondrement, décombres, mort, ruines, désolation, bouleversement, chaos, colère, impuissance, désespoir.

Et surtout, surtout, basculement, folie. / Anéantissement. (65-6)

Les mots que sélectionne alors la narratrice construisent un champ sémantique de la ruine et du bouleversement qui, dans leur polysémie, peuvent traduire une réalité investie par la subjectivité et corrélativement débordant d'opacité et de trouble : " anéantissement » (vocable fort pour désigner la catastrophe extrême dans ses effets) possède un double sens (destruction matérielle complète et abattement moral total) comme justement «bouleversement » et surtout «tremblement de terre » qui autorisent ce passage du propre au figuré, du matériel à l'intime. Partant, regard et analyse de l'énonciatrice se portent sur la colère, l'impuissance de l'homme, son effondrement et non plus sur le mouvement destructeur de la terre. 
Le langage humain dont la narratrice revendique l'usage traduit le séisme dans des "mots délétères » (66) qui de façon paroxystique produisent de la corruption, sont capables d'altérer : ils introduisent de la folie laquelle, dans l'univers romanesque de Maïssa Bey, est présentée comme une force de perturbation ou de résistance aux versions officielles et aux règles de la société « ce modèle de cohésion, de cohérence et d'infinie harmonie, sans la moindre dissonance » (34).

Dans cet antagonisme des discours s'esquisse donc la possibilité de percevoir la puissance subversive du langage humain grâce à sa visée poétique. L'auteure joue sur les ressemblances des lieux et des personnages réels et fictionnels mais l'espace textuel est revendiqué comme lieu de fictions et de potentialités sémantiques et poétiques. La narration met ainsi en avant sa propre capacité de " corruption » de la représentation du réel en introduisant d'autres perspectives qui peuvent contester les discours objectifs et officiels notamment ceux de l'Histoire parce que la secousse de la terre impose, pour toujours, une certaine familiarité avec la peur et la mort, fracasse non seulement la terre mais toutes les certitudes plaçant l'homme dans la logique des "si » (de l'irréel du passé), du doute, du questionnement, du non savoir. Le roman du séisme crée un ordre fictionnel où tout « est plausible, possible » (63) ; la fabulation même, rendue légitime par l'inédit qu'ouvre le séisme, fonctionne comme mode de connaissance et d'éclairage du monde et de l'homme dans sa fragilité. Car, grâce à cette réalité brutale, tout redevient signe qui nécessite une autre posture de l'homme : questionnant sa fragilité, il prend conscience de son arrogance, de son aveuglement, du « caractère dérisoire, de l'inconsistance et de l'absurdité de tout ce qui leur semblait essentiel, tellement primordial, à peine quelque secondes plus tôt » (107). Les hommes découvrent que la terre leur est « insoumise » et qu' " elle peut, quand elle le veut, où elle le veut et seulement si elle le veut, s'ouvrir, se cabrer, les expulser » ; elle écrit sur la peau de leurs mensonges les signes de leurs inéluctables défaites » (113). L'homme, victime d'un " mal de terre », est placé devant une « écriture du vide et de l'absence » (64).

En cela, ce roman de Maïssa Bey prend immédiatement place dans une tradition (francophone) de la représentation du séisme au Maghreb : L'œil et la nuit de Laâbi (1969), Agadir de Khair-Eddine (1967), plus récemment encore Le Jour du séisme de Nina Bouraoui (1980) sont des textes de fureur et de souffle explorant des modalités d'écriture et un imaginaire libéré, propices à une lecture de l'être mais aussi de la Cité. La représentation référentielle n'est pas une priorité dans cette tradition maghrébine qui déplace la description vers la méditation et/ou le questionnement idéologique, élargissent les contours de la ville par la prise en compte du pays. La violence avec laquelle le Maghreb est entré dans l'Histoire et les nombreuses crises qu'il connait après les indépendances sont figurées par l'image du séisme 
et la destruction de villes-symboles; ainsi du « cadavre de la ville » s'établit le diagnostic du mal de l'individu et du collectif mais s'esquisse aussi pour le peuple de survivants l'horizon d'un avenir à partir de ce « séisme venu à venir » nous dit Laâbi $(1969,46)$. Le motif diégétique du séisme est donc utilisé pour sa force symbolique car il traduit « cette rébellion du silence, venu chercher dans les pogroms de l'anonymat d'autres nappes de silence pour ramener la terre à son inorganisation originelle » (46). Le texte littéraire, dans cette lignée romanesque du Maghreb, peut servir à exposer alors tout à la fois le spectacle de la ville détruite, territoire mémoriel bouleversé par les secousses de la terre et de l'Histoire et « la vision du cataclysme dans sa force de révélation » (46).

Surtout ne te retourne pas assume les diverses résonances politiques et poétiques de cette tradition littéraire, mais traduit aussi un cheminement scripturaire propre à Maïssa Bey. Le dessein de cette dernière est, constamment, de faire entendre la voix des femmes dans un contexte social d'exclusion et de réclusion. L'affirmation du sujet féminin nécessite la mise en place de stratégies de questionnement et de résistance, la capacité à raturer l'existant aliénant pour faire émerger des contre-discours et une autre vérité. Le point de vue féminin est omniprésent dans Surtout ne te retourne pas grâce d'abord à la focale très complexe utilisée pour traduire la vision du séisme et ses répercussions : une narratrice dédoublée et un concert de voix féminines construisent une polyphonie subversive dans un espace social qui leur impose habituellement silence et domination. Les espaces diégétiques et énonciatifs sont investis par une communauté de femmes, de tous âges et de toute origine sociale qui complète le récit dédoublé de la narratrice au double "Je ». C'est par ces voix de femme et notamment celle d'Amina/Whabiba qu'émerge la représentation du séisme à travers une écriture lacunaire en termes informatifs mais aussi fragmentée. Mimesis et mathesis ne sont guère recherchées dans les descriptions qui, d’une façon épurée, évoquent la ville victime et ses cicatrices, et dressent le portrait d'une population hagarde qui apprend à sonder sa douleur sociale et identitaire.

L'effacement du nom de la ville « Boumerdès » dans le texte permet d'ailleurs d'occulter complètement l'espace référentiel et d'en faire un support métaphorique. La fragmentation de l'écriture, privilégiée alors, introduit des brèches, ouvre des interstices d'où émergent des discours souterrains, notamment ceux qui ont longtemps été retenus, étouffés dans une telle société oppressante et répressive. Le fragment évite le discours totalisant voire totalitaire, et enregistre les fluctuations des associations d'idées, des bribes aléatoires de souvenirs et de constructions oniriques voire mensongères. L'écriture fragmentaire adaptée à l'éclatement de la terre ; il permet une puissance d'évocation qui agit aussi sur le lecteur, l'obligeant à marquer des temps d'arrêt et à questionner cette esthétique de lambeaux de 
textes. Il implique de l'énonciateur et de son interlocuteur, nous dit Catherine Henri-Ménassé,

une exigence d'attention au multiple, au complexe, au paradoxal, invitant à la rupture de l'univocité et installant la notion d'ouverture, d'inachèvement, d'incertitude, d'ébranlement et de chaos, au cœur même de ce qui fait notre capacité à penser le monde. En ce sens [l'écriture fragmentaire] est acte de résistance. $(2014,40)$

L'aspect lacunaire et fragmenté, rendu cohérent par le phénomène tellurique imprévisible et destructeur que l'écriture s'efforce de restituer, tire sa puissance et sa logique d'un discours interne en tension, celui d'un sujet en crise.

Car ce n'est pas la représentation qui compte mais le sujet féminin regardant, focale qui subit l'onde de choc dans sa chair, qui déambule dans l'espace détruit. Par son regard, le sujet féminin advient comme conscience et subjectivité ; il intériorise le séisme en le reflétant ou inversement. La narratrice traversant la ville « défaite, décomposée, désagrégée, disloquée » (14) exprime progressivement son paysage intérieur, qui n'est que "souffrance », " plaie » comme la terre. Son regard ne se pose pas sur les décombres et la poussière mais reproduit la rumeur de la secousse qui est « hurlement de femme » justement. Des « entrailles ouvertes » de la terre blessée émane notamment une odeur qui s'insinue en elle : «Elle envahit ma bouche, mes narines, se coule dans mes cheveux. Millimètre par millimètre, elle s'incruste. [...] A mon tour je suis corrompue » (14). Le texte peut alors filer la métaphore de la crise identitaire et traduire le phénomène naturel en psyché malade, en corps blessé, atteint en son sein par ce mouvement imprévisible de la terre qui fonctionne comme un hasard nécessaire, phénomène de synchronicité au sens jungien du terme. Le séisme dont on ne cherche pas à connaitre les causes (registre de la science ou de la religion dont l'énonciatrice montre soit les limites de la perspective soit les aberrations) instaure une perte de repères : il offre l'occasion de se détourner de ce paysage urbain enseveli et des vestiges d'un ancien ordre. Il instaure donc le chaos créatif qui fait venir le temps de l'inédit et de la renaissance. Il ramène l'homme et la femme au cœur du lieu dévasté à un état de chaos originel où tout redevient possible.

\section{Expérience collective de femme et « fin d'un monde »}

Le séisme est ainsi ce moment du « basculement » de l'homme dans un temps dilaté et un autre espace renversé où « le ciel semble être posé de guingois au-dessus des décombres » (63), les survivants faisant dès lors l'expérience d'un «après » qui signe non pas «la fin 
du monde » (avec des connotations religieuses) mais « la fin d'un monde » (64), celui précisément de la Cité avec son ordre social, politique, religieux qui assigne hommes et surtout femmes à une place précise dans une logique patriarcale et masculine. De l'espace ravagé par le séisme, émergent notamment les voix de femmes qui, piégées par l'imprévisible, entrent en résistance pour improviser, inventer la vie, leur identité. De ce non lieu qu'est la ville raturée, au plutôt du camp de sinistrés réplique d'un " tiers espace » au sens où l'entend Alexis Nouss (2005) et Homi Bhabha (1990), il devient possible pour les femmes de poser un nouveau regard sur la réalité, de s'arroger le droit de relire l'Histoire et de prendre la parole pour contester les normes sociales et discursives qui les ont réduites au silence. La vulnérabilité et la précarité réveillent les énergies et les rêves et génèrent l'agentivité des femmes qui vont se débarrasser de toutes les entraves patriarcales et religieuses.

L'expérience collective des femmes du camp 8 que relate Maïssa Bey est d'abord réappropriation de la marge dans laquelle elles sont traditionnellement reléguées. Le camp de sinistrés est un lieu de mise à l'écart, construit «à la périphérie du village, en marge de toute vie et assez loin de toute habitation » (137) mais aussi «lieu étrange " " réceptacle de toutes les douleurs et de tous les recommencements ». Ici se lisent ou se reproduisent d'abord toutes les brutalités, misères et discriminations qui touchent tout autant la femme que le peuple algérien dans son ensemble. Mais c'est là aussi que le séisme les « a fait basculer dans une autre dimension, une autre relation au temps et à la réalité des choses » (176) et qu'il devient alors possible de créer un autre équilibre où les femmes réorganisent la vie pour échapper « aux prédicateurs et tous ceux qui profitent de la vulnérabilité des sinistrés. Pour reprendre en main, enfermer à nouveau dans des postures, des exclusions et des dominations » (128). Les femmes mises en scène vont éviter cette manipulation car sans papier, sans parents, « chiens sans collier » (101) elles sont délivrées à présent de toute autorité tutélaire aliénante, notamment celle de l'homme. Regroupées autour de Dada Aïcha qui crée une famille matriarcale, les adolescentes Nadia et Amina échappent d'une part à la « généalogie » masculine et « stérile » qui enferme physiquement dans la cellule familiale, puis d'autre part, à l'enfermement « intérieur », produit par les leçons enseignées par la mère, la " Grande Officiante » complice de l'autorité patriarcale. Elles rejettent tous les interdits et les règles d'obéissance et de soumission qui les condamnaient à n'être que cette « inhumaine entité » qui représente, selon S. de Beauvoir, la femme modèle construite par le système patriarcal et pour le seul profit de celui-ci.

De même, Sabrina et Khadija sortent des rôles et des fonctions que la société leur imposait « avant » le séisme. Sabrina « c'est son nom de guerre. La guerre qu'elle mène contre la misère. Avec pour 
seules armes son corps, son insolence et sa détermination » (115) vit la prostitution comme une action émancipatrice qui lui rend son autonomie et sa liberté, qui lui permet de satisfaire des rêves nobles et de prendre en charge sa famille. Quant à Khadija, propriétaire d'un salon de coiffure au nom éloquent « Le jardin parfumé ${ }^{3}{ }^{3}$ elle se livre à une action thérapeutique et révolutionnaire avec ses séances de coiffure et d'esthétique offertes à toutes les compagnes du camp ; toutes cassent ainsi l'image de femmes chosifiées, sexualisées, utilisées et s'affirment par des actes et des paroles tout en ayant conscience de la condamnation sociale dont le poids et la violence sont régulièrement rappelés. Elles manifestent une rébellion envers les normes, créent surtout de l'estime de soi dans un projet social et altruiste où le sujet féminin peut s'épanouir et non plus seulement « survivre » (128). Dans leur geste de rébellion qui vise la transformation de la manière dont est régi le réel social, elles se constituent en sujet pensant et agissant, s'exprimant librement et faisant leurs propres choix.

Ces personnages féminins mises en scène par la romancière algérienne refusent donc d'adopter les statuts sociaux féminins qui les placent sous la loi du père puis de l'époux, d'être des filles de, femmes de et sœurs, épouses et mères, ${ }^{4}$ qui ne peuvent décider, faire, agir que dans une économie patriarcale; elles se font alors écarter ou s'écartent d'elles-mêmes de leur société d'origine ; elles sont les « audacieuses » ou les «effrontées » parce qu’ " elles piétinent allègrement et sans vergogne lois et codes sociaux » selon le discours de la société bien-pensante; elles sont aussi les «folles» ou les « notes discordantes » qui mettent la société traditionnelle en « intranquillité ». Cette folie leur permet en effet d'affronter la société, de déjouer ses règles et de s'assumer dans leur singulière autonomie. C'est dire aussi que, dans la folie (étiquette que leur colle la société pour les blâmer et les accuser d'atteinte aux codes et aux rôles sociaux prescrits de longue date), ces femmes puisent les forces vives sommeillant en elles, affirment un « je » puissant fait de volonté et d'actes et surtout « se déchaînent. Et [...] à force de se déchaîner, finissent par s'en aller, enfin libérées, enfin libres » (33-4). Elles refusent d'être le sujet figé à une place définitive par la société. Par ces écarts identitaires et de postures sociales, elles réussissent également à montrer et démonter tous les mécanismes d'enfermement et d'aliénation ; notamment le mécanisme d'aliénation produit par l'intériorisation de l'idée de «faute » (chez les jeunes filles comme Amina et Nadia qui se doivent d'être des filles «sans histoire » mais aussi de façon plus

3 Renvoi au titre du célèbre traité d'érotologie écrit au XVème par l'écrivain Cheikh Nefzaoui.

4 Voir Isabelle Boisclair $(2000,181)$ qui, à partir de l'univers fictionnel, examine les statuts de la femme dans le régime patriarcal. 
générale chez toutes les femmes, comme l'induisent les discours religieux) $;^{5}$ autre mécanisme d'aliénation celui du «dressage » et de la surveillance permanente de la femme par le tuteur ou ses substituts dans cette société totalitaire panoptique qui construit le « féminin » et empêche l'écart moral et social.

Le concept d'agentivité pour décrire les actions des personnages féminins de ce roman permet de sortir des dualités homme/femme, bourreau/victime, actif/passif et permet à l'auteure de proposer au lecteur l'idée qu'un sujet féminin est autant constitué que constitutif, qu'il lui est toujours possible de réagir, d'opposer à un pouvoir son propre pouvoir afin de transformer les règles qui dictent son existence et l'enferment dans une vulnérabilité infériorisante. L'agentivité des femmes rendue possible par le désordre ouvert par le séisme se manifeste donc par une réorganisation des relations de pouvoir qui légitiment et performent la domination masculine. Chez Maïssa Bey, les femmes ainsi marginalisées et qui s'affirment en tant qu'agent interprétatifs permettent alors de subvertir radicalement l'Histoire dominante portée par les discours politiques et sociaux officiels : elles disent leur vérité, elles énoncent elles-mêmes ce qu'elles vivent et donnent leur propre version des faits et du monde, réévaluent les discours officiels et ouvrent de nouvelles perspectives. Il leur est, du reste, une nécessité de se voir elles-mêmes et de mettre en discours cette réalité de femmes avec leurs propres mots pour effacer ceux des préjugés, ceux des paroles rabattues, des injonctions de toute sorte que l'énonciation reproduit mais en les saturant d'ironie. Et même les histoires - « Des histoires du temps d'avant. Des histoires comme aiment à en raconter les grands-mères » (87) - que raconte la vieille dada Aïcha, mémoire vive du passé colonial entre autres, ont autant de valeur que la grande histoire officielle faite d'ombres et de silences voire de mensonges. Dans tous les interstices du texte en lambeaux et de cette marge que constitue le camp jaillissent leurs paroles de femmes qui traduisent une dissidence mais d'abord aussi une expérience singulière et légitime. C'est donc bien à une entreprise de reconquête de soi et de la parole que se livrent les protagonistes de Maïssa Bey à partir de cette révolte de la terre « insoumise » qui est la métaphore de la révolte des femmes.

5 « N'est-elle pas à l'origine même du séisme et de la colère divine », laisse entendre ironiquement Maïssa Bey pour mieux condamner cet arbitraire du statut féminin : alors «il est recommandé aux femmes de se couvrir pour ne pas exciter les instincts les plus bas présents en chaque homme, et ne pas attirer les foudres divines sur la communauté des croyants [...] on ne peut arriver qu'à la conclusion suivante : nous femmes, par notre perfidie, notre insoumission, notre esprit de rébellion et notre inconséquence innée, étions certainement à l'origine de la mort de milliers d'innocents » (110). 


\section{Un être " hypogé »}

Le séisme fracasse donc non seulement la terre mais l'ordre social et autorise des expériences féminines inédites et collectives ; il rend possible également l'aventure intérieure en révélant le « je hypogé » (119) de la narratrice. Le récit rétrospectif qu'elle mène de sa perspective singulière est matière à explorer de nouveaux horizons intérieurs, à explorer l'être souterrain libéré par les forces d'un séisme symbolique. Amina commente ainsi son récit : «ma révolte et mon besoin d'errance et d'oubli [...] se nourrissent de trop de mensonges, de trop de silences [...] et surtout de la sensation de n'être jamais à ma place, où que j’aille » (119). Sa fugue puis son amnésie traduisent à la fois un déplacement identitaire et une crise de l'être trop longtemps brimé et soumis à la loi de la norme et d'un passé dont il lui faut faire table rase. Par le séisme intériorisé, Amina ouvre en elle un espace de potentialités, de latences d'être qu'il lui faudra explorer, assumer, imposer au mépris du moule qu'impose l'existence collective ; " Je suis venue au monde, dit-elle, dans un tournoiement de poussière, un jour de cris, de ciels retournés, de peur, de chaos, d'effondrements et de décombres, un lendemain de fin du monde, tout au long d'une infime et terrifiante contraction de la terre. / Depuis ce jour, on m’appelle Wahida, la seule et peut-être même l'unique. Désormais tout est plausible. Et peut-être possible » (112). L'expérience intériorisée du séisme exprime passions et pulsions qui ont lézardé l'être de surface fabriqué par l'éducation, nourri de mensonges et de silences résignés. Colère, haine, rage venue du plus profond de son être étouffé et brimé ont donné à Amina la force ou l'impulsion nécessaire à la révolte, seule attitude de refus et d'opposition capable de conduire cette jeune Parque vers sa « Mystérieuse Moi » enfouie. En quittant le domicile familial, celle-ci prend son destin en main car ainsi que le justifie Amina « tout ce qui advient à chacun d'entre nous obéit à une logique et parfois même à une volonté qui est en nous mais dont nous ignorons tout » (23). Cette justification a des allures de formules nietzschéennes; elle semble inviter à une actualisation de tous les possibles en soi, à une restauration de la pleine force des instincts créateurs.

Si l'identité personnelle se construit à travers une série de crises, selon Claude Dubar (2010), elle advient également de la capacité du sujet à l'inventer, à effectuer un geste créateur en somme. La crise révèle et confronte la personne à elle-même pour l'obliger à réfléchir et à changer, à s'inventer elle-même, avec les autres. S'inventer, pour la narratrice, consiste bien d'abord à jeter le masque social, cette ar-

6 En archéologie, un hypogée est une construction souterraine et plus spécifiquement une tombe creusée dans le sol. 
mure élaborée sur reniements successifs. L'invention de soi se poursuit ensuite dans le souci d'aller à la rencontre des autres. Amina interrompt justement le récit de son errance identitaire pour se tourner vers le vécu de ceux qui ont partagé sa vie dans le camp « car ce sont eux, et eux seuls qui m'ont permis de m'accrocher, de reprendre pied, de retrouver ce qui restait d'humain et de sensé en moi » (65) explique-t-elle ; plutôt que d'interruption, sans doute faudrait-il parler de bifurcation de son récit vers autrui car, dans ce détour, c'est bien au-devant de son moi propre qu'elle avance continûment. Cette étape est capitale dans l'élaboration de son être, d'une part parce qu'elle rencontre des femmes capables de la replacer dans de nouvelles relations familiales et sociales : "C'est ainsi que, en quelques jours, j'ai changé de nom, d'origine, de statut et que, sans trop de difficultés, je suis devenue l'aînée d'une famille dont presque tous les membres, virtuels ceux-là, avaient eu la bonne idée de disparaître, corps et biens le jour du tremblement de terre» (97) ; d'autre part parce qu'elle découvre de nouveaux modèles féminins en tout point opposés à celui de sa mère. Naïma, Khadidja et même la jeune Nadia offrent des exemples de résistance à la loi des hommes, notamment dans la passion qu'elles mettent à vivre en toute liberté et autonomie. Au contact des femmes autres, elle apprend des façons différentes de vivre sa condition de femme. La théoricienne Lena Lindhoff $(2003,163)$, faisait remarquer justement que le sujet féminin a besoin d'inscrire sa quête d'identité dans des structures intersubjectives de femmes. Les relations entre femmes donnent la possibilité d'une nouvelle identification en fonctionnant comme des miroirs. Ces nouveaux modèles féminins constituent le socle d'une prise de conscience de l'importance de la dissidence pour s'inventer autre.

L'étape la plus décisive dans l'invention de soi pour Amina reste néanmoins celle qui consiste à aller à la rencontre de cet autre dissimulé en elle. Il s'agit d'effectuer une démarche intérieure que la romancière algérienne matérialise dans ces différents romans par la scène du miroir dans laquelle le personnage effectue un franchissement d'être. Amina, nue, face au miroir, « à la fois regardée et regardante » (197) se réapproprie son corps « étrange, étranger » et advient comme sujet qui développe une relation d'altérité avec celle qu'elle était avant le séisme. Par ce face-à-face du moi et de l'autre en elle, elle manifeste son refus définitif d'une identité figée et normative. Dans cette expérience de soi à soi, la construction identitaire suppose pour le personnage de Maïssa Bey aussi de faire revivre «la petite fille » en soi dont la société a effacé les traces et qui est dépositaire pourtant de l'« identité première» $(2010,89)$; il s'agit ensuite «d'aller jusqu'au bout de soi », d'écouter ses désirs, de retrouver la jouissance du corps, d'éprouver le monde autrement qu'à travers le regard social, de façon authentique et libre. La conquête du corps est une victoire aussi bien symbolique qu'existentielle dans le parcours 
identitaire des femmes. Christine Detrez (2009) a très bien montré dans son étude, «A corps et à cris : résistances corporelles chez les écrivaines maghrébines », la place centrale qu'occupe la thématique du corps dans les écritures de femmes du Maghreb et les différentes déclinaisons de ce thème dans le registre de la résistance aux injonctions sociales. Maïssa Bey expose effectivement le corps féminin pour mieux dévoiler l'aliénation subie par les femmes mais aussi leur geste de résistance par et pour la réappropriation de leur corps confisqué.

Ainsi, écouter la voix intérieure et la voix des autres sont des étapes dans la construction identitaire féminine ; cette écoute de l'altérité mobilise l'instance énonciatrice de ce récit polyphonique et interdialogique qui guette le surgissement de l'être relégué « dans des fonds souterrains, trop lointains, trop sombres pour que quelqu'un ait l'idée de s'y aventurer » $(2018,66)$. Le récit qui semble surgir de la terre/femme bouleversée par le séisme produit ainsi une multiplication de discours de femmes qui donnent à entendre la passion de vivre et d'être femme en Algérie, tout en détruisant les discours autoritaires qui les ont aliénées. Le tremblement de terre est un choc salutaire qui conduit l'être féminin vers le questionnement de la place et de l'identité que la société patriarcale lui a assignées au nom d'une prétendue fragilité physique, psychique et morale, une « vulnérabilité » artificiellement construite qui la place en situation de silence et de dominée. La représentation symbolique de l'onde de choc que produit le séisme permet donc à Maïssa Bey de renouveler le pari d'une écriture de femme engagée qui s'insurge contre une forme d'injustice et d'indignité faites aux femmes mais propose aussi courageusement une réponse possible par l'acceptation de situation d'incertitudes et d'invention de soi.

\section{Bibliographie}

Beauvoir, S. de (1949). Le deuxième Sexe. Paris : Gallimard.

Bey, M. (2001). Cette Fille-là. Paris : Éditions de l'Aube.

Bey, M. (2005). Surtout ne te retourne pas. Paris: Édition de l'Aube. L'Aube poche.

Bey, M. (2018). Nulle autre Voix. Paris : Éditions de l'Aube.

Bhabha, H. (1990). «The Third Space ». Rutherford, J. (ed.), Identity: Community, Culture, Difference. London : Lawrence and Wishart, 207-21.

Boisclair, I. (2000). « Laure Clouet, femme de personne ». Joubert, L. ; Hayward, A. (éds), La vieille Fille, lecture d'un personnage. Montréal : Triptyque, 83-98.

Detrez, C. (2009). «A corps et à cris : résistances corporelles chez les écrivaines maghrébines». Oran : Algérie. https://halshs.archives-ouvertes. $\mathrm{fr} /$ halshs -00974685 .

Dubar, C. (2010). La Crise des identités. Paris : PUF. 
Lila Lamrous

Maïssa Bey : romancière de l'agentivité de femmes

Hekman, S. (1995). «Subject and Agent. The Question for Feminism ». Kegan Gardiner, J. (ed.), Provoking agents: Gender and Agency in theory and Practice. Urbana: University of Illinois Press, 194-206.

Henri-Ménassé, C. (2014). «René Char - Maurice Blanchot : la métaphore et le fragment ». Le Coq-Héron, ERES, 4, 219, 36-45. https://www.cairn.info/revue-le-coq-heron-2014-4-page-36.htm\#.

Kaouah, A. (2004). « Maïssa Bey: la parole conquise ». Notre Librairie. Revue de littérature du Sud, 155, 28-31.

Laâbi, A. (1969). L'œil et la nuit. Rabat: SMER.

Lindhoff, L. (2003). Einführung in die feministische Literaturtheorie. Stuttgart : Verlag J.B. Metzler.

Nouss, A. (2005). Plaidoyer pour un monde métis. Paris : Textuel. Collection Essais et documents. 


\title{
Les catastrophes naturelles dans les romans caribéens d'expression française
}

\author{
Alessia Vignoli \\ Instytut Romanistyki, Uniwersytet Warszawski, Polska
}

\begin{abstract}
The notion of 'disaster' pervades the Caribbean thought. The common origin of the Caribbean region, the European colonization, caused two disasters: the extermination of Native Americans and the deportation of African slaves. The union between nature and the oppressed people against the oppressor resulted in the creation of an environmental conscience that the Caribbean literature has often expressed. This essay will investigate the common points shared by some Haitian, Martinican and Guadeloupean authors in the writing of natural hazards. It will show that, despite the diversity that marks the Caribbean, there is a repetition of common features that proves its geopoetic unity.
\end{abstract}

Keywords Disaster literature. French-Caribbean Literature. Haitian Literature. Natural disasters. Ecoliterature.

Sommaire 1 Introduction. - 2 Les catastrophes naturelles dans la pensée caribéenne. - 3 Projets de reconstruction, résistance individuelle et collective. - 3.1 Du roman paysan au roman post-sismique haïtien. - 3.2 Cyclone et culture de résistance. - 4 Enjeux sociopolitiques de la mise en fiction de la catastrophe. - 4.1 Catastrophes et esthétique 'spiraliste' dans le milieu rural et urbain haïtien. -4.2 Chronique d'un désastre politique et social. - 5 Exprimer le retour du refoulé. - 5.1 Un cyclone refoulé. - 5.2 Le séisme comme catharsis. -6 Conclusion.

$\begin{array}{lll} & \text { Peer review } & \\ \text { Edizioni } & \text { Submitted } & 2020-07-30 \\ \text { Ca'Foscari } & \text { Accepted } & 2020-09-09 \\ & \text { Published } & \text { aaaa-mm-dd } \\ & \text { Open access } & \end{array}$

() 2020 | ()( Creative Commons Attribution 4.0 International Public License 


\section{Introduction}

Dans le présent travail nous nous proposons de donner un panorama de la représentation de la catastrophe naturelle dans des romans appartenant aux littératures caribéennes d'expression française. Nous aborderons quelques œuvres d'auteur.e.s haïtien.n.e.s (Jean-Euphèle Milcé, Jean-Claude Fignolé, Pierre Clitandre, Évelyne Trouillot), martiniquais (Raphaël Tardon) et guadeloupéen.n.e.s (Daniel Maximin, Gisèle Pineau, Maryse Condé) afin de mettre en évidence les points de convergence qui caractérisent la mise en fiction de l'aléa naturel (sécheresses, inondations, cyclones, tremblements de terre, éruptions volcaniques) aux Antilles françaises et en Haïti. Les littératures caribéennes sont en effet porteuses de préoccupations communes par rapport à la thématique de la catastrophe, malgré le caractère composite des territoires d'origine des romans évoqués. Nous verrons que la catastrophe peut être exploitée par l'écrivain.e pour dénoncer des inégalités sociales. Elle peut aussi donner voix à une résistance individuelle ou collective ou réveiller chez les personnages du récit des souvenirs du passé.

Compte tenu des différences du point de vue de la langue et surtout des expériences historiques, ${ }^{1}$ les littératures caribéennes ont souvent exprimé les liens qui se sont créés entre les habitants et la nature tropicale, hostile au colonisateur européen. Notre parcours nous permettra de montrer que, dans ce corpus hétérogène, il est possible de repérer, en regard d'un ensemble de traits similaires et divergents, des esthétiques diversifiées, associées à une 'littérature de la catastrophe' qui ne cesse de s'écrire au-delà des frontières, à la croisée des cultures.

\section{Les catastrophes naturelles dans la pensée caribéenne}

Selon le romancier cubain Antonio Benítez-Rojo, les facteurs mis en place d'habitude pour décrire cette région composite constituent les obstacles principaux que l'on rencontre quand on étudie la Caraïbe : sa situation insulaire et archipélique, son instabilité politique, son hétérogénéité culturelle, son syncrétisme religieux, l'isolement réci-

Le présent article a été réalisé grâce à la subvention du projet Literackie obrazowanie katastrof naturalnych w prozie haitańskiej i antylskiej (nr. 2017/27/N/HS2/00671) accordée par Narodowe Centrum Nauki, Polska (Centre National de Recherche Scientifique, Pologne).

1 Ainsi, parmi les pays caribéens de langue française, il est important de distinguer Haïti de la Martinique et de la Guadeloupe car leurs histoires, aux origines coloniales semblables, sont sensiblement différentes, l'Indépendance d'Haïti de la France datant de 1804 . 
proque des îles et l'absence d'une historiographie commune ([1989] 2001, 1). On peut même parler d'une difficulté à nommer cet espace qui se reflète dans la confusion persistante entre 'Antilles' et 'Caraïbe', deux mots qui ont une origine linguistique différente, sans parler du terme anglais, West Indies, hérité de l'époque coloniale. ${ }^{2}$ Malgré ces distinctions, Benítez-Rojo affirme qu'il faut envisager la Caraïbe dans sa globalité géoculturelle. L'écrivain cubain défend l'idée d'une conception cyclique de la temporalité qui correspond à une façon spécifiquement caribéenne de vivre l'histoire synchroniquement, à savoir qu'une phase historique donnée n'efface pas celle qui la précède. Il associe cette répétition au caractère insulaire de la région et, surtout, à l'expérience de la plantation, commune à tous les territoires caribéens. Il s’agit, selon lui, d'une répétition implacable des dynamiques économiques et sociales appartenant au mécanisme de la plantation ([1989] 2001, 203). En se référant explicitement à Gilles Deleuze et Félix Guattari, ${ }^{3}$ Benítez-Rojo évoque la nature rhizomatique de la région caribéenne, ce qui n'est pas sans rappeler la «poétique de la relation » et le concept d'« identité-rhizome » théorisés par le Martiniquais Édouard Glissant. ${ }^{4}$ Benítez-Rojo parle de « méta-archipel » pour définir l'espace caribéen ; contrairement à un archipel 'ordinaire', le « méta-archipel » n'a pas de limites ni de centre, il est un espace chaotique à l'intérieur duquel il y a une île qui se multiplie sans cesse ([1989] 2001, 9).

Deux catastrophes majeures ont frappé la région caribéenne après l'arrivée des Européens sur les territoires habités par les peuples autochtones. Au génocide des Amérindiens ${ }^{5}$ a fait suite la traversée transocéanique des navires remplis d'esclaves africains qui ont dû s'adapter au travail dans les plantations et, en même temps, faire corps avec la nature tropicale pour se tourner contre le maître européen. Sur ces terres de " métissages imposés par les vols de l'histoire » (Maximin 2006, 15), la nature a joué un rôle central dans la lutte des opprimés contre les oppresseurs. Comme l'explique l'écrivain guadeloupéen Daniel Maximin, la surpuissance de l'élément naturel à l'époque coloniale mettait tous les individus à l'épreuve, sans épargner les Européens qui se montraient vulnérables à ses déchaînements $(2006,93)$. La nature caribéenne a pu être ainsi associée aux révoltes, imprévisibles et destructrices, qui ravageaient fréquem-

2 Comme le rappelle Virginie Turcotte, 'Antilles' est le nom donné à ces territoires par les colonisateurs, alors que 'Caraïbe' trouve son origine dans le mot karib qui désignait un des peuples autochtones et sa langue $(2010,24)$.

3 Voir Deleuze, Guattari 1980.

4 Voir Glissant 1990.

5 Le terme 'génocide' est utilisé par plusieurs spécialistes pour se référer au massacre des Amérindiens. Voir Fombrun (1992, 96-7) et Fouchard (1972, 109-10). 
ment leurs habitations et plantations. À partir de la fin du XVe siècle, les contacts entre les Européens et les peuples de la Caraïbe ont provoqué des dégâts irréversibles dans les écosystèmes de la région. ${ }^{6}$ Malcom Ferdinand constate que ces dégâts ont été à la base d'une "révolution écologique coloniale » qui a marqué les modes de vie des populations caribéennes, leur rapport à la terre et à l'environnement $(2015,65)$.

Dans la littérature caribéenne, on remarque une forte présence de la représentation du cyclone. L'écrivain cubain Alejo Carpentier accorde un rôle de premier plan au cyclone dans El siglo de las luces (Le siècle des lumières, 1962) alors que le poète barbadien Kamau Brathwaite le place au centre de sa réflexion sur l'identité caribéenne (Deckard 2016, 28-9). Le cyclone est récurrent chez d'autres poètes caribéens, comme le Jamaïcain Ishion Hutchinson (After the Hurricane, 2016) ou la Portoricaine Loretta Collins Klobah (After Hurricane Lenny, Carriacou, 2011). On peut mentionner aussi Against the Grain (2008), un texte autobiographique où Edward A. Markham, originaire de Montserrat, évoque le passage du cyclone 'Hugo' en 1989, et le roman Land of Love and Drowning (2014) où Tiphanie Yanique, née aux Îles Vierges des États-Unis, narre la vie de trois générations d'une famille confrontée à plusieurs cataclysmes. Si l'on prend en considération la présence du tremblement de terre dans la pensée caribéenne, on peut observer que l'isotopie du séisme soustend la parole défaite et hachurée des deux poètes martiniquais Aimé Césaire et Édouard Glissant (Shelton 2015, 1623). Quant aux volcans, ils ont une fonction spécifique dans l'imaginaire caribéen et l'éruption volcanique est une métaphore très parlante chez Aimé Césaire, Frantz Fanon, Édouard Glissant et Vincent Placoly. Selon Mark D. Anderson, la géographie volcanique de la Caraïbe possède un statut symbolique rattaché à la construction des identités individuelles et nationales $(2011,107-8)$. Dans son étude de la métaphore du volcan dans Cahier d'un retour au pays natal (1939) de Césaire, J. Michael Dash souligne le rôle central du feu sortant du volcan qui exprime une force puissante et purificatrice. Selon Dash, le mouvement ascensionnel de la conflagration imprègne l'imagination de Césaire et figure la nécessité de changements politiques radicaux $(1998,68)$.

Ces exemples montrent que l'écriture de la catastrophe s'étend sur l'ensemble des pays caribéens et que, souvent, le thème du désastre peut donner naissance à la parole des peuples opprimés qui expriment leur résistance contre la domination. Comme le constate Françoise Simasotchi-Bronès, les littératures caribéennes sont le lieu

6 L'érosion des terres à la suite de l'abattage des bois, préconisé par l'administration coloniale en vue d'augmenter la surface de la plantation sucrière, en est un exemple patent. Voir Barthélémy (2000, 58-61 et passim). 
d'expression d'une conscience écologique, née de la relation entre l'individu dominé et son environnement :

Écolittératures, nées dans l'imminence du désastre, elles [les littératures caribéennes] révèlent ou rappellent la charge de résistance et d'émancipation portée par la conscience écologique spontanée des populations précarisées et subalternisées (Amérindiens, esclaves africains, etc.) dans la géohistoire néo-américaine (2020, 292-3).

\section{Projets de reconstruction, résistance individuelle et collective}

Certains auteurs semblent vouloir mettre l'accent sur le dépassement du trauma engendré par la catastrophe et sur la possibilité d'une reprise en main et d'un recommencement collectif. Dans deux romans appartenant à notre corpus d'analyse, Les jardins naissent de l'écrivain haïtien Jean-Euphèle Milcé et L'Île et une nuit du Guadeloupéen Daniel Maximin, la catastrophe entraîne plusieurs conséquences positives : la mise en œuvre des projets de reconstruction, l'émergence d’une nouvelle pensée citoyenne et le rétablissement individuel et collectif après le désastre.

\subsection{Du roman paysan au roman post-sismique haïtien}

La littérature haïtienne est riche en exemples de représentations de cataclysmes de tout genre, avec un intérêt particulier pour l'observation de la dégradation écologique. Bien avant le tremblement de terre du 12 janvier 2010, qui a très durement endommagé l'environnement haïtien, plusieurs auteurs ont proposé dans leurs romans une réflexion sur les dégâts causés par deux phénomènes en particulier : la sécheresse et la déforestation. La tradition littéraire haïtienne du roman paysan, inaugurée par La montagne ensorcelée (1931) de Jacques Roumain, se charge du devoir de représenter la condition paysanne et les problématiques associées à la terre avec un intérêt pour la dimension ethnographique du milieu rural. Dans son étude des rapports entre le discours romanesque et la réalité paysanne, Marie-Denise Shelton souligne que plusieurs romans consacrés au milieu rural décrivent les conséquences du « drame de la terre $»^{7}$ qui pèsent sur la vie du paysan haïtien : l'érosion des mornes, les catastrophes naturelles, l'iso-

7 C'est le titre aussi d'un roman de Jean-Baptiste Cinéas (Le drame de la terre, Cap-Haïtien, Imprimerie du Séminaire adventiste, 1933). 
lement, l'ignorance et la pauvreté $(1993,55)$. Parmi les romans qui abordent le thème du "drame de la terre », Gouverneurs de la rosée (1944) de Jacques Roumain occupe une place particulière. L'auteur y met en valeur l'importance de la solidarité entre les paysans et la nécessité du travail collectif (coumbite en créole haïtien) pour surmonter le drame économique et social. Il raconte les efforts des villageois pour trouver de l'eau afin de sortir d'une longue période de sécheresse et dénonce l'érosion des mornes provoquée par le déboisement massif pratiqué par les paysans eux-mêmes. Selon Malcom Ferdinand, Roumain est l'un des premiers écrivains « à avoir réussi à penser ensemble l'exigence de préservation des ressources naturelles avec les luttes pour l'égalité et la liberté d'un peuple » $(2015,70)$.

La pratique du travail collectif des paysans haïtiens réapparaît dans sa version contemporaine dans le roman post-sismique Les jardins naissent de Jean-Euphèle Milcé, publié en 2011. Dans ce court roman narré souvent sur un mode ironique, l'auteur met en relief un projet quasi utopique : la reconstruction de Port-au-Prince. Il imagine la coopération des habitants qui travaillent ensemble pour transformer la ville détruite en une " capitale-jardin » (Milcé 2011, 49), en faisant pousser des espaces verts au milieu des décombres. Parmi les principaux acteurs de cette tentative pour revitaliser la ville figurent Daniel, un Haïtien expulsé du Canada à cause de sa situation d'immigré irrégulier, et Marianne, une Française au service du CICR (Comité International de la Croix Rouge) suisse. Milcé met en valeur la part active des Haïtiens dans la reconstruction de leur pays, idée rarement acceptée dans les projets gérés par les ONG internationales. ${ }^{8}$ L'opération d'ensemencement des potagers, telle qu'elle est imaginée dans ce roman, montre la nécessité de donner aux Haïtiens un rôle de premier plan pour bâtir l'avenir du pays :

Le projet s'est mué en lutte adoptée et portée par des populations qui n'ont cessé de réclamer, en plus du droit de vote, celui de mettre leur grain de sel dans les plans du futur. (Milcé 2011, 98)

Des travaux récents en écocritique, en particulier ceux de Serenella Iovino et de Bruno Latour, montrent que le dépassement de la catastrophe dans le milieu urbain est possible si l'on adopte une perspective fondée sur une pensée 'bioéthique' qui intègre la mémoire de la ville et l'imagination littéraire. ${ }^{9}$ Milcé propose une solution quelque peu utopique pour la sortie de la paralysie après le séisme, qui n'est pas complètement désincarnée ou illusoire. Elle oppose à une approche technocratique et au concept paternaliste de 'résilience' une alternative

. Voir Iovino 2015, 2016 et Latour 2015. 
'verte', c'est-à-dire l'idée de renouer avec la tradition haïtienne des jardins créoles. ${ }^{10}$ Cette idée est mise en avant en faveur d'une approche environnementale qui allie la vie matérielle, sociale et politique de la ville avec son environnement. L'espace post-sismique de Port-au-Prince, celui d'une extrême désolation matérielle et humaine, se transforme en une 'biopolis' planifiée et mise en œuvre par les Haïtiens. La solution romanesque proposée par Milcé est en même temps ironique car elle dévoile une distorsion entre les projets humanitaires introduits de force par les ONG et les besoins réels du pays. En ce sens, en tissant des liens entre la conscience environnementale et l'esthétique littéraire, Les jardins naissent donne vie à un projet social qui incite à l'action et à l'évolution de la pensée écologique (Blanc, Chartier, Pughe 2008, 17).

\subsection{Cyclone et culture de résistance}

La représentation littéraire de la catastrophe naturelle peut aussi mettre en valeur le rôle de la résistance individuelle face au désastre, comme le montre le roman L'Île et une nuit (1995) de l'écrivain guadeloupéen Daniel Maximin, organisé autour d'un cyclone. ${ }^{11}$ Déjà connu et redouté par les Amérindiens, ${ }^{12}$ le cyclone ou l'ouragan, comme il serait plus approprié de l'appeler dans le contexte caribéen, ${ }^{13}$ est un phénomène météorologique sensiblement différent des éruptions volcaniques et des tremblements de terre, par le fait qu'il vient de l'extérieur (hors de l'île) et qu'il est perçu comme plus menaçant car d'origine inconnue. De plus, sa trajectoire est souvent imprévisible. Pourtant, le lien entre création et destruction, thème récurrent dans les œuvres de Maximin, appartient aussi à ce phénomène, comme le montre l'origine du mot 'ouragan', qui remonte aux peuples amérindiens du Mexique et de la Caraïbe. Les Maya et les Taïnos appelaient 'Huracan' un dieu créateur et, en même temps, maléfique car il avait le pouvoir de déchaîner de forts vents sur la terre et de provoquer des inondations (Emanuel 2005, 18).

10 Sur le savoir-faire des paysans haïtiens, notamment sur leurs techniques de mise en valeur du jardin créole, voir Lévy 2004, 80-1.

11 Il s’agit de 'Hugo' qui a frappé la Guadeloupe en 1989 (Britton 2010, 111).

12 «C'est précisément sous la plume du Découvreur Christophe Colomb que se trouve la première évocation de la plus fantasque des catastrophes naturelles - l'ouragan. Il s'agit, bien entendu, de cet 'huracan', en parler amérindien, qui s'opposa au dessein de l'Amiral de la Mer Océane de donner fond dans l'île promptement baptisée Dominique. C'était le dimanche 3 novembre de l'An 1493 » (Yacou 2007, 9).

13 «Les cyclones tropicaux prennent des noms différents selon la mer ou l'océan où ils naissent. On les appelle 'typhons' (typhoon en anglais) dans le Pacifique, 'Baguia' aux Philippines, willy-willies près des côtes de l'Australie, ou 'ouragans' (hurricanes en anglais) dans l'océan Atlantique nord et sur la mer des Caraïbes » (Chaboud, s.d.). 
Dans L'Île et une nuit, le cyclone peut difficilement être intégré au paysage guadeloupéen et il est décrit dans toute sa force aveugle et destructrice. ${ }^{14}$ Le personnage principal, Marie-Gabriel, se prépare à résister au désastre, enfermée dans sa vieille maison. La lutte entre la femme et le cyclone se déroule pendant une nuit, au cours de sept heures auxquelles correspondent sept chapitres. La tempête fait revivre des voix du passé qui appartiennent à des personnes lointaines, chères à Marie-Gabriel. La femme, seule face à la puissance déchaînée de la nature, fait appel à des éléments protecteurs qui créent une sorte de « communauté intertextuelle » (Britton 2010,123) :

les amis, les livres, Adrien, la mémoire de la naissance et de l'enfance, la musique, le conte et la légende. (Chaulet-Achour 2000, 51)

L'Île et une nuit est un roman de combat individuel où la résistance contre le malheur tient symboliquement dans le choix de Marie-Gabriel de rester dans sa maison et d'attendre l'arrivée du cyclone en solitaire, pendant « une veillée de survie » (Maximin 1995, 31). Malgré de nombreuses références à des catastrophes qui ont réellement eu lieu et bien que l'auteur se livre parfois à leur description, l'accent est mis sur le comportement humain face à l'événement et sur la résistance de l'individu mais aussi de toute la communauté atteinte par le cyclone :

il n'y avait d'humain à préserver dans tout cyclone que le naturel de la résistance des humains. (Maximin 1995, 93)

Il convient de souligner à cet égard que L'Île et une nuit se termine par une opposition qui, chez Maximin, signale plutôt une ambivalence, entre le désastre et la création, la destruction entraînée par l'ouragan et la résistance qui préfigure une reconstruction :

$\mathrm{Au}$ dehors, le cyclone déchaîné assurait les finitions de la catastrophe. Et pourtant, elle entendait déjà sourdre la résistance de l'île au travail sous le masque du désastre en cours. (Maximin 1995, 172)

Le cyclone est le symbole par excellence de la conception cyclique de la temporalité exprimée par Daniel Maximin dans son œuvre romanesque. La nature, en véritable actant du récit, impose ses rythmes et ne recule pas devant les aléas du contrôle de l'homme ; au contraire, elle 'se rebelle' périodiquement, comme le montrent les dernières saisons cycloniques, particulièrement violentes (voir Rozières 2017). En

14 Maximin remarque pourtant que la force destructrice du cyclone n'est pas forcément négative : "Ils ne font pas que de la destruction. Ainsi le cyclone abat les mauvais arbres, fait des coupes claires qui permettent de régénérer les forêts » $(2006,25)$ 
même temps, l'imaginaire apocalyptique qui parcourt le roman est remis en question par l'auteur à travers la représentation qu'il donne du phénomène naturel. En effet, chez Maximin le cyclone ne figure pas la fin du monde : au contraire, son passage s'insère dans la dynamique d'un éternel recommencement, donnant lieu à une vision de la Caraïbe qui rappelle celle de Benítez-Rojo, beaucoup plus proche de l'idée de 'chaos' que de celle d"apocalypse' (Britton 2010, 114).

\section{Enjeux sociopolitiques de la mise en fiction de la catastrophe}

Comme le remarque Peter Utz, la littérature peut remplir une fonction d'avertissement et dénoncer le comportement de l'homme, qui agit trop souvent en maître des lieux :

elle s'insurge contre une civilisation qui exclut la nature, incapable de la percevoir autrement que comme une menace hostile et catastrophique. ([2013] 2017, 18)

La littérature met aussi en garde contre les inégalités au sein d'une société, souvent préexistantes, qui deviennent encore plus évidentes après une catastrophe. Cette fonction d'avertissement qui revient à la littérature de la catastrophe est bien illustrée dans deux romans haïtiens, Les possédés de la pleine lune de Jean-Claude Fignolé et Cathédrale du mois d'Août de Pierre Clitandre, et un roman martiniquais, La Caldeira de Raphaël Tardon. Malgré les divergences dues aux pays de provenance des auteurs, aux désastres représentés et aux différentes esthétiques déployées, ces exemples montrent bien que la mise en fiction de la catastrophe peut avoir une dimension sociale.

\subsection{Catastrophes et esthétique 'spiraliste' dans le milieu rural et urbain haïtien}

Fignolé et Clitandre abordent de façon complexe le rapport entre une communauté et son environnement en ayant recours à une esthétique dite 'spiraliste'. ${ }^{15}$ Dans Les possédés de la pleine lune, publié en 1987, Fignolé met en scène l'univers mystérieux des Abricots, village de pêcheurs situé dans le sud-ouest d'Haïti. Dans cette « chro-

15 Selon ses principes, le rapport au réel repose principalement sur l'hybridation des genres, la fragmentation du récit et les techniques répétitives des contes qui créent une atmosphère onirique, voire surréaliste (Chemla 2015, 36). 
nique villageoise où la réalité et les mythes se confondent » (Chemla 2015, 38), l'écrivain décrit une succession de catastrophes d'origine humaine et naturelle. Parmi les catastrophes dues aux hommes, les faits réels et surnaturels se mêlent : la découverte d'un cadavre, la disparition d'un corps, mais aussi la présence menaçante d'une « bête à sept têtes ", monstre mythologique présent aussi dans le folklore caribéen qui, dans le texte, représente sur un mode allégorique la dictature duvaliériste (Parisot 2018, 167). Fignolé brosse le portrait de la vie paysanne et de ses difficultés, montrant l'immobilité des habitants face aux cataclysmes de toute espèce qui s'abattent sur le village et dévastent les récoltes :

Entre une récolte perdue et une autre à moitié dévastée par les rats ou par la sécheresse, par les averses ou par les sauterelles, nous végétons. (Fignolé 1987, 32)

Le village des Abricots est décrit comme un lieu

rongé par la pluie acide, calciné par une sécheresse, balayé par trois cyclones, décoloré par décision de la bête à sept têtes. (Fignolé 1987, 97)

Au milieu de cet univers apocalyptique, la terre, personnifiée, souffre comme les habitants du village. La pluie est accueillie comme un miracle par les paysans car elle signifie le retour à la vie et aux activités liées à la culture des champs. Pourtant, souvent les pluies trop abondantes provoquent des inondations meurtrières. Comme dans les contes narrés par plusieurs grand-mères qui apparaissent dans le roman, cyclones, sécheresses et épidémies se répètent dans un mouvement cyclique, symbole d'une spirale inéluctable d'où les paysans des Abricots semblent ne pas pouvoir sortir.

Dans Les possédés de la pleine lune, les catastrophes s'enchaînent et accablent l'existence des Haïtiens dans le milieu rural. De même, la menace de l'apocalypse caractérise aussi la vie dans le milieu urbain, en particulier dans les bidonvilles de Port-au-Prince, comme on peut le voir dans Cathédrale du mois d'Août de Pierre Clitandre, publié en 1980. Clitandre y raconte l'exode des paysans contraints à émigrer vers la capitale à cause de la centralisation des activités provoquée par une décision administrative. Yolaine Parisot considère que l'esthétique 'spiraliste' de Clitandre « abolit les frontières entre le cauchemar et la réalité » $(2018,176)$. En effet, l'univers représenté dans Cathédrale du mois d'Août est dominé par la présence menaçante de la fin du monde, préfigurée par le climat d'oppression qui règne sur le bidonville et par des désastres d'origine naturelle qui sont le miroir de la dégradation de tout un écosystème. Une isotopie de l'apocalypse balise le récit où plusieurs catastrophes se suc- 
cèdent : des épidémies de peste et des cycles de sécheresse, brusquement interrompus par des pluies torrentielles qui provoquent des inondations. Comme dans Les possédés de la pleine lune de Fignolé, chez Clitandre les pluies entraînent des victimes et des dégâts matériels dans le bidonville :

Il avait plu durant tout un jour sans arrêt et l'avalasse avait emporté vers la mer des lits aux ressorts brisés, des tôles rouillés, des planches de bicoques effondrées et autres objets hétéroclites, sous les cris angoissés des habitants de la zone. Le lendemain, sous un soleil de zombi jaloux, ces derniers découvrirent des cadavres d'hommes, de femmes et de chiens, obstruant la bouche d'égout et flottant sur une eau sale. Personne ne les reconnut ou les pleura. (Clitandre [1980] 2013, 19)

Après chaque avalasse, 'pluie torrentielle' en créole haïtien (Valdman, Iskrova 2007), des cadavres flottent sur l'eau boueuse et une odeur de mort se répand parmi les bicoques du bidonville ; les gens paniquent, convaincus qu'une épidémie de peste va bientôt éclater.

Si la représentation des catastrophes naturelles peut contribuer à une illustration, sur un ton apocalyptique et sur un mode allégorique, des existences écrasées par la répression politique et la dégradation de l'environnement, elle peut aussi faire revivre, a posteriori, des conflits sociaux qui peinent à disparaître, comme le montre le Martiniquais Raphaël Tardon dans son roman La Caldeira.

\subsection{Chronique d'un désastre politique et social}

Dans La Caldeira, publié en 1948, l'éruption de la Montagne Pelée, survenue en 1902, est un élément central de la narration. Le roman, malheureusement oublié par la critique, présente la lutte électorale de 1902 où les Blancs affrontent les Mulâtres. ${ }^{16}$ Loin d'être une simple description des derniers jours de la ville de Saint-Pierre, ancienne capitale de la Martinique, et de ses habitants, La Caldeira est un récit aux fortes implications morales. L'auteur y décrit le préjugé de couleur ainsi que l'éternelle lutte entre les Mulâtres et les Békés (Blancs descendant des familles des premiers colons), en partie responsables de la mort des Pierrotins, coincés dans Saint-Pierre à cause du second tour des élections législatives du 11 mai 1902 - date qui sous aucun prétexte ne pouvait être repoussée. Tardon dénonce ainsi la sottise

16 Les élections législatives opposaient le planteur Fernand Clerc (Parti républicain progressiste), Louis Percin (Parti radical socialiste) et Joseph Lagrosillière (Parti socialiste ouvrier). Voir Ursulet 1997, 121-48. 
cachée derrière la rivalité politique et sociale qui est à l'origine de la décision des autorités de ne pas évacuer la ville. Le message de l'auteur est clair : la responsabilité humaine est évidente car les autorités auraient pu éviter la catastrophe humaine. Dans La Caldeira, une description détaillée du déroulement de la catastrophe alterne avec la représentation de la société de Saint-Pierre, de ses vices et de son racisme, visibles derrière certains aspects de la vie quotidienne. L'éclatement de la Montagne Pelée est décrit par l'auteur à travers une narration presque cinématographique où le suspense joue un rôle central : après une succession de signes avant-coureurs et de décès mystérieux aux teintes grand-guignolesques, le volcan écrase Saint-Pierre avec toute sa puissance. L'effet de cette technique de retardement, à l'instar d'un disaster movie hollywoodien, est la sidération face à une catastrophe qui ne devait pas se produire avec une telle ampleur. L'investissement du roman dans le social est aussi manifeste : la catastrophe met fin à la hiérarchie traditionnelle, elle nivelle les différences et rend tous les individus identiques. D'où des effets d'humour noir qui mettent en pièces toute distinction de race :

Adieu Saint-Pierre, lumière des Antilles. De toutes les Antilles, quarante mille personnes ensevelies sous les décombres, surprises dans la rue, ou projetées loin en mer sous le choc de la nuée, tel était le tribut électoral payé à la mort. Un charnier pour un siège au Parlement. La mort gagnait sur tous les tableaux. C'était elle la triomphatrice de la bagarre politique et raciale. Pour le prouver d'irréfutable façon, elle étendit dans les rues, sur la Savane, sur le boulevard, trois mille cadavres. [...] Pas un de plus ; sur les quarante mille qu'elle emporta. Aucun de ces cadavres ne put être identifié : ils étaient tous noirs, scalpés, épilés, défigurés; sans nez, sans lèvres, et nus. Blancs, octavons, quarterons, hybrides, mulâtres, chabins, câpres, nègres, ils étaient tous noirs, carbonisés, uniformément noirs, et sans la moindre caractéristique ethnique. (Tardon [1948] 1977, 293-4)

Comme le souligne Jack Corzani $(1999,96)$, Tardon mentionne de façon méticuleusement ironique les catégories établies sur une base prétendue scientifique par l"hématologie raciste' à partir de Moreau de Saint-Méry. ${ }^{17}$ Or le racisme, inhérent à la société de Saint-Pierre, survit après la catastrophe. Dans la dernière scène du roman, le $\mathrm{Mu}$ lâtre Perrier rend visite à son demi-frère Joubert à l'hôpital de Fortde-France, mais il est toujours considéré comme un intrus par son père blanc. Le roman de Tardon, qui place la responsabilité humaine au centre de la catastrophe, se termine sur une note pessimiste car 
l'éruption destructrice n’a pas réussi à briser le préjugé de couleur. Cette conclusion de Tardon est confirmée par les sources documentaires, parmi lesquelles le témoignage de Césaire Philémon :

Beaucoup de gens croient naïvement que l'horrible catastrophe du 8 mai 1902 entraînera - entre autres conséquences - la disparition du stupide préjugé à cause des affreux malheurs subis en commun par tous les enfants du pays. - Erreur. $(1930,126)$

\section{$5 \quad$ Exprimer le retour du refoulé}

Après la catastrophe et déclenchés par celle-ci, des souvenirs tapis au fond de la mémoire peuvent remonter à la surface. Dans les romans que nous avons choisis pour illustrer cette fonction de la catastrophe, L'Espérance-macadam de Gisèle Pineau et Absences sans frontières d'Évelyne Trouillot, il s'agit d'épisodes traumatiques liés à l'enfance des personnages ou de secrets de famille inavouables. Le désastre fait ainsi revivre un passé refoulé que l'on croyait à jamais oublié.

\subsection{Un cyclone refoulé}

Les catastrophes naturelles sont souvent les déclencheurs de l'action dans l'œuvre de Gisèle Pineau, auteure d'origine guadeloupéenne née à Paris. L'Espérance-macadam, publié en 1995, s'ouvre par la description du paysage guadeloupéen après le passage du cyclone 'Hugo', en 1989. Le même événement est repris à la fin du roman, conformément au mouvement circulaire qui rappelle la nature périodique du cyclone. Parallèlement, un autre cyclone est évoqué tout au long de la narration : celui qui a ravagé la Guadeloupe en 1928. La protagoniste, Éliette, n'a que huit ans quand le cyclone de 1928 s'abat sur sa maison. Tout ce qu'elle sait de cet événement lui a été raconté par sa mère Séraphine, qui lui en a parlé pendant des années pour dissimuler une vérité qui porte sur le passé de la famille. En effet, l'histoire racontée par Séraphine contient beaucoup de non-dits au sujet du « Passage de La Bête », selon l'expression dont elle se sert pour nommer la catastrophe. Celle-ci a laissé un « grand tourbillon » (Pineau 1995, 25) dans la tête d'Éliette et a rendu folle Séraphine. Éliette reste plongée dans le silence pendant trois ans après le cyclone et retrouve peu à peu sa voix grâce à la relation qu'elle noue avec le nouveau compagnon de sa mère. Devenue adulte, elle ne parvient pas à se souvenir de cette nuit ensevelie dans les « décombres de sa mémoire » (Pineau 1995, 126) et vit dans un « passé-toujours-présent » (64), enfermée dans son trauma.

En réalité, l'évocation du cyclone de 1928 est un subterfuge dont se sert Séraphine pour masquer la violence perpétrée par son com- 
pagnon sur leur fille Éliette. Cette superposition du phénomène naturel et du viol se répète dans l'histoire racontée par Angela, jeune fille qui trouve protection chez Éliette après avoir dénoncé son père Rosan, qui abusait d'elle depuis des années :

Combien de fois s'était-il jeté pis qu'un cyclone sur le corps d'Angela. Bête sauvage ! (Pineau 1995, 252)

Quand Éliette entend l'histoire d'Angela, les souvenirs traumatiques liés au viol qu'elle a subi en 1928 remontent à la surface. Soixante ans après, elle comprend que la poutre qui l'a traversée quand elle avait huit ans " avait un visage, des yeux, des dents longues » (Pineau $1995,219)$ et qu'il s'agissait de son père biologique, surnommé Ti-Cyclone dans son enfance. À cause de ses blessures, Éliette ne peut pas avoir d'enfants et son désir de maternité, amputé par la violence du père, va naître seulement avec l'arrivée d'Angela.

Régine Jean-Charles explique que le passage de 'Hugo' en 1989, la dévastation et le trauma qu'il a occasionnés ont permis à Pineau de trouver un lexique capable de décrire la brutalité du viol et de l'inceste $(2014,101-2)$. Pineau a ainsi pu faire un parallèle entre les effets du viol sur le corps de la femme et les conséquences du passage du cyclone sur le paysage. Pour Éliette, après soixante ans passés « avec un cyclone niché en dedans d'elle » (Pineau 1995, 280), 'Hugo' signifie une délivrance. Les pluies torrentielles qui accompagnent le cyclone sont vécues comme une purification pour le corps et l'âme de la femme violée qui se dresse face aux vents menaçants et se met à danser, emportée par le son des tambours provenant d'une des cases du quartier. Juste quelques heures avant l'arrivée de 'Hugo', Éliette rend visite à sa marraine, qui connaît toute la vérité au sujet de la violence subie par la femme en 1928. La marraine refuse de raconter l'épisode du viol, consciente du fait qu'Éliette a désormais accès à la vérité :

Éliette, ma fille, tu connais déjà toute l'histoire. Laisse aller ce cyclone et comprends que la vie n'est pas une rumination éternelle. Il y aura d'autres cyclones, quantités. Et personne peut rien contre ça. [...] Et il faudra bien rester par en bas et puis se relever, rebâtir, panser les plaies, regarder pour demain l'espérance et replanter toujours, l'estomac accoré par la faim (Pineau 1995, 297).

La marraine réaffirme le caractère circulaire du cyclone et la nécessité de reconstruire, à chaque fois, après la destruction. Ses mots semblent vouloir souligner que la violence de la nature, comme celle perpétrée par l'homme, n'a pas de fin et revient toujours. 


\subsection{Le séisme comme catharsis}

La thématique des secrets de famille dévoilés par la catastrophe naturelle est aussi abordée par l'écrivaine haïtienne Évelyne Trouillot dans Absences sans frontières, publié en 2013. Le roman raconte la vie de Gérard, qui a quitté Haïti pour New York en situation illégale, et son rapport avec sa fille de dix-sept ans, Géraldine, restée à Portau-Prince. Gérard l'assiste de loin, en lui envoyant de l'argent, mais il participe aussi à sa formation culturelle. Il n'a jamais vu sa fille car il est parti avant sa naissance, et leur relation s'est soudée uniquement à distance. Moline, la mère de Géraldine, est décédée et l'enfant a grandi avec sa grand-mère Gigi et la sœur de celle-ci, Tanza.

Trouillot aborde la question des effets du séisme de janvier 2010 sur l'individu à travers le personnage de Gigi ; la catastrophe frappe intimement la vieille femme et fait remonter à la surface des souvenirs anciens. Ce processus est déclenché par une nouvelle bouleversante : le séisme modifie brusquement la situation de Gérard car, grâce à un changement dans la politique d'immigration aux ÉtatsUnis, les Haitiens peuvent demander le TPS (Temporary Protected Status) et rentrer librement en Haïti. La possibilité du retour de Gérard, dix-neuf ans après son départ, signifie aussi le retour d'un passé refoulé fait de mensonges et de dissimulation. Gigi a des cauchemars qui font surgir une vérité inavouable sur la naissance de Géraldine. La catastrophe naturelle qui s'abat sur le pays est doublée d'un drame intime dont Gigi est la victime principale :

Tout annonçait un autre séisme, quelque chose de profond, de dangereux et d'immuable, auquel elle ne pourrait pas échapper. (Trouillot 2013,151)

À travers l'évocation des souvenirs de Gigi, l'on apprend que sa fille, Moline, avait fait une fausse couche dont Gigi n'avait jamais parlé à Gérard de crainte qu'il arrête d'envoyer de l'argent à la famille pour financer les études de Géraldine. Qui plus est, Gigi a sournoisement contraint Moline à coucher avec un homme amoureux de Tanza. Gérard ignore qu'il n'est pas le père biologique de sa fille, née de cette rencontre fortuite organisée par Gigi. Le séisme va précipiter la révélation de ce secret. Gigi, rongée par les remords à des années de distance, meurt quelques jours avant le retour de Gérard,

avec son secret intact et jamais avoué, forte et ambitieuse, désireuse que sa fille et sa petite-fille échappent à la misère qu'elle avait connue enfant et dans sa jeunesse. (Trouillot 2013, 238-9)

Tanza raconte la vérité à Géraldine qui comprend avoir grandi au milieu des mensonges. Dans Absences sans frontières, le tremblement 
de terre joue un rôle de déclencheur de souvenirs tapis dans la mémoire et remis en vie. Il joue aussi un rôle cathartique car la quête identitaire, circonscrite par l'urgence, rend possible la reconquête de la dignité et une présence au monde au-delà des frontières.

\section{Conclusion}

Dans notre parcours à travers un corpus hétérogène comprenant des romans d'auteur.e.s haïtien.n.e.s, guadeloupéen.n.e.s et martiniquais, nous avons présenté plusieurs exemples d'écriture de la catastrophe issus du « méta-archipel » caribéen, en focalisant en focalisant notre attention en particulier sur des romans en langue française. En évoquant les travaux théoriques d'Antonio Benítez-Rojo, Édouard Glissant et Daniel Maximin, nous avons signalé les liens entre la géographie et la littérature et entre l'aspect destructeur du désastre et la résistance qui s'organise immédiatement après. En abordant la question de l'écriture de la catastrophe naturelle aux Antilles françaises et en Haïti, nous avons souligné l'importance de la conception cyclique du temps et de l'histoire qui sous-tend la pensée caribéenne ; il s'agit d'un dispositif spatiotemporel imposé par la géographie (isolement réciproque des pays caribéens à cause de leur situation insulaire) et par les circonstances historiques (héritage commun de la plantation esclavagiste).

En guise de conclusion, nous voudrions mentionner un roman où le « méta-archipel » caribéen s'ouvre au monde. Il s'agit d'En attendant la montée des eaux de l'écrivaine guadeloupéenne Maryse Condé, paru en 2010. L'action du roman se déroule dans différentes régions de la planète, d'abord en Afrique où est né le protagoniste, le docteur Babakar Traoré, jusqu'à la Caraïbe - Guadeloupe et Haïti - en passant par le Canada et le Moyen Orient. En se référant à de nombreux désastres à l'échelle globale, Condé veut mettre en avant une " solidarité cosmopolite » où s'articule une pensée sur la souffrance partagée par plusieurs pays (Brant 2015, 57-63). La structure 'à spirale' du roman rappelle, selon Daniel Brant, la conception cyclique de la temporalité élaborée par Daniel Maximin dans sa trilogie guadeloupéenne $(2015,61)$ mais aussi l'esthétique 'spiraliste' des Haïtiens Jean-Claude Fignolé et Pierre Clitandre et la répétition cyclique de la violence de l'homme et de la nature illustrée par Gisèle Pineau. La vision transcaribéenne et, plus généralement, transnationale du désastre qui est celle de Maryse Condé rappelle la démarche que nous avons entreprise pour montrer que la mise en fiction de la catastrophe naturelle, dans le roman caribéen d'expression française, peut s'articuler autour de quelques axes communs : les enjeux éthiques et politiques de la représentation du désastre, la mise en place d'une résistance individuelle ou collective et la remémoration d'événements traumatiques. 


\section{Bibliographie}

\section{Corpus d'analyse}

Clitandre, P. [1980] (2013). Cathédrale du mois d'Août. Port-au-Prince : Éditions Ruptures.

Fignolé, J.-C. (1987). Les possédés de la pleine lune. Paris : Seuil.

Maximin, D. (1995). L'île et une nuit. Paris : Seuil.

Milcé, J.-E. (2011). Les jardins naissent. Montréal : Éditions les 400 coups.

Pineau, G. (1995). L'Espérance-macadam. Paris : Stock.

Tardon, R. [1948] (1977). La Caldeira. Fort-de-France : Desormeaux.

Trouillot, É. (2013). Absences sans frontières. Montpellier : Éditions Chèvrefeuille étoilée.

\section{Corpus secondaire}

Carpentier, A. (1962). Le siècle des lumières. Paris : Gallimard.

Collins Klobah, L. (2011). The Twelve-Foot Neon Woman. Leeds : Peepal Tree Press.

Hutchinson, I. (2016). House of Lords and Commons. New York: Farrar, Straus and Giroux.

Markham, E.A. (2008). Against the Grain : A 1950s Memoir. Leeds : Peepal Tree Press.

Yanique, T. (2014). Land of Love and Drowning. New York : Riverhead Books.

\section{Critique}

Anderson, M.D. (2011). Disaster Writing: The Cultural Politics of Catastrophe in Latin America. Charlottesville : University of Virginia Press.

Barthélémy, G. (2000). Créoles-Bossales : conflit en Haïti. Petit-Bourg : Ibis Rouge.

Benítez-Rojo, A. [1989] (2001). The Repeating Island. The Caribbean and the Postmodern Perspective, Second Edition. Durham ; London : Duke University Press.

Blanc, N. ; Chartier, D. ; Pughe, T. (2008). « Littérature \& écologie : vers une écopoétique ». Écologie et politique, 36(2), 15-28.

Brant, D. (2015). Geographies of Suffering : the Literature of Catastrophe in the Francophone Caribbean [PhD thesis]. Urbana-Champaign : University of Illinois at Urbana-Champaign.

Britton, C. (2010). The Sense of Community in French Caribbean Fiction. Liverpool : Liverpool University Press.

Chaboud, R. (s.d.). « Cyclones tropicaux ». Encyclopædia Universalis. http:// www.universalis.fr/encyclopedie/cyclones/.

Chaulet-Achour, C. (2000). La trilogie caribéenne de Daniel Maximin. Analyse et contrepoint. Paris: Karthala.

Chemla, Y. (2015). Littérature haïtienne 1980-2015. Delmas : C3 Éditions.

Corzani, J. (1999). «La fortune littéraire de la 'catastrophe de Saint-Pierre': entre commémoration et mythification: le jeu des idéologies ». Yacou, A. (éd.), Les catastrophes naturelles aux Antilles. D'une Soufrière à l'autre. Paris: Karthala, 75-97. 
Dash, J.M. (1998). The Other America: Caribbean Literature in a New World Context. Charlottesville: University of Virginia Press.

Deckard, S. (2016). «The Political Ecology of Storms in Caribbean Literature ». Campbell, C.; Niblett, M. (eds), The Caribbean : Aesthetics, World-Ecology, Politics. Liverpool : Liverpool University Press, 25-45.

Deleuze, G. ; Guattari, F. (1980). Capitalisme et schizophrénie 2 : Mille plateaux. Paris : Minuit.

Emanuel, K. (2005). Divine Wind. The History and Science of Hurricanes. New York: Oxford University Press.

Ferdinand, M. (2015). «La littérature pour penser l'écologie postcoloniale caribéenne ». Multitudes, 60(3), 65-71.

Fombrun, O.R. (1992). L'Ayiti des Indiens (analyses et textes d'historiens). Portau-Prince : Fondation 92.

Fouchard, J. (1972). Langue et littérature des aborigènes d'Haïti. Paris : Éditions de l'école.

Glissant, É. (1990). Poétique de la relation. Paris : Gallimard.

Iovino, S. (2015). Ecologia letteraria. Una strategia di sopravvivenza. Milano: Edizioni Ambiente.

Iovino, S. (2016). Ecocriticism and Italy. Ecology, Resistance, and Liberation. London : Bloomsbury Academic.

Jean-Charles, R.M. (2014). Conflict Bodies: The Politics of Rape Representation in the Francophone Imaginary. Columbus: Ohio State University Press.

Latour, B. (2015). Face à Gaïa : huit conférences sur le nouveau régime climatique. Paris : La Découverte.

Lévy, J.J. (2004). Entretiens avec Georges Anglade. L'espace d'une génération. Montréal : Liber.

Maximin, D. (2006). Les fruits du cyclone: une géopoétique de la Caraïbe. Paris : Seuil.

Moreau de Saint-Méry, M.L.E. (1797). Description topographique, physique, civile, politique et historique de la partie française de l'isle Saint-Domingue, tome 1. Philadelphie : chez l'auteur.

Parisot, Y. (2018). Regards littéraires haïtiens. Cristallisations de la fiction-monde. Paris : Classiques Garnier.

Philémon, C. (1930). Galeries Martiniquaises : population, mœurs, activités diverses et paysages de la Martinique. Paris: Ateliers Printory.

Rozières, G. (2017). « Ouragans Maria, Harvey, Irma, Jose... la faute au réchauffement climatique ? C'est plus compliqué que ça... ». Le Huffington Post. http://www.huffingtonpost.fr/2017/09/20/ouragans-maria-harvey-irma-jose-la-faute-au-rechauffement-climatique-cestplus-complique-que-ca_a_23215989/.

Schuller, M. (2016). Humanitarian Aftershocks in Haiti. New Brunswick : Rutgers University Press.

Shelton, M.-D. (1993). Image de la société dans le roman haïtien. Paris : L'Harmattan.

Shelton, M.-D. (2015). Éloge du séisme: le tremblement de terre en littérature. E-book. Paris : L'Harmattan.

Simasotchi-Bronès, F. (2020). « Roman francophone caribéen et pensée écologique ». Gauvin, L. ; Fonkua, R. ; Alix, F. (éds), Penser le roman francophone contemporain. Montréal : Les Presses de l'Université de Montréal, 280-93.

Turcotte, V. (2010). Lire l'altérité culturelle dans les textes antillais. Québec: Presses de l'Université du Québec. 
Alessia Vignoli

Les catastrophes naturelles dans les romans caribéens d'expression française

Ursulet, L. (1997). Le Désastre de 1902 à la Martinique : l'éruption de la Montagne Pelée et ses conséquences. Paris : L'Harmattan.

Utz, P. [2013] (2017). Culture de la catastrophe. Les littératures suisses face aux cataclysmes. Carouge : Éditions Zoé.

Valdman, A. ; Iskrova, I. (eds) (2007). Haitian Creole-English Bilingual Dictionary. Bloomington : Indiana University.

Yacou, A. (2007). «Introduction ». Jno-Baptiste, P. ; Yacou, A. (éds), Les risques majeurs aux Antilles: approche culturelle et prévention sociale. Paris : Karthala, 9-10. 



\title{
La critique environnementale dans les récits animaliers de René Maran
}

\author{
Lia Milanesio \\ Università Ca' Foscari Venezia, Italia
}

\begin{abstract}
This article aims at analysing René Maran's five animal novels. In these texts, Maran criticizes the colonial system not only for its cruelty to the native population, but also for its ecological violence against the bush and its non-human inhabitants. In particular, this research will be focusing on the author's ability to abandon a human (and colonial) point of view in order to adopt an animal one. On the one hand, this new subjectivity - as well as Maran's comprehension of indigenous naturalist society - allows the writer to condemn the colonial period from an ecocritical perspective. On the other hand, it provides evidence of the existence of culture among the beasts of his novels. Finally, this article will also prove that it is thanks to their culture that Maran's animals will try to resist the colonial-centred environment and ideology.
\end{abstract}

Keywords Environmental Humanities. Animal Studies. Postcolonial Studies. Francophone Literature. René Maran.

Sommaire 1 Introduction. - 2 Le point de vue des bêtes de la brousse. - 3 La société naturaliste et la société occidentale. - 4 Le rôle de la culture animalière pour se défendre des colons. -5 Conclusion.

$\begin{array}{lll} & \text { Peer review } \\ & & \\ \text { Edizioni } & \text { Submitted } & 2020-08-12 \\ \text { Ca'Foscari } & \text { Accepted } & 2020-09-25 \\ & \text { Published } & 2020-12-22 \\ & \text { Open access } & \end{array}$

(ㄷ) 2020 | () Creative Commons Attribution 4.0 International Public License 


\section{Introduction}

René Maran est le premier écrivain qui dénonce et critique le système colonial français. Il présente les aspects les plus brutaux de la colonisation dans ses nombreux romans, essais et poèmes. Dans cet article, il s'agira de valoriser un aspect important de ses romans animaliers, qui n'a pas suscité l'attention des critiques : la présentation de la violence exercée sur le système naturel de l'Oubangui-Chari de 1927 à 1953, sur les animaux de la brousse et sur l'environnement. L'exploitation des territoires colonisés se cache derrière la 'mission civilisatrice', dont le but est de justifier la colonisation à travers la prétendue amélioration de la condition des populations indigènes, sous l'aspect culturel, technologique, religieux et également agricole. Il s'agira, donc, de réinterpréter cet assujettissement colonial que l'auteur nous décrit sous la forme d'une dégradation de la brousse (Graham, Tiffin 2015).

La vision écologique de Maran est liée à sa prise de notes sur le terrain : c'est un homme de lettres, avide de savoir, curieux envers toute nouveauté qui se présente à lui. Avec précision, il étudie les us et coutumes des populations indigènes qu'il rencontre lors de son séjour en Afrique. De plus, il se consacre à l'observation et à l'annotation des comportements des animaux et des liens existant entre eux, les hommes et le milieu naturel. Dès lors, l'utilisation de l'espace africain comme argument pour souligner de façon originale et tragique l'oppression de la colonisation française résulte de son « devoir d'écrivain français » (Maran 1938, 24) et de sa passion pour l'étude. Djouma chien de brousse (1927), Le Livre de la brousse (1934), Bêtes de la brousse (1941), Mbala l'éléphant ([1947]) et Bacouya, le cynocéphale (1953) : voilà autant de livres de Maran animés par la description de la violence sur l'environnement.

L'article s'articule en trois parties. La première se penchera d'abord sur la question du roman colonial. Il s'agit de comprendre si les œuvres de Maran appartiennent ou non à ce genre. Ensuite, en analysant le point de vue des bêtes dans les récits animaliers, il s'agira de comprendre comment le racisme environnemental des Européens se dessine et comment René Maran donne une vision globalisante des colonies dans ses récits. La deuxième section sera consacrée au concept de société naturaliste, d’impérialisme environnemental et enfin de trauma social vécu par les animaux. La troisième partie mettra en exergue l'utilisation de la culture chez les animaux comme outil contre la domination coloniale et comme gardienne des leurs identités. En particulier, il s'agira d’analyser comment René Maran décrit l'apprentissage de cette culture et de quels éléments elle se nourrit. 


\section{Le point de vue des bêtes de la brousse}

La première question à laquelle cet article entend répondre est : à quel genre les romans de René Maran appartiennent-ils ?

Il faut d'abord esquisser les caractéristiques du roman colonial. La langue de ce type de roman est marquée par l'étrangeté' : l'on trouve des emprunts, des approximations et des calques des langues indigènes (griot, tam-tam, case, balafon). L'utilisation d'éléments exotiques est, d'une part, un choix de l'auteur pour représenter le monde des colonies afin de charmer son lecteur avec des images lointaines. D'autre part, ce type d'éléments reflète l'idéologie des lecteurs du temps (XIXe siècle) qui est imprégnée des motifs et des préjuges de la propagande coloniale. Le résultat est donc une image pittoresque et déformée du monde indigène, où les Noirs apparaissent sauvages, belliqueux, menteurs, bestiaux (Maran 1927, 67-8) et où le noyau de la narration est la justification de la mission colonisatrice de l'Empire français.

Or, il faut prendre en considération trois aspects pour saisir l'identité des romans de Maran : le style de l'écriture, les connaissances et les intentions de l'auteur, ses personnages.

Premièrement, la langue de Maran est littéraire et érudite. Dans Hommage à René Maran (1965), René Violaine félicite son ami pour la " pureté de style » (Senghor et al. 1965, 33) de ses romans et Maran lui répond que la « belle langue » française est sa passion et « un de [s]es dadas » (33). Le style élevé est accompagné par une présence rare du français tirailleur et de mots indigènes. En effet, l'auteur enrichit la vraisemblance de ses romans par des éléments de réalisme tels que des mots comme nini, mata, yabao, dène-dzam, boundjou, ${ }^{1}$ véritables expressions des populations indigènes. La langue française s'enrichit donc du lexique africain afin de traduire efficacement le cadre culturel du récit. Ce qui différencie les romans de Maran des romans coloniaux est la représentation des colonies faite d'un point de vue inspiré par une meilleure compréhension de la vie des indigènes (Irele 2014, 435). Dans les romans coloniaux, les colonisateurs sont ceux qui donnent à la narration leur point de vue. Au contraire, dans les romans de René Maran, l'auteur donne une représentation culturelle et sociale la plus précise possible. Cela détermine un changement du point de vue qui passe des colons aux bêtes de la brousse douées de parole et qui racontent la vraie Afrique coloniale. Ainsi, René Maran est un auteur qui s'éloigne de la tradition coloniale et donne naissance au roman africain francophone (cf. Irele 2014, 427-45).

1 Il s'agit d'imprécations en langue banda et du mot utilisé par les indigènes pour se référer aux Blancs, 'boundjou', qui sont utilisés par Maran dans ses romans. Cf. Maran $1941,170-2 ; 1927,54-7,77,94 ;[1947], 51-5$. 
Par ailleurs, Pierre Mille écrit dans son article publié dans Le Temps en 1909 qu' $^{\prime}$

[u]ne œuvre de littérature coloniale [...] serait celle qui eût été produite dans un pays où [...] un de ces Européens qui [...] y aurait vécu les seules années où l'on possède une sensibilité, où on pénètre dans leur essence la nature et les hommes. (Mille 1909)

Mille souligne l'authenticité du contenu des romans coloniaux. En effet, René Maran explique dans la préface de Batouala (1938) que son but est la représentation de la vérité objective : aboutir au récit de ce qu'il a vu et vécu, et dénoncer les «abus, [les] malversations et [les] atrocités qui [...] abondent » (Maran 1938, 18) dans les pays colonisés. Pour que ses romans soient « aussi exactement que possible, la reconstruction de la vie » (Senghor et al. 1965, 131) de brousse, Maran se documente sur le monde indigène et naturel de manière minutieuse.

Enfin, la focalisation interne et les dialogues entre personnages noirs et blancs sont rares. Cela permet à Maran d'éviter de mettre en opposition le français de France des fonctionnaires et le petit noir des indigènes tel que le font les écrivains des romans exotiques. Dans les romans coloniaux de René Maran, quand les Noirs parlent, ils le font dans un français courant et correct, à l'exception des tirailleurs sénégalais. René Maran choisit de faire parler ses personnages principaux, les bêtes, dans un français standard. Ce faisant, les discours de dénonciation paraissent, sur le plan langagier, aussi dignes d'autorité que les discours de légitimation coloniale.

Les récits animaliers donnent au lecteur la possibilité d'accéder à un monde nouveau : celui des bêtes de la brousse de l'Oubangui-Chari. Dans ces récits présentés à la troisième personne par un narrateur externe, les instances dialogiques sont importantes : elles se présentent sous forme de discours directs et de discours rapportés donnant accès aux pensées et aux réflexions de la faune. Djouma chien de brousse (1927) est le premier roman colonial animalier qui se focalise sur le milieu canin. C'est dans les romans suivants que Maran présente l'ensemble du royaume animalier. C'est à travers la polyphonie des voix des animaux, trait formel qui se présente en premier lieu dans Bêtes de la brousse (1941), que l'auteur dresse une vision externe sur les rapports entre les colons et les colonisés et sur la dégradation environnementale. Au chien, s'ajoutent les cynocéphales, les phacochères, les lions, les rhinocéros, les pythons, les charognards, les fourmis, les coqs, les hippopotames, les buffles, les éléphants, les caïmans et les panthères.

Pour comprendre comment se construit la dénonciation de la colonisation dans les romans animaliers, il faut expliquer les sujets impliqués dans l'écriture de René Maran : l'auteur/narrateur, le lec- 
teur et les héros. D'abord, le narrateur externe des romans subit l'influence de l'auteur : René Maran est un homme de culture occidentale et un officier colonial qui croit au pouvoir civilisateur de la colonisation. Sa position ressort dans l'utilisation de formules et commentaires qui indiquent la sympathie et la proximité de Maran envers les bêtes et les Noirs. Le narrateur commente donc et utilise des mots qui reflètent l'ambiguïté d'un homme qui croit au système dont il fait l'expérience, mais dont il saisit le mauvais fonctionnement. En outre, le lecteur idéal est le public de langue française influencé par la culture coloniale et les officiers coloniaux. Cela implique un auditoire favorable à la colonisation. La stratégie de Maran est donc d'introduire des animaux qui soutiennent d'abord la colonisation, mais qui, après un parcours d'éveil, prennent conscience des faits. Page après page, le lecteur fait l'expérience de l'inefficacité de la domination française et développe une pensée critique sur l'empire colonial. Enfin, les héros : les victimes du système colonial. Il s'agit de l'ensemble de la faune, une communauté externe par rapport aux groupes sociaux impliqués dans la colonisation, les Européens et les Africains. La voix des Blancs est complètement effacée de la narration : René Maran élimine les mots de la classe dominante qui sont élitistes, éternels et, n'admettant pas un échange d'idéaux, éteignent la lutte sociale (Volosinov 1980). Apparemment, les animaux ne présentent qu'une vision partielle des sujets impliqués dans la période coloniale. En réalité, leur polyphonie donne voix aux points de vue multiples des colonisés, de la nature et de l'auteur ; ce dernier représente le colon philanthrope. Ainsi, d'une représentation fragmentée et partielle, Maran donne une vision globalisante et critique du monde colonial. En outre, la polyphonie des bêtes de la brousse sert à protéger l'auteur de ses accusations envers le système colonial : il prend ses distances et n'assume pas la responsabilité des critiques présentées. En effet, le point de vue du narrateur/auteur s'exprime à travers les consciences des personnages animaliers. La narration à la troisième personne et l'utilisation du discours direct et indirect permettent de limiter la présence de l'auteur et d'aboutir à un certain degré de mimesis. René Maran utilise des formules comme 'telles étaient les pensées de...' ou 'ces réflexions faites...' pour introduire ou pour achever les dialogues intérieurs des personnages présentés sous forme de discours rapportés. Ces types de dialogues montrent les deux idéologies qui cohabitent dans l'animal : les valeurs de la classe dominante (les colons) et celles de la classe faible (les bêtes). En particulier, les personnages de Bacouya et de Doppélé expriment un clivage entre ces deux voix. Dès lors, la classe dominante s'impose non seulement de façon physique sur le territoire, mais aussi de façon idéologique. La prise de conscience des aspects négatifs du système colonial se réalise parfois comme dialogue sous forme de question/ réponse à une seule voix. L'animal dresse une confrontation entre la 
culture coloniale avec ses valeurs et ses stéréotypes et la réalité de la brousse dans laquelle il vit (Maran 1953, 14-17). Le personnage découvre l'absurdité et l'incompatibilité des principes occidentaux avec son idéologie de la brousse et retrouve son équilibre intérieur grâce à l'harmonie entre ses pensées et l'idéologie de la classe animalière à laquelle il appartient.

Ainsi, l'auteur met en scène la relation de force entre les colonisateurs et l'espace colonisé en donnant une représentation spatiale du conflit culturel et environnemental. Dans les terres colonisées, une des problématiques qui ressort est le nombre des habitations des colons qui augmente, ce qui enlève de l'espace vital aux animaux, aux plantes et aux indigènes. Le paysage change et avec lui la vie de ses habitants. René Maran donne voix à la société de la brousse qu'on peut définir comme 'société naturaliste' (Dounias 2007) et dont il saisit l'essence : l'harmonie existante entre chaque élément de la brousse. Grâce à cette intuition, René Maran décrit la faune comme à la fois la victime de la violence coloniale et le miroir des sociétés indigènes qui subissent les exactions de ce système : pour les deux, il s'agit d'esclavagisme et d'exploitation. Les animaux de l'auteur ont le pouvoir de montrer le clivage culturel et en même temps de montrer que chaque action venant de l'homme provoque des conséquences directes sur l'environnement. De même, la question écologique dont les bêtes sont les porte-parole constitue un argument supplémentaire contre l'oppression coloniale.

René Maran met en exergue, d'une part, la profonde connaissance du territoire de la part des indigènes et, d'autre part, le désintérêt des Occidentaux envers l'habitat. Cette insouciance amène à la méconnaissance du monde indigène : les colons font des choix erronés qui mettent en danger les hommes noirs et leur environnement.

Eh bien, ils [les Noirs] avaient eu beau s'écarter de ces endroits malsains où pullulaient des mouches voraces, les « commandants » les y avaient replacés de force, et les y avaient maintenus à coups de fusil. (Maran 1927, 91)

La connaissance de l'espace naturel s'avère essentielle pour qu'on puisse le respecter et qu'il puisse survivre. De même, l'exploitation des ressources naturelles est conditionnée par l'incompréhension du territoire. C'est le cas de la production de caoutchouc : cent kilogrammes de racines de caoutchouc

travaillés pendant quinze jours, à raison de douze heures par jour [...] ne produisait qu'un kilo de mauvais caoutchouc. (164-5)

De plus, le salaire pour ce dur travail est d'un sou par jour. En d'autres termes, l'exploitation territoriale des colons va de pair avec leur igno- 
rance envers les pratiques agricoles des indigènes et leur violence raciale. En effet, les Occidentaux ont exalté le mythe de l'« inépuisable fertilité » (Tourte 2005, 297) des territoires de l'Empire colonial français. Toutefois, cette légende laisse bientôt la place à la déception. La pratique indigène prévoit des cultures itinérantes, idée qui est en contraste avec le style de vie sédentaire de l'Occident. La culture continue du territoire épuise les éléments nutritifs, comme l'azote, le phosphore et le potasse présents dans le terrain pour la correcte nutrition des plantes (397) et mène à la stérilité du sol africain. Dans les récits de Maran, la colonisation s'explique, alors, à travers les mots : exploitation, travail forcé et destruction de l'écosystème. Il s'agit alors de 'racisme environnemental' que le philosophe Deane Curtin définit comme

[1]a connexion [...] de la race et de l'environnement où l'oppression de l'un est connectée, et soutenue par l'oppression de l'autre. (Ferdinand 2014, 4)

René Maran montre donc au lecteur que les animaux et le milieu naturel nécessitent la protection et le respect. L'auteur revendique une nouvelle colonisation et ses romans ont pour but de

intéresser la France à ses colonies et d'y créer une opinion publique qui contrebalance l'autocratie des gouverneurs. (Senghor et al. 1965, 19)

En effet, ce qui ressort de sa mise en parole de la faune est que l'homme blanc est la seule bête à mettre en danger les autres animaux de la brousse. Toutefois, il faut garder à l'esprit que René Maran est en faveur de la colonisation : son objectif est moins de mettre fin au système colonial que de le rendre juste.

\section{La société naturaliste et la société occidentale}

La culture d'une société donnée ressort du cadre naturel dans laquelle elle se trouve. L'interaction humaine avec l'environnement crée des connaissances, des savoirs et des concepts spécifiques (Gaffuri 2018). Le système anthropologique paraît différent pour les Africains et pour les Occidentaux. Deux visions se dessinent : d'une part la vision 'anthropocentrique' liée à la colonisation ; de l'autre part celle 'écocentrique' typique des sociétés naturalistes. Autrement dit, le système anthropocentrique occidental différencie, dans l'ensemble de l'environnement, les hommes des autres sujets non-humains. En revanche, les populations indigènes n'appliquent pas cette division : leur système apparaît accueille le vivant, le non-vivant, le visible, 
l'invisible, l'humain mais aussi le non-humain (Onwudinjo 2015, 510). L'homme est donc partie intégrante de la nature et du milieu broussard. En d'autres termes, il existe une interdépendance entre chaque hôte de la brousse. Grâce au point de vue des bêtes de la brousse, le lecteur comprend l'écart de la vision des colons et des indigènes envers l'engagement écologique. À leur arrivée dans le territoire subsaharien, les colonisateurs ont vu un espace 'sauvage', c'est-à-dire qu'il n'était pas au diapason avec la vision occidentale de l'espace naturel. La mise en valeur du territoire, aux yeux des colonisateurs, s'accomplit à travers leur «impérialisme environnemental » (Lassi 2013, 6) :

[1]'agriculture doit être la première opération de mise en valeur des pays neufs. (Tourte 2005, 12)

Ce sont les mots utilisés en 1904 par Joseph Chailley-Bert, fondateur de l'Union Coloniale Française. Il déclare qu'il faut passer des cultures traditionnelles indigènes aux 'cultures riches' occidentales qui répondent aux exigences de la demande du marché européen. Autrement dit, il s'agit de « cultures coloniales » (Tourte 2005, 19). D'où le fait que la 'plantation coloniale' se réfère à

une entreprise agricole réalisant une emprise permanente [...] et dont la finalité est la recherche du gain monétaire [...] par la production [...] de denrées végétales exportées. (20-1)

Les colonisateurs suppriment les cultures autochtones et s'engagent dans l'organisation et l'encadrement des indigènes afin d'optimiser la production. Selon l'idéologie coloniale, seuls des 'sauvages' peuvent habiter dans un environnement sauvage et la seule voie pour les améliorer consiste à agir sur les deux. L'homme occidental se reconnait, en effet, en ce qu'il est capable de manipuler et d'organiser la production d'un territoire donné. Le mode d'appropriation du territoire africain et l'exploitation du sol traduit un rapport de force qui est exercé par l'élite colonisatrice. Un nouveau changement depuis la création des plantations est le double sentiment de ravissement et de peur de la part des 'petits animaux', c'est-à-dire les oiseaux. La culture du mil et du maïs attire de nombreux volatiles qui se nourrissent de ces plantes. Cette situation, apparemment heureuse, s'apprête en réalité à détruire l'harmonie de la brousse. Les oiseaux attirent les 'grandes bêtes' (phacochères, panthères, lions) qui de fait déterminent soit l'abandon de cette source de nourriture de la part des volatiles, soit le choix de rester et de subir le stress dû à la peur et la menace de la présence des animaux féroces $(1953,136-7)$. Un facteur nouveau influence le sentiment d'angoisse envers les individus Blancs : les bêtes «ne se sentaient plus chez elles nulle part » ([1947], 27). La faune se 
sent exclue et se voit chassée de son territoire par des individus qui exigent d'être les premiers occupants ayant le droit d'imposer leur propre loi (Maran 1953, 164). Les animaux et les hommes réagissent, alors, avec les mêmes émotions (peur, exclusion) et les mêmes actions (fuir, se rendre). De plus, la nature est représentée comme être vivant capable de ressentir des émotions et de réagir aux événements comme un être humain. Par exemple, la brousse crie et s'agite comme si elle se trouvait soumise à la torture :

[1]a brousse, torturée par l'ouragan, hurlait et se démenait sous la pluie. (Maran 1941, 18)

Afin de souligner l'aspect vivant de chaque élément de l'environnement, Maran accompagne le nom en langue indigène du nom commun pour le définir : 'Donvorro, la tornade', 'Ipeu, la lune' et 'Lolo, le soleil'. De même, il utilise le nom indigène et le nom commun pour toutes les bêtes de ses récits ('Bokorro, le serpent python'). En nommant les animaux, souvent, l'auteur utilise le nom en langue indigène tout seul avec la fonction de nom propre ('Bokorro'). Alors, tout sujet de la brousse, aussi bien vivant que non-vivant, acquiert le même niveau que l'homme : il possède un nom propre ; il éprouve des émotions et les manifeste de différentes manières; il est en relation et en communication avec le reste de la nature. De fait, toute hiérarchie dans le royaume naturel entre hommes, animaux et éléments naturels n'existe plus.

Or, la mise en valeur du territoire selon la vision colonisatrice provoque deux conséquences terribles sur l'environnement. En premier lieu, la brousse n'avait jamais été si « [n]ue, dure, sèche, craquelée, crissante, poudreuse, farouche » (Maran 1953, 112) avant l'arrivée des Blancs et leur exploitation du sol. En second lieu, l'augmentation de l'espace pour les plantations et les cases pour accueillir les colons ont amené à la « dévor[ation] sans pitié » (Maran 1934, 12) de la brousse. En effet, face à l'excessive exploitation du sol, Jean Dybowski, qui prend part au premier voyage agronome en A.E.F (18911892), dit :

notre action a été [...] nuisible et coupable [...]. Nous avons détruit, par des récoltes abusives, les ressources naturelles. (Tourte 2005, 38)

René Maran n'est donc pas le seul à souligner cette problématique. Le dessèchement du sol est une des majeures préoccupations des experts qui s'intéressent, au XXe siècle, de la région de l'Afrique centrale. Toutefois, leur souci ne concerne pas le bien-être des populations autochtones, mais plutôt les néfastes conséquences au niveau économique (Tourte 2005, 292 et ss.). Quels sont les facteurs introduits par les colons qui ont mené au dessèchement? L'extension 
des surfaces cultivées (qui est déjà de facto un enjeu du dessèchement) procède du déboisement de la brousse, qui produit de même un contrecoup dans la circulation des eaux ; la construction de barrages ; l'augmentation du rendement du sol et la fertilisation (Tourte 2005, 393-6). Pourtant, la sécheresse en tant qu'elle représente une métaphore prédominante dans les romans de l'auteur dénonce l'inefficacité de la mission civilisatrice et de son idéologie colonisatrice. L'appropriation du territoire de la part des colons provoque plusieurs effets néfastes sur l'écosystème. Par exemple, la fuite des hommes déclenchée par les « répressions [...] qui vont probablement pleuvoir sur les villages » (Maran 1927, 93).

L'occupation de nouvelles aires dont les tribus ne connaissent pas le territoire est le fruit de la peur, de la faim et du travail forcé. Pour ces populations, il s'agit d'abandonner leurs connaissances bâties sur une " expérience héréditaire, fondée sur l'observation la plus exacte » $(1927,91)$ qui leur permettaient de vivre en harmonie avec la nature environnante. Au contraire, lors d'un déplacement, l'équilibre entre les villageois et la brousse est détruit et il faut le reconstruire à travers la connivence avec le nouvel espace naturel. L'équilibre est doublement mis en cause : non seulement par les changements apportés par les Blancs, mais aussi par les hommes noirs qui, en se déplaçant, s'installent dans des lieux qui, de fait, étaient occupés et contrôlés par les animaux :

[c]haque pas qu'il [l'homme noir] faisait en avant rétrécissait le domaine des bêtes de la brousse. ([1947], 27)

Tout élément de la brousse est dépourvu de ressources pour réagir à l'invasion de la colonisation et, de fait, la seule bouée de sauvetage pour la brousse est « qu'elle s'habitue malgré tout à leur [des Blancs] présence » $(1941,72)$. Cela signifie que détruire l'environnement et l'harmonie existante comporte une conséquence nihiliste sur le système de la brousse. En d'autres termes, l'identité de la brousse est de plus en plus effacée par la présence du Blanc qui impose sa présence physique, culturelle et économique.

La différence la plus importante entre les sociétés naturalistes et les sociétés occidentales consiste d'un côté dans le fait de vivre dans un tout en harmonie avec son environnement et de l'autre côté dans l'exploitation du territoire dans un but productif et donc économique. D’ailleurs, dans ses romans animaliers, René Maran souligne plusieurs fois la violence et l'absurdité de l'utilisation des armes à feu par les colons. Les sociétés naturalistes admettent l'abattage des bêtes pour faire face au besoin de subsistance des individus. La chasse au feu s'effectue seulement pendant la saison sèche et la préparation à cet événement s'accompagne de célébrations et de rituels. René Maran consacre vingt-quatre pages $(1934,187-211)$ à la description de 
cette récurrence. Il souligne l'importance de la connaissance de la faune. Les hommes imitent par leurs danses les mouvements et les comportements de plusieurs animaux : ils sont des êtres vivants sacrés et respectés. Or, les indigènes les chassent à l'aide des sagaies, des chiens, du feu et du rythme des tams-tams. Tuer une seule bête dure plusieurs heures, voire plusieurs jours. Au contraire, les colons, grâce aux armes, peuvent tuer de nombreuses bêtes dans des temps restreints. L'abattage des animaux n'est pas une nécessité de survie pour les Blancs, mais « [leur] principal amusement [...] et [leur] seule occupation » $(1941,165)$. L'homme blanc détient alors un pouvoir d'extermination que l'homme noir ne possède pas et qu'il n'utilise pas, puisque l'emploi d'une telle violence contredirait les principes des sociétés naturalistes. Ainsi, par exemple, « l'éléphant [...] devenait d'une rareté singulière » $(1927,101)$, non seulement à cause de la consommation de viande, mais aussi à cause du commerce de l'ivoire. Les études de G.A. Bradshaw nous aident à expliquer les conséquences sur la communauté de ces animaux. Elles traitent du changement des comportements des éléphants suite à un trauma social. Ces bêtes s'organisent selon des sociétés matriarcales et ont leurs bases sur des rapports d'attachements sociaux qui les amènent à former des groupes avec des individus profondément liés entre eux. Or, si l'on regarde la chasse indigène, la perte d'un seul individu du troupeau des éléphants apporte un trauma plus supportable que le choc de perdre plusieurs membres du groupe dans la même journée. Bradshaw écrit :

Wild elephants are displaying symptoms associated with human PSTD : abnormal startle response, depression, unpredictable asocial behavior and hyperaggression. (Bradshaw 2005, 807) ${ }^{2}$

Le stress provoqué par des traumas cause donc une agressivité inattendue qui traduit une forme de résistance qui aide à tolérer l'oppression colonisatrice. René Maran décrit et donc témoigne de ces comportements. Un exemple se trouve dans Mbala l'éléphant, où Mbala ayant vu plusieurs abattages par les armes à feu dans son troupeau, réagit de cette façon :

[s]on humeur s'en était ressentie. Il s'était mis à haïr l'homme, qu'il fût noir ou blanc de peau [...]. Il chargeait, au moindre bruit, dans la direction du bruit entendu. Méthode excellente! Des hommes noirs de peau avaient pâti. Nombreux étaient ceux qu'il avait eu la joie de réduire en bouillie. ([1947], 24-7)

2 «Les éléphants sauvages démontrent des symptômes associés avec des syndromes post-traumatiques humaines : réaction de sursaut anormale, dépression, comportement asocial imprévisible et hyper agression » (traduction de l’Auteure). 
Mais comment expliquer les comportements violents des Blancs vis à vis des animaux ? La réponse réside dans une seule phrase :

[i]ls tuaient pour le plaisir de tuer, pour le plaisir d'affirmer à tous, à la face de la création, la petitesse de leur taille et la puissance de leurs moyens de destruction. (28)

D’un côté, le regard des bêtes ne discerne que la violence et la cruauté de l'homme blanc qui impose aux hommes noirs de « crever à la peine pour nourrir [...] leur lucrative paresse » $(1927,60)$ dans les plantations et de l'autre, les Occidentaux ne saisissent que le pouvoir productif et donc économique du territoire colonisé.

L'harmonie entre les Africains et les éléments de la brousse est fondamentale : ce type de lien crée un réseau conscient de l'aide mutuelle entre les hommes et les animaux. D’ailleurs, dans la chasse au feu, le chasseur reconnait l'aide fondamentale du chien lors de cette pratique et de même son rôle à l'intérieur du village, car les chiens ont le devoir de le protéger et les chiots de couleur jaune ou blanc donnés à d'autres ethnies engagent un lien d'alliance ou d'amitié entre villages. Le chien est lié au chef du lignage, car les deux sont porteurs de 'fécondité', qualité fondamentale pour la réussite des activités à travers des techniques apprises par plusieurs rituels (Dounials, Mesnil 2017, 632). Le chien, de sa part, reconnaît dans l'homme un sujet de référence pour des situations difficiles. Par exemple, dans une scène de Djouma chien de brousse (1927), la chienne Mbimé apporte à son patron Batouala un chiot piqué par un scorpion : la chienne fait confiance aux connaissances médicales des hommes de sa tribu qui utilisent notamment des remèdes naturels à base d'herbes (Maran 1927, 25-7). La relation entre le maître et son chien explique comment se traduit de façon pratique et quotidienne l'idée de 'société naturaliste'. D'ailleurs, avant l'arrivée des colonisateurs, la société de la brousse se montre respectueuse de l'environnement à travers des pratiques éco-durables. Non seulement, les colons endommagent le système naturel, mais aussi le système culturel. Par exemple, les populations indigènes utilisaient les bergeries $^{3}$ pour élever les cabris et les protéger des panthères. L'abattage d'un nombre élevé de panthères produit des changements dans l'élevage des cabris, y compris la perte de l'utilisation des bergeries, pratique répandue dans l'ex-colonie de l'Oubangui-Chari.

3 Une 'bergerie' est « une véritable maison, construite en terre et couverte d'un toit de chaume. La porte se composait d'une série de troncs de bois entassés les uns sur les autres et maintenus par deux gros poteaux » (Dounials, Mesnil 2017, 635). 


\section{Le rôle de la culture animalière pour se défendre des colons}

Dans les romans de René Maran, les sentiments et les valeurs des animaux sont mis en relief : par exemple, à leur sujet il parle de félicité, de bonne volonté, de méfiance et de patience (Maran 1941, 9-11). L'auteur montre également la faculté de penser des bêtes en parlant, dans ses récits, de pensées, de ruminations, de méditations et de jugements. De plus, chaque animal emploie sa façon de penser qui est influencée par la culture de sa race. Le serpent Bokorro explique la modalité de réflexion de son espèce :

[1]es serpents [...] ont des méthodes de réflexion bien à eux. Il leur faut être en état de grâce pour procéder à cette opération de l'esprit. Or ils ne parviennent à l'être que le ventre plein. Il se terrent alors et se lovent dans le trou où ils habitent, dorment en digérant et méditent en dormant. (Maran 1941, 168)

René Maran donne un aperçu de la culture animalière ou de la brousse. D'ailleurs, la culture est un produit de la nature et de ses habitants, les deux choses étant liées. Par exemple, à propos de sa forêt natale, l'éléphant Mbala dit que

[s]es moindres coins et recoins faisaient partie de ses fibres, de son être. ([1947], 11)

Ainsi, la culture de l’homme influence celle de la faune : Doppélé le charognard récite un conte « entendu raconter par le banda » (73). Il comprend, en effet, la langue des indigènes, comme les autres bêtes de la brousse. Néanmoins, l'échange culturel à travers les mots s'avère unidirectionnel : seulement des hommes vers les animaux, car les hommes ne comprennent pas les langages de la brousse. Pourtant, les indigènes ont étudié les bêtes et leurs habitudes et ont construit leur culture ainsi que leur tradition autour des animaux et de la brousse.

Le milieu naturel exerce une influence sur la culture de ses hôtes. Alors, comment se transmet la culture entre les bêtes de René Maran ? Les hommes apprennent à l'aide des contes, des maximes, des jeux que les enfants rencontrent dans leurs premières années. Pour les animaux de Maran, l'acquisition d'informations sur l'environnement s'établit à travers les mêmes moyens. Les bêtes grandissent avec des idées et des préjugés envers les autres animaux et envers les hommes qui sont définis et transmis par leur groupe d'appartenance ou, plus en général, par le royaume animalier. Il y existe même des stéréotypes : les rhinocéros sont insociables et méfiants ; les hommes noirs menteurs ; les taons agaçants et les tiques tenaces. Alors, tels que les hommes, les animaux apprennent de façon sociale à travers 
l'imitation des comportements, à travers l'apprentissage des contes et des histoires et ils apprennent de même plusieurs langues.

La culture de la brousse des bêtes présente des caractéristiques intrinsèques. D’abord, l'aspect qui ressort le plus dans les romans de René Maran est l'importance de la fonction éducative des contes. Souvent, les histoires racontées par le charognard Doppélé se terminent par des maximes avec des leçons de plusieurs types. Nombreuses sont celles qui dispensent des enseignements de vie tels que :

si mieux vaut boire, quand on meurt de soif, eau boueuse que de ne pas boire du tout, mieux encore vaut boire, dès qu'on peut le faire, eau limpide qu'eau boueuse (1941, 66-7).

Ou encore : « grain de mil ne deviendra jamais grain de maïs » (211). Parfois, les histoires racontent l'origine de certaines rivalités à l'intérieur du règne animal. Dans Bêtes de la brousse (1941, 106 et ss.), Mbala l'éléphant raconte pourquoi les hippopotames et les éléphants ne vivent plus ensemble comme auparavant et accompagne l'histoire avec de mythes et de légendes sur les deux espèces; en outre, les chansons ont un rôle central pour le partage des savoirs et des enseignements parmi les bêtes $(1941,129)$. Comme pour les indigènes, les chansons peuvent aussi se lier à un rite ou à une pratique. Ici, une des chansons des charognards :

tous les animaux de la brousse [...] reconnurent l'une des strophes du chant si beau, si noble, si pur dont les charognards ont fait leur chant de chasse. $(1941,142)$

Le chant s'avère une forme de protestation et naît d'une motivation agressive. L'éthologue Irenäus Eibl-Eibesfeldt soutient que l'homme, lorsqu'il est dans l'impossibilité de combattre physiquement et matériellement, doit trouver d'autres moyens pour lutter. Les animaux de Maran, tel que les indigènes, voient en la chanson dans une langue inconnue à l'ennemi une voie pour régler les questions avec les Blancs ; de plus, elle accroît l'union du groupe.

Enfin, les bêtes apprennent l'identité du groupe auquel elles appartiennent et les rôles que chaque individu doit jouer (par exemple, les charognards sont chargés de mettre les animaux au courant de la situation de la brousse). Ils apprennent les devoirs de chaque individu à travers une routine précise qui se répète chaque jour. Toutefois, pendant la colonisation, leurs habitudes sont menacées par l'homme blanc. Voici les mots des hippopotames :

[i]l est dur de laisser derrière soi, peut-être pour toujours, les lieux où l'on a su se créer tant de douces habitudes ! (1941, 23-4). 
D'ailleurs, cette nouvelle menace provoque des changements dans l'éducation des animaux nouveaux nés, et René Maran montre les préoccupations des groupes animaliers. Les nouvelles générations sont élevées dans la crainte et la méfiance envers les hommes, surtout envers les Blancs. Sous l'influence de la peur et de l'angoisse pour la présence des hommes, se raconter les histoires et chanter les chansons liées aux traditions et à la culture de la faune s'avère une résistance culturelle, intellectuelle et idéologique pour éviter d'être absorbés dans la culture coloniale qui s'impose, comme nous l'avons vu, aussi bien sur les hommes que sur les animaux. En effet, l'éducation décrite par Maran est de type collectif : transmettre et répéter les contenus et les valeurs socio-culturelles exige la participation de la communauté entière : chaque individu ou groupe transmet une partie du savoir qui sert à former la mémoire collective de la vie (Lassi 2013, 77).

René Maran tient compte de chaque élément de la société naturaliste, des hommes, des animaux, mais aussi de l'environnement. Il montre également la participation des éléments naturels aux événements de la brousse. Dans les récits animaliers, le mauvais temps représente un allié des indigènes et des bêtes. Les éléments récurrents sont la pluie, le vent, la tornade et l'orage. Ils ont pour objectif de mettre en difficulté les Blancs et de créer un péril de source naturelle auquel, selon la nature, ils ne sont pas prêts à faire face. Pourtant, l'homme blanc a construit des cases à la manière occidentale qui protègent contre le mauvais temps, ou encore, si les plantations sont détruites, les colons vont exiger plus de travail des Noirs. La nature échoue dans ses tentatives de faire obstacle aux colonisateurs ou aggrave l'exploitation des indigènes. C'est ce que pense Mbala l'éléphant face à l'orage :

[t]erre et ciel essayèrent vainement de se coaliser, afin de mieux résister aux assauts dont ils étaient l'objet. [...] On ne savait néanmoins où commençait la terre, où finissait le ciel. [...] Des rafales de grêle bourrelaient le sol submergé. [...] Et l'ouragan continuait à se démener dans le vide que bouleversaient ses sautes d'humeur, tandis que la pluie, de plus en plus drue, de plus en plus compacte, de plus en plus farouche, servait de trait d'union entre ciel et brousse. (Maran [1947], 139)

René Maran décrit donc une société qui se sert de sa culture pour garder et protéger son identité face à la tyrannie culturelle des colonisateurs. 


\section{Conclusion}

Dans ses récits animaliers, René Maran s’engage non seulement à la dénonciation des errements de la colonisation envers les indigènes, mais il consacre aussi son écriture au témoignage des déséquilibres créés au niveau environnemental. Ce type de double dénonciation résulte de la décision de raconter les faits du point de vue de ceux qui ont subi la colonisation, et non pas par les indigènes, mais par les bêtes. En leur donnant la parole, Maran réussit à illustrer d'une manière nouvelle le processus de la colonisation. Ce sont donc les histoires d'une société opposée à la société occidentale qui souffre et lutte contre les colonisateurs à travers la mémoire de ses propres origines et de sa propre culture.

René Maran démontre que, avant la colonisation, les habitants de la brousse, hommes et animaux, ont vécu dans une harmonie et un équilibre qui témoignent de la cohabitation heureuse entre les hommes et la nature, dans un système qui utilise les ressources naturelles dans et pour le meilleur équilibre possible.

\section{Bibliographie}

\section{Fuvres de René Maran}

Maran, R. (1927). Djouma chien de brousse. Paris : Albin Michel.

Maran, R. (1934). Le Livre de la brousse. Paris : Albin Michel.

Maran, R. (1941). Bêtes de la Brousse. Paris : Albin Michel.

Maran, R. (s.d.) [1947]. Mbala l'éléphant. Illustrations G. Barret. Paris : Éditions Arc-en-ciel.

Maran, R. (1953). Bacouya, le cynocéphale. Paris : Albin Michel.

\section{Critique}

Bakhtine, M. (1977). Le Marxisme et la philosophie du langage. Paris : Les éditions de minuit.

Bédia, J.-F. (2008). «Écrire l'humanité par l'animalité : une stratégie narrative d'intertextualité dans le roman africain francophone ». Francofonía , 17, 6376. http://www.redalyc.org/pdf/295/295116120๑4.pdf.

Bonner, J.T. (1980). Evolution of culture in Animal. Princeton : Princeton University Press.

Bradshaw, G.A. (2005). «Elephant Breakdown ». Nature Publishing Group, 433, 807. https://doi.org/10.1038/433807a.

Degras, A. (2012). Akotara. Un tryptique consacré aux Gbayas du Nord-Ouest Centrafricain. Venafro : Poligrafica Terenzi.

De Loughrey E. ; Handley G.B. (eds) (2011). Postcolonial Ecologies. Oxford : Oxford University Press.

Dounias, E. et al. (éds.) (2007). Le Symbolisme des animaux. Paris : IRD Éditions.

Eibl-Eibesfeldt, I. (1977). Amore e odio, aggressività e socialità negli uomini e negli animali. Milano: Mondadori. Oscar saggi Mondadori. 
Ferdinand, M. (2014). «Écologie politique et pensées postcoloniales. Tentatives du 'postcolonial ecocriticism' ». Colloque Penser l'écologie politique: sciences sociales et interdisciplinarité (Université Paris Diderot, 13-14 janvier 2014). https://halshs.archives-ouvertes.fr/halshs-02289655.

Gaffuri, L. (2018). Racconto del territorio africano. Milano : Lupetti.

Garrard, G.(ed.) (2014). Ecocriticsm. Oxford : Oxford University Press.

Genette, G. (1976). Figure III. Torino : Einaudi.

Graham, H. ; Tiffin H. (2015). Postcolonial Ecocriticism : literature, animals, environment. London; New York: Routledge.

Irele, F.A. (2014). « Du roman colonial français au roman francophone postcolonial. René Maran le précurseur ». McDonald, C.; Suleiman, S.R. (éds), French Global. Une nouvelle perspective sur l'histoire littéraire. Paris : Garnier, 42745. Classiques Jaunes 644.

Joubert, J.-L. et al. (éds) (2006). « Notre librairie». Indispensables animaux, 163. Saint-Etienne : Dumas-Titoulet Imprimeurs.

Laland K.N. ; Hoppitt W. (2003). «Do Animals Have Culture?». Evolutionary Anthropology Issues News and Reviews, 12(3), 150-9. https://doi. org/10.1002/evan.10111.

Lassi, É.-M. (2013). Aspects Ecocritiques de l'imaginaire africain. Oxford : African Books Collective.

Maran, R. (1933). «Légendes de l’Oubangui-Chari ». Les Euvres Libres. Paris : Arthème Fayard et Cie, 147.

Maran, R. (2017). Batouala. Paris : Magnard. Coll. Classiques \& Contemporains.

Mille, P. (1909). « Littérature coloniale ». Le temps, 19 août.

Nixon, R. (2011). Slow Violence and the Environmentalism of the Poor. London: Harvard University Press.

Onwudinjo, K. (2005). « A Critical Perspective on the Image of the Environment in Tanure Ojaide's The Tales of The Harmattan ». International Journal of Humanities and Cultural Studies, 1, 505-18.

Senghor, L.S. et al. (1965). Hommage à René Maran. Paris : Présence africaine.

Tisserant, Ch. (1930). « Plantes à filasse employées par les Bandas du Haut-Oubangui (Afrique Équatoriale Française) ». Revue de botanique appliquée et d'agriculture coloniale, 101, 10-15. https://www.persee.fr/doc/jatba_0370-3681_1930_num_10_101_4818.

Tourte, R. (2005). Histoire de la recherche agricole en Afrique Tropicale Francophone. Vol. 4, La période coloniale et les grand moments des jardins d'essais 1885-1890-1914-1918. Roma : FAO.

Van den Avenne, C. (2005). « Passer d'un monde à l'autre, d'une langue à l'autre. Lecture de deux incipits d'Ahmadou Kourouma ». Mochet M.-A. et al. (éds), Plurilinguisme et apprentissage. Mélanges Daniel Coste. Lyon : ENS Éditions, 237-46.

Volosinov, V.N. (1980). Il linguaggio come pratica sociale. Bari : Dedalo Libri. 



\title{
La Peste d'Albert Camus : une analyse de la société coloniale algérienne à travers le prisme de l'épidémie
}

Alessia Berardi

Università Ca' Foscari Venezia, Italia

\begin{abstract}
March 2020: as Coronavirus continues spreading across the globe, the wellknown novel La Peste (1947) by Albert Camus appeals to new readers all over the world. This article offers a new modest reading of La Peste by adopting an approach which emerges from the intersection between Environmental Humanities and Postcolonial Studies. We will explore to what extent the plague is an allegory of human suffering and isolate the different levels on which the allegory may work. Furthermore, we will focus on the link between the representation of the plague outbreaking in Oran and the French-Algerian, multiethnic society. What will the 'epidemic fiction' reveal about social structures and practices in the context of colonial Algeria's last years of existence, and how? The representation of the epidemic seems to mirror the inequalities of that colonial society that is not depicted in the novel, and yet it reminds the reader that societies are constructed and, thus, can be improved.
\end{abstract}

Keywords AlbertCamus. Postcolonial Theory. Epidemic Fiction. Plague. French Algeria.

Sommaire 1 Introduction. - 2 Oran et la peste: une épidémie allégorique? - 3 L'épidémie et l'échec de l'universalisme. - 4 Conclusion.

$\begin{array}{lll} & \text { Peer review } \\ & \text { Submitted } & 2020-06-30 \\ \text { Edizioni } & \text { Accepted } & 2020-09-30 \\ \text { Ca'Foscari } & \text { Published } & 2020-12-22 \\ & \text { Open access } & \end{array}$

(c) 2020 | (ㅇ) Creative Commons Attribution 4.0 International Public License 


\section{Introduction}

Comme le montrent les données d'Edistat, ${ }^{1}$ l'éclat de l'épidémie de coronavirus en Europe au début de 2020 a relancé les ventes de $L a$ Peste, le célèbre roman d'Albert Camus publié pour la première fois en 1947. La presse italienne, française et anglophone reconnaît, voire légitime la nature palliative d'une telle lecture en ces temps d'urgence sanitaire : le récit d'épidémie semble contribuer à rendre supportable la véritable épidémie en cours. Les milieux académiques ne sont pas 'immunisés' contre le charme de la littérature d'épidémie. D'un côté, les études actuelles continuent de problématiser les modalités de représentation de l'épidémie (cf. Murat 2020) ; de l'autre, elles ouvrent de nouvelles pistes de réflexion dans les plus récents domaines interdisciplinaires tels que les humanités médicales (cf. Nash 2019) ou les humanités environnementales. C'est sous cet angle-ci que nous étudierons La Peste. En particulier, nous nous mettrons à l'écoute du narrateur de l'épidémie, le docteur Rieux, ainsi que de l'écrivain Albert Camus, pour analyser la société multiethnique oranaise des années 1940 que la mise en scène de la peste permet d'entrevoir.

Cette analyse s'inscrit avant tout dans le débat autour de l'algérianité' d'Albert Camus, qui s'est élevé à la suite de la publication posthume de son roman le plus autobiographique, Le Premier Homme, en 1994. Bien que Camus soit généralement reconnu comme un écrivain appartenant au canon littéraire français, il nous semble important de réencadrer certaines de ses œuvres dans le contexte de sa région natale, l'Algérie française, et ceci pour dévoiler à la fois les paradoxes et les richesses d'une production littéraire qui est en tension constante entre la métropole française et les marges coloniales, l'Occident' et l'Orient'. Les humanités environnementales, et notamment les études sur l'épidémie et sur le récit d'épidémie, ouvrent de nouvelles pistes de réflexion à l'intérieur de ce débat. Dans le cadre de l'écocritique, l'épidémie est notamment un problème d'espace social : à cause du risque d'infection, non seulement le fléau menace-t-il la sphère communautaire, mais il dessine également des « cartographies du soin et de l'isolement » qui révèlent l'appartenance de classe, de genre, de race etc... des individus (Lowe 2014, 304). En outre, la réflexion sur l'épidémie peut s'inscrire dans l'un des thèmes principaux de l'écocritique, à savoir le rapport (non forcément d'opposition) entre la nature et la culture, l'environnement et la société (Zapf 2010, 136). Nous verrons donc comment La Peste d'Albert Camus est une œuvre qui peut répondre aux sollicitations et des études postcoloniales et des humanités environnementales.

1 Le graphique d'Edistat peut être consulté au lien suivant : https://twitter.com/ edistat_actu/status/1234450836538957824. 
Dans un premier moment, nous étudierons la portée allégorique de l'épidémie de peste d'Oran par rapport aux communautés indigènes franco-algériennes. Il s’agira de mettre en évidence les contradictions que soulève une lecture allégorique du roman lorsqu'il est question des «Arabes $»{ }^{2}$ sans pour autant discréditer la voix auctoriale qui propose, elle-même, une telle interprétation. Pour ce faire, nous nous servirons de quelques passages de La Peste ainsi que des déclarations d'Albert Camus. Dans un deuxième moment, nous ne prendrons en considération que le contenu du roman et, en particulier, les déclarations de son narrateur, le docteur Rieux. Enfin, à travers la révision de la littérature scientifique sur l'épidémie qui s'est développée au sein des humanités environnementales, nous reformulerons l'opposition culture/nature et nous montrerons que l'épidémie de La Peste est un phénomène politique et social, et qu'il est donc légitime de s'interroger sur les contradictions de la société soi-disant universaliste que le roman esquisse.

\section{Oran et la peste : une épidémie allégorique ?}

Le seuil du roman d'Albert Camus est occupé par une épigraphe tirée de Robinson Crusoe (1719), de l'écrivain anglais Daniel Defoe : «Il est aussi raisonnable de représenter une espèce d'emprisonnement par une autre que de représenter n'importe quelle chose qui existe réellement par quelque chose qui n'existe pas » (Camus 2006, 33). Cet élément péritextuel donne au public la clé de lecture du roman : il l'invite à interpréter les événements de l'histoire qui suivra comme étant des révélateurs de faits réels. C'est dans sa lettre de réponse aux accusations de Roland Barthes qu'Albert Camus explicite de quelle vraie épidémie il s'agit (285-7). Barthes a vu dans $L a$ Peste la promotion d'une «morale antihistorique » (286) ; le romancier réplique alors non seulement que «La Peste [...] a comme contenu évident la lutte de la résistance européenne contre le nazisme » (286), mais aussi que son roman peut « servir à toutes les résistances contre toutes les tyrannies » (286). Autrement dit, Camus déclare exploiter le récit d'épidémie pour raconter la montée du nazisme et la Résistance, ainsi que pour dénoncer toute forme de tyrannie, de quelque base idéologique dont elle se nourrisse. Dans ce sens, La Peste répond donc à des exigences littéraires morales (Marx 2020).

2 Albert Camus désigne les membres des communautés indigènes d'Algérie par l'ethnotype d'« Arabes ». L'emploi de ce terme a animé le débat académique : bien que ce soit un terme désormais dépourvu de connotation raciste à l'époque où Camus l'utilise (Foexlee 2009, 36), c'est vrai qu'il ne tient pas compte de l’hétérogénéité des peuples autochtones. Nous avons donc choisi d'employer le même terme qu'Albert Camus tout en rappelant au lecteur ses limites : dorénavant, il sera donc indiqué entre guillemets. 
Le choix de se servir du récit d'épidémie pour mettre à nu l'oppression et le mal s'inscrit dans une forte tradition littéraire dont Camus n'est pas le dernier représentant. C'est depuis l'Antiquité que les fléaux ont été chargés d'un poids allégorique important, quand il était commun de croire que l'épidémie était une punition divine (Voisine-Jechova 2001, 267). La reconnaissance et l'acceptation de la portée symbolique de l'épidémie sont probablement dues à l'essence même de l'épidémie : c'est un ennemi invisible qui s'abat sur l'humanité sans préavis et sans explication, d'où le pouvoir évocatoire qu'elle suscite chez l'écrivain (Stephanson 1987, 225).

Malgré l'ancrage de La Peste dans ce schéma narratif conventionnel, l'allégorie développée par Albert Camus a suscité de nombreuses perplexités auprès des critiques, que Jennifer Cooke (2009, 31-2) a récapitulées. Premièrement, plusieurs chercheurs reprochent à l'écrivain d'avoir choisi un phénomène naturel tel qu'une épidémie pour dénoncer le(s) totalitarisme(s) ; nous reviendrons sur cette considération par la suite. Deuxièmement, certains critiques pensent qu'une interprétation exclusivement allégorique du roman rend difficile la formulation d'autres potentielles analyses, et qu'il serait superficiel de ne faire confiance qu'aux déclarations de l'auteur, qui d'ailleurs reposent sur la réception des lecteurs : " La preuve en est que cet ennemi qui n'est pas nommé, tout le monde l'a reconnu, et dans tous les pays d'Europe » (Camus 2006, 286; italique ajouté). Enfin, l'allégorie réduirait le potentiel de la littérature d'engendrer un " témoignage fictionnel », susceptible de combler aussi bien les vides du récit historique officiel que ceux des témoignages personnels partiels.

En ce qui concerne notre problématique, c'est-à-dire la représentation de la société multiethnique franco-algérienne, l'allégorie joue un rôle essentiel : l'acceptation de la lecture allégorique par parti pris pourrait limiter, voire empêcher un discours engagé du point de vue postcolonial. En effet, l'allégorie autorise des incongruités que l'adhérence au réel tel quel ne permettrait pas ; la première d'entre elles est l'absence de la communauté indigène dans une ville coloniale algérienne telle qu'Oran l'est. Si la dimension allégorique de La Peste est admise, Oran peut passer pour une ville de la France métropolitaine en raison de la description qui en est faite.

Voici l'incipit du roman :

Les curieux événements qui font le sujet de cette chronique se sont produits en 194., à Oran. [...] À première vue, Oran est, en effet, une ville ordinaire et rien de plus qu'une préfecture française de la côte algérienne. (Camus 2006, 35)

Certainement, le roman est présenté moins comme une fiction, que comme une "chronique » : les événements sont proposés comme s'ils s'étaient véritablement passés. Ainsi, dans un premier moment 
et malgré le paratexte auctorial, le lecteur n'hésite pas à associer la « ville ordinaire » dont il est question dans le roman à la vraie ville d'Oran en Algérie française. Comme le constate Michel Murat, « nous sommes dans le domaine du vraisemblable » (2020, s.p.).

Cependant, du moment où Albert Camus affirme qu'il ne croit pas « au réalisme en art » dans sa lettre à Barthes (Camus 2006, 286), des doutes surgissent quant à la factualité de la ville d’Oran. À bien voir, la comparaison entre l'Oran de La Peste et les Orans que décrit Albert Camus dans sa non-fiction - nous pouvons par exemple mentionner l'essai « Petit guide pour des villes sans passé » du recueil L'Été (Camus 2006, 593-6) - montre que la ville du roman de 1947 est une ville imaginaire (Berardi 2020, 59-61). L'Oran de La Peste ne partage donc que le nom et la position géographique de la véritable ville d'Oran (Carroll 2007, 53) ; loin d'être une ville méditerranéenne telle que les cités décrites dans l'essai, l'Oran de La Peste paraît plutôt comme une ville métropolitaine, moderne et aliénante.

Dans une telle ville - européenne, dans les années 1940 -, il ne serait pas étonnant de ne pas rencontrer de membres de la communauté indigène si le narrateur ne laissait pas échapper un renvoi à leur existence à deux reprises. Dans le célèbre passage où Rambert demande à Rieux de lui signer un certificat de santé, les deux personnages se promènent dans « les ruelles du quartier nègre » (Camus 2006, 90). Or, dans le vocabulaire colonial l'expression « village nègre » désigne une zone de la ville où résident notamment des indigènes (Ansel 2012, 76). Il est donc clair que, malgré sa dimension allégorique, l'Oran de La Peste n'est pas habitée exclusivement par des citoyens français. Ceci est confirmé également par l'évocation explicite des « Arabes ».

L'épisode dont il est question se déroule dans la première partie du roman, quand la peste n'a pas encore été reconnue. Rambert, journaliste parisien en mission à Oran, interrompt les consultations du docteur Rieux pour l'interviewer :

Il enquêtait pour un grand journal de Paris sur les conditions de vie des Arabes et voulait des renseignements sur leur état sanitaire. Rieux lui dit que cet état n'était pas bon. Mais il voulait savoir, avant d’aller plus loin, si le journaliste pouvait dire la vérité.

'Certes, dit l'autre.

- Je veux dire : pouvez-vous porter condamnation totale?

- Totale, non, il faut bien le dire. Mais je suppose que cette condamnation serait sans fondement.'

Doucement, Rieux dit qu'en effet une pareille condamnation serait sans fondement, mais qu'en posant cette question, il cherchait seulement à savoir si le témoignage de Rambert pouvait ou non être sans réserves.

'Je n'admets que les témoignages sans réserves. Je ne soutiendrai donc pas le vôtre de mes renseignements. 
- C'est le langage de Saint-Just', dit le journaliste en souriant.

Rieux dit sans élever le ton qu'il n'en savait rien, mais que c'était le langage d'un homme lassé du monde où il vivait, ayant pourtant le goût de ses semblables et décidé à refuser, pour sa part, l'injustice et les concessions. (Camus [1947] 2006, 41; italique ajouté)

La présence des «Arabes » à Oran est clairement formulée. Si Rieux - qui est le seul narrateur et organisateur du récit (Ansel 2002, 48) - inclut cette conversation dans sa chronique, il nous semble évident qu'elle doit avoir un certain poids à l'intérieur du roman et pour l'auteur même. À travers ce passage, Albert Camus montre en effet qu'il est conscient des problèmes affligeant les communautés indigènes des départements algériens, mais en même temps qu'il n'est pas disposé à en parler dans l'espace restreint du roman. La voix du narrateur reproduit la voix auctoriale : Rieux refuse de témoigner des conditions des «Arabes » de crainte que les déclarations qu'il prononce ne soient pas relatées de manière intégrale et objective, « sans réserves », de même que Camus refuse de s'attarder sur les questions coloniales dans une œuvre de fiction destinée au public métropolitain (Ansel 2012, 18) qui, comme le journaliste parisien Rambert, ne pourrait pas «porter condamnation totale » (Camus 2006, 41). C'est hors de la fiction, et précisément dans ses reportages de 1939 pour le journal Alger Républicain, que l'écrivain dénonce les conditions de vie des communautés autochtones (307-36).

Cette interprétation est soutenue par les déclarations d'Albert Camus lui-même. En réponse aux remarques de l'écrivain berbère algérien Mouloud Feraoun qui déplore l'absence d'indigènes dans $L a$ Peste (Chaulet Achour 2013), Camus affirme que :

pour les mettre en scène, il faut parler du problème qui empoisonne notre vie à tous, en Algérie ; il aurait donc fallu écrire un autre livre que celui que je voulais faire. Et pour écrire cet autre livre d'ailleurs, il faut un talent que je ne suis pas sûr d'avoir. (Feraoun 1969,54$)$

Dans ces lignes, Camus reconnaît qu'il existe un « problème » mortel en Algérie, bien qu'il n'évoque pas explicitement les politiques coloniales françaises. Or, d'après l'écrivain, la dénonciation de ce fléau requiert un espace autonome qu'il n'est pas en mesure de garantir, d'où l'effacement des «Arabes » dans La Peste. Il semble donc que l'oppression française des communautés indigènes ne puisse pas être représentée allégoriquement par le biais de l'épidémie de peste d'Oran, ce qui est en conflit avec les affirmations précédemment observées de Camus qui prétend ne pas avoir nommé aucun « visage » de la « terreur » pour « pouvoir mieux les frapper tous » (Camus 2006, 287). 
En somme, de nombreuses contradictions surgissent d'une lecture plus ou moins allégorique de l'épidémie de La Peste. D'une part, l'interprétation allégorique du fléau se heurte et contre l'évocation de la communauté indigène dans le roman et contre certaines des déclarations auctoriales ; de l'autre, une lecture exclusivement référentielle de la « chronique » ne suffit pas à appréhender les motivations de l'absence d'« Arabes ». Il est évident que ce paradoxe dérive de la position historiquement difficile d'Albert Camus, qui d'après les mots d'Edward Said (2000, s.p.) est « un homme moral dans un monde immoral » : pied-noir de naissance et parisien adopté, tout en plaidant pour les droits des indigènes, il ne parvient jamais à renier la légitimité de l’Algérie française, subissant ainsi les attaques et de sa propre communauté et des intellectuels français de gauche. Cependant, puisque notre objectif ici n'est pas de juger de l'implication morale de Camus dans la décolonisation de l'Algérie, il nous suffit de constater que aussi bien une lecture littérale de La Peste qu'une lecture allégorique entraine des paradoxes concernant la représentation des communautés indigènes franco-algériennes. Loin d'essayer de les dissoudre, nous continuerons donc notre réflexion par l'ouverture d'une perspective différente.

\section{L'épidémie et l'échec de l'universalisme}

Nous avons déjà mentionné l'un des reproches principaux qui ont été adressés à Albert Camus, c'est-à-dire le choix de recourir à l'image de l'épidémie pour dénoncer l'oppression idéologique. En effet, comparer les fascismes à la peste d'Oran équivaut à renier l'intervention de l'homme et dans la montée des intolérances et dans le combat contre celle-ci, l'épidémie étant imprévisible et inexplicable : «On était obligé seulement de constater que la maladie semblait partir comme elle était venue » (Camus 2006, 220). L'image de la peste prive l'homme d'un quelconque rôle dans la prévention et dans la résistance à l'oppression, car tout effort humain contre le fléau serait vain : le nombre des contagions augmente et diminue sans une raison apparente, et ceci malgré l'engagement de la formation sanitaire volontaire dirigée par Rieux.

L'épidémie d'Oran est donc considérée exclusivement comme un phénomène naturel inéluctable, mais contre lequel l'homme peut quand même s'acharner, tel qu'un nouveau Sisyphe en révolte (Bonk 2010, 16). Se définissent ainsi deux dimensions, l'une en contraste avec l'autre : il existe d'abord une dimension «naturelle » liée à la peste, à laquelle s'oppose ensuite une dimension humaine et sociale. Lorsque Tarrou affirme que le microbe est naturel, alors que « le reste [est] un effet de la volonté » (Camus 2006, 209), Giuliana Lund $(2011,135)$ croit que : 
[Albert Camus] implies that disease [...] has an environmental cause. Health, on the other hand, arises from the rebel's determination to reject this norm and assert human values in place of the violence and amorality of nature. ${ }^{3}$

Le discours s'articule donc autour du couple binaire humain/non-humain, le second élément étant un « Autre » à démanteler afin de sauvegarder le bien-être de la société. Dans ce sens, le discours peut s'inscrire dans l'une des problématiques principales de l'écocritique, qui est le rapport plus ou moins conflictuel entre l'homme et l'environnement, la culture et la nature (Glotfelty 1996, XX).

Cependant, ce que cette interprétation et ses effets ne prennent pas en considération est la nature intrinsèquement sociopolitique de l'épidémie. Le professeur transdisciplinaire Ed Cohen $(2011,16)$ rappelle en effet qu'une épidémie est essentiellement " humaine » en raison de l'étymologie même du mot : du grec epi, 'sur', et dêmos, 'peuple', une épidémie se distingue d'une épizootie, car elle ne s'abat que sur l'homme, et

what makes human life special is the political character which qualifies it as « human » in the first place. ${ }^{4}$

Une épidémie n'est donc pas seulement un phénomène naturel, biologique ; c'est aussi - et peut-être surtout - une affaire humaine, autrement dit, sociale et politique. C'est alors grâce à la dimension essentiellement humaine de l'épidémie que Macs Smith $(2016,194)$ peut affirmer que le choix d'Albert Camus d'exploiter la peste comme allégorie des totalitarismes n'entraîne pas nécessairement la déresponsabilisation des hommes face à l'injustice.

L'épidémie secoue en somme la vie sociale de l'homme. Elle ne s'abat pas seulement sur l'individu comme une maladie quelconque, mais plutôt sur la collectivité, et ceci à cause de son caractère contagieux (Cohen 2011, 16). Le narrateur de La Peste, en qualité de médecin ainsi que de simple citoyen d'Oran, semble être conscient de la dimension sociale de l'épidémie, comme le montre la transition du « je » au « nous » qu'entraîne la maladie dans les passages suivants. Jusqu'au moment où les institutions sanitaires et l'opinion publique ne reconnaissent et n'assument pas l'épidémie en cours, le citoyen est encore un individu qui mène une vie quasiment ordinaire - malgré

3 « [Albert Camus] insinue que la maladie [...] a une origine naturelle, alors que la santé émerge de la détermination de l'homme révolté de rejeter cet état naturel et d'affirmer les valeurs humaines à la place de la violence et de l'amoralité de l'environnement » (traduction de l'Auteure).

4 «Ce qui rend spéciale et justement 'humaine' la vie des êtres humains, c'est son essence politique » (traduction de l'Auteure). 
les rats qui meurent dans la rue - et dont les émotions sont strictement personnelles bien qu'il vive en contact constant avec les autres :

Apparemment, rien n'était changé. Les tramways étaient toujours pleins aux heures de pointe, vides et sales dans la journée. Tarrou observait le petit vieux et le petit vieux crachait sur les chats. Grand rentrait tous les soirs chez lui pour son mystérieux travail. Cottard tournait en rond et M. Othon, le juge d'instruction, conduisait toujours sa ménagerie. Le vieil asthmatique transvasait ses pois et l'on rencontrait parfois le journaliste Rambert, l'air tranquille et intéressé. Le soir, la même foule emplissait les rues et les queues s’allongeaient devant les cinémas. (Camus 2006, 77)

Le narrateur est un observateur externe qui se limite à décrire ce qu'il voit lors de ses déplacements de travail dans la ville, d'où l'emploi de la troisième personne qui crée un écart entre le narrateur et les autres personnages mentionnés. En outre, les verbes - tous à l'imparfait - ainsi que les adverbes de temps accentuent le caractère routinier des activités et des loisirs des habitants d'Oran. Au contraire, lorsque l'état de peste est déclaré et que la ville est fermée, le narrateur met l'accent sur le caractère soudain et irréversible des événements à travers l'emploi du passé simple ; en outre, il parle à la première personne du pluriel et évoque une collectivité qui partage les mêmes sentiments :

À partir de ce moment, il est possible de dire que la peste fut notre affaire à tous. Jusque-là, malgré la surprise et l'inquiétude que leur avaient apportées ces événements singuliers, chacun de nos concitoyens avait poursuivi ses occupations, comme il l'avait pu, à sa place ordinaire. [...] Mais une fois les portes fermées, ils s'aperçurent qu'ils étaient tous, et le narrateur lui-même, pris dans le même sac et qu'il fallait s'en arranger. C'est ainsi, par exemple, qu'un sentiment aussi individuel que celui de la séparation d'avec un être aimé devint soudain, dès les premières semaines, celui de tout un peuple. (Camus 2006, 78 ; italique ajouté)

La répétition de l'adjectif ou adverbe " tout », « tous » insiste sur l'impartialité de la peste : elle peut s'abattre sur qui que ce soit. Par la suite, les événements semblent le confirmer : aussi bien des hommes que des femmes attraperont la peste, ainsi que des enfants et des gens âgés, des riches et des pauvres. D'ailleurs, c'est parce qu'elle frappe sans aucune distinction sociale que la peste possède un très fort potentiel littéraire (Vitaux 2010, 177).

La plupart des Oranais qui tombent malades restent anonymes : ils ne sont évoqués que par les chiffres de contagion du bulletin quotidien de Joseph Grand. Ils constituent donc un seul «personnage 
collectif » qui subit les supplices de la peste et, éventuellement, la mort (Stevenson 2017, 35). Il nous semble que l'idée d'un «personnage collectif » visé par la peste est proche de la notion d'« Homme » qui est développée dans La Déclaration des Droits de l'Homme et du Citoyen de 1789 et sur laquelle est fondée la société universaliste française. D’une manière générale, l'universalisme à la française proclame l'égalité entre les individus au nom d'une idée abstraite d'être humain (Wihtol de Wenden 2007, 49). La différence (d'ethnie, de genre, de classe, de religion, etc.) n'est pas reconnue par l'universalisme. Dans ce sens, la peste est universaliste dans la mesure où elle ne fait pas de discrimination : devant elle, tout individu peut succomber. Ce que le narrateur Rieux donne à voir à travers l'évocation répétitive des chiffres des décès est que la cible de la peste est l'« Homme », c'est-à-dire un être humain qui n'existe pas, mais qui est susceptible de représenter tout être humain vivant.

Or, l'universalisme à la française et l'idée d'« Homme » qui en est au centre ont soulevé bien des perplexités, ainsi que l'ont fait La Peste et l'égalité que le roman met en scène. D’après bien des critiques, l'universalisme français se veut égalitaire tout en favorisant implicitement la discrimination à cause du caractère exclusif de la définition d'« Homme » qu'il a façonnée : certains individus ne sont pas considérés comme étant dignes de bénéficier des droits « universels » car ils ne répondent pas aux critères que cette définition prévoit. Il s’agit d'un paradoxe qui est particulièrement évident si nous pensons à la colonisation française, quand la République - bâtie sur les réflexions universalistes des Lumières - forge une définition d'« Homme » basée sur l'opposition entre l'homme blanc occidental et l'indigène noir primitif. Le sens moral supérieur du premier lui impose de " civiliser » le second, justifiant ainsi la colonisation à travers une forme abusive de bonne foi qui est la mission civilisatrice (Gallissot 2006, 14).

L'épidémie de La Peste agit de manière analogue. Tout en étant présentée comme impartiale, la peste frappe exclusivement la communauté européenne d'Oran. L'universalisme semble ainsi s'adresser à une catégorie limitée d'individus qui rentrent dans la définition occidentale d'« Homme ». Les « Arabes », au contraire, sont exclus du récit, et ceci bien que leurs conditions de vie soient «mauvais[es] » (Camus 2006, 41) et qu'ils soient donc plus prédisposés à la maladie et à la contagion (Lund 2011, 144). Dans une certaine mesure, La Peste témoigne donc du plus grand paradoxe de l'universalisme à travers la mise en scène d'une épidémie qui est censée s’abattre sur tout homme, mais qui se révèle discriminatoire.

Le choix d'évacuer les «Arabes » du récit de la peste d’Oran ne peut être imputé qu'au docteur Rieux, qui en est le seul narrateur intradiégétique, quoiqu'en cachette. Il nous semble que l'adoption de la focalisation interne suscite des perplexités liées à l'exclusion de la communauté indigène du récit : premièrement, la partialité du point 
de vue de Rieux ; deuxièmement, sa prétention de parler au nom de tout citoyen oranais. Il s'agit de deux aspects qui sont strictement liés l'un à l'autre.

Afin de dissimuler son implication directe dans les événements, le narrateur Rieux se cache derrière l'emploi de la troisième personne. Il n'avoue qu'il est l'« auteur » du récit que lorsque celui-ci « touche à sa fin » (Camus 2006, 243) ; et pourtant, bien qu'il ait enfin dévoilé sa stratégie, il refuse de se servir du pronom «je » pour relater les derniers événements liés à l'épidémie, ainsi que pour formuler la morale que le public devra retenir (244-8). Ce choix dérive de la nécessité de proposer aux lecteurs un document auquel ils peuvent faire confiance, et de faire en sorte qu'ils partagent intégralement le message final qui y est proposé. L'objectif du narrateur Rieux est de rédiger une « chronique » qui soit aussi fiable qu'un témoignage à la première personne (Cooke 2009, 27) ; il l'accomplit en prenant les distances du personnage Rieux à travers l'emploi de la troisième personne. Rieux est un témoin qui se veut « fidèle » et «objectif » (Camus 2006, 243) : il aspire à la véridicité et à l'impartialité. Cependant, d'après Yves Ansel $(2002,48)$, il n'y parvient pas : « il refuse les données brutes de l'histoire : il sélectionne et oriente les faits, les ordonne, leur donne une forme ». À titre d'exemple, Ansel (59) montre que la manière dont Rieux se sert des sources extérieures - telles que le journal de Tarrou ou les cahiers de Grand - est biaisée : le narrateur porte explicitement un jugement plus ou moins négatif sur les documents qu'il propose aux lecteurs, les subordonnant ainsi à sa propre narration. En d'autres termes, Rieux n'octroie qu'à lui-même le privilège de façonner la version officielle des événements d'Oran, et ceci parce qu'il se sent investi d'une mission : il est « appelé à témoigner » (Camus 2006, 243), et il ne s'y soustrait pas.

Pour accomplir son devoir, Rieux croit « [devoir] parler pour tous » (Camus 2006, 244). Or, cette affirmation pose un problème d'autorité : qui a accordé un tel pouvoir à Rieux ? Personne que lui-même. Il se sent légitimé parce que, nous l'avons déjà dit, il croit que ses sentiments sont les sentiments de tout un peuple devant la cécité de la peste. En outre, Rieux justifie sa prise de parole à travers sa profession de médecin, qui lui a permis de rendre visite à bien des Oranais : il se croit « bien placé pour rapporter ce qu'il [a] vu et entendu » (243). De nombreux chercheurs ont vu dans ces affirmations la preuve de l'échec de l'universalisme (voir Lund 2011, 137 ; Smith 2016, 201) : Rieux personnifierait l'intellectuel européen et eurocentriste qui se croit autorisé à parler au nom de l'« Autre », lui enlevant ainsi tout espace de parole et le réduisant au silence. Or, cette interprétation néglige l'évidence qu'en fait Rieux ne parle pas au nom de l'« Autre » parce que, simplement, il n'inclut pas l'« Autre ». L'universalisme de Rieux n'est valide que pour la communauté européenne d'Oran parce que c'est la seule communauté dont il relate les angoisses. 
Rieux ne se permet donc pas de parler au nom des «Arabes » et c'est peut-être celle-ci la raison pour laquelle il décide de les évacuer tout court de sa « chronique ». Un tel choix pourrait s'expliquer par l'impossibilité de «porter condamnation totale » (Camus [1947] 2006, 41) que nous avons précédemment évoquée. Dans ce sens, Rieux s'opposerait à toute déclaration sur l'état sanitaire de la population indigène ainsi qu'il refuserait de mettre en scène un fléau et une résistance qui ne sont pas les siens et qu'il ne pourrait pas condamner entièrement en raison de son identité pied-noire. En effet, lorsque Rieux éclaircit au lecteur son rôle de narrateur et de témoin, il avoue qu'il a « gardé une certaine réserve, comme il convient à un témoin de bonne volonté » (Camus [1947] 2006, 243). Le mot « réserve » est l'un des mots-clés de la conversation entre Rieux et Rambert. Bien qu'il puisse s'agir d'un cas fortuit, il n'est pas impossible de supposer que, par cet écho, le narrateur Rieux souhaite faire allusion encore une fois à sa position inconfortable quand il s'agit de la communauté indigène : sa « bonne volonté » ne suffit pas à mettre en scène et les Français d'Algérie et les Algériens indigènes, d'où les restrictions qu'il se donne lui-même. Nous pouvons ainsi conclure que l'exclusion des « Arabes » est certainement problématique ; pourtant, elle autorise une lecture du roman qui préserve Rieux de l'accusation d'hypocrisie.

\section{Conclusion}

L'objectif de cet article a été de révéler le lien entre l'épidémie et la société multiethnique oranaise dans La Peste d'Albert Camus. Nous sommes parti d'un constat : les événements du roman se déroulent dans une ville franco-algérienne ; pourtant, aucun indigène algérien n'est inclus dans le récit.

Nous avons premièrement étudié la nature allégorique de la peste car c'est de celle-ci que dépend l'ambiguïté de la (non-)représentation de la communauté indigène oranaise. Nous avons mis en lumière à la fois la défaillance de l'allégorie et l'imprécision du vraisemblable, dépendant probablement des positions idéologiques d'Albert Camus. Ensuite, nous avons mis l'accent sur l'essence intrinsèquement politique et sociale de l'épidémie, en montrant ainsi que, dans La Peste, il n'existe pas de dualité culture/nature. Enfin, à travers l'analyse de la voix narratoriale, nous avons étudié le rapport entre l'épidémie et la représentation de la collectivité, et nous avons montré que la peste d'Oran et l'universalisme républicain français suivent des schémas discriminatoires analogues.

En guise de conclusion, il ne reste qu'à rappeler que cette étude ne se veut pas une attaque contre Albert Camus pour avoir été contradictoire ou pour ne pas avoir mis en scène les communautés indigènes algériennes. Nous avons plutôt voulu décrire et tenter d'ex- 
pliquer de telles contradictions, sans la vanité de juger l'écrivain et l'homme. Puisque c'est exclusivement le roman qui a fait l'objet de cette étude, nous voulons conclure en mettant l'accent sur sa valeur littéraire. En fin de compte, le succès que La Peste a retrouvé aujourd'hui aux temps du coronavirus montre que le roman a été en mesure de transcender les frontières de temps et d'espace dans lesquelles il a été conçu, et ceci grâce à un récit qui peut être interprété à la fois de manière allégorique et littérale. Dans ce sens, il serait intéressant d'analyser si, parmi les pays qui ont connu une augmentation des ventes du roman d'Albert Camus, c'est aussi l'Algérie qui apparait, un pays qui n'est pas encore totalement à l'aise avec le « silence » de l'écrivain sur l'indépendance (Leperlier 2017, 71).

\section{Bibliographie}

\section{Fuvres de Albert Camus}

Camus, A. (2006-2008). CEuvres Complètes [dorénavant désignées par OC]. 4 vols. Edité par J. Lévi-Valensi et R. Gay-Crosier. Paris : Gallimard. Bibliothèque de la Pléiade.

En particulier, nous avons pris en considération les textes suivants:

Camus, A. [1938] (2006). «Petit guide pour des villes sans passé ». L'Été. OC, vol. 3, 593-6.

Camus, A. [1939] (2006). « Misère de la Kabylie ». Actuelles III. Chroniques algériennes 1939-1958. OC, vol. 4, 307-36.

Camus, A. [1947] (2006). La Peste. OC, vol. 2, 31-289.

Camus, A. [1955] (2006). "Lettre à Roland Barthes sur La Peste». OC, vol. 2, 285-8.

\section{Critique}

Ansel, Y. (2002). «La Peste des Carnets au roman ». Littérature, 128, 46-64. https://doi.org/10.3406/litt.2002.1773.

Ansel, Y. (2012). Albert Camus, totem et tabou. Politique de la postérité. Rennes: Presses Universitaires de Rennes.

Berardi, A. (2020). La subalternité dans "L'Étranger", "La Peste" et "Le Premier Homme" d'Albert Camus. Une analyse postcoloniale et de genre [thèse de master]. Venise: Università Ca' Foscari. http://hdl. handle. net/10579/16801.

Bonk, R. (2010). « Medicine as an Absurdist Quest in Albert Camus' The Plague ». Eä. Journal of Medical Humanities \& Social Studies of Science and Technology, 2(1), 1-22. http://www.ea-journal.com/art2.1/Medicine-asan-Absurdist-Quest.pdf.

Carroll, D. (2007). Albert Camus the Algerian: Colonialism, Terrorism, Justice. New York: Columbia University Press. 
Chaulet Achour, C. (2013). «Albert Camus face à la question algérienne ». Histoire coloniale et postcoloniale. https://histoirecoloniale.net/Albert-Camus-face-a-la-question.html.

Cohen, E. (2011). "The Paradoxical Politics of Viral Containment; or, How Scale Undoes Us One and All ». Social Text, 29(1), 15-35. https://pdfs. semanticscholar.org/e 02 c/e54305c48e46bacd263fcae39fe2b2a47ab1.pdf.

Cooke, J. (2009). «Writing Plague: Transforming Narrative, Witnessing, and History ». At the Interface/Probing the Boundaries, 55, 21-42. https://doi. org/10.1163/9789401206716_004 .

Feraoun, M. (1969). Lettres à ses amis. Paris : Éditions du Seuil.

Foxlee, N. (2009). "'Arabes', 'Algériens' et autres appellations dans le discours camusien ». Bulletin de la Société des Études Camusiennes, 88, 358. https://www.academia.edu/4010871/_Arabes_Algériens_et_ autres_appellations_dans_le_discours_camusien.

Gallissot, R. (2006). La République française et les indigènes : Algérie colonisée, Algérie algérienne (1870-1962). Paris : Éditions de l'Atelier.

Glotfelty, C. (1996). «Introduction. Literary Studies in an Age of Environmental Crisis ». Glotfelty, Cheryll ; Fromm, H. (eds), The Ecocriticism Reader : Landmarks in Literary Ecology. Athens ; London: University of Georgia Press, XV-XXXVII.

Leperlier, T. (2017). « Camus et la 'littérature algérienne' : une notion stratégique dans l'espace littéraire francophone ». French Politics, Culture \& Society, 35(3), 68-90. https://bit.ly/3rjhmKb.

Lowe, C. (2014). «Infection ». Environmental Humanities, 5(1), 301-5. https:// doi.org/10.1215/22011919-3615559.

Lund, G. (2011). «A Plague of Silence : Social Hygiene and the Purification of the Nation in Camus's La Peste ». Symposium : A Quarterly Journal in Modern Literatures, 65(2), 134-57. https://doi.org/10.1080/00397709.20 11.573737.

Marx, W. (2020). "Ce que la littérature nous apprend de l'épidémie ». Fabula. Atelier de théorie littéraire. https://www.fabula.org/atelier.php?Ce_ que_la_litterature_fait_a_l_epidemie.

Murat, M. (2020). «La peste comme analogie». Fabula. Atelier de théorie littéraire. https://www.fabula.org/atelier.php?La_peste_comme_ analogie.

Nash, W. (2019). The Health Humanities and Camus's "The Plague". Kent : The Kent State University Press.

Said, E. [1993] (2000). "Albert Camus, ou l'inconscient colonial ». Le Monde diplomatique. https://www.monde-diplomatique.fr/2000/11/ SAID/2555.

Smith, M. (2016). « What Dies in the Street: Camus's La Peste and Infected Networks ». French Forum, 41(3), 193-208. https://www.jstor.org/stable/10.2307/90001133.

Stephanson, R. (1987). «The Plague Narratives of Defoe and Camus : Illness as Metaphor ». Modern Language Quarterly, 48(3), 224-41. https://doi. org/10.1215/00267929-48-3-224.

Stevenson, L. (2017). Le savoir médical dans "La peste" d'Albert Camus, "La quarantaine" de J.M.G. Le Clézio et "Les trois médecins" de Martin Winckler [thèse de maîtrise]. Ottawa : Département de Français, Faculté des Arts, Universi- 
té d'Ottawa. https://ruor.uottawa.ca/bitstream/10393/35842/3/ Stevenson_Laura_2017_thèse.pdf.

Vitaux, J. (2010). "La rémanence de la peste dans l'imaginaire collectif». Vitaux, J., Histoire de la peste. Paris: Presses Universitaires de France, 149-87. https://www.cairn.info/histoire-de-la-peste-9782130584094-page-149.htm.

Voisine-Jechova, H. (2001). " La peste comme interrogation existentielle : parallèles et anti-parallèles entre Lagerkvist et Camus ». Revue de littérature comparée, 298(2), 263-74. https://doi.org/10.3917/rlc.298.0263.

WihtoldeWenden, C. (2007). «Debating Cultural Differencein France ».Raymond, G. (ed.), The Construction of minority identities in France and Britain. London : Palgrave Macmillan. https://doi.org/10.1057/9780230590960_4.

Zapf, H. (2010). «Ecocriticism, Cultural Ecology, and Literary Studies ». Ecozon@, European Journal of Literature, Culture and Environment, 1(1), 136-47. https://opus.bibliothek.uni-augsburg.de/opus4/frontdoor/ deliver/index/docId/57938/file/332-608-1-PB.pdf. 



\title{
Ruy Duarte de Carvalho's Neo-Animist Project: An African Approach to Posthumanism
}

\author{
Alice Girotto \\ Università Ca' Foscari Venezia, Italia
}

\begin{abstract}
The literary project of the Angolan writer Ruy Duarte de Carvalho, from the collection of poems Chão de oferta (1972) to the metafictional trilogy Os filhos de Próspero (2009), along with his work as an anthropologist, filmmaker and intellectual, is animated by a research for a conjunction between subject, geology, landscape and politics, which rejects Western epistemological categories and their underlying powers. At the end of his life, this trait of his personality and work took the form of an unfinished neo-animist project, based on a decalogue in which he criticises the humanist paradigm for the sake of recovering an "equilibrium politics" relying on "[o]ther paradigms set aside and hidden from consideration because they originate in cultures dominated or annihilated by the Western world". The aim of this essay is both to trace the origins of Carvalho's neo-animist project in his early poetry and narrative and connect it with a broader African criticism, both literary and philosophical, in order to include his thought within the African approach to posthumanism.
\end{abstract}

Keywords Neo-animism. Posthumanism. Ecology of knowledges. Southern epistemologies. Angolan literature. Ruy Duarte de Carvalho.

$\begin{array}{lll} & \text { Peer review } & \\ \text { Edizioni } & \text { Submitted } & 2020-07-15 \\ \text { Ca'Foscari } & \text { Accepted } & 2020-09-11 \\ & \text { Published } & 2020-12-22 \\ & \text { Open access } & \end{array}$

(c) 2020 | ()(1) Creative Commons Attribution 4.0 International Public License 
In a 2005 talk at the University of Coimbra, given in occasion of the presentation of his second novel, the Angolan writer Ruy Duarte de Carvalho, reasoning around the historical time in which his multifaceted work was born and evolved, declared it as being

um tempo em [que] a modernidade e as suas expressões se projectam na negação do seu próprio projecto.......... em que a fé e a confiança no projecto humanista, renascentista e iluminista de colocar a regulação do mundo ao alcance do homem através da ideologia totalizante e totalitária do progresso e da complexificação tecnológica, e de uma verdade e de uma liberdade que dão cobertura a toda a ordem de arbitrariedades e de crimes, se negam como saída e resolução óbvias para os problemas da espécie.

(Carvalho 2008, 24-5)

a time in [which] modernity and its expressions project themselves in the negation of their own project.... in which the faith and confidence in the Humanism, Renaissance and Enlightenment project of putting the regulation of the world within man's means through the totalizing and totalitarian ideology of progress and technological complexification, and of a truth and a freedom that act as a front for any kind of arbitrariness and crime, negate themselves as obvious way out and solution for the species' problems. 1

With these words he highlighted a common thread underlying his whole literary project, first as a poet and then as a novelist, and indeed his own life and work as an anthropologist, filmmaker and intellectual, that is a restless research for a conjunction between subject, geology, landscape and politics, which, in a postcolonial fashion, rejects Western epistemological categories and the powers they are the mirror of (Carvalho 2019). As stated earlier in the same text, the explanation for this attitude must be traced back to his own experience as a

sujeito em situação no contexto de uma configuração social e formal, política, fora da geografia humana e física que [o] viu nascer. (Carvalho 2008, 20) ${ }^{2}$

1 All the quotations from Ruy Duarte de Carvalho's works, except those from his NeoAnimist Decalogue, and from Boaventura de Sousa Santos's A cruel pedagogia do vírus are translated into English by the Author of the essay.

2 As a matter of fact, these words are also a proof of the deep influence of post-modern anthropology on Carvalho's literary work for the extreme relevance in it of the ethnographer's own subjectivity and perception within his/her field research (Miceli 2011, 15). 
situated subject in the context of a social and formal (political) configuration outside the human and physical geography that saw [him] to be born.

A hybrid subject, indeed, but in the opposite direction in comparison with the hybridity postulated by Bhabha (1994): in fact, Ruy Alberto Duarte Gomes de Carvalho (1941-2010) was born in Portugal and moved to Angola with his family in his early childhood, being among the more than 170,000 Portuguese that between 1945 and 1960 emigrated there, pushed by the colonization politics of the Salazarist regime and attracted by the growing wealth produced by the coffee-driven economy of the colony (Castelo 2007, 177). Realizing his own 'Angolanness' after reading Luandino Vieira's most known work Luuanda (1965), ${ }^{3}$ he took part in Angola's freedom fight and finally became an Angolan citizen in 1975. From 1975 to 1981 he directed films for the State television and Film Institute of the newly independent country. In 1982, with the film Nelisita, he graduated at the School of Advanced Studies in Social Sciences in Paris and in 1986 obtained his PhD in social anthropology and ethnology, with a thesis about the production of cultural difference between fishermen in Luanda's coast. From 1987 he taught social anthropology at the universities of Luanda in Angola, São Paulo in Brazil and Coimbra in Portugal, and developed his field inquiries mainly with pastoralist and agro-pastoralist societies in South-western Angola and North-western Namibia. It is precisely in this context that his metafictional trilogy Os filhos de Próspero (2009) was born and is mainly set - a 'South in the South' which is not surprisingly and programmatically the subject of the poem that opened his career as a writer ${ }^{4}$ and the geography to which he definitively and irreversibly consecrated himself since, at least, the ' 70 s. $^{5}$ And also his neo-animist project, developed at the end of his life and left unfinished, arose from the lengthily ruminated on observations made during his field work among the Kuvale, being thus a testimony of an "ecology of knowledges" (Santos, Nunes, Meneses 2007) that has as its cornerstone a peculiar "Southern epistemology" (Santos 2009).

Carvalho's neo-animist project was conceived, as described by the author's son in a publication resulted from a commemorative congress about his work held in Lisbon in 2015, as an investigation to

3 Among his other influences in this sense, he cited also Viriato da Cruz, Aires de Almeida Santos and Ernesto Lara Filho, Angolan writers and nationalists.

4 I am referring here to "O sul" (The South), the first poem of Ruy Duarte de Carvalho's first poetry collection Chão de oferta (1972).

5 References to this consecration can be found in other poems of Chão de oferta and in the short stories of Como se o mundo não tivesse leste (1977). 
be carried out by a team of philosophers, anthropologists, filmmakers and other intellectuals on the limits and contradictions of humanism and the criticism it had received until that moment in various historical periods and contexts, on the one hand, and on animist practices, on the other, to get a concrete understanding of them and of their alterity to humanism and Western categories; to this theoretical groundwork an action programme with its own manifesto and movement would follow. The two sides of the project are made clear in the very first point of his Neo-Animist Decalogue, written in 2009 and published few months before his death:

1 - Although coming from an animist conception that everything in the world has a soul that each existence expresses according to its body and substance, the term neo-animism occurs to name an action program made to question the humanist paradigm that dominates and conducts the march of the world, enlarged to the whole human species' practices and responsibilities but exclusively under the imperative and enforced grammar made by a small part of it. To the Neo-Animists the proposal and the implementation of said program is the first task. This program, however, does not seek to only take references coming from the grammar of the animist paradigm but also from all the cultural or civilizational paradigms from past and present everywhere in the world where human species has produced or is producing interrogations, reflections, inventions, concepts, rules and ways of action. (Carvalho 2010)

The very first sentence puts this decalogue at that crossroads, or convergence, between post-humanism and post-anthropocentrism that for Braidotti (2019, xi) defines posthuman thought. By recognizing that each existence has a soul and through the terminology used for referring to human beings, that is as a 'species' - assuming thus that it is but one among many others -, Carvalho can be said to integrate post-anthropocentrism as a critic of the human supremacy over the whole planet, which derives from granting to the anthropos "an ontological privilege in the large majority of the historical accounts on the human" (Ferrando 2019, 54). Yet the emphasis, not only in this opening paragraph but all along the decalogue, is on the "imperative and enforced grammar" of only a small part of human beings, namely European and, more broadly, Western culture and discourse. Sinking their roots in the humanist, universalist ideal of 'man as measure of all things', they "turned into an exceptionalist civilizational standard" (Braidotti 2019, xii) and claimed for themselves the exclusivity of determining, from the top of a hierarchical scale, the "ways, measures and disposals necessary to life in society" (Carvalho 2010) all over the world, disrespectful towards other paradigms. This ech- 
oes the foundations of postcolonial thought and studies, whose specific contribution to the genealogy of posthumanism intended in its three developments (critical, cultural and philosophical posthumanism) is indeed quite relevant among the other "studies of the difference" (Ferrando 2019, 2). It is at least since Cesaire's 'humanist' bourgeois and Fanon's Europe "rushing to her doom" (Fanon [1961] 1963, 9) that the limits and contradictions of the founding myths of Western power, in other words its "'spiritual' exhaustion" (Carvalho 2019, 14), are questioned and belied. ${ }^{6}$ Actually, the first justified, supported, absolved, legitimized, nurtured and exercised "the crime in itself, the crime against man, [...] the humiliation of man as such" (Césaire [1955] 2001, 36) identified with Nazism, ${ }^{7}$ while the second one was a continent "where they are never done talking of Man, yet murder men [...] in all the corners of the globe" (Fanon [1961] 1963, 311).

But if we accept Braidotti's previously mentioned definition of the broader, non-hyphenated posthumanism, and even more precisely Ferrando's post-humanist, post-anthropocentric and post-dualist philosophical posthumanism, the contrast between it and the still anthropocentric anticolonial post-humanism is patent. Fanon's words are crystal clear in this sense: to "try to resolve the problems to which Europe has not been able to find the answers [...] there is no question of a return to Nature" (Fanon [1961] 1963, 314) but rather of "try[ing] to set afoot a new man" (316). ${ }^{8}$ As poignantly highlighted by Iheka (2019b), the focus, in Fanon's but also other anticolonial and postcolonial intellectuals' writings, remains on human beings, in a certain sense confirming the colonialist dualism that opposed nature and culture, the latter being the human's modifying touch on the first that, even if devoid of the so inherently colonial domination trait, still configures itself as the core action of a humanist homo faber. Iheka's argument is not only an acute examination of the texts of the postcolonial 'founding fathers', but also a critique of what he reads as a distinct absence in later postcolonial studies that based their analysis on those seminal texts, particularly as far as African literary works are concerned:

6 Cf. also the archaeology of the definitions of 'Blackness' and 'race' made by Mbembe $(2017,2)$; other references to Western founding myths and "social teleologies", with particular reference to development as an expression of Western episteme, cf. Sarr 2019.

7 The connection between humanism and eurocentrism, and between this and the hierarchical and racial concept of humanity promoted by both colonialism and Nazi-fascism, is well evidenced in Mellino's (2014) introduction to the latest Italian translation of Discourse on Colonialism (Mellino 2014).

8 The anthropocentric character of anticolonial thought must not be generalized, however, as Mbembe's reference to Césaire's poetry proves: "Some saw in the Black Man the salt of the earth, the vein of life through which the dream of a humanity reconciled with nature, and even with the totality of existence, would find its new face, voice, and movement" $(2017,7)$ 
If human imbrication with nonhumans has always been a feature of African societies and the literary expressions that emerge from them, why then has African literary criticism not paid much attention to the interlinkages and their ecological significance? Why have human concerns remained at the heart of literary criticism in Africa? (Iheka 2018, 6)

His remarks are based on the assumption, which is central in his Naturalizing Africa. Ecological Violence, Agency, and Postcolonial Resistance in African Literature, that Africa is maybe the most important place to understand "human imbrication with nonhumans", not just from the ecocritical perspective that has become paramount in the recent "environmental turn in African literary studies" (Iheka 2019a, 203), ${ }^{9}$ but more significantly in consideration of "the primacy of nature in African cultural systems" and its "imbrications with Homo sapiens in Africa's ecologies" (203) - an aspect highlighted also by Moolla (2016, 9), who emphasizes the long-term presence and agency of the natural world and animals in African cultural forms, being as they are the condition of existence of cosmologies, texts and artistic expressions of the latter, when compared to the much more recent appearance of ecocritical scholarship.

These imbrications and primacy of nature - animals and plants, but also landscapes and climate elements (rocks and waters, winds and rains), in a word "earth-beings" (Cadena 2010, 336) or "otherthan-human beings" (341) - in relation to the human, which are the premises of Ruy Duarte de Carvalho's neo-animist project, are a constant presence in his literary works from the very beginning, which can hence be considered an important contribution to African posthumanism. The title of his first poetry collection, Chão de oferta (Land of offer), modulated in the title and recalled in the verses of the poem "A terra que te ofereço" (The earth I offer you), combines in itself maybe the most archetypal of all other-than-human beings (the earth, the land) and an act, a practice that inherently and anthropologically distinguishes the human from all the other species and beings (the offer). In "Nas praias do sul" (In Southern beaches) there is a sort of exchange of reciprocal attributes, in particular relating to fertility, between the human element (a feminine 'you') and nature: ${ }^{10}$

9 Ecocriticism focuses in particular on the intersections between social and ecological issues and violence; among the scholars who adopt this lenses to interpret African literary texts, cf. Caminero-Santangelo 2014; 2015.

10 It is, indeed, a trait that can be observed also in the previously mentioned poem, which follows in the collection: "da lua que transportas | com a sólida | e materna nudez do horizonte" (Carvalho 2005, 20) (of the moon that you carry | with the solid | and maternal nakedness of the horizon). 
instalas-te embrião

no útero da rocha

e inventas-te filha

do rosto do mar.

(Carvalho 2005, 15)

you settle embryo

in rock's uterus

and invent yourself daughter

of sea's face.

Inanimate beings such as the 'rock' and the 'sea' have the power to generate a human offspring thanks to usually mammalian organs and functions, in the same way as the moon that menstruates ("menstrua-se a lua" - Carvalho 2005, 15). On the contrary, human organs and body are referred in their mineral substance, made of air and salt as they are:

circulam-te as brisas e teus ossos

de sal

filigranam-se iminentes de puro mar.

(Carvalho 2005, 15)

breezes circulate in you and your bones

made of salt

imminent filigree of pure sea.

We cannot talk of a personification in the classical (and Eurocentric) sense with which we understand this figure of speech, that is the attribution to inanimate objects of prerogatives proper to the human, which consequently end up acting as human beings - and of a nondefined figure of speech complementary to personification. ${ }^{11}$ Actually, we are in front of an intermingling that can be interpreted in the light of Iheka's "aesthetics of proximity" $(2018,21)$, namely in its second connotation, of a nearness "brought about by similarities and shared characteristics" (22) and that implicates "multispecies entanglement [...] the enmeshment of human and nonhuman lives" (23).

There is another poem of this collection in which the aesthetics of proximity emerges in a particularly gritty fashion: "Chagas de salitre" (Saltpeter sores), a tragic ode to the hard and cruel history of

11 We cannot talk here, for example, of 'objectification' as this is a concept of the field of social philosophy that, moreover, has a negative meaning (cf., for example, Mbembe 2001 ; 2017), while the attribution to human beings of prerogatives proper to inanimate beings as a figure of speech, in "Nas praias do sul", is neutral. 
slavery and colonial subjugation. Here, an action proper of the vegetable kingdom is attributed to organic but human material (“[...] vegetar | da urina e do suor | da carne virgem [...]" - Carvalho 2005, $22 ;[\ldots]$ the vegetate | of urine and sweat | of virgin flesh [...]), while human beings, or their remains, are reduced to an anonymous flowing of waters: "marés vazantes de gente amordaçada" (22) (tides of the gagged at a low ebb) and "[...] rios renovados de cadáveres" (23) (rivers refitted with corpses). Lastly, the nineteenth parts and thirty-seven stanzas of "Novembrina solene - Transmudação das águas" (Solemn November rain - Waters transmutation) stage the enchantment of the sequence of seasons in Southern Angola, in which the bush, the acacia, the cactus take the scene; in which the cycle of animals' life succeeds with its migrations, selections of the weak, herds that get lost; in which everything follows the rhythm of dewy mornings, low clouds and the direction of the wind. It is the triumph of natural elements:

\author{
Era novembro \\ um mês de cargas raras \\ húmido ardor \\ goma indecisa \\ sobressalto de ar.
}

De atenção às nuvens e à direcção do vento

consulta às luas e à ligeira referência

de um alado brilho de insecto

precursor

de um novembro a derramar-se em chuva morna.

(Carvalho 2005, 41)

\author{
November was \\ a month of rare downpours \\ humid ardour \\ uncertain ngoma \\ unease of the air. \\ Of attention to clouds and the direction of wind \\ enquiry into the moon and the slight reference \\ of a winged insect shine \\ harbinger \\ of a November slopping out in warm rain.
}

The only human traces are some tools (here the drums, the ngoma), actions (paying attention, enquiring, visiting), body parts (hands, feet, mouths) and few others; a presence, however, that at no time stands out and that enacts a real "equilibrium politics" (Carvalho 2010).

This concept is crucial when we talk about African cultural systems: it is the "mutual belonging (cobelonging) to a common world" 
discussed by Mbembe (2017, 1), a "system of exchange, reciprocity, and mutuality [in which] humans and nonhumans [are] silt for one another" (181). ${ }^{12}$ The Cameroonian philosopher traces back the birth of this concept of connection between beings to ancient Africa, explaining it as a celebration of life and as an act of care towards a both fragile and hostile environment, in order for it to be propitious to the development of life itself, born out of a seed. This relationship between the human and the environment needed to be constantly negotiated:

[N]ature was a force in and of itself. One could not mold, transform, or control nature when not in harmony with it. And this double labor of transformation and regeneration was part of a cosmological assembly whose function was to consolidate the relationships between humans and the other living beings with which they shared the world. (Mbembe 2017, 180-1)

"As águas do Capembaua” (Capembaua's waters), one of the three short narratives that compose Ruy Duarte de Carvalho's first prose work Como se o mundo não tivesse leste (As if the world had no East), depicts exactly the search for restoring the "cosmological assembly" and its harmony disrupted by a deliberate act of men. The narrative is divided in two parts, in which the story of the establishment of a fazenda in South-western Angola, of a long period of drought, of a South African killed by a leopard and of the coming back of the rain is told from the two points of view of $\mathrm{R}$, a white manager of the fazen$d a$, and José, a black (presumably Kuvale) foreman in the same fazen$d a$ and member of a pastors' clan affected by its establishment. The story is told by a narrator that seems to overlap with the author's figure, inaugurating here that auto-fictional mode of narration that will later characterize all Carvalho's novels and that brings about a circular relationship between life and text (Miceli 2011, 27).

In the narrative, the centrality of nature - which in Carvalho, as we have already seen, is often substantiated not in single animals, plants or inanimate beings, but in a more comprehensive 'landscape' or 'geography' - can be immediately understood from the brief introduction to the twofold story, where the narrator relates the circumstance in which he came to know José's version of it; it is the narrative itself, the words that humanly make sense of the world, that 'demands' an exact reference to the surrounding environment: "[E]nquanto a narrativa se vertia, as frases demandavam, nas anha-

12 Sarr, too, talks of the second legacy of Africans after the first, ancestral one for the whole Homo sapiens species as "a different perspective of social life, emanating from other mythological universes and lending it to the common dream of life, balance, harmony, meaning" $(2019,15)$ in a time of crisis of the technical paradigm and civilization. 
ras, a exacta referência a que aspiravam" (While the narrative poured, sentences demanded, in the savannahs, the exact reference to which they aspired, Carvalho [1977] 2003, 14). ${ }^{13}$ Moreover, since the beginning we know about the active role played by a leopard, which at the end of the story kills a seemingly randomly appeared South African character whose death could have some connections, according to the narrator, with R's rush fleeing from the fazenda. Lastly, the description of the drought, a distinct section of the first part of the short story, deploys on the pages devoted to it the role played by each element in this cyclically repeating tragedy: the arrogant ostentation of the sun, "[a] soberba passividade da lua, a demitir-se de ordenar as águas" (the superb passivity of the moon, which resigns from commanding waters, Carvalho [1977] 2003, 30), the earth that does not participate to this suspension of time and continues its movement and the movement of life, the grass that darkens, the cattle that dies, men and women that break themselves of their habits to try and find a different way for surviving. The juxtaposition of all the elements establishes their respective place in this comprehensive picture; humans come last and are dependent on the signs given by all the other elements to take their decisions.

But it is in the second part of the short story, the one reconstructed by the narrator from José's interpretation of the events (that is, from a Kuvale point of view), that we understand the cosmological order within which the whole story must be read and the final "reorganização do cosmos e [...] reintegração dos homens no mecanismo das funções astrais" (reorganization of cosmos and [...] reintegration of men in the mechanics of astral functions, Carvalho [1977] 2003, 65). This is possible thanks to a precise attitude, adopted by the narrator himself, which implies broadening the perspective that the whites usually have on reality to encompass a wider consideration in terms of time and space; these are dimensions that must be 'auscultated' complicitly in order to be able to translate the energies deeply hidden in them and, thus, see the whole sequence of events as "uma harmoniosa e lógica articulação de forças inseridas num conteúdo de serena continuidade, sem sobressalto ou estranheza" (a harmonious and logic articulation of forces inserted in a content of serene continuity, without unease or strangeness, Carvalho [1977] 2003, 53). While in the first part of the story the only, somehow mysteriou-

13 In another passage, the facts occurred in the story are illustrated not only by characters, but also by geography ("as personagens e a geografia que ilustravam os casos" - Carvalho [1977] 2003, 21) and the development of R's adventure is determined by the conflicts and contradictions proper of a semi-arid region, so by all the elements, human and nonhuman, that equally contribute to environmental equilibrium: "A acção desenrola-se numa região semi-árida. E toda a aventura de $\mathrm{R}$ decorre de conflitos e de contradições ligados a isso" (24). 
sly connected events appear to be the South African's death, R's fleeing from the fazenda and an invasion of its delimited, irrigated land by pastors and their herds after one year and a half of drought, José's tale testifies broader relationships among facts and (present and past) living beings:

'[S]e abririam para nós os sinais de que a ordem se alterava nas razões mais fundas, onde se excede a limitada interferência dos homens vivos'. (Carvalho [1977] 2003, 58-9)

'Signals would open for us that the order was altering in its deepest reasons, where the limited interference of living humans is surpassed'.

The transfer of the local clan's most important habitational centre (onganda) from its original, ancestral establishment due to the delimitation of the fazenda land leaves an intimate wound in the clan's chief, who dies shortly after; with him, the sacred cow that represents him in the hierarchy of animal belongings inside the clan almost immediately dies, too; the leopard appears as a sign of ancestors' rejection of remedial sacrifices; and the rain stops to fall. The only solution to this inauspicious chain of events envisioned by the subsequent diviners consulted by the clan is the introduction of the whites, who are responsible for the initial offence, that is the onganda transfer, into the sacred mechanisms that govern existence. Actually, their immunity to the occult forces that had always regulated not only the blacks' lives, but the entire cycle of existence, had always allowed them the privilege of not respecting neither any kind of law nor human and nonhuman beings, and of considering themselves the legitimate conquerors and lords of the land and of everything inhabiting it. But this exclusivity now puts them in the position of finally entering "no último reduto do poder dos negros" (in the ultimate redoubt of the black's power, Carvalho [1977] 2003, 67); this is why, when R decides to hunt the leopard during the last night of his stay at the fazenda, José suggests that he, a white indeed but (before this) a man respected by the pastors, brings with him a Boer ambiguously appeared in the area few weeks before. This would lead to the end of the drought (and of the story):

Perseguida a onça, abate-se o animal sobre o sul-africano e cumpre-se a vontade dos defuntos. As contas estão saldadas e a chuva chove, abundante e clara. (Carvalho [1977] 2003, 70)

Chased the leopard, the animal kills the South-African and fulfils the dead's will. The bill is paid off and the rain falls, abundant and light. 
The intersection, in "As águas do Capembaua", between what I called the 'Kuvale point of view', which brings together all living beings in its consideration, and the narrator's positive attitude towards a wider perspective, able to embrace a non-Western paradigm of interpretation of reality, anticipates in the literary text what would be later defined the epistemological practice of the "ecology of knowledges", that is

an invitation to the promotion of non-relativistic dialogues among knowledges, granting 'equality of opportunities' to the different kinds of knowledge engaged in ever broader epistemological disputes aimed both at maximizing their respective contributions to build a more democratic and just society and at decolonizing knowledge and power. (Santos, Nunes, Meneses 2007, xx)

In Carvalho's short story, it would be impossible for the narrator himself - even before his narrative act to actually take place - to fully understand the complex framework represented in the text without the dialogic attitude implied by the ecology of knowledges, which requires both the rational, 'Western' explanation of the facts (R's account) and the non-Western one (the Kuvale point of view). The latter is interpreted through other epistemological lenses and derives from different prior knowledge. These, in turn, come from a worldview, or cosmology, that is alternative to Western (and Eurocentric, though allegedly universally valid) modern science, and in their difference they represent an epistemological challenge to its dominance within the realm of the knowledge produced by human beings all around the world. In this sense these alternative knowledges and cosmologies are "Southern epistemologies" because, in their act of resisting their own suppression and claiming their righteous place on the same playing field of modern science, they question the epistemological premises of the global North dominance over the global South ${ }^{14}$ in the form of colonialism, justified through the equation between (the 'right') knowledge and power. The operation of giving voice, through the ecology of knowledges, to these "[o]ther paradigms set aside and hidden from consideration because they originate in cultures dominated or annihilated by the Western world" (Carvalho 2010) is essential to deactivate the epistemological privilege of scientific knowledge, one funded on the drastic separation of nature from society. ${ }^{15}$ The link that con-

14 The global South, which partially but not necessarily coincides with the geographic Southern hemisphere of the earth, is defined by Santos $(2009 ; 2020)$ as a political, social and cultural space-and-time experienced by those subject to capitalism in its colonial and patriarchal relationship with the world.

15 Santos, Nunes and Meneses talk, in this respect, of "substituting a monoculture of scientific knowledge" (2007, xlviii). It is not casual their use of terms referring to 
nects indigenous, non-Western, Southern epistemologies' struggle to survive the hegemonic, colonial, subalternizing attempts to destroy their cosmologies, and their relationship with nature, which they do not consider as a resource to be exploited and set aside from the human, civilized world, appears thus to be the only possible "way out and solution for the species' problems", the only one able to ensure, at the same time, the "reproduction and maintenance of the species and personal expression and knowledge" (Carvalho 2010).

Ruy Duarte de Carvalho's neo-animist project had the purpose of overcoming, in a posthumanist fashion, Western "'spiritual' exhaustion". It was written at a time, in 2009, when this depletion had but begun to show its material signs, in the form of the financial crisis started the year before in the United States and of the Great Recession that followed. Reading it (and all Carvalho's work) again in these days, in the meantime and shortly after the lockdown enforced by the outbreak of a pandemic caused, as many experts affirm, by the consequences of the imposition of Western economic and societal model on the entire planet and its disruption of ecosystem balances, reveals how much needed and vital the contribution of an African, non-aligned and reflexive intellectual is, in order for us, human beings from any part of the Earth, to "assuma[mos] uma posição mais humilde no planeta que habita[mos]" (Santos 2020, 31) (assume a humbler position on the planet we are living on) and

[nos] habitue[mos] a duas ideias básicas: há muito mais vida no planeta do que a vida humana, já que esta representa apenas 0,01\% da vida existente no planeta; a defesa da vida do planeta no seu conjunto é a condição para a continuação da vida da humanidade. (31)

get accustomed to two basic ideas: there is much more life on the planet than human life, since this represents just $0.01 \%$ of the existing life on the planet; defending the planet life in its entirety is the condition for humanity to continue to live.

Because, as Ruy Duarte de Carvalho concluded his decalogue,

10 - we are all together, all in the same boat, all the men and all that exists in the whole universe. And if there are other univers-

nature and environment ('ecology', 'monoculture') in relation with knowledge, as they recognise biodiversity as both a 'contentious area' and a concrete example of an ecology of knowledges, because the discourses produced on ecosystems, living species and organisms are not a prerogative of hegemonic scientific institutions and alternative discourses produced mainly in the global South are part of the network of knowledge on biodiversity. 
es, so will them, still, be with us on the same boat. And god is not an entity... it is the totality of a non-understandable creative process in the becoming which each one of us, Person, Animal, Rock, Grass, Star, Asteroid, Wind, Blow and Whisper, Pain and Grief, Joy and Glory, is an unbreakable and inseparable part... (Carvalho 2010)

\section{Bibliography}

Bhabha, H.K. (1994). The Location of Culture. London; New York: Routledge. https://doi.org/10.4324/9780203820551.

Braidotti, R. (2019). "Preface. The Posthuman as Exuberant Excess". Ferrando, F. (ed.), Philosophical Posthumanism. London: Bloomsbury Academic, xi-xvi.

Cadena, M. de la (2010). "Indigenous Cosmopolitics in the Andes. Conceptual Reflection beyond 'Politics'”. Cultural Anthropology, 25(2), 334-70. https://doi.org/10.1111/j.1548-1360.2010.01061.x.

Caminero-Santangelo, B. (2014). Different Shades of Green. African Literature, Environmental Justice, and Political Ecology. London; Charlottesville: University of Virginia Press.

Caminero-Santangelo, B. (2015). "Witnessing the Nature of Violence. Resource Extraction and Political Ecologies in he Contemporary African Novel”. DeLoughrey, E.; Didur, J.; Carrigan, A. (eds), Global Ecologies and the Environmental Humanities. Postcolonial Approaches. London; New York: Routledge, 226-41.

Carvalho, L. (2019). "Ruy Duarte de Carvalho e o neoanimismo". Lança, M. (ed.), Diálogos com Ruy Duarte de Carvalho. Lisboa: BUALA (Associação Cultural); Centro de Estudos Comparatistas (Faculdade de Letras - UL), 14-18.

Carvalho, R. Duarte de [1977] (2003). Como se o mundo não tivesse Leste. Lisboa: Edições Cotovia.

Carvalho, R. Duarte de (2005). Lavra (Poesia reunida 1970-2000). Vol. 1, Chão de oferta. Lisboa: Edições Cotovia.

Carvalho, R. Duarte de (2008). "Falas \& vozes, fronteiras \& paisagens... escritas, literaturas e entendimentos... (2005)". Carvalho, R. Duarte de, A câmara, a escrita e a coisa dita... fitas, textos e palestras. Lisboa: Edições Cotovia, 11-26.

Carvalho, R. Duarte de (2009). Os filhos de Próspero. 3 voll. Lisboa: Edições Cotovia.

Carvalho, R. Duarte de (2010). Neo-Animist Decalogue. https://www.buala.org/en/ruy-duarte-de-carvalho/neo-animist-decalogue.

Castelo, C. (2007). Passagens para África. O povoamento de Angola e Moçambique com naturais da metrópole. Porto: Afrontamento.

Césaire, A. [1955] (2001). Discourse on Colonialism. New York: Monthly Review Press.

Fanon, F. [1961] (1963). The Wretched of the Earth. New York: Grove Press.

Ferrando, F. (2019). Philosophical Posthumanism. London: Bloomsbury Academic. https://doi.org/10.5040/9781350059511.

Iheka, C. (2018). Naturalizing Africa. Ecological Violence, Agency, and Postcolonial Resistance in African Literature. Cambridge: Cambridge University Press. https://doi.org/10.1017/9781108183123. 
Iheka, C. (2019a). "African Literature, Audience and the Search for the (Non) Human". Adejunmobi, M.; Coetzee, C. (eds), Routledge Handbook of African Literature. London; New York: Routledge, 203-14. https://doi. org/10.4324/9781315229546-14.

Iheka, C. (2019b). "African Literature, Ecological Interconnections, Theoretical Entanglements". Cambridge Journal of Postcolonial Literary Inquiry, 6(2), 300-4. https://doi.org/10.1017/pli.2018.57.

Mbembe, A. (2001). On the Postcolony. Berkeley; Los Angeles: University of California Press.

Mbembe, A. (2017). Critique of Black Reason. London; Durham: Duke University Press. https://doi.org/10.1215/9780822373230.

Mellino, M. (2014). "Introduzione. La furia di Caliban: nell'occhio della grande tempesta". Césaire, A., Discorso sul colonialismo seguito da Discorso sulla negritudine. Verona: Ombre corte, 7-47.

Miceli, S. (2011). Contar para vivê-lo, viver para cumpri-lo. Autocolocação e construção do livro na trilogia ficcional de Ruy Duarte de Carvalho [dissertation]. Lisboa: Universidade de Lisboa.

Moolla, F.F. (2016). Natures of Africa. Ecocriticism and Animal Studies in Contemporary Cultural Forms. Johannesburg: Wits University Press. https://doi. org/10.18772/22016069131.

Santos, B. de Sousa (2009). "Para além do Pensamento Abissal. Das linhas globais a uma ecologia de saberes". Santos, B. de Sousa; Meneses, M.P. (eds), Epistemologias do Sul. Coimbra: Almedina, 23-72.

Santos, B. de Sousa (2020). A cruel pedagogia do vírus. Coimbra: Edições Almedina.

Santos, B. de Sousa; Nunes, J.A.; Meneses, M.P. (2007). "Opening Up the Canon of Knowledge and Recognition of Difference". Santos, B. de Sousa (ed.), Another Knowledge Is Possible. Beyond Northern Epistemologies. London: Verso, xix-lxii.

Sarr, F. (2019). Afrotopia. Minneapolis: University of Minnesota Press. https:// doi.org/10.5749/j.ctv105bb1g. 



\section{Note della Redazione}

Come affermano Robert S. Emmett e David E. Nye, l'affermarsi delle environmental humanities (scienze umane ambientali) a partire dagli inizi del nuovo millennio dimostra una sempre più forte consapevolezza che le crisi ecologiche del nostro pianeta non hanno bisogno (solo) di risposte di ordine tecnologico, ma anche di interventi di natura politica e culturale (Emmett and Nye 2017, 1). Non è un caso che, nel suo influente saggio The Great Derangement, Amitav Ghosh affermi che la crisi climatica sia, prima di tutto, una crisi della cultura e dell'immaginazione (Ghosh 2016, 9). Quello delle environmental humanities è un campo altamente interdisciplinare, che si fonda e dà una nuova cornice alle ricerche dei decenni precedenti nell'ambito degli studi letterari, dell'antropologia, della filosofia, degli studi di genere, della storia e della geografia, per citare alcune delle aree di studio coinvolte. Inoltre, mette in contatto questi saperi (e altri ancora) con le scienze naturali come la biologia, la geologia e la climatologia. Si tratta di un campo, infine, in cui trovano una naturale prosecuzione una serie di affermate prospettive teoriche: non ultima quella degli studi postcoloniali, che sempre più frequentemente uniscono i loro tradizionali interessi di ricerca all'ecocritica e all'ecologia, specialmente attraverso una serie di prospettive radicali come quella della giustizia ambientale e climatica e quella degli indigenous studies.

Le environmental humanities sono l'orizzonte di riferimento di questo dossier tematico del Tolomeo, declinate secondo la specificità e gli interessi di ricerca della rivista: l'analisi delle forme del testo postcoloniale in una prospettiva interdisciplinare (la parola scritta in conversazione con le arti visive e le scienze sociali) e una robusta prospettiva comparatistica e multilinguistica. Gli interventi qui presentati combinano l'analisi del testo letterario, e delle sue intersezioni con l'ecologia, il non-umano, i disastri naturali, la violenza ecologica e coloniale, e la giustizia climatica, con gli strumenti degli studi di genere, della stilistica, della sociologia, oltre che, natural- 
mente, della teoria postcoloniale e dell'ecocritica. Sono ampiamente rappresentate le due principali aree linguistiche di cui si occupa il Tolomeo (francofonia e anglofonia), ma fanno la loro presenza anche altri contesti linguistici e culturali (l'Angola e il Meridione italiano).

La sezione francofona del dossier si apre con l'intervento di Lila Ibrahim-Lamrous, incentrato sull'analisi del romanzo di Maïssa Bey, Surtout ne te retourne pas. L'articolo adotta un approccio interdisciplinare che mette in dialogo gli studi di genere sia con le environmental humanities sia con il discorso postcoloniale. Nel libro il terremoto che colpisce la città algerina di Boumerdès - evento storico realmente accaduto nel maggio 2003 - viene investito di un'importante funzione simbolica: da un lato la città in rovine rispecchia la sofferenza individuale della protagonista; dall'altro lo smottamento che ha devastato il paesaggio urbano rimanda metaforicamente al crollo delle norme sociali. Ė camminando tra i detriti di un mondo fisico e ideologico distrutto dal sisma che il personaggio prende via via coscienza della condizione femminile in Algeria: la catastrofe diventa l'occasione per iniziare un viaggio alla ricerca di sé nel tentativo di riappropriarsi del proprio passato e della propria agency.

Di catastrofi naturali si occupa anche il contributo successivo, nel quale Alessia Vignoli fa un'ampia rassegna delle narrazioni del disastro all'interno del panorama letterario caraibico moderno e contemporaneo. Quella che viene proposta è una visione transculturale delle letterature francofone di Haiti, Martinica e Guadalupa, che preferisce evidenziare i numerosi punti di contatto tra i vari Paesi piuttosto che soffermarsi sulle loro singole specificità. La percezione ciclica del tempo, che sembra essere comune a tutte e tre le realtà insulari, è il punto di partenza da cui si dirama la riflessione di Vignoli, che individua tre grandi assi tematici per l'analisi del suo corpus: l'aspetto etico e politico della rappresentazione del disastro; la messa in atto di una sorta di resistenza collettiva e individuale; il ruolo della memoria in relazione a eventi traumatici.

Il terzo saggio del dossier ci porta dall'altra parte dell'Atlantico, nello specifico nell’Ubangi-Sciari della prima metà del Novecento. Lia Milanesio rilegge la rappresentazione del colonialismo nei romans animaliers di René Maran da un punto di vista ecocritico. In queste opere lo scrittore martinicano anticipa il discorso postcoloniale denunciando le violenze perpetrate dal colonialismo francese sulla brousse e i suoi abitanti. Legittimati da una presunta missione civilizzatrice, i danni a tutto l'ecosistema - flora, fauna e popolazioni locali - sono raccontati dal punto di vista degli animali, vittime dimenticate della colonizzazione. Al modello antropocentrico europeo gli animali contrappongono un tipo di società ecocentrica che elimina ogni carattere gerarchico dal rapporto tra esseri viventi ed elementi naturali. Benché ne critichi gli aspetti più controversi attraverso la voce delle bestie della giungla, Maran non condanna nettamente il 
colonialismo francese, anzi: Milanesio fa notare che l'autore rimarrà un convinto sostenitore dell'impresa africana.

Anche Alessia Berardi incentra la sua riflessione su un contesto coloniale, quello della città di Orano del «194...». Il suo intervento, che chiude la parte francofona del dossier, s'interroga infatti sulla possibilità di reinterpretare La Peste di Albert Camus in una prospettiva ecocritica. La posizione ambigua di Camus sulla questione algerina, così come l'assenza degli Arabi nel romanzo, rende problematica la lettura allegorica della peste come simbolo universale di oppressione. Gli studi recenti legati alle environmental humanities lasciano da parte la funzione metaforica dell'epidemia per concentrarsi sulla sua dimensione sociale: da una parte la peste non conosce barriere e coinvolge indiscriminatamente tutta la comunità; dall'altra l'isolamento che porta con sé marca e accentua le discriminazioni sociali. La lettura dell'epidemia come fenomeno politico e sociale permette, secondo Berardi, di evidenziare le numerose contraddizioni della società del romanzo, che, pur volendosi universale, mostra i tratti di una collettività assai poco inclusiva.

L'intervento di Alice Girotto ci conduce invece nell'ambito delle letterature lusofone, attraverso un'analisi dell'opera dello scrittore angolano Ruy Duarte de Carvalho. Di questa figura eclettica - è stato antropologo e cineasta oltre che scrittore e intellettuale - Girotto esplora le tracce di un incompiuto progetto 'neoanimista', i cui echi si ritrovano anche nella sua poesia, scritti e opere precedenti, che sonda i limiti e le contraddizioni dell'umanesimo occidentale e offre una visione alternativa del rapporto tra uomo e entità animali, vegetali e 'inanimate'. Girotto mette in relazione il progetto di Carvalho con il postumanesimo di matrice occidentale, con pensatori postcoloniali, ma soprattutto con filosofia e critica panafricana, collocando la sua opera all'interno di un approccio specificatamente africano al postumanesimo.

In parte simile, nel ricostruire una visione ecologica di uno specifico autore, è l'intervento di Esterino Adami, che esplora la raccolta di saggi Making India Awesome dell'indiano Chetan Bhagat, romanziere in lingua inglese e prolifico giornalista per testate sia anglofone sia in Hindi. I saggi di Bhagat affrontano varie tematiche di ordine politico e sociale come la pulizia degli spazi pubblici e privati e il cibo; Adami, facendo uso di strumenti teorici che vanno dagli studi postcoloniali alla stilistica, ne identifica le strategie persuasive, l'ambiguo framework ideologico, caratterizzato da una sostanziale adesione ai concetti di sviluppo e di nazione tradizionalmente intesi, e la visione dell'ecologia e dell'ambiente che ne consegue. L'analisi di Adami ci mostra come linguaggio, retorica e ambiente si intersechino in un contesto postcoloniale globalizzato come quello della nuova India.

Anche il saggio di Luigi Cazzato e Antonella D’autilia si occupa di sviluppo e delle sue ricadute ecologiche e coloniali, adottando un ap- 
proccio distintamente comparativo, interdisciplinare e transmediale. Il saggio costruisce un percorso centrato sulle acciaierie tarantine, sviluppate dagli anni sessanta in poi, e i disastri ecologici che ne sono nati. L'intervento parte da riflessioni animate da resoconti di viaggiatori inglesi nel Sud Italia, dalla teoria della world-ecology e del Capitalocene di Jason Moore e dagli studi decoloniali; colloca poi il caso di Taranto all'interno di un più generale modello di sviluppo e modernizzazione di matrice coloniale, pur nelle sue specificità legate al contesto italiano e, più specificamente, meridionale. Dopo una ricostruzione storica e sociologica del rapporto della città con l'industria siderurgica e le sue implicazioni ecologiche e coloniali, il saggio offre vari esempi di azioni di resistenza ecoartistica (mostre, fumetti e graphic novel) all'azione distruttiva capitalocenica in atto a Taranto.

Il saggio di Chiara Xausa è centrato invece sul romanzo Young Adult di Cherie Dimaline The Marrow Thieves, ambientato in un Canada distopico, distrutto dal cambiamento climatico e in cui la maggior parte della popolazione ha perso la capacità di sognare, con l'eccezione delle popolazioni indigene, il cui midollo ha la capacità di curare questa perdita e che per questo viene forzatamente estratto dalle forze governative. Xausa discute il romanzo nell'ottica di criticare, da un lato, le varianti egemoniche ed eurocentriche del dibattito sull'Antropocene, che non tengono conto dell'impatto differenziato delle catastrofi ecologiche e climatiche; e dall'altro forme di narrazione distopica che, in modo analogo, ignorano la prospettiva della giustizia climatica che è di primaria importanza per le popolazioni indigene. Xausa mostra come il romanzo di Dimaline, di contro, crei un nuovo modello di letteratura eco-distopica per raccontare una storia di oppressione e violenza coloniale - specialmente nella sua natura di 'violenza lenta', nelle parole di Rob Nixon - diretta verso le popolazioni indigene, invece che una storia di catastrofe ecologica 'universale'.

Il saggio di Isabella Marinaro effettua invece un'analisi stilistica ed ecocritica del racconto dello scrittore cinese-americano Ha Jin, intitolato «A Tiger Fighter is Hard to Find». Tramite un'analisi dei pronomi personali del racconto, Marinaro analizza le strategie con cui l'autore dispiega la sua ironia verso le vicissitudini di una sventurata produzione cinematografica, incaricata dalla propaganda governativa cinese di produrre un film in cui un eroe di film d'azione sconfigge una tigre a mani nude. L'analisi stilistica fa emergere una critica della violenza politica ed ecologica portata avanti dal regime cinese, di cui finiscono per essere vittime sia gli esseri umani che lavorano alla produzione sia la tigre stessa.

Chiude il dossier il saggio di Nicola Pilia, che affronta uno dei testi canonici della letteratura postcoloniale anglofona (specie in ottica ecocritica): The Hungry Tide di Amitav Ghosh, ambientato nella regione indiana dei Sundarbans. Il saggio di Pilia si fonda, a livello 
teorico, su un'analisi dei concetti di dwelling e dispossession, cruciali per leggere il romanzo di Ghosh, e su quello di 'violenza lenta', che è utilizzato anche nell'articolo di Xausa. All'interno di questa cornice, Pilia rilegge l'evento centrale del romanzo - il massacro di Morichjhãpi, di cui viene inoltre ricostruito il contesto storico di riferimento - come un modello per comprendere storie contemporanee di eco-rifugiati e migranti climatici.

Lucio De Capitani, Silvia Boraso

Università Ca' Foscari Venezia

per la redazione del Tolomeo

\section{Editorial notes}

As Robert S. Emmett and David E. Nye have claimed, the success of the environmental humanities from the beginning of the millennium has shown growing awareness that the ecological crisis facing the planet requires not merely a technological response, but political and cultural action too (Emmet and Nye 2017, 1). It is no coincidence that Amitav Ghosh, in his important essay The Great Derangement, claims that the climate crisis is, before anything else, a crisis of culture and the imagination (Ghosh 2016,9). The Environmental Humanities constitute a highly interdisciplinary field which draws on, and provides a new framework for, previous decades' research in literary studies, anthropology, philosophy, gender studies, history, and geography, to name just a few. They also provide a bridge to the natural sciences, biology, geology, and climatology. The field has offered a natural home to a series of well established theoretical research areas, not least of which postcolonial studies, which have increasingly combined their traditional research interests with ecocriticism and ecology, through a series of radical approaches such as environmental and climate justice, and indigenous studies.

The environmental humanities provide the backdrop to this special issue of Tolomeo, in the context of the journal's specific research interests: the analysis of the forms of postcolonial texts from an interdisciplinary perspective (the written word in conversation with the visual arts and the social sciences) and a robust comparative, multilingual perspective. The contributions presented here combine textual analysis, and its interconnections with ecology, the non-human, natural disasters, ecological and colonial violence, and climatic justice, with the instruments of gender studies, stylistics, sociology, not to mention postcolonial theory and eco-criticism. The two principal language areas with which Tolomeo is concerned (francophone and anglophone) are well represented, but so are other linguistic and cul- 
tural contexts (Angola and the south of Italy).

The francophone section opens with Lila Ibrahim-Lamrous's contribution centred on the analysis of the novel Surtout ne te retourne pas by Maïssa Bey. The writer adopts an interdisciplinary viewpoint in which gender studies dialogue with the environmental humanities as well as with postcolonial discourse. The novel recounts the earthquake which hit the Algerian city of Boumerdès in May 2003, and which is given an important symbolic dimension: the ruined city mirrors the individual suffering of the protagonist, while the landslide which has devastated the urban landscape is a metaphor for the collapse of social norms. Walking through the ruins of a physical and ideological world destroyed by the earthquake the protagonist gradually becomes aware of the female condition in Algeria: the catastrophe offers the opportunity to begin a voyage of self discovery as she attempts to retake possession of her own past and her own agency.

The next contribution is also concerned with natural disasters, as Alessia Vignoli presents a wide range of disaster narratives within the panorama of modern and contemporary Caribbean literature. She offers a transcultural vision of the francophone literatures of Haiti, Martinique and Guadeloupe, preferring to highlight the numerous points of contact between the countries rather than to dwell on their specific individual traits. The cyclical perception of time, which seems to belong to all three insular realities, is the starting point for Vignoli's reflection, which identifies three major thematic strands with which to analyse her corpus: the political and ethical aspect of the representation of disaster; the emergence of a kind of individual and collective resistance; and the role of memory in relation to traumatic events.

The third paper takes us to the other side of the Atlantic, to Ubangi Shari at the beginning of the twentieth century. Lia Milanesio re-examines the colonialism of René Maran's romans animaliers from an eco-critical perspective. In these works Maran anticipates postcolonial discourse by denouncing the violence perpetrated on the brousse and its inhabitants by French colonialism. Justified by the supposed mission of civilisation, the damage wreaked on the entire ecosystem - flora, fauna, and local populations - are seen through the eyes of the animals, the forgotten victims of colonization. In contrast with the anthropocentric European model they belong to a kind of ecocentric society which has eliminated any hierarchical relationship between living creatures and natural elements. Although he criticises the most controversial aspects (of the European model) through the voice of the animals of the jungle, Maran does not explicitly condemn French colonialism, on the contrary: Milanesio notes that he was to remain a convinced supporter of the African adventure.

Alessia Berardi also offers a reflection on a colonial context, that of the city of Oran in "194...". Her contribution, which concludes the 
francophone section, raises the possibility of reinterpreting Camus' La Peste in an ecocritical perspective. Camus' ambiguous position with regard to the Algerian question, and the absence of Arabs in the novel, makes it difficult to interpret the allegorical reading of the plague as a universal symbol of oppression. Recent studies grounded in an environmental humanities approach leave aside any metaphorical function of the epidemic and concentrate on the social dimension; the plague knows no barriers and affects all communities indiscriminately, but at the same time the isolation that it entails underlines and accentuates social discriminations. The interpretation of the epidemic as a social and political phenomenon makes it possible, Berardi suggests, to highlight the numerous contradictions in a society depicted in the novel which, although seeing itself as universal, displays the characteristics of a collectivity which is far from inclusive.

Alice Girotto's contribution is an excursion into lusophone literature, through an analysis of the work of Angolan writer Ruy Duarte de Carvalho, an eclectic figure, anthropologist and filmmaker as well as writer and intellectual. Girotto explores the traces of an unfinished 'neoanimist' project that can be found in his poetry, which explores the limits and contradictions of western humanism and offers an alternative vision of the relationship between humans and animal, vegetable and 'inanimate' entities. Girotto links Carvalho's project with western posthumanism, with postcolonial thinkers, and especially with philosophy and pan-African criticism, locating his work in a specifically African approach to posthumanism.

In a similar vein, in its reconstruction of the ecological vision of a specific writer, is the contribution by Esterino Adami. It explores the collection of essays Making India Awesome by the Indian writer Chetan Bhagat, a novelist who writes in English, and a prolific journalist for both the Anglophone and Hindi press. Baghat's essays tackle a variety of themes dealing with political and social issues, such as the cleaning of public and private spaces and food; Adami makes use of theoretical instruments ranging from postcolonial studies to stylistics, and identifies the strategies of persuasion, the ambiguous ideological framework, characterized by the large scale adhesion to traditionally understood concepts of development and nation, and the vision of ecology and the environment which is its consequence. His analysis shows us how language, rhetoric and environment intersect in the globalised postcolonial context which is the new India.

The article by Luigi Cazzato and Antonella D'autilia is also concerned with the ecological and colonial fall out of development, adopting a distinctive approach which is comparative, interdisciplinary and transmedial. It is centred around the steelworks of Taranto which date back to the 1960s, and the ecological disasters which they caused. It starts with reflections inspired by the accounts of English travellers in southern Italy, Jason Moore's theory of world ecolo- 
gy and Capitolocene, and decolonial studies. Taranto is seen as part of a more general model of development and modernisation with a colonial matrix, albeit specifically linked to the Italian context, and, more specifically, the south of Italy. After a historical and sociological reconstruction of the city's relationship with the steel industry and its ecological and colonial implications, the paper offers examples of ecoartistic resistance (exhibitions, cartoons, and graphic novels) to the destructive capitolocenic forces being exerted on Taranto.

Chiara Xausa's paper is centred on the young adult novel by Cherie Dimaline The Marrow Thieves, which is set in a dystopic Canada, destroyed by climate change, and where most of the population has lost the ability to dream, with the exception of the indigenous peoples, whose bone marrow has the capacity to cure this loss, and so for this reason is forcibly extracted by the government. Xausa discusses the novel with a view to criticising the hegemonic Eurocentric variants of the debate on the Anthropocene which take no account of the differentiated impact of ecological and climatic catastrophes, as well as the forms taken by a dystopic narrative which, in a similar way, ignore any perspective of climate justice which is of fundamental importance to the indigenous peoples. Xausa shows how DimalinÈs novel creates a new model of eco-dystopic literature which relates a story of colonial oppression and violence - especially in its nature of 'slow violence', in the words of Rob Nixon - aimed at the indigenous populations, rather than the story of a 'universal' ecological catastrophe.

The article by Isabella Marinaro is a stylistic and ecocritical analysis of Chinese-American writer Ha Jin's short story entitled "A Tiger Fighter is Hard to Find". Through an analysis of personal pronouns in the story, Marinaro examines the strategies with which the author wields his irony against the misadventures of an unfortunate film production which has been given the task by the Chinese governmental propaganda machine to make an action film in which the hero defeats a tiger with his bare hands. The analysis lays bare a critique of the political and ecological violence which has been promoted by the Chinese regime, and to which both the humans working on the production, and the tiger itself, fall victim.

The volume concludes with an essay by Nicola Pilia, who writes about one of the canonical texts of postcolonial Anglophone literature (especially from an eco-critical perspective): The Hungry Tide, by Amitav Ghosh, set in the Indian region of the Sundarbans. Pila's essay is based, on a theoretical level, on an analysis of the concepts of dwelling and disposession, crucial to understand Ghosh's novel, and that of 'slow violence', which is also used in the article by Xausa. Within this framework, Pilia invites us to re-read the central event of the novel - the massacre of Morichjhãpi, and its reconstructed historical context - as a model for understanding contemporary stories 
of eco refugees and climate migrants.

Lucio De Capitani, Silvia Boraso

Ca' Foscari University of Venice

Editorial Board, Il Tolomeo

English translation by David John Newbold

Ca' Foscari University of Venice

\section{Notes de la rédaction}

Comme le soulignent Robert S. Emmett et David E. Nye, depuis le début du nouveau millénaire, l'émergence des humanités environnementales ("environmental humanities" dans le champ anglo-saxon) montre qu'une prise de conscience croissante des crises écologiques de notre planète nécessite non seulement des réponses technologiques, mais aussi des interventions politiques et culturelles (Emmett et Nye 2017, 1). Ce n'est pas un hasard si, dans son influent essai The Great Derangement, Amitav Ghosh affirme que la crise climatique est avant tout une crise de la culture et de l'imagination (Ghosh 2016, 9). Le domaine des humanités environnementales est en soi de nature hautement interdisciplinaire, scientifiquement fondé et à même de conférer un nouveau cadre à la recherche des décennies précédentes dans les domaines des études littéraires, de l'anthropologie, de la philosophie, des études de genre, de l'histoire et de la géographie, pour n'en citer que quelques-uns. Elle met également ces connaissances (et d'autres connaissances) en lien avec les sciences naturelles telles que la biologie, la géologie et la climatologie. Enfin, c'est un domaine dans lequel un ensemble de perspectives théoriques établies trouvent un prolongement naturel : notamment celle des études postcoloniales, qui combinent de plus en plus leurs intérêts de recherche traditionnels avec l'écocritique et l'écologie, en particulier par le biais d'un ensemble de perspectives radicales telles que la justice environnementale, la justice climatique et les études indigènes.

Les humanités environnementales constituent l'horizon de référence de ce dossier thématique de Tolomeo, décliné selon la spécificité et les intérêts de recherche de la revue : l'analyse des formes du texte postcolonial dans une perspective interdisciplinaire (l'écrit en conversation avec les arts visuels et les sciences sociales) et une solide perspective comparative et multilingue. Les articles présentés ici associent l'analyse du texte littéraire et ses intersections avec l'écologie, le non-humain, les catastrophes naturelles, la violence écologique et coloniale et la justice climatique, avec les outils des études de genre, de la stylistique, de la sociologie mais aussi avec ceux 
de la théorie postcoloniale et de l'écocritique. Les deux principales sphères langagières de Tolomeo (francophone et anglophone) sont largement représentées, mais d'autres environnements linguistiques et culturels (Angola et Italie du Sud) figurent également dans ce nouveau numéro.

Le volet francophone de notre dossier s'ouvre avec la contribution de Lila Ibrahim-Lamrous qui se penche sur l'analyse du roman de Maïssa Bey, Surtout ne te retourne pas. L'article adopte une approche interdisciplinaire qui met en dialogue les études de genre avec les humanités environnementales et le discours postcolonial. Dans l'ouvrage en question, le tremblement de terre qui a frappé la ville algérienne de Boumerdès - un événement historique qui s'est réellement produit en mai 2003 - est investi d'une importante fonction symbolique : d'une part, la ville en ruines reflète la souffrance individuelle de la protagoniste ; d'autre part, le glissement de terrain qui a dévasté le paysage urbain fait métaphoriquement référence à l'effondrement des normes sociales. C'est en parcourant les débris d'un monde physique et idéologique détruit par le tremblement de terre que le personnage prend peu à peu conscience de la condition féminine en Algérie : la catastrophe devient l'occasion d'entamer un voyage à la recherche d'elle-même pour tenter de reprendre possession de son passé et de son agence.

Les catastrophes naturelles font également l'objet de l'article que propose Alessia Vignoli : un large aperçu des récits de la catastrophe dans le panorama littéraire moderne et contemporain des Caraïbes. Ce panorama prend la forme d'une conception transculturelle des littératures francophones d'Haïti, de la Martinique et de la Guadeloupe, qui préfère mettre en évidence les nombreux points de contact entre les différents pays plutôt que de s’attarder sur leurs spécificités individuelles. La perception cyclique du temps, qui semble être commune aux trois réalités insulaires, est le point de départ à partir duquel s'articule la réflexion d'Alessia Vignoli. Trois axes thématiques principaux sont mise en exergue pour l'analyse de son corpus : l'aspect éthique et politique de la représentation de la catastrophe ; la mise en œuvre d'une sorte de résistance collective et individuelle ; le rôle de la mémoire par rapport aux événements traumatisants.

Le troisième article du dossier nous fait traverser l'Atlantique et nous transporte dans l'Oubangui-Sciari de la première moitié du XXe siècle. Lia Milanesio relit d’un point de vue écocritique la représentation du colonialisme dans les romans animaliers de René Maran. Dans ces ouvrages, l'écrivain martiniquais anticipe le discours postcolonial en dénonçant les violences perpétrées par le colonialisme français sur la brousse et ses habitants. Légitimés par une prétendue mission civilisatrice, les dégâts causés à l'ensemble de l'écosystème - flore, faune et populations locales - sont racontés du point de vue des animaux, victimes oubliées de la colonisation. Le monde 
animal est mis en contrepoint du modèle anthropocentrique européen avec un type de société écocentrique qui élimine tout caractère hiérarchique de la relation entre les êtres vivants et les éléments naturels. Bien qu'il critique ses aspects les plus controversés par la voix des bêtes de la jungle, Maran ne condamne pas clairement le colonialisme français, bien au contraire : Milanesio souligne que l'auteur restera un fervent partisan de l'entreprise africaine.

Alessia Berardi axe également sa réflexion sur un contexte colonial, celui de la ville d'Oran en «194...». Son discours, qui clôt la partie francophone du dossier, s'interroge sur la possibilité de réinterpréter La Peste d'Albert Camus dans une perspective écocritique. La position ambiguë de Camus sur la question algérienne, ainsi que l'absence des Arabes dans le roman, rendent problématique la lecture allégorique de la peste comme symbole universel de l'oppression. Les études récentes liées aux humanités environnementales laissent de côté la fonction métaphorique de l'épidémie pour se concentrer sur sa dimension sociale : d'une part, le fléau ne connaît pas de barrières et implique indistinctement toute la communauté ; d'autre part, l'isolement qu'il entraîne marque et accentue la discrimination sociale. La lecture de l'épidémie comme phénomène politique et social permet, selon Berardi, de mettre en évidence les nombreuses contradictions de la société du roman. Cette dernière, bien qu'universelle, présente les traits d'une communauté assez peu inclusive.

Alice Girotto, quant à elle, nous fait entrer dans la sphère de la littérature lusophone, à travers une analyse de l'œuvre de l'écrivain angolais Ruy Duarte de Carvalho. De cette figure éclectique - il fut anthropologue et cinéaste, mais aussi écrivain et intellectuel - Girotto explore les traces d'un projet «néo-animiste» inachevé, dont on retrouve également les traces dans sa poésie, ses écrits et ses œuvres antérieures, qui sonde les limites, les contradictions de l'humanisme occidental et propose une conception alternative de la relation entre l’homme et les entités animales, végétales et «inanimées». Girotto relie le projet de Carvalho au post-humanisme occidental, aux penseurs postcoloniaux, mais surtout à la philosophie et à la critique panafricaines, en inscrivant son travail dans une approche spécifiquement africaine du post-humanisme.

L'article d'Esterino Adami, qui explore la collection d'essais Making India Awesome de l'Indien Chetan Bhagat, romancier en anglais et journaliste prolifique pour les journaux anglais et hindi, est en partie similaire à la reconstitution d'une vision écologique d'un auteur particulier. Les essais de Bhagat abordent diverses questions politiques et sociales telles que la propreté des espaces publics et privés ou la nourriture. Adami mobilise des outils théoriques allant des études postcoloniales à la stylistique pour identifier leurs stratégies de persuasion, leur cadre idéologique ambigu. Selon l'auteur, cette ambiguïté repose aussi bien sur une adhésion substantielle aux 
concepts de développement et de nation conçus dans une perspective traditionnelle que sur la conception de l'écologie et de l'environnement qui en découle. L'analyse proposée par Adami nous montre comment la langue, la rhétorique et l'environnement se croisent dans un contexte postcolonial mondialisé comme celui de la nouvelle Inde.

L'article de Luigi Cazzato et Antonella D'autilia traite également de développement industriel, de ses effets écologiques et coloniaux, en adoptant une approche spécifiquement comparative, interdisciplinaire et transmédiatique. L'article construit un parcours centré sur les aciéries de Tarente qui se sont développées à partir des années 1960, et les catastrophes écologiques qui en ont découlé. L'essai part de réflexions animées par des rapports de voyageurs anglais en Italie du Sud, par la théorie de l'écologie mondiale, par le Capitalocène de Jason Moore et par des études décoloniales. L'article envisage ensuite le cas de Tarente dans un modèle plus général de développement et de modernisation de la matrice coloniale dans sa spécificité liée au contexte italien et, plus spécifiquement au sud de l'Italie. Après une reconstruction historique et sociologique de la relation de la ville avec l'industrie sidérurgique, de ses implications écologiques et coloniales, l'essai met en avant différents exemples de résistance éco-artistique (expositions, bandes dessinées et romans graphiques) à même de lutter contre l'action dévastatrice du Capitalocène à Tarente.

L'article de Chiara Xausa se concentre quant à lui sur le roman Young Adult de Cherie Dimaline, The Marrow Thieves, qui se déroule dans un Canada dystopique, détruit par le changement climatique et où la plupart de la population a perdu la capacité de rêver, à l'exception des peuples indigènes, dont la moelle a la capacité de guérir cette perte. Indigènes qui sont soustraits de force de leur communauté par les forces gouvernementales. Xausa traite du roman pour critiquer d'une part, les variantes hégémoniques, eurocentées du débat sur l'Anthropocène (variantes qui ne tiennent pas compte de l'impact différencié des catastrophes écologiques et climatiques) et pour critiquer d'autre part, les formes de narration dystopique qui, de manière similaire se soucient peu d'une justice climatique pourtant fondamentale pour les peuples indigènes. Xausa montre comment le roman de Dimaline crée en revanche un nouveau modèle de littérature éco-dystopienne pour raconter, plutôt qu'une histoire de catastrophe écologique «universelle», une histoire d'oppression et de violence coloniale - surtout dans sa nature de «violence lente», selon les mots de Rob Nixon - dirigée vers les peuples indigènes.

La contribution d'Isabella Marinaro propose une analyse stylistique et écocritique de la nouvelle de l'écrivain sino-américain $\mathrm{Ha}$ Jin, intitulée «Un combattant tigre est difficile à trouver». À travers une analyse des pronoms personnels utilisés dans le récit, l'auteure analyse les stratégies par lesquelles l'auteur déploie toute son ironie 
face aux vicissitudes d'une production cinématographique malchanceuse, commandée par la propagande du gouvernement chinois pour produire un film dans lequel un héros de films d'action bat un tigre à mains nues. L'analyse stylistique révèle une critique de la violence politique et écologique exercée par le régime chinois, dont finissent par être victimes aussi bien les êtres humains travaillant à la production que le tigre lui-même.

Enfin, le dossier est clôturé par l'article de Nicola Pilia, qui traite, principalement dans une perspective écocritique, de l'un des textes canoniques de la littérature postcoloniale anglophone : The Hungry Tide d'Amitav Ghosh, dont l'action se situe dans la région des Sundarbans en Inde. L'essai de Pilia se fonde, sur le plan théorique, sur une analyse des concepts d'habitation et de dépossession, essentiels à la lecture du roman de Ghosh, et sur celui de "violence lente», également mobilisé dans l'article de Xausa. Dans ce cadre, Pilia conçoit l'événement central du roman, le massacre de Morichjhãpi (dont le contexte historique est également reconstitué), comme un modèle pour comprendre les histoires contemporaines d'éco-réfugiés et de migrants climatiques.

Lucio De Capitani, Silvia Boraso Université Ca' Foscari de Venise pour la rédaction de Il Tolomeo Traduction française par Yannick Hamon

Université Ca' Foscari de Venise

\section{Bibliografia / Bibliography / Bibliographie}

Emmett, R.S.; Nye, David E. (2017). The Environmental Humanities. A Critical Introduction. Cambridge (MA); London: The MIT Press.

Ghosh, A. (2016). The Great Derangement: Climate Change and the Unthinkable. Chicago; London: University of Chicago Press. 



\section{Varia}





\title{
Mélancolie de Janvier
}

\section{Yves Chemla}

Université Paris Descartes, France

\begin{abstract}
The question of the nationalism of the Haitian writer Louis-Joseph Janvier (1855-1911) is more complex than it seems on the surface. An all-pervading theme in most of his texts, the nationalist imperative takes on different forms, the majority of which are based on facts which occurred at the time Janvier was writing. Janvier tries to exhaust the subject by establishing the facts and by challenging beliefs. He desperately tries to portray a positive, benevolent, welcoming image of Haitian nationalism, but as is often the case, this figure is also made up of contradictory aspects, which have been present since its emergence at the time of the French Revolution, and which undoubtedly found one of its first political expressions at the battle of Valmy, in September 1792. Janvier has thus to overcome the dissatisfaction that accompanies Haitian nationalism for its underlying exclusiveness. These paradoxical relationships of the author with the national founding ideal undergo significant changes throughout his career as an essayist, pamphleteer and novelist, diplomat and politician, ranging from the severe judgment, even contempt, for those who dispute the basis of this requirement to the radical criticism of an ideology which reveals its dark and deadly face. The proposed contribution seeks first to identify, through texts of various origins, the themes, the facts or just the simple echoes of this idea of nation that seems to run through Janvier's text.
\end{abstract}

Keywords Haiti. Nationalism. Louis-Joseph Janvier. Paradox.

$\begin{array}{lll}\text { Puty } & \text { Peer review } & \\ \text { Edizioni } & \text { Submitted } & 2020-03-23 \\ \text { Ca'Foscari } & \text { Accepted } & 2020-09-01 \\ & \text { Published } & 2020-12-22 \\ & \text { Open access } & \end{array}$

(c) 2020 | () Creative Commons Attribution 4.0 International Public License 
La question du nationalisme de Janvier (1855-1911) est plus épineuse qu'elle ne le semble en apparence. Saturée dans la plupart des textes, l'exigence nationaliste se décline de différentes façons, la plupart adossées à des faits liés à l'actualité. Janvier cherche à épuiser les argumentaires, en nouant les savoirs, en dénouant les croyances. Il tente d'installer une figure positive, c'est-à-dire bienveillante et accueillante du nationalisme haïtien, mais comme c'est souvent le cas, cette figure est aussi faite d'oppositions. Il est comme en butte à quelque chose qui ne prend pas : le nationalisme haïtien est fondé lui aussi sur de l'exclusion. Cette contribution s'attache à identifier d'abord, grâce à plusieurs textes les axes, les faits ou mêmes les seules traces de cette idée de nation qui parcourt le texte de Janvier. Ce sont donc avant tout des hypothèses qui sont proposées. Il apparaît ainsi que dans la plupart des textes de Janvier qui relèvent de la science politique ou de l'histoire, l'idée nationaliste est présentée comme une évidence informée par les études qu'il a suivies, en particulier à Paris. En revanche, comme on le constatera, dans le roman Une Chercheuse, cette idée est présentée comme le signe même de la dégradation à la fois sociale et morale. C'est cette variation des significations, sans doute liée à une variation de genre d'écriture et de projet, qui retient l'attention. C'est enfin un nœud rarement considéré dans ce qu'il faut approcher comme un élément de l'histoire des idées. Celle-ci exige une contextualisation importante, à même de permettre au lecteur d'apprécier la façon dont Janvier élabore sa pensée nationaliste et la critique de celle-ci, puis comment il passe de l'essai à la fiction décentrée et délocalisée, pour mieux parvenir à évoquer la situation haïtienne. Enfin, Janvier, s'il connaît les classiques de la littérature française, connaît aussi ceux d'Haïti, en particulier les textes de l'historien Madiou. C'est avec tout cet outillage, qu'il convoque à plusieurs reprises dans son œuvre, qu'il va affirmer la dégradation de l'idée de nation, en raison de son incomplétude et surtout de sa facticité.

La question nationale, l'identification de la notion de nation, Janvier ne la pose pas indépendamment de l'histoire de l'idée de nation, c'est-à-dire de son envers désormais évacué par la dynamique de la révolution haïtienne et de la lutte pour l'indépendance : l'ancien régime. Il faut repenser, relire le texte de Gusdorf consacré à l'histoire de l'idée de nation, et remonter aux temps les plus lointains pour comprendre le dépoussiérage violent auquel les révolutionnaires ont participé et qu'ils ont induit, et qui est passé par Saint-Domingue. C'est cette construction idéale qui informe aussi la pensée haïtienne. De même, il semble qu'on ne souligne jamais assez dans le cadre haïtien, comment les consciences sont passées d'une révolte contre la servitude à celle pour l'indépendance, ce qui n'est pas tout à fait la même chose, l'une n'entraînant pas nécessairement l'autre. Sans doute aussi, est-ce bien leur articulation qui a rendu nécessaire la victoire, en même temps que la nature des forces en présence. Le contrôle de la 
violence de tous contre tous dans l'ancien régime féodal, et qui assure des soumissions ascensionnelles, est au cœur de la réflexion sousjacente d'Une Chercheuse : le narrateur y rappelle à propos de Foncine, présenté justement comme le résidu d'une fin de race, le Capitulaire de Quierzy, par lequel Charles le Chauve institua la noblesse héréditaire, en 877. Le cri lancé par Kellermann à Valmy signifie le ralliement à une idée vraiment neuve pour le coup.

Il faut bien rappeler qu'en constituant les États Généraux en Assemblée nationale, les députés français retirent à la royauté sa sacralité, car ils affirment la supériorité de leur autorité à celle du roi. Mutation des valeurs, mutation de l'autorité, mutation de l'exercice du pouvoir, mutation du dire politique : le débat gagne le centre de la parole, et de l'écrit jusqu'à la saturation. Les mots doivent convaincre et persuader. La condition politique devient celle de l'exercice de la parole agissante.

Et puis, il y a cette surprise dans Une Chercheuse : l'échec radical du nationalisme, qui se réduit à une machine à fabriquer du désir et qui est en réalité le magasin de la mort, comme le rappelle le docteur Qulb à Edriss, qui va mourir, et pour rien, sinon un acte de langage, ou une déclaration convertie en babiole diplomatique. Le nationalisme se transforme dans le texte de Janvier, si sourcilleux pourtant, et si exigeant, en une triste passion qui conduit à la disparition de trois êtres aimants et aimés, dont l'un n'est pas encore né. C'est de cette tristesse qu'est tissé le roman. Pour essayer d'en avoir une vue claire, il faut remonter dans des aspects particulièrement opaques de cette histoire et de ses discours.

Pour parvenir à interpréter l'espérance et l'idéologie nationalistes telles qu'elles se manifestent dans le discours de Janvier, il importe assurément de revenir aux sources de ce mot, et de remonter le temps. L'idée nationaliste a une histoire identifiée et désormais balisée. C'est une enfant de la Révolution française, qui va la légitimer tout au long du XIXème s., notamment lors des Révolutions européennes de 1848 et du « Printemps des peuples ». C'est d'abord une proclamation en forme de cri de guerre, lancé à Valmy, par un officier âgé, François Christophe Kellermann, issu de la noblesse. Né en 1735, il est militaire depuis l'âge de 15 ans et il a adhéré à la Révolution. Dans un geste qui ne laisse pas d'étonner chez un officier tenu pour médiocre tacticien par Napoléon, il lance ce cri qui marque à la fois sans doute la fin de la Révolution, et l'acte même du changement de régime. Ce cri et le geste de retirer le couvre-chef est repris par les soldats. Ce cri fait marquer le pas à l'armée prussienne considérée alors comme la meilleure du monde. Gusdorf (1987, 117-55) a montré combien ce geste marque une césure entre deux temps, et signale que la monarchie ancienne est devenue telle : la canonnade et l'enthousiasme du 20 septembre 1792 à Valmy battent en brèche la légitimité des monarchies traditionnelles et inaugurent celle du régime 
institué par la Révolution française. Le 21 septembre, le lendemain, la nouvelle convention nationale proclame la République. Comme l'écrit Thiesse (1991-2001, 12), « La véritable naissance d'une nation, c'est le moment où une poignée d'individus déclare qu'elle existe et entreprend de la prouver ».

«Vive la nation! » : ce qui est célébré et appréhendé sur le mode de la sommation est doublement étrange. D'abord en ce que cette injonction se détermine comme évidence d'une entité dont le moins qu'on puisse affirmer est qu'elle est particulièrement rétive à une définition stable ; ensuite parce qu'elle en appelle à la vie de ce qui du point de vue de l'étymologie a quelques traits quand même de la naissance commune. C'est d'une façon certaine à une double célébration qu'en appelle le geste qui eut lieu à Valmy : celui de cette entité nouvelle, affective, qui suscite une émotion réitérée depuis, et que celle-ci soit dotée de la faculté de la vie. Et cela semble si soudain :

Des virtualités éparses dans l'ordre de la pensée et du langage cristallisent d'un seul coup : un univers de vocables, de sentiments et de valeurs se trouve frappé de déchéance et remplacé par un tout autre système d'évidences. Sous les yeux de l'Europe ébahie, une autre France remplace la France de toujours, en vertu d'une mutation à peu près instantanée. (Gusdorf 1987, 120)

Elle affirme qu'il y a révolution, c'est-à-dire trajectoire orbitale, encore qu'on ne revienne justement pas au point de départ, puisque la dimension du temps est ainsi générée : ce n'est pas le retour du même ou bien au point de départ. C'est un renversement, ou plutôt une naissance, déjà celle de la république et de son calendrier : le 1er vendémiaire an I prend la place du 22 septembre 1792. L'émergence de cette idée de nation fait sortir du cadre de pensée de la féodalité, qui veut que les populations suivent le temps imposé par l'église ainsi que les mouvements imposés par les seigneurs : « un mariage soumet les Bretons à la couronne de France», rappelle par exemple Gusdorf $(1987,123)$. Certes. Le cri de Valmy est élevé comme triple exigence : d'une solidarité organique, d'une conscience collective, d'un droit à l'existence, pour reprendre les expressions du même penseur. Et même si la société inégalitaire est sans cesse reconduite, une façon de décrire et d'ordonner la société est périmée par ce cri. Demeurent cependant des différences de rangs, de richesse. Demeure aussi l'aristocratie, recréée par Napoléon. Il y a également une nouvelle hiérarchie ecclésiastique. Mais la nation fabriquait du projet commun, tandis que sentiment national et conscience nationale gagnaient toute l'Europe. Nombreux furent ceux qui étant à Valmy en auront une vue à peu près distincte : Francisco Miranda, par exemple, dont une statue est érigée dans le village. Le buste de Simon Bolivar orne également le village. L'écho du cri retentit aux Amériques 
donc. On peut aisément glisser de ce retentissement jusque dans les campagnes de la partie française de Saint-Domingue qui vont devenir haïtiennes. Mais aussi, au nom de cette nation qui prétend à la supériorité sur l'Europe, voire sur une partie du monde car elle promeut la fraternité, le cri va aller se déformant. Ça commence avec la Terreur, avec la guerre civile en Vendée, ça continue avec le caporalisme et la glorification de la Grande Armée, et puis au nom même des nations, on le sait, l'impérialisme militaire napoléonien sera abattu.

D'une certaine façon, ce délabrement est l'inentendu du cri qui en appelle à la célébration de la naissance et de l'énergie qu'elle supporte et qu'elle transmet : naissance, vie, mais alors aussi l'inverse comme un possible, comme un risque, ou comme une attente : l'exclusion du projet commun. C'est comme cela que retentit le mot « nation ».

Pourtant, il ne faut pas le perdre de vue : c'est un peu comme une passion révolutionnaire qui se joue avec le cri de Valmy, et cette passion sera souvent reconduite, comme un trait inhérent à son espérance, que rappelle Robespierre dans le discours du 18 pluviôse an II :

à quelques nuances près de perfidie ou de cruauté, tous les tyrans se ressemblent. Pour nous, nous venons aujourd'hui mettre l'univers dans la confidence de vos secrets politiques, afin que tous les amis de la patrie puissent se rallier à la voix de la raison et de l'intérêt public ; afin que la nation française et ses représentants soient respectés dans tous les pays de l'univers où la connaissance de leurs véritables principes pourra parvenir ; afin que les intrigants qui cherchent toujours à remplacer d'autres intrigants soient jugés par l'opinion publique sur des règles sûres et faciles. ${ }^{1}$

En germe, le discours de la nation porte celui de l'exclusion : ce qui n'est pas la nation doit en être rejeté à l'extérieur, éliminé, éradiqué, comme une peste. La passion de la nation est entière, et c'est bien le point aveugle avec lequel il faut sans relâche négocier. La référence à la nation est exclusive, y compris dans ses attributs, par exemple la langue. On se souvient du rapport de l'abbé Grégoire du 16 Prairial an II «Sur la nécessité d’anéantir le patois et d'universaliser l'usage de la langue française » et qui contient ces mots qui ne furent pas sans conséquence, on s'en doute, sur la difficile construction d'Haïti, tant la renommée de l'auteur était importante : « les Nègres de nos colonies, dont vous avez fait des hommes, ont une espèce d'idiome pauvre comme celui des Hottentots, comme la langue franque, qui, dans tous les verbes, ne connoît guères que l'infinitif » (Grégoire 1794, 3).

1 Robespierre 1794, disponible à la page : https://ihrf.univ-paris1.fr/enseignement/outils-et-materiaux-pedagogiques/textes-et-sources-sur-la-revolution-francaise/robespierre-discours-du-18-pluviose-an-ii/. 
Pour le moment, à Saint-Domingue, c'est progressivement le moment du ralliement, de l'exigence de projet commun. Il faudra attendre le débarquement et la tentative de réinscription de l'esclavage par le corps expéditionnaire pour que celui-ci soit constitué en ennemi commun, et que les différentes factions s'allient, en particulier après l'enlèvement et l'incarcération de Toussaint-Louverture puis d'André Rigaud au fort de Joux, à quelques cellules l'un de l'autre. Dans le Mémorial, Napoléon n'a nulle conscience de l'événement qui s'est accompli à Saint-Domingue : la passion de la nation française a été aveuglante au point que la passion nationale haïtienne a été occultée. Dans le Mémorial, Napoléon traite uniquement des affaires militaires, et de la répartition des groupes désignés par la « couleur », reprenant les caractérisations courantes. Il met en revanche sur le compte des planteurs l'exigence de l'expédition, et blâme Leclerc de n'avoir pas fait preuve d'un grand sens politique et tactique. La dernière remarque concernant Saint-Domingue mérite pourtant qu'on s'y attarde, car elle marque une reconnaissance de facto de la légitimité nationale, par le biais de l'économie :

Le système colonial que nous avons vu est fini pour nous ; il l'est pour tout le continent de l'Europe ; nous devons y renoncer et nous rabattre désormais sur la libre circulation des mers et l'entière liberté d'un échange universel. (Las Cases 1842, 688)

Donc un pays est bien né, et avec lequel le commerce eût été possible.

Mais au-delà du plaidoyer pro domo et de l'hagiographie courante du Mémorial, il convient de revenir sur l'idée nationale telle qu'elle s'est exprimée à Saint-Domingue-Haïti. Gageons alors que progressivement la position de Janvier gagnera en lisibilité.

Avant de devenir une question de nationalité et de prérogatives, la nation est centrale en Haïti à partir du moment où les révoltes d'esclaves prennent de l'ampleur et où l'exigence est de parvenir à créer un front commun. On sait les tergiversations, les alliances et les retournements d'alliances jusqu'au moment où tous les partis forment une coalition qui finit par l'emporter. Le préambule de la Déclaration d'Indépendance ${ }^{2}$ crée d'abord un " pays », là où il y avait des origines et des intérêts différents et divergents. Le lexique est particulièrement intéressant : «Indigènes d'Hayt i », «Puissances étrangères », 《pays indépendant »; « la Proclamation traite des mots » : « il faut par un dernier acte d'autorité nationale assurer à jamais l'empire de la liberté dans le pays qui nous a vu naître ». C'est une proclamation des égaux : "Citoyens, mes Compatriotes », qui demeure néan-

2 Disponible à l'adresse : http://www.nationalarchives.gov.uk/dol/images/examples/haiti/०००1.pdf. 
moins insatisfaite : « le nom français lugubre encore nos contrées ». La suite présente la seule occurrence du terme «nation » de la déclaration, et il résonne bizarrement :

Tout y retrace le souvenir des cruautés de ce peuple barbare ; nos lois, nos mœurs, nos villes, tout porte encore l'empreinte française ; que dis-je, il existe des français dans notre Isle, et vous vous croyez libres et indépendans [sic] de cette République qui a combattu toutes les nations, il est vrai ; mais qui n’a jamais vain$\mathrm{cu}[\mathrm{que}]^{3}$ celles qui ont voulu être libres.

L'affirmation traite ainsi en paradoxe le constat des violences républicaines : elle attribue la qualité de nations à celles qu'ont combattues les républicains français et seules celles qui aspiraient à la liberté ont été, ou pas, vaincues... ce qui laisse le lecteur songeur. En même temps le caractère initial de cette déclaration est revendiqué. Mais pas de qualité voire d'attribut de Nation au corps du peuple d'Haïti.

On ne saurait cependant pas tenir comme une évidence cette déclaration d'Indépendance : l'étonnement, voire une forme d'hébétude, semble affecter tous les acteurs de l'événement et qui s'empressent de prêter serment. On l'a assez répété : les Haïtiens ont combattu pour l'indépendance des États-Unis d'Amérique, et donc parmi les officiers comme Christophe, la réalité de celle-ci est acquise. Il est possible de se séparer de l'emprise politique, économique et commerciale de la métropole. C'est aussi une aspiration de nombreux planteurs, on le sait. Mais à Saint-Domingue, ce n'est pas le seul enjeu. L'indépendance va de pair avec l'abolition de l'esclavage, déjà obtenue en 1793 et que l'expédition Leclerc vient remettre en cause. L'indépendance est une étape de la libération, et pas une fin en soi, même si elle le devient. Cette évidence actuelle modifie les points de vue, et elle est l'objet d'insistance dans la déclaration. Mais point de nation dans cet assemblage. Lapsus ? Trop plein d'évidence ? Impossibilité de rassembler tant il y a de nations différentes parmi les Indigènes ? Car si le terme d'indigène désigne la personne originaire du pays où elle se trouve, alors on est loin du compte. La question de la nation est d'abord un problème à résoudre. Et faire appel à elle, cela revient à rester dans le déclaratif. On rappelle que dans l'imaginaire du vodou la distinction de nation, en fonction des origines africaines, est d'importance.

Rares sont les mentions de la nation en vérité. La Constitution de 1805 en porte trois particulièrement signifiantes :

3 Ajout manuscrit sur le texte imprimé, d'origine inconnue, et qui modifie considérablement la signification du texte... 
- Article 12 : «Aucun blanc, quelle que soit sa nation, ne mettra le pied sur ce territoire, à titre de maître ou de propriétaire et ne pourra à l'avenir y acquérir aucune propriété » ;

- Article 25 : « Le gouvernement assure sûreté et protection aux nations neutres et amies qui viendront entretenir avec cette île des rapports commerciaux, à la charge par elles de se conformer aux règlements, us et coutumes de ce pays »;

- Article 28 : « Au premier coup de canon d'alarme, les villes disparaissent et la nation est debout ».

La nation, c'est l'autre, sauf à partir du moment où Haïti est en danger. La nation, alors, est en armes.

Les mentions de la nation sont très rares également chez Vastey et à notre connaissance ne renvoient pas à la nation haïtienne, mais aux autres, comme dans les expressions " tribunal des nations », « la grande et magnanime Nation britannique $»{ }^{4}$ On trouve également une mention éloquente dans le premier numéro, programmatique, de L'Abeille Haytienne, présentation rédigée par Jules Solyme Milscent :

Concitoyens! Nous formons une nation libre : veillons à notre conservation. En favorisant le progrès des lumières, nous nous rapprochons d'une époque qui, en forçant les peuples à nous respecter et à nous estimer, détruira pour toujours, l'espoir de nos ennemis. ${ }^{5}$

C'est reconnaître ainsi combien est délicate l'affirmation de la nation, en particulier dans la situation que connaît Haïti dans ces années de scission de la terre en trois entités distinctes : le Royaume du Nord, la République, la République paysanne de la Grande-Anse (1807-1819). Les Haïtiens sont avant tout « peuple ». Le vrai problème, et de taille, est que l'article 14 de la constitution de 1805 ne sera jamais accepté, ni même réellement entendu, malgré les protestations de conformité :

Toute acception de couleur parmi les enfants d'une seule et même famille, dont le chef de l'État est le père, devant nécessairement cesser, les Haïtiens ne seront désormais connus que sous la dénomination générique de Noirs ».

On sait que la réalité a démenti cette aspiration, et que la question du projet commun de société s'est aussi fracassée sur la ligne de couleur.

4 De Vastey 1814, respectivement dans l'Adresse au Roi et l'Introduction.

5 L'Abeille Haytienne. Journal politique et littéraire, 1, Port-au-Prince, 7 juillet 1817, 7. http://catalogue.bnf.fr/ark:/12148/cb32680720w. 
Louis Joseph Janvier s'est longuement penché sur cette exigence et sa propre conception de la nation haïtienne a sans doute été affectée par ses expériences.

Ce qui est central semble moins la nation, entité abstraitement définissable, que la nationalité. C'est du moins le sentiment trouble qu'éprouve le lecteur avec l'ouvrage de Janvier consacré à l'histoire des constitutions d'Haïti. Mais le trouble, le lecteur familier de l'historiographie haïtienne l'a déjà éprouvé. Janvier arrive après une succession d'ouvrages qui remettent en cause la vox populi.

Il convient de remonter encore le temps et de s'arrêter à un texte important. Texte fondateur, L'Histoire d'Haïti de Thomas Madiou trace les contours de cette nation haïtienne introuvable. L'insistance de l'auteur à saturer d'exemples et d'anecdotes les rappels de l'extermination initiale ne doit pas faire perdre de vue que, pour lui, c'est bien évidemment la question de l'indigénat qui est posée. Il n'y a plus d'indigènes en Haïti, à peine seulement quelques traces. Madiou affirme de surcroît pour sa propre identité une ascendance amérindienne, lointaine, ce qui lui permet d'étreindre dans sa propre personne les différentes composantes qui ont participé à la genèse anthropologique d'Haïti. Janvier aussi affirme une telle ascendance. Demeure le constat tragique : s'il y avait nation, elle a disparu. Et il faut quand même imaginer le trouble qui se saisit de ceux qui ont remplacé ces disparus. Janvier aussi sera porteur du même trouble, comme on l'a montré dans l'article initialement consacré à son œuvre (cf. Chemla 2005, 7-36).

Dans le même souci d'identifier ce qui fait défaut au projet commun d'une introuvable nation haïtienne, il faut revenir à la haine inexpiable entre Noirs et Gens de couleur, et à la guerre dite des Couteaux (1799-1800), aux très nombreuses exactions et aux logiques d'exterminations mises en œuvre pendant ce conflit interne. Le souvenir en restera longtemps gravé dans la conscience haïtienne, alors qu'il a été longtemps ignoré par les études consacrées à Haïti par les chercheurs étrangers. Le projet commun est longtemps défaillant. Un exemple, extrait de L'Histoire d'Haïti de Madiou, permet d'appréhender en partie cette défaillance consubstantielle. Il restitue les points de vue des partisans de Rigaud pendant la guerre des Couteaux, en particulier dans l'hyperbole du discours haineux, pendant la préparation du siège de Jacmel en 1799, la ville étant un camp retranché des partisans de Rigaud :

La détermination de vaincre ou de mourir était peinte sur les traits de chaque soldat. La haine la plus implacable contre l'autorité de Toussaint Louverture se manifestait dans les rangs ; chacun voyait en lui le protecteur du colon blanc, le bourreau des hommes de couleur, et l'ennemi de la liberté des noirs. Les femmes elles-mêmes, noires et jaunes, étaient pleines de courage, et se montraient déterminées à partager tous les dangers du soldat. (Madiou 1847, 7) 
Au-delà de l'opposition entre les deux chefs Rigaud et Toussaint, au-delà de la ligne de couleur à prétention anthropologique, les oppositions semblent si radicales qu'elles en deviennent insurmontables. Madiou insiste dans cette partie de son Histoire sur les différences d'attitude entre les principaux officiers, parvenant progressivement à opposer les postures de Dessalines à celle de Christophe, préfigurant ainsi le moment de la séparation, et de la fin du projet commun. Toute la question est de parvenir à qualifier correctement ces conflits récurrents : s'agit-il d'une guerre civile à l'intérieur d'une colonie ou bien d'un conflit entre plusieurs, deux déjà, nations distinctes, et qui s'excluent ? Considérer que ce questionnement n'affecte pas Janvier serait une vue de l'esprit, incomplète et pour le coup, teintée de l'écran des logiques réductrices de l'étranger. Cette logique de disjonction, aussi bizarre qu'elle puisse se présenter, semble inhérente à l'État haïtien.

Il est un autre texte de Madiou qui permet d'approcher cette question. Restée dans de la famille jusqu'en 2017, l'Autobiographie de Thomas Madiou est de surcroît particulièrement intéressante dans ce contexte si complexe, parce qu'elle permet de mieux comprendre l'articulation entre la réflexion et la construction du politique, comme gestion d'un État en manque ou peut-être, comme l'auteur le suggère, comme un État manqué, dans le contexte déterminé par le paiement de l'indemnité aux planteurs, et l'endettement chronique de ce même État. Ainsi, encore, tout remonte aux années qui ont précédé la déclaration d'Indépendance. Et les conséquences en sont désastreuses encore, et l'autorité de Madiou n'eût sans doute pas pâti si les Haïtiens avaient pu disposer de ce texte plus tôt :

Ceux qui possèdent ne sont aux yeux du peuple que des bandits heureux ; ainsi les serviteurs dans les maisons privées, les journaliers dans les montagnes et dans les villes, enfin la classe des prolétaires nourrit-elle contre les chefs de maison d'abord une haine implacable puis un mépris réel qui n'est nullement déguisé, car celui chez lequel le prolétaire est engagé n'est vraiment presque à coup sûr qu'un brigand masqué sous de beaux vêtements. Aussi le peuple frappe-t-il fort dans les troubles sociaux et politiques. Dans les tourmentes révolutionnaires, il n'a pas besoin de connaître la couleur de son chef, il s'inquiète peu s'il est noir, blanc de peau ou mulâtre pourvu qu'il l'autorise à piller. Mais son intime confiance politique est dans le chef noir. (Madiou [2017], 93)

Ce premier constat d'une pérennisation de la haine sociale est redoublé de la mise en accusation du groupe social et phénotypique dont relèverait Madiou, mais dont il se sépare en se plaçant au service de l'État, comme historien et comme ministre responsable. Le tableau qui ressort est particulièrement inquiétant, et a sans doute structuré la description sociale jusqu'à récemment : 
Les hommes éclairés qui donnent la plupart l'exemple des malversations ont été les premiers corrupteurs du peuple qui pense qu'il dispose de son bien quand il dévaste les propriétés privées ; cependant il faut bien reconnaître qu'il y a quelquefois des victimes innocentes, des familles qui avaient gagné leur bien-être à la sueur de leur front, mais ce sont des exceptions. Les plus instruits, presque tous, ne se livrent aux malversations que parce qu'ils n'ont pas foi en l'avenir de leur nation indépendante, ni même de la société. Ils se hâtent de faire grand profit de leur position pour pouvoir se retirer à l'étranger. Bien d'entre eux disent qu'on ne doit parvenir au pouvoir que pour s'enrichir ou exercer des vengeances. Ces malversations et déprédations appauvrissent le trésor public et empêchent d'améliorer en aucune façon l'état matériel du pays. Depuis 1804 jusqu'à ce jour (1868), rien ne se répare ; les ruines s'amoncellent ou pour mieux dire, les traces des ruines ont même disparu tant le pillage fonctionne avec persévérance, lentement, il est vrai, mais sans cesse, on dirait des animaux rongeant les os des morts. (Madiou [2017], 94)

Né en 1855, Louis Joseph Janvier a ce délitement d'un État haïtien problématique sous les yeux. Il aura des mots très durs sur la guerre de tous contre tous, sur les généraux « d'arrondissements », mais aussi contre l'indécence économique et sociale d'une bourgeoisie corrompue, dans Le Vieux Piquet en particulier (Janvier 1884).

C'est en partie sur ce soubassement que va s'élaborer le discours de Janvier touchant à la fibre patriotique et à la définition d'une nation jusque-là introuvable, sinon dans les situations d'alarme. Les publications de Janvier s'échelonnent en trois étapes, on le rappelle succinctement : d'abord, en 1881, il publie sa thèse de médecine. Entre 1882 et 1886, il entreprend un travail sur les causes internes et externes du mépris à l'égard d'Haïti. En 1889, il publie le roman Une Chercheuse, qui semble être au cœur de son approche. Enfin, entre 1905 et 1908, les trois ouvrages publiés tiennent de l'action politique, en vue d'être élu. Janvier meurt assez jeune, à 55 ans, en 1911, probablement épuisé par une activité intellectuelle qui l'a occupé nuit et jour.

L'ouverture discursive est la fameuse thèse de médecine consacrée à la Phtisie pulmonaire - Causes - Traitement préventif. L'approche que mène Janvier, en accord avec les théories du temps est avant tout sociale et hygiéniste : étudier les causes de la phtisie, c'est

aborder une question qui tient autant à la médecine qu'à l'économie d'une nation. La phtisie est une cause de dépopulation. Par elle, la société, minée dans ses réserves, tombe en décadence, en sénilité. L'homme se doit conserver non seulement pour lui-même, non seulement pour sa famille, mais avant tout pour la patrie. (Janvier 1881, XVI) 
La santé n'est bonne qu'au regard d'un projet commun et national, en tous les cas patriotique. Le relevé des causes de la maladie met en place une géographie sociale, des profondeurs des sous-sols, à l'élévation en altitude. Tout un imaginaire se déploie qui articule les causes de la maladie à la descente dans les profondeurs à la fois urbaines et sociales :

Des faits nombreux ne laissent aucun doute sur l'influence que la lumière exerce sur l'organisme. Les individus qui vivent dans l'obscurité respirent moins ; ils ont les chairs molles, bouffies, infiltrées ; leurs tissus sont atones et sujets à l'hydrohémie : ${ }^{6}$ tels les prisonniers dans les cachots non ensoleillés, les marins de la cale et de la cambuse, les concierges, les ouvriers qui travaillent au-dessous du niveau du sol, ceux qui habitent les caves. Ces malheureux privés des bienfaisants rayons du soleil meurent le plus souvent de scrofule et de phtisie. (Janvier 1881, 114)

En Haïti même, cette répartition spatiale est reproduite par la configuration géographique : aux villes, dans les espaces de bord de mer et parfois marécageux, où se déroulent vie commerciale et vie politique, ainsi que les plaisirs de la chair, la phtisie est une marque courante. En revanche, dans les hauteurs, où travaillent réellement les paysans, la phtisie est rare :

Ainsi en Haïti [...] île montagneuse par excellence, assez grande et assez élevée pour que ses côtes seulement soient balayées par les vents de la mer, la phtisie est très rare dans les campagnes.

Le montagnard haïtien, toujours vif et gai, alerte et dispos, fort et intelligent, travaillant à sa guise, - assez mais pas trop -, libre, maître de son champ, bien nourri et bien soigné, content de peu d'ailleurs, est rarement atteint de maladies chroniques. Connaissant peu les causes morales déprimantes, toujours au grand air, aimant la danse, l'équitation, la chasse et la pêche, y excellant, vivant large et bien enfin, il ne meurt presque jamais de phtisie ${ }^{7}$

À l'inverse, dans les villes, la situation est déplorable :

La phtisie n'est pas très rare à Port-au-Prince, la capitale, ville bâtie sur le bord de mer, moitié sur un terrain d'alluvion, moitié sur un terrain crétacé, grand centre d'affaires commerciales, où la vie

6 Présence d'eau en quantité anormalement élevée dans le sang.

7 Janvier 1881, 31. Une note à ce texte vante l'hygiène corporelle des paysans. Plus loin (143), la vêture des paysannes est elle aussi proposée comme la confirmation d'une hygiène corporelle exemplaire. 
de bureau, des grands comptoirs, des grandes administrations, retient les hommes captifs tout le jour, où l'on a toutes les facilités et toutes les séductions d'une vie de trop peu d'exercices physiques et le soir peut-être quelque peu libertine. (Janvier 1881, 31)

C'est cette cartographie que les ouvrages de Janvier vont affiner et préciser. Cependant, on relève l'insistance de Janvier pour une bio-politique avant la lettre. Le texte de sa thèse lui permet d'élaborer une pensée tournée vers l'hygiénisation de la société, et vers la définition d'une modernité sociale à même d'envisager un projet de société. Ainsi, la sexualité (masculine, il faut le rappeler) est traitée sous l'angle non du plaisir, mais de la nécessité. La sexualité féminine, quant à elle, doit être régulée dans le mariage, sous peine de la voir participer au dérèglement. La thèse de Janvier trace ainsi les contours d'un projet politique : bâtir une nation saine, et capable d'échapper aux atermoiements et à la médiocrisation. C'est une nation dynamique, capable d'exercices physiques, en particulier pendant l'adolescence ou la «tuberculisation » est la plus sensible. L'exemplarité de l'élévation haïtienne, la vie des paysans dans les hauteurs par opposition aux urbains dépravés, l'engagement à la régulation de la sexualité libèrent du fléau phtisique (Janvier 1881, 260-1), et augmente la liberté de la population saine et hygiénisée. Il y a évidemment une antithèse :

L'homme de quarante ans qui, de gaieté de cœur et sans nul souci de la dignité humaine, s'adonne à la boisson, au tabac et aux femmes, mène une vie molle, lâche et efféminée, cet homme-là est un être peu utile et peu intéressant. Il est justement puni si, au fond de la coupe orgiaque, il trouve ce qu'il a cherché : la mort, - cette maladie dont on ne guérit pas ». (Janvier 1881, 262)

Ce sont les derniers mots du texte de cette thèse à visée essentiellement politique. De notre point de vue, ce texte inaugural affirme en creux la perception du fait haitien par Janvier, au regard d'un environnement mental et culturel qui justement n'est pas haïtien, mais français, évidemment. L'opposition entre les deux va rapidement permettre de mettre en relief une série de paradoxes, comme l'articulation douloureuse notamment entre la décrépitude haïtienne, avérée, et le souci de défendre coûte que coûte l'exemplarité de cette présence au monde rendue possible par d'âpres combats (cf. Chemla 2005).

Dans un premier temps, Janvier mène l'offensive contre les détracteurs. On retiendra ici d'abord essentiellement l'ouvrage publié en pro- 
testation contre l'article considéré comme insultant de Léo Quesnel. ${ }^{8}$ La lettre de Janvier et sa réponse à la réponse de Quesnel constituent un argumentaire à la fois historique et anthropologique. Il y passe en revue la plupart des assertions du plumitif, et y répond presque point par point : les Haïtiens réalisent le programme de la Révolution française, et malgré l'âpreté des guerres, ne gardent pas rancune aux Français. Ils sont informés par la culture savante française. Sur le terrain de la race, il faut en finir avec les pseudo catégories décrites par Moreau de Saint-Méry, enfin. Et surtout, il ne faut plus considérer les différences phénotypiques depuis le mode de la blancheur. Quant à la proximité avec l'état de nature, une série d'évolutions marquantes a fait reculer cet état dans les lointains. Évolution intellectuelle, évolution morale, évolution religieuse ont été des facteurs d'élévation. Ce que montre l'argumentaire de Janvier est bien que l'haïtianité ne saurait être exclue du concert des peuples, et que cette haïtianité a réussi l'exploit d'abolir le système de «castes » qui a prévalu dans la société créole :

C'est à peine si l'œil sagace de l'homme d'État, ce psychiâtre [sic] d'une nation, peut rencontrer et observer dans les moments de crises politiques aiguës (élections législatives, élection présidentielle) des cas sporadiques de cette curieuse et singulière maladie de l'intelligence qu'on appelle le préjugé de couleur. Cela ne se peut plus observer que chez quelques rares esprits bornés, superficiels, ignorants ou monstrueusement pervers quoique éclairés, ou encore dans quelques âmes faibles, emplies de visions ou de chimères et affolées par la peur de dangers imaginaires. (Janvier 1882, 50)

La société haïtienne, selon Janvier, ne générerait pas d'exclusion, contrairement à ce qu'affirme Quesnel.

En même temps, Janvier rappelle que les nouvelles mainmises coloniales se traduisent par la disparition des populations indigènes, comme ce fut le cas dans l'île originelle qui aura été le laboratoire de l'extermination. Ce n'est pas tant de l'exclusion dont il faut se prévaloir que de l'éloignement, considéré comme un gage de protection contre l'emprise de l'autre qui installe la plupart du temps un échange aux termes inégaux. Puis le texte pivote, et à partir d'une longue étude sur le mariage, étude critique aux nombreuses références savantes, dotée d'une longue citation de Schopenhauer, Janvier dresse un constat de l'institution, pour en rappeler l'inanité, sinon comme un bio pouvoir avant la lettre. La polygamie, en effet, permet de peupler

8 Revue politique et littéraire du 21 janvier 1882, selon les indications de Janvier. Il n’a pas été possible de consulter l'original, l'année 1882 de la Revue Bleue, autre titre de la revue, est manquante au catalogue de la BNF (juillet 2020). 
un pays rapidement. Le mariage de raison ou de convenance n'est en effet pas propice à l'amélioration de la patrie :

Le cœur et la nature n’ayant pas présidé à ces unions, n’ayant pas signé au contrat, n'ayant pas été prié à ces noces, se retirent aussi quand doit venir l'enfant ; aussi celui-ci naît-il souvent rachitique, phtisique, mal bâti, égoïste et devient traitre à sa famille, traitre à son pays au moment du danger. (Janvier 1882, 70)

En conclusion de cet argumentaire, dont les moments sont rappelés en fin d'article, comme pour en faciliter la mémorisation, Janvier affirme que sa certitude est acquise qu'Haïti se fera d'elle-même, c'est-à-dire par les Haïtiens : « Haïti farà da se » (Janvier 1882, 85).

Un peuple noir devant les peuples blancs..., publié en 1883 reprend une partie de ces arguments en les circonstanciant à partir des allégations de Cochinat. Ouvrage connu, dont on a déjà traité la substance, en particulier les fondements de l'argumentaire de Cochinat, il est ouvert par une longue série de citations touchant à la nation ainsi que la patrie.

Là encore, il est utile d'examiner quelques éléments contextuels. C'est en 1882 que Renan publie Qu'est-ce qu'une nation? Le retentissement est important dans toute l'Europe, en raison certes de la renommée de son auteur, mais sans doute aussi parce que les conflits récents entre États européens ont mis à mal la notion : le capitalisme triomphant, la technicisation en marche, le caractère total et meurtrier des affrontements, mettent quelque peu à mal le sentiment national, même si en France il est conforté par l'espérance de la reconquête de l'Alsace et de la Lorraine. Et puis les conquêtes impériales et l'entreprise en cours des colonisations exigent sans doute un examen nouveau. Renan actualise la notion, dont il montre qu'elle est floue, et qu'elle ne saurait servir de principe politique, mais que, de fait, elle ouvre un horizon, ou indique une direction. Il en rappelle les origines révolutionnaires : "C'est la gloire de la France d'avoir, par la Révolution française, proclamé qu'une nation existe par ellemême » (Renan 1882, 10).

L'essentiel est donc bien dans la dynamique de l'autonomie et le fait que la nation se refonde sans cesse, puisant ses forces de renouvellement en son sein.

Mais de là à parvenir à répondre de façon positive à la question, il apparaît que le paysage est contrasté. La conférence, d’une tonalité particulièrement moderne, va montrer que la définition de la nation est improbable, voire introuvable. Janvier ne peut que prêter attention à ce discours idéaliste de Renan, qu'il critique par ailleurs. Renan passe au crible les différents marqueurs habituellement utilisés, pour leur dénier toute efficacité : l'histoire, la race, la religion, le commerce, dont les communautés d'intérêt transcendent les fron- 
tières, la géographie. Il n'existe pas de frontières naturelles, et déclarer le contraire c'est accepter d'aller vers le pire :

La vérité est qu'il n’y a pas de race pure et que faire reposer la politique sur l'analyse ethnographique, c'est la faire reposer sur une chimère. [...] En d'autres termes, les origines zoologiques de l'humanité sont énormément antérieures aux origines de la culture, de la civilisation, du langage. (Renan 1882, 15)

Parmi les marqueurs mal utilisés et propices eux aussi au pire, il y a la langue, qui est une formation particulièrement arbitraire :

Les langues sont des formations historiques, qui indiquent peu de choses sur le sang de ceux qui les parlent, et qui, en tout cas, ne sauraient enchaîner la liberté humaine, quand il s'agit de déterminer la famille avec laquelle on s'unit pour la vie et pour la mort. (Renan 1882, 21)

Alors, quid de la nation? Passé le moment critique, la recherche d'une définition devient délicate. Renan ne cache pas la difficulté : la nation est approchée comme un «principe spirituel » pas nécessairement pérenne. Les nations peuvent disparaître, ou bien simplement cesser d'être le moteur d'une espérance. Car c'est bien sur ce terrain que la nation peut-être prend sens : la nation est un présent, une présence dans le présent, c'est-à-dire un héritage et un projet. « Dans le passé un héritage de gloire et de regrets à partager, dans l'avenir un même programme à réaliser » (Renan 1882, 27).

C'est dans cette dynamique, encore une fois, que la nation construit pleinement la signification de sa présence continuée : la nation se manifeste dans le consentement à la solidarité, au sacrifice (du passé déjà, et de ce qu'il faut détruire pour permettre l'irruption de l'avenir), à l'abdication de l'individu au profit de la communauté, au vivre ensemble. Mais la nation se manifeste aussi par la consultation des populations. La dictature, et Renan est sans doute un des premiers penseurs du politique dans ce sens, ruine l'idée même de la nation, même et surtout s'il s'en fait un emblème, ou pire, une enseigne guerrière. Ce «principe spirituel » demeure néanmoins suffisamment imprécis pour permettre aussi des interprétations fondées sur un racialisme assez courant dans les années 1880-1900. Janvier est aussi informé par ce type d'argumentaire et son propre texte en est souvent marqué.

Revenons à La République d'Haïti et ses visiteurs 1840-1882 (Janvier 1883). L'ensemble au titre imposant et informatif, est placé sous l'égide d'une figure féminine, la matrie. L'ouvrage est lui-même imposant. Sa préface est en grande partie constituée de citations, d’auteurs le plus souvent contemporains de Janvier, mais aussi d'anciens, qui célèbrent 
l'attachement à la patrie, et méprisent son abandon. Ainsi le terrain est préparé ensuite pour un déferlement lui aussi imposant : l'épuisement de la figure du préjugé. À plusieurs reprises, Janvier rappelle ainsi les conditions d'émergence du fait haïtien, comme sa préservation et sa consolidation. Il rappelle les contradictions des discours critiques sur Haïti : ils sont en général muets sur les problèmes dénoncés quand ils ont cours en France ou en Europe. Il fait de Cochinat une sorte de repoussoir à toute prise de parole critique à l'égard du fait haïtien, surtout quand cette critique est perçue comme injuste, voire insultante. La technique du pamphlet est au point pour Janvier : il reformule le discours de l'insulte, en montre l'inanité et le caractère dérisoire ou mensonger, et justifie par ce moyen-là de renvoyer l'adversaire dans le silence, en l'insultant. Le lecteur est sensible au caractère littéraire de la diatribe, presque poétique, et qui souvent quitte son objet, dans une envolée vers des images volontiers évocatrices :

Croyez-vous donc que c'est du sang de mollusque, du sang blanc, du sang de homard qui circule en nous ?... Si vous êtes une huitre, croyez-vous que je doive forcément vous ressembler ? ... Croyezvous que je doive me laisser cracher au nez, laisser défigurer mon frère, mes amis, les miens que vous traitez de vaniteux, d'ivrognes, de voleurs, de lâches, de tellement paresseux et inconscients qu'ils dormiraient dans l'eau ? Puis-je laisser insulter à ma famille, à la famille agrandie, c'est-à-dire à ma patrie, sans sentir bouillonner tout mon être et bondir dans mes artères ce vieux sang de combattant qui m'étouffe ? (Janvier 1883, 481)

Ainsi, attenter à l'intégrité d'un des membres, c'est attenter à la «patrie ». Encore une fois, on attendrait ici l'autre mot, celui de nation, ou bien la reprise de celui de matrie.

Le nationalisme de Janvier est peut-être sans doute imprononçable, comme l'est l'objet désirable d'une origine commune, d'une naissance commune. La nation, c'est peut-être alors cela : la naissance qualifiée depuis une " même » origine. C'est un songe, alors, car la qualification de l'identité de lignage s'apparente plus à un mythe qu'à la réalité. L'idée nationaliste s'approche peut-être par certains attributs, celui du sang, celui du désir d'une société. Par-delà une prétendue ligne de couleur, qui pourtant revient si souvent sous sa plume, il rejoint ainsi le constat mené et énoncé secrètement par Madiou dans son Autobiographie. La littérature haïtienne est très souvent aux prises avec le trouble dès qu'elle s'empare de cette question, ainsi que nous l'avons montré autrefois (cf. Chemla 2003, 36, 61, 89, 161-3), dès lors que les origines sont identifiées à partir d'un lieu.

En 1884, il publie Haïti aux Haïtiens, dont le titre est déjà une déclaration nationaliste, et qui comme c'est souvent le cas, témoigne d'une charge d'anxiété, entraînée par la perte du sentiment d'identi- 
té. Il s'en prend aux menées qui visent à déliter le pays, à le mettre à l'encan, ou en coupe réglée, à l'image de l'Égypte, qui à force d'investissements et d'emprunts se retrouve en quasi faillite, et donc livrée aux banquiers. Composé de cinq articles destinés au journal La Nation, cet ouvrage dont le premier article, «Les Gloutons et les Candides » est daté de 10 jours avant la mort de Madiou, qui avait récusé la légitimité des révoltés. Janvier dénonce les tentatives de démembrement et rappelle que ce sont les paysans haïtiens eux-mêmes qui doivent industrialiser leur exploitation de la terre. Il dénonce aussi la duplicité des puissances coloniales :

Par le passé, on peut préjuger de l'avenir. On a cherché à nous humilier ; on nous dépouille et on nous pille; on nous a mis et on nous met chaque jour le poignard sous la gorge ; on nous a menacés et on nous menace dans notre indépendance parce que nous avons une dette de quarante millions ; on a colporté partout que nous étions des sauvages pour nous intimider et de nous mieux rançonner ; ceux qui nous léchaient la main chez nous nous appelaient singes en Europe. (Janvier 1884b, 15)

Certes, et s'il s'agit bien d'une déclaration que l'on éprouve comme nationaliste, on ne parvient pas aisément à concevoir ce qu'il en est de la nation. Dans d'autres passages, ce sont les rapports entre la France et Haïti qui sont valorisés, sur le plan de la formation intellectuelle. Et par celle-ci, c'est bien l'exemplarité haïtienne qui est mise en avant, dans un dépassement d'une définition restrictive de la nation, et en opposition frontale avec l'économie fondée sur la rapine :

Il y a une grande Europe : celle qui continue Diderot, Condorcet, Grégoire, la Grande Constituante et la Convention ; c'est celle des philosophes, des émancipateurs, des aimeurs ; celle de Michelet, de Schœlcher, de Pierre Laffite [sic, pour Laffitte] ; celle-là nous dit : Nous vous émancipons le cerveau pour que vous vous mettiez un jour à la tête de la race noire. (Janvier 1884b, 31)

L'enjeu est de parvenir à résister à la dépossession.

En 1884, Les Affaires d'Haïti 1883-1884 (Janvier 1973) étendent la réflexion à partir des événements militaires qui ont secoué Haïti pendant l'année 1883 (mars-octobre). La tentative de prise du pouvoir dirigée par Boyer Bazelais, fondateur en 1870 du parti libéral a été un échec malgré des combats importants, lors de la chute de Miragoâne, pendant laquelle il est mort, ce qui a entraîné la capitulation de ses partisans. La ligne de partage politique entre le parti libéral et son rival, le parti national, fondé en 1879, est avant tout une ligne de couleur. C'est à partir de ce clivage que Janvier va donner une approche de ce qu'il conçoit au sujet de la nation. 
La première étape consiste à exclure les traîtres et les renégats, qui ne se reconnaissent pas dans l'histoire du surgissement de la nation haïtienne :

Un jeune peuple a besoin de sa bonne réputation pour prospérer, pour grandir. Il doit impitoyablement clouer au pilori de son histoire tous les félons, tous les renégats ; il doit flétrir à jamais, pour l'enseignement des générations futures, les noms des insensés et des traîtres qui, à l'étranger, se constituent les assassins de l'honneur collectif. (Janvier 1973, 9)

Le problème est analysable à l'aune du préjugé de couleur. Ainsi, relevant dans la presse française des remarques racistes au sujet du président Salomon, il rappelle que la tyrannie n'est pas l'apanage des Noirs :

Tibère, Néron, Héliogabale, Pierre le Cruel, Charles IX, Henri VIII, César Borgia furent des tyrans dont l'Europe occidentale ne saurait oublier les noms. Ils n'étaient pas des nègres. Je défie qu'on cite le nom d'un seul chef noir qui les ait égalés en férocité. Il serait grand temps qu'on fût juste, indulgent envers la race noire ; qu'on l'innocentât surtout de tous les crimes et forfaits dont il était de mise autrefois de charger la mémoire de ses types les plus éminents ou les plus célèbres. (36 note 2 )

Le préjugé de couleur est un attribut majeur des libéraux, qui sont avant tout des clairs, et qui sont coupable d'une erreur de taille :

Il serait à désirer qu'un Haïtien, quelle que soit la couleur de sa peau, écrivit, spécialement pour son pays, un livre court et précis où il serait démontré que le mulâtre est un hybride et qu'il n'existe pas de race mulâtre ; que noirs et mulâtres devraient vouloir se qualifier de 'nègres', qu'en Europe et en Amérique le mulâtre et le nègre ne font qu'un; qu'aux États-Unis et en France, par exemple, on ne fait aucune distinction entre un mulâtre et un nègre instruits ; qu'on n'en fait non plus aucune entre un mulâtre et un noir ignorants ; qu'en tant qu'on établisse distinction entre un nègre instruit et un mulâtre ignorant, l'avantage est tout entier en faveur du nègre. (47 note 1$)$

En fait, ceux qui se définissent comme libéraux ne sont guère plus que des antinationaux et qui n'obéissent réellement à aucune règle :

"Que les antinationaux ne viennent pas parler de principes, de libéralisme : ils n'ont pas de principes sociaux et ne sont nullement des libéraux. C'est ce que les artisans, les paysans sentent très 
bien, et voilà pourquoi le pouvoir des chefs mulâtres n'a jamais été que précaire ; voilà pourquoi ils n'ont jamais pu bâtir que sur du sable. Ils n'auront jamais la confiance du vrai peuple, s'ils ne prouvent, au préalable, par toute une existence de labeurs, par une conduite pleine de respect pour les lois du nombre et pour la loi écrite, que le préjugé de couleur ne fit jamais partie de leur bagage politique. Puissent-ils me comprendre pour le plus grand bien de la patrie. (21 note 1)

Là où la pensée de Janvier se fait réellement déterminante et argumentée, c'est quand il affirme que le préjugé de couleur est un paravent de la haine sociale. Il anticipe les longues analyses qu'il va publier dans l'ouvrage consacré aux constitutions d'Haïti et à la critique du Code rural promulgué sous la présidence de Boyer. La nation haïtienne est dépossédée de ses terres par ceux-là mêmes qui devraient les défendre, ce qui reconduit les oppositions violentes entre travailleurs et propriétaires souvent auto-institués. Les paysans du Nord en ont fait les frais autrefois :

Ces paysans, qu'on a appelés piquets, et dont les revendications étaient entièrement justes et bien fondées, furent massacrés ou dispersés par les troupes régulières que les gouvernants réactionnaires, qui siégeaient à Port-au-Prince, envoyèrent contre eux. Jusqu'à aujourd'hui, en Haïti et à l'étranger, des publicités mal renseignés ou peu sincères continuent d'insulter à la mémoire de ces vaillants prolétaires. De 1843 à 1883, la situation continua d'être déplorable pour les paysans. Ils travaillaient sur des terres qui étaient détenues par de soi-disant propriétaires, dont les droits étaient souvent contestables et quelquefois absolument problématiques et qui, pourtant, s'emparaient audacieusement de la moitié et même des deux tiers de leurs récoltes. (56)

Si la terre passait réellement dans les mains des paysans, ce serait la fondation de la citoyenneté, et non plus la continuation du « demi-servage » (43)

Pour donner réalité à la nation, il est nécessaire de tenir une dynamique que ne connaît pas Haïti : il faut à la fois cohésion mais aussi vertu. Certaines nations donnent modèle à la cohésion, qui s'actualise en projet constant, alors que le pouvoir en Haïti n'a jamais été le socle de l'État, et a été considéré comme une fin en soi, ce qui annule l'espérance d'une nation :

La cohésion intellectuelle est chose capitale et la discipline est chose supérieure. Les Prussiens savent se soumettre les uns aux autres : de là leurs triomphes. La discipline coercitive, celle qui donne à l'enfant l'habitude de se conformer aux lois ; le sentiment 
d'allégeance et de loyauté (loyalisme anglais) qui existe aussi dans une démocratie où l'on croit à la liberté, à l'égalité, au peuple ; l'esprit de nationalité qui lie tous les hommes ensemble dans une nation, leur donne un grand respect de l'honneur du pays, tout cela manque absolument aux prétendus libéraux haïtiens. Ils ne savent pas obéir et aspirent tous au commandement, encore qu'ils ne fassent absolument rien pour le mériter, pour le tenir de ceux qui en sont les véritables dispensateurs. Ils ne comprennent pas le bourgeois ; ils n'ont jamais deviné l'artisan. Quant au paysan, ils l'ignorent. Ils se figurent que les montagnards n'ont rien dans le cerveau, ce en quoi ils se trompent grossièrement. De l'histoire de leur pays, ils savent à peine les noms ou les renient ; les idées du passé, celles du présent et de l'avenir, on dirait qu'un brouillard les leur dérobe. Ils sont aveugles et sourds. Quos vult perdere Jupiter dementat. ${ }^{9}$ Un esprit superstitieux dirait qu'ils expient le crime du Pont-Rouge. Moi je dis qu'ils expient les attentats contre la justice et le droit, tous les mensonges que leurs pères et eux ils ont commis de 1801 à 1883. Une fatalité effrayante et mystérieuse pèse sur eux. Le peuple se venge. Il est destin, Fatum. (59 note 1)

Ce n'est qu'à partir du moment où le projet est partagé qu'il peut être mis en œuvre, et permettre de faire vivre le corps de la nation :

La nation, c'est l'État vivant, c'est l'être, le corps ; le gouvernement, c'est l'état pensant et agissant, c'est le cerveau, l'esprit. Le citoyen instruit doit respecter le corps et respecter le cerveau tout en signalant à celui-ci ses défaillances, tout en travaillant patriotiquement et pacifiquement à l'empêcher de commettre des fautes qui pourraient être préjudiciables non seulement aux générations vivantes, mais encore aux générations futures. Ce devoir, un des plus glorieux parce qu'il est un des plus difficiles, il doit le remplir en tout temps, en tout lieu, même au péril de sa vie. (60 note 1)

En même temps, force est de constater que la plupart de ces considérations sur la constitution de la nation se présentent dans le texte de façon presque latérale : la plupart sont en notes.

Après avoir relevé les obstacles à la reconnaissance de la nation haïtienne en raison des préjugés extérieurs et intérieurs, analysés les causes politiques et sociales de cette impossibilité, Janvier interroge les fondements mêmes de l'État, à partir de l'étude de ses constitutions, dont Haïti a connu pléthore entre 1800 et son époque. En 1886, il publie une longue étude qui demeure à ce jour une mine de renseignements : Les Constitutions d'Haïti, 1801-1885 (Janvier 1886a).

'Jupiter rend fou ceux qu'il veut perdre'. 
Demeurent ainsi une succession de constitutions pendant la durée du XIXème s., ce qui dénote une singulière difficulté à bâtir le projet d'un État, de gouvernements et d'une administration œuvrant dans la durée. Là encore, l'articulation avec le principe spirituel et renanien commun fait immédiatement défaut. Dès les premières pages, le trouble est installé : " L'histoire d'une nation raconte son existence depuis ses origines, comme l'observation médicale raconte la maladie depuis sa naissance » (Janvier 1886a, II). La comparaison est pour le moins bizarre. Si la matrie conçoit la nation, ce serait comme une de ces maladies pernicieuses qui sapent la vitalité de l'enfant. Et cette maladie serait déjà présente dans le corps maternel. Ce serait à considérer évidemment depuis la figure génocidaire initiale, bien sûr, qui a rendu « cette terre qui avait bu le sang de Kaonabo et d'Anakaona » stérile au projet politique (Janvier 1883, 634). Il y a ainsi une grande cohérence de l'imaginaire nationaliste, malgré les contradictions apparentes.

Pourtant, ce que relève Janvier est bien une sorte de volonté politique à empêcher la construction de l'unité :

C'est au code rural de 1826, qui créait dans un pays qu'on prétendait égalitaire deux nations dans la nation, l'une suçant l'autre, c'est au code rural de 1826 qu'il faut remonter pour trouver une explication au pillage des villes renversées par le tremblement de terre de 1842, les origines des révoltes de paysans qui eurent lieu entre 1843 et 1847, des événements du mois d'avril 1848 et, sous Geffrard, celle des révoltes des montagnards de Jérémie, de la prise d'armes d'Adoubi, c'est-à-dire de la colère latente du paysan contre le citadin, du prolétaire contre le bourgeois, faisant explosion à chaque moment propice. (Janvier 1886a, 152)

Et Janvier dépasse le seul constat. Il trace des lignes de progression :

La nation haïtienne sera heureuse lorsqu'elle sera paisible, paisible quand elle sera éclairée, éclairée le jour où à la politique sentimentale, conservatrice des causes des conspirations, elle préférera la politique scientifique, celle qui les prévient ; elle sera riche et respectée le jour surtout où elle fera de la politique démocratique, de la politique des majorités d'intérêts, de la politique du plus grand nombre. Hors de là, ce sera toujours le gâchis ou même le chaos, la stagnation, ou même le recul. (Janvier 1886a 265)

Il en arrive ainsi à identifier ainsi ceux qu'il considère comme les membres de la nation réelle : " La Constitution de 1846 avec les amendements de 1859 et de 1860 ne changea rien au sort des classes laborieuses qui constituent le substratum, la vraie base de la nation » (Janvier 1886a, 277). 
Le nationalisme tient alors au primat de la nation sur ce qui n'est pas elle. L'approche sociologique est ainsi déterminante dans les textes et dans l'argumentaire, et permet de tracer une ligne de partage radicale.

Ce n'est cependant encore pas aussi simple : toute l'œuvre de Janvier à la fois rend compte de ce constat, dans la lignée des essayistes et des penseurs qui l'ont élaboré progressivement - en particulier Madiou - en même temps qu'il le déconstruit, notamment dans sa critique souvent acerbe de l'impossibilité de bâtir un projet. Dans Le Manuscrit de mon ami, le narrateur désabusé au centre du roman, et comme un double de l'auteur et de ceux de sa génération, reprend ce constat, et l'étend à une véritable description sociale : " L'Histoire d'Haïti est l'histoire de l'écrasement de l'énergie individuelle par les Pouvoirs Publics - lesquels n'ont jamais voulu qu'une chose : l'éga-

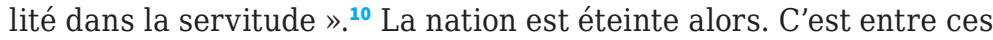
extrêmes que gravite la pensée de la nation.

Une Chercheuse va donner à lire le roman de cette complexité. Le roman va aussi mettre en évidence ce qu'on qualifie de mélancolie de Janvier : l'état de tristesse profonde qui l'affecte pendant la dernière partie de son existence.

C'est une forme de désenchantement qui prévaut quant à l'exposition de l'action nationaliste dans le roman.

Une partie du roman de Janvier peut être lue comme une étude anthropologique des conditions d'exercice d'un pouvoir dont l'objet le plus occulte, mais aussi le plus essentiel, est la menée impérialiste. Loin de s'éloigner radicalement de la topique haïtienne, il semble bien que l'auteur la mette progressivement en perspective, en l'inscrivant dans la question de l'expansion coloniale, dont Haïti est une des victimes potentielles, parmi tant d'autres. C'est aussi par là que le projet romanesque trouve aussi son véritable espace : plusieurs auteurs déclarent, en effet, que Janvier aurait dû se contenter de l'écriture de l'essai et du pamphlet, que Le Vieux Piquet, par exemple, a une stricte valeur démonstrative, et que la fiction n'ajoute rien à ce que l'on sait des conditions de vie misérables des paysans. Jean Jonassaint a bien montré, au contraire, que ce court texte est sans doute à l'origine du genre de l'« audience », et que Justin Lhérisson en est sans doute le premier héritier. Dans Une Chercheuse, il fait tomber les masques de la respectabilité, et la fiction parvient à pointer du doigt l'innommable et l'impensé de la science politique. Le roman montre une galerie de personnages liés de quelque façon au pouvoir. Ils sont rarement montrés sous un jour positif. (Chemla 2005, 27 ss.) 
Tout le roman repose sur des pratiques de décentrement et de déplacement, du moins du point de vue des personnages centraux, Mimose et Edriss. C'est même le projet romanesque qui est lui-même décentré : Haïti, dont Janvier est un défenseur infatigable, n’est traitée qu'à la marge dans le roman, mais précisément à travers l'onomastique de la galerie quasi zoologique de portraits des personnels politiques que reçoit Mimose dans le salon qu'elle tient à Paris. La disqualification de ce personnel est sans doute courante, puisqu'on la retrouve dans l'Autobiographie de Madiou, à cette époque inédite, on le rappelle. Mais en même temps, au premier niveau de la narration, ce sont quand même les personnages français qui sont montrés sous un jour sans grandeur. Ainsi, au début du roman, les conciliabules des habitantes de Bonneuil témoignent de la médiocrité d'esprit de celles-ci. La plupart des amants ou des relations, y compris d'extraction populaires, de Mimose sont ainsi disqualifiés en raison de leur étroitesse d'esprit et de leur avidité. La nation française est peu structurée, sinon par la médiocrité : que ce soit celle de la noblesse, et là encore à propos de Foncine, le mari décati de Mimose, Janvier évoque une nouvelle fois le Capitulaire de Quierzy, pour rappeler que la transmission des titres est une décision humaine, ou bien que ce soit la composante bourgeoise ou populaire, aucune ne trouve grâce aux yeux du narrateur. Le texte regorge de signes de distinctions en revanche pour le couple : dans ses vêtures, ses plaisirs, sa culture, ses manières de table. Dans le chapitre initial, la description du mobilier, des instruments de musique et surtout des bijoux hyperbolisent cette aspiration de Mimose en particulier pour l'extraordinaire et l'exception. Il y a du Monte-Cristo en Mimose : l'identité initiale s'est arasée, puis a été occultée. Mais c'est bien elle qui informe le personnage social. Le personnage d'Edriss n'est pas en reste : ce portrait du Levantin, portrait plutôt très positif, quoiqu'à la fin entraîné par une passion qui n'est pas réellement sienne, doit inciter à réfléchir sur la situation haïtienne : les Levantins installés en Haïti ne sont pas toujours traités correctement, à cette époque. Janvier montre que leur nationalisme n'est pas moins réel. Sauf qu'il se porte sur la patrie quittée. $\mathrm{Ou}$ bien, c'est aussi possible, c'est bien l'auteur lui-même qui conçoit une conception désormais variable du nationalisme.

Mais les chapitres qui racontent les atermoiements politiques français, le mépris des puissances européennes pour le droit des nations, comme pour les lois de la guerre pourtant décrétées par ces mêmes puissances, inverse la réalité : la « civilisation » dont elles assurent porter le flambeau pour éclairer le monde de leurs lumières est un leurre, qui sert de piège à la pensée des peuples traités en subalternes. La différence entre ces puissances et le despotisme oriental tient à la culture, et à la capacité à produire du savoir. Il s'ensuit une déclaration désabusée des personnages qui vivent le bombardement d'Alexandrie, Edriss et son camarade Qalb, également médecin. C'est ce dernier qui réduit l'idéal nationaliste à peu de chose : 
Tiens, regarde... continua Qalb, avec un rire amer, en montrant à Gazy le cadavre d'un soldat dont la tête avait été emportée par un biscaïen, tiens, regarde, c'est le peuple qui se bat et qui meurt... ce fétichiste !... Au fond, mon vieux Gazy, toi et moi, nous sommes aussi de grands sots. Que faisons-nous ici ? Presque rien. Toi... tu salues les boulets... et voilà. Ils nous arrivent très bien et nos artilleurs peuvent à peine riposter. (Janvier 1888, 350)

Ce composé de désinvolture, de ressentiment, de haine de soi, de colère aussi, énonce également le désaveu de l'idéologie qui a mené les deux jeunes hommes là où ils sont, l'idéal nationaliste.

Qalb en fait une analyse qui le réduit à une passion triste, sans objet littéralement, dont le cadavre du soldat décapité devient l'emblème. Le nationalisme exige des étayages puissants, notamment en matière d'éducation et de leviers sociaux. Les textes ne cessent de déplorer leur absence, au point que cette déploration est devenue un stéréotype du roman haïtien. Le reporter dans la fiction à la pénombre intellectuelle égyptienne révèle de la part de Janvier une pensée plus globale qu'il n'y paraît d'abord. La longue digression sur l'état politique de l'Égypte qui occupe le chapitre XIII intitulé «Égypte et France » révèle chez Janvier une capacité de décentrement peu commune, mais qui se rattache à une tradition identifiée chez les cadres haïtiens de la diplomatie, ${ }^{11}$ ou chez un activiste comme Bénito Sylvain (1868-1915), cadet de Janvier, chantre du panafricanisme. Janvier élargit la focale haïtienne et la ramène à son propre temps, celui des menées impériales et des colonisations de l'âge des industries. La matrice de la libération haïtienne, les moments glorieux, la Crête à Pierrot, Vertières, ne sont plus d'usage, et ne rendent pas compte d'une lecture efficace du présent.

Mais en même temps, il ne saurait lui échapper que remettre en question l'aspect fondateur de tel ou tel épisode du récit national pourrait causer du tort à sa carrière en Haïti. Le contexte politique français retient son attention, en particulier le discours du préjugé, de la part de Gambetta et de ses affidés (cf. Janvier 1888, 340). C'est toute l'organisation du monde qui est atteinte par la morsure de la guerre et du bombardement, et en particulier la notion de « civilisation » par ailleurs intégrée puissamment dans la construction rationnelle de la nation, comme Janvier le montre dans son élaboration de la notion. On connaît l'anathème de Qalb : " Ils appellent cela de la civilisation. C'est de la barbarie retournée » (Janvier 1888, 347). Voilà pour l'impérialisme des puissances colonisatrices. Mais les gouvernements égyptiens qui ont rendu la catastrophe possible, comme dans de nombreuses situations - et Janvier songeait à la Tunisie, mais aussi toute son œuvre renvoie à l'attentisme haïtien évidemment : 
Le gouvernement est pourri de préjugés ; il se défie des savants ; il déteste particulièrement ceux qui ont le malheur d'être jeunes; il hait les penseurs et les écrivains. D'ailleurs il ne lit pas. Il croit en Allah, aux derviches, à la routine, à la vieillesse et il boit de l'eau. À force d'entendre ses phrases boursouflées et vides, de constater sa nullité ou sa trahison, je suis devenu pessimiste et révolutionnaire. Il se figure que des vieillards qui n'ont jamais rien lu, ni rien étudié et qui sont incapables de réflexions profondes, ont plus d'expérience personnelle que des jeunes gens érudits qui ont étudié tous les siècles et qui, par conséquent, possèdent l'expérience accumulée de tous les pays. N'est-ce pas singulier ? (Janvier 1888, 349)

Cette réflexion peut facilement caractériser tel ou tel chef d'État haïtien, souvent au XIXème siècle un militaire sorti du rang. Ce qui faisait la gloire d'un Dessalines, esclave illettré et révolté qui faisait écrire ses proclamations par Boirond-Tonnerre n’a plus été d'actualité, passé le temps des guerres de libération.

Pour bien saisir cette courbe discursive et argumentative de la représentation de la nation à la fois comme idéal et comme acte jamais mis en œuvre, il est nécessaire de bien prendre en charge le caractère déceptif du roman chez Janvier, déjà avec Le Vieux Piquet, qui est une lodyans sur un lit de mort, une longue réprobation en même temps que la déception en regard du projet de 1804. Une Chercheuse fait de la passion nationale et nationaliste une passion triste : elle annule toute perspective de bonheur et de fondation. Elle tue jusqu'à l'espérance de la naissance. Janvier passe plus de trente ans en Europe sans doute à ressasser cette figure. ${ }^{12}$ Les seuls écrits de Louis Joseph Janvier ensuite sont destinés à présenter un projet présidentiel, qui échouera. Il n'est plus vraiment polémiste, il analyse, décrit, nomme. C'est finalement quelque chose comme l'évidence nationale qui semble peu à peu lui échapper. Dans Les Affaires d'Haïti, pour valoriser le gouvernement de Salomon, il avait écrit :

Une nation noire vivant de sa vie propre, ayant des lois particulières codifiées, une magistrature, des tribunaux, un système financier, des douanes, des journaux, un système électoral, un parlement, une littérature fille de la littérature française, tel est l'intéressant et curieux tableau qui nous est offert par la république d'Haïti. (Janvier 1886b, 96)

12 Il épouse une dame Windsor. Interrogé en privé quelques temps avant sa disparition, le poète français Ludovic Janvier, son petit-fils, m'a raconté que sa mère, la fille de Louis Joseph, se rendait souvent en Grande-Bretagne. Je n'en sais pas plus. 
On le sait, cela n’a jamais vraiment eu cours. Et le dernier avatar du nationalisme haïtien, et qui a rassemblé sur sa personne les figures de maître des corps, maître des paroles et maître des esprits, dans une pseudo-synthèse d'une pseudo-haïtianité, François Duvalier, a montré combien pouvait devenir étroite la voie d'un nationalisme d'inspiration noiriste. C'est un danger qui, de notre point de vue, n'a pas échappé à Louis Joseph Janvier, et qui l'a conduit à réserver son enthousiasme nationaliste. Et c'est dans un roman que cette révision se manifeste.

\section{Bibliographie}

\section{Fuvres de Louis-Joseph Janvier}

Janvier, L.J. (1881). Phtisie pulmonaire - Causes - Traitement préventif. Paris : A. Parent.

Janvier, L.J. (1883). Un peuple noir devant les peuples blancs (étude de politique et de sociologie comparée). La République d'Haïti et ses visiteurs 1840-1882. Réponse à M. Victor Cochinat et à quelques autres écrivains. Paris : Marpon et Flammarion.

Janvier, L.J. (1884a). Le Vieux Piquet; scène de la vie haïtienne. Paris: A. Parent. Janvier, L.J. (1884b). Haïti aux Haïtiens. Paris : A. Parent.

Janvier, L.J. (1886a). Les Constitutions d'Haitti 1801-1885. Paris : Marpon et Flammarion.

Janvier, L.J. [1885] (1973). Les Affaires d'Haïti 1883-1884. Paris : Marpon et Flammarion. [Port-au-Prince: Panorama].

Janvier, L.J. (1888). Une Chercheuse. Paris : Marpon et Flammarion.

\section{Critique}

Chemla, Y. (2003). La Question de l'autre. Matoury: Ibis rouge.

Chemla, Y. (2005). «Louis-Joseph Janvier, écrivain national », in « Lectures et écritures haïtiennes ", numéro thématique, Francofonia, 49, 7-36.

Dorsinville, M.A. (2006). Mémoires de la décolonisation. Montréal : Mémoire d'encrier.

Grégoire, Henri (abbé Grégoire) (1794). Rapport sur la nécessité et les moyens d'anéantir les patois et d'universaliser l'usage de la langue française. Suivi du décret de la Convention nationale, présenté lors de la séance du 16 prairial an II (3 juin 1794). Paris : Convention nationale.

Gusdorf, G. (1987). "Le cri de Valmy ", in "Eléments pour une théorie de la nation ", numéro thématique, Communications, 45, 117-55. https:// doi.org/10.3406/comm.1987.1671; https://www.persee.fr/doc/ comm_0588-8018_1987_num_45_1_1671.

Hibbert, F. [1910] (2013). Le Manuscrit de mon ami. Jacmel : édition de La Dodine (en feuilleton dans le quotidien Le Matin). Format ePub.

Las Cases, E. de (1842). Mémorial de Sainte-Hélène. Paris : Ernest Bourdin Éditeur. 
Madiou, Th. (1847). Histoire d'Haïti, tome 2. Port-au-Prince : Imprimerie de Jh Courtois.

Madiou, Th. [2017] (s.d.). Autobiographie. Port-au-Prince : Éditions Henri Deschamps.

Renan, E. (1882). Qu'est-ce qu'une nation ? = Conférence faite en Sorbonne, le 1 mars 1882. Paris : Calmann Lévy.

Robespierre, Maximilien de (1794). Rapport sur les principes de morale politique qui doivent guider la Convention nationale dans l'administration intérieure de la République, fait au nom du Comité de salut public, le 18 pluviôse, l'an $2 e$ de la République, imprimé par ordre de la Convention nationale (18 pluviôse an II-5 février 1794). https://ihrf.univ-paris1.fr/enseignement/ outils-et-materiaux-pedagogiques/textes-et-sources-surla-revolution-francaise/robespierre-discours-du-18-pluviose-an-ii/.

Thiesse A.-M. (1991-2001). La Création des identités nationales. Europe XVIIIeXIXe siècle. Paris : Éditions du Seuil.

Vastey, Baron de (1814). Le Système colonial dévoilé. Cap-Henry : P. Roux imprimeur du Roi. 


\title{
Sur les pas de Louis-Philippe Dalembert. Un hommage à la carrière du " gavroche caraibe »
}

\author{
Silvia Boraso \\ Università Ca' Foscari Venezia, Italia
}

\begin{abstract}
In 1982, the Haitian writer Louis-Philippe Dalembert published his first collection of poetry, Évangile pour les miens. It was the beginning of a prolific, multiform, and successful career. During the next thirty years, he has published four other poetry collections, four collections of short stories, ten novels, and numerous essays. Intended as a tribute to Dalembert's literary work, this article will try to describe the evolution of his production in verse and prose. In particular, three recurrent themes will be discussed: 1) the elaboration in his first texts of a system of rememorating strategies that will lead to the formulation of the notion of 'pays-temps'; 2) the use of an urban setting, namely the borough, to convey the collective values of the community; 3) the birth, in his late publications, of a universal poetics transcending any type of border.
\end{abstract}

Keywords Louis-Philippe Dalembert. Haitian literature. Vagabondage. Migrant literature. Universal poetics.

Sommaire 1 Introduction. -2 « on my mind haiti ». -3 Le quartier comme nombril du monde. -4 Le texte : une passerelle vers l'Autre. -5 Conclusion.

$\begin{array}{lll} & \text { Peer review } \\ & \text { Submitted } & 2020-06-28 \\ \text { Edizioni } & \text { Accepted } & 2020-08-07 \\ \text { Ca'Foscari } & \text { Published } & 2020-12-22 \\ & \text { Open access } & \end{array}$

(C) 2020 | (a) Creative Commons Attribution 4.0 International Public License 


\author{
étranger \\ des lieux de l'homme \\ je cherche le temps \\ qui pourrait \\ m'habiter \\ l'autre rive du rêve \\ (Dalembert 2000, 54)
}

\title{
1 Introduction
}

C'est dans une petite île de la Caraïbe de 76.192 km², plus précisément dans cette partie occidentale qui est devenue, en 1804, la République d'Haïti, que commence le long voyage de l'écrivain Louis-Philippe Dalembert. Né à Port-au-Prince en 1962, Dalembert quitte bientôt son pays natal pour se vouer à un vagabondage incessant qui l'emmènera au fil des années aux quatre coins du monde : à Paris d’abord, puis à Rome, à Jérusalem, en Amérique latine et en Afrique méditerranéenne ensuite, enfin de nouveau à Paris, sans compter ses nombreux retours en Haïti intercalés entre un déplacement et l'autre. Saturée de cette constante pérégrination, sa production en vers et en prose restitue sur la page, notamment dans la variété de points de vue et dans l'hétérogénéité de formes et de contenus, les expériences disparates vécues par l'auteur sur son chemin. L'imaginaire qui s'ensuit est constitué par une série de paysages qui s'enchaînent, peuplés de gens de cultures et d'ethnies composites habitant une dimension spatio-temporelle floue située entre l'ici et l'ailleurs, le temps présent et le temps passé. Un univers littéraire que Dalembert a parfait pendant toute sa carrière, commencée il y a presque quarante ans par la publication, en Haïti, de son premier recueil de poèmes, Évangile pour les miens (1982). Depuis lors, son parcours d'écrivain a été aussi riche qu'éclectique : quatre autres recueils de poésie paraîtront, suivis par quatre recueils de nouvelles, dix romans - dont le dernier, Mur Méditerranée (2019) a reçu, entre autres, le Prix de la langue française octroyé par l'Académie française -, un roman-document et un récit, auxquels il faut ajouter les nombreux textes publiés à l'intérieur d'ouvrages collectifs. ${ }^{1}$ Cet article se propose de retracer le parcours littéraire de l'auteur en essayant de décrire l'évolution de son œuvre depuis ses débuts jusqu'à nos jours. En particulier, trois étapes seront identifiées : 1) la création, à partir de la thématique

1 Pour une liste complète, voir la Bibliographie. Bien que cet article ne soit voué qu'à la production littéraire de Dalembert, il faut accorder ici une mention spéciale à sa contribution à la théorisation et à la diffusion de la culture et de la littérature caraïbes, dont témoignent ses nombreux essais, collaborations et directions d'ouvrages spécialisés. Pour des approfondissements, consulter sa notice biobibliographique sur le site île en Ille: http://ile-en-ile.org/dalembert/. 
du retour au pays natal, d'un dispositif de la mémoire qui amènera Dalembert à la formulation du concept de pays-temps ; 2) la mise en récit, dans un scénario urbain porteur des valeurs de la communauté, des destins individuels qui y prennent racine ; 3) les trajectoires spatio-temporelles de la migration comme représentation d'une poétique de l'universel au-delà de toute frontière.

\section{2 « on my mind haiti »}

Pour comprendre le parcours littéraire de Louis-Philippe Dalembert, il faut revenir à la case départ, en Haïti, ce « tout petit pays | qui a planté sa gueule | [...] dans la chair de la mer caraïbe » (Dalembert 2017b, 9-10). Que ce soit sous le pseudonyme de Salbounda - pays imaginaire dont la capitale, Port-aux-Crasses, s'apparente beaucoup à la ville de Port-au-Prince et constitue le scénario privilégié des premiers romans et contes de l'écrivain $-^{2}$ ou qu'elle soit à peine évoquée dans le récit, ${ }^{3}$ Haïti demeure une présence constante à l'intérieur de ses œuvres. N'ayant pas été obligé, contrairement à la plupart des écrivains haïtiens de la diaspora, de s'enfuir de son pays, Dalembert quitte Haïti le cœur léger de qui peut toujours décider d’y revenir, mais sans jamais envisager pour autant la possibilité d'un retour définitif. Bien que volontaire, ce choix entraîne pourtant, par son caractère inéluctable, un inexorable sentiment de nostalgie qui se traduit, dans la vie de l'auteur, par de nombreux retours au pays natal et, dans sa production en vers et en prose, par la permanence de l'île en tant que lieu physique et allégorique à la fois :

on ne laisse pas ce pays

ni même ne s'en va

de cette terre

de cette femme

sortir peut-être

et encore

(Dalembert 2017b, 103-4)

2 À titre d'exemple, on peut nommer les deux premiers romans, Le Crayon du bon Dieu n'a pas de gomme (1996) et L'autre face de la mer (1998) ainsi que les contes « Délices port-aux-crassiennes », «Macaronade » et «Le songe d'une photo d'enfance » (Dalembert 1993).

3 Même quand les histoires se déroulent ailleurs, on peut toujours relever la présence de la Caraïbe dans le récit : Jean, jeune narrateur de Rue du Faubourg Saint-Denis (2005) qui vit à Paris, et Azaka, protagoniste de Ballade d'un amour inachevé (2013) qui s'est installé dans les Abruzzes en Italie, partagent tous les deux des origines caribéennes; avant de se rendre en Afrique, Laurent, l'un des personnages de Noires blessures (2011), séjourne brièvement en Haïti dans le cadre d'un projet humanitaire. 
Lointaine dans l'espace et dans le temps, Haïti devient la dépositaire au sens large de la période heureuse de l'enfance, cette époque bénie que Dalembert a vécu dans « l'émerveillement et un certain nombre de manques » (Chemla 2011, 386) et qui exerce depuis toujours une fascination inaltérable bâtie sur la tension « entre l'ici et le là-bas, [...] entre aujourd'hui et hier » (386). Ce décalage constituerait l'indice majeur de l'impossibilité de réhabiter les lieux de l'existence passée qui serait, selon l'auteur, une enfilade d'étapes successives - les " pays-temps » $-{ }^{4}$ qui ne peuvent être vécues qu'une seule fois. Ce qui n'empêche pas pourtant la plupart de ses personnages, aussi bien ceux de sa poésie que de sa prose, de se livrer à une recherche assidue de ce passé qu'ils sont censés avoir perdu pour toujours. On assiste alors à une alternance d'allers et de retours les ramenant aux endroits familiers de leur enfance, circonscrits en grande partie à un milieu ouvertement caribéen, dont la récurrence à l'intérieur de l'œuvre dalembertienne est le témoignage le plus frappant de la volonté, voire de la nécessité de la part de l'auteur de dire sa terre natale. ${ }^{5}$

Cette récursivité a viré, au fur et à mesure que l'auteur ainsi que ses personnages s'éloignaient de l'île, vers une évocation plus discrète dépassant le binôme " pays natal/pays d'accueil » tant d'un point de vue idéologique que d'un point de vue narratif (Pessini 2012, 156). En effet, Haïti a longtemps coïncidé avec le pays, au sens littéral, et le temps, au sens figuré, de la mémoire, en se situant au centre d'une oscillation temporelle entre le passé et le présent, oscillation qui est devenue l'une des marques distinctives de l'esthétique dalembertienne. Caractérisée par un emploi massif du flashback, sa production romanesque est constellée de récits analeptiques qui constituent souvent les piliers sur lesquels l'entière architecture narrative est axée. À ce « regard rétrospectif » dans le temps (Gannier 2018, 91) s’accompagne aussi un mouvement parallèle dans l'espace mimant le retour en arrière des personnages. C'est le cas, par exemple, du protagoniste du Crayon qui rentre à Salbounda après des longues années d'exil à la recherche des souvenirs de son enfance.$^{6}$ Cependant, ce repli sur le passé s'avère bientôt décevant lorsqu'il découvre que la réalité présente ne correspond plus à la topographie retenue par sa mémoire :

4 Notoire est la définition de « pays-temps » par laquelle son premier roman se clôt : « tu recherches un autre pays. Celui que dans tes pérégrinations en terre étrangère tu as nommé le pays-temps. Celui qu'on n'habite jamais qu'une seule fois. Comme le fleuve d'Héraclite. Cette terre d'au-delà des races et des nationalités » (Dalembert 1996, 271). Pour des approfondissements, voir Gannier 2018 et Ghinelli 2005.

5 Sur l'analyse de ce thème dans les premiers romans de Dalembert, voir Pessini 2018, 21-2.

6 De même, dans Autre, Grannie se remémore son passé lorsqu'elle se rapproche du bord de mer, où elle s'attardait, enfant, à regarder l'océan, à cette époque-là synonyme d'espoir et maintenant, à ses yeux de vieille femme désabusée, source de chagrin. 
L'homme n'osa pas pénétrer dans la cour de l'ancienne maison familiale qui, vue de l'extérieur, lui parut ridiculement petite, alors que ses souvenirs en faisaient une citadelle. Tout aussi insignifiante la véranda, qui exigeait des efforts titanesques de ses frêles bras rien que pour se hisser sur une balustrade d'environ un mètre cinquante de haut. Si ce n'était que ça. En fait, elle lui semblait une vulgaire galerie d'une de ces constructions sauve-qui-peut du tiers monde. Mais, se dit l'homme pour se consoler, peut-être gardait-elle les échos de ses jeux, des parties de foot à quatre, des empoignades féroces qui s'ensuivaient, des tentatives de réconciliation, pleines d'orgueil et de naïveté. Elle fut la seconde déception sur le chemin de la mémoire, après la profonde désolation ressentie en se promenant dans les rues de Port-aux-Crasses, sa ville natale. (Crayon, 1996, 15-16)

Une fois constaté que le retour physique à Salbounda, c'est-à-dire Haïti, ne donne pas accès au pays-temps de l'enfance et s'avère donc

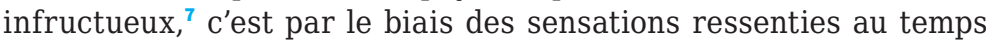
présent, souvent déclenchées par des évènements particuliers ramenant à la surface des souvenirs qu'ils croyaient ensevelis pour toujours, ${ }^{8}$ que les personnages parviennent à récupérer des fragments de leur passé.

Si avant l'accent était mis sur le topos du retour aux lieux de l'enfance, qui étaient pour l'auteur ceux de Port-au-Prince, à un certain moment Dalembert semble se détacher de sa terre natale; il commence à centrer plutôt sa production romanesque sur la présentation narrative des événements, en accordant une attention particulière au champ temporel du récit premier. À partir de Rue - le premier roman qui coupe net avec l'ambiance caribéenne - l'écrivain développe effectivement un modèle narratif qui, tout en faisant preuve d'une créativité remarquable, propose toujours un récit-cadre situé dans le hic et nunc de l'histoire. Les personnages sont présentés aux lecteurs

7 Dalembert décrit ainsi l'expérience du retour : «Ce décalage entre le réel et ce que notre mémoire en a retenu semble encore plus profond quand on rentre d'un long départ. Au fond, c'est notre regard qui a changé. [...] [O]n n’habite pas un espace géographique, un pays, une ville, mais le Temps. Ce Temps est fait de pays successifs qu'on n'habite jamais qu'une fois, et seul » (Ghinelli 2005, 124-5).

8 C'est ce qui arrive, par exemple, au protagoniste des Dieux voyagent la nuit (2006). Après avoir assisté à un manger-les-anges à New York, les souvenirs liés à ses expériences enfantines du vaudou lui reviennent à son esprit d'adulte. De même, dans Ballade, Azaka se souvient soudainement de sa précédente mésaventure sous les débris lorsqu'il assiste à un nouveau tremblement de terre. Si les procédés qui donnent accès à la remémoration du passé ne changent pas, ce qui varie, par contre, est la nature des épisodes qui remontent ainsi à la surface : souvent, ces derniers sont liés à des moments de bonheur, mais parfois ils peuvent aussi cacher, comme c'est le cas pour Azaka, des traumatismes dont l'individu a essayé de se débarrasser (voir Borst 2009, 3). 
dans leur état présent qui est, quand ce n'est pas celui d'un vagabond sur la terre, tout du moins loin d'être statique. ${ }^{9}$ Cet ancrage du récit aux circonstances actuelles de l'action permet à l'auteur de camper une puissante alternance présent-passé qui deviendra la marque de fabrique de son style. D'un côté, cette plongée cadencée dans la mémoire confère aux personnages, souvent éloignés de leurs origines, une conscience majeure de leur identité culturelle (c'est le cas, par exemple, du protagoniste des Dieux, qui s'aperçoit, à travers les souvenirs évoqués par ses expériences présentes, de l'importance du vodou dans son passé). De l'autre côté, cette oscillation, que Dalembert structure magistralement, détermine le déclenchement du processus herméneutique qui consent au public de comprendre les comportements des personnages aujourd'hui par la connaissance de ce qui leur est arrivé hier. ${ }^{10}$

Encore, dans les textes plus récents on peut détecter un glissement en avant au niveau de l'histoire racontée : l'action s'étale dans un mouvement progressif qui projette déjà les personnages dans leur futur, tandis qu'avant on assistait souvent à une régression vers leur terre natale. On peut repérer un ultime exemple de cette stratégie narrative dans le dernier roman de l'auteur, Mur, où le passé des trois protagonistes scande leur trajet de la Libye à Lampedusa, le récit se faisant une véritable passerelle entre ce qu'elles ont laissé derrière elles et ce qui les attend à l'avenir. Ce que l'auteur propose est encore une fois un " voyage dans le temps ", à travers un " monde dont les dimensions s'étirent » (Pessini 2018, 34), impliquant toujours à la base l'idée de départ. Géographiquement, l'endroit d'où les trois protagonistes de Mur viennent perd de son importance - non d'un point de vue culturel mais d'un point de vue strictement topographique ; ce qui compte est que ces femmes - comme d'ailleurs la totalité des personnages dalembertiens, divisés entre ceux qui sont partis et ceux qui ont toujours rêvé de le faire (Cacchioli 2018, 51) -, se déplacent constamment, même après avoir atteint leur destination initiale. Les vagabondages de ces gens - de nationalités, langues, religions et cultures disparates dont les chemins s'entrecroisent - ${ }^{11}$ partagent tous, quelle que soit la motivation qui les a poussés à l'errance, le même caractère délibéré, leur départ étant le résultat d'un

9 Pour n'en rester qu'à Jean, le narrateur de Rue, il est présenté en fugitif essayant d'échapper aux policiers dans les rues de Paris.

10 Les raisons d'agir de Laurent dans Noires ne s'expliquent qu'à la lumière de ce qui lui est arrivé dans son enfance; dans Ballade, l'apparemment incompréhensible peur du noir d'Azaka est après justifiée par le fait qu'il a passé, lorsqu'il était petit, des jours entiers enseveli sous les décombres avant d'être sauvé par les secouristes.

11 Cette multiplicité, qui caractérise tous les textes de l'écrivain et qui se traduit par autant de pluralités d'écriture, insère son ouvrage dans le sillage de l'« écriture nomade » (Famin 2011, 177). 
libre choix. ${ }^{12}$ C'est le même choix que Dalembert a fait lorsqu'il a laissé Haïti pour ne jamais y revenir et rester, départ qui a scellé pour toujours la séparation physique et métaphorique de l'auteur d'avec sa terre natale (Ghinelli 2005, 128). Depuis lors, son regard s'est posé sur une multiplicité de lieux et de gens différents, dont les influences se sont ajoutées à sa sensibilité caribéenne. L’acquisition de cette subjectivité composite par laquelle l'écrivain parvient à interpréter passé et présent, ici et ailleurs, se traduit dans ses œuvres par la reproduction de « cartographies sensorielles » bariolées qui se projettent, à travers un caléidoscope de points de vues différents, sur les paysages et les communautés de son imaginaire (Marinho 2018, 182).

\section{Le quartier comme nombril du monde}

Les communautés décrites dans les textes de Dalembert - à l'intérieur desquelles on voit une nuée de personnages interagir entre eux - ont toujours ce caractère populaire que l'on ne peut que comparer à celui du quartier de Bel Air dont l'auteur est originaire et dont il a cherché la couleur locale dans tous ses déplacements successifs. ${ }^{13}$ Que ce soit au bord de la mer caraïbe (Crayon, Autre) ou dans le centre d'une ville cosmopolite comme New York ou Paris (Dieux, Rue), dans ses romans et dans ses contes le quartier constitue le carrefour du monde où tout se passe. Il fonctionne en tant que microcosme qui abrite souvent, au milieu d'une masse d'individus bizarres, l'enfance des jeunes protagonistes dans une représentation urbaine qui prend des teintes de rêve et de cauchemars à la fois (Pessini 2012, 294). Si les conditions économiques de ces endroits, où normalement les couches sociales les moins aisées résident, ne permettent pas à Dalembert d'en camper un portrait idyllique, c'est néanmoins à l'intérieur de cet amalgame d'êtres humains désabusés mais solidaires qui habitent ces lieux que l'on assiste à l'intégration de l'individu :

Tous ces gens se côtoient, se méprisent, fraternisent, se brassent allègrement, au hasard du feu sanguin ou des comptes bancaires, communient d'un seul élan autour du Wharf, poteau-mitan de ralliement, leur conscience à eux Salboundais de première, deuxième, troisième génération, car peu d'entre eux peuvent revendiquer d’avoir été là depuis toujours, d’avoir été présents au baptême de

12 Dalembert met souvent en avant cet aspect : ses personnages « 'sont partis comme ça, pour partir...' Par choix. Sans qu'ils aient été poussés par qui que ce soit, ni par quoi que ce soit » (Ghinelli 2005, 127).

13 Sur le sujet, voir son interview pour Île en Île au lien : https://www.youtube.com/ watch?v=-legTclkeUw. 
cette terre, hormis peut-être les Zambos des montagnes du Bahoruco, ces nègres aux yeux bridés et aux pommettes saillantes, métis de Karibs et d'Africains. (Crayon, 1996, 62)

Cette « faune », qui englobe l'individu dans un élan de solidarité humaine surmontant n'importe quelle barrière socio-culturelle, est le seul véritable ancrage à la communauté sociale qui reste pour les vagabonds de l'univers dalembertien. Ces quartiers de la ville caribéenne font écho, entre autres, au Faubourg Saint-Denis, le quartier parisien « [b]ruyant et coloré » $(R u e, 10)$ où « Arabes », «Yougos de Sangatte » et «Blacks » (17) se côtoient et dont la multiethnicité lui a valu le sobriquet moqueur d'Onu, ainsi qu'au petit cercle d'étrangers qu'Azaka et sa femme ont formé à Cipolle, le village italien où ils vivent (Ballade).

Lorsqu'ils abandonnent l'appui réconfortant que la communauté leur offre, les personnages perdent l'un des points de repère de leur existence. Dans leur errance ils éprouvent ce sentiment de nostalgie typique de qui a quitté sa terre natale ainsi qu'une sensation constante d'isolement, auquel s'accompagne un sentiment « d'abandon, de trahison du vieux rêve de révolution lié au lieu/temps natal » (Ghinelli 2005, 129). Si Dalembert dépeint, par ses choix toujours autodéterminés, un prototype de héros libre, cet « étranger en marche sur la terre » est pourtant « drapé dans sa solitude » (Dalembert 2017b, 13), l'obligeant à rechercher constamment un nouveau recoin où s'implanter. Qu'ils retournent au quartier de leur enfance, comme le fait le protagoniste du Crayon, ou qu'ils s'acclimatent dans l'ambiance cosmopolite de la ville nouvelle, comme c'est le cas pour les étrangers du Faubourg Saint-Denis (Rue), les gens qui peuplent le récit sont tous à la poursuite de relations humaines qui puissent les rapprocher à la fois de leur terre (natale et d'accueil) et de leur identité. C'est seulement dans ces liens qui recréent un réseau social accueillant, similaire à celui de leur enfance, que les individualités des personnages - et de l'auteur - parviennent à retrouver leur place dans le monde :

un homme a tendu la main

un homme sans l'étoffe d'un héros

un homme ni tout à fait toi

ni tout à fait moi

[...]

il a tendu sa main

humaine

changé les nuages de l'hiver

en un gros soleil d'été

et d'un poing rageur

a gommé les frontières

(Dalembert 2017b, 35-6) 
Cet accent posé sur la solidarité entre les hommes peut être décelé aussi dans les œuvres en prose. Pour n'en prendre qu'un exemple, c'est par l'intervention de deux personnages en particulier qu'Azaka, bien que loin de son pays et de sa famille, s'intègre dans la petite communauté des Abruzzes italiens d'où sa femme est originaire. Ce qui ne signifie pas que ce processus d'inclusion soit immédiat et encore moins évident. Pour que cela advienne, en effet, l'esprit d'initiative de l'étranger, pourtant nécessaire, ne suffit pas et c'est toujours par l'intermédiaire d'un membre de la communauté qu'il peut avoir accès au cercle restreint des locaux. Pour Azaka, cet agent médiateur est constitué dans un premier temps par la figure du patron et puis par celle de sa femme, deux figures qui l'aident à entrer respectivement dans la vie publique et dans la vie privée de Cipolle.

Expatriés caribéens vivant à Paris, haïtiens installés à New York, noirs intégrés dans les communautés montagnardes de l'Italie, encore juifs d'origines polonaises émigrés en Haïti, tous contribuent à former cette « mosaïque » (Vignoli 2016, 30) d’identités hétéroclites que Dalembert fait se croiser et se recroiser dans un mélange de chemins qui s'enchevêtrent. Ce qui compte alors, ce n'est plus tant le lieu où ils atterrissent, mais la rencontre, l'échange humain comme nouveau point de départ fournissant à l'individu l'occasion de s'intégrer à l'intérieur de sa nouvelle réalité. Sans, bien évidemment, gommer ses origines ! Dans sa vie comme dans ses œuvres, Dalembert a opté pour un modèle d'intégration culturelle malléable, que l'on pourrait décrire, empruntant la métaphore de l’arbre caraïbe proposée par Alba Pessini, comme caractérisé par « une identité caraïbe mobile, changeante mais, malgré tout, forte. Ce n'est donc pas la solidité des racines qui est mise en avant mais le bruissement des branches » $(2012,289)$. Le quartier animé de Port-aux-Crasses se voit donc substitué par une constellation de faubourgs disséminés dans le monde qui évoluent avec les déplacements des personnages, mais qui rappellent toujours dans leur vivacité la saveur du bord de mer. ${ }^{14}$ Ces renvois caribéens se retrouvent dans toute la production littéraire de l'auteur et se concrétisent dans la présence d'images récurrentes comme celles, entre autres, de l'île et de l'eau. Que ce soit l'île de la Tortue (L'Île du bout de rêve, 2003), l'Île de France (Rue) ou l'île de Lampedusa (Mur), ${ }^{15}$ l'île a toujours

14 Cet éloignement du décor caribéen est un choix esthétique et idéologique à la fois, comme l'affirme Dalembert dans un entretien : " pour moi, cette saturation du paysage haïtien dans les œuvres, relève de cette longue tradition de littérature engagée, plus largement de la littérature politique, dans lesquelles s'inscrit la littérature haïtienne. L'Île du bout des rêves répond alors à un projet : écrire au-delà de cette saturation. Rue du Faubourg Saint-Denis prend en charge aussi bien la langue que le paysage géographique et humain, qui n'ont rien à voir avec Haïti. Cela rend alors possible la présence d'Haïti de manière moins obsessionnelle, avec moins de pathos » (Chemla 2011, 385-6).

15 Sur le thème de l'insularité, voir Pessini 2008 et Famin 2011. 
joué un rôle central dans la poétique de l'auteur, provoquant, par les promesses tacites évoqués par ses rives, un désir presque irrésistible d'horizons nouveaux. Pour les rejoindre, les protagonistes doivent se confronter à des étendues d'eau séduisantes et effrayantes à la fois. Si au début de sa carrière d'écrivain cette mer, qui ne pouvait qu'être la mer Caraïbe, offrait un éventail de possibilités infinies, ses romans étant principalement axés sur ce qui précédait « l’appareillage définitif vers l'ailleurs » (Pessini 2012, 21), dans sa production plus récente, elle s'érige en obstacle à surmonter avec un récit qui se focalise surtout sur ce qui arrive aux personnages après leur départ. Les multiples trajectoires que ces derniers dessinent dans leurs pérégrinations à travers l'Atlantique (Avant que les ombres s'effacent, 2017) et la Méditerranée (Mur) marquent le passage du départ à l'étape successive, qui n'est pourtant jamais le point d'arrivée définitif.

\section{$4 \quad$ Le texte : une passerelle vers l'Autre}

Cette perpétuelle errance des personnages reflète le vagabondage sans fin de l'auteur et a donné lieu, dans sa production littéraire, à un complexe enchevêtrement de références intertextuelles provenant de sources et de cultures diverses qui vont de la Bible aux chefsd'œuvre de la littérature mondiale ainsi qu'au folklore caraïbe, mais pas seulement. ${ }^{16}$ Ces influences ont toujours joué un rôle de premier plan dans le texte dalembertien - notamment dans l'emploi récurrent de l'épigraphe - mais c'est avec Rue que l'écrivain parvient à mettre en place un véritable dialogue entre son récit à lui et $L a$ vie devant soi de Romain Gary (Étienne 2018). Ce roman marque aussi pour Dalembert une sorte de fracture avec ses œuvres précédentes. Outre l'aspect ouvertement interactionnel, en effet, Rue introduit pour la première fois dans l'imaginaire de l'auteur un décor autre que caribéen, en étalant un ensemble bariolé de cultures, langues et ethnies diverses visant à briser les stéréotypes liés au concept universel d'intégration (Étienne 2018, 147-8). Pour ce faire, l'auteur confère à Jean, le jeune narrateur homodiégétique du récit, la maîtrise d'un langage hétéroclite qui enchaîne et combine par sa créativité les différentes voix résonnant dans le faubourg :

Dalembert [...] confère à son personnage une langue à la fois décalée et réaliste, parfois brutale dans ses assertions, mais qui rend possible de dire les oppositions et les confrontations, une langue

16 Parmi les citations savantes de l'île Dalembert inclut, par exemple, quelques paroles d'une chanson des Nomadi, groupe musical très connu en Italie dont le nom, « nomades », renvoie significativement à l'idée de vagabondage qui lui est chère. 
rapide et souple qui parvient à épouser les contours de l'émotion [...] à dire le monde avec beaucoup d’acuité. (Chemla 2005)

Ce monde se retrouve unifié dans l'espace textuel où le français abrite, sans aucune dissonance, les mots de l'italien, de l'espagnol, de l'hébreu, de l'arabe et de l'allemand, dans un retentissement de langages qui fait écho aux voyages de l'auteur autour du globe : " Ma quête, c'est d'essayer de renouveler la langue, de faire en sorte, si possible, que chaque langue corresponde à une histoire donnée » (Gyssels, Cooreman 2008, 102). ${ }^{17}$ Dans ses ouvrages, cela se traduit par un « vagabondage langagier [...] simultanément diatopique, diachronique et diastratique » qui reflète la « nature déambulatoire » des personnages (Marinho 2018, 186) et qui amuse le lecteur par sa vivacité.

Cette langue - comme la rue, d'ailleurs - se fait alors le lieu privilégié qui réunit toutes les expressions de la multiplicité et qui renvoie à une certaine idée de «permanence » (Théodat 2018, 115). Ultime expression de l'interaction humaine, elle cimente les liens entre les individus et mène à bien tout processus d'enracinement. Par exemple, dans Ballade, l'intégration d'Azaka ne s'achève que parce qu'il maîtrise bien l'italien ; dans Avant, le docteur Ruben commence son assimilation à la population haïtienne par sa connaissance du français, qu'il a appris en lisant significativement De l'égalité des races humaines de l'essayiste haïtien Anténor Firmin, mais il l'accomplit définitivement seulement lorsqu'il arrive à parler le créole qui lui permet d'avoir accès à une communauté plus élargie que celle de l'élite. À ces expériences positives et réussies, Dalembert oppose des épisodes brutaux de déracinement qui ont souvent affaire à des conditions de voyage misérables, à la limite de la décence humaine, où l'aspect de la diversité linguistique n'est jamais mis de côté. Dans Mur (mais cela vaut également pour l'Autre), la barrière de la langue contribue à la déshumanisation des migrants formant cette masse anonyme et muette de silhouettes recroquevillées les unes sur les autres. Réduits à l'état de bétail, ne montrant plus aucun signe de solidarité humaine, séparés par la méfiance et incapables de communiquer dans ce baragouin incompréhensible de langages différents, les

17 Cette réponse constitue une rare exception, car Dalembert a toujours été réfractaire à tout classement et questionnement machinal sur la langue, souvent de routine pour les écrivains francophones. En se figurant la langue française en tant qu'une « damoiselle inaccessible », la contribution piquante qu'il a publié en 2006 sur Libération traduit bien son sentiment: "J'aime à considérer que notre rapport ne regarde que toi et moi. Qu'à vouloir ou devoir trop souvent le légitimer, on passe à côté de l'essentiel. Qu'au moment où on se donne l'un à l'autre, dans l'intimité de nos joies et de nos peines, tu es de moi et moi de toi. Cela seul compte. Mais ce regard de l'autre, interrogeant nos gestes. Sondant l'intensité de tes orgasmes. Son voyeurisme tenace qui, à trop se fixer sur nos attouchements, délaisse, ô paradoxe, le fruit de ces amours. J'aimerais tant qu'on nous foute la paix quand on baise ! » (2006b). 
hommes et les femmes enfermés dans la cale retrouvent une partie de leur humanité lorsqu'ils s'unissent contre la milice qui contrôle le bateau. C'est la parole qui permet de recréer une sorte d'espace politique commun (Théodat 2018, 123) à travers le chant de Chochana et le discours de Semhar, protagonistes du roman, ${ }^{18}$ qui réveillent dans leurs esprits la dignité qu'ils avaient perdue.

Construit sur une "poétique de l'hybridité des langues et des genres » (Vignoli 2016, 29) qui a été rapprochée de l'enracinerrance postulée par Jean-Claude Charles et qui « ne connaît pas la soustraction, mais l'addition » (2001), le texte dalembertien se fait une passerelle sur le monde qui relie non seulement l'ici et l'ailleurs, le passé et le présent, mais aussi mon identité à moi et celle de l'Autre. En réunissant dans un ensemble cohérent ces différents plans de la diversité, l'imaginaire qui en résulte reproduit au niveau littéraire la « synthèse » culturelle vécue au niveau biographique par l'auteur :

Le but n'est pas de faire étalage d'une quelconque maîtrise des langues, mais de dire ce que je suis. Sans tricher. En ce sens, on peut parler d'une synthèse qui s'est faite en moi : Synthèse entre les voyages plus ou moins longs, ce que je nomme « mon vagabondage »; entre les différents lieux où j’ai vécu : Port-au-Prince, Paris, Rome, Jérusalem, qui m’habitent encore lors même que je les ai laissés. Synthèse entre l'enfant, l'adolescent que j'ai été et l'adulte que je suis devenu. Entre le croyant d'hier, élevé dans le respect strict du sabbat, et l'agnostique d'aujourd'hui. (Snoussi 2003)

De ces étapes qui se superposent mais qui ne s'excluent pas réciproquement dérive la nécessité chez Dalembert de représenter une certaine idée de l'universel, qui se traduit dans ses dernières œuvres par une urgence mettant l'auteur au service de la société humaine. Le texte littéraire constitue alors pour l'écrivain l'occasion pour tendre sa main vers l'Autre au-delà de toute frontière :

Ce qui importe pour moi c'est une certaine transversalité. C'est de trouver des passerelles d'une culture à une autre, d'une histoire à une autre, le lieu où les êtres humains peuvent se rencontrer sans être pour autant concurrents, où homme et femme peuvent se rencontrer sans être concurrents. Comme le soulignait Fanon, quand on dit du mal des Juifs, quelque part on dit aussi du mal des Noirs. (Gyssels, Cooreman 2008, 104)

18 Ce n'est pas par hasard que les protagonistes, dont l'exceptionnalité leur permet de raccommoder les liens interrelationnels que les conditions minables des migrants ont brisés, sont polyglottes et n'ont aucun souci à communiquer entre elles. 
Ce côté engagé de l'auteur s'est reversé et dans sa prose et dans ses vers. Des débris de Sarajevo à la guerre libanaise (En marche sur la terre, 2017), du Kouto- - le massacre des Haïtiens en terre dominicaine ordonné par le gouvernement Trujillo (Autre) - à la révolution cubaine (Le roman de Cuba, 2009), de la traite des esclaves (Autre) au massacre des Juifs (Avant), en passant par la dénonciation de la pauvreté qui touche les quartiers populaires (Crayon, Autre, Rue) et des conditions terribles que les migrants doivent endurer (Mur), Dalembert a su décrire les plaies affligeant l'humanité tant à l'échelle locale qu'à l'échelle globale, se faisant ainsi le porte-voix des humbles de la terre.

\section{Conclusion}

Dans cette contribution nous avons essayé de retracer l'itinérance physique et poétique de Louis-Philippe Dalembert, qui a acquis dans le temps une dimension planétaire (Marinho 2018, 191) et qui a amené à une évolution considérable sa production littéraire. En partant de l'analyse des lieux clés de son imaginaire - d'abord Haïti, pays et temps à la fois, habité par le souvenir d'une enfance heureuse, ensuite les quartiers du monde, ces microcosmes où les individus interagissent au-delà de leur diversité - cette réflexion s'est par la suite concentrée sur l'universalité de l'écriture dalembertienne, qui est passée d'une dimension géographiquement circonscrite à une ouverture complète sur le monde. D'où cette poétique du vagabondage, étrangère à toute idée de frontière et qui a conféré à l'auteur, éternel « gavroche caraïbe », le rôle de «passeur d'imaginaires » cosmiques (Marinho 2018, 179).

\section{Bibliographie}

\section{Fuvres de Louis-Philippe Dalembert}

Dalembert, L.P. (1982). Évangile pour les miens. Port-au-Prince : Choucoune.

Dalembert, L.P. (1989). Et le soleil se souvient (suivi de) Pages cendres et palmes d'aube. Paris: L'Harmattan.

Dalembert, L.P. (1993). Le songe d'une photo d'enfance. Paris : Le Serpent à Plumes Éditions.

Dalembert, L.P. (1996). Le Crayon du bon Dieu n'a pas de gomme. Paris : Éditions Stock.

Dalembert, L.P. (1998). L'autre face de la mer. Paris : Éditions Stock.

Dalembert, L.P. (2000). Ces îles de plein sel, et autres poèmes. Yvry-sur-Seine : Silex; Nouvelles du Sud.

Dalembert, L.P. (2003). L'île du bout des rêves. Paris : Bibliophane-Daniel Radford. 
Dalembert, L.P. (2005a). Poème pour accompagner l'absence. Montréal : Mémoire d'encrier.

Dalembert, L.P. (2005b). Rue du Faubourg Saint-Denis. Monaco : Éditions du Rocher.

Dalembert, L.P. (2006a). Les dieux voyagent la nuit. Monaco : Éditions du Rocher.

Dalembert, L.P. (2006b). "Une damoiselle inaccessible». https://www.liberation.fr/hors-serie/2006/03/16/-une-damoiselle-inaccessible-_32960.

Dalembert, L.P. (2007). Histoires d'amour impossibles... ou presque. Monaco : Éditions du Rocher.

Dalembert, L.P. (2009). Le roman de Cuba. Monaco : Éditions du Rocher.

Dalembert, L.P. (2010). « Potoprens nan sewom / Porto Principe con la flebo ». Il Tolomeo, 13(2), 30-2.

Dalembert, L.P. (2011). Noires blessures. Paris : Mercure de France.

Dalembert, L.P. (2013). Ballade d'un amour inachevé. Paris : Mercure de France.

Dalembert, L.P. (2017a). Avant que les ombres s'effacent. Paris : Sabine Wespieser Éditeur.

Dalembert, L.P. (2017b). En marche sur la terre. Paris : Éditions Bruno Doucey.

Dalembert, L.P. (2019). Mur Méditerranée. Paris : Sabine Wespieser Éditeur.

\section{Critique}

Borst, J. (2009). «Violence et mémoire dans le roman haïtien contemporain ». Publif@rum,10,1-11.http://www.publifarum.farum.it/index.php/ publifarum/article/view/179/1042.

Cacchioli, E. (2018). « Départs volontaires, faux départs, retours impossibles dans l'œuvre de Louis-Philippe Dalembert ». Pageaux 2018a, 51-68.

Charles, J.C.(2001). «L'enracinerrance ». Boutures, 1(4), 37-41. http://ile-enile.org/jean-claude-charles-lenracinerrance/.

Chemla, Y. (2005). «Littérature du faubourg ». http://www.ychemla.net/ fic_doc/dalemb_faubo1.html.

Chemla, Y. (2011). «Louis-Philippe Dalembert. Quelques pistes pour arpenter le monde ». Ménard 2011, 385-93.

Chemla, Y. (2018). «La poésie de Louis-Philippe Dalembert ». Pageaux 2018a, 197-226.

Cooreman, G. (2010). «Migration clandestine dans L'autre face de la mer de Louis-Philippe Dalembert». Gyssels, K. ; Ledent, B. (éds), Présence africaine en Europe et au-delà. African presence in Europe and beyond. Paris : L'Harmattan, 215-34.

Cooreman, G. (2018). « Migration clandestine vers le 'premier monde' (l'Amérique, l'Europe). Les boat people haïtiens dans L'Autre Face de la mer de Louis-Philippe Dalembert ». Pageaux 2018a, 71-90.

Diard, D. (2018). « Entre l'ici et l'ailleurs. Louis-Philippe Dalembert l'aède vagabond ». Loxias-Colloques, 9. http://revel.unice.fr/symposia/actel/index.html?id=1017.

Étienne, A. (2018). «Rue du Faubourg Saint-Denis de Louis-Philippe Dalembert. De Romain Gary à Haïti ou une nouvelle cartographie pour l'idéal républicain français ». Pageaux 2018a, 141-60.

Famin, V. (2011). «L'Autre Face de la mer de Louis-Philippe Dalembert ou les récits de la dualité caribéenne ». Ménard 2011, 177-88. 
Gannier, O. (2018). « Retour au pays-temps de Grannie (Louis-Philippe Dalembert : Le crayon du bon Dieu n'a pas de gomme, L'Autre Face de la mer, "Les dieux voyagent la nuit") ». Pageaux 2018a, 91-108.

Ghinelli, P. (2005). Archipels littéraires. Chamoiseau, Condé, Confiant, Brival, Maximin, Laferrière, Pineau, Dalembert, Agnant. Montréal : Mémoire d'encrier.

Gyssels, K. ; Cooreman, G. (2008). « Autour du Faubourg Saint-Denis : une causerie avec Louis-Philippe Dalembert ». /l Tolomeo, 11(1), 98-106.

Marinho, M. (2018). "L'arbre à palabres, paysages imaginaires. Regards sur une traduction brésilienne de Louis-Philippe Dalembert ». Pageaux 2018a, 179-96.

Ménard, N. (éd.) (2011). Écrits d'Haïti. Perspectives sur la littérature haïtienne contemporaine (1986-2006). Paris : Khartala.

Pageaux, D.-H. (éd.) (2018a). Louis-Philippe Dalembert. Entre vagabondage et humanisme. Paris : L'Harmattan.

Pageaux, D.-H. (2018b). "'Persistance de l'humain'. Pour une lecture de Louis-Philippe Dalembert ». Pageaux 2018a, 227-53.

Pessini, E. (2008). « Présences insulaires dans l'œuvre de Louis-Philippe Dalembert ». Imbroscio, C. et al. (éds), Des îles en archipel. Flottements autour du thème insulaire en hommage à Carminella Biondi. Frankfurt Am Main : Peter Lang, 67-82.

Pessini, A. (2012). Regards d'exil. Trois générations d'écrivains haïtiens. Saarbrücken : Presses Académiques Francophones.

Pessini, A. (2018). «L'arpentage du monde dans l'œuvre de Louis-Philippe Dalembert ». Pageaux 2018a, 17-35.

Puig, S. (2018). «Ancrages et déplacements chez Louis-Philippe Dalembert : le cas de "Rue du Faubourg Saint-Denis" ». Pageaux 2018a, 129-40.

Snoussi, M. (2003). "À propos de L'île du bout des rêves ». http://africultures.com/a-propos-de-lile-du-bout-des-reves-3080/.

Théodat, J.-M. (2018). «Autogéographie du Faubourg ». Pageaux 2018a, 111-27.

Vignoli, A. (2016). «Louis-Philippe Dalembert, 'vagabond jusqu'au bout de la fatigue' ». Il Tolomeo, 18, 29-40. http://doi.org/10.14277/2499-5975/ Tol-18-16-3. 



\title{
Le transfert culturel chez Ousmane Sembène : du péritexte auctorial au péritexte traduit
}

\author{
Cristina Schiavone \\ Università degli Studi di Macerata, Italia
}

\begin{abstract}
The aim of the contribution is to show, through examples taken from the analysis of paratextual elements, the liminal space lying outside the fiction, how the cultural diversity, of which Ousmane Sembène's opus is the expression, is treated first by the author and in a second step by the Italian translator, mediating the intercultural dialogue. This research also provides a few useful suggestions for a 'good' translation of sub-saharian Francophone literatures.
\end{abstract}

Keywords Paratext. Cultural diversity. Translation. Sub-saharian Francophone literatures. Sembène Ousmane.

Sommaire 1 Introduction. - 2 Distance culturelle et diglossie littéraire : le(s) défi(s) de l'écrivain et du traducteur. - 3 Analyse péritextuelle. - 4 Conclusion.

$\begin{array}{lll} & \text { Peer review } \\ & \text { Submitted } & 2020-11-11 \\ \text { Edizioni } & \text { Submitted } & 2020-11-26 \\ \text { Ca'Foscari } & \text { Published } & \text { aaaa-mm-dd } \\ & \text { Open access }\end{array}$

() 2020 | ()(1) Creative Commons Attribution 4.0 International Public License 


\section{Introduction}

Le problème du transfert culturel ${ }^{1}$ a longtemps occupé et parfois préoccupé les écrivains subsahariens de langue française. Cette préoccupation est assez évidente surtout chez les pionniers de ces littératures dont le lectorat était pour la plupart européen francophone mais non initié aux cultures africaines. Par conséquent, ces œuvres présentent souvent une dimension ethnographique et didactique qui l'emporte quelquefois sur la dimension poétique. Elle se manifeste surtout par des intrusions d'une instance autre que le narrateur qui s'adresse parfois de manière récurrente au lecteur virtuel. Ce qui transforme l'écrivain en un traducteur au sens étymologique du terme : à savoir en l'instance qui transporte, transmet un message le plus souvent de type informatif-explicatif, dans l'intention d'éviter ainsi le risque de 'trahison' due à la méconnaissance de la part du destinataire de la charge de certains éléments spécifiques des cultures si peu connues du public occidental.

Quant à la question de la traduction des littératures africaines en langue française dans d'autres langues européennes, la problématique de la différence culturelle, qui est d'ailleurs intrinsèque à toute traduction, y devient encore plus cruciale à cause de sa complexité, car ces littératures sont le résultat d'une première traduction, dans le sens large du terme (Bandia 2001), ou mieux d'une transposition de la langue-culture première des écrivains vers le français, langue importée en Afrique subsaharienne et imposée avec la colonisation au début du XXème siècle. Il s'agit, en fait, de littératures qualifiées aussi d'hétérolingues (Grutman 2002, 331)² et exophones (Bokiba 2007).

Par conséquent, ce phénomène souligne la fonction centrale remplie par l'écrivain subsaharien, à savoir sa fonction médiatrice, c'està-dire d'agent de communication interculturelle, déjà à partir du contexte même du texte original. ${ }^{3}$ À cette médiation s'ajoute, dans le texte traduit, celle du traducteur, qui, lui aussi, joue un rôle de passeur entre l'univers du texte source et celui du texte cible et qui doit mettre en œuvre toute une série de stratégies aptes à garantir la fi-

1 Michel Espagne souligne un aspect important en définissant cette notion : " Tout passage d'un objet culturel d'un contexte dans un autre a pour conséquence une transformation de son sens, une dynamique de resémantisation [...]. Transférer, ce n'est pas transporter, mais plutôt métamorphoser, et le terme ne se réduit en aucun cas à la question mal circonscrite et très banale des échanges culturels. C'est moins la circulation des biens culturels que leur réinterprétation qui est en jeu » $(2013,1)$

2 Grutman 2002, 331 : « le texte littéraire est un espace où peuvent se croiser plusieurs (niveaux de) langues ». Myriam Suchet a développé la notion d'hétérolinguisme de Grutman et l'a appliquée aussi à la traduction $(2009,2014)$.

3 Bokiba 2007, 113 : «L'écrivain [exophone] est ainsi l'acteur privilégié d'une dynamique interculturelle». 
délité au texte original et en même temps la lisibilité et l'efficacité au niveau pragmatique du texte d’arrivée (Bokiba 2007, 114). La distance culturelle et la complexité du texte justifieraient donc le recours à un complément d'explication offert par les espaces situés aux marges du texte de fiction : le paratexte.

Cette contribution vise d'abord à montrer par des exemples avec quelles modalités la diversité culturelle, dont les œuvres d'Ousmane Sembène ${ }^{4}$ sont l'expression, est traitée par l'instance auctoriale. Deuxièmement, cette réflexion vise à illustrer quel type de médiation les traducteurs italiens mettent en œuvre dans leur acte de communication traductive. L'étude se base sur l'analyse de certains espaces non fictionnels, appartenant notamment au péritexte. Par péritexte, sous-catégorie paratextuelle, on entend :

tous les éléments paratextuels qui entourent, enveloppent et accompagnent le texte dans un espace qui reste compris à l'intérieur de la publication éditée, sur le même support et donc indissociable à lui. (Frías 2010, 289) 5

L'œuvre de Sembène est un terrain propice pour ce genre d'étude, car ses romans,nouvelles et récits sont caractérisés par la présence significative d'éléments paratextuels autoriaux, notamment des notes de bas de pages et plus souvent les annotations intratextuelles. Les traductions italiennes, de leur côté, présentent, elles-aussi, un appareil paratextuel qui mérite d'être observé de près et comparé avec celui de la version originale.

\section{Distance culturelle et diglossie littéraire : le(s) défi(s) de l'écrivain et du traducteur}

La question de la difficulté à restituer dans la langue cible la charge culturelle transmise par la langue source dans le processus de traduction des œuvres littéraires subsahariennes destinées à un public européen non francophone, mérite réflexion. Tout traducteur qui aborde des textes dans le domaine des littératures africaines, devrait nécessairement posséder des compétences non seulement strictement linguistiques, à savoir lexico-grammaticales, mais aussi textuelles, pragmatiques, sociolinguistiques, historiques et culturelles au sens large, contexte d'origine de l'écrivain africain. Le traducteur doit saisir et prendre en compte toute la complexité du monde plurilingue et pluri-

4 Écrivain et cinéaste sénégalais, né à Ziguinchor en 1925 et décédé à Dakar en 2007.

5 José Juste Frías est le fondateur de la discipline de la 'paratraduction' et de l'école de Vigo (Espagne). 
culturel qui est un trait typique de la plupart des réalités du continent d'où émerge l'œuvre à traduire car il s’agit très souvent d'une œuvre qui naît, de par son histoire, dans un espace de l’hétérogène : « La littérature euro-africaine est caractérisée par des formes hybrides qui mélangent les traditions autochtones et occidentales » (Bandia 2001). En effet, ce sont des littératures qui sont nées dans des contextes coloniaux et postcoloniaux, mais nourries de traditions orales riches et bien enracinées. Par conséquent, elles présentent des phénomènes de vernacularisation et de diglossie littéraire : «Traduire dans une langue européenne une réalité africaine originaire d'une culture-source orale conduit au phénomène de diglossie littéraire » (Bokiba 2007, 113). ${ }^{6}$

Ce phénomène investit entre autres l'aspect du rythme et la musicalité de l'écriture littéraire reproduisant l'effet d'oralité (Tine 1985, 114), ${ }^{7}$ des composantes que le traducteur ne peut pas ne pas prendre en compte quand il aborde la traduction de textes littéraires subsahariens où l'oralité est un élément très important (Meschonnic 1982).

Sur les plans lexical et syntaxique, les littératures africaines sont caractérisées par l'introduction massive de xénismes, d'emprunts, de calques des langues-cultures africaines, langue première des écrivains. La présence de ces interférences linguistiques et culturelles, souvent nombreuses dans le texte, peut parfois nuire à la bonne réception du contenu. Ici,le péritexte peut intervenir pour jouer un rôle comparable à un dictionnaire interstitiel ${ }^{8}$ qui compense l'opacité des implicites culturels du texte (Mbow 2011, 65).

C'est dans cette optique que les éléments paratextuels des textes africains se révèlent importants également en traduction : l'introduction ou la préface, la postface et surtout les notes (situées en bas de page ou en fin de texte), par leur force pragmatique, remplissent une fonction de médiation entre l'univers de l'œuvre et le monde du lecteur.

Un dernier aspect qui souligne la distance culturelle entre les cultures africaines et les cultures occidentales est la différence sur le plan du style communicatif.

La question la plus commune qu'un traducteur se pose est : comment rendre, faire émerger dans la langue d'arrivée toute la complexité de cet espace pluriculturel, toute cette richesse dont l'œuvre

6 À propos de la notion de 'diglossie littéraire', on renvoie aussi à Giordan, Ricard 1976. En réalité, Grutman préfère utiliser le terme 'hétérolinguisme' plutôt que 'diglossie', qu'il considère une notion à la connotation politique, tout comme 'bilinguisme' (Grutman 1997, 37).

7 « Pour le public africain, l'ethno-texte [...] fonctionne souvent comme un clin d'œil, et de ce fait permet d'établir une connivence immédiate entre l'auteur et l'auditoire 'implicite' ». Quant au lecteur occidental, il est souvent installé d'une manière explicite dans l'espace de l'ethno-texte, et il apparaît toujours comme un narrataire. C'est à lui que s'adresse en priorité tout un métalangage, tout un appareil explicatif » (Tine 1985, 114).

8 Ce mot est emprunté à Galisson $(1998,257)$. 
subsaharienne est porteuse, sans diminuer la valeur esthétique du texte et tout en gardant le plaisir de la lecture ? Pour citer un exemple relatif à la proxémie : comment rendre dans la langue cible la symbolique de la gestualité africaine, certains silences, certains regards, certains mouvements des yeux, la manière de mettre et d'enlever le foulard des femmes sénégalaises, tous des messages indirects porteurs d'informations essentielles sur la psychologie des personnages ou bien qui sont parfois moteurs de l'histoire?

En effet nous avons affaire à des cultures qui ont un style de communication indirecte à un degré assez élevé, à savoir des cultures où le paraverbal a un poids significatif qu'on ne peut pas négliger. ${ }^{9}$

Tel est le défi et en même temps la condamnation du traducteur des textes littéraires africains !

Ainsi, la difficile mission du traducteur est celle de rendre transparent d'abord à son esprit le message, la symbolique des culturèmes (Lungu-Badea 2009, 19) du texte source et ensuite trouver un traduisant correspondant fidèle et/ou efficace dans le texte cible.

Genette, dans son essai fondamental, Seuils, ${ }^{10}$ appelle « auxiliaires » les éléments paratextuels, que l’on considère généralement accessoires. Par contre, ces éléments peuvent remplir des fonctions multiples d'une certaine importance, surtout dans les textes africains, si bien que des spécialistes après Genette ont revu cette idée de 'marginalité' du paratexte. ${ }^{11}$ De plus, son importance est certifiée, en général, par la présence du pacte de lecture situé déjà à partir du premier élément paratextuel qui est le titre.

\section{Analyse péritextuelle}

Notre analyse se focalise sur certains éléments faisant partie de l'appareil paratextuel, notamment des éléments qui appartiennent à la catégorie du 'péritexte' : préfaces et/ou postfaces, notes infrapaginales et glossaires. Il s'agit de lieux importants du transfert et de la négociation linguistique et culturelle, lieux où l'effort de médiation de l'écrivain et du traducteur, parfois aussi du préfaceur et/ou postfaceur, est plus visible que dans d'autres éléments paratextuels.

En ce sens, l'œuvre de Sembène nous a semblé constituer un corpus idéal pour ce genre de recherche, de par sa richesse et sa varié-

9 Sur cet aspect, on revoie au modèle de communication interculturelle de Ting-Toomey, Dorjee 2019.

10 «Un discours fondamentalement hétéronome, auxiliaire, voué au service d'autre chose qui constitue sa raison d'être, et qui est le texte » (Genette 1987, 16).

11 Cf. parmi les spécialistes du sujet : Frías 2010 ; Lane 2005 ; Dürrenmatt 2004 ; Elefante 2012. 
té au niveau péritextuel, et aussi parce que cet écrivain est parmi les premiers et rares auteurs subsahariens dont quatre textes ont été traduits en italien dans une période qui va de 1978 à 1990 :

- (1960). Les bouts de bois de Dieu. Banty mam Yall. Paris : le livre contemporain (roman).

- (1990). Il fumo della savana. Introduzione di D. Maraini, traduzione di CG. Marolda. Roma : Edizioni Lavoro (romanzo).

- (1962). Voltaïque. La noire de... Paris : Présence africaine (nouvelles).

- (1991). La Nera di.... Traduzione, note e postfazione di L. Cenerini. Palermo : Sellerio (racconti).

- (1966). Le Mandat précédé de Véhi Ciosane. Paris : Présence africaine (récits).

- (1978). Il vaglia. Traduction de C. Brambilla. Milano : Jaca Book (romanzo breve).

- (1979). Vehi-Ciosane ossia Bianca-Genesi. Introduzione e traduzione di C. Brambilla. Milano : Jaca Book (romanzo). ${ }^{12}$

En ce qui concerne l'approche par l'analyse du paratexte, les études de Gérard Genette sont bien sûr une référence incontournable, mais aussi celles d'autres spécialistes qui ont repris et approfondi ces études comme José Yuste Frías, Philippe Lane et Patrick Marot.

Le paratexte remplit plusieurs fonctions. Il peut avoir une fonction exégétique et/ou métadiscursive, contribue à clarifier la narration, à traduire des mots et expressions qui pourraient être obscurs au destinataire, à authentifier le récit et commenter le réel. Il peut aussi avoir une vocation humoristique de contrepoids. Patrick Marot distingue cinq fonctions du texte liminaire : fonctions auctoriale, pragmatique, didactique, herméneutique, monumentale (Marot 2010, 9).

En tout cas, la dimension pragmatique, que Genette avait d'ailleurs déjà soulignée et d'autres spécialistes après lui, ${ }^{13}$ domine sur les autres. Le paratexte est un acte linguistique pourvu d'une force illocutoire car il a à la fois une visée informative et la capacité d'orienter la réception, à savoir d'influencer la lecture (Lane 2005, 185).

Pour ce qui est du péritexte, sur quoi notre étude s'appuie, tout ce qui est présent de la première à la quatrième page de couverture rentre dans cette sous-catégorie : nom de l'auteur ou plutôt son ou ses pseudonyme(s), titre et sous-titre, colophon, dédicaces, épigraphes, préfaces, postfaces quatrième de couverture, collection. À ces éléments, on peut ajouter le nom du traducteur, une figure qui n'a pas été prise en compte par Genette, mais qui le sera ensuite, englobée et

12 Si les deux brèves romans sont à l'origine publiés dans un seul volume, dans l'édition italienne ils sont publiés à la distance d'un an l'un de l'autre.

13 Notamment Frías 2010; Lane 2005; Pier 1989. 
abordée par d'autres spécialistes du paratexte en traduction comme Chiara Elefante, José Yuste Frías et Philippe Lane.

Le tableau qui suit réunit des éléments péritextuels des traductions italiennes des œuvres de Sembène :

\begin{tabular}{lllll}
\hline Collection & Titre & 4ème de couverture & Préface/Introduction & Postface \\
\hline $\begin{array}{l}\text { Difronte } \\
\text { e attraverso }\end{array}$ & Ilvaglia & Extrait du roman & Non & Non \\
\hline $\begin{array}{l}\text { Difronte } \\
\text { e attraverso }\end{array}$ & $\begin{array}{l}\text { Vehi- } \\
\text { Ciosane } \\
\text { ossia } \\
\text { Bianca- } \\
\text { Genesi }\end{array}$ & $\begin{array}{l}\text { Présentation de } \\
\text { Sembène et de l'œuvre }\end{array}$ & $\begin{array}{l}\text { Oui. Présentation de } \\
\text { l'auteur et de ses œuvres }\end{array}$ & Non \\
\hline $\begin{array}{llll}\text { Il lato } \\
\text { dell'ombra }\end{array}$ & $\begin{array}{l}\text { Ilfumo } \\
\text { della } \\
\text { savana }\end{array}$ & $\begin{array}{l}\text { Présentation de } \\
\text { Sembène et de son }\end{array}$ & $\begin{array}{l}\text { Oui. Introduction } \\
\text { d'écrivain (autorité } \\
\text { reconnue dans le marché } \\
\text { éditorialitalien) }\end{array}$ & Non \\
\hline La memoria & $\begin{array}{l}\text { La Nera } \\
\text { di... }\end{array}$ & $\begin{array}{l}\text { 2 présentations de } \\
\text { Sembène: } 1 . \text { Aperçu } \\
\text { de ses œuvres et de sa } \\
\text { poétique. } \\
\text { 2. Bio-bibliographie }\end{array}$ & Non & $\begin{array}{l}\text { Oui. } \\
\text { Remarques } \\
\text { de la } \\
\text { traductrice } \\
\text { fin volume }\end{array}$ \\
\hline
\end{tabular}

La plupart des préfaces ou introductions des éditions italiennes examinées montrent une tendance généralisée à exalter l'œuvre et l'écrivain, à tracer un parcours de lecture qui très souvent tend à déborder vers une interprétation du texte et ont souvent un but didactique (Schiavone 2016, 155). Bref, il s'agit de textes où le discours argumentatif domine sur le discours informatif et descriptif. Par contre, dans la préface du roman Il fumo della savana, ce n'est pas du tout le cas. Il s'agit de la voix d'une écrivaine, Dacia Maraini, qui présente une œuvre littéraire sans prétention d'analyse critique ou d'orientation de lecture. Elle ne fait que partager ses souvenirs d'un séjour au Sénégal et d'une lecture précédente de Sembène, à savoir Voltaïque. Dacia Maraini exprime ses émotions et sensations et aussi le pouvoir évocatoire de l'écriture de Sembène :

Immediatamente, appena comincio a leggerlo, ritrovo quella prosa, quell'atmosfera, quel misto di simpatia bonaria e di crudele precisione che caratterizza lo stile dello scrittore senegalese. Con la suggestione di quegli odori, quei suoni, quelle immagini africane seppellite nella memoria. (Sembène 1990, VII)

La préface est intégrée par une note de la directrice de la collection, Itala Vivan, qui offre, d'ailleurs, en quatre pages, des infor- 
mations bio-bibliographiques de l'auteur, une contextualisation de l'œuvre dans le panorama historique et littéraire subsaharien, et aussi quelques clés de lecture du roman.

À propos des notes, la présence presque imposante d'annotations intratextuelles en forme d'incises entre parenthèses multifonctionnelles est un des traits saillants dans la presque totalité des œuvres originales du corpus examiné.

Il s'agit de vraies intrusions d'une voix distincte du narrateur omniscient, responsable d'annotations, de commentaires, ou bien de descriptions de détails visuels de la 'scène' qui sont typiques des didascalies de l'écriture théâtrale ou cinématographique (scénario). ${ }^{14} \mathrm{De}$ temps en temps, cette instance s'adresse directement au lecteur virtuel comme si tous les deux étaient présents à l'intérieur du même cadre énonciatif, en lui donnant des instructions de lecture et même de prononciation (notes à valeur didascalique) :

- Qu'est-ce qu'on joue, Oncle (entendez Chéri) lui demanda celle qui venait derrière lui. (Voltaïque, 10)

Momutu (prononcez Momoutou) (Voltaïque, 194)

Prononcer : Thiosane (Véhi Ciosane, 17 note 1)

Ce procédé de l'instance notatrice reproduirait la situation d'énonciation caractéristique de la tradition orale, où le public destinataire est toujours pris en compte, témoin de l'acte de parole du narrateur-griot et souvent interpellé par ce dernier, parfois encore impliqué comme témoin de l'action racontée (homodiégétique).

À propos des notes, nous avons procédé à un inventaire de type quantitatif et qualitatif pour l'ensemble des œuvres analysées, grâce à la création d’un modèle de schéma adapté à notre corpus à partir de celui élaboré par Fučiková $(2011,146)$.

\begin{tabular}{lll}
\hline & Texte source & Texte cible \\
\hline Titre & Le Mandat & Ilvaglia \\
\hline Nombre de notes & 25 sur 79 pages & 50 sur 89 pages \\
\hline Type de note & Auctoriale $: 25$ & Auctoriale $: 20$ \\
& Allographe $: 0$ & Allographe $(\mathrm{NdT}): 30$ \\
\hline Collocation de la note & En bas de page $: 1$ & En bas de page $: 50$ \\
& Intratextuelle $: 24$ & Intratextuelle $: 0$ \\
\hline
\end{tabular}

14 Ce qui montre aussi une autre facette du créateur Sembène : celle du cinéaste. 
Cristina Schiavone

Le transfert culturel chez Ousmane Sembène : du péritexte auctorial au péritexte traduit

\section{Fonction de la note}

1. Traduction

2. Métalinguistique

(didascalique)

3. Explication (encyclopédique)

4. Référence (historique, intertextuelle, culturelle)
Exemples:

1. Nda: canari contenant de

l'eau potable. (116)

2. 0

3. 0

4. 0
Exemples:

1. Garni : nobile (29)

2. Voi : interiezione di sorpresa, indignazione e ammirazione

(48)

3. Volof: $:^{15}$ principale lingua di comunicazione in Senegal (23)

4. CFA : franco della Comunità Finanziaria Africana, in corso dal 1945 negli stati appartenenti alla Comunità Francese (23)

\begin{tabular}{|c|c|c|}
\hline Glossaire & Non & Non \\
\hline & Texte source & Texte cible \\
\hline Titre & Véhi Ciosane ou Blanche-Genèse & Bianca Genesi \\
\hline Nombre de notes & 44 sur 101 pages & 69 sur 115 pages \\
\hline Type de note & $\begin{array}{l}\text { Auctoriale : } 44 \\
\text { Allographe : } 0\end{array}$ & $\begin{array}{l}\text { Auctoriale : } 30 \\
\text { Allographe }(\mathrm{NdT}): 39\end{array}$ \\
\hline Collocation de la note & $\begin{array}{l}\text { En bas de page : } 5 \\
\text { Intratextuelle : } 39\end{array}$ & $\begin{array}{l}\text { En bas de page : } 69 \\
\text { Intratextuelle : } 0\end{array}$ \\
\hline $\begin{array}{l}\text { Fonction de la note } \\
\text { 1. Traduction } \\
\text { 2. Métalinguistique } \\
\text { (didascalique) } \\
\text { 3. Explication (encyclopédique) } \\
\text { 4. Référence (historique, } \\
\text { intertextuelle, culturelle) }\end{array}$ & $\begin{array}{l}\text { Exemples: } \\
\text { 1. Malaika: anges (30) } \\
\text { 2. Ciosane: Prononcer: } \\
\text { Thiosane (17) } \\
\text { 3. Navétanekat: paysan se } \\
\text { louant pendant le navet: } \\
\text { l'hivernage (39) } \\
\text { 4. Lallal: chez les Wolofs, } \\
\text { les Sérères, les Mandès on } \\
\text { accueille l'hôte en étalant les } \\
\text { plus beaux pagnes sous ses } \\
\text { pieds (38) }\end{array}$ & $\begin{array}{l}\text { Exemples: } \\
\text { 1. Saytanè : satana (64) } \\
\text { 2. Griote : Pronunciare griot. } \\
\text { Femminile di griot. Vedi nota } \\
\text { 3(38) } \\
\text { 3. Henné : pasta fatta con } \\
\text { polvere alcanna, usata per il } \\
\text { trucco degli occhi (39) } \\
\text { 4. Diambur-diambur: un tempo } \\
\text { schiavi di varia origine, sotto la } \\
\text { diretta dipendenza del re, di cui } \\
\text { formavano la milizia. Colmati } \\
\text { di favori, si consideravano di } \\
\text { casta superiore. Oggi il nome } \\
\text { significa « uomo libero » (15) }\end{array}$ \\
\hline \multirow[t]{2}{*}{ Glossaire } & Non & Non \\
\hline & Texte source & Texte cible \\
\hline Titre & $\begin{array}{l}\text { Les bouts de bois de Dieu. Banty } \\
\text { Mam Yall }\end{array}$ & Il fumo della savana \\
\hline Nombre de notes & 81 sur 379 pages & 104 sur 312 pages \\
\hline
\end{tabular}

15 'Volof' est égal à 'wolof'. La traductrice respecte la graphie du texte d'origine. 
Cristina Schiavone

Le transfert culturel chez Ousmane Sembène : du péritexte auctorial au péritexte traduit

\begin{tabular}{|c|c|c|}
\hline Type de note & $\begin{array}{l}\text { Auctoriale : } 81 \\
\text { Allographe : } 0\end{array}$ & $\begin{array}{l}\text { Auctoriale : } 46 \\
\text { Allographe }(\mathrm{NdT}): 58\end{array}$ \\
\hline Collocation de la note & $\begin{array}{l}\text { En bas de page : } 73 \\
\text { Intratextuelle : } 8\end{array}$ & $\begin{array}{l}\text { En bas de page : } 104 \\
\text { Intratextuelle : } 0\end{array}$ \\
\hline $\begin{array}{l}\text { Fonction de la note } \\
\text { 1. Traduction } \\
\text { 2. Métalinguistique } \\
\text { (didascalique) } \\
\text { 3. Explication (encyclopédique) } \\
\text { 4. Référence (historique, } \\
\text { intertextuelle, culturelle) }\end{array}$ & $\begin{array}{l}\text { Exemples: } \\
\text { 1. Samaras : sandales } \\
\text { 2. Eskaï Allah! : exclamation } \\
\text { d'étonnement adressée à Dieu. } \\
\text { 3. taparquats: sorte de massue } \\
\text { dont les ménagères se servent } \\
\text { pour repasser les vêtements. } \\
\text { 4. Place du ler septembre: } \\
\text { nommée ainsi par les ouvriers } \\
\text { en souvenir de leur première } \\
\text { tentative, en 1938, qui fut } \\
\text { réprimée et échoua }\end{array}$ & $\begin{array}{l}\text { Exemples: } \\
\text { 1. Nghenghe : frusta in } \\
\text { bambara. (16) } \\
\text { 2. Wa lahi: frequente } \\
\text { interiezione rafforzativa, in } \\
\text { originale nel testo; [...]. (59) } \\
\text { 3. Flamboyant: Indigofera } \\
\text { ingens, albero originario delle } \\
\text { Antille dalla vivace fioritura } \\
\text { rossa, che cresce in numerosi } \\
\text { paesi tropicali. (9) } \\
\text { 4. Hadji : titolo dovuto ai } \\
\text { musulmani credenti che hanno } \\
\text { compiuto il viaggio rituale alla } \\
\text { Mecca almeno una volta nella } \\
\text { vita (69) }\end{array}$ \\
\hline Glossaire & Non & Oui : 37 entrées \\
\hline
\end{tabular}

\begin{tabular}{lll}
\hline & Texte source & Texte cible \\
\hline Titre & Voltaïque & La nera di... \\
\hline Nombre de notes & 21 sur 210 pages & 58 sur 137 pages \\
\hline Type de note & Auctoriale $: 21$ & Auctoriale $: 17$ \\
& Allographe $: 0$ & Allographe $(\mathrm{NdT}): 38$ \\
& & Auteur $+\mathrm{NdT}: 3$ \\
\hline
\end{tabular}

Collocation de la note En bas de page : 1

Intratextuelle: 20

En bas de page : 1

Intratextuelle : 14

Fin de texte : 43

\section{Fonction de la note}

1. Traduction

2. Métalinguistique

(didascalique)

3. Explication (encyclopédique)

4. Référence (historique, intertextuelle, culturelle)

\section{Exemples:}

1. Sabars: tam-tams (19)

2. Oncle : entendez Chéri. (10)

3. Fédération : fédération du

Mali-Soudan-Sénégal. (30)

4. Jusqu'au XVIIème siècle, on payait outre la pacotille des aunes de cauris, puis on remplaça les aunes de cauris par des barres de fer. Il est écrit aussi que dans d'autres marchés la monnaie d'échange avait été de tout temps des barres de fer (199-200)

\section{Exemples:}

1. Mousses: i gatti (79)

2. Zio : intende caro (147)

3. Sabar: riunione animata dalla musica del tam-tam (147)

4. Soundiata Keita: nel

XIII secolo ha liberato i Mandinghi dalla dominazione di Soumangourou Kanté, re Sosso. La leggenda si è impadronita della sua figura di eroe che l'infanzia paralitica non destinava a grandi imprese. (147)

\begin{tabular}{lll}
\hline Glossaire Non Non \\
\hline
\end{tabular}


Ces fiches montrent tout d'abord la présence imposante de l'écriture notulaire auctoriale dans le texte source, de par l'importance du nombre d'occurrences et aussi par sa collocation à l'intérieur du texte, ce qui contribue à l'établissement d'une hybridation générique, car il insère un 'autre' discours à l'intérieur même de la narration (Gauthier 2015, 2). Dans le texte traduit, les notes auctoriales intratextuelles, sauf quelques exceptions, sont presque toutes déplacées ou en bas de page, ou à la fin du texte. Le traducteur recourt souvent à la stratégie de l'explicitation et de l'amplification. Il ajoute notamment des compléments d'informations historiques et culturelles en intégrant la note auctoriale ou en créant d'autres notes, ou, comme dans le cas du roman Il fumo della savana, en ajoutant un glossaire à la fin du texte.

Le cas de sabar dans Voltaïque constitue un exemple d'explicitation et d'amplification à la fois :

\section{T.S. Sabar : tam-tams (Voltaïque, 19)}

T.C. Sabar : riunione animata dalla musica del tam-tam. (La nera di..., 147)

Il arrive aussi que le traducteur italien, pour expliciter l'information contenue dans la note auctoriale, se l'approprie en l'adaptant aux exigences du lecteur virtuel italien :

T.S. Missé Dézean : Monsieur Déjean, directeur de la Compagnie. (Les Bouts de bois de Dieu, 210)

T.C. Sinor Dezan : Trascrizione della pronuncia senegalese del nome del direttore della Compagnia, signor Déjean (N.d.T.) (Il fumo della Savana, 173)

Nous nous arrêterons de manière plus approfondie et ponctuelle sur l'analyse comparée du recueil de nouvelles Voltaïque et de sa traduction italienne, La Nera di..., car cette publication de l'éditeur italien Sellerio représente un cas isolé par rapport à la tendance générale des traductions précédentes. ${ }^{16}$ Cette œuvre se détache des autres traductions du corpus en ce qui concerne la stratégie éditoriale de l'époque et aussi du point de vue du traitement du paratexte dans la partie qui concerne la traductrice.

D’abord, la collection 'La memoria', dans laquelle a été placée, n’a aucune référence géographique ou culturelle spécifique, car le sujet l'emporte sur l'origine géographique ou la nationalité de l'auteur. En

16 Sur ce point, cf. Schiavone 2016. À propos des titres des œuvres de Sembène, nous avons analysé Les bouts de bois de Dieu. Banty mam Yall dans une autre publication de Schiavone 2008. 
effet, dans la même collection, qui compte 231 titres, apparaissent aussi des titres de grands écrivains tels que Kipling, Defoe, Voltaire, Anatole France, Gide, Tourgueniev, Sénèque, Plutarque, etc.

En deuxième lieu, l'édition italienne ne présente aucune introduction, ni de notes de bas de page. Ainsi, le lecteur, en principe, ne risque d'être l'objet d'aucun conditionnement dans son activité de décodification et d'interprétation du texte littéraire, car toute explication est reportée dans une section placée à la fin de l'œuvre par des renvois au glossaire et à une sorte de postface.

À titre d'exemple, dans le tableau suivant est reporté l'appareil notulaire auctorial comparé de Voltaïque et La nera di...

\begin{tabular}{|c|c|}
\hline Voltaïques. La noire de... (notes intratextuelles) & La Neradi... ${ }^{17}$ \\
\hline Oncle (entendez Chéri) & $\begin{array}{l}\text { Zio : intende « caro ». La moglie chiama il marito } \\
\text { zio, perché nel contesto matriarcale africano } \\
\text { si chiede al marito di avere verso la sposa la } \\
\text { stessa generosità e lo stesso slancio che uno zio } \\
\text { ha verso le nipoti. (note fin de texte) }\end{array}$ \\
\hline $\begin{array}{l}\text { Fatou (appellation qu'on donne dans certains } \\
\text { milieux aux femmes illettrées) }\end{array}$ & $\begin{array}{l}\text { Fatou (appellativo che in certi ambienti si dà } \\
\text { alle donne illetterate) }\end{array}$ \\
\hline Sabar (tam-tams) & $\begin{array}{l}\text { Riunione animata dalla musica del tam-tam. } \\
\text { (note fin de texte) }\end{array}$ \\
\hline $\begin{array}{l}\text { Fédération (fédération du Mali... Soudan- } \\
\text { Sénégal) }\end{array}$ & $\begin{array}{l}\text { Federazione (Federazione del Mali-Sudan- } \\
\text { Senegal) }\end{array}$ \\
\hline Dimasse (dimanche) & $\begin{array}{l}\text { Alterazione fonetica per dimanche (domenica) } \\
\text { (note fin de texte) }\end{array}$ \\
\hline Déded (non, non) & No, no (note fin de texte) \\
\hline Vav (Oui) & Sì (note fin de texte) \\
\hline $\begin{array}{l}\text { J'ai trouvé ces bouts de lettres. J'ai ressemblé } \\
\text { ce que j'ai pu. [note de bas de page] }\end{array}$ & $\begin{array}{l}\text { Ho trovato questi brani di lettera. Ho raccolto } \\
\text { quel che ho potuto. [note de bas de page] }\end{array}$ \\
\hline Dieunahs (les rats) & Dieunahs (i topi) \\
\hline $\begin{array}{l}\text { Inekeiv (ce mot ne signifie pas exactement le } \\
\text { malin ni le rusé, mais les deux à la fois) }\end{array}$ & $\begin{array}{l}\text { Inekeiv (questa parola non significa } \\
\text { esattamente il maligno }{ }^{18} \text { o l'astuto ma le due } \\
\text { insieme) }\end{array}$ \\
\hline mousses (chats) & Mousses (i gatti) \\
\hline $\begin{array}{l}\text { (à défaut d'eau, les ablutions au sable sont } \\
\text { permises) }\end{array}$ & $\begin{array}{l}\text { (in mancanza d'acqua, sono permesse le } \\
\text { abluzioni con la sabbia) }\end{array}$ \\
\hline
\end{tabular}

17 Dans ce tableau, les notes du texte cible dont on a respecté la même collocation que dans le texte source, ont été reportées sans aucune indication. Par contre, pour les notes déplacées dans d'autres espaces du paratexte, on a indiqué leur place entre crochets.

18 Le choix de 'maligno' pour traduire 'malin' mériterait un commentaire, mais ce type de réflexion sortirait du contexte de notre analyse. 


\begin{tabular}{|c|c|}
\hline $\begin{array}{l}\text { (Il faut retenir que dans un cas pareil, la femme } \\
\text { doit tout rendre) }\end{array}$ & $\begin{array}{l}\text { (Bisogna ricordare che in un caso simile la } \\
\text { moglie deve restituire tutto) }\end{array}$ \\
\hline (En toute logique, dans ce milieu, il avait raison) & $\begin{array}{l}\text { (Secondo la logica, in quell'ambiente, aveva } \\
\text { ragione) }\end{array}$ \\
\hline \multirow{2}{*}{$\begin{array}{l}\text { (il faut savoir que Saër est moitié voltaïque, } \\
\text { moitié sénégalais. Mais il ne porte pas de } \\
\text { balafres) }\end{array}$} & $\begin{array}{l}\text { (Bisogna sapere che Saer è per metà voltaico, } \\
\text { per metà senegalese. Ma non ha sfregi) }\end{array}$ \\
\hline & Balafres > sfregi (note fin de texte) \\
\hline I.F.A.N. (Institut français d'Afrique Noire) & (Istituto Francese per l’Africa Nera) \\
\hline Momutu (prononcez Momoutou) & Momutu [dans le texte] \\
\hline $\begin{array}{l}\text { Barres de fer (jusqu'au XVIIle siècle, on payait } \\
\text { outre la pacotille des aunes de cauris, puis on } \\
\text { remplaça les aunes de cauris par des barres de } \\
\text { fer. Il est écrit aussi que dans d'autres marchés } \\
\text { la monnaie d'échange avait été de tout temps } \\
\text { des barres de fer). }\end{array}$ & $\begin{array}{l}\text { Sbarre di ferro (fino al diciassettesimo secolo si } \\
\text { pagava oltre che con la paccottiglia, con aune } \\
\text { di cauri, poi si sostituirono le aune di cauri } \\
\text { con sbarre di ferro. Ė anche scritto che in altri } \\
\text { mercati le sbarre di ferro erano state sempre } \\
\text { moneta di scambio). }\end{array}$ \\
\hline Maître de langue (interprète) & Maestro di lingue (interprete) \\
\hline $\begin{array}{l}\text { Acquerats (les acquerats étaient les commis } \\
\text { indigènes, reconnaissables à leur uniforme, } \\
\text { aux couleurs de la nation pour laquelle ils } \\
\text { opéraient. Les gens les appelaient couramment } \\
\text { «chasseurs d'esclaves») }\end{array}$ & $\begin{array}{l}\text { Acquirenti (gli acquirenti erano dei commessi } \\
\text { indigeni, riconoscibili dalla loro uniforme con i } \\
\text { colori della nazione per la quale operavano. La } \\
\text { gente li chiamava comunemente « cacciatori di } \\
\text { schiavi ») }\end{array}$ \\
\hline $\begin{array}{l}\text { Un arbuste (ce que les Oulofs nomment } \\
\text { Bantamaré. Ses feuilles sont antiseptiques) }\end{array}$ & $\begin{array}{l}\text { Un arbusto (che i Wolof chiamano Bantamaré. } \\
\text { Le sue foglie sono antisettiche) }\end{array}$ \\
\hline
\end{tabular}

On peut remarquer que les notes auctoriales ont tantôt la fonction de traduction du wolof ou de l'arabe vers le français, tantôt celle d'information et d'explication, de référence historique et/ou culturelle.

Si dans le texte original elles sont dans leur totalité, à l'exception d'une seule, à l'intérieur même du texte, dans la traduction italienne elles sont rarement dans le texte : dans la majorité des cas elles sont déplacées à sa fin, dans une section appelée « Note». Cette section comprend des entrées supplémentaires ajoutées probablement par la traductrice dans l'intention de faciliter l'accès à des éléments de la culture sénégalaise et arabo-musulmane. En outre, dans le tableau ci-dessus il est possible d'observer que certaines notes auctoriales du texte source ont été reprises par la traductrice qui les a amplifiées dans le texte cible (par exemple, la première note). En général, il s'agit surtout d'un complément d'informations culturelles et historiques que la traductrice suppose être inconnues de son lecteur virtuel, tel que griot, sabar, calebasse, timis, kora, marabutto, pagne, legos, cadi, caid, taleb, tafsir, bilal et d'autres. L'appareil des notes de fin de texte, formé de 43 entrées, est suivi d'une sorte de postface, appelé « Nota », de neuf pages sous forme de remarques, rédigé par la traductrice, qui repère trois aspects importants des nouvelles dans 
trois brefs chapitres : la tradition orale dans l'écriture, les voix de la narration, le héros noir. De plus, la traductrice ajoute quelques lignes consacrées aux problèmes de réception du texte de la part d'un lectorat dont le profil correspondrait fondamentalement aux habitués de la littérature française. Elle signale l'effet de surprise déterminé par les écarts du français standard motivé par la présence des interférences avec le wolof : phraséologismes, ellipses, métaphores, signes diacritiques, etc.

La traductrice déclare aussi que là où cela a été possible, les écarts ont été respectés dans la traduction italienne dans le but de rappeler au lecteur que la francophonie ne s'identifie pas avec le français et que cette traduction est l'exemple concret d'une expérimentation.

Là où elle remarque qu'il serait intéressant de repérer de manière détaillée les écarts du français au wolof, la traductrice ouvre très discrètement un horizon de recherche sur le plan du contact et de l'hybridation linguistique, effet de l'interaction entre langue française et langue wolof (et nous ajouterions aussi l'arabe, qui est une autre langue très présente dans ces textes) $:^{19}$ et encore, à propos de la culture de l'oralité, les phénomènes de vernacularisation et de diglossie linguistique et littéraire. Pourtant, elle suppose en même temps que le lecteur de ce texte (lecteur virtuel) n'est pas censé avoir cette exigence, en arrêtant ainsi tout de suite son élan :

le richieste che si ipotizzano per il lettore di questo testo non rendono necessaria tale ricerca di carattere linguistico. $(1991,161)$

C'est justement sur ce point que la traductrice, en donnant une certaine représentation du lecteur virtuel de cette œuvre, de ses compétences linguistiques et culturelles et de ses exigences, en arrive à donner aussi une image de soi (ethos) et de sa propre éthique de la traduction.

\section{Conclusion}

Cette enquête a été menée à partir de l'analyse comparée de certains éléments du péritexte dans les œuvres de Sembène et dans ses traductions italiennes. L'examen s'est ensuite arrêté sur la comparaison entre Voltaïque et La nera di..., car sa version italienne présente des particularités qui méritaient plus que dans les autres textes d'être

19 Le Sénégal est un pays à majorité musulmane, par conséquent l'arabe classique est utilisé dans la pratique religieuse. Mais certaines expressions en arabe sont très fréquentes aussi dans la conversation et en français et en wolof ou dans d'autres langues sénégalaises. 
analysées en détail. D’après une étude quantitative et qualitative, qui ne prétendait pas être exhaustive, nous avons voulu attirer l'attention sur le fait que dans la traduction italienne des littératures caractérisées par une distance culturelle significative comme celle de l’Afrique subsaharienne, le péritexte se révèle un adjuvant très important dans un acte de communication qui se voudrait égalitaire entre une culture source et une culture cible, adjuvant dont on ne peut pas encore se passer. En effet, même dans les publications italiennes les plus récentes, si préfaces et postfaces ont tendance à disparaître, les médiations par le biais des notes sont bien présentes et en bon nombre, plus récemment avec une certaine préférence pour le glossaire et les notes en fin de texte, comme dans le cas de la publication plus récente de La nera di... C'est d'ailleurs un peu la tendance, qui se fait de plus en plus récurrente, de respecter la liberté interprétative du lecteur. Ceci dans le but de ne pas interrompre l'illusion de la fiction et le flux de la lecture et donc de garder 'le plaisir du texte' littéraire, raison d'ailleurs pour laquelle la pratique de l'écriture notulaire est souvent stigmatisée. En plus, le glossaire et les notes en fin de texte ont un autre atout par rapport à la note de bas de page et encore davantage par rapport à la note intratextuelle, car ils permettent de s'étendre dans des explications plus détaillées, comme nous avons pu le remarquer par exemple dans le cas de la première entrée du glossaire de La nera di..., où il existe un écart assez important avec celle de la version originale.

Une deuxième observation tirée de cette analyse quantitative et qualitative, est que la fonction du péritexte dans le texte africain est multiple : surtout informative sur le plan du cadre référentiel, souvent didactique, mais aussi pragmatique car, nolens volens, elle oriente la lecture et l'interprétation du texte.

De son côté, le péritexte en traduction est une instance de reconnaissance et de légitimation importante pour l'écrivain francophone subsaharien et donc pour la culture source mais c'est aussi de légitimation du traducteur qui finit par sortir de l'anonymat, de cette invisibilité à laquelle il est le plus souvent « condamné » (Venuti 1995).

Le péritexte en traduction permet, en outre, de faire un portrait du lecteur virtuel « (cultivé, ignorant, idéologiquement proche ou lointain du destinateur, etc.) » (Bokobza Kahan 2014-2016, 2) avec qui le responsable des notes, souvent le traducteur, entretient une sorte de dialogue. Il peut aussi permettre de tracer un profil du traducteur, de son ethos et de son éthique. Les remarques finales de la traductrice de La nera di..., les choix des entrées pour les notes et pour le glossaire ont montré non seulement les compétences linguistiques et culturelles de la traductrice, mais aussi sa déontologie et son positionnement idéologique, sa subjectivité et sa force illocutoire à partir du profil qu'elle trace du lecteur virtuel du texte.

Le traducteur des littératures subsahariennes devrait garder toujours à l'esprit, et l'exemple de Sembène Ousmane en est la preuve, 
que depuis leur naissance, pour ces littératures en langue française, on peut parler d'écriture frontalière ou même migrante au sens large (Declercq 2011, 308), car il s’agit de littératures à la croisée de deux ou plusieurs mondes et cultures (Diop 2012) qui donnent lieu à des textes 'hybrides' (Bhabha 1994). La présence si consistante d'éléments paratextuels témoigne que pour tout écrivain subsaharien l'acte d'écrire en français est un acte de traduction-médiation entre ces mondes.

Finalement, le traducteur doit toujours être bien conscient, dans l'acte traduisant, que la langue est strictement liée à l'identité, même plus : ${ }^{20}$ elle est une émanation de l'identité. Aussi, il doit être conscient que, comme Paul Ricœur le rappelle bien à travers la notion d'hospitalité langagière et en s'inspirant à la pensée d'Antoine Berman : «Traduire, c'est à la fois habiter dans la langue de l'étranger et donner hospitalité à cet étranger au cœur de sa propre langue » (Ricœur 1998, 15). ${ }^{21}$

De son côté, Oustinoff met en garde le traducteur sur des aspects incontournables quand on aborde la traduction des textes subsahariens :

Encore faut-il ne pas faire les mêmes « constructions mentales » dans l'autre langue ou ne pas prêter à l'étranger des « visions du monde » qui ne sont pas les siennes. [...] Ce rayonnement des langues dominantes a cependant un revers, celui d'un sentiment de supériorité qui est la négation même du respect de la diversité culturelle dont parle la Déclaration de l’Unesco. (Oustinoff 20032007, 120)

La traduction doit donc être littérale (Berman 1984) et bien tempérée (Oustinoff 2003-2007, 123) car la mission majeure est celle de « Féconder le Propre par la médiation de l'Étranger » (Berman 1984, 16). Aussi : « il s'agit ici de se convaincre de la nécessité d'adhérer à l'idée qu'une œuvre traduite [...] doit être étudiée, sans complexe et pureté identitaire, comme participant au rayonnement de la langue d'arrivée » (Bokiba 2007, 115). ${ }^{22}$

Enfin, le traducteur, lui aussi dans sa fonction d'agent de communication interculturelle, a une grande responsabilité et un grand pouvoir : celui de contribuer à une expansion des frontières linguistiques

20 Michaël Oustinoff : « les langues ne constituent pas des instruments interchangeables mais [...] elles sont au contraire au fondement même de l'identité » $(2007,114)$.

21 Par « hospitalité langagière » Ricœur entend ce processus de médiation où « le plaisir d'habiter la langue de l'autre est compensé par le plaisir de recevoir chez soi [...] la parole de l'étranger » (Ricœur 2004, 20).

22 Cf. Wuilmart 2006. 
et culturelles de sa propre culture, qui mène à l'affirmation identitaire et à la représentation d'une culture autre. Dans le même ordre d'idées, Oustinoff confirme que « la fonction première de la traduction est [...] d'être la grande médiatrice de la diversité » (Oustinoff 2007, 124), mais à condition que l'acte traduisant vise le maintien et la survie dans le texte cible de l’hétérogénéité intrinsèque du texte source. Pour le dire avec les mots du grand écrivain anglophone Chinua Achebe, à condition que la langue d'arrivée se charge du poids de l'expérience culturelle autre, celle de l'univers de la postcolonie africaine. ${ }^{23}$ Tel est le vrai défi dont le traducteur des littératures subsahariennes doit prendre conscience.

En attendant que le lecteur italien se familiarise davantage avec les cultures africaines, la présence imposante du péritexte, des notes spécialement, si on veut la considérer un mal, ${ }^{24}$ pour le moment est un mal nécessaire, car il joue sans aucun doute un rôle essentiel dans un dialogue des cultures qui se voudrait égalitaire.

\section{Bibliographie}

\section{Fuvres de Ousmane Sembène}

Sembène, O. (1960). Les bouts de bois de Dieu. Banty mam Yall. Paris : le livre contemporain.

Sembène, O. (1962). Voltaïque. La noire de... Paris : Présence Africaine.

Sembène, O. (1966). Le Mandat précédé de Vehi Ciosane ou Blanche Genèse. Paris : Présence Africaine.

Sembène, O. (1978). Il vaglia. Trad. di C. Brambilla. Milano : Jaca Book.

Sembène, O. (1979). Véhi-Ciosane ossia Bianca Genesi. Trad. di C. Brambilla. Milano: Jaca Book.

Sembène, O. (1990). Il fumo della savana. Introduz. di Dacia Maraini, trad. di C.G. Marolda. Roma : Edizioni Lavoro.

Sembène, O. (1991). La Nera di... Traduzione, note e postfazione di L. Cenerini. Palermo : Sellerio.

23 Allusion aux paroles de l'écrivain nigérian Chinua Achebe : « Bear the burd of another experience » se référant au phénomène d'appropriation de l'écrivain africain de la langue importée par le colonisateur européen. Cf. Ashcroft, Griffiths, Tiffin 2007, 16).

24 Dans les milieux de la critique littéraire et de la traductologie, on considère souvent la note une présence 'gênante' où même un signe de l'échec du traducteur. Nicolas Gauthier affirme que l'écriture infrapaginale sacrifie la fiction du récit. Il cite Honoré de Balzac, selon qui la note auctoriale est un « coup d'épingle qui désenfle le ballon du romancier » (Gauthier 2015, 1). Pour Genette (1987, 337), la note est un « coup de pistolet référentiel dans le concert fictionnel ». Umberto Eco, de son côté, en arrive à affirmer : «Ci sono delle perdite che potremmo definire assolute. Sono i casi in cui non è possibile tradurre, e se casi del genere intervengono, poniamo, nel corso di un romanzo, il traduttore ricorre all'ultima ratio, quella di porre una nota a piè di pagina - e la nota a piè di pagina ratifica la sua sconfitta. » $(2003,95)$ 


\section{Critique}

Ashcroft, B. ; Griffiths, G. ; Tiffin, H. (2007). Postcolonial Studies. Key Concepts. 2nd edition. New York: Routledge.

Bandia, P. (2001). « Le concept bermanien de l'étranger dans le prisme de la traduction post-coloniale ». TTR, 14(2), 123-39.

Bandia, P. (à paraître). « Multilinguisme et hétéroglossie littéraire : le point sur l'homogénéisation en traduction ». Interfrancophonies.

Berman, A. (1984). L'épreuve de l'étranger. Culture et traduction dans l'Allemagne romantique. Paris: Gallimard.

Berman, A. (1999). La traduction et la lettre ou l'auberge du lointain. Paris : Seuil.

Bhabha, H. (1994). The Location of Culture. London; New York : Routledge.

Bokiba, A.-P. (2006). Le paratexte dans la littérature africaine francophone. Paris: L'Harmattan.

Bokiba, A.-P. (2007). « La traduction littéraire, vecteur d'interculturalité ». Synergies Chili, 3, 111-17.

Bokiba, A.-P. (2009). « Le français et les langues partenaires: les enjeux de l'édition bilingue ». Cheymol, M. (éd.), Littératures au Sud. Paris : Les archives contemporaines, 83-8.

Bokobza Kahan, M. (2014-2016). «Image d'auteur ». socius : ressource sur le littéraire et le social, 1-8. http://ressources-socius.info/index.php/ lexique/21-lexique/197-image-d-auteur.

Declercq, E. (2011). « ‘Écriture migrante’, 'littérature (im)migrante’, ‘migration literature' : réflexions sur un concept aux contours imprécis ». Revue de littérature comparée, 3, 339, 301-10. https://www.cairn.info/revue-delitterature-comparee-2011-3-page-301.htm

Diop, B.B. (2012). «Écrire entre deux langues. De Doomi Golo aux Petits de la guenon ». Repères-Dorif, 2. https://www.dorif.it/ezine/ezine_articles.php?art_id=40.

Dürrenmatt, J. (2004). « Ce que les notes disent de la fiction ». Lavocat, F. (éd.), Usages et théorie de la fiction: le débat contemporain à l'épreuve des textes anciens (XVI-XVIIIe siècles). Rennes: Presses universitaires de Rennes, 23956. Interférences.

Eco, U. (2003). Dire quasi la stessa cosa. Esperienze di traduzione. Milano : Bompiani.

Edema, A.B. (2004). « Les xénismes dans les romans africains : entre citations, traduction et créativité lexicale ». Le Français en Afrique, 19, 226-43.

Elefante, C. (2012). Traduzione e paratesto. Bologna : Bononia University Press.

Espagne, M. (2013). « La notion de transfert culturel ». Revue Sciences/Lettres, $1,1-9$.

Frìas, J.Y. (2010). «Au seuil de la traduction : la paratraduction ». Naaijkens, T. (ed./éd.), Event or incident/Evénement ou incident. Bern : Peter Lang, 287316.

Fučiková, M. (2011). «Le discours préfaciel et les notes dans les textes de P. Chamoiseau : statut et fonction du paratexte ». Alvès, A. ; Pourchet, M. (éds), Les médiations de l'écrivain : les conditions de la création littéraire. Paris: L'Harmattan, 137-51.

Galisson, R. (1998). Le dictionnaire de noms de marques courants. Essai de lexiculture ordinaire. INaLF (CNRS). Paris : Didier érudition.

Gauthier, N. (2015). « Un masque derrière un masque : la note de bas de page dans les mystères urbains ». Médias 19. http://www.medias19.org/index.php?id=17805. 
Genette, G. (1987). Seuils. Paris : Seuil.

Giordan, H. ; Ricard, A. (éds) (1976). Diglossie et littérature. Bordeaux : Maison des Sciences de l'homme d'Aquitaine.

Grutman, R. (1977). Des langues qui résonnent. L’hétérolinguisme au XIXème siècle québécois. Québec: Fides.

Grutman, R. (2002). «Les motivations de l'hétérolinguisme : réalisme composition, esthétique ». Brugnolo, F. ; Oriolo, V. (a cura di), Eteroglossia e plurilinguismo letterario, vol. 2. Roma : Il Calamo, 329-49.

Gyssels, K. (2007). « Les crises du 'postcolonial' ? Pour une approche comparative ». Revue internationale de politique comparée, 14(1), 151-64.

Lane, P. (2005). «Pour une reconception linguistique du paratexte ». Lane, P. (éd.), Des discours aux textes: modèles et analyses. Rouen: Publications des Universités de Rouen et du Havre, 183-205.

Lungu-Badea, G. (2009). «Remarques sur le concept de culturème ». Translationes, $1,15-78$.

Marot, P. (éd.) (2010). Les textes liminaires. Toulouse : Presses Universitaires du Mirail. CRIBLES essais de littérature.

Mbow, F. (2011). «Paratexte et visée de l'énonciation romanesque en littérature africaine ». Glottopol, 18, 52-67.

Meschonnic, H. (1982). Critique du rythme. Lagrasse : Verdier.

Ngom, O. (2018). « Peut-on se baigner deux fois dans le même fleuve? À propos de l'auto-traduction de Doomi Golo de Boubacar Boris Diop ». E.L.A., $46,45-57$.

Oustinoff, M. (2003-2007). La traduction. Paris: PUF.

Pier, J. (1989). « Pragmatique du paratexte et signification ». Études littéraires, 21(3), 109-18.

Ricœur, P. (1998). «La marque du passé ». Revue de Métaphysique et de Morale, 1, 7-31.

Ricœur, P. (2004). Sur la traduction. Paris : Bayard.

Risterucci-Roudnicky, D. (2008). Introduction à l'analyse des œuvres traduites. Paris : A. Colin.

Schiavone, C. (2008). "Plurilinguismo e francofonia in Senegal : contatto, interferenza e mediazione linguistico-culturale nello spazio francofono ». Interfrancophonies, 2, 1-36, http://www.interfrancophonies.org/ schiavone_08.pdf.

Schiavone, C. (2016). "Les seuils du roman subsaharien francophone : le péritexte entre texte originel et traduction italienne ». Bisconti D. ; Fabiani D. (éds), Regards croisés France-Italie : langues, écritures et cultures. Macerata: EUM, 147-60.

Shreiber, M. (2007). «Transfert culturels et procédés de traduction : l'exemple des réalias ». Lombez C. ; Von Kulessa R. (éds), De la traduction et des transferts culturels. Paris : L'Harmattan, 185-94.

Suchet, M. (2009). Outils pour une traduction postcoloniale. Littératures hétérolingues. Paris: Ed. des archives contemporaines.

Suchet, M. (2014). L'Imaginaire hétérolingue. Ce que nous apprennent les textes à la croisée des langues. Paris : Classiques Garnier.

Tine, A. (1985). « Pour une théorie de la littérature africaine écrite ». Présence Africaine, 134-135, 99-121.

Ting-Toomey, S. ; Dorjee, T. (2019). Communicating across cultures. 2nd edition. New York: The Guilford Press. 
Cristina Schiavone

Le transfert culturel chez Ousmane Sembène : du péritexte auctorial au péritexte traduit

Tymozco, M. (1999). « Postcolonial writing and literary translation ». Bassnett, S. ; Trivedi, H. (éds), Post colonial translation. Theory and practice. London; New York: Routledge, 19-39.

Venuti, L. (1995). The Translator's Invisibility: A History of Translation. London : Routledge.

Wuilmart, F. (2006). «La traduction littéraire : source d'enrichissement de la langue d'accueil ». RiLUne, 4, 141-50. 


\title{
Littérature des Français du Maghreb et métissage linguistique Les cas de Jean Pélégri
et Jean-Pierre Koffel
}

Anna Zoppellari

Università degli Studi di Trieste, Italia

\begin{abstract}
The literary work of Jean Pélégri and Jean-Pierre Koffel presents two different ways of rendering the richness of linguistic mixing in colonial and post-colonial Maghreb. For both of them, it is a question of making the various communities living in the area talk by building a bridge that seems to have its origin in the Mediterranean poetics of the École d'Alger, but which tends to integrate into a profoundly Maghreb literature. Both use a French language marked by the different languages that characterise the Maghreb. However, in Pélégri this attempt originates from the need to respond to the drama of the war and the separation from Algerian territory; in Koffel it starts from the need to respect the codes of the police, making the everyday life and the familiarity of the Moroccan and French speakers of Morocco, but opening up to a linguistic analysis that crosses the lines of the text, almost wanting to mix didactic motivations and meta-textual play.
\end{abstract}

Keywords Pélégri. Koffel. Maghreb. Colonial literature. École d’Alger.

Sommaire 1 Introduction. - 2 Français d'Algérie et Français du Maroc. Une approche culturelle commune? - 3 Deux poétiques similaires ? - 4 Pour conclure.

$\begin{array}{lll} & \text { Peer review } & \\ & \text { Submitted } & 2020-11-20 \\ \text { Edizioni } & \text { Accepted } & 2020-11-26 \\ \text { Ca'Foscari } & \text { Published } & 2020-12-22 \\ & \text { Open access } & \end{array}$

(C) 2020 | @( Creative Commons Attribution 4.0 International Public License 


\section{Introduction}

Le travail sur et à travers la langue constitue un enjeu fondamental de la conscience identitaire qui s'inscrit dans les œuvres littéraires, et comme identification d'un lien étroit avec la norme linguistique, et comme volonté de marquer une distance avec cette même norme. En préfaçant le monumental Français dans l'espace francophone publié sous la direction de Didier de Robillard et de Michel Beniamino en 1993, Albert Valdman rappelait que l'une des implications de l'étude linguistique du français de la francophonie est l'étude des « relations avec les autres idiomes avec lesquels il coexiste », ainsi que « la manière dont il est perçu par divers groupes sociaux » (11). Dans le cas des Français du Maghreb, le français a eu une fonction identitaire assez complexe, voulant signifier à la fois un lien avec l'Hexagone et une particularité par rapport à Paris. En situation coloniale, au moment où le français était la seule langue officielle de l'Algérie, du Maroc et de la Tunisie, le parler des Franco-algériens avait pris « des aspects particuliers » (Lanly 1970, 10) dont l'élément fondamental était, pour Lanly, l'acquisition d'« un vocabulaire nouveau relatif aux choses, aux institutions, aux mœurs et usages du pays » (10). Ce parler franco-algérien vivait en contiguïté avec d'autres langues (l'arabe, le berbère, les français régionaux, les autres langues européennes parlées par des colons d'origine non française, etc.) qui avaient été à la base d'interférences linguistiques dans le français parlé couramment par les colons. Jeanne Duclos a parlé à ce propos d'un « cosmopolitisme européen » $(1992,5)$ et d'une langue française caractérisée par « une coloration particulière » (5). Sur la lignée de Jeanne Duclos, nous éviterons de passer en revue les composantes de ce parler franco-algérien et nous allons retenir l'appellation, assez générale, de «français d'Algérie » (5) car, dans son ampleur, elle permet de couvrir à la fois le registre populaire (normalement appelé 'pataouète') et un registre plus ample qui considère aussi la présence de « mots de l'Administration, des institutions arabes, etc. » (5). Au-delà de toute désignation, ce qui, pour nous, est important, c'est de mettre en évidence que ce français colonial a eu une fonction identitaire pour les Français d'Algérie et du Maghreb originaires des pays européens de la Méditerranée et ayant acquis la nationalité française à la suite des différentes lois promulguées en France durant l'entreprise coloniale. Si le processus de francisation des Européens d'Algérie a été étroitement lié au processus de scolarisation, ${ }^{1}$ il n'a pourtant pas empêché la création d'un véritable « dialecte algérien » (Lanly 1970, 22). Ce dia-

1 Lanly souligne que la scolarisation a touché, au moins « jusqu'en 1944 [...] surtout [...] les Européens », de sorte qu'« en 1954 [...] [1']'assimilation' des nationalités est presque achevée » (Lanly 1970, 13). 
lecte est attesté dans la littérature algérianiste et ne manque pas de toucher les textes de l'École d'Alger. Les œuvres de Louis Bertrand, de Robert Randau, d'Auguste Robinet, dit Musette, d'Edmond Brua et d'autres constituent un exemple intéressant de cette production qui s'est liée à la création d'une conscience identitaire des Français d'Afrique du Nord (notamment d'Algérie). Selon Lanly, ce mixage linguistique avait laissé de côté l'élément arabe. Non que soient absents dans le français d'Afrique du Nord les mots venant de l'arabe (dialectal et classique), mais, s'il est vrai que le substrat arabe a participé à la formation du parler des colons d'Afrique du Nord, Lanly insiste sur l'idée que « la plus grande part des modifications enregistrées par le français colonial est l'œuvre des 'colons' eux-mêmes : elle résulte de la rencontre des Français avec des étrangers qui se sont progressivement francisés » (22). Des recherches plus récentes se sont cependant intéressées à la fonction du français dans les communautés arabophones et berbérophones du Maghreb (colonial et indépendant) et ont reconnu à cette langue une fonction véhiculaire centrée sur la fonction poétique et éthique de la représentation de la pluralité langagière dans l'œuvre de deux écrivains français du Maghreb ; notre recherche reste un peu en marge de ces questions mais s'ancre sur ces études concernant la pluralité linguistique au Maghreb aux niveaux diachronique et synchronique.

L'intérêt des œuvres littéraires de Jean Pélégri et de Jean-Pierre Koffel réside dans leur volonté de donner au mélange linguistique qu'ils mettent en scène une valeur plus ample que celle de la représentation du parler d'une seule communauté, et de construire, au niveau linguistique, sinon la représentation d'un monde pluriel et profondément nord-africain, un pont entre les communautés.

\section{Français d'Algérie et Français du Maroc. Une approche culturelle commune?}

Jean Pélégri naît à Alger en 1920 (« l’année de Dib » comme il le soulignait souvent), dans une ferme de la Mitidja appelée «Haouch el Kateb » (nom prophétique pour sa signification : la Ferme de l’Ecrivain) et située entre l'Arba, Sidi-Moussa et Boughara (ancienne Rovigo). Son père est un colon appartenant à une famille arrivée en Algérie lors de la première vague coloniale, sa mère la fille d'un militaire. L'un de ses oncles, Gustave Pélégri, ingénieur agronome, passionné de botanique, avait construit une grande pépinière dans sa ferme de Sidi-Salem et avait participé à la création du Jardin d'Essai d'Alger. Jean étudie au Lycée d'Alger et obtient une licence de philosophie à l’Université de la même ville. Pendant ce temps, son père doit abandonner la ferme suite à la ruine financière de la famille. Le jeune Jean devient professeur dans plusieurs lycées et collèges de la métropole 
(Hénin-Liétard, Ajaccio). Puis il revient en Algérie et il est nommé au collège du Champs-de-Manœuvres à Alger du $1^{\text {er }}$ octobre $1953 \mathrm{au}$ 21 juin 1956 (Le Boucher 1997, 162). Il sera ensuite professeur à Paris, ville où il vit jusqu'à sa mort survenue en 2003. Toute sa vie durant, il a entretenu un lien fort avec sa terre d'origine tant et si bien que la totalité de son œuvre publiée est consacrée à l'Algérie ou à l'immigration en France.

Jean-Pierre Koffel naît à Casablanca en 1932. Son père est d'origine alsacienne, son parrain est italien; pour cette raison, JeanPierre sera à la fois surnommé 'boche' et 'macaroni'. Quand JeanPierre a 9 ans, sa famille va vivre à Marrakech, où il restera jusqu'en 1962. Il commence en 1954 une longue activité de professeur de français et d'inspecteur au Maroc, pays où il restera toute sa vie, à l'exception de sa dernière année d'activité professionnelle (1992-1993), quand il devra aller vivre à Paris pour l'année obligatoire en France.

Ces notations biographiques nous proposent deux personnalités différentes, mais liées par une condition similaire, celle des Français nés au Maghreb pendant la colonisation, ayant vécu à cheval entre la colonisation et l'acquisition de l'indépendance et vivant la situation paradoxale du 'colon libéral', telle qu'elle a été définie par $\mathrm{Al}$ bert Memmi dans le Portrait du colonisateur. Pour tous les deux, la période de l'acquisition de l'indépendance est une expérience dramatique et nécessaire ; néanmoins, pour Pélégri elle correspond à une séparation presque définitive du terroir et pour Koffel à un enracinement encore plus fort au pays de naissance. Pour tous les deux, le sentiment de refus du colonialisme est total, même si la situation historique et les choix existentiels sont opposés. Pélégri avait pris position contre le régime colonial bien avant la guerre de libération algérienne ; Hervé Sanson a précisé comment Jean Pélégri avait été sensibilisé " aux injustices flagrantes commises envers les Arabes » (Sanson 2007, 44) depuis la fin de la Seconde Guerre Mondiale : " le retour au pays de ses anciens camarades de jeu ayant participé à la libération de la France et la reprise de l'humiliation quotidienne, le grave déficit de reconnaissance malgré les services rendus à la Patrie opèrent une mutation essentielle chez le jeune pied-noir » (44). Lors du déclenchement des luttes pour l'indépendance algérienne et des troubles au Maroc, Koffel s'était engagé sans ambiguïté pour l'indépendance de l'Algérie et des deux protectorats français d'Afrique du nord. En 1958, après la conquête de l'indépendance marocaine, il signera la " motion des $481 »,{ }^{2}$ avec laquelle un groupe de Français du Maghreb foncièrement anticolonialistes demandent l'indépendance immédiate de l'Algérie. Cela dit, il nous semble important de conduire

2 Pour le développement d'un anticolonialisme humanitaire en France et dans les colonies, voir Julien 1979. 
une approche des deux écrivains à partir d'une même perspective en fonction des similitudes qui traversent leur œuvre.

\section{Deux poétiques similaires?}

Avant de nous intéresser aux similitudes qui traversent leurs œuvres, il convient d'en analyser les différences fondamentales. Chez Pélégri, le recours à une langue parlée (ou considérée comme telle) trouve son expression la plus complète dans Le Maboul, le livre de la crise et du renouveau. Dans d'autres textes, Pélégri se pose le problème de la langue du peuple algérien, mais ce n'est qu'avec le Maboul que ses tentatives atteignent le niveau le plus intéressant au point de vue du style. Avec Koffel, le recours à un langage métissé et bariolé est présent dans plusieurs de ses œuvres et nous avons alors affaire à la fois à la langue des pieds-noirs et à la langue des Marocains. Une autre différence se pose au niveau du domaine littéraire, qui est sans autres spécifications chez Pélégri, et s'inscrit dans les marges du concept chez Koffel, écrivain polygraphe, mais qui est surtout connu pour ses polars. Encore, Koffel publie à partir des années 1990 et dans un Maroc désormais indépendant depuis plusieurs décennies, tandis que Pélégri écrit les textes qui nous intéressent alors que l’Algérie est en train de se libérer.

Malgré la différence des conditions, nous considérons qu'il existe des similitudes importantes, notamment dans l'approche tout à fait comparable que les deux écrivains ont de la condition socio-linguistique du français en Afrique du Nord, leur but n'étant pas en fait de délimiter un champ identitaire pour les Français de l'Afrique du Nord, mais de représenter une condition linguistique de pluralité et de mixité à partir de la langue française. De ce point de vue, la critique a souvent mis en relief comment, pour Jean Pélégri, le recours à un français 'pluriel' se fait sur la voie de la rencontre avec l'autre et de l'acceptation de la voix de ce dernier dans le processus narratif. Déjà en 2000, la critique avait rappelé que la langue du Maboul est construite à partir de la volonté de retrouver le langage des deux communautés - arabe et française - qui cohabitaient dans l'Algérie coloniale, cette pluralité linguistique que le jeune Jean avait connue depuis son enfance. "Le Maboul », a affirmé l'écrivain, " est écrit à partir de l'arabe dialectal » (Pélégri 1984, 22). Plus tard, l'écrivain précisera que le personnage qui est à l'origine du héros du Maboul est quelqu'un qui « pense en arabe, mais parle en français » (Pélégri 2007, 134). Nous sommes donc loin de la fonction identitaire d'une langue plus ou moins vernaculaire qui identifie une communauté et nous devons rechercher les raisons de cette expérience linguistique dans la tension éthique qui est à la base de l'ensemble de l'œuvre de Pélégri. Publié en 1963, à savoir une année après l'indépendance 
de l'Algérie, ce roman se ressent de la situation dramatique des dernières années de la lutte pour la libération. On y perçoit une prise de conscience qui sera à la base de la volonté d'écrire «à partir des paroles des uns et des autres » (Pélégri 1990, 48). Ce parcours d'empathie avec l'autre avait été long et, bien sûr, jamais accompli totalement. Le premier moment comporte la rédaction de textes « sur l'injustice coloniale » (Pélégri 1990, 48), la lecture de quelques textes significatifs (La grande maison de Dib, Coran), la naissance d'une « volonté d'écoute » (Pélégri 1990, 48) jusqu'à l'acquisition de la parole des autres. Les Oliviers de la justice, sous ses deux formes, d'abord de roman en 1959 et de film ensuite (1962), constitue à ce niveau l'œuvre charnière où la conscience éthique se traduit en écoute comme le révèlent le poids des personnages arabes et l'accueil de leur parole. Cette étape est presque contemporaine de la rédaction du poème «Les Paroles de la rose ». Présenté comme transcription des mots de Fatima, la domestique arabe des Oliviers de la justice, ce poème prend pour l'écrivain la fonction d'approche du peuple algérien et de sa parole. Pour la première fois, l'écrivain devient le kateb, le scribe qui « assis à l'ombre d'un mur, devant ses plumes et son écritoire, [...] rédige sous la dictée de ceux qui ne savent pas écrire » (Pélégri 1990, 48). Ces deux tentatives d'approche d'une 'parole algéroise' n'atteignent pas cependant la complexité stylistique du Maboul, roman qui se construit à partir du personnage de Slimane (le maboul, autrement dit le fou, du titre), ${ }^{3}$ et où l'instance narrative extradiégétique (toujours à la troisième personne) laisse volontiers la parole à un personnage qui parle parfois à la première personne, parfois à la deuxième. ${ }^{4}$ Dans les deux cas qui précèdent le Maboul, nous avons affaire à une écriture qui reste dans sa substance à l'intérieur d'un français volontairement correct, bien que simplifié. Le tissu linguistique du Maboul, présente plusieurs lignes qui vont de l'utilisation répandue de la métaphore au recours à un lexique et à des expressions prises dans différents niveaux de langage, ainsi que dans différentes langues, ou encore à la désagrégation de l'unité temporelle et narrative du récit : le lexique français est parsemé de mots arabes et berbères, ${ }^{5}$ quelques mots espagnols sont à relever, sans que leur présence soit importante, plusieurs expressions sont enfin calquées sur l'arabe. Un autre élément significatif est constitué par des signes graphiques qui semblent vouloir suggérer une instance

3 Pour la signification du mot Maboul et de sa fonction symbolique à l'intérieur du texte, nous renvoyons à Sanson 2007 et à Zoppellari 1995.

4 Sur cette conscience indéterminée, où les trois personnes s'alternent sans solution de continuité, nous renvoyons à Zoppellari 1995.

5 Nous avons retenu 4 mots berbères et 89 arabes, avec plus de 100 occurrences au total. 
narrative double, l'alternance entre italique et romain, ou des graphismes qui pourraient rappeler, sans les reproduire avec exactitude, des usages espagnols (point d'interrogation - non renversé - en début de phrase). ${ }^{6}$ Les premières critiques qui suivent la publication du Maboul soulignent la particularité d'une langue basée sur « des procédés qui brisent la syntaxe courante du français écrit » (Memmi 1969, 260) ; les éditeurs de l'Anthologie des Écrivains français du Maghreb signalent la présence de phrases qui apparaissent comme des «traductions de l'arabe » (260). André Wursmer publie, en 1964, un article dans Les Lettres françaises où il aborde, le premier sans doute, la question du langage de Slimane dans le Maboul. Sans entrer dans une analyse foncièrement linguistique, Wursmer pose la question du ton et de sa force. "Pélégri a atteint le ton juste » écrit-il, « son Slimane vit, il parle sa langue » (Wurmser in Le Boucher 2000,194). Pélégri, de son côté, parle « d’une expérience particulière de possession » (cit. in Le Boucher 2000, 194). En 1966, Mohammed Did déclare qu’avec ce texte Pélégri avait " créé à son usage une autre langue française » (Dib 1965, 57). Ce qui est significatif, c'est que, dans cette quête d'une langue commune, l'écrivain semble vouloir construire non pas la langue identitaire d'une communauté, mais une langue commune construite sur base française et avec des calques et des recours au lexique des langues de substrat. D’une manière générale, on peut dire que la discussion sur la langue du Maboul se fait autour de la question entre relation de la langue de base (le français) et le substrat (la critique semble s'intéresser peu au fait que ce substrat soit l'arabe dialectal, l'arabe classique ou encore le berbère). La critique reconnaît en général l'effort de l'écrivain qui travaille le français à partir de calques sur l'arabe pour donner la parole à ceux qui ne l'avaient pas et rendre la complexité culturelle et linguistique du terroir. Le même Pélégri écrira des pages très intéressantes sur la pluralité linguistique de son pays natal dans $M a$ mère, l'Algérie et dans Documents sur le déluge où il précisera comment « l'histoire de Slimane [...] nécessitait le recours à un langage nouveau » (Pélégri 1965, 60). Il se sert de nombreux codes typiques de la littérature des voyageurs français et aussi des écrivains français du Maghreb (utilisation de mots arabes et berbères, mots pris dans d'autres langues de l’Europe méditerranéenne, simplification des structures linguistiques françaises), mais il s'adonne à une tentative véritable d'interpénétration des systèmes linguistiques. L'effet général est celui d'une étrangeté langagière qui traduit la stratifica-

6 Sanson $(2007,46)$ avance, quant à lui, l'interprétation suivante : « Le point d'interrogation précède la phrase interrogative elle-même : à la source est la question. Cette nécessité d'en passer par l'autre et sa langue s'articule à l'unicité de la référence, à la singularité d'un espace orienté, l'espace algérien ». 
tion du langage. Un aspect intéressant de cette stratégie de mixage linguistique est que les mots 'étrangers' ne sont mis en évidence par aucun signe graphique (guillemets ou italique) et, parmi ceux-ci, les mots arabes ou berbères ne sont pas accompagnés de traduction, ni dans le texte ni en bas de page, ni à la fin du texte. Il est vrai que le contexte permet le plus souvent de comprendre la signification de ces mots, mais cette insertion dans le fil du discours fait penser à la volonté de mettre en scène le polyglottisme et le babélisme de base.

Face à une écriture qui naît dans l'urgence d'une crise historique et semble s'alimenter du sentiment de dépossession, l'œuvre de Koffel ne connaît pas une genèse aussi teintée de couleurs foncées. Plusieurs facteurs contribuent à rendre moins dramatique la prise de parole, tels les choix politiques du jeune Jean-Pierre au temps des luttes d'indépendance, le contexte culturel différent au moment de la publication des œuvres, le choix du genre littéraire, ainsi que le fait que l'écrivain s'était, désormais, bien intégré au tissu social et économique du Maroc. Koffel écrit - ou en tout cas publie - la plus grande partie de son œuvre entre la fin du XXème et le début du XXIème siècle et, s'il est vrai qu'il a été un écrivain polygraphe (romans, poèmes, nouvelles, essais, édition de recueils, études linguistiques), il s'adonne surtout à l'écriture de romans policiers. Sur le plan de la nationalité littéraire, il faut signaler quelques hésitations de la critique envers l'identification du groupe auquel il appartiendrait. Estelle Maleski a discuté le statut français ou francophone de l'écrivain. La chercheuse a déclaré son incertitude en raison de la nationalité française de l'écrivain, mais elle s'est résolue à le considérer comme un écrivain foncièrement francophone du fait qu'il a toujours vécu au Maroc. Par ailleurs, nous ne pouvons oublier que cet écrivain, qui avait été radié de l'enseignement français au temps de la signature de la motion des 481, et qui a dû attendre 1969 pour y être réintégré, a toujours aimé se définir un écrivain pied-noir et un «Français du Maroc » (Maleski 2003, 320), en acceptant, non sans ironie, sa condition intermédiaire.

Pour ce qui est des œuvres qui nous intéressent, nous avons affaire à une production littéraire dont le but fondamental est de procurer le plaisir immédiat du lecteur, mais qui n'oublie pas de poser un regard attentif, critique et ironique à la fois, sur la société marocaine. Aussi les stratégies narratives tiennent-elles compte tout d'abord de ce présupposé qu'on pourrait définir sous le signe classique de 'plaire et instruire', là où le plaisir est lié à la volonté d'écrire des textes amusants et l'instruction à la volonté de donner un aperçu corrosif d'une société en transformation, surtout en ce qui concerne sa classe moyenne. Il faut d'ailleurs considérer le développement, non négligeable, du roman policier au tournant du siècle et la fascination de la représentation pessimiste d'un monde contemporain où les forces négatives semblent prendre le dessus. Notons encore ra- 
pidement que les héros de Koffel sont le plus souvent des anti-héros et que la police n'arrive pas toujours, ou arrive le plus souvent en retard, à la solution des enquêtes. C'est encore Malenski qui reconnaît que l'œuvre littéraire de cet écrivain s'insère dans le sillage de l'évolution du genre au Maghreb pour sa volonté d'« engage[r] [...] la fiction policière dans une perspective presque documentaire, offrant une tribune à un discours critique engagé se faisant la plupart du temps le vecteur d'une mise en accusation des individus jugés responsables de la crise » (Maleski 2003, 304). Koffel lui-même a plusieurs fois revendiqué son amour pour la littérature noire ainsi que son importance dans le panorama international et son but qui est de " faire réfléchir sur une société et [de] s’amuser beaucoup. Amuser, en caricaturant, en soulevant des ridicules [...], en jouant de bons tours aux salopards objectifs » (Maleski 2003, 322-3). Ces déclarations sont pour nous importantes pour leurs implications dans les choix linguistiques et stylistiques. D’un point de vue général, nous avons affaire à une écriture dont le but est de donner l'effet de réel à travers la langue, en utilisant plusieurs niveaux de styles et, bien sûr, en faisant recours à un lexique et à une morphosyntaxe variés, pour reproduire une situation de pluralité langagière : français familier et populaire, expressions arabes ou berbères, italianismes, hispanismes, tout contribue à donner l'idée d'un métissage en acte. Chaque personnage utilise son style et son langage, mais, dans l'ensemble, nous avons l'image d'une société plurielle, construite à partir de plusieurs identités qui ont, chacune, leur histoire et leur voix.

Le but est de rendre compte, tout en recourant à la langue française, d'un large éventail de langues et de dialectes parlés au Maroc. En général, on retrouve ainsi des variations et des stratégies discursives du français contemporain, du français colonial, la contamination lexicale par le français populaire ou familier, l'argot métropolitain, les dialectes du midi (provençal, toulousain, marseillais), les calques ou les adaptations (phonétiques et/ou graphiques) de mots espagnols et italiens, ainsi que l'adoption de mots originaires du sabir, de l'arabe et du berbère. L'écrivain veut « parler français à la surface, et, sous la couche, arabe dialectal » en utilisant un langage qui ne serait « totalement et spontanément accessibles qu'à des Marocains francophones » (Koffel 2003, 324).

Ce mélange linguistique naît d'une préoccupation à la fois poétique et éthique : il s'agit de donner un aperçu de la situation linguistique qui caractérise le territoire marocain. Soulignons, au passage, que cette approche reproduit, au niveau fictionnel, les préoccupations scientifiques des études qui ont caractérisé la fin du siècle dernier et le début du nouveau siècle, et qui ont abordé la situation linguistique du Maghreb dans sa complexité diachronique et synchronique en mettant en évidence « l'importance du plurilinguisme [et] la labi- 
lité des frontières linguistiques » (Dakhlia 2004). ${ }^{7}$ Sans être directement lié à l'œuvre de Koffel, ce nouveau contexte scientifique et culturel constitue néanmoins un présupposé important pour comprendre la valeur symbolique des problématiques linguistiques mises en œuvre dans les textes narratifs.

Sans entrer dans l'analyse approfondie des textes, nous renvoyons à l'utilisation du berbère marocain dans Rapt à Inezlane ; de la darija et de l'espagnol dans l'aventure rocambolesque de deux jeunes étudiants marocains qui ramènent le cadavre de leur grand-mère au Maroc (C'est ça que Dieu nous a donné, 2003) ou dans l'intrigue internationale Pas de visa pour le paradis d'Allah (1997); du français des colons, teint de pataouète ou de sabir, dans Des Pruneaux dans le tagine (1996); du français des immigrés maghrébins de France, du français populaire, du verlan et du $\mathrm{FCC}^{8}$ dans Nous l'appellerons Mehdi (1994) ; du français des pieds-noirs rapatriés et des anciens d'Algérie dans ce même roman. D'une manière générale, ce qui est significatif, c'est que, paradoxalement, les éléments lexicaux 'étrangers' sont le plus souvent mis en évidence par l'auteur avec l'italique, ou expliqués étymologiquement non seulement dans des notes à la suite du chapitre mais aussi à l'intérieur des dialogues ; les explications acquièrent ainsi un statut narratif et métalinguistique à la fois. Autrement dit, les mots arabes, berbères, ou d'autres langues méditerranéennes, sont soumis à une procédure que l'on peut qualifier de didactique et dont le but est de mettre au centre de l'attention du lecteur la pluralité langagière qui caractérise et a caractérisé le Maroc ; cet aspect revêt d'ailleurs un intérêt certain car il pose le problème du lecteur implicite et des objectifs de l'écrivain.

Si par exemple l'expression Allahou akbar ou le mot oued n'ont pas besoin d'explication, la phrase "c'est cela que Dieu nous a donné », qui donne le titre au roman para-homonyme, est à l'origine d'une longue disquisition qui explique son contexte culturel :

On lui avait servi quasi sur un plateau d'argent les sésames ouvretoi sans quoi elle n'eût pu quitter sa chambrette - ce qu'elle eût d'ailleurs fort bien admis. "C'est cela que Dieu nous a donné », aimait-elle à dire, phrase où s'exprimait toute la résignation qu'on lui avait inculquée. (Koffel 2001, 34)

Ainsi que cette note à la suite du chapitre :

7 Nous renvoyons, à ce propos, aux études menées par l'équipe de l'EHESS et de l'IRMC (Tunis), mais aussi à Dakhlia 2004. L'intérêt de ces dernières recherches est qu'elles s'ancrent dans la volonté de rendre compte du pluralisme langagier au Maghreb, mais aussi de prendre en compte l'héritage colonial et son côté dramatique encore ressenti comme présent dans la conscience langagière.

8 Français Contemporain des Cités. 
La phrase, «c'est ça que Dieu nous a donné » est 'hadchi lli aatana llah', avec variante 'aatani' (m'a donné, à moi, un attributaire plus personnalisé, qui se fond quand même dans le nous collectif, lequel désigne l'ensemble des hommes, ou, plus simplement, la communauté musulmane). Dans certaines régions, on peut ajouter, par dérision : « Ou ssouq », c'est-à-dire : 'Et le souk'. (Koffel 2001, 34)

Les différents textes sont ainsi étoffés de véritables glossaires à fonction divulgatrice et méta-textuelle.

L'écrivain reconnaît l'importance de ce mosaïque linguistique, signe de sa position d'intermédiaire et revendication de la pluralité culturelle du Maroc et du Maghreb :

Oui, je mêle les langues et j'assume un double héritage culturel : la culture classique occidentale, qui prend ses sources dans le grec et le latin et la culture populaire marocaine qui prend ses sources dans l'arabe et le berbère (le tamazight). (Koffel 2003)

\section{Pour conclure}

L'analyse des œuvres narratives de Jean Pélégri et de Jean-Pierre Koffel permet de mettre en valeur une approche comparable de deux questions de fond de la littérature issue du Maghreb : rapport à la norme linguistique et question identitaire. Chez Pélégri, le métissage linguistique représente l'extrême tentative de mettre en scène un lien avec un espace algérien vécu comme perdu à jamais ; chez Koffel, la mise en scène d'un français travaillé par la pluralité des registres de styles et par l'utilisation d'expressions prises dans l'arabe et dans le berbère parlés au Maroc constitue une tentative de représenter un espace quotidien et familier. Une différence fondamentale doit être cependant trouvée au niveau de l'approche et de la pratique de cette pluralité : si l'œuvre de Pélégri peut être considérée comme une tentative qui à la fois réhabilite et s'éloigne d'un pseudo-sabir ${ }^{9}$ qui caractérise une certaine littérature coloniale,,$^{10}$ celle de Koffel présente une approche plus analytique et pseudo-scientifique de la pluralité linguistique. Koffel étudie les différents niveaux de langage des locuteurs qu'il côtoie sans donner à cette pluralité une valeur véritablement éthique : chaque personnage a un langage bien à lui et à lui seul, et c'est au total l'image d'un patchwork linguistique qui se dégage de ses textes ; Pélégri essaie de donner la parole à l'autre et

9 Pour la définition de pseudo-sabir, nous revoyons à Perego 1968, 602-4.

10 Pour approfondir la question des différentes langues présentes dans l'Afrique du Nord au temps de la colonisation, nous renvoyons à Costantini 2018. 
c'est pour le personnage arabe qu'il adopte un langage particulier et libérateur. Chez les deux écrivains, néanmoins, le recours à un langage métissé s'inscrit dans la constitution de poétiques semblables à celle, méditerranéenne, des auteurs de l'École d'Alger, mais elles tendent à s'intégrer dans une littérature foncièrement maghrébine.

\section{Bibliographie}

\section{Fuvres de Jean-Pierre Koffel}

Koffel, J.-P. (1994). Nous l'appellerons Medhi. Casablanca : Le Fennec. Koffel, J.-P. (1996). Des Pruneaux dans le tagine. Casablanca : Le Fennec. Koffel, J.-P. (1997). Pas de visa pour le paradis d'Allah. Casablanca : Le Fennec. Koffel, J.-P. (1998a). La Cavale assassinée. Casablanca : Traces du Présent. Koffel, J.-P. (1998b). Rapt à Inezlane. Casablanca : Le Fennec. Rééd. 2002. Koffel, J.-P. (1999). L'Inspecteur Kamal fait chou blanc. Casablanca : Le Fennec. Koffel, J.-P. (2001). C'est ça que Dieu nous a donné. Rabat : Marsam. Koffel, J.-P. (2003). «Entretient ». Maleski 2003, 478-90.

\section{Fuvres de Jean Pélégri}

Pélégri, J. (1952). L'Embarquement du lundi. Paris : Gallimard. Rééd. Paris : Sindbad 1997.

Pélégri, J. (1959). Les Oliviers de la justice. Paris : Gallimard.

Pélégri, J. (1963). Le Maboul. Paris: Gallimard.

Pélégri, J. (1965). « Huit documents du déluge ». Afrique, 48, 60-4.

Pélégri, J. (1967). Les Monuments du déluge. Paris : Christian Bourgois.

Pélégri, J. (1972). Le Cheval dans la ville. Paris: Gallimard.

Pélégri, J. (1984). «Interview accordée à la Revue CELFAN ». CELFAN, 3(3), 20-3.

Pélégri, J. [1989] (1990). Ma mère l'Algérie. Arles : Actes Sud (suivi de « Les paroles de la rose ", 8 poèmes de 1956).

Pélégri, J. (1999). Les Étés perdus. Paris : Le Seuil.

Pélégri, J. (2007). «Interview ». Expressions maghrébines, 6(2), 133-50.

\section{Critique}

Costantini, A. (2018). « De la littérature dite sabir. Regards coloniaux divers sur l'Autre ». Annali di Ca' Foscari. Serie occidentale, 52, 141-74. http://doi. org/10.30687/Ann0c/2499-1562/2018/01/०07.

Dakhlia, J. (éd.) (2004). Trames de langues: Usages et métissages linguistiques dans l'histoire du Maghreb. Nouvelle édition. Rabat : Institut de recherche

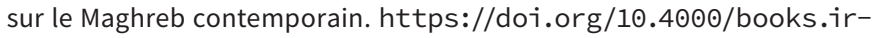
mc. 1446.

Dib, M. (1965). « Le Maboul rétablit l'équilibre avec L'Etranger ». Afrique, 48, 57.

Duclos, J. (1992). Dictionnaire du français d'Algérie. Français colonial, pataouète, français des Pieds-Noirs. Paris : Bonnetton.

Julien, Ch.-A. (1979). Une pensée anti-coloniale-Positions 1914-1975, avec la collaboration de M. Morsy. Paris : Sindbad.

Lanly, A. (1970). Le français d'Afrique du Nord. Étude linguistique. Paris: Bordas. 
Le Boucher, D. (2000). Jean Pélégri l'Algérien ou Le Scribe du Caillou. Paris ; Alger : Marsa. Algérie Littérature / Action 37-38.

Maleski, E. (2003). Le roman policier à l'épreuve des littératures Francophones des Antilles et du Maghreb [thèse de doctorat]. Bordeaux: Université Michel de Montaigne-Bordeaux III. http://www. Limag.com/Theses/Maleski.htm.

Memmi, A. (1957). Portrait du colonisé précédé du portrait du colonisateur. Préf. de J.-P. Sartre. Paris : Buchet-Chastel.

Memmi, A. (1969). Anthologie des écrivains Français du Maghreb. Paris : Présence Africaine.

Perego, P. (1968). « Les sabirs ». Martinet, A. (éd.), Le langage. Paris : Gallimard, 597-607.

Robillard, D. de ; Beniamino, M. (éds) (2010). Le français dans l'espace francophone. Paris: Honoré Champion.

Sanson, H. (2007). « Jean Pélégri ou le piège de la langue : relater la relation ». Zoppellari 2007, 43-50.

Zoppellari, A. (1995). « La terre et l'écriture. Analyse du Maboul de Jean Pélégri ». Ruhe, E. (Hrsg.), Europas islamische Nachbarn : Studien zur Literatur und Geschichte des Maghreb, Bd. 2. Würzburg: Königshausen \& Neumann. Zoppellari, A. (éd.) (2007). Jean Pélégri. Expressions maghrébines, 6(2), 1-178. 



\title{
Language Colonization and English Hybridization: The Use of Irish English Lexis in Twentieth Century Irish Drama
}

\section{Fabio Luppi}

Università degli Studi Roma Tre, Italia

\begin{abstract}
Irish English, albeit a variant of the language of the colonizer, can be considered an important identitarian element in twentieth century Irish literature. By taking into account Irish English terms in a selection of Anglo-Irish plays (with particular focus on the titles), this paper examines the lexical choices that contribute to rendering cultural, geographical and political meanings - some of which are derogatory, patronizing and pejorative. The conclusion, with reference to Brian Friel's Translations, reflects on the implications of the dominance of English in Ireland following its replacement of Irish Gaelic. The various loanwords and calques from Irish Gaelic found in these plays thus acquire a cultural and political significance that is specific to the Irish context.
\end{abstract}

Keywords Irish English. Drama. Lexis. Language Colonization. Cultural Identity.

Summary 1 English Linguistic Imperialism and Ireland. - 2 Geographical Connotations: Belonging to a Place. - 3 Derogatory and Patronizing Words Referring to People's Actions / Behaviours. - 4 Language and / Is Politics. - 5 Language, Progress and Identity. - 6 Conclusions.

$\begin{array}{lll} & \text { Peer review } & \\ & \text { Submitted } & 2020-08-12 \\ \text { Edizioni } & \text { Accepted } & 2020-09-20 \\ \text { Ca'Foscari } & \text { Published } & 2020-12-22 \\ & \text { Open access } & \end{array}$

(C) 2020 | (a) Creative Commons Attribution 4.0 International Public License 


\section{$1 \quad$ English Linguistic Imperialism and Ireland}

English political and economic dominance in Ireland was matched by a cultural and linguistic exploitation with the imperialistic aim of assimilating the Irish ethnic group which was to be absorbed into the dominant (English) culture and language, while possibly remaining subordinate to the colonizers. By the end of the 19th century, this also produced two literatures of modern Ireland that, paradoxically, emerged from the translation movement - the translation of early Irish literature into English (cf. Cronin 1996, 138). Mediating between the two languages,

translation [meant] an act of pillage and conquest, or [maybe] a bisociative shift towards a newer, more exciting synthesis. (Cronin 1996, 142)

From the Renaissance, and the Republican experience (with Oliver Cromwell), knowledge of English was increasingly necessary to

deal with local administrations and courts of law, to follow national politics and to communicate with landlords who were mainly English-speaking Protestants. (Binelli 2010, 38)

As bilingualism became a necessity, Irish became more and more marginal. Undoubtedly, the National Schools System of 1831, which had banned Irish from schools, had an enormous impact. This trend increased significantly during the Great Famine, when whole swathes of the population died from starvation and thousands of families (many of whom were Irish Gaelic speaking) were forced to emigrate. In these years (1845-1849) at least 1.5 million native speakers either emigrated or died. Furthermore, as Eagleton (1995, 12) reports, while there were still many areas of rural Ireland after the famine where Irish was spoken, it was often eschewed as it was thought to bring bad luck. Moreover,

children who spoke their native tongue were routinely punished and made to wear a "tally stick" around their neck which counted the number of times they had accidentally reverted to their mother tongue. This led to the stereotyping of the Irish language as backwards and associated with the lower classes. (Ní Scolláin 2017, 240)

In conclusion,

the major reason for the advance of English and the contraction of Gaelic - the two processes were practically if not necessarily 
linked - was the desire to survive in the modern world, or better still to improve one's lot. (MacDonagh 1983, 104)

In Ireland, English was increasingly perceived as "a fateful necessity for progress" (MacGiolla Chríost 2005, 101) and "a symbol for opportunity and success" (Filppula 2002, 9).

The Empire recognized British Standard English as the sole variety for administrative and official purposes, while the two main regional variants in Ireland - Ulster-English in the North and HibernoEnglish in the South - had no official recognition, though they were spoken and extensively used in literature. Hiberno-English was heavily influenced in its vocabulary, pronunciation and syntax by Irish Gaelic, that found some redress over the language of the colonizer, adapting it to the new colony and its linguistic needs. This was the case with the emergence of culture-bound words - terms encoding typical or valid information within a specific culture (place and time). ${ }^{1}$ Indeed, the assumption that there is a fundamental link between language and culture is what underpins the present paper.

However, Irish Gaelic could also influence the language of the colonizer:

Even in the parts of Ireland where Irish has long been extinct its unconscious influence still controls the usage of speakers of English. (Bliss 1984, 150)

Furthermore,

Hiberno-English has evolved a vocabulary of its own that reflects every aspect of life in Ireland. (Wall 2001, 13)

The inclusion of Irish loanwords in Irish English had been an ongoing process in the bilingual Irish context since the Middle Ages. Though Irish-born speakers could not seamlessly shift between Irish and English, they could combine

elements from both languages for the purpose of mutual comprehension by the Irish and English populations. (Cesiri 2012, 39)

Thus, even though Irish Gaelic had to bow to English as the dominating language, it also had its linguistic rearguard revenge, hybridizing English, and enriching the language of the colonizers through borrowings, calques and loanwords that were common in the Irish English variant. 
Nevertheless, English was increasingly perceived in the last century as a means of getting on, and necessary when encountering the practicalities of public life. The symbolic dominance of English over its next-door colony/neighbour did not prevent a strong identitarian linguistic sense from persisting, even in the English variety defined as Hiberno-English, Anglo-Irish or Irish English. With the Celtic Revival the question of language became culturally crucial - with George Moore celebrating Irish as a language fresh from the Middle Ages and W.B. Yeats noting that English was the language of newspapers and materialism.

By providing an analysis of occurrences of Irish English words from a selection of Irish plays written in different moments of twentieth century, this paper aims to demonstrate how lexical choices imply deliberate political, cultural and social meanings. The examples taken into consideration can be divided into five categories:

1. loans from Irish (in texts adapted to English orthography);

2. retention of $M E$ or EModE terms (words that had become obsolete in England);

3. English words with a different meaning to the one they had in England (often influenced by Irish);

4. words borrowed from other varieties of English (generally from Scots or northern English dialects);

5. hybrid forms with an English stem and an Irish suffix. ${ }^{2}$

In the following sections I will provide non-systematic illustrations of these items.

\section{Geographical Connotations: Belonging to a Place}

The use of words of Irish origin with strong geographical connotations denotes intentional stress on national belonging and identity. This is exemplified in the titles of two masterpieces of Irish theatre: John Millington Synge's In the Shadow of the Glen (1903) and Marina Carr's more recent By the Bog of Cats (1998). A glen is "a secluded narrow valley" (Merriam-Webster Dictionary) or a "deep narrow valley, especially among mountains" (Cambridge Dictionary). Its origin is "late 15c., from Scottish, from Gaelic gleann 'mountain valley' (cognate with Old Irish glenn, Welsh glyn)" (Online Etymology Dictionary) and, more specifically, "from Scottish Gaelic \& Irish gleann, from Old Irish glenn" (Merriam-Webster Dictionary). The word is not simply synonymous with 'valley'. Indeed, had Synge desired, he would have undoubtedly chosen the more common English word. On 
the contrary, the author's specific intention was to connotate the setting geographically.

Carr's By the Bog of Cats echoes Synge's title, even if it cannot be considered a precise equivalent, as the word bog is English and so can refer to English places too. However, the etymology of the word is Irish Gaelic: the Middle English bog actually derives from the Irish and Scottish Gaelic bogach (soft, boggy ground) from the Old Irish bog (meaning soft). Though the word bog might technically be excluded from our discourse as it only obliquely comes from Gaelic, its use in Carr's title recalls Synge's title in both its rhythm and structure. Both titles open with a preposition of place indicating the setting, and they both exploit the same syntagmatic sequence comprising a head ("shadow" / "bog") and a post-modifier: a prepositional phrase modifying the noun ("glen" / "cats"). This is a particularly appealing choice with regard to titles. In a list of Irish plays staged and written in the twentieth century, these two titles are the only instances (excluding proper names of places or people) that follow this structure and include geographical (though unspecified) references. Significantly, the only similar titles in the aforementioned list of approximately 1,000 plays listed in the well-documented website www. irishplayography.com are:

- The Moon in the Yellow River (Denis Johnson, 1931) where the reference to a place is vague and there is no Irish origin to the terms used. While it might be argued that there is a geographical connotation, the title is, in fact, a quote from Ezra Pound and does not refer to specific Irish contexts;

- The Grand House in the City (Brinsley MacNamara, 1936), which does not contain a reference to an Anglo-Irish word, though the expression Grand House clearly indicates the Big Houses of the Ascendancy in Ireland;

- Down the Heather Glen (Joseph Tomelty, 1953) where, despite the reference to glen, there is no real post modifier;

- I'm Getting out of this Kip (Heno Magee, 1972), a title that is different from those discussed here. The interesting feature here is the use of the Irish slang kip, meaning either a "dump of a place" or "to sleep". Its Irish origin and early use in fictional works are of interest, and are worth quoting:

It was first recorded in the middle of the eighteenth century as an Irish slang term for a brothel. The earliest example known is from Oliver Goldsmith's The Vicar of Wakefield. As Goldsmith was Irish, educated in Dublin, the implication is that the word was first used in that city. It has long continued to be used there in that way." ${ }^{3}$ 


\section{Derogatory and Patronizing Words Referring to People's Actions / Behaviours}

Two other interesting play titles including Gaelicisms refer to people's actions. In so doing they contribute to giving a negative view of Irish society, focusing on elements that convey a sense either of dishonesty or of irresponsible behaviour. The first example of a play with such a title is Dion Boucicault's The Shaugraun (1874). The Anglo-Irish word shaugraun (also spelt shaughraun, shoughraun, shaughrawn) comes from the Irish seachrán, also seacharán, meaning a wanderer, a vagabond, though it can refer to wandering mentally too. It can also be used in the form of to be on the shaugraun ('to wander' cf. Dolan 2006, 205). ${ }^{4}$ The title character of Boucicault's play is a roguish poacher and very much a comic figure - he is not a negative character, although he responds to the stereotype of the Irish hustler with his typical Stage Irish or Paddywhackery. It is an image that is informed by patronizing and at times contemptuous colonial attitudes.

Another play title that exploits an Irish English term is R.J. Ray's The Gombeen Man (1913), gombeen (man) being a Hiberno-English term used to describe small grocers, hucksters or moneylenders, who profit from the misfortunes of others during times of shortage. ${ }^{5}$ Its origin is the Irish word gaimbín, meaning monetary interest. The term originally referred to a moneylender and then became associated with the shopkeepers and tradespeople who exploited the starving during the Famine by selling much-needed food and goods on credit at ruinous interest rates. It is thus a pejorative word, and, significantly, has given rise to the expression Gombeneesim and Gombeenery. As the term has evolved and acquired a specific meaning in a precise historical context, it should be viewed from a cultural rather than an etymological perspective (bearing in mind Raymond Williams' seminal Keywords). The word gombeen man has been

more recently applied to a small-town or rural entrepreneur involved in a number of enterprises, and to the type of political and economic opportunist who emerged following the struggle for Irish independence. (Welch 1999, 186)

The word stands for a criminal, a usurer, but also for the typical person who made a fortune at the time when Ray was writing his play. This clearly indicates that the author himself was drawing a negative portrait of a particular Irishman, a fraudster taking advantage of others' misfortunes. In historical periods such as the Great Fam- 
ine or the years preceding Irish independence, such dishonest, or simply selfish, conduct reflects an anti-nationalist attitude, and demonstrates disrespect for the safety and welfare of the community. A similar case is found in Edward F. Barret's play The Grabber (1918), a grabber being a person who "took over the land of an evicted tenant farmer, esp. during the Land War, 1879-82 (pej.)” (Wall 2001, 189). Its source is evidently English ${ }^{6}$ but its distinctive use in Ireland is closely linked to a specific moment of Irish history.

Both Boucicault's The Shaugraun and Ray's The Gombeen Man - referring to people and their anything but edifying behaviour - exploit negative colonial stereotypes of supposedly typical subaltern (colonized Irish) characters. The former adopts an apparently benevolent (though actually patronizing) attitude towards the title character. The latter realistically portrays the miseries of the new Irish landowners who did not manage to keep hold of their properties, falling victim to opportunistic moneylenders. Here the use of Irish English apparently helps to consolidate negative stereotypes, suggesting that certain misconduct is typically Irish ${ }^{7}$ and conversely stigmatizing certain characters who do not support Ireland in times of uncertainty. Such stereotypes have long been the focus of sociolinguistic studies. Labov defines them as

variants that are readily perceived and commented on (rightly or wrongly) as salient in the speech of particular social or ethnic groups, nationalities, etc. (Labov in Furkó 2013, 124)

The term is similarly defined in social psychology, that is, as a set

of beliefs shared by in-group members about how one's own and other groups are characterized by certain traits or behavioural tendencies which may be positive or negative. (Bourhis, Maass 2005, 1587)

It is clear that the Irish English variant is used here as a way to

categorize events, infer intent, and derive expectations about what [stereotyping] is likely to ensue. (Gumperz 1982, 130) ${ }^{8}$

6 This particular word has remained almost unchanged since Proto-Indo-European (source also of Sanskrit, Avestan, and Old Persian) through to Germanic languages and hence English (cf. Online Etymology Dictionary: https://www.etymonline.com/ search?q=grab).

7 However, despite the use of stereotyped Irish characterization, Boucicault revolutionized the way Irish people were portrayed on the British stage. The author's cautious political position towards Irish Nationalism clearly emerges from such characterization. One more necessary remark is that his plays were not specifically meant for an Irish audience.

8 Cf. Furkó 2013, 125. 
Another title based on an idiomatic expression to refer to a person is Lennox Robinson's The Whiteheaded Boy (1916). White-head (-headed, cann-a-bawn, ceann ban) means 'favourite, i.e., it is a calque (or loantranslation) from the Irish ceann-bhán, meaning white (fair) head. ${ }^{9}$ In the play, the young man Denis Geoghegan - who has always been overprotected as the youngest child of the family - tells his older brothers that he wants to live his life his own way. Denis feels that he needs to be self-sufficient, without any family demands. "I want to be independent" he says, and adds later, "I only want to be able to do what I like with my own life" (Robinson 1982, 112). His brother Duffy comments on this, adopting a telling simile:

Free?... Bedad, isn't he like old Ireland asking for freedom, and we're like the fools of Englishmen offering him every bloody thing except the one thing? (Robinson 1982, 114)

The relationship between Denis and his family mirrors Ireland's claim for independence and British rule. The title, like that of Boucicault's play, presents a stereotype of something that is typically Irish. The connotations are that Ireland itself cannot yet be self-sufficient: it is like a young boy, the youngest of the family, who must be protected / directed - and implicitly patronized and controlled.

\section{$4 \quad$ Language and / Is Politics}

Language is politics: two other examples from Synge's Playboy of the Western World (1907) can be quoted here. They both refer to words that do not derive from Gaelic, but are of particular interest when used in a specifically Irish context as they communicate political ideas and intent. In order to be fully understood, these terms require the reader / audience to be aware of a specific historical period. The female protagonist, Pegeen Mike, says that if she had a brave fiancé like Christy Mahon in her house, she "wouldn't be fearing the loosed khaki cut-throats, or the walking dead" (Synge 2008, 78). While it can easily be inferred that the expression khaki cut-throats refers to violent people of some sort, the reader might think that the 'khaki' refers to the uniform of convicts or some other kind of persona non grata. In fact, Italian translators have often mistaken or simplified this item, interpreting it as a reference to criminals and their prison uniform. They failed to grasp, or perhaps decided that it was impos-

9 Cf. Wall 2001, 363-4. A similar title is Dion Boucicault's The Colleen Bawn (1860). This expression means a girl, a young woman, deriving from the Irish cailín bán, meaning fair girl (cf. Dolan 2006, 60; Wall 2001, 116-17). 
sible to convey, that 'khaki' is a reference to British soldiers. When the play was written, the connotations were so clear that Lady Gregory (one of the directors of the Abbey Theatre) mentioned a request from the English censor to remove all reference to it from the text because it was considered derogatory to His Majesty's Armed Forces (Gregory 1977, 55). The expression, therefore, makes Pegeen Mike's political stance crystal clear.

In the same text, Pegeen Mike and others use a similar expression that also has political overtones: peeler. ${ }^{10}$ According to Collins dictionary, this is a (old-fashioned) British and Irish slang word for policeman, derived from the surname of Sir Robert Peel, ${ }^{11}$ founder of the Royal Irish Constabulary, one of the two police forces active in Ireland from 1822 until 1922. This expression occurs twelve times in Synge's play, and it is also mentioned by Christy in an utterance that is very similar to the one quoted above. He says:

Oh, glory! It's late for knocking, and this last while I'm in terror of the peelers, and the walking dead. (Synge 2008, 83)

Considering the contexts in which the word occurs, and the adjectives associated with it, what clearly emerges is that (British) authority is seen with mistrust and fear (the word fear and its synonyms are associated with the expression peeler on three occasions), ${ }^{12}$ but also defiance: Christy's father was taken to an asylum for "battering peelers" (82) while a man Pegeen admired is said to have "knocked the eye from a peeler" (70). Generally speaking, however, the term signals punitive authority. ${ }^{13}$ It is thus possible to conclude that in an Irish context both terms (khaki cut-throats and peeler) are extremely partisan and have strong connotative meanings that go beyond any simple reference to the thing described. Indeed, such expressions position the characters who use them politically.

11 Significantly, both slang terms for policemen, "Bobby" and "Peeler", derive from his name: Robert and Peel. Peel also established the Metropolitan Police Service in London in 1829.

12 "The peelers is fearing him" (Synge 2008, 78); "If they're not fearing you, itself, the peelers, in this place [...]" (78).

13 Christy's antagonist tells him, "Come on to the peelers, till they stretch you now" (Synge 2008, 119); Christy himself tells his father that the crowd wants to take him to the police: "they're taking me to the peelers to have me hanged for slaying you" (120) 


\section{$5 \quad$ Language, Progress and Identity}

When comparing English to Irish, a linguistic controversy with two opposing factions often arises: those in favor of the new language, who see it as a symbol of progress, and those who view it as a Trojan horse, helping the Empire to usurp - by means of language substitution - the Irish cultural identity. This controversy is made explicit in two other twentieth century Irish plays: George Shiels' The New Gossoon (1930) and Brian Friel's Translations (1980).

Luke Cary - the protagonist of Shiels' play - is the new (modern) gossoon (lad). He is sadly conscious of his origins: an old and morally conservative nation, albeit a young one. A sociolinguistic analysis of the title of this play reveals more than one might expect. The word gossoon is the anglicization of the Irish gasún or garsún, (supposedly from the Old French garçun, doublet of garçon).

[I]t has been fancifully claimed that the French root of this word may indicate the practice of Anglo-Norman gentry calling their Irish serving boys garçon. (Dolan 2006, 112)

[Yet,] the true Irish word is gossure, derived from gos [gas], a branch, and ur, [úr] 'young', and means literally a young shoot. (Dolan 2006, 104)

The fact that the lad is "new" means that he represents modernity. Indeed, the whole plot turns on the conflict between old and new generations. Peter Cary, the protagonist's uncle, is the representative of the old generation called in to rein in the youngsters (i.e., his nephew) and put a brake on corrupting progress, as represented by machinery, new means of transport, leisure and clothes brought in from the city. As he exclaims,

This country's going to hell at a hundred miles an hour! Petrol and pictures and potheen ${ }^{14}$ and jazz and doles and buses and bare legs and all sorts of foreign rascalities. (Shiels 1954, 259)

Luke's mother Ellen also constantly uses words that stress age differences, such as "my old-fashioned ears" (203) or when referring to herself as "the old-fashioned fool mother" (207). Moreover, Ellen highlights the different terms used by young and older generations:

14 The word potheen is also interesting here. An Irish English word deriving from the English word pot, it originally referred to the illegal Irish drink made from potatoes and brewed in the mountains. It was then used to describe any illegally distilled spirits. Potheen (poitín) was traditionally distilled in a small pot; the term is a diminutive of the Irish pota, meaning "pot" (cf. Binelli 2010, 47). 
"Sally calls you a rotter. But I call you by the old Irish name - a rascal!" (207). It is not quite clear why Ellen says that the word rascal is Irish. What is clear is that she also plays the game of code switching to highlight the difference between what is new and what is old. And so significantly, the old name she uses is - in her view - Irish. For her and many others, things that are Irish are positive, while English words represent novelty and corruption.

While it is true that Luke represents immaturity, he also represents a chance for renewal, and although Peter and Ellen stand for wisdom, this also means stasis. Other characters render this apparently neat opposition in less obvious ways: Ned Shay, the serving man, says that

the changes I see are all for the better. I like to see the big bus stopping at the end of the road to lift the kids for school. (Shiels 1954, 260)

Similarly, Sally Hamil, Luke's fiancée, is described as young and modern: the stage directions describe her as a twenty-year-old, wearing a "bright jumper and coloured skirt", with "newly waved" hair and "smoking a cigarette" (193). Yet it turns out that she is a responsible and serious young woman, the only one capable of putting Luke - and his carefree but aimless life - straight. In The New Gossoon, despite the common view that the young generation seems modern and careless, the young couple finally ends up following a conscientious disciplined life based on hard work and sanctified by marriage. In this case, the contraposition between Irish and English is softened by a happy ending.

The situation described in Friel's Translations is much more complex. Furthermore, it can also provide theoretical underpinning to the examples under discussion. The play is built upon the same clash between modernity and the past, again reflected in the languages of colonizer and colonized. It is set in 1833 in the fictional Irish-speaking town of Baile Beag (supposedly in County Donegal). Two British soldiers, Lieutenant Yolland and Captain Lancey - in charge of mapping the Baile Beag area - are working with Owen, an Irishman. Owen has been taken on as an interpreter to help the soldiers rename local place-names by translating them into English, adjusting them semantically and/or phonetically as necessary. Significantly, Owen gives much credit to this operation:

my job is to translate the quaint, archaic tongue you people persist in speaking into the King's good English. (Friel 2016, 442) 
Hugh (the headmaster of the local hedge school ${ }^{15}$ and Owen's father) is a wise man who loves languages (including Irish, Latin and Ancient Greek), but he is also resigned to the British operation of changing place-names. One of his speeches is worth quoting, in that he paraphrases from George Steiner's After Babel in a crucial passage:

You'll find, sir, that certain cultures expend on their vocabularies and syntax acquisitive energies and ostentations entirely lacking in their material lives. [...] But remember that words are signals, counters. They are not immortal. And it can happen - to use an image you'll understand - it can happen that a civilization can be imprisoned in a linguistic contour which no longer matches the landscape of... fact. (Friel 2016, 457-9)

This very much mirrors Steiner:

In certain civilizations there come epochs in which syntax stiffens, in which the available resources of live perception and restatement wither. Words seem to go dead under the weight of sanctified usage; the frequence [sic] and sclerotic force of clichés, of unexamined similes, of worn tropes increases. Instead of acting as a living membrane grammar and vocabulary become a barrier to new feeling. A civilization is imprisoned in a linguistic contour which no longer matches, or matches only at certain ritual, arbitrary points, the changing landscape of facts. (Steiner 1980, 21-2)

Maire, the local milkmaid and a student at the hedge school, also states explicitly that in her view Irish Gaelic is an obstacle to progress. She quotes the moderate Irish leader Daniel O'Connell who said that "the old language is a barrier to modern progress" (Friel 2016, 437). According to Owen and Maire, English is a symbol of progress and such imperialistic linguistic domination is for the better. However, real linguistic understanding, based on real linguistic (and sociolinguistic) awareness, is found in the character of Yolland. He realizes that a language is not an abstract code, but is imbued with the culture that has produced it:

Even if I did speak Irish I'd always be an outsider here [...] I may learn the password but the language of the tribe will always elude me [...] The private core will always be... hermetic. (Friel 2016, 456)

15 Hedge schools were small informal illegal schools for children of non-conforming faiths (Catholic and Presbyterian). They emerged in the $18^{\text {th }}$ century as a response to the prohibition of Catholic education in Ireland. Significantly, Friel's play is set in a hedge school, and its subject is the defense of Irish culture against a cultural (and linguistic) colonialism. 
Given that language is rooted in a cultural tradition, there will always be an idiolect that remains obscure to the non-native speaker (cf. McGrath 1989, 41). Yolland anticipates Lotman's concept of semiotic space, or semiosphere (coined in 1984), where communication is seen as a unified mechanism (if not organism) implicit in a community that shares not only a linguistic code, but also the cultural means to decode all the signs that contribute, along with language, creating a cultural sphere. The perception of the world varies according to the language used, and even the same language in a variant form such as Irish English may well imply a different perspective of, and referentiality to, reality. This explains Hugh's comment that

it is not the literal past, the 'facts' of history, that shape us, but images of the past embodied in language. (Friel 2016, 490)

Yolland also realizes that "something is being eroded" (459) with the British operation of anglicizing place names and substituting Irish with English: this 'something' is the layered meaning that words have acquired over the centuries.

\section{Conclusions}

As we have seen, language is perceived as a fundamental identitarian feature expressed as a linguistic variant endowed with borrowings, calques and Irish English terms that necessarily refer to a specific Irish context. Sometimes it is also possible to find almost imperceptible traces in certain Irish English words that are permeated by the Gaelic substratum. These may have thus acquired a dual (or more complex) meaning that might well be opaque to nonIrish speakers. Such words create an ambiguity between Standard English and Irish English, thus producing specific nuances, which require investigation. Declan Kiberd provides an example of this, quoting from Synge's Riders to the Sea (1904). When a character (Bartley) leaves for a long and dangerous journey and does not take food with him, Nora (the protagonist) comments:

And it's destroyed he'll be going till dark night, and he after eating nothing since the sun went up. (Synge 2008, 20)

The adjective destroyed in Irish English has a milder meaning than in Standard English as it is influenced by Gaelic. In Gaelic it would merely mean

being 'destroyed with hunger, thirst, work, etc.' a sense which normally denotes great discomfort. (Kiberd 1993, 81) 
In English the word evokes much darker images, of dissolution and even death. In this context Synge uses a word that when read as Irish English does not sound particularly negative, but if read as Standard English prophetically foregrounds the inauspicious events of the whole play.

To conclude, we might paraphrase Loreto Todd's final remarks in her seminal work The Language of Irish Literature. Although Todd refers to broader linguistic features and not just to lexis, she notes that the selection of Irish English words is not a superficial linguistic choice, but the "expression of a world-view and a signal of national and cultural identity" (Todd 1989, 145) that is often opposed to colonialist or anti-nationalist language.

\section{Bibliography}

Binelli, A. (2010). "Irish English and Dublin English in Damien Dempsey’s Lyrics". Palusci, O. (ed.), English But Not Quite. Locating Linguistic Diversity. Trento: Tangram Edizioni Scientifiche, 37-55.

Binelli, A. (2013). Lingua, semiologia e traduzione dall'inglese. Trento: Tangram edizioni scientifiche.

Bliss, A.J. (1984). "English in the South of Ireland". Trudgill, P. (ed.), Language in the British Isles. Cambridge: Cambridge University Press, 135-51.

Bourhis, R.Y.; Maass, A. (2005). "Linguistic Prejudice and Stereotypes. No. 155". Ammon, U.; Dittmar, N.; Mattheier, K.J.; Trudgill, P. (eds), Sociolinguistics: An International Handbook of the Science of Language and Society. Berlin; New York: Walter de Gruyter, 1587-602.

Cesiri, D. (2012). Nineteenth-Century Irish English: A Corpus-Based Linguistic and Discursive Analysis. Lampeter: The Edwin Mellen Press.

Cronin, M. (1996). Translating Ireland. Translation, Languages, Culture. Cork: Cork University Press.

Dolan, T.P. (2006). A Dictionary of Hiberno-English: The Irish Use of English. Dublin: Gill and Macmillan.

Eagleton, T. (1995). Heathcliff and the Great Hunger. Studies in Irish Culture. London; New York: Verso.

Filppula, M. (2002). The Grammar of Irish English: Language in Hibernian Style. London: Routledge.

Friel, B. (2016). Collected Plays 2. London: Faber and Faber.

Furkó, B.P. (2013). "Irish-English Stereotypes. A Variational Pragmatic Analysis". Acta Universitatis Sapientiae, Philologica, 5(2), 123-35.

Garvin, T. (1987). "The Politics of Language and Literature in Pre-independence Ireland". Irish Political Studies, 2, 49-63.

Gregory, A. (1977). Interviews and Recollections. Edited by E.H. Mikhail. London: Springer.

Gumperz, J. (1982). Discourse Strategies. Cambridge: Cambridge University Press.

Kiberd, D. (1993). Synge and the Irish Language. London: Palgrave Macmillan.

Labov, W. (1972). Sociolinguistic Patterns. Philadelphia: Pennsylvania University Press. 
Lotman, Y.M. (1984). "O semiosfere". Sign Systems Studies (Trudy Po Znakovym Sistemam), vol. 17. Tartu: University of Tartu Press, 5-23.

MacDonagh, O. (1983). States of Mind: A Study of Anglo-Irish Conflict, 1780-1980. London: Allen and Unwin.

MacGiolla Chríost, D. (2004). The Irish Language in Ireland: From Goídel to Globalisation. London: Routledge.

McGrath, F.C. (1989). "Irish Babel: Brian Friel's Translations and George Steiner's After Babel". Comparative Drama, 23(1), 31-49.

Ní Scolláin, N. (2017). "Stereotypes and Linguistic Prejudices in Ireland". Dąbrowska, A.; Pisarek, W.; Stickel, G. (eds), Stereotypes and linguistic prejudices in Europe. Contributions to the EFNIL Conference 2016 in Warsaw. Budapest: Research Institute for Linguistics, Hungarian Academy of Sciences, 239-46.

Owens, C.D.; Radner, J.N. (eds) (1990). Irish Drama, 1900-1980. Washington DC: Catholic University of America Press.

Richards, S. (2016). "'We Were Very Young and We Shrank from Nothing'. Realism and Early Twentieth-Century Irish Drama". Grene, N.; Morash, C. (eds), The Oxford Handbook of Modern Irish Theatre. Oxford: Oxford University Press, 105-20.

Robinson, L. (1982). Selected Plays of Lennox Robinson. Gerrard Cross; Bucks: Colin Smythe.

Shiels, G. (1954). Three Plays: Professor Tim. Paul Twyning. The New Gossoon. Toronto: Macmillan.

Steiner, G. (1980). After Babel: Aspects of Language and Translation. Oxford: Oxford University Press.

Synge, J.M. (2008). The Complete Works of J.M. Synge. London: Wordsworth Editions.

Tymoczko, M. (2014). Enlarging Translation, Empowering Translators. London: Routledge.

Todd, L. (1989). The Language of Irish Literature. London: Macmillan.

Welch, R. (1999). The Abbey Theatre, 1899-1999: Form and Pressure. Oxford: Oxford University Press.

\section{Dictionaries}

De Bhaldraithe, T. (ed.) (1959). English-Irish Dictionary. Dublin: Angum.

Dineen, P. (ed.) (1904). Folklóire Gaedhilge Agus Béarla. Dublin: Irish Text Society.

OED (Oxford English Dictionary).

Quin, E.G. (ed.) (1983). Dictionary of the Irish Language: Based Mainly on Old and Middle Irish Materials. Compact ed. Dublin: Royal Irish Academy.

Toner, G., et al. (eds) (2007). eDil: Electronic Dictionary of the Irish Language. Online: Royal Irish Academy.

Wall, R. (2001). An Irish Literary Dictionary and Glossary. Gerrards Cross: Colin Smythe. 

Interviste | Interviews | Interviews Documenti | Documents | Documents 



\title{
Jacques Stephen Alexis au Moulin d'Andé : L'espace d'un scintillement
}

\author{
Kathleen Gyssels \\ Université d'Anvers, Belgique
}

Cherchant les exogénèses ${ }^{1}$ pour Le Dernier des Justes d'André Schwarz-Bart, je tombe sur un fait inattesté dans les nombreuses publications le concernant : l'auteur du Dernier des Justes et de La Mulâtresse Solitude, co-auteur de romans devenus des classiques caribéens comme Un plat de porc aux bananes vertes et co-auteur de Simone Schwarz-Bart (Pluie et vent sur Télumée Miracle)² est passé par un lieu symbolique pour la genèse de toute une littérature postShoah, postcoloniale et post-mémoire (selon le concept de Marianne Hirsch). Il s'agit du Moulin d'Andé, dans l'Eure. Cet ermitage d'ar-

1 Toute modification entre les versions différentes que connaît un manuscrit jusqu'à sa publication finale. Il n'y a pas moyen de consulter les versions antérieures pour Le Dernier des Justes, toutes étant détruites ou perdues. Quant aux brouillons des manuscrits de Simone, elle avoue ne pas savoir taper et c'est donc André qui aurait livré les versions préparatoires de ses deux romans publiés sous son nom à elle. Nous sommes face à des archives forcément parcellaires, à des ébauches fragmentaires, vérité qui dérange d'autant plus que le chercheur a un corpus romanesque et théâtral (essentiellement) publié ensemble et séparément. Les deux auteurs ont choisi de ne pas lever le voile sur la coécriture. La plupart des avant-textes faisant défaut, il devient difficile, voire impossible, d'avoir une approche génétique.

2 Signé par Simone Schwarz-Bart, paru au Seuil l'année même de La Mulâtresse Solitude, en 1972. A travers des détails comme «la veillée de neuf jours et de neuf nuits » pour Reine Sans Nom, la grand-mère de Télumée, l'on devine l'influence de l'auteur d'origine juive. La veillée d'un défunt y dure exactement neuf nuits et neuf jours, contrairement à la veillée antillaise. Lire Gyssels 2020a.

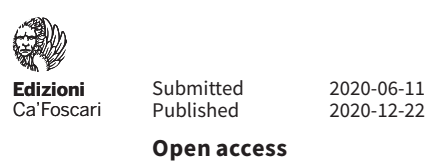

(c) 2020 | ()(i) Creative Commons Attribution 4.0 International Public License 
tistes de tout bord, de cinéastes et de dramaturges a également servi d'atelier d'écriture pour Jacques Stephen Alexis, me témoigne avec une grande passion Suzanne Lipinska, l'hôtesse du lieu.

Or, tout au long de ma recherche sur des auteurs antillais comme Edouard Glissant, haïtiens comme René Depestre, africains américains comme Richard Wright ou encore auteurs francophones d'origine juive comme Georges Perec ou André Schwarz-Bart (Gyssels 2014), le nom du Moulin d'Andé restait étrangement occulté dans les publications académiques, les mémoires et biographies des auteurs. Pourtant, tous sont passés par ce « lieu de tous les possibles».

Dès lors, le propos de cet article est avant tout de mettre sur la carte le méridien artistique inégalé qu'est le Moulin d’Andé pour la scène artistique d'après-guerre, et d'illustrer l'impact du passage et du séjour d'auteurs originaires des ex-colonies. Parmi eux, beaucoup d'artistes tentant de transcender leurs traumatismes et de pallier à une réception parfois baisée de leurs œuvres. J'aimerais suggérer ici que cette " résidence d'écrivains » et d'artistes (musiciens, peintres, cinéastes et dramaturges) avant la lettre soit déclaré une espèce de "Parlement d'écrivains », tant la parole muselée, la création contestée, et la théorie précurseur s'y retrouvaient. Trente ans avant la fondation du Parlement d'écrivains ${ }^{3}$ par des auteurs en exil, menacés de fatwa, à Strasbourg puis à Aubervilliers, le Moulin d'Andé accueillit dans l'immédiat après-Congrès 1956 à la Sorbonne un réseau d'intellectuels international et interdisciplinaire. Je ne ferais ici qu'illustrer deux illustres hôtes, l'Haïtien Jacques Stephen Alexis et André Schwarz-Bart. ${ }^{4}$

Seul Michel Séonnet, inconnu dans les rangs des 'Caribéanistes', découvrit l'importance de cette résidence d'écrivains avant la lettre, étape marquante pour la trajectoire de l'auteur haïtien Jacques Stephen Alexis :

Avant de commencer, le compose [sic] sembla se ramasser sur luimême, comme s'il lui fallait rassembler dans un dernier effort les morceaux d'un édifice dont il n'était pas totalement sûr. Tout cela pouvait se raconter de tellement de façons différentes ! Enfin, au bout d'un moment, il s'élança. Quel silence ! L'univers se serait-il refermé autour d'un vieux moulin campé sur un bras de la Seine ? L'univers aurait-il fini par se réduire à cette chambre située au-dessus de la grande roue de bois aux aubes brisées qui, ne tournant plus, laisse sans frein se dérouler sous elle le gron-

3 https://www.lemonde.fr/archives/article/2003/06/12/le-parlement-international-des-ecrivains-s-autodissout_323712_1819218.html.

4 Voir aussi ma communication sous presse dans les Actes du Colloque Université de Caen (novembre 2019) : Gyssels à paraître. 


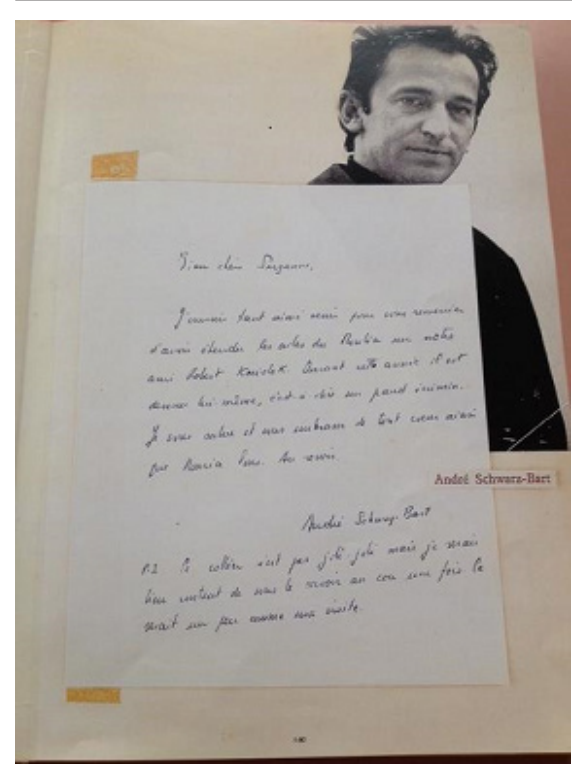

Figure 1 Le mot d'André Schwarz-Bart à Suzanne Lipinska. Volume 1 du Livre d'Or. @ Suzanne Lipinska. Transcription : «Très chère Suzanne, J'aurais tant aimé venir pour vous remercier d'avoir étendu les ailes du Moulin à notre ami Robert Kociolek. Durant cette année il est devenu lui-même, c'est-à-dire un grand écrivain. Je vous salue et vous embrasse de tout cœur ainsi que Maurice Pons. Au revoir. Post Scriptum : Le collier n'est pas joli joli mais je serais bien content de vous le voir au cou une fois... Ce serait un peu comme une visite ».

dement apaisé d'une eau verte et peu rapide ? Il n'y a que le bruit de l'eau pour faire pièce au silence. La Seine ! Rien à voir avec les emportements de l'Artibonite ! Le Moulin d'Andé ! Aucun rapport avec l'effervescence de Port-au-Prince! Alexis est à sa table. Il ne la quitte que pour manger ou pour quelques rapides discussions avec d'autres hôtes comme lui de passage, quelques-uns ont aussi participé au Congrès des écrivains et artistes noirs. Mais ce ne sont que parenthèses, pauses, rares. (Séonnet 1983, 83)

Séonnet signale que la rédaction de L'espace d'un cillement, le premier volet de la trilogie, germa dans un moulin campé sur la Seine pas très loin de Rouen. Ecrit en une seule lancée, dans l'espace de quelques semaines, le romancier choisit d'ouvrir son roman par l'exil et l'éventualité d'un retour au pays natal, peiné par la séparation d'Haïti.

Dernier des Justes (1959) s'y rendit, accompagné par son protégé, Robert Kociolek. Sans que ne sache la date exacte de sa visite, il importe qu'il a pu y croiser le chemin d'autres auteurs, des plus illustres tels les frères Lanzmann, Ionesco, Edouard Glissant (qui ne le signale nulle part) et aux illustres inconnus, tels, Paul Niger - alias Albert 
Béville - si l'on doit en croire une autre dédicace « à mme Lipinska ». Tous ont séjourné à des intervalles différents dans cet atelier d'écrivains en Normandie. L'Africain Américain Richard Wright, quant à lui, y avait écrit l'essentiel de son Black Boy, pendant que Georges Perec y occupait pendant cinq ans la chambre « Jeanne d'Arc » pour écrire $L a$ Disparition. Rien que pour ces deux célébrités fuyant les phares médiatiques, le Moulin d'Andé a servi d'abri et d'atelier d'écriture, loin du Paris bruyant et étouffant par les petites guerres intestines entre artistes rivaux, ou tout simplement à l'invitation de fidèles du Moulin. De surcroît, certains auteurs avaient été sérieusement lésés par des critiques assassines de leurs ouvrages ${ }^{5}$ ou avaient souffert la censure.

L'auteur d'une autobiographie d'une enfance affreuse dans le Deep South et le fondateur de l'OuLiPo se sont donc croisés dans ce havre de paix. Quant à Richard Wright, il s'installa définitivement dans un petit village près du Moulin où je me rendis pour découvrir avec déception qu'aucune plaque ne rappelle le domicile du grand auteur, ami de Sartre et de de Beauvoir, et dont l'œuvre a été étudiée par Albert Memmi et Frantz Fanon, parmi tant d'autres. Wright y était venu, comme Alexis et Depestre, après le Premier Congrès des Ecrivains noirs à la Sorbonne, en 1956. Marqué à vie par l'univers ségrégué et raciste, Wright alla trouver en ces écrivains de toutes nationalités et couleurs confondues une confrérie soudée. Celui qui allait devenir une figure tutélaire pour la génération de Toni Morrison, James Baldwin, et de nombreux autres écrivains de couleur, d'auteurs juifs (Albert Memmi s'appuie sur l'œuvre de Wright pour ses essais qui feront date) et afrodiasporique, avait élu domicile dans cet espace nourricier, loin du Quartier latin et son nombrilisme. Traumatisé par l'esclavage et le racisme, Wright s'entendait bien avec ces auteurs de nationalité différente, s'exprimant en différentes langues, venus d'horizons divers mais qui avaient tous épousé l'idéologie communiste pour briser les chaînes de l'oppression (noire, juive).

Tous ces auteurs et artistes, cinéastes de la Nouvelle Vague (Truffaut) et régisseurs du théâtre de l'absurde (Ionesco), anthropologues

5 Le négationniste Robert Faurisson s'en prit à « André Schwarz-Bart, escroc littéraire ", article qu'il mit à jour sur son blog (http://www. robertfaurisson.blogspot. it) après l'avoir publié dans Dubidando (décembre 2006), l'année même de la mort de l'auteur, fin septembre. Je me demandais avec effroi si Schwarz-Bart a pris connaissance de cette accusation. Dans l'hypothèse qu'il ait pu en prendre connaissance, elle peut l'avoir blessé si profondément qu'il en a suspendu beaucoup d'écrits restés dans ses tiroirs. Pour plus d'infos sur Faurisson : https://dagobertobellucci.wordpress. com/page/2315/?app-download=windowsphone.

Faurisson eut l'impudence de rassembler l'ensemble de ses articles dans Faurisson 2011. Comparée à « l'Affaire Schwarz-Bart » (l'accusation de plagiat), cette allégation est autrement plus violente et perturbante. D'où la suspension non pas de l'écriture, mais de la publication. Schwarz-Bart avait foison de projets restés inachevés à sa mort, d'opéras (avec Marcel Delaistier) à des pièces de théâtre. Sur l'œuvre théâtrale avortée, lire Gyssels 2018. 
(Georges Condominas, « Condo » pour les amis, et Roger Bastide) et éditeurs (Maurice Nadeau et François-Régis Bastide), ont pu croiser la route de Jacques Stephen Alexis. Bref, ce «lieu de tous les possibles » que Suzanne Lipinska se plaît à rappeler dans Le Moulin d'An$d e^{6}{ }^{6}$ un collectif avec les témoignages de ceux et celles qui rendent hommage à l'Association culturelle et artistique privée, se doit de figurer dans une histoire des idées de la France au-delà des frontières étriquées ("race », « religions », « nation », « langue »), plus particulièrement depuis l'après-guerre jusqu'à la fin des années '90. Pour une histoire des lettres postcoloniales, d'une République d'une littérature et de la scène mondiale multiculturelle, le Moulin d'Andé est un repère et une repaire, bien que plusieurs de ces grands noms ait négligé de le signaler... Si petit à petit, les journalistes (pourtant familiers des noms précités) et les mémorialistes (notamment de René Depestre $)^{7}$ commencent à découvrir le lieu de mémoire, le nom de Glissant, de Schwarz-Bart, n'y figurent pas parmi les célébrités y associées (Marin la Meslée 2020, 72-4).

Alexis reste en effet l'auteur de l'exil, ayant vécu l'essentiel de sa vie hors d'Haïti pays natal qu'il dut fuir à cause de son opposition au régime duvaliériste. L'hésitation constante entre deux voies, entre deux sens, entre deux motifs le poursuivra toute sa vie : fuite devant une menace, toujours sur le départ et désireux de revenir au lieu. Ces mouvements de pendule ne sont pas étrangers à André Schwarz-Bart qui ne cessa de déménager...

Que Schwarz-Bart ait pu rencontrer cette phalange métisse au Moulin d'Andé m'importe pour un nombre infini de raisons, qui ne sont pas toutes littéraires au demeurant : Jacques-Stephen Alexis, René Depestre, Georges Perec aussi dont l'écriture consonne à plusieurs endroits avec celle du Dernier des Justes. Voire même, qu'il ait tiré profit des conversations avec ces confrères et consœurs séjournant là-bas, que leurs causeries aient pu résulter dans l'un ou l'autre ourlet, revers, ou écho dans ses romans remarquablement bien réussis, comme dans ses romans restés inédits à sa mort en 2006 et que Simone Schwarz-Bart ensemble avec quelques proches qu'elle remercie en note et dans les interviews prend la lourde responsabilité d'éditer et de présenter au Seuil comme «les volumes du cycle » romanesque. ${ }^{8}$

\section{Le Moulin d'Andé (1992).}

7 René Depestre publia en 2018, aux éditions Odile Jacob, Bonsoir tendresse. Autobiographie (avec une préface de Marc Augé, et un avant-propos de Jean-Luc Bonniol). Ce dernier mentionne plusieurs séjours au Moulin d'Andé dans la chronologie de celui qui, à mes humbles yeux, demeure une des figures les plus originales de l'avant-garde artistique et intellectuelle francophone, le Nestor autrement immortel.

8 Lire mes articles en ligne sur L'Étoile du matin (2009), L'Ancêtre en Solitude (2015) dans entre autres Il Tolomeo, Africultures, Image et Narrative. 
Comme Alexis, il s'agit d'auteurs ayant finalement publié peu, mais quelle grandeur, quelle indémodable force, quel esprit humaniste ! L'auteur de trois romans, Compère général soleil (1955), Les Arbres musiciens (1957), L'Espace d'un cillement (1959) et d'un recueil de contes et nouvelles, Romancero aux étoiles (1960), a pu rencontrer Schwarz-Bart de la même façon que ce dernier a pu y s'entretenir avec Perec, Beckett (dont la muse et compagne Barbara Bray deviendra la traductrice des romans de Simone Schwarz-Bart), Maurice Pons, et plus tard Nancy Huston, pour ne nommer que quelques illustres hôtes. Autant il s'agit de résidents remarquables, ayant participé à des séminaires et master class, des rencontres transatlantiques entre par exemple les féministes de premier plan Monique Wittig et Françoise Basch et leurs consœurs américaines, autant le Moulin d'Andé demeure une archive inestimable, réseau nulle part attesté dans l'Histoire de la littérature et de la critique, du monde de l'édition et de l'Histoire des idées de la France et de la francophonie, voire des sciences humaines.

Ce silence m'interpelle. Comment expliquer que ni Alexis, Depestre, Wright ou Schwarz-Bart n'ont mentionné leur visite à Maurice Pons et Suzanne Lipinska alors même qu'il a pu s'agir de moments, voire de tournants décisifs dans leurs carrières respectives ? Et pour ce qui concerne l'importante mutation d'une France coloniale à une République postcoloniale, comment ne pas interroger davantage que la capitale française demeure apparemment pour ces mêmes auteurs le Centre, alors que c'est dans la marge, en périphérie, que les « lignes » bougent, que les regards aient pu changer, les perspectives s'inverser? De fait, l'on peut toutefois postuler qu'ils y ont trouvé des compagnons artistes et écrivains grâce à qui leur œuvre a pu gagner en densité et profondeur. En effet, si tous quatre ont connu un immense succès, c'est aussi en partie à cause des échanges avec des confrères et consœurs dans ce havre de paix.

Fait inattesté dans les publications et le docufilm, ${ }^{9}$ dans sa bibliographie et ses spécialistes, tels que M. Dash, pour ne donner que cet exemple féru d'Alexis, la résidence au Moulin d'Andé d'Alexis (et la même observation vaut pour Depestre qui dédie affectueusement plusieurs ouvrages à Maurice Pons) ${ }^{10}$ a contribué sans doute au fait

9 À travers ce long métrage, tourné, entre autres, en Haïti, aux États-Unis, en France, à Cuba et au Canada, Antonin part sur les traces d'Alexis et à la recherche de tous les témoins encore vivants. L'admiration d'Antonin pour ce grand écrivain transpire tout au long du documentaire, construit avec beaucoup de délicatesse. Des voix et des images, parfois d'époque, portent bien le personnage. https://sites.google.com/site/berroueetoriol/system/errors/GeneralAttachment.

10 Une autographe adressée à Maurice Pons, dans Encore une mer à traverser (2005), une autre à Lipinska dans un volume de la collection "Poètes d'aujourd'hui » lui consacré (1985), en font foi. C'est bien tardivement, dans la biographie de Jean-Luc Bonn- 
qu'ils soient devenus des classiques de la littérature haïtienne, et par extension caribéenne. Mort en avril 1961 à son retour au pays natal, dans des circonstances mystérieuses, cette figure imposante du $20^{\mathrm{e}}$ siècle haïtien est devenue le martyr du duvaliérisme, sa mort tragique sur laquelle les versions varient étant imputée aux Tontons Macoutes. Que de versions du retour clandestin d'exil et de la capture de Jacques Stephen Alexis ! Est-il mort à Fort Dimanche, la lugubre prison des Duvalier, aux Casernes Dessalines ou au Môle St. Nicolas ? Selon des témoignages, il aurait été fusillé ? Ce qui est certain, c'est qu'il a été capturé et porté disparu le 21 avril 1961, à la veille de ses 40 ans.

De même, la sombre mémoire de persécutions, massacre et torture taraude l'œuvre schwarz-bartienne et celle de Richard Wright. Quant à René Depestre, il forme avec ces deux-là un réseau de compagnons de route parmi les nombreux amis et amies qu'il a salués à plusieurs reprises.

Car ces trois auteurs avaient en commun, outre l'horreur de l'esclavage et l'univers ténébreux de la Plantation, monde concentrationnaire sous les Tropiques, leur engagement communiste, soit leur farouche opposition aux idéologies capitalistes. Dans Une faim d'égalité, ${ }^{11}$ Richard Wright témoigne de la terreur permanente de vivre en tant que Noir dans le Deep South régi par des capitalistes yankees. Monté à New York à cause du racisme au quotidien, Wright est venu se réfugier en France. Grâce à l'accueil mémorable de Sartre et de de Beauvoir, de Claude Lanzmann et de Gertrude Stein, il devint vite l'écrivain consacré. Dissident de l'Amérique capitaliste au point d'être suivi par le FBI, Wright embrasse l'idéologie communiste jusqu'aux déboires de Staline et l'invasion des Russes à Prague au « Printemps 1968». Les rapproche l'engagement, de même que la rencontre de Depestre et d'Alexis avec Mao Tse Tong.

Alexis, quant à lui, caractérisait Haïti, son pays semi-féodal et semi-colonial dans un non-État où les individus ont beaucoup plus tendance à s'attacher à des sauveurs qu'à des idées politiques précises et à la lutte de masses structurellement organisée. En 1961, Alexis participa aux grandes réunions communistes dans le bloc de l'Est, à Moscou et à Pékin. Il participe au congrès des partis communistes de plus de 80 pays à Moscou. Sa plaidoirie en faveur de la résolution des rivalités entre la Chine et l'Union Soviétique, qui affectent

iol, qu'il est fait enfin mention du Moulin. Bonniol établit la chronologie et pour la première fois, Depestre mentionne ce Moulin d’Andé ; cf. Depestre 2018 (Préface).

11 Protégé de Simone de Beauvoir et de Sartre, Wright vit ses nouvelles et romans quasiment simultanément traduits et publiés par Gallimard. À Paris, l'œuvre de Richard Wright avait été introduite au curriculum grâce à René Favre, l'époux de Geneviève Favre qui ont formé toute une génération de chercheurs dans la littérature noire américaine (et caribéenne). Lire Gyssels 2020b. 
les mouvements de gauche en Amérique Latine et dans la Caraïbe, est mémorable. De même, Depestre sera de la partie à cette même conférence, pendant que Richard Wright joignit le parti communiste en 1932 : toute la mouvance anticolonialiste et antiraciste était de gauche, tout au long des années 60 et 70. Bien que Depestre comme Aimé Césaire rompent avec le communisme, avec la politique de la Guerre Froide avec la censure de nombreuses voix rebelles, ils resteront fidèles à la lutte contre les inégalités qui rive juifs et Noirs, à la doctrine d'égalité des classes et de "races» (Gyssels 2008). Juif laïque, André Schwarz-Bart embrassa lui aussi le communisme, jusqu'à ce qu'il se dessille les yeux avec l'Affaire Slansky : les déboires avec le communisme qu'il a essuyés, lui et d'autres Bundistes et membres des différentes fractions communistes juives. Ces branchements sont relatés dans L'Étoile du matin. ${ }^{12}$

Alexis, Wright, Depestre, Schwarz-Bart, un autre trait permet encore de les souder. Le réalisme merveilleux, certes, moins présent chez le romancier américain. C'est ici qu'on touche à une autre spécificité caribéenne : pour Schwarz-Bart, le 'réalisme merveilleux' servira de contrepoids à l'intolérable poids du réel, au présent inénarrable (insupportable de douleur, voire de torture). La veine se marie aussi sans faille avec les leçons d'anthropologie qui éclairent les différentes croyances de différentes communautés. Il tira grandement profit des anthropologues qui gravitaient autour de Pons : Georges Condominas (Condo, pour les amis), Jean Pouillon (voir Pons 2002, 29-32), Roger Bastide, Claude Lévi-Strauss et un intellectuel 'sans domicile fixe' qui, sans qu'il n'ait fait escale dans l'Eure, participa aux débats et aux séances de réécritures, appelés 'pilpuls' entre Polonais (juifs ou non) en errance à Paris autour d'un roman en formation. Car la genèse d'un roman monumental, plusieurs fois remanié qui sortira finalement sous le titre Le Dernier des Justes, doit beaucoup à ces conversations et ces élucidations de version en version, celles-ci, au nombre de quatre, ayant été détruites par l'auteur.

$\mathrm{Au}$ Moulin d'Andé, une certaine conception d'œuvre unique, c'està-dire attribuée au seul génie singulier d'un individu spécifique, avait été reconsidérée et dûment nuancée. Autrement dit, le succès phénoménal du premier Goncourt après-Shoah pourrait en partie tout au moins avoir bénéficié de conseils et de retouches, de recommandations et de questionnements des premiers lecteurs de brouillons et de premières ébauches (parmi lesquels l'équipe autour de Jean Lacouture au Seuil) qui restent malheureusement pour la recherche

12 Sorti à titre posthume par Simone Schwarz-Bart, Seuil, 2009, sous le seul nom d'André. Les autres titres posthumes portent leurs deux noms. Il s'agit de L'ancêtre en Solitude (2015) et d'Adieu Bogota (2017). Un dernier « volume » du « cycle antillais », portant sur l'Afrique devrait voir le jour en 2021. 
inconnus, sciemment ou pas. Ainsi, un premier assistant était l'anthropologue dilettante, juif déboussolé doté d'un bagage culturel impressionnant, Abrasza Zemsz (1908-1979). ${ }^{13}$ Comme le déclare Richard Marienstras dans Etre un peuple en diaspora (1975, 131-42), Le Dernier des Justes avait connu plusieurs versions. Seule la dernière avait la légende des lamed-waf comme une idée structurant le roman historique en 'bible' de la judaïté européenne à travers le temps.

Enfin, il reste un détail capital dans la biographie de quelques illustres invités au Moulin : l'ouverture sur l'Autre, sur l'Autre culture, celle du Livre et de l'esprit juif laïque, de l'humanisme sans frontières qui leur vint par leurs conjointes... L'Africain Américain Richard Wright a été marié à deux reprises à des Polonaises de surcroît juives, pendant que l'Haïtien René Depestre vit également avec son épouse de culture juive, et que Schwarz-Bart s'est retiré à la maison Souvenance, à Goyave, avec Simone Brumant qui prit son nom d'écriture. Couples en création, couples mixtes, il s'agit d'auteurs « Luftmensch » qui s'enracinent dans d'autres lieux, " citoyens du monde », et ouverts sur les confluents de cultures et de communautés.

Comme l'a bien vu Séonnet, l'auteur haïtien avait un dernier roman en tête : Coquelicots, en référence aux fleurs qui illuminent les prés verts d'Andé à l'été :

L'espace d'un scintillement. La lumière joue un rôle central dans l'imaginaire de tout poète et tout écrivain. Surtout quelqu'un originaire des Antilles ou d'Haïti sait les multiples réverbérations de la lumière solaire ou lunaire sur le reflet de l'eau. Jacques Stephen Alexis a été particulièrement sensible aux jeux des miroitements et des scintillements car ils ponctuent l'éphémère qu'il cherche à faire durer, à capter, à transcrire dans l'œuvre romanesque. La dernière vision du rivage de la terre natale qui s'éloigne s'imprime dans la mémoire et y demeure. C'est une image qui ne disparaîtra jamais. Viendrait-elle à s'estomper, les yeux n'auraient-ils plus la force de l'affronter, les mots sont là qui la raniment. Dans chaque livre, l'image affleure comme un récif à la fois dangereux et bienvenu : la terre n'est pas loin ! Obsédante et revivifiante pourtant, lumineuse douleur, celui qui s'en va la recueille précieusement : il

13 Voir Gyssels 2019. Or, il se trouve que ces modifications capitales sont venues d' Abrasza Zemsz, selon Elisabeth Brami (née en 1946 à Varsovie), fille du peintre Emanuel [Tolek] Proweller. Elle-même psychologue clinicienne et écrivaine, elle était gamine lorsque Schwarz-Bart venait à la maison, le manuscrit en poche, discuter des ajouts et des coupures à apporter au chef-d'œuvre en devenir, en compagnie de Marienstras et de Zemsz, parmi d’autres. Voilà la face cachée de mon auteur vénéré : non seulement il s'est gardé coi sur Abrasza Zemsz qui se suicida le 8 septembre 1979, se défenestrant de l'Hôtel André Latin, dans la rue Gay-Lussac (et non pas sur le Bvd Saint-Germain, comme le pensent Plougastel et Schwarz-Bart 1959, 87), mais aussi ses proches préféraient garder le silence. 
suffirait d'inverser le mouvement, de passer le film à l'envers, et le retour serait là, déjà présent dans l'instant qui s'éloigne et exile. Celui qui part ne s'en va que pour mieux revenir, comme nous le montre superbement une page de la prose lyrique d'Alexis.

Et Séonnet de terminer sur une belle page d'anthologie :

«En avant toute !... »

L'Églantine se redresse vivement sur les coudes, à plat ventre, elle regarde les lumières du port qui scintillent, les colliers électriques qui frissonnent au col des montagnes, l'échiquier embrasé des mille et une fenêtres de la ville, enfin les taches du souvenir : 'La Frontière' et son auréole qui poudroie le campanile de Sainte-Anne, le Bel-Air et ses plaques de teigne sous les rares étoiles, les deux bras implorants de la cathédrale, cette immense verdeur du Champ-de-Mars, enfin la montagne bossue, piquetée de coups de bec par les saisons et l'immémoriale misère des hommes s'écaillant sur le bourrelet infectueux des faubourgs, tout s'éloigne dans une lenteur vertigineuse, rêve et réalité, lieux de paix, chambres de torture, électricité spectrale des lampadaires et les rues et les toits et l'adieu farfelu des arbres convulsionnaires. ${ }^{14}$ (Italique dans l'original)

Rendons aux lieux d'inspiration, lieux abreuvant l'imagination, toute leur signifiance ; rendons-leur toute leur résonance en situant désormais le Moulin d'Andé d'où sont partis les courants fertiles de mémoires imbriqués, vivier irriguant diasporas noire et juive qui se devrait par conséquent d'être un repère dans l'histoire littéraire caribéenne, et dans l'histoire de la France artistique d'après-guerre tout court. 


\section{Bibliographie}

\section{Fuvres de Jacques Stephen Alexis}

Alexis, J.S. (1955). Compère Général Soleil. Paris : Gallimard.

Alexis, J.S. (1957). Les Arbres musiciens. Paris: Gallimard.

Alexis, J.S. (1959). L'espace d'un cillement. Paris : Gallimard.

Alexis, J.S. (1960). Romancero aux étoiles. Paris: Gallimard.

Alexis, J.S. (1971). "La Belle Amour humaine 1957 ». Europe, 49.501 (janvier), 20-7.

Alexis, J.S. (2017). L'étoile Absinthe. Paris : Zulma.

\section{Critique}

Couffon, Claude (1985). René Depestre. Paris : Pierre Seghers. Poètes d'aujourd'hui 252.

Depestre, R. (2005). Encore une mer à traverser. Paris : Gallimard. Collection Vermillon, La Table Ronde.

Depestre, R. (2018). Bonsoir tendresse. Autobiographie. Préface de Marc Augé ; avant-propos de Jean-Luc Bonniol. Paris : Éditions Odile Jacob.

Faurisson, R. (2011). Écrits révisionnistes (2005-2007), vol. 6. Édition Privée HorsCommerce. Akribeia.

Gyssels, K. (2008). «André Schwarz-Bart: héritiers et héritage dans la diaspora noire ». Pardès, 44(1), 149-73. https://www.cairn.info/revuepardes-2008-1-page-149.htm.

Gyssels, K. (2009). « De L'étoile du soir à L'étoile du matin : le dernier roman schwarz-bartien ou l'art de la ré-apparition ». MicRomania, 71(4), 3-15.

Gyssels, K. (2013). «A Schwarz-Bart à Auschwitz et Jérusalem ». Image et narrative, $14(2)$. http://www.imageandnarrative.be/index.php/imagenarrative/article/view/310.

Gyssels, K. (2014). Marrane et marronne : la co-écriture réversible d'André et Simone Schwarz-Bart. Leyde: Brill.

Gyssels, K. (2015a). "Simone et André Schwarz-Bart, L'ancêtre en solitude ». OSO : Surinamese linguistics, literature and history, 1-2, November, 19-21.

Gyssels, K. (2015b). «Work in Progress : l'œuvre posthume d'André SchwarzBart ». Africultures, 14 juillet 2015. http://africultures.com/work-inprogress-loeuvre-posthume-dandre-schwarz-bart-13079/.

Gyssels, K. (2017). « Schwarz-Bart, André ; Schwarz-Bart, Simone (2017). "Adieu Bogota”. Paris : Éditions du Seuil, 265 pp. Suite - et fin ? - du cycle antillais schwarz-bartien ».IITolomeo, 19, 327-38. http://doi.org/10.14277/24995975/Tol-19-17-22.

Gyssels, K. (2018). « Who Owns Schwarz-Bart? Écrivains dans le tiroir ». Dalhousie French Studies, 112, 121-39.

Gyssels, K. (2019). « Portrait of an Authentic Schnorrer; Abrasza Zemsc in Richard Marienstras' Memory ". Journal of Jewish Identities, 19, 197-209.

Gyssels, K. (2020a). «Femme-chamane entre 'tsaddik' et 'quimboiseuse' : l'interface anthropoétique dans l'œuvre schwarz-bartienne (cycle antillais / ashkénaze) / Between 'tsaddik' and 'quimboiseuse': The Anthropoetic Interface in the Schwarz-Bart Novels / Mulher-xamã entre tsaddik e quimboiseuse : a interface antro-poética na obra schwarz-bartiana (ciclo antilhano/ asquenazista) ». Caligrama. Revista de Estudos Românicos, nº spécial sur les 
Littératures latino-américaines d'expression française (Haïti, Guadeloupe, Martinique et Guyane), 25(3). http://dx.doi.org/10.17851/2238$3824.25 .3 .77-100$.

Gyssels, K. (2020b). L'antillectuel Léon-Gontran Damas, vers une France décoloniale. Amsterdam : Brill.

Gyssels, K. (à paraître). «'Tous nomades !' Pour un méridien normand ». Schneider, A. ; Jeannin, M. ; Calvet, Y. ; Cleren, M. (éds), L'Écriture de la migration dans la littérature et le cinéma contemporains pour adultes et pour enfants = Actes du Colloque Université de Caen (novembre 2019). Roma : Edizioni Universitarie Romane. Collection Voix de la Méditerranée.

Le Moulin d'Andé (1992). Préface Suzanne Lipinska. Paris : Quai Voltaire.

Marienstras, R. (1977). Être un peuple en diaspora. Paris : Maspéro, 1977. Réédité avec une préface d'Élise Marienstras, postface Pierre Vidal-Naquet. Paris : Éd. Amsterdam, 2008.

Marin la Meslée, V. (2020). «Au Moulin d’Andé ». Le Point, 2501, 30 juillet, 72-4. Pons, M. (2002). « Notre ami Jean Pouillon ». Les Temps Modernes, 620-1, 29-32. Schwarz-Bart, A. (1959). Le Dernier des Justes. Paris : Seuil.

Schwarz-Bart, S. (1972). Pluie et vent sur Télumée Miracle. Paris : Seuil.

Schwarz-Bart, A. ; Schwarz-Bart, S. (1967). Un plat de porc aux bananes vertes. Paris : Seuil.

Schwarz-Bart, A. ; Schwarz-Bart, S. (1972). La Mulâtresse Solitude. Paris : Seuil. Schwarz-Bart, A. ; Schwarz-Bart, S. (2015). L'ancêtre en solitude. Paris : Seuil. Schwarz-Bart, A. ; Schwarz-Bart, S. (2017). Adieu Bogota. Paris : Seuil.

Séonnet, M. (1983). Jacques-Stephen Alexis ou le voyage vers la Lune de la belle amour humaine. Toulouse : Pierres Hérétiques.

Wright, R. (1979). Une soif d'égalité. Paris : Gallimard. 


\section{An Ecocritical Journey Through the Narrative of the Canadian Novelist Alissa York: How Literature Explores Environmental Problems}

Adriana Cerminara

Università degli Studi di Torino, Italia

Alissa York was born to Australian immigrant parents in Northern Alberta, Canada, in 1970. Her family moved to Canada (Athabasca, Alberta) from Australia. Experiencing nature through outdoor activities, along with numerous stories she heard from her father about wildlife in Australia and its national mythology, played an essential role in her early life. York's family moved to Victoria, Vancouver Island, in 1977 and after graduating from high school, she moved to Montreal, Quebec, where she studied English Literature at McGill University. She studied there for two years, yet she did not complete her degree. Some years later, York decided to attend a master's degree in creative writing. York became full-time faculty at Humber's celebrated School for Writers in the fall of 2017. She has been teaching and mentoring writers since 2007. York's interest in our relations with our surroundings, with our fellow human beings, with the natural world, and all creatures that live together on this planet permeates her writing. Alissa York's internationally acclaimed novels include Mercy (2008), Effigy (2009), Fauna (2011) and The Naturalist (2016). York is also the author of the short fiction collection, Any Given Power (1999). The present interview focuses mainly on her personal and professional background and her two most recent novels The Naturalist and Fauna.

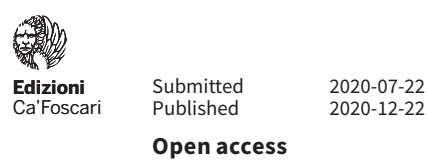

(c) 2020 | ()( Creative Commons Attribution 4.0 International Public License 
ADRIANA CERMINARA How does your narrative work relate to your personal life? When did you start feeling close to the themes that you develop in your novels?

ALISSA YORK Although my father was teaching several things, one of them was the so-called outdoor education. We spent a lot of time outdoor: camping, fishing, canoeing, hiking. That combined with a lot of stories that we heard about wildlife in Australia, which is very different from the one in Canada, but it is also quite an important part of the national mythology of that country. Added to that, we always had pets in the home (cats, dogs). I love animals still in the way that children do. A lot of my early memories involve animals, whether they might be pets or wild animals. I was always very attuned to that element of life. Nevertheless, I mostly lived in cities as I grew up.

A.C. Reading your novels, I found it difficult to identify your writing style as belonging to just one literary movement. How would you define yourself as an author? Which literary movement do you feel you belong to?

A.Y. I consider myself a writer in Realism, although people have commented that my works have a kind of, what they call, Northern Gothic influence which I can relate too. There are also elements of extreme realism, Naturalism, that makes into my works at times. There are elements of Ecocriticism. I must say, none of them is deliberately chosen. It is all reflexion of my concerns, of my world view and most importantly it is what the characters of each novel require. What gives me the idea to begin a novel is always a character that I have in mind and I try to build the stories around it, inserting other characters with their own personal stories. I am always following the story and the characters and never imposing any kind of traditions, theoretically, although all of these things make their way in through one's world view, beliefs, what you notice, what you focus on.

A.C. I have found the mixing of different narrative forms in your novels very compelling: i.e. the notebook in The Naturalist and the blog in Fauna. How do these narrative forms influence the storytelling?

A.Y. In those cases, that usually happens because a first-person voice has asserted itself in one way or another. For example, in The Naturalist, Walter's notebook grew out of a couple of directions; I was becoming aware that I wanted Walter's voice, or better, Walter's voice was beginning to take shape by itself in relation to the research I was doing. In other words, I was reading about this XIX Century naturalist who had gone to the Amazon, so in that kind of tradition, in that context that fits with the character and, at the same time, I became fascinated with the field of notebooks as a form of writing itself. It only made sense 
to me, also exciting and funny, to write my own field notebook from the XIX Century. Of course, the kind of voice that comes about it has to be responsive to all the parameters: he is male, he is from a certain time, he is of a certain age, he is of a certain background, he has various concerns, he has different personality traits and all of that has to be reflected in the voice, otherwise it is not genuine. The same can be said for the letters or blog posts, I am always trying to reflect many aspects of the characters to make an immersive experience for the reader, where they can fully believe that this voice belongs to that character. Some of the story would present itself as third-person.

A.C. Your stories are stories of love. What role does love play in your novels?

A.Y. I believe my novels are always about love. Various characters try to love and be loved and very often failing as we do. However, we still try. Love is such an essential driving force for almost all humans.

A.C. When writing or before writing, is there a particular message that you want to communicate to your readers?

A.Y. No, it never starts that way. I don't have a particular message that I want to communicate to my readers before starting writing. This is one of the interesting things when you are discussing your work, when it already has a life and it has become something. You cannot quite describe what it actually is because what it actually is, it begins with something that grows. All the research that one does, it does not go directly into the novel, but it is all part of that process of imagining that world sufficiently to be able to get gown on the cage. The message makes itself. I truly believe that when people want to write literary fiction and think they are in the driver's seat in terms of the message, they tend to write a piece that is not strong as it could be. Because it is not a direct form. Art itself is not a direct form. It's done through 'the showing' rather than 'the telling', which is something that we do over and over in creative writing. You must do the work to show it rather than just tell about it. That is the moment in which the mysterious happens.

A.C. The leading question here is which role does literature play in environmental discourse and in shaping human beings' behaviour towards nature and other creatures. In your opinion, which role does literature play in the whole discourse?

A.Y. Literature plays a central role. Reading literary fiction increases empathy. I knew this to be true long before; most leaders of literary fiction know that. They know it in their blood, in their bones. It's one of the things that they require from literature, it's that deep sense of connection. It often applies to our 
fellow human beings but depending on the literature it can connect to much more.

A.c. Do you believe your novels can be read from an ecocritical point of view and they can contribute to the environmental discourse?

A.Y. Yes! I know that people already do, and it is wonderful to think of that going on for various reasons. One of which is the joy to be read closely and carefully after all the work that goes into a book. It is so wonderful to think of people reading it so closely and carefully and with such a consideration. I feel that the Arts in general have a huge role to play in addressing a number of these huge issues that we have to deal with.

A.C. While reading, one has the feeling that your stories are perfectly intertwined; they include flora, fauna, and the human world. Although sitting on my chair, I found myself travelling among new landscapes, stories, and fantastic creatures that I did not know were existing in this world. [About The Naturalist.] Writing about the Brazilian Amazon rainforest is, somehow, a call to the world of the beauty we are losing day by day?

A.Y. The novel ended up absolutely being a novel that is seen as a call to the world to become aware of the landscapes that we are losing, and the wildlife that during the XIX Century were still there. However, it came more from other ways. I was applying for a fellowship and that fellowship asked that you developed yourself and your practice as an artist. So, it wasn't so much projected as it developed. I asked myself: "What can develop myself?". The answer came back right away: The Amazon. I have always been fascinated by it and it was a huge topic, a huge setting, utterly overwhelming. Of course, what comes through, the more you learn about it, you just have a sense of it being a centre of a life force in the world. When you are reading about it and you also go there, that sense feels palpable. So, the fact that we are disregarding the value of that place along with so many other wild places in the world is potentially even more upsetting. This is because, if not there, if we not value there, where then? I don't know the answer to that, but, again, that is how it found its way naturally. There are great concerns that find their way in the novel, but they are also in the air. With such an intertwined setting, it was very challenging to write it. This is how it works: you take the reader into that particular world.

A.C. Within The Naturalist, anthropology plays an essential role. In your opinion, to what extent can the killing of animals be justified by anthropological reasons?

A.Y. In many cultures the killing of animals can either be in balance or not in balance. When capitalism comes to the max, usually after not very long that goes out of balance. What you kill 
and for what reasons, how much, when and how you do it, whether there is any element of sacredness or understanding of the sacredness of life sustained with the process or not. If all those questions around the practice do not lead to any sense of the sacredness of life, it becomes compartmentalized.

A.C. [About Fauna] Since architecture is shaping and determining the future of some species, what role does urban planning play in the novel?

A.Y. The building of cities is dangerous for so many animals. There are a few species to thrive to find their way across the city. I admire animals that can manage to survive in the face of what we do to the landscape. For example, Toronto is on a major bird migration path, a significant amount of those birds that migrate are killed due to those buildings. The numbers are over $1 \mathrm{mln}$ birds per year. On the other hand, some of those wild birds have become sufficiently compromised to become extinct and there will be more. People fightto turn off the lights at night that draw nightflyer birds, but they still resist. They want to keep all those lights on, burning energy and killing birds through the night for no reason. I suppose because of what those building mean to them, they are symbols of permanence or power. So, what is that you want? A space that is devoid of life except for human life? It's really important that we change how we think. People who don't care about ruining a certain landscape do not value the land but do not value first the lives of people who may live there, and second that plenty of creatures live there.

A.C. I saw Guy Howell in Fauna as an open-minded person who fears neither people nor animals. He embraces people's wrecked stories and rescues animals in his sanctuary. Do you think that having more people like Guy would make the world a better place?

A.Y. Yes, I do. Guy is the kind of hero in the book. The book focuses on all their lives, but he is a kind of centre. He runs a place that is basically a sanctuary for human beings and for animals. Maybe he is not concretely touching any lives, but the way he moves through the world is exemplary. In addition, the role that literature plays in his life as well it is an essential component to the shaping of this character. He evolved through the sanctuary where he lived. With many thanks to the author for her generosity. Adriana Cerminara's Interview was included in her MA thesis on an ecocritical reading of the works of Alissa York, University of Turin. 


\section{Bibliography}

York, A. (2010). Fauna. Toronto: Vintage Canada, Random House Canada.

York, A. (2016). The Naturalist. Toronto: Vintage Canada, Random House Canada. 


\section{La vérité sort de la bouche du cheval, un roman pour découvrir l'altérité Quelques mots avec Meryem Alaoui}

Martina Gennari

Università degli Studi di Macerata, Italia

Meryem Alaoui, écrivaine née à Casablanca, ayant grandi au Maroc et vivant actuellement aux États-Unis, publie son premier roman, intitulé La vérité sort de la bouche du cheval, en 2018, remportant le Prix littéraire Beur FM Méditerranée - TV5Monde. Toutefois, est-il possible d'inclure ce roman dans l'univers littéraire beur? Jmiaa, protagoniste de l'histoire, ne partage pas le même dilemme identitaire généralement vécu par les personnages des romans beurs, car elle ne connaît pas les difficultés liées à l'intégration en France. Même dans la langue adoptée par l'auteur, on ne retrouve pas les mêmes caractéristiques des romans beurs, à savoir la pratique du « français contemporain des cités » associée à un besoin identitaire et d'autodétermination dans la société, généralement jugée hostile par les personnages. Néanmoins, on constate l'utilisation d'un langage familier, truffé d'expressions issues de la sphère orale, parfois crues, à travers lequel Meryem Alaoui raconte la vie de Jmiaa, une prostituée de Casablanca, qui change totalement après la rencontre avec Chadlia, surnommée Bouche de cheval, une réalisatrice marocaine émigrée en Hollande, à la recherche d'une actrice pour son film qui se déroule dans le quartier où vit Jmiaa. Cette opportunité transformera pour toujours la vie de la protagoniste.

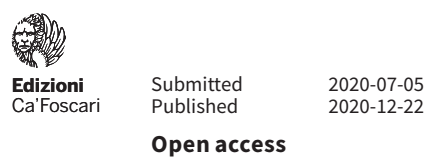

(C) 2020 | ()(i) Creative Commons Attribution 4.0 International Public License 
Personnage à l'esprit vif, plein d'énergie, extrêmement déterminé et qui parle sans ambages, Jmiaa guide le lecteur dans une ville vibrante de vie, de bruits, de couleurs et de parfums, complexe et parfois contradictoire, mais qui est aussi un reflet de son caractère. Ainsi, le lecteur découvre le quotidien d'une réalité différente en plongeant dans un quartier populaire de Casablanca, en se familiarisant avec ses nombreux personnages, mais surtout, en instaurant un contact avec Jmiaa, la narratrice de l'histoire. À travers des expédients stylistiques, notamment celui de l'apostrophe, Jmiaa s'adresse directement au lecteur tout au long de l'histoire et partage avec lui ses propres émotions, ses angoisses, ses inquiétudes et ses désirs de revanche.

Dans le roman, la langue française et la langue marocaine cohabitent en harmonie et équilibre, et le code-switching transporte constamment le lecteur d'une culture à l'autre, en lui donnant la précieuse opportunité de découvrir, grâce au glossaire final, les traditions et les aspects culturels de la culture maghrébine. Le glossaire peut être considéré comme l'élément le plus emblématique de cette ouverture vers l'altérité, car il ne s'agit pas seulement d'un outil de nature linguistique, mais plutôt de nature culturelle. On y retrouve, par exemple, les noms de certains chanteurs ou de personnages liés au monde du spectacle qui appartiennent à la culture populaire maghrébine, mais aussi des lieux géographiques et des plats typiques de la tradition marocaine.

La vérité sort de la bouche du cheval représente un exemple très significatif d'une narration contemporaine qui permet de créer un rapport avec l'altérité et les spécificités d'une culture autre.

MARTINA GENNARI Le prix littéraire que vous avez remporté vous situe dans le panorama de la littérature francophone, notamment, dans celui de la littérature beur. Est-ce que vous partagez cette « étiquette » ou est-ce que vous vous sentez « coincée »?

MERYEM ALAOUI Non, je ne partage pas cette étiquette, parce que, par définition, le « beur » est quelqu'un qui a une origine étrangère à la France et qui a grandi en France, donc quelqu'un d'origine maghrébine mais qui a grandi en France. Or, moi je suis marocaine, je suis née au Maroc, j’ai grandi au Maroc. Ce terme ne s'applique pas à moi !

M.G. La ville de Casablanca peut être considérée comme un élément autobiographique parce que vous y avez grandi. Quel est le rôle de cette ville dans le roman, dans la vie de Jmiaa et dans votre vie, aussi?

M.A. Je ne m'étais pas posé la question en écrivant, mais, en fait, en interagissant avec les gens, en voyant l'accueil que le livre a reçu, les questions qu'on m’a posées, je me suis rendu compte que Casablanca est comme un personnage dans l'histoire. Il 
s'agit d'une ville très présente : elle a des attributs physiques, elle a son énergie, elle a une psychologie, elle est, vraiment, comme un personnage du roman, pour moi. Et je ne m'étais pas du tout rendu compte. C'est la ville dans laquelle j'ai grandi, que je connais le mieux. J'ai déjà habité dans d'autres villes, mais, Casablanca, est celle dans laquelle il est plus facile, pour moi, de me reconnecter. Quand j'arrive à l'aéroport de Casa, tout de suite, j'ai l'impression que je suis chez moi. Il y a d'autres endroits où je peux avoir ce sentiment d'être chez moi, mais ce qu'il y a, peut-être, en plus, à Casablanca, est cette compréhension intuitive de ce qui se passe autour.

M.G. Pourquoi vous avez décidé d'émigrer aux États-Unis ?

M.A. J'ai émigré aux États-Unis, principalement, à cause de mon mari. Il a fondé un magazine qui s’appelle «Tel Quel», qui existe toujours, mais qui n'est plus sous la même forme, ni sous la même ligne éditoriale. On a travaillé ensemble pendant un certain nombre d'années et puis on a commencé à avoir des problèmes politiques, donc on a décidé de partir.

M.G. Un des aspects les plus intéressants de votre roman est celui de la langue. Qu'est-ce que cela signifie, pour vous, le choix d'écrire en français et quelles en sont les implications ? Est-ce que vous vous sentez, en quelque sorte, traductrice de vousmême ?

M.A. Oui et non. Non, parce que le choix du français est un choix qui s'est imposé de lui-même, parce que c'est la langue que j'écris le mieux, celle dans laquelle j'arrive à exprimer le plus précisément ce que j'ai envie de dire. Donc, quand je cherche un mot, une expression pour exprimer une émotion particulière, une tournure, ou un rythme, la langue dans laquelle j'arrive à trouver exactement ce que je cherche est le français. Donc, dans ce sens-là, je ne suis pas traductrice de moi-même, parce que la langue française est l'outil que je manie le mieux.

Oui, dans un autre sens, parce que l'histoire se déroule à Casablanca, un milieu qui est entièrement arabophone. Donc, dans ma tête, pour coller au mieux aux personnages, je pensais à beaucoup de répliques qui venaient en arabe, d'expressions qui venaient en arabe, de comparaisons qui venaient en arabe et je les traduisais ou adaptais en français. C'est, peut-être, aussi cela qui contribue à la création d'une langue un peu bizarre, même parce que Jmiaa est un personnage qui est exclusivement arabophone !

M.G. En lisant votre roman, j’ai considéré le glossaire final comme une ouverture vers le monde arabo-musulman pour le lecteur non arabophone. Est-ce qu'il s'agit d'une interprétation correcte? 
M.A. Oui, le glossaire est effectivement destiné au lecteur non-marocophone, car même un arabophone n'aura pas nécessairement les éléments de culture ou de langage pour interpréter le référentiel de Jmiaa.

M.G. Le titre du roman parle d'une « vérité qui sort de la bouche du cheval ». De quelle vérité s'agit-il ?

M.A. En effet, je ne pensais pas que l'éditeur allait garder ce titre ! Pour moi, il fallait un titre au manuscrit, alors j'ai travaillé sur des expressions du livre. J'ai un vieil ami retraité qui travaillait dans l'édition à l'époque et donc on lui a envoyé des propositions de titre. Il a dit que «La vérité sort de la bouche du cheval» c'était un bon titre et je l'ai envoyé, en pensant que l'éditeur allait faire son travail, mais, en fait, il l'a gardé ! C'est à l'interprétation du lecteur ! Il y a un proverbe français qui dit que « la vérité sort de la bouche des enfants », parce que tout ce qui sort de la bouche d'un enfant, normalement, correspond à la vérité. Donc, il peut y avoir cette liaison entre la bouche du cheval et la bouche des enfants !

M.G. À un certain point du roman, Jmiaa part pour l'Amérique, un continent qui représente un rêve dans son imaginaire. Estce que cela est lié au mythe de l'Occident comme réalité idéale, parfois utopique, dans l'imaginaire des migrants ?

M.A. Oui, c'est vrai. Ici, au Maroc, mais aussi dans beaucoup de pays, on a ce fantasme dans nos têtes qui nous fait penser que l'Occident est magnifique, une réalité où tout fonctionne, où l'argent coule à flots. Il y a ce mythe et beaucoup de personnes défavorisées au Maroc veulent partir parce qu'ils ont l'idée de ce rêve qui ne colle pas à la réalité, mais qui est impossible de déloger. La perception de l'Amérique est encore pire, parce que si l'Europe est magnifiée comme si elle était une sorte d'Eldorado, les États-Unis sont perçus comme un film, directement !

M.G. Une image qui m’a frappée vers la fin du livre est celle du « pont ». Jmiaa voit « un pont construit dans les airs. Comme une image qui n'existe pas, sauf dans les mirages de ton esprit ». Est-ce que cela a une valeur métaphorique, celle, par exemple, d'un pont qui lie des mondes et des cultures ?

M.A. Oui, cela a une valeur métaphorique, mais je pense plutôt que c'est celle de l'élévation. Le pont est au-dessus du monde et sa hauteur fait sortir Jmiaa de sa condition en l'élevant pendant un moment, la fait rêver, la place, pendant cet instant, dans un monde où tout est possible, y compris les grands rêves.

M.G. Le portrait que vous offrez de Jmiaa est celui d'une femme à la personnalité très forte, libre et indépendante, qui ne se soumet pas aux hommes. Est-ce que vous la considérez une exception dans l'époque où elle vit? 
M.A. Je n'ai pas l'impression qu'elle constitue une exception. Jmiaa a des caractéristiques de plusieurs femmes que je connais, des femmes avec son énergie. Il y a beaucoup de gens, ici au Maroc, qui sont liés à la tradition musulmane, mais qui vivent différemment. On est un pays musulman dans la constitution, mais dans la vraie vie on est beaucoup plus libres que la plupart des pays arabes. Il est vrai que, dans la plupart des cas, les gens se définissent comme musulmans, mais, dans les actions, ce n'est pas toujours comme ça : il y a des gens qui boivent, des gens qui ne respectent pas toujours leur mariage, les gens vivent ! Il s'agit d'arbitrages personnels. Beaucoup de gens ne font pas les prières, mais on se dit « Ce n'est pas grave! Ce n'est pas très méchant! Par contre, il faut qu'ils jeûnent ». On peut boire de l'alcool et en même temps ne pas accepter de voir une prostituée dans la rue. Ce sont des arbitrages personnels que chacun choisit. Il s'agit d'une forme de liberté et de prison en même temps et chacun choisit la condition dans laquelle il veut se mettre. À propos de Jmiaa, je pense que les femmes, au Maroc, ont assez de caractère !

M.G. Pour conclure, souvent, en lisant votre roman on a l'impression de voir les scènes qui se déroulent sous nos yeux, grâce aux descriptions très animées de la vie des quartiers de Casablanca. Est-ce qu'une adaptation cinématographique de ce roman peut rentrer dans vos projets futurs?

M.A. J'avais un projet d'adaptation qui était en cours, mais qui n'a pas abouti, principalement pour des raisons financières. En effet, quand j’ai fini d'écrire et que j’ai été publiée, l'adaptation cinématographique était, pour moi, un projet naturel, une continuation logique. Cela ne s'est pas fait tout de suite, mais peut être que ce n'est juste pas le moment ! 

Recensioni | Reviews | Comptes rendus 



\section{Sandra Federici}

L'entrance des auteurs

africains dans le champ

de la bande dessinée

européenne de la langue française (1978-2016)

Alessandro Scarsella

Università Ca' Foscari Venezia, Italia

Recensione di Federici, S. (2019). L'entrance des auteurs africains dans le champ de la bande dessinée européenne de la langue française (1978-2016). Paris: L'Harmattan, 2019, 353 pp.

Uscendo nella «Collection Logiques Sociales, Série: Études Culturelles, Dirigée par Bruno Péquignot», il presente contributo di Sandra Federici colloca immediatamente l'investigazione sul fumetto in un ambito di attenzione storico-sociologico, più che letterario. Questo sia detto preliminarmente, a conferma dell'oscillazione dell'oggetto d'indagine tra metodologie e punti vista alternativi e talora irriducibili, in forza altresì della natura radicalmente interdisciplinare della bande dessinée e delle narrazioni grafiche in generale. Notevole tuttavia l'intersezione, nel metodo utilizzato dall'autrice nel mettere ordine all'interno di corpus di fenomeni e testi pressoché sterminato, tra sociologia e comparatistica letteraria, percepibile nell'affiancare al concetto di campo di Bourdieu, le riflessioni su centro e periferia di Pascale Casanova e sul concetto di 'antinomia' elaborato da Paul Dirkx nel merito della ricezione di un premio Nobel anoma-

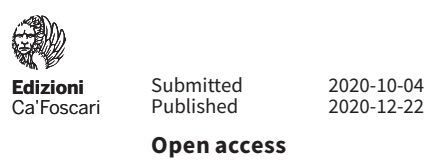

(C) 2020 | ()( Creative Commons Attribution 4.0 International Public License 
lo e conflittuale come Claude Simon, esponente di una francofonia sradicata e difficile:

Un concept utile pour penser les trajectoires des auteurs est celui d'antinomie, développé par Paul Dirkx à la fois dans une perspective générale (à propos de Claude Simon, en ce cas) et en relation avec les «littératures périphériques ».

D’un point de vue général, l'antinomie se définit comme l'« antagonisme entre une force qui favorise l'hétéronomie et une autre qui tend à l'autonomie », la " coexistence entre une dépendance envers des ressources exogènes et une raison d'être [...], la voie autonome que [l'auteur] s'est tracée lui-même ». (239)

Questi principi soccorrono particolarmente laddove una periferia, ovvero una comunità subalterna, genera strategie culturali conformi a una penuria di capitale simbolico e a un atteggiamento eteronomo che si rivela ancora e per lungo tempo tributario di un rapporto di dipendenza univoca dal 'centro'. Si può quindi ben comprendere l'analogia con gli interrogativi suscitati dal Nobel a Simon, dal momento che il problema di riscrivere i rapporti di forza all'interno dei valori estetici riaffiora puntualmente quando dalla periferia determinati autori e valori irrompono nel canone, così il fumetto e la novella grafica africana fanno prepotente ingresso «dans le champ de la bande dessinée européenne de la langue française», dalla fine degli anni Settanta a oggi, come recita il titolo del libro, costituendo valore, modificando il giudizio dei lettori e quindi trovando accoglienza nell'idiocanone della bande dessinée. Vieppiù congruente appare l'applicazione di questo modo di vedere le cose, a prescindere dalle regole del gioco postcoloniale, a produzione e ricezione della bande dessinée, genere e linguaggio di per sé coinvolto nell'interazione tra livelli culturali gerarchici ed eterogenei. In tal senso l'ampio lavoro di Sandra Federici si configura come modello per investigazioni analoghe che prendano le mosse da determinate circoscrizioni territoriali e regionali, quantunque meno estese dell'Africa francofona.

Di questi autori si parla nella seconda parte del volume; e sono: Barly Baruti, Pat Masioni, Marguerite Abouet ai quali sono dedicati paragrafi monografici); quindi i contributi più eccentrici, decorrenti già dall'inizio del Terzo millennio, e di più ardua classificazione, di Joe Daly, Anton Kannemeyer, Conrad Botes, Karlien de Villiers, Pahé, Christophe Edimo.

Era infatti necessario ricostruire con acribia il processo editoriale complesso che si pone all'origine del boom della bande dessinée africaine. Se il lieto fine attuale si identifica con la promozione estetica del prodotto grafico, solo la storia dell'editoria intesa in accezione più larga e connessa all'evoluzione dei processi comunicativi e del mercato può rappresentare la base più solida per la comprensione e l'interpretazio- 
ne degli oggetti semioticamente ibridi. In tal senso si giustifica e si deve ritenere opportuno lo squilibrio sussistente tra le due parti del volume («Approches générales» - «Le champ européen de langue française et l'entrance des auteurs africains», 39-230; «Trajectoires d'auteurs», 233-314) il cui rapporto risulta essere di oltre il doppio contro la metà. Si consideri anche la difficoltà obbiettiva di procedere a una concreta analisi testuale della bande dessinée e del fumetto in generale, in quando prodotto contestualizzabile con buona credibilità, ma non 'testualizzabile' in base alle tecniche consuete di critica letteraria. Nondimeno le annotazioni della Federici alla presentazione dell'opera dei singoli protagonisti si rivelano promettenti, interrompendosi quasi si direbbe sul più bello nella lettura dei temi e dei motivi, nonché nell'individuazione di ricorrenze e di stilemi quali appaiono con buona probabilità tanto connessi a biografemi indissolubili dall'esperienza individuale, quanto immessi nella tradizione del fumetto. Esemplare per questa angolazione la presentazione di tre maestri, Barly Baruti (già attivo negli anni Ottanta/Novanta), Pat Mansioni (entrambi congolesi) e Marguerite Abouet (ivoriana): «auteurs qui ont été retenus en fonction de leur position relativement avantageuse dans le champ»(18-19). Soffermandoci sul secondo e terzo nome citati, coerente con questa evidenza, si apprezza come Federici, dopo aver ricostruito l'itinerario pilota di Baruti, ancora indefinito tra africanità e talento individuale, indichi invece l'approdo di Pat Masioni a una forma di equilibrio tra la personale immagine pubblica di prestigiosa mediazione interculturale conseguita e la propria produzione. Il passaggio dal debutto in patria, all'affermazione attraverso la dimensione del fumetto migrante e il decisivo riconoscimento conseguito negli USA, rappresenta un paradigma eloquente:

Cas unique parmi les auteurs de l'Afrique subsaharienne francophone, Masioni réussit à publier à l'enseigne d'un grand éditeur états-unien, à savoir DC Comics ; plus précisément, il s'agit de sa filiale Vertigo, indépendante pour sa ligne éditoriale. Il a dessiné les épisodes n 13 (2009) et 14 (2010) de la série écrite par Joshua Dysart Unknown Soldier, ainsi que le tome Unknown Soldier Easy Kill, co-dessiné avec Alberto Ponticelli. ${ }^{1}$ La publication dans l'une des maisons d'édition américaines les plus respectées, spécialisée dans les œuvres destinées aux adultes, du genre horreur ou fantastique, globalement de bonne qualité, lui a donné une importante légitimation. (274)

1 Piace segnalare, a riprova della condivisione ad ampio spettro locale e oltre l'ambito francofono dei valori della graphic novel, l'edizione italiana: Un omicidio semplice, Milano: Planeta DeAgostini, 2013. Federici rammenta come nella progettazione del lavoro a un certo punto Dysart, trovandosi nella necessità di rimpiazzare Ponticelli, indisponibile per il completamento delle tavole, scegliesse deliberatamente un africano che era stato testimone della guerra civile. 
Mercato e collaborazioni artistiche di rilievo sono dunque all'origine dell'accesso di Masioni nel mainstream della narrazione grafica. Diversa strategia, ma analogamente vincente, va osservata nel percorso di Marguerite Abouet, autrice legata al molteplice ambito tematico dell'emigrazione, della nazione nativa, dell'istituzione e del genere, secondo una logica che le consente di mantenere aperto un duplice canale di comunicazione con l'Europa e con l'Africa. Come riepiloga Federici:

Le parcours de Marguerite Abouet illustre ainsi la nécessité de négocier les positions à prendre en considération, tantôt en cédant aux pressions du champ socio-politique pour jouer la partition du témoin d'origine migrante, tantôt en lui opposant son image d'écrivaine d'histoires simplement humaines (et urbaines) qui passionnent tout type de public. L'auteure oscille en fonction de cette contradiction antinomique avec l'objectif d'arriver à une « coexistence entre une dépendance des ressources exogènes et une raison d'être particulière devenue [...] dans le cas d'un créateur singulier, la voie autonome qu'il s'est tracée lui-même ». (290)

Le frasi tra virgolette sono desunte da un'intervista rilasciata della stessa sceneggiatrice di Aya de Yopougon (2005). In assenza di vera letteratura secondaria su espressioni recenti e ancora in cerca di inquadramento epistemologico, le testimonianze dirette degli autori appaiono come una fonte attendibile certamente. Si tratta di materiale elaborato da Sandra Federici con padronanza e strumenti di analisi che nel complesso propongono il protocollo adeguato e le istruzioni per l'uso per ulteriori ricerche da condurre, lo si ripeta, sul genere letterario grafico in 'campi' differenziati e in aree linguistiche diverse. Un fatto però colpisce, come la frizione antinomica e il conflitto, che si descrivono a monte di traiettorie tanto complesse, possano risolversi con l'asserzione della dignità monografica acquisita da un autore e che le testimonianze intenzionali dell'autore sembrano giustificare. In questo senso il passaggio dalla bande dessinée al roman graphique sembra aver presupposto l'intersezione di elementi autofinzionali più o meno espliciti. Andare oltre il biografismo autoriale si prefigura come la meta auspicabile per la nuova critica del fumetto. 


\title{
Luigi Gaffuri Racconto del territorio africano
}

\author{
Silvia Boraso \\ Università Ca' Foscari Venezia, Italia
}

Recensione di Gaffuri, L. (2018). Racconto del territorio africano. Letterature per una geografia. Milano: Lupetti, 318 pp.

Frutto di un lavoro di ricerca durato quasi due decenni, il saggio Racconto del territorio africano. Letterature per una geografia, pubblicato nel 2018 presso Lupetti, raccoglie le riflessioni che Luigi Gaffuri ha formulato nel corso della sua attività di geografo sul rapporto che unisce la geografia alla letteratura. Gli ultimi anni hanno visto fiorire una vasta serie di studi interdisciplinari che hanno dimostrato quanto l'individuazione di possibili collegamenti tra le due discipline sia un terreno di ricerca ancora tutto da esplorare, un serbatoio da cui trarre prospettive critiche nuove e originali. Gaffuri s'inserisce in questa scia ed elegge come oggetto della propria analisi le rappresentazioni letterarie di un territorio, quello africano, ancora oggi alle prese con le amare conseguenze di un passato coloniale che ne ha plasmato non solo la conformazione geofisica ma anche la configurazione degli immaginari ad essa collegati. Nel tentativo di dimostrare come la geografia e la raffigurazione dello spazio partecipino attivamente alla costruzione della dimensione coloniale all'interno del romanzo, il libro prende in esame tre grandi capolavori della letteratura mondiale: Tempo di uccidere (1947) di Ennio Flaiano, La mia Africa (1937) di Karen Blixen e Cuore di tenebre (1899) di Joseph Conrad. Pur tenendo conto delle peculiarità di ciascun testo, ciò che emerge dalla lettura proposta - a cavallo tra studi postcoloniali, analisi letteraria ed esperienza di terreno - è la presenza di una «geografia dell'abuso e dell'asimmetria» (30) che si cela dietro alle raffigurazio-

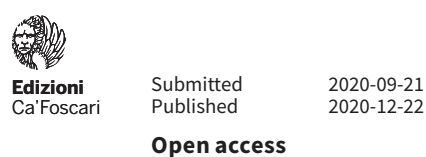

(C) 2020 | ()( Creative Commons Attribution 4.0 International Public License 
ni dell'Altro africano e che perpetua una visione eurocentrica dell'Africa avente le sue origini agli inizi dell'età moderna.

Dopo una breve ma puntuale Prefazione di Massimo Fusillo, «Spazio, paesaggio, territorio: tra geografia e letteratura», il volume si apre con due capitoli introduttivi, uno dedicato alla definizione della metodologia adottata, l'altro contenente una vasta panoramica sulla storia della disciplina. La prospettiva a cui Gaffuri ricorre per le sue osservazioni è quella della geografia culturale su base semiotica, la quale interroga il territorio come testo e lo interpreta «come tessitura di segni nella cui trama s'innestano le conflittualità latenti tra diversi attori e nel cui ordito si svelano le loro strategie di potere» (62). In questa cornice l'autore chiarisce poi cosa intende quando parla di territorio, nella consapevolezza che qualsiasi definizione adottata porta comunque in sé una certa dose di ambiguità. Secondo Gaffuri:

il territorio è l'ambito sociale che nasce dall'azione trasformativa dell'uomo sulla natura, sullo spazio fisico, piegando cognitivamente e concretamente l'ambiente ai propri fini per garantire la riproduzione biologica delle comunità insediate e la riproduzione delle loro condizioni di vita e di lavoro. Mettendo al centro il territorio, si sposta l'attenzione dall'ambiente naturale al ruolo degli uomini e delle società, svolto nella loro qualità di attori geografici dotati di intenzioni e finalità. (66; corsivo nell'originale)

Questo approccio permette di reinterpretare il binomio uomo-natura in una nuova luce: la nozione di territorio non presuppone l'opposizione dell'individuo all'ambiente naturale, ma invita piuttosto a riflettere sui legami che li uniscono. Nel saggio questi ultimi sono individuati negli atti di territorializzazione che l'uomo opera sulla natura che lo circonda e sono riletti in ottica postcoloniale allo scopo di determinare le strategie impiegate dagli stati imperialistici europei per la conquista dell'Africa. Nello specifico le strategie di territorializzazione:

hanno a che fare con l'appropriazione cognitiva dello spazio naturale e con la conseguente manipolazione intellettuale del territorio che si condensa e codifica nei nomi di luogo o, più in generale, assume le forme di una rappresentazione figurativa o discorsiva; [...] si concretizzano nell'appropriazione fisica dello spazio e del territorio, generando una materialità costruita; [...] creano contesti operativi, convenzionalmente definiti come strutture territoriali, formazioni geografiche nelle quali è possibile portare a compimento programmi di qualche rilevanza sociale.

Tali atti stabiliscono tre diversi tipi di controllo sulla superficie terrestre e sul territorio già creato dagli uomini, vale a dire rispettivamente un controllo simbolico, un controllo pratico e un controllo sensivo. (67) 
La proposta di Gaffuri è di cercare d'individuare questi atti all'interno della fitta rete di rappresentazioni che compongono l'opera letteraria. Per riuscirci, il geografo decide di setacciare le descrizioni del paesaggio presenti nei tre testi selezionati, al fine di portare alla luce il complesso dispositivo narrativo che chiama «racconto del territorio». È nel paesaggio infatti che l'uomo è in grado di percepire, mediante i prismi della propria cultura, i rapporti tra l'ambiente naturale e l'ambiente costruito (73); considerare la sua funzione all'interno del testo come quella di semplice cornice narrativa sarebbe quantomeno riduttivo.

Questo quadro iniziale, propedeutico all'analisi testuale, è seguito dalle tre sezioni riservate ai singoli romanzi. In quella consacrata a Tempo di uccidere, fanno da perno alla riflessione proposta i processi di territorializzazione che hanno caratterizzato la colonizzazione italiana dell'Abissinia. Flaiano, che aveva fatto parte del contingente inviato dal governo fascista e che era rimasto profondamente segnato da quella che aveva vissuto come un'esperienza aberrante, traccia un ritratto del territorio dal quale traspare vividamente la collisione di due entità culturali opposte, in contrasto anche nel modo di rapportarsi alla terra. Allo sguardo del colonizzatore, che proietta sui luoghi una visione puramente geometrica dello spazio, viene a contrapporsi la concezione fluida di tempo e spazio dell'uomo africano, ancora estraneo al razionalismo che sarà imposto successivamente dalla cultura occidentale. Il saggio mette in evidenza come la diversa percezione del territorio che hanno il protagonista italiano e le popolazioni locali sia uno degli elementi centrali della struttura narrativa, costruita su un sistema di opposizioni binarie che lascia trapelare le posizioni anti-imperialistiche dell'autore.

Sicuramente più coloniale è invece la visione di Karen Blixen, a cui è dedicato il capitolo successivo del volume. In un interessante compendio tra cartografia e letteratura, Gaffuri individua nelle descrizioni de La mia Africa il riflesso di una geografia della dominazione (168) che trova le sue origini nell'atteggiamento di superiorità dei colonizzatori bianchi. La scrittrice danese, ricca proprietaria terriera che ha vissuto in Kenya gran parte della sua vita prima di tornare in Europa, condivide le posizioni della propria casta e le riversa in maniera più o meno consapevole nella prosa descrittiva de $L a$ mia Africa: «L'insistenza sui panorami, sugli scenari, sugli orizzonti, sulla prospettiva rivela infatti un meccanismo di costruzione simbolica soggiacente, a matrice topografica, dal quale è difficile liberarsi» (175). Si può riscontrare un altro segno evidente del punto di vista coloniale dell'autrice nelle pagine che dedica a Nairobi: effigie di tecnologia e ricchezza, la città è apprezzata per la sua somiglianza con le più grandi capitali europee, evocate in tono nostalgico come massimi simboli di civiltà. Malgrado Karen Blixen abbia una visione prevalentemente aristocratica dell'ambiente, nel romanzo è possibi- 
le rintracciare alcuni elementi che richiamano una prospettiva keniota autoctona. Ne sono un esempio le lunghe descrizioni riservate all'imprevedibilità della pioggia: il susseguirsi dei periodi di siccità e di precipitazioni detta i ritmi vitali delle popolazioni locali, che vedono nella fertilità del terreno non una fonte di guadagno ma il presupposto per la propria sopravvivenza.

Il capitolo seguente del saggio è dedicato infine a Cuore di tenebre di Joseph Conrad. In questa sezione Gaffuri s'interroga sul rapporto tra territorio e nomos: gli atti di territorializzazione operati dal governo belga in Congo sono interpretati alla luce del declino, già a fine Settecento, dello Jus Publicum Europaeum, concetto politico elaborato da Carl Schmitt basato sulla divisione netta del mondo tra Europa e non-Europa. In particolare, la riflessione viene focalizzata sull'eredità geografica lasciata dall'imperialismo nelle ex colonie africane. Lo studioso invita gli specialisti a farsi carico della denuncia della dinamiche di sfruttamento umano e territoriale che ancora oggi caratterizzano i rapporti asimmetrici tra Europa e Africa, e a rinnegare quella branca della critica occidentale che per anni le ha passate sotto silenzio. Questo discorso prosegue poi nella sezione conclusiva del libro, dove Gaffuri analizza le rappresentazioni territoriali dell'Africa all'interno di testi subsahariani. Il ribaltamento di prospettiva mostra come la territorializzazione - eccessiva o, al contrario, insufficiente - operata dalle potenze europee continui in qualche modo a influenzare le politiche agrarie e urbanistiche dei paesi africani, penalizzandone lo sviluppo sociale, politico ed economico.

Il manuale si chiude con l'intervento di Eleonora Fiorani, «Dalla letteratura all'arte africana contemporanea», che prende in esame un ricco ventaglio di raffigurazioni del territorio all'interno di dipinti, installazioni, statue e foto di artisti africani. In particolare, l'autrice sottolinea come sia possibile individuare nel superamento delle categorie binarie - che dividono gli autori in coloro che ricercano la tradizione degli antenati e coloro che aderiscono al paradigma metropolitano - l'atto di libertà estrema che affranca gli artisti contemporanei dalle rigidità e dai pregiudizi di un certo tipo di critica postcoloniale.

Partendo dall'analisi delle raffigurazioni dell'Africa in tre grandi romanzi coloniali per arrivare all'illustrazione del paesaggio nelle ultime forme artistiche d'espressione africana, il saggio Racconto del territorio africano. Letterature per una geografia fornisce al lettore un ottimo modello metodologico ed evidenzia tutte le potenzialità offerte da un approccio interdisciplinare alla geografia e alla letteratura. Con questo libro Gaffuri ha il grande merito di emancipare il territorio dal suo ruolo secondario di sfondo: le rappresentazioni territoriali, investite di una nuova funzione narrativa, permettono al testo di manifestare le complesse dinamiche sociali e culturali su cui poggia l'interazione uomo-ambiente. 


\title{
Langue française et arts du spectacle à Montréal : aperçu de deux parcours biographiques
}

\author{
Cristina Brancaglion \\ Università degli Studi di Milano, italia
}

\begin{abstract}
In 2018 the biographies of two women who have shaped and animated the city's theatrical life since the 1930s were published in Montréal: one, Madame JeanLouis Audet, dedicating herself to the training of actors through her diction courses, the other, Monique Miller, playing key roles in the Montreal and the international theatre scene. The paths of the two women are outlined in two volumes - signed respectively by Muriel Gold and Pierre Audet - rich in iconographic documents that allow to relive some important episodes of Québec's sociolinguistic and cultural history. The aim of this contribution is to highlight how the two writings can be useful sources for the study of some of the changes that marked the advent of the 'Révolution tranquille': the first signs of women's emancipation, the emergence of Québec's cultural production, the advent of television and its correlations with the other performing arts (theater, radio, cinema), the role of some actors in Québec's linguistic, ideological and artistic evolution
\end{abstract}

Keywords French language. Diction. Theater. Madame Audet. Monique Miller.

Sommaire 1 Introduction. - 2 Madame Audet: une pionnière du théâtre à Montréal. - 3 Monique Miller : le fil d’Ariane du théâtre au Québec.

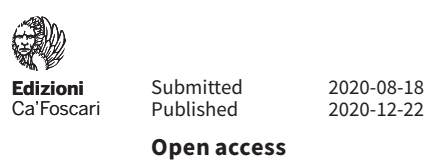

(C) 2020 | ()(B) Creative Commons Attribution 4.0 International Public License 


\section{Introduction}

L’année 2018 a vu paraître à Montréal deux biographies qui offrent aux lecteurs intéressés au Québec l'opportunité de (re)découvrir la vie de deux femmes qui ont participé à l'évolution culturelle de cette communauté francophone avant et après la Révolution tranquille $:^{1}$ Madame Jean-Louis Audet, enseignante de phonétique et diction qui a formé plusieurs générations de professionnels de la parole entre les années 1930 et 1960, et Monique Miller, grande artiste montréalaise et ancienne élève de Madame Audet. La lecture de ces ouvrages permet de se plonger dans l'histoire complexe d'une époque en pleine transformation et de l'observer de l'intérieur, en suivant les existences croisées des deux protagonistes.

Les deux paragraphes qui structurent cette contribution visent d'une part à faire connaître deux personnalités féminines qui ont contribué à façonner l'histoire linguistique et culturelle du Québec et d'autre part à mettre en lumière l'intérêt de ces deux volumes biographiques, qui offrent au lecteur l'opportunité de porter un regard nouveau sur l'histoire culturelle récente de Montréal et du Québec, reconstruite à travers des documents et des témoignages peu connus ou inédits.

Les deux femmes au centre de ces biographies sont liées par un rapport de maîtresse à élève, mais aussi par la personne de Pierre Audet, précieuse source d'information pour l'autrice du premier volume, puisqu'il s'agit du petit-fils de Madame Audet, et de l' auteur du deuxième ouvrage, dédié à une grande actrice à laquelle il est lié depuis son enfance.

\section{Madame Audet : une pionnière du théâtre à Montréal}

La première biographie, signée par Muriel Gold, reconstruit la vie d'une enseignante de diction qui s'est vouée pendant toute sa carrière à la cause du français dit 'universel'. Comme le met en lumière le sous-titre du volume, elle a participé aux premières expériences radiophoniques et a joué un rôle important dans la vie théâtrale : Plus fort ! L'histoire de Madame Audet. Pionnière du théâtre et de la radio québécoises, amoureuse de la langue française.

Muriel Gold, qui a été directrice artistique du Centre Saidye-Bronfman entre 1972 et 1980, a commencé à s'intéresser à la carrière de

1 L'expression 'Révolution tranquille' désigne le processus de modernisation politique, culturel, économique de la société québécoise mis en place dans les années 1960 sous le gouvernement de Jean Lesage. Cf. « La modernisation du Québec (1960-1981) », Histoire du français au Québec, http://www.axl.cefan.ulaval.ca/francophonie/HISTfrQC_s4_Modernisation.htm. 
Madame Jean-Louis Audet (de son vrai nom Yvonne Duckett) au début des années 1970, pendant les recherches pour sa maîtrise en théâtre à l'Université Mc Gill, consacrée aux écoles de théâtre pour enfants de Montréal. Après avoir publié les résultats de son travail dans deux articles parus dans les années 1990 (Gold 1991, 1994), l'autrice a récemment repris sa documentation pour en confectionner une biographie. Elle veut ainsi donner suite à un vœu formulé en 1990 par la journaliste et romancière québécoise Denise Bombardier, une des anciennes élèves de Madame Audet (Gold 2018, 9).

Le volume est donc une reconstruction documentaire de la vie de Madame Audet et de son entourage, dans laquelle Gold s'autorise quelques procédés de fiction caractéristiques du genre biographique (Andrès 2005 ; Vigneault 2013) sans avoir recours à la dimension réflexive qui ferait pencher le texte vers l'essai. Gold y décrit la vision de la langue prônée par Madame Audet, et parfois encore défendue par ses anciennes élèves, une vision corrective ciblée sur l'usage dit 'standard', qui est aujourd'hui assez controversée. Après environ un demi-siècle de recherches (socio)linguistiques qui ont contribué à mieux faire connaître la variation sociale et géographique du français, en mettant en valeur sa richesse fonctionnelle au sein de chaque communauté francophone, ${ }^{2}$ ces discours orientés vers la norme 'internationale', c'est-à-dire hexagonale (Corbeil 2007, 306), peuvent susciter de vives réactions. ${ }^{3}$

De ce point de vue, le volume de Muriel Gold se présente comme le tableau d'une époque révolue. Son intérêt principal demeure dans la quantité d'informations et de documents iconographiques qui y sont publiés, issus essentiellement d'archives privées. Cela rend particulièrement précieuse cette publication qui met à disposition d'un large public une quantité de photos, de programmes de spectacles et d'extraits de presse autrement presque inaccessibles. La page des « Remerciements » révèle que la documentation a été fournie essentiellement par des proches de Madame Audet : son petit-fils Pierre

2 À ce sujet l'on pourrait renvoyer à une bibliographie très vaste ; je me limiterai à rappeler la mise en valeur du français comme langue « pluricentrique » par Pöll (2005) et le volume de Reinke, Ostigy (2016) qui décrit la variation interne du français québécois ainsi que les attitudes linguistiques de ses locuteurs.

3 Une polémique s'est déchaînée entre l'automne 2018 et l'automne 2019 suite aux participations de Denise Bombardier à la populaire émission Tout le monde en parle. Les critiques de Mme Bombardier sur la qualité de la langue parlée dans les communautés francophones canadiennes minoritaires a déclenché une bataille dans la presse et sur les réseaux sociaux. On lira par exemple l'article de Patrick Lacelle paru le 7 octobre 2019 sur le site de Radio-Canada «Ici Nouveau-Brunswick » (https://ici.radio-canada.ca/nouvelle/1334595/denise-bombardier-tout-le-monde-en-parlelangue-francais) et la tentative de réconciliation de Paul Bourgeault publiée le 10 octobre 2019 sur la version en ligne du journal Le Devoir (https://www.ledevoir.com/ opinion/libre-opinion/564477/denise-au-pays-de-tout-le-monde-en-parle). 
Audet, les autres petits-enfants Audet et « les vedettes québécoises de la scène et de l'écran, qui [...] ont offert gracieusement leur temps pour évoquer leurs souvenirs de la grande dame avec laquelle ils ont étudié il y a longtemps » (6). L'« Avant-propos » de John Ripley, ancien directeur de thèse de Muriel Gold, permet de comprendre l'importance des témoignages des célébrités québécoises :

[d]e nombreux artistes de la scène reconnaissent leur lourde dette à son endroit ainsi qu'une sorte d'émancipation créative moins définie, mais également importante, issue de son engagement dans son art et envers le bien-être de ses élèves en tant qu'acteurs et personnes. Curieusement, très peu avait été écrit au sujet de cette femme remarquable. L'essentiel de ce que l'on savait d'elle avait été transmis et continuait d'être transmis oralement (7)

Muriel Gold retrace dans une brève « Préface » (9-10) sa découverte de Madame Audet en 1972 et y déclare sa fascination pour cette « grande dame » (10) disparue deux ans plus tôt, qu'elle n'a donc pas eu la chance de rencontrer. Les données biographiques antérieures à l'ouverture du studio de diction de Madame Audet sont brièvement récapitulées dans l'« Introduction » (11-13) où le lecteur découvre le vrai nom de Madame Audet, Yvonne Duckett, et apprend qu'elle est née à Sorel le $1^{\text {er }}$ octobre $1889,{ }^{4}$ d'une mère québécoise et d'un père d'origine irlandaise. Le volume est ensuite organisé en trois parties qui s'ensuivent selon un critère chronologique.

Gold reconstruit tout d'abord l'histoire de la famille Duckett (1431) en mettant en valeur, entre autres, l'ancêtre William Duckett - le grand-père d'Yvonne, qui émigra au Canada en s'embarquant comme clandestin à Dublin en 1820 - et Richard Joseph, père d'Yvonne, qui finit par établir la famille Duckett à Montréal, et qui était bilingue. Ensuite, le chapitre intitulé « Rousse et combative » (22-31) retrace l'enfance et la jeunesse d'Yvonne Duckett, jusqu'à son mariage et à sa formation comme enseignante de diction (1889-1933). Ici Muriel Gold met justement en relief la difficile situation des femmes à cette époque, quand le droit à l'éducation et à la reconnaissance socio-professionnelle n'était pas encore reconnu (Descarries 2011). Yvonne se heurte à ces difficultés dès l'âge de 11 ans :

elle en veut furieusement à son père d'avoir refusé de l'envoyer au collège comme ses trois frères aînés. [...] son père, comme beau-

4 À cause probablement d'une faute de frappe, à la p. 11 l'on indique l'année 1898 ; la date correcte cependant apparaît à la p. 22 et trouve confirmation à la p. 117 dans une photo qui reproduit l'inscription sur le monument à la mémoire de la « Marraine » situé au cimetière Côte-des-Neiges de Montréal. 
coup d'hommes de son temps, ne juge pas nécessaire qu'une fille soit initiée aux classiques. Ainsi, seuls les mâles de la famille obtiennent l'avantage de fréquenter le collège Sainte-Marie, un collège jésuite privé pour garçons. (23)

Yvonne poursuit ses études en autodidacte, et après son mariage avec le dentiste Jean-Louis Audet - suite auquel elle modifie son nom, selon un « changement d'identité habituel à l'époque » (25) - elle s'inscrit aux cours de phonétique pour adultes du Conservatoire Lassalle (1926), obtient son diplôme d'Élocution française à l'Université de Montréal (1930), commence à collaborer avec la radio CKAC et avec la Société du Bon parler français. Finalement elle parvient à ouvrir son propre studio de diction (1933), malgré l'opposition de Georges Landreau, directeur du Conservatoire Lassalle, qui « considère l'étude de la phonétique comme son domaine » et qui :

en tant qu'homme des années 1930, [...] juge odieux qu'une femme affiche son intelligence, son ambition et son enthousiasme de manière aussi flagrante. Très rares sont les femmes mariées qui continuent à travailler après leur mariage ; continuer à travailler après avoir donné naissance à des enfants est encore plus impensable. Bien des gens considèrent que les femmes qui travaillent menacent les valeurs familiales traditionnelles de l'Église catholique. (30-1)

L'on sait que le projet de Madame Audet était celui de rendre accessible l'enseignement de la diction aux enfants de toutes les classes sociales (Gendron 2014). Gold soutient en outre que le modèle visé n'était « pas le français 'à la française', mais une 'voix de chez nous' bien parlée, bien prononcée » (31) : malheureusement ces affirmations, remarquables pour ceux qui s'intéressent à l'évolution de la norme orale au Québec, ne sont pas référencées; elles traduisent sans doute l'attitude de Madame Audet aux débuts de son activité, avant de subir l'influence des linguistes français.

Le dynamisme de Madame Audet est bien mis en lumière dans le troisième chapitre, qui emprunte son titre à une célèbre chanson appartenant au rituel quotidien de ses élèves : « Le beau palais de dame tartine » (32-41). Gold y décrit le studio de la rue Saint-Hubert, la participation de quelques enfants qui allaient devenir des célébrités (Gaétan Labrèche, Pierre Dagenais), les concerts et les émissions radiophoniques pour enfants dirigées par Madame Audet, parmi lesquelles Radio Petit-Monde, " la première émission radiophonique au Québec (et sans doute au Canada) présentée par des enfants, pour des enfants » (39), lancée en octobre 1933. Mais la série qui «attirera des milliers d'auditeurs québécois et fera connaître les acteurs et le Studio Saint-Hubert de Madame Audet » (41) est Les Aventures de Madeleine et Pierre, à laquelle Gold consacre le chapitre 4, qui en 
reprend le titre (42-7). Cette émission financée par The Kellogg Company of Canada a été dirigée par Madame Audet entre 1938 et 1949 et a vu la participation de ses deux fils André, scénariste, et Jean-Marc, comédien. Ce radio-feuilleton, situé dans le village québécois fictif de Mont-Tranquille, se composait d'épisodes de 15 minutes qui étaient diffusés sur les ondes de CKAC et de CHRC. L'histoire de cette émission radiophonique, et des mises en scènes théâtrales qui en ont été tirées, a été reconstruite et documentée par Galarneau (2006). Muriel Gold souligne la dimension éducative de ces productions, qui investit aussi la correction linguistique :

Toujours soucieuse d'enseigner le bon parler, Madame Audet veillait à introduire, sans avoir l'air d'y toucher, des corrections à la prononciation. Par exemple, quand Ti-Coune [personnage anglophone] disait "Viens donc icitte », un autre personnage le reprenait : « Non, on dit ici ». (47, italiques dans le texte)

Malgré cela, quelques aspects de la variation régionale du français parlé au Canada étaient mis en valeur, grâce notamment au talent de Jean-Marc :

Marco [Jean-Marc] joue dans Madeleine et Pierre pendant dix ans. Comme son frère excelle dans le maniement des dialectes, André crée pour lui des personnages de différentes régions du Québec, comme Zéphirin, un pêcheur gaspésien, et d'autres issus de communautés autochtones. (43)

Quant aux contenus des cours de phonétique que Madame Audet dispensait aux enfants (et à leurs mères, qui devaient les accompagner), ils peuvent être reconstruits grâce aux informations et aux réflexions que Madame Audet elle-même a publiées dans son manuel de diction : Les Monologues du Petit-Monde, daté 1938 à la fin du volume, et souvent réédité. Gold, en s'appuyant sur une réédition de 1958, s'en sert pour décrire la méthode d'enseignement de Madame Audet et le répertoire de textes qu'elle proposait aux enfants plus jeunes (de 3 à 11 ans : ch. 5, « La cage aux oiseaux », 50-7) et aux adolescents (ch. 6 : "Une missionnaire de la langue française », 58-63). Gold ne manque pas d'introduire quelques anecdotes utiles pour se faire une idée plus précise de cette femme, assez exigeante du point de vue de l'enseignement, mais très généreuse avec ses élèves :

Le réfrigérateur des Audet était toujours accessible : les enfants grimpaient les marches jusqu'à la cuisine et prenaient dans le frigo la nourriture qui faisait cruellement défaut chez eux. Pour ménager la fierté de ces enfants et celle de leurs parents, Madame Audet prétendait que l'école recevait des bourses. En réalité, elle 
n'obtint jamais le moindre sou du gouvernement. « Développer le talent, c'est ça mon salaire ! La culture avant l'économie ! » disait-elle. (56)

Ce trait de son caractère a été mis en honneur en 1989 par l'acteur et metteur en scène Albert Millaire, qui a intitulé à Madame Audet un fonds de secours pour les élèves en difficulté du Conservatoire d'art dramatique de Montréal (105).

L'entourage de Madame Audet est présenté dans la deuxième partie du volume de Muriel Gold (64-96), qui offre au lecteur des informations sur les anciens élèves de Madame Audet devenus ensuite des vedettes, mais aussi sur ses collaborateurs et sur sa famille, jusqu'à l'évocation de la «fin tragique » d'André Audet, décédé à 37 ans, en 1952, après avoir ingéré une solution toxique.

Dans la troisième et dernière partie du volume (97-114), Gold se soucie de mettre en relation le programme de correction linguistique de Madame Audet avec les objectifs de "La Révolution tranquille » (ch. 11 : 97-104). Les chercheurs en études québécoises découvrent ici une donnée intéressante, qui marque le lien existant entre le programme éducatif de Madame Audet et celui qui est considéré comme le déclencheur de la querelle du joual, le frère Untel $:^{5}$

Les préoccupations de Madame Audet en matière de réforme de l'éducation étaient en parfaite adéquation avec les visées du gouvernement provincial de Jean Lesage. La politique « d'éducation pour tous » propre à la Révolution tranquille tirait son origine des inquiétudes quant à la qualité du français parlé au Québec. Madame Audet avait un allié solide en la personne du frère Jean-Paul Desbiens (connu sous le nom du Frère Untel), qui n'hésitait pas à dénoncer le système d'éducation dans des lettres colligées dans son ouvrage intitulé Les Insolences du Frère Untel. (98)

L'histoire de Madame Audet se termine par l'évocation des prix et hommages qu'elle a reçus : une réception d'honneur à l'occasion de ses 25 années d'enseignement en 1957 et l'attribution de la médaille de l'Alliance française en 1965. Le dernier chapitre, « Une pionnière de la langue » (ch. 13, 108-14) est une sorte de récapitulation des multiples activités de Madame Audet, de ses idées sur l'enseignement du français oral et, plus en général, de sa vision du Canada. Enfin, dans un «Épilogue » (115-17) et trois « Annexes » (118-34) Gold offre des informations sur les lieux de Montréal qui sont liés à la mémoire de Madame Audet et sur les membres plus jeunes de la famille Audet,

5 Sur cette querelle linguistique et, plus généralement, sur la question du joual, cf. Bouchard 2002, Corbeil 2007, Desbiens 2010. 
parmi lesquels Pierre Audet, petit-fils de Madame Audet et auteur de l’autre biographie présentée dans cette contribution.

\section{Monique Miller : le fil d'Ariane du théâtre au Québec}

Pierre Audet a sans doute reçu comme héritage familial le talent de l'écriture. Sa biographie Monique Miller, Le bonheur de jouer $-{ }^{6} \mathrm{au}$ contraire du volume de Muriel Gold, qui était très proche d'un ouvrage académique - révèle un travail de mise en fiction assez soigné, qui emmène le lecteur à travers l'histoire culturelle du $\mathrm{XX}^{\mathrm{e}}$ siècle québécois de façon très naturelle. La vie et la carrière de cette grande actrice y sont retracées à travers 48 chapitres non numérotés qui se présentent comme des tableaux thématiques disposés selon un ordre chronologique. Les célébrités des arts du spectacle deviennent des personnages réels et intimes, qui ne manquent pas de s'exprimer quelquefois à travers des dialogues rédigés dans un style évoquant la spontanéité de la langue orale. Le biographe, qui glisse occasionnellement dans sa prose des éléments lexicaux nous rappelant son appartenance québécoise, reconstruit la vie de Monique Miller en croisant les nombreux témoignages recueillis par un grand nombre d'entretiens avec des acteurs et créateurs qu'il a interviewés (273-4).

Pierre Audet ouvre son livre par une sorte d'hommage à son père André, le fils aîné de Madame Audet disparu tragiquement en 1952 (cf. supra). André Audet avait été auteur et adaptateur de nombreux textes pour enfants et ses écrits étaient retapés à la machine par une jeune fille de quinze ans qui rêvait de devenir une grande actrice et qui avait trouvé chez les Audet une seconde famille : Monique Miller. Ce travail de transcription, «sur des feuillets superposés, séparés les uns des autres par de minces papiers carbone » (30) a été un entraînement efficace au métier d'actrice :

Elle exécute minutieusement ce travail en découvrant le texte à première vue. Les dialogues surgissent sous ses doigts. Une phrase en quelques clic-clacs... retour au chariot... la réplique déboule sur la ligne suivante. Machinalement, un peu comme si c'était elle qui écrivait, elle se familiarise avec les personnages, leurs motivations, les différents niveaux de langage correspondant à leur statut ou leur personnalité. Elle s'initie au point de vue de l'auteur en tapant les didascalies, les indications d'action et d'intention. Sans compter les notes de mise en ondes, les timings, les effets sonores, les choix de musique. Cet exercice d'observation et

6 Les renvois à ce volume à l'intérieur de ce paragraphe se feront par la simple indication des pages entre parenthèses. 
de précision est à la base de sa formation et lui vaudra l'admiration de ceux qui travailleront avec elle plus tard. (31)

Fascinée par l'émission Les Aventures de Madeleine et Pierre, Monique décide de prendre des cours de diction avec Madame Audet et reste captivée par cette femme extravagante qui lui offre bientôt la possibilité de jouer des petits rôles dans des émissions radiophoniques, à une époque où « la radio [...] est un haut lieu d'effervescence créatrice. Et une manne pour les comédiens » étant donné qu'on y diffusait « des feuilletons écrits par les meilleurs auteurs » (27).

Tout au long du volume, l'on peut retrouver les étapes principales de la vie de Monique Miller : les moments charnières de sa vie sentimentale, à commencer par son mariage avec François Gascon, qui est à l'origine de son éloignement de Madame Audet (76-7) ; la naissance de son fils Patrice, en 1955, et la difficile conciliation entre la famille et le travail ; les tourments liés aux autres relations qui ont fait suite à la fin de ce premier mariage ; son engagement dans le mouvement de grève de Radio-Canada en 1958 (évènement reconstruit aux pages 108-10) ; sa participation active à la campagne pour le premier référendum en 1980 ; les pertes douloureuses de ses proches, de ses collègues et amis. Ces épisodes plus ou moins privés sont incorporés à la narration de sa carrière, qui la révèle active dans plusieurs arts du spectacle : de la radio, au théâtre, au cinéma, à la télévision elle est toujours présente sur les scènes nationales et internationales, en étonnant le public tant dans des rôles du répertoire classique que dans des pièces et adaptations québécoises ou dans des expérimentations plus modernes. Le lecteur la suit de spectacle en spectacle et peut ainsi se rendre compte de la richesse de la vie culturelle du Québec et au Québec dans ces années.

En assistant aux succès de Monique Miller, l'on peut retrouver les œuvres principales qui ont marqué la naissance d'une production ancrée dans la réalité québécoise. En 1952, à l'âge de 18 ans, elle joue dans l'adaptation cinématographique de la pièce Tit-Coq de Gratien Gélinas, devenu une grande vedette au Québec avec ses Fridolinades, qui « adapt[aient] le burlesque américain à la réalité canadienne-française » (59). L'année suivante, Monique connaît un succès international avec le rôle de Ciboulette dans Zone, une pièce à intrigue policière située dans la réalité urbaine montréalaise, pour laquelle Marcel Dubé «a écrit les dialogues en québécois, un parler populaire structuré » (72). En 1955 elle participe à la série télévisée Cap-aux-Sorciers de Guy Dufresne, qui met en valeur la culture régionale de la côte de Charlevoix et la « langue savoureuse » de ses habitants : "Monique découvre avec ravissement un langage poétique, truffé des expressions imagées de Port-au-Persil » (85). En 1968, elle incarne le personnage d'Élise dans l'adaptation du Pygmalion de George Bernard Shaw par Eloi de Grandmont, une «ga- 
mine du Faubourg à m'lasse » (155) qui s'exprime en joual. Grandmont trouve en effet que cette pièce :

prête mieux à une adaptation au Québec qu'en France. L'écart entre le français normatif et le joual est aussi grand que celui qui existe entre le Queen's English et le parler cockney de Cheapside. (155)

La pièce choque le public du TNP (Théâtre National Populaire) de Montréal, mais Monique défend ce choix, elle trouve qu'il « reflète le drôle de bilinguisme pratiqué par bien de Montréalais cultivés qui passent indifféremment du bon français au joual, selon les circonstances ». Du point de vue de la récitation, elle souligne que «le joual demande à être articulé avec autant de précision que le bon français» (156).

Quelques semaines plus tard, le joual entre en scène avec Les Belles-Sœurs de Michel Tremblay. Pierre Audet explique le bouleversement que ces deux représentations comportent dans la pratique théâtrale :

Avec Pygmalion, et surtout Les Belles-Sœurs, commence le déversement du joual dans la dramaturgie québécoise. Plusieurs acteurs formés à la grande école classique avaient toujours eu le souci de ressembler aux acteurs français. Ils connaissent alors une longue traversée du désert. Même le parler québécois de Marcel Dubé tombe en désuétude. La mode est à une langue relâchée, à l'improvisation, aux créations collectives sans l'aide d'un dramaturge. Les élèves du Conservatoire ne rêvent que de jouer du Michel Tremblay. L'École nationale de théâtre ouvre grand les écluses, on cesse d'y donner des cours de diction. (157)

Ce changement cependant ne nuit pas à la carrière de Monique Miller : elle « naviguera sans effort à travers toute cette turbulence langagière » (157) et aura aussi quelques occasions d'interpréter des personnages de Michel Tremblay, le plus récent étant la vieille $\mathrm{Al}$ bertine dans une reprise d'Albertine en cinq temps en 2014 (255-8).

Dans les années 1990, elle avait en outre joué Mme Félix dans Montréal P.Q., un téléroman de VLB (Victor-Lévy Beaulieu) qui « évoque les mœurs du Québec très religieux de l'après-guerre, quand le vice et la vertu semblaient cohabiter sans se toucher » (203). Les choix linguistiques de l'auteur posaient des difficultés aux comédiens :

il ajoute à son vocabulaire des mots-valises, des « mal entendus », des néologismes de son cru, comme «malaucoeuré ». [...] Pour mémoriser, les comédiens fonctionnent habituellement par association. Tel mot renvoie à tel mot et tel mot à telle image. Mais VLB 
s'amuse à déconstruire tout ça. (204-5)

Face à ces obstacles, Monique « devient [...] la gardienne du texte, insiste pour que tous le disent tel quel » (205) en confirmant ainsi sa grande adaptabilité, un trait de sa personnalité qui est bien mis au clair à travers les mots d'Annick Bergeron :

elle était capable de s'aventurer dans des esthétiques dramatiques différentes. C'est pour ça qu'elle a autant joué dans sa vie. Et autant de choses différentes. Je ne sais pas si nous, les plus jeunes, on aura la chance de toucher à une aussi grande variété de répertoires. (214)

C'est sans doute grâce à cela que Monique Miller a su s'intéresser à la nouvelle génération, la comprendre, la soutenir :

À la mi-cinquantaine, Monique ne veut pas regarder le train passer. Elle choisit de rester jeune avec les jeunes, tout en restant fidèle aux acteurs de sa génération. Elle se passionne plus que jamais pour le nouveau théâtre. Elle va à la rencontre des auteurs, des acteurs après les spectacles. Elle se manifeste avec la nouvelle génération pour continuer à évoluer avec elle.

Ce faisant, elle devient une espèce d'aiguilleuse dans le métier. Quand elle découvre un nouveau talent [...] elle prend le «mégaphone Miller » et tout le monde du métier le sait le lendemain. (199)

Cette ouverture d'esprit lui permet d'être toujours présente sur la scène et lui vaut une carrière immense. Comme le dit l'acteur Jean-François Casabonne, " pour raconter le théâtre d'ici, il faut dérouler Monique », puisqu'elle représente « le fil d'Ariane » du théâtre québécois (269). Elle sait en conserver des souvenirs précis, à tel point que sa culture et sa mémoire sont devenues légendaires : elle les a toujours nourries en étudiant à fond les textes et en s'imposant un grand effort de documentation et conservation :

« Téléphone à Monique. C'est sûr qu'elle le sait. » Cette phrase revient souvent dans la bouche d'Albert Millaire, de Michel Tremblay ou d'André Gagnon, quand l'un d'eux a besoin de se rappeler d'un évènement artistique ou d'un fait qui concerne leurs propres carrières. Elle a un immense bagage culturel dans lequel elle peut aller puiser à tout moment à la vitesse de l'éclair. Des événements de l'histoire du Québec, du théâtre, de la vie personnelle de ceux avec qui elle a travaillé. Même les noms et dates de naissance des enfants de tous ses amis. C'est le mystère Monique. (235)

Le thème de la mémoire est d'ailleurs un sujet important pour Mo- 
nique, ainsi que pour son biographe, qui nous transmet cet appel contre l'appauvrissement de la mémoire collective :

Au Québec du Je me souviens, dit Monique, la mémoire a quatre ans. Si t'as rien fait en quatre ans, t'es dans l'oubli complet. Il n'y a plus aucun respect pour ce qui a été fait avant. Tout est dans l'instantané. On est dans le bruit des nouvelles affaires. Next! (234)

Cette biographie, ainsi que celle de Madame Audet, sont finalement de véritables travaux de conservation, et de transmission, de la mémoire d'une portion de l'histoire québécoise. Tout lecteur curieux, tout chercheur, saura y glaner des informations percutantes sur les métiers de la scène, sur les spécificités des différents genres proposés à la radio, au théâtre ou à la télévision et sur les changements produits par l'évolution de ces différents médias au cours du $\mathrm{XX}^{\mathrm{e}}$ siècle et même au-delà. On pourra s'amuser à chercher de petites anecdotes curieuses qu'il est impossible d'évoquer dans le cadre de cette contribution, comme celle qui révèle l'origine de la chanson Mon Pays de Gilles Vigneault ou celle qui dévoile l'ancêtre commun du duo (Monique) Miller et (Albert) Millaire (Audet 2018, 104, 137-8). Les documents iconographiques qui enrichissent les deux volumes agrémentent la lecture et permettent de se faire une idée plus claire des personnalités, des milieux évoqués. C'est le bonheur de revivre une époque à travers les yeux de deux témoins privilégiés.

\section{Bibliographie}

Andrès, B. (2005). «L'essai biographique : incarner l'archive ». Voix et Images, 30(2), 67-78. https://doi.org/10.7202/@11244ar.

Audet, P. (2018). Monique Miller. Le bonheur de jouer. Montréal : Les Éditions Libre Expression.

Bouchard, C. (2002). La langue et le nombril. Une histoire sociolinguistique du Québec. Montréal : Fides.

Corbeil, J.-C. (2007). L'embarras des langues. Origine, conception et évolution de la politique linguistique québécoise. Montréal : Québec Amérique.

Desbiens, J.-P. (2010). De quoi ont-ils peur? Onze lettres insolites du frère Untel au "Devoir". Présentation, commentaires et notes de L.-A. Richard; préface de B. Descôteaux. Québec : Les Presses de l'Université Laval.

Descarries, F. (éd.) (2011). Ligne du temps de l'histoire des femmes au Québec. Réseau québécois en études féministes (RéQEF) et Conseil du statut de la femme (CSF). http://www. histoiredesfemmes.quebec/.

Galarneau, D. (2006). L'Univers de Madeleine et Pierre. Outremont : Carte blanche.

Gendron, J.-D. (2014). La modernisation de l'accent québécois. De l'accent traditionnel au nouvel accent: 1841-1960. Esquisse historique. Contribution à l'histoire de la prononciation du français au Québec. Québec : Les Presses de l'Université Laval. 
Gold, M. (1991). "A Missionary of the French Language through Drama: Madame Jean-Louis Audet (1890-1970) ». Theatre Research in Canada / Recherches théâtrales au Canada, 12(1). https://journals.lib.unb.ca/ index.php/TRIC/article/view/7277.

Gold, M. (1994). « Le Petit-Monde de Madame Audet (1933-1969) ». L'Annuaire théâtral : revue québécoise d'études théâtrales, 6, 165-92.

Gold, M. (2018). Plus fort ! L'histoire de Madame Audet. Pionnière du théâtre et de la radio québécoises, amoureuse de la langue française. Montréal : Bouquinbec.

"La modernisation du Québec (1960-1981) ». Histoire du français au Québec. http://www.axl.cefan.ulaval.ca/francophonie/HISTfrQC_s4_ Modernisation.htm.

Pöll, B. (2005). Le français langue pluricentrique ? Études sur la variation diatopique d'une langue standard. Frankfurt am Main ; Berlin ; Bern [etc.] : Peter Lang.

Reinke, K. ; Ostigy, L. (2016). Le français québécois aujourd'hui. Berlin ; Boston: De Gruyter.

Vigneault, R. (2013). «La subjectivité comme vérité. Réflexions sur l'essai biographique ». Dion, R. ; Regard, F. (éds), Les nouvelles écritures biographiques: la biographie d'écrivain dans ses reformulations contemporaines. Lyon : ENS Éditions. https://doi.org/10.4000/books.enseditions. 4508. 



\section{Alessandra Ferraro "Représentations littéraires de la différence culturelle »}

Fabiana Fianco

Università Ca' Foscari Venezia, Italia

Review de Ferraro, A. (éd.) (2017). «Représentations littéraires de la différence cultu-

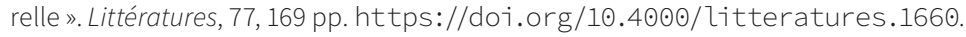

Dans un monde globalisé où les barrières géographiques, socio-politiques et culturelles semblent désormais en voie de disparition, la rencontre avec l'Autre paraît un processus parfaitement ancré dans notre réalité. Le numéro 77 de Littératures, dirigé par Alessandra Ferraro, remet pourtant en question l'acceptation inconditionnelle d'une altérité culturelle qui n'est pas toujours facile à discerner et à respecter. La problématique autour de laquelle se constitue le dossier est extrêmement claire et reconnaît à la littérature une place privilégiée : «Les textes littéraires peuvent-ils fournir des clés pour affronter le 'choc des civilisations' qui paraît inévitable dans le monde contemporain ? ». C'est à partir de ce questionnement que les articles réunis essaient d'interroger le rôle que la littérature a pris dans la représentation de la différence culturelle au fil du temps. La crise qui - d'après Nicola Gasbarro - «traverse l'ensemble de notre savoir » est intelligemment affrontée sans s'arrêter à une seule perspective ou approche méthodologique et sans limites chronologiques : du Moyen-Âge jusqu'à l'époque postcoloniale, la rencontre avec l'altérité est analysée tant du point de vue historico-anthropologique que linguistique, sociologique et proprement littéraire, offrant ainsi au lecteur plusieurs angles de lecture.

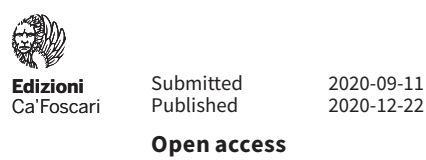

(C) 2020 | ()( Creative Commons Attribution 4.0 International Public License 
L'ouverture du dossier par Nicola Gasbarro - «Un nouveau récit anthropologique et littéraire ? » (17-29) - confronte d'emblée les lecteurs à un paradoxe postmoderne au cœur du choc des civilisations : d'un côté, la mondialisation aurait complexifié les relations culturelles; de l'autre, elle n'aurait pas éliminé la difficulté concrète de coexistence et promu le renforcement d'une perspective inclusive. Gasbarro propose alors de remplacer les grandes théories du savoir scientifique par « la pragmatique du savoir narratif » en s’appuyant sur le pouvoir social et symbolique du métarécit, entendu comme un « petit récit » du quotidien qui bouscule la grande histoire et l'esprit universel. L'analyse de sa structure lui permet d'identifier un système organisé de codes culturels spécifiques dont les dynamiques dépendent de la relation variable entre réel et imaginaire, ainsi que d'une certaine complexité diachronique et synchronique des éléments qui le composent. À travers l'analyse comparative et différentielle des métarécits, Gasbarro en présente une classification pour définir la diachronie des civilisations et la variété de leurs perspectives pratiques. Pour l'auteur, le métarécit littéraire peut devenir une anthropologie du nouvel imaginaire mondial et un « instrument indispensable pour relancer l'histoire » d'un monde postmoderne qui éprouve encore le désir de raconter la diversité.

Après avoir posé les bases d’un conflit historique qui est loin d'avoir été résolu aujourd'hui, la contribution de Sergio Cappello, "Différences et altérité dans le récit idyllique médiéval : Floire et Blancheflor » (31-46), nous projette au milieu du XIIème siècle. À travers une analyse cohérente et complète, l'auteur présente le récit comme la possible réécriture d'un conte arabe et compare la version médiévale avec les récits épiques traditionnels. Si dans ces derniers c'est la conversion religieuse qui domine et qui marque une réduction sensible de l'altérité, dans le récit idyllique médiéval prévalent au contraire le métissage, l'adoption de valeurs étrangères et l'acceptation de la différence. Le conflit entre l'Orient andalous et l'Orient arabo-musulman se purifie donc dans " une œuvre porteuse d'une critique culturelle interne » où le mariage scelle un pacte de réciprocité sans meurtre ni rivalité.

L'Orient reste au centre de l'article d'Adone Agnolin, « Le rite et le lieu de l'autre. L'accomodatio jésuite au Malabar » (57-65). En explorant le domaine asiatique de la colonisation espagnole et portugaise, Agnolin se focalise sur la représentation de l'Orient par le filtre de l'expérience missionnaire des jésuites. Tiraillés entre la nécessité de suivre leurs principes théologiques et doctrinaux ainsi qu'un processus d'adaptation aux sociétés locales, les missionnaires ont réussi à concilier la complexité rituelle locale avec la culture occidentale. Selon Agnolin, l'inclusion de l'altérité religieuse des indigènes a été possible grâce à l'accomodatio jésuite : la médiation réalisée sur le plan d'une « ritualité sacramentelle » aurait donc permis une identi- 
fication opérationnelle entre deux univers apparemment conflictuels. L'auteur ne s'arrête pas là et souligne que la querelle sur les accommodements a été également au centre de la philosophie de Voltaire et d'Antoine-Marin Le Mierre. Agnolin argue que c'est la complexité de l'univers des Lumières qui a instauré une nouvelle accomodatio ancrée dans la communion entre le général et le spécifique des civilisations indigènes.

De contexte colonial il est encore question dans « Ici et là-bas : la Nouvelle-France et la France dans Lettres au cher fils d'Élisabeth Bégon » (67-77). Dans ce texte, Elisabeth Nardout-Lafarge analyse la correspondance de l'aristocrate québécoise entre 1748 et 1753. Elle en tire l'un des premiers témoignages de la Nouvelle-France à travers une base manuscrite originale qui vient de prouver sa valeur littéraire. D’après Nardout-Lafarge, Élisabeth Bégon illustre une « identité édifiée entre colonie et métropole », mais qui éprouve des difficultés sociales énormes tant au Canada qu'en France. L'analyse de la correspondance reflète un décalage culturel qui s'opère dans une tension continuelle entre deux pays qui lui permettent néanmoins de se redécouvrir sur le plan identitaire. L'auteure observe que c'est le contexte colonial qui déclenche un processus de différenciation culturel réalisé à l'épreuve du quotidien plutôt qu'au niveau de l'expression des idées ou de la création d'un récit.

En allant au-delà des domaines français et francophones, Cristina Pompa analyse la construction d'une identité symbolique et nationale au Brésil dans « Raconter l'Indien. Quelques exemples dans la littérature brésilienne » (79-100). L'altérité indigène est interrogée en fonction d'un discours national remontant à l'époque coloniale et devenant de plus en plus fondamental entre le XIX ${ }^{\text {ème }}$ et le XX X̀me $^{\text {en }}$ siècle. L'exploration et l'évolution de l'image de l'Indien au fil du temps permettent à Pompa de suivre deux pistes de recherches - les problèmes ethniques du Brésil et la question identitaire - à l'aide d'un patrimoine narratif et collectif hétérogène et diversifié : du domaine strictement littéraire, elle puise dans un réservoir anthropologique, éducatif et proprement culturel. Dans un article exhaustif et riche en références textuelles, Pompa nous apprend que malgré la place ambiguë souvent reconnue à l'Indien, ce dernier reste un élément crucial pour la reconnaissance du Brésil en tant que pays multiculturel.

Le panorama culturel et littéraire du dossier s'étend jusqu'à l'Afrique subsaharienne avec la contribution « Regards obliques : les représentations linguistiques de différence dans le roman subsaharien d'expression française » (101-15). Valeria Sperti s'intéresse aux dispositifs discursifs et énonciatifs qui manifestent des procédés d'altérisation du français et de différenciation culturelle qui découlent d'une manipulation volontaire de la part des auteurs subsahariens. Le corpus étudié est bien ciblé : les extraits analysés sont tirés des «nouvelles écritures africaines » de Kourouma, Lopès, Sassine 
et Labou Tansi publiées à partir des années 1980. Sperti analyse ainsi l'indigénisation des langues occidentales au moyen du " code-switching », l'usage social de la parole, l'hétérolinguisme et les relexifications qui mettent en évidence les tropicalités des personnages affichés. Une bonne partie de l'article est consacré à L'État honteux de Sony Labou Tansi qui est étudié sous un angle assez captivant : l'adéquation de la première version du roman aux instances hexagonales et le concept de "tropicalité » sous forme de figures stylistiques et discursives. Par sa contribution, Sperti conclut que les distorsions hybrides que les auteurs subsahariens font subir à leur langue détruisent « la fixité de la différence » et de l'altérité, tout en gardant un œil sur la tradition africaine.

Sylvie Vignes nous fait voyager du contexte africain à celui du Québec contemporain dans «Chemins vers soi en passant par l'autre : poétique de la différence chez Monique Proulx » (117-26). Les textes choisis - Les Aurores montréales, Champagne et Ce qu'il reste de moi - sont savamment interprétés à la lumière d'un entrecroisement entre langues, ethnies et cultures multiples, ce qui rend ses romans porteurs d'une véritable «poétique de la différence ». Caractérisés par l'ouverture aux autres et le refus de l'exclusion ou de l'assimilation, les œuvres analysées affichent des personnages qui incarnent ce que Vigne définit comme une « impitoyable et incessante guerre des mondes ». Elle redécouvre ainsi en Proulx une écrivaine qui exploite sa plume pour montrer avec insistance que la rencontre avec l'Autre, au sens anthropologique, fonde toute culture - y compris la culture québécoise - et contribue à la construction d'une identité saine.

Le dossier sur la représentation de la différence culturelle se clôt sur une lecture inédite de « L'éternelle différence juive dans Pour en finir avec la question juive de Claude Grumberg » (127-40). À travers une excellente analyse discursive du dialogue entre le Juif natif et le Voisin antisémite, Jean-Paul Dufiet argue que face à la question de l'identité juive, Grumberg montre un vide définitionnel et « l'impuissance de deux discours opposés ». Grâce aux théories de Lévinas et à la mise en relief de l'humour de la pièce, Dufiet explique le paradoxe qui en est au cœur : Grumberg ne veut pas donner une définition de l'être juif ou du non-être juif, mais essaie plutôt de dessiner une identité juive «fractionnée et conflictuelle », car « lui chercher un contenu unique c'est se heurter à la pluralité ». L'analyse de l'échange théâtral révèle efficacement que Glumberg ne réduit pas l'altérité au même et qu'il exprime, au fond, les différentes manières et possibilités d'être Juif.

Ce numéro de Littératures présente également un inédit par Pierre Samson, « De messie à homme chevauché : le nous révélé par l'autre » (143-9) et une étude libre par Vivien Matisson, " Recourir à la détestable beauté des phrases : les enjeux de 'l'inflation verbale' dans la prose de Louis-René des Forêts » (153-64). Si la contribution de Ma- 
tisson dévoile au plus près « le sentiment de la langue » propre à des Forêts, celle de Samson est plus au diapason avec les articles qui précèdent : en expliquant comment il a réussi à façonner une représentation romanesque fiable et crédible du Brésil sans y être jamais rendu, l'écrivain québécois nous rappelle qu'au-delà de toute différence culturelle, c'est l'essence d'un personnage qui compte, ainsi que le courage de «tourner le dos à cette étrange conception d'une vérité universelle».

Que ce soit au moyen d'une perspective anthropologique, socio-historique, linguistique ou littéraire, toutes les contributions du numéro 77 de Littératures constatent que la différence culturelle est loin d'être un sujet que nous pouvons considérer «épuisé ». La thématique choisie est explorée sous plusieurs formes littéraires et à différentes époques, en essayant de donner une vision globale d'une différence culturelle qui évolue au cours des siècles. Si quelques-uns des articles semblent plutôt théoriques, la plupart privilégie des références textuelles qui aident à mieux saisir l'argumentation proposée. L'originalité du numéro repose certainement sur la multiplicité de pays pris en considération - France, Québec, Brésil, Afrique - ce qui stimule et encourage l'approfondissement de la représentation de la différence culturelle dans d'autres littératures pour une étude comparative. Sans jamais réduire le concept de « différence » à une seule définition ou modalité de manifestation, ce dossier de Littératures semble suggérer que l'inévitable « choc des civilisations » du monde contemporain peut être positivement exalté par le moyen le plus évident mais encore peu exploité : la littérature. 



\section{Francis-Joachim Roy Les chiens}

\section{Silvia Boraso}

Università Ca' Foscari Venezia, Italia

Review de Roy, F.-J. (2019). Les chiens. Montreuil : Le Temps des Cerises éditions, 232 pp.

Né à Port-au-Prince en 1923 et mort en France en 1969, Francis-Joachim Roy est un écrivain et intellectuel haïtien qui a voué sa vie et sa carrière littéraire à la dénonciation des crimes et des injustices qui ont ravagé sa terre natale depuis son indépendance, en 1804, jusqu'à nos jours, s'intéressant notamment à la condition de la classe paysanne et s'opposant fermement au régime duvaliériste. Au niveau personnel, ce militantisme dévoué lui vaudra l'exil à l'étranger - qu'il passera en France, à Paris, où il s'installera dans les années 1940 et d'où il poursuivra sa lutte acharnée contre la corruption de la classe dirigeante haïtienne. Au niveau littéraire, son dévouement coûtera à l'auteur un anonymat presque total parmi ses compatriotes : son seul roman, Les chiens, demeure toujours inédit dans son pays d'origine. Publié pour la première fois chez Robert Laffont en 1961, ce texte est resté longtemps méconnu, voire inconnu, tant par le public que par la critique. En 2019 il apparaît sous un jour nouveau grâce à l'édition particulière du Temps des Cerises qui a le mérite de reporter à l'attention des spécialistes ainsi que d'un lectorat plus large l'un des chefs-d'œuvre de la littérature haïtienne. En effet, le récit fait non seulement preuve d'un engagement politique et social estimable de la part de Roy, mais il révèle aussi un talent littéraire extraordinaire dont il ne nous reste malheureusement que ce seul échantillon.

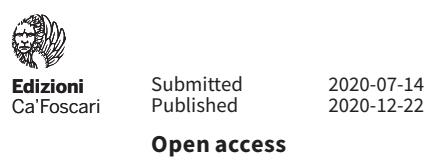

(C) 2020 | ()( Creative Commons Attribution 4.0 International Public License 
Inspiré par la tentative de putsch du Général-Président Paul Eugène Magloire en 1956, Les chiens raconte l'histoire d'une dizaine de personnages dont les chemins se croisent au cours d'une seule journée dans les rues de Port-au-Prince. Dans un style qui reprend la tradition haïtienne de la lodyans, Roy campe une mosaïque de scènes différentes montrées à l'intérieur d'un long plan séquence qui suit de près les péripéties des protagonistes. Mais c'est justement l'arrivée des chiens dans la capitale qui va déterminer le glissement d'une scène à l'autre. D'une présence initiale sporadique, ces bêtes se font de plus en plus nombreuses et arrivent à prendre possession à la fois de la ville et du récit : non seulement le roman s'ouvre sur leur apparition inattendue dans Port-au-Prince et se clôt par leur départ subit, mais leur marche vers le centre-ville va également scander le rythme de la narration, ce qui fait de ces figures animalières apparemment secondaires le véritable pilier autour duquel s'articule le roman. Dans le texte, ces animaux vont revêtir des valeurs allégoriques différentes qui vont évoluer au fur et à mesure que les événements s'enchaînent. Comme le propose dans la postface à cette édition Alessandro Costantini, professeur de Littératures francophones à l'Université Ca' Foscari de Venise qui avait déjà consacré aux Chiens de Roy un long article, ${ }^{1}$ ces chiens assument tant le rôle d' « antagonistes de l'homme » (216) que la fonction symbolique de «représentation de l'humanité misérable » (216) et de «métaphore d'une collectivité » (217).

Le passage de trois chiens « maigres, couverts d'une poussière immémoriale » (7) devant l'entrée de la boutique où Cocobé est assis attire son attention. Ce dernier, l'une des figures principales du portrait social croqué par l'écrivain, surveille attentivement les mouvements de l'étrange groupe canin et interprète la nature exceptionnelle de l'événement comme un présage, les chiens n'étant que des « prends garde » (13) envoyé par le sort. L'atmosphère qui hante la ville, en effet, ne laisse rien présager de bon à l'horizon, sensation amplifiée par la confusion qui semble régner dans les rues principales, plus que d'habitude congestionnées par le trafic. À travers les multiples déplacements des personnages qui passent d'un quartier à l'autre, Roy nous décrit la capitale haïtienne comme une ville étouffée par des relents pestilentiels provenant des déchets amassés partout et assourdie par le vacarme épouvantable produit par l'engorgement de gens, d'animaux et de voitures :

À la hauteur de l'Abattoir, une odeur épouvantable de déjections remplissait l'air. La brise, presque étale à cette heure de la mati-

1 Costantini, A. (2007). " Des chiens et des hommes : de la métonymie individuelle à la métaphore collective (Les chiens haïtiens de F.-J. Roy et les autres) ». Interculturel/ Francophonies, 12, 77-121. 
née, la brassait avec la fumée âcre qui se rabattait sur le quartier, partant [d]es montagnes d'ordures que le Service d'hygiène $\mathrm{Pu}$ blique déverse, au long des années, face à la ville, et qui se consument lentement lorsque leur propre pourrissement les fait monter à la température interne du tas. Sur un sol d'immondices se tient le marché au charbon. Ânes, mulets et chevaux, tous noirs, y enfoncent jusqu'au jarret et une population de zombis, dont on ne distingue que les dents blanches et les gencives rouges, y végètent dans la crasse, dormant à même le sol, vêtus de sacs vides ou dans des ajoupas coniques dont les palmes couvertes de poussière font penser à une parodie infernale du dimanche des Rameaux. (50)

Ce cadre pitoyable va être secoué par une tension croissante jaillie de nulle part et par une inquiétude générale qui s'empare soudainement de la population port-au-princienne, désormais accoutumée aux troubles bouleversant de temps à autre leur ville, une population qui ne peut qu'attendre que la tempête s'abatte en espérant qu'elle ne soit pas trop violente :

Le nègre de Port-au-Prince est comme ça. Dès qu'il y a quelque chose dans l'air, il le sent et, ce qu'il y avait dans l'air ce matin, le chauffeur ne saurait dire ce que c'est, sinon que c'était un jour à ne pas bêtiser avec les gens. (49)

Dans un crescendo d'agitation marqué par l'enchaînement de plus en plus resserré de leurs arrivées successives, les chiens pointent à l'improviste hors de ce paysage urbain de la misère et s'érigent en représentants des malheureux de la ville auxquels ils ressemblent aussi bien au niveau physique qu'au niveau comportemental :

Devant lui, incroyablement maigres, trottinaient quatre chiens. L'un d'eux avait la queue coupée au ras des fesses. Le second levait haut les pattes comme un ivrogne. Le troisième avait dû être noir. Ce qui lui restait de pelage était gris et il avait un épi blanc sur le front. Quand la voiture de Barsac le doubla, il fit un bond de côté et montra les dents. Il avait l'air, avec son museau effilé, ses minces lèvres bises et ses gencives pâles, de l'image même de la haine et de l'impuissance. (42-3)

En partageant cette « haine » et cette « impuissance » avec la majorité pauvre de la population, au début du récit les chiens adoptent une attitude résignée face aux hommes qui les harcèlent et qui s’avèrent être significativement les puissants de la ville. Pour n'en produire que quelques exemples, c'est un soldat qui renverse délibérément par sa voiture un chien trottinant au bord de la route (27) ; c'est un gendarme qui ignore complètement le cadavre d'un animal écrasé (15) 
qui va s'ajouter aux milliers d'autres entassés sur des trottoirs déjà bondés de déchets. Les animaux comme les hommes semblent accepter passivement les injustices perpétrées par les figures au service du pouvoir, des gendarmes et des politiciens qui profitent de leur misère et qui se divertissent en les tuant sans merci.

« Mais dans la lumière diaprée des Tropiques, vingt-quatre heures c'est beaucoup » (209). Quand le découragement semble prendre le dessus, Roy offre aux lecteurs et aux personnages un moment d'espoir qui dans l'espace d'une journée va remettre en ordre la roue du destin. En effet, au moment où les individus perdent leur dignité d'êtres humains et s'abaissent à un état bestial, les chiens s'érigent en « vieilles Érinyes, chiennes cruelles qui veillent à punir les méchants » (209). Significatif à ce sujet, l'épisode qui se produit dans le bureau de Lefranc, cousin du Président du pays et membre du gouvernement, marque un véritable tournant à l'intérieur du récit. Lefranc et Janine, sa belle secrétaire qu'il désire ardemment, regardent deux chiens s'accoupler devant leur fenêtre ; la concupiscence de l'homme et le consentement impudent de la femme, qui semble finalement céder à ses avances ne serait-ce que pour son gain personnel, se reflètent dans la lascivité des animaux et contribuent à la représentation d'une classe politique dégradée, guidée exclusivement par ses passions charnelles et par ses propres intérêts.

Contre ces abus, les chiens abandonnent leur attitude résignée et se dressent en protecteurs de la communauté, empêchant ainsi pour une fois aux puissants de la ville de prendre le relais. Pour accentuer ce glissement de signification, Roy sature le récit d'un lexique militaire servant à désigner l'avancée inexorable des animaux vers les centres du pouvoir. L'emploi de termes tels que « contingent » (115), « rangs », « parade », « défilait », « marche » (122) renforce l'idée implicite dans le roman que la véritable force ne peut que dériver de la collectivité - canine au niveau narratif, humaine au niveau symbolique - et renvoie explicitement au combat acharné auquel les animaux se livreront contre la milice présidentielle. À la fin du roman, une fois la victoire remportée, les chiens s'en iront comme ils étaient venus, discrètement, restituant la ville libérée à ses habitants.

Dans un style à mi-chemin entre le récit oral et le tournage cinématographique, Les chiens de Roy présente au public une histoire que l'auteur a définie comme une « farce énorme et tragique » (209) caractérisée par un étalage flagrant des vices humains, dont la corruption et la violence de la classe politique ne sont que l'expression la plus évidente. Ce serait toutefois une erreur de penser à son roman comme à un portrait larmoyant des conditions misérables des habitants de Port-au-Prince : ce que Roy offre aux lecteurs ainsi qu'à ses compatriotes est une occasion de se racheter par le biais d'une allégeance collective sporadique, peut-être invraisemblable mais puissante, aux yeux du destin. Ne serait-ce que pour vingt-quatre heures. 


\section{Daniel-Henri Pageaux Lectures Indiaocéanes. Essais sur les francophonies de l'Océan Indien}

Sara Pagone

Università degli Studi di Trieste, Italia

Review de Pageaux, D.-H. (2016). Lectures Indiaocéanes. Essais sur les francophonies de l'Océan Indien. Paris: Maisonneuve, 349 pp. Itinéraires poétiques, Itinéraires critiques 51.

Daniel-Henri Pageaux avait déjà mis en évidence à quel point la recherche dans le domaine francophone doit « examiner les rapports entre langue française et langues créoles, même si (surtout si) se posent de complexes questions et pas mal de cloisons à abattre : la construction identitaire d'une littérature créolophone dans un contexte 'francophone' institutionnalisé, l'étude des relations fortement asymétriques, la question des genres 'littéraires' en créole » (Itinéraires comparatistes. Paris : Maisonneuve, 2014, 1: 281). En se limitant à un espace géographique défini, qui comprend l'île Maurice, La Réunion, Madagascar et l'île Tromelin, l'auteur se place dans cette perspective en nous livrant Lectures Indiaocéanes. Essais sur les francophonies de l'Océan Indien. Il donne ainsi une présence non négligeable à la littérature de ces îles devenues des colonies françaises et qui vivent une condition de double culture et mériteraient plus de poids dans le panorama littéraire actuel. Cette tentative révèle sans aucun doute la richesse de l'ouvrage. Le comparatiste analyse minutieusement les productions littéraires de ces territoires dans lesquels on peut retrouver des traces, respectées ou subverties, de modèles

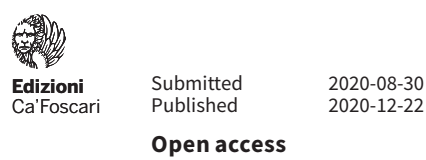

(C) 2020 | ()( Creative Commons Attribution 4.0 International Public License 
littéraires européens du siècle des Lumières à l'époque contemporaine. Pageaux surligne magistralement les preuves de contacts entre les écrivains de l'aire indiaocéane et les auteurs occidentaux ainsi que les aspects innovants et spécifiques de cette francophonie. D’ailleurs, la francophonie n'est pas « comme un cheval de Troie de l'eurocentrisme ou, pire encore, d'un universel déguisé en pseudo-impérialisme culturel »(13). L'identité plurielle des auteurs considérés, autochtones mais aussi français, contribue à stimuler l'analyse de Pageaux. Le comparatiste ajoute que son objectif n'est pas de dresser une histoire de cette littérature : il s'est efforcé plutôt d'identifier, de préserver et de valoriser la singularité de chacune des œuvres dont il propose la lecture. Toutefois, il remarque la présence commune de quelques grands thèmes tels le marronnage, l'enfance perdue, l'exil et la recherche de la dignité humaine.

Son examen commence à l'île Maurice, colonie française de 1715 à 1810, devenue ensuite possession de la couronne britannique jusqu'à son indépendance en 1968. L'île a une remarquable tradition littéraire dans laquelle Vicram Ramharai voyait déjà trois phases. Le texte le plus célèbre est bien sûr Paul et Virginie de Bernardin de Saint-Pierre, écrivain de France métropolitaine qui situe son roman dans la lointaine Île de France du XVIIIe siècle et devient, avec ce texte, l'un des pères fondateurs du préromantisme. Malgré le cadre exotique, la société idyllique et la force des sentiments amoureux contrariés, Paul et Virginie présente une vision pessimiste de l'existence humaine. Il est significatif que Pageaux le mentionne au tout début de son ouvrage : dans le roman on retrouve certes les aspects du préromantisme européen mais aussi ceux de la littérature populaire, coloniale et militante, la technique du feuilleton et la liaison entre le mythe et la réalité qui reviennent ponctuellement dans les œuvres qui suivent. Il en est ainsi dans Sidner ou les dangers de l'imagination de Barthélemy Huet de Froberville, homme de lettres et soldat français, qui arrive à contrecœur sur l'île comme militaire. Son œuvre est le «premier roman en français publié dans l'hémisphère sud » (17). Le thème d'un amour difficile rapproche trois grands auteurs : Lucien Brey, né en France, mais qui publie en 1878 Ratsitatane de Lucien Brey ou les Mystères de Port-Louis, la première œuvre mauricienne consacrée à l'analyse de la société de l'île ; Pierre Benoît qui publie, en 1948, Jamrose, l'histoire d'amour entre Virginie Willoughby et Paul de Jamrose en mettant en scène des contrastes familiaux qui font penser au Romeo et Juliette de Shakespeare et en contextualisant cet amour dans les luttes entre Français et Anglais pour le contrôle de l'île ; et, enfin, Marcel Cabon, écrivain mauricien qui peut paraître comme « l'un des pionniers du mauricianisme » (163).

En abordant la littérature de La Réunion, Pageaux précise qu'elle s'exprime aussi bien en français qu'en créole réunionnais. L'île est devenue une colonie française en 1649 et a obtenu le statut de département d'outre-mer en 1946. Afin de comprendre les aspects de 
la littérature réunionnaise, il convient de considérer séparément les genres littéraires. Pour ce qui concerne la poésie, Pageaux fait mention d'Alain Lorraine et de Carpanin Marimoutou. La production romanesque de l'île est dominée par les contemporains Anne Cheynet et Alex Gauvin. La première est une écrivaine polyédrique que passionnent les mots de l'« oraliture », toujours attentive à sauvegarder l'histoire, élément fondateur de la société réunionnaise. Alex Gauvin affronte, quant à lui, la question de la nourriture : ses œuvres ne sont pas seulement une nourriture romanesque, mais aussi et surtout une nourriture pour l'âme. Il est, par ailleurs, l'un des grands noms qui dominent le milieu littéraire réunionnais actuel depuis qu'il a fait paraître à la fin des années 1970 un manifeste pour la défense de la langue créole. Les lectures consacrées à Alain Lorraine et à Anne Cheynet sont d'une pertinence particulière puisqu'elles mettent en lumière à quel point les deux écrivains retravaillent ou subvertissent les références littéraires européennes et constituent « une évolution (une rupture ?) dans l'idée même de littérature » (12).

C'est Madagascar qui constitue l'avant-dernière étape de ce périple dans le monde de l'Océan Indien. L'île a commencé à susciter les intérêts des empires coloniaux européens à la fin du XIXe siècle. Lorsque les Britanniques y renoncent définitivement, les Français en profitent pour en faire d'abord un protectorat puis une colonie. Au début des années 1950, c'est la France qui entame des réformes en faveur de l'indépendance de l'île, proclamée officiellement en 1960. Les langues officielles sont le malgache et le français. La langue malayo-polynésienne donne vie à une création artistique très variée où se retrouvent des modes d'expression orale traditionnelle qui s'intègrent à la modernité du chant, du théâtre, du cinéma et des formes écrites; création qui coexiste avec la production francophone. $\mathrm{Pa}$ geaux retrace enfin l'histoire, à partir de 1761, d'un îlot, lorsqu'il fut le théâtre d'un naufrage survenu à un navire français et présente l'espace insulaire comme hostile et franchement inhospitalier, une " véritable antithèse de toutes les îles » (8). Le souvenir de ce drame et la question éthique d'une promesse non tenue sont repris dans Les naufragés de l'île Tromelin d'Irène Frain, roman publié en 2009.

L'intérêt du volume de Pageaux est, entre autres, d'ouvrir de nouvelles perspectives d'analyse de la Littérature indiaocéane. À partir de la nécessité de mener l'étude des francophonies, « entendue comme processus interculturel, 'dialogue des cultures' » (13), Pageaux réalise un travail méticuleux de comparaison entre la littérature européenne et la littérature de l'Océan Indien. L'auteur médite enfin sur l'écriture d'un deuxième volume pour donner une juste attention aux écrivains qu'il a dû écarter. Il est dès lors d'autant plus significatif de songer avec Pageaux à la rédaction de Lectures Indiaocéanes II, afin que celles-ci puissent contribuer à un enrichissement ultérieur des études de la francophonie de l’Océan Indien. 



\section{"Univers de la radio ». Ponts, 19,2019}

Anna Michieletto

Università Ca' Foscari Venezia, Italia

Review de «Univers de la radio ». Ponts, 19, 2019.

Ce volume de Ponts, consacré en grande partie aux Notes de lecture, contient trois articles concernant l'univers de la radio dans la francophonie et un article sur la représentation du modèle noir dans la peinture européenne du XIXème siècle à nos jours.

Alessandra Ferraro retrace le parcours professionnel et artistique d’Anne Hébert au Canada et en France à travers ses contributions radiophoniques. Au début de sa carrière, Hébert travaille beaucoup pour la radio, qui « lui permet de diffuser son œuvre de création » (14) refusée par les éditeurs. Elle collabore avec la CBV (Canadian Broadcasting Villeneuve) de sa ville, Québec, qui transmet ses contes dialogués de 1950 à 1952 le samedi en début de soirée. En 1953, elle passe à l'ONF (Office National du Film), où elle deviendra la première femme francophone scénariste télévisuelle. Elle apprend ainsi l'écriture pour la télévision et la radio, dont l'amour lui vient, petite, d'entendre la voix de son père, chroniqueur. Pendant cette période, Hébert fait partie d'un groupe d'intellectuels pour qui « l'alliance entre radio et littérature peut jouer un rôle important dans un Canada arriéré au point de vue culturel » (16) ; pour elle « l'écriture radiophonique a représenté la partie la plus consistante de sa production littéraire » (17).

À partir de 1967, Hébert, qui entretemps a gagné des prix importants, est régulièrement invitée aux émissions littéraires radiophoniques, cette fois en France. En 1978, France Culture transmet sa

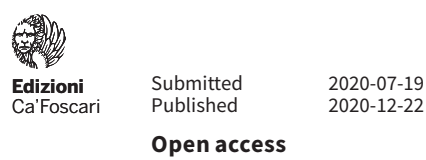

(C) 2020 | ()( Creative Commons Attribution 4.0 International Public License 
pièce radiophonique L'Île de la demoiselle. Contrairement à d'autres écrivains qui donnent «un échantillon de leur production antérieure » (20) ou parlent d'un sujet qui les intéresse, elle choisit donc d'« exploiter à fond les possibilités qu'offre la mise en onde à l'expression littéraire » (20). La pièce relate la vie de Marguerite de Nontron, jeune fille qui part au Canada sous le règne de François Ier, lors de la première tentative de colonisation. Elle donnera son nom à l'île où elle passe deux ans en exil, abandonnée avec son amoureux par Robert de Roberval, directeur de l'expédition dont elle était la pupille.

Ferraro analyse en détail l'écriture d'Hébert, qui respecte les règles de la radiodiffusion : pour favoriser un choix adéquat des voix, elle déclare immédiatement l'âge des personnages, par ailleurs limités en nombre ; elle utilise de nombreuses didascalies qui aident la mise en onde ; elle recherche des effets de réalisme langagier. Cela montre bien sa maîtrise du code de l'écriture radiophonique. Ferraro retrace aussi les étapes successives d'adaptation du texte, en comparant le premier tapuscrit et le script de l'émission, ainsi que des documents de Georges Godebert, collaborateur et réalisateur responsable de la diffusion. Elle signale la division en séquences et la réduction des interventions du narrateur. Hébert conserve pourtant l'orthographe phonétique originelle, fondamentale pour souligner « l'ancrage régional et social des personnages » (25). L'auteure de l'article entrevoit un parallélisme entre le choix de la Nouvelle-France et de la période historique du drame d'un côté et la vie d'Hébert qui voyage comme l'héroïne de l'autre. Les femmes du passé, d’ailleurs, « hantent l'imaginaire » (27) de l'écrivaine telles que des « voix sans corps » (28) auxquelles l'auteure donne la parole, comme cela a bien été évoqué par la mise en onde radiophonique.

Marie-Christine Jullion et Ilaria Cennamo abordent la radio comme vecteur d'identité culturelle dans la politique canadienne. La radio canadienne a en effet toujours convoyé un « projet politique et culturel centré sur le rayonnement de l'identité de son Pays, notamment face aux États-Unis » (33). Jullion et Cennamo analysent en particulier le paragraphe 3 de la Loi sur la radiodiffusion de 1991, aussi bien que des documents du site web officiel du CRTC (Conseil de la radiodiffusion et des télécommunications canadiennes), organisme public indépendant de réglementation et surveillance des médias. Le paragraphe 3 de la Loi conçoit la radio comme promoteur de la culture identitaire nationale dans son organisation et sa programmation. Il « déclare les objectifs principaux du système et détermine le rôle de la Société Radio-Canada et du réseau d'entreprises impliquées en rapport avec la création de la programmation » (35)

Le CRTC promeut la dualité linguistique, la souveraineté et la diversité du Canada multiculturel par rapport aux États-Unis et de la radio canadienne par rapport à Internet. Les auteures soulignent le rôle joué à partir de la Révolution Tranquille (années 1960) pour la 
culture québécoise. L'univers lexical renvoie souvent au Canada et les langues minoritaires aussi sont valorisées : tout citoyen peut se reconnaître dans cet « imaginaire socio-discursif » (41). Les services télévisés complètent la programmation des stations de radio.

Jullion et Cennamo évoquent ensuite trois éléments du site web francophone de Radio Canada qui mettent en œuvre la législation analysée : « la fonction de réglage régional de l'information » (44) qui permet de personnaliser les contenus et le guide-horaire et de créer un compte-utilisateur ; la présence d'une zone consacrée aux parents, d'une autre pour les enfants et d'une pour les jeunes; la rubrique « Espaces autochtones ». Les auteures, tout en souhaitant un approfondissement de la recherche et du débat, reconnaissent la capacité d'adaptation de la radio face à l'actuelle hybridation des discours médiatique, institutionnel et promotionnel et sa relation avec le web.

Francesca Paraboschi étudie la représentation de l'usage de la radio et la valeur qui lui est attribuée dans Le Nègre et l'Amiral de Raphaël Confiant, roman martiniquais publié en 1988. Elle a choisi cet ouvrage parmi les trois consacrés par l'auteur à la période de la Seconde Guerre mondiale et au régime Vichyste de l'amiral Robert à cause justement de l'importance du rôle de la radio.

Dans ce livre, à Fort-de-France l'annonce radiophonique de l'éclatement de la guerre suit un procédé propre au réalisme magique en faisant référence à un épisode remontant à l'année précédente. Le héros est un crieur qui prône tout de suite l'enrôlement au secours de la mère patrie. La " chaîne de transmission des informations » (55) passe par l'oralité dans les quartiers populaires comme le Morne Pichevin, où la compréhension du français est douteuse : " la langue créole riche en inventions relève d'une veine démiurgique à même de restituer la réalité en une forme plus captivante » (55) et la parole « détermine plus que toute annonce à la radio » (56). La radio catalyse pourtant l'attention des héros, notamment la BBC pour écouter De Gaulle, mais les stations sont mal captées et les messages tellement mal compris que beaucoup se laissent tromper par la propagande vychiste. La réalité en résulte inimaginable et trop différente des souvenirs scolaires et du patriotisme contre la perfide Albion. Amédée, ancien professeur, comprend ainsi plus qu'il n’arrive à expliquer à son auditoire de presqu'illettrés marqués par l'asservissement colonial. On le voit bien lors d'une collecte pour le régime, qui décrit d'ailleurs la Martinique telle qu'une île paradisiaque, sans évidemment mentionner la misère qui y règne.

Le style énonciatif de Confiant, fortement oralisé, est basé sur « radio-bois-patate » ou « radio-bombe-sirop » (63) pour reproduire la réalité des bas quartiers. Le conditionnement culturel colonial français et une scolarisation insuffisante transforment la géographie et les personnages historiques cités par la radio au gré de la fantaisie. Le « désir d’héroïsme » (67) et « l'énergie fabulatrice de la parole 
noire » (67) l'emportent sur le manque d'action en Martinique. Le seul à comprendre que les Américains n’ont pas débarqué sur l'île, par exemple, est en effet l'indien Vidrassamy.

Éloignement, lassitude, irréalité et enchantement font percevoir une « fausse guerre » (70) face à la routine de la misère, voilà pourquoi on loue la parole nègre en créole et l'invention linguistique. L'annonce radiophonique de la fin de la guerre est mentionnée dans le roman, mais l'enthousiasme est nuancé par la souffrance de Philomène, qui, après plusieurs mois de sévices endurées sur un navire, apprend que son amant s'est suicidé. Le roman inachevé de ce dernier est finalement brûlé et «Confiant semble thématiser ses craintes et ses soucis dans la rédaction de son premier roman en français » (75). L'auteur arrive cependant, à travers son nouveau procédé narratif, à reproduire la réalité du parler populaire et à décoloniser « un pan d'histoire peu connu » (76) de sa terre natale.

Liana Nissim, dans son article Épiphanies Noires, confronte le catalogue de l'exposition Le modèle noir de Géricault à Matisse (2019) et les actes du colloque ayant eu lieu à Milan en 2015 Le ricchezze dell'Africa (Les richesses de l'Afrique) « sur les monstrations relatives au continent africain dans les expositions internationales du XIXe siècle à 2015 » (81).

Dans les tableaux chronologiques du catalogue de l'exposition de Paris, Nissim remarque qu'en Europe les modèles noirs sont presque toujours d'origine caribéenne. La colonisation de l'Afrique noire, sous prétexte de « mettre fin au trafic négrier et à l'esclavage local » (83), suit en effet la traite, l'esclavage en Amérique, les soulèvements des esclaves, la première et la deuxième abolition de l'esclavage (1794 et 1848) et entretemps l'indépendance d'Haïti (1804-1825), ce qui explique peut-être cette « confusion assimilatrice » (83). Les artistes noirs du cirque dans les tableaux de Degas et de Toulouse-Lautrec (fin XIXème siècle) viennent encore des Caraïbes, tandis que dans la première moitié du XXème siècle c'est le tour des afro-américains qui introduisent le jazz à Paris.

Le regard de l'artiste occidental s'avère en général dévalorisant, du racisme scientifique au «statut ambigu de l'ethnologie » (87) des années 1930.

Nissim remarque aussi dans le catalogue « un certain décalage entre les images de l'exposition et les textes qui les accompagnent » (88). C'est le cas de la Vénus hottentote et du succès des spectacles « ethniques » du XIXème siècle en Amérique et en Europe et des zoos humains, où l'on exhibait les Noirs comme s'ils étaient des animaux « exotiques » de zoo, cités par contre dans L'Africa esposta. Il s'agissait d'expositions anthropologiques qui en disaient davantage des sociétés qui les organisaient que des « autres » exhibés. Flaubert nous en a laissé un commentaire en 1853, où il a reconnu la faim dans le regard des Noirs qu'il voyait, comme le rappelle le petit livre italien. 
L'exposition Le Modèle noir a comme but la « recodification partagée [...] d'une socialité inclusive » (91) au XIXème siècle, pour que les Noirs arrivent « à créer un espace esthétique propre, moderne, noir et parisien » (91). Selon Nissim, une telle recherche ne devrait pas refuser les pratiques « vues aujourd'hui comme négatives ou honteuses » (91), mais plutôt les insérer dans leur cadre historique. La postface du catalogue, appréciée de l'auteure, reconnaît en effet qu'il faut « montrer pour déconstruire » (93), reconnaître les démarches du passé pour être capable de changer le présent et sa mentalité.

Pour conclure, on peut donc affirmer que la radio s'avère un dispositif capable de promouvoir un lien unique et spécial avec l'identité nationale, comme cela arrive dans le cas du Canada francophone. Elle peut cependant opposer sa vérité à celle du bouche-à-oreille, comme dans le roman de Confiant. La question du langage aussi s'impose : la radio requiert des spécificités, ainsi que le démontrent l'écriture et la mise en onde de la pièce L'île de la demoiselle d'Anne Hébert.

Dans un contexte complètement différent, mais toujours afférent à la représentation, la peinture et la réflexion qu'elle inspire, et que l'on retrouve dans les catalogues ou les actes des colloques, peuvent véhiculer des messages capables soit de renforcer soit de déconstruire l'image des Noirs, africains ou afro-américains. 



\section{Fawzia Zouari Molière et Shéhérazade}

Sara Alouani

Università di Verona, Italia

Review de Zouari, F. (2018). Molière et Shéhérazade. Paris: Cent Mille Milliards \& Descartes \& Cie, 164 pp.

Née en 1955 à Dahmani, un petit village maraboutique dans la région du Kef, en Tunisie, dans une famille où le père était Cheikh et la mère la savante du clan, Fawzia Zouari grandit dans une communauté très liée à ses racines, aux traditions musulmanes et aux ancêtres, d'où il était très difficile de sortir et, en même temps, où l'on ne pouvait pas rentrer sans appartenir au clan. En fait, c'est justement pour éviter l'indignation des membres de sa famille que l'écrivaine publie son premier roman autobiographique, J'ai épousé un français (2010), sous le pseudonyme d'Aïcha Kessler.

Après avoir abordé plusieurs fois la thématique de l'étrangéité dans ses romans, comme par exemple Ce pays dont je meurs (1999), dans l'essai Molière et Shéhérazade, Zouari analyse un thème bien connu tel que l'utilisation de la langue du « colon » avec une audace exquisément personnelle et explique méticuleusement le voyage qui l'a amenée à utiliser, contre tout schéma religieux, un idiome considéré par la culture musulmane comme la langue « des infifidèles ». (12).

Cet essai est une réflexion introspective où Zouari évoque ses liens familiaux, dévoile les détails des traditions maraboutiennes qui caractérisent ses origines et explique les difficultés liées au choix de la langue. Molière et Shéhérazade est divisé en petits chapitres qui sont plus précisément des interrogations et des sujets que l'auteure aborde parfois en utilisant des images très fortes de son enfance pour rendre encore plus clair son rapport avec la langue arabe et, en même temps, son grand amour pour la langue française. Quand

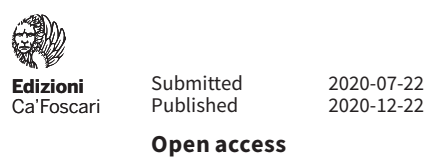

(C) 2020 | ()( Creative Commons Attribution 4.0 International Public License 
elle parle d'amour c'est aussi au sens propre, car elle était « tombée amoureuse » (52) de son professeur de français et n'arrêtait pas de lire et relire Madame Bovary. Ce roman marquera profondément son adolescence car il lui permettra de vivre intensément une vie différente, en dehors de son clan : celle d'Emma. Et pendant longtemps, Zouari a considéré cette fascination exercée par le professeur comme responsable de lui avoir « fait aimer la langue de l'étranger » (52).

Pourtant, le choix d'écrire en français, explique Zouari, est quelque chose de plus profond qu'un amour d'adolescente. En fait, au tout début de son essai, elle explique sa « peur » d'écrire en arabe, une réticence qui cache un sentiment de trahison envers sa tradition et encore plus spécifiquement envers sa mère. L'arabe est la « langue de Dieu » (10), la langue du « Kitab » (40), le livre sacré par excellence, c'est à dire le Coran. D'un côté, affirme Zouari, l'arabe est la langue de Celui qui est « le seul inventeur de récits » (32). Ainsi personne ne devrait oser raconter et inventer des histoires en utilisant l'alphabet qui a été chuchoté au Prophète par l'ange Gabriel. De l'autre côté, il y a la peur d'utiliser une langue étrangère pour dévoiler les sujets les plus intimes : « exhiber les codes de la tribu et ses secrets » (83) pourrait «mettre dans l'embarras Dieu » (84) et sa famille.

À travers les pages de cet essai, Zouari explore le parcours qui lui a permis de percevoir le français comme étant quelque chose de plus qu'une langue maternelle : une « langue utérine » (121), c'est-à-dire une langue innée et primordiale comme si elle l'avait absorbée « alors que maman [la] portait encore » (121). Cette prise de conscience lui permet finalement de publier en 2016 Le corps de ma mère, un récit entièrement consacré à la vie de sa génitrice, sans craindre de dévoiler ses secrets. Zouari affirme même protéger de cette manière l'image de sa mère face à Dieu, en faisant « en sorte que Dieu n'en sache rien, lui faisant passer les frontières incognito, cachée sous la langue étrangère » (110).

Cette assertion met implicitement en évidence la volonté de préserver sa mère de la désapprobation de sa communauté islamique, en la cachant derrière un voile langagier imaginaire qui n'est pas « sacré ».

Toujours est-il que la musicalité de la langue arabe, de la « berceuse » (15) que sa mère lui chantait et des récitations du Coran auxquelles elle assistait pendant son enfance reste bien gravée dans son écriture. C'est ainsi que pour elle, écrire la culture arabe en français devient un compromis entre deux langues qui se fondent, en cherchant « à traduire la langue qui existe dans chaque écrivain » (131).

Zouari conclut alors son essai en affirmant que la langue avec laquelle elle écrit est sûrement très difficile à accepter, surtout par les éditeurs, qui exigeraient sans doute un style plus normé. Elle refuse toutefois opiniâtrement d'écrire dans une forme qui n'est pas la sienne, car elle a « [s]on français » (143) : ce qui réaffirme tout le sens de son argumentation. 


\section{Agnès Schaffauser Salim Bachi}

Sara Alouani

Università di Verona, Italia

Review de Schaffauser, A. (éd.) (2019). Salim Bachi. Paris: L'Harmattan, 266 pp. Autour des écrivains maghrébins.

Nous nous trouvons ici face à un recueil sans précédents, dans le sens qu'il n'existe pas, à ce jour, une collection d'essais et d'études entièrement consacrée aux œuvres de Salim Bachi. En outre, pris en considération par la critique littéraire internationale, les romans de cet auteur « atypique » (12), comme le définit Agnès Schaffauser, « font l'objet d'une grande attention médiatique et académique » (12).

Né à Alger en 1971 dans une famille qui «n'était pas traditionaliste ni religieu[se] » (226), comme il l'explique dans l'entretien inédit avec Schaffauser publié à la fin du recueil, Salim Bachi décide de s'installer à Paris en 1997 où il commence son parcours d'écrivain et c'est ainsi qu'en 2001, il publie son premier roman, Le chien d'Ulysse. Son chef d'œuvre reçoit le Prix Goncourt et le prix de la vocation 2001 et c'est avec l'analyse de ce roman par Jaouad Serghini que s'ouvre la section Études au singulier du volume. La division du recueil en deux grandes parties souligne l'envergure et la méticulosité de ce travail ainsi que l'extrême complexité de la production littéraire de Bachi, qui nécessite une analyse profonde non seulement en explorant ses œuvres individuellement mais aussi en les comparant entre elles. C'est ce qui se passe dans la deuxième partie du volume, Études au pluriel.

Une grande attention est accordée au sujet de la pluralité d'identités présentes dans la production de l'écrivain franco-algérien, qui re-

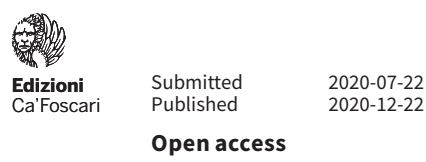

(C) 2020 | ()( Creative Commons Attribution 4.0 International Public License 
jette toute étiquette, comme l'explique Schaffauser en reprenant les mots de Roger Célestin : Salim Bachi, en fait, est considéré par celui-ci comme « juste un écrivain » qui ne peut rentrer dans aucune catégorie spécifique (12). Les nombreuses identités de Bachi, qui ne sauraient ne pas rappeler Les identités meurtrières maaloufiennes, comme l'affirme Serghini, sont mises en évidence par la « pluralité de voix » (42) des narrateurs présents dans Le chien d'Ulysse. Cette «polyphonie » (42) est une caractéristique particulière des écrits de Bachi et on la retrouve aussi dans Le silence de Mahomet, où il raconte la vie du Prophète à travers la voix de ses femmes et de ses compagnons Abu Bakr et Khaled Ibn al Walid. Cet aspect est bien exploré dans l'essai de Carine Bourget, qui considère le choix de Bachi d'utiliser quatre narrateurs comme une technique pour « accentuer différents aspects de [...] la personnalité du prophète » (129) et, par conséquent, les différentes identités.

L'« altérité » (36) est sagement mise en évidence par Bachi grâce également à l'utilisation de la mythologie juxtaposée aux images du présent, comme dans le cas de Cyrtha, la ville «protagoniste » du roman Le chien d'Ulysse et de La Kahena, son deuxième récit. Bernard Aresu, dans son essai, trouve des allusions à « l'antique Cirta » et aux « Syrtes d’Ulysse » (71), tandis qu'elle est présentée dans les deux romans comme une ville algérienne imaginaire, où les faits se déroulent, et qui, toujours selon Aresu, pourrait évoquer «la Quasantina moderne [...] Annaba ou Alger » (71).

Le symbolisme et les références aux plus grands auteurs comme Ovide, Shakespeare et Joyce, omniprésents dans les récits de Bachi, soulignent l'immense bagage culturel et littéraire de l'auteur. Cette connaissance historique est aussi mise en évidence par Bachi dans son écriture, car il ne manque pas de présenter en détail des biographies, toujours réinterprétées selon son style, en rendant hommage à des personnages illustres comme le politicien Jérôme Bertagna, le diplomate Aristide de Sousa Mendes, l'écrivain Albert Camus et le poète latin Ovide. Ces biographies sont bien analysées dans ce recueil par plusieurs contributeurs.

L'intertextualité est une autre caractéristique de l'écriture de Bachi, méticuleusement analysée dans ce recueil, et qui dévoile sa connaissance minutieuse des textes classiques qui l'influencent. On peut trouver des exemples dans plusieurs romans, en particulier dans ceux qui traitent du terrorisme. Dans Tuez-les tous Bachi fait des références précises à Hamlet : le héros shakespearien, explique Yves Davo, y est vu comme « le premier avatar qui [...] défin[it] le personnage » (98). Dans Moi, Khaled Kelkal on retrouve des allusions à Robinson Crusoé de Defoe, car, comme Imane Terhmina l'affirme dans son essai, la situation de confusion intérieure du terroriste Khaled est comparée par l'auteur à celle du naufragé (137).

Le roman maghrébin prend une forme très particulière et variée chez Bachi : son écriture est identifiée par Jaouad Serghini comme « 
le carrefour du métissage et de l'interculturel » (33). Les œuvres de Salim Bachi sont caractérisées par une hétérogénéité non seulement dans le style mais aussi dans les sujets traités, dont la décennie noire en Algérie, le terrorisme, la religion, l'exil, l'identité, le deuil. La prochaine étape, affirme Bachi dans l'entretien inédit inclus dans le recueil, consistera à " faire parler les femmes », avec quelques figures particulièrement captivantes (229).

Les analyses présentées dans ce volume font donc ressortir toute la richesse d'une écriture qui n'a pas été pleinement appréciée de la critique à sa juste valeur, malgré l'attribution initiale du prix Goncourt. En fait, plusieurs contributions renvoient à des entretiens inédits avec l'auteur, ce qui souligne une pénurie d'essais et des critiques à son égard. Ce volume constitue donc une lecture précieuse, et non seulement pour les spécialistes de Bachi, de cette voix francophone entre plusieurs mondes qui mérite d'être étudiée en profondeur. 



\section{Michela A. Calderaro Sheer Bliss: A Creole Journey}

Amanda T. Perry

Montreal, Concordia University and Champlain College-Saint Lambert

Review of Calderaro, M.A. (2020). Sheer Bliss: A Creole Journey. Kingston, Jamaica: The University of West Indies Press, 2020, 174 pp.

Sheer Bliss: A Creole Journey, recently published by the University of West Indies Press, represents the culmination of Italian scholar Michela A. Calderaro's decades-long effort to rescue creole writer Eliot Bliss from obscurity. It is also an unusual text, eschewing many of the norms of academic writing to allow readers to follow along, step by step, as Calderaro's curiosity about Bliss transforms into an intensive research project. In the process, Calderaro reveals the extensive networks that allow researchers to operate and the underrecognized support systems that nurture writers in the first place.

The author of Saraband (1931) and Luminous Isle (1934), Eliot Bliss is, as Calderaro outlines in her preface, difficult to categorize: at once modernist, feminist, and queer, stranded between British and Caribbean literary traditions. Like many modernists, Bliss writes semiautobiographical novels that foreground the internal worlds of their protagonists over dramatic events and depict society on the brink of radical change. Her work also features women who reject the conventional path of marriage and children, with certain passages implying their homosexuality. Intriguingly, this queer subtext actually renders Bliss' characters less radical than the author herself, as she pursued romance with women throughout her life. Calderaro hypothesizes that this reticence in fiction may stem from Bliss's ties to the

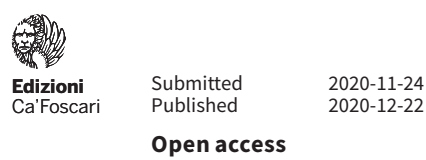

(C) 2020 | ()( Creative Commons Attribution 4.0 International Public License 
Caribbean, as she laboured under the weight of stereotypes about hypersexualized creole women. Bliss was born in Jamaica in 1903 to British parents, spending part of her childhood there and returning to the island for two years as a young adult. Her second novel, Luminous Isle, frankly depicts the racism of white colonial society in Jamaica and features a protagonist torn between cultures, who does not feel entirely European but is also unable to fully identify with the Caribbean's Black population. In this regard, Bliss explores similar thematic terrain to the better known creole writer Jean Rhys, and Calderaro unearths evidence that the two were close friends.

For the uninitiated, Sheer Bliss's first chapter give some idea of the texture of Bliss's work, analyzing her two published novels in connection to Rhys's Wide Sargasso Sea. At the same time, Calderaro details the fortuitous nature of her discovery: she found a copy of Saraband in a second hand bookstore while working on another project. Rather than reconstruct the chronology of Bliss's life, the book largely follows Calderaro's own research process, moving from Bliss's published novels to an investigation of her diaries at the University of Tulsa to a long dialogue with Patricia Allan-Burns, Bliss's lifelong companion whom Calderaro met and befriended after several years of research. This structure is both eccentric and compelling, as Calderaro includes email exchanges with librarians and researchers and information about her struggles to secure funding. Some of her efforts are ultimately frustrated: for instance, a note mentioning a third novel by Bliss, The Albatross, triggers years of searching, only for Calderaro to conclude that the book must never have been published. Then again, Calderaro unearths entirely unsuspected treasures, finding the manuscript to the unpublished novel Hostile Country and two unpublished collections of poetry. While Calderaro provides several excerpts from Bliss's poetry and has published the collection online, readers may find themselves wanting more in respect to Hostile Country, as they are forced to make due with a brief description of the novel's premise and its first few lines. Above all, however, these false leads and surprising turns effectively demonstrate that the research process is far from linear.

Why, exactly, Eliot Bliss is worth rescuing as a writer is a question that haunts the book, and Calderaro does not fully engage with its implications. In the course of her research, she unearths a whole network of queer women writing in England in the 1930s, providing short portraits of Anna Wickham, Patience Ross, and Kay Burdekin, among others. All are potential candidates for the kind of treatment Calderaro affords Bliss. Why work on Eliot Bliss in particular? Is it the literary quality of her work, the originality of her perspective, her influence on Jean Rhys? Does she complicate our understanding of queer writing or Caribbean literature in fundamental ways? After all, the vast majority of writers and books are forgotten, with only 
a rare few attracting a sustained readership. While Calderaro does not make her case explicitly, her work doubles as a form of advocacy. In this regard, her great triumph is not only the present volume but Emma Garman's 2019 column on Bliss in Paris Review, which credits Calderaro for "almost single-handedly sav[ing Bliss] from oblivion" while simultaneously introducing the writer to a larger audience (Garman 2019).

Ultimately, as much as the book is a portrait of Eliot Bliss, it also becomes one of Patricia Allan-Burns, Bliss's lifelong companion. Calderaro begins the text with a meeting between her and the elderly Allan-Burns, slowly filling in the context in subsequent chapters. Allan-Burns met Bliss when she was nineteen and stayed with her for sixty years, providing emotional and financial support until Bliss's death in 1990. The woman Calderaro refers to as Pat worked in a factory and took on extra jobs while Bliss, largely incapacitated by illness and frequently depressed, continued trying to write. Calderaro is obviously enchanted with Allan-Burns, providing long descriptions of their encounters as she negotiates the ethics of prying into Bliss's private life in the company of her partner. At times, readers may even find themselves siding with Allan-Burns against the putative subject of the book. When she comments on not having been intellectual enough for Bliss, and the tragedy of her companion finishing her life far removed from London's literary circles, Calderaro forcefully reminds her: "It was difficult for you too" (107). AllanBurns provides a striking example of the overlooked support that writers rely on, and her tendency to elevate Bliss's needs above her own calls into question the cult of literary genius.

At the same time, Calderaro unveils the support that her own research has required. She is generous with her praise for the many librarians, archivists, fellow scholars, and well-intentioned individuals that enabled her to overcome various barriers: a lack of university research funds, the scattered nature of her archives, the sponsorship needed to gain access to some libraries. This maneuvering takes place against the backdrop of Bliss's persistent poverty. Her marginalization in the 1930s, when she no longer had nice enough clothes to attend literary events, was only compounded with time, and Allan-Burns describes petitioning for aid with the Writer's Society to help pay for her companion's nursing home. There is room for deeper reflection here, regarding the disjunction between cultural capital and financial resources, the material positions of professors in comparison to the writers they study, or simply how expensive archival research can be. As it stands, Calderaro's book will provide a jolt of recognition for most scholars, and acts as a tribute to the many people that make books happen.

Bliss is slowly attracting a larger following, with Evelyn O'Callaghan's early work on the writer (O'Callaghan 1993), which provided Calderaro 
with the springboard for her project, now complemented by readings from Natasha Omise'ke Tinsley (2010), Emma Sterry (2017), and Elizabeth McMahon (2016). Yet Calderaro is still the main scholar working on Bliss, and her new monograph vastly increases the amount of information available about the life of this long-neglected writer. Sheer Bliss is unconventional, to be sure, and unlikely to satisfy readers that are theoretically inclined, but for anyone interested in learning more about Bliss, the book's wealth of empirical information renders it invaluable.

\section{Bibliography}

Garman, E. (2019). "Feminize Your Canon: Eliot Bliss". Paris Review, 12. https://www.theparisreview.org/blog/2019/03/12/feminize-yourcanon-eliot-bliss/.

McMahon, E. (2016). Islands, Identity and the Literary Imagination. London: Anthem Press.

O'Callaghan, E. (1993). Woman Version: Theoretical Approaches to West Indian Fiction by Women. New York: St. Martin's Press.

Sterry, E. (2017). The Single Woman, Modernity, and Literary Culture: Women's Fiction from the 1920s to the 1940s. London: Palgrave Macmillan.

Tinsley, O.N. (2010). Thiefing Sugar: Eroticism between Women in Caribbean Literature. Durham (NC): Duke University Press. 


\section{Sara Florian Caribbean Counterpoint: The Aesthetics of Salt in Lasana Sekou}

Lucio De Capitani

Università Ca' Foscari Venezia, Italia

Review of Florian, S. (2019). Caribbean Counterpoint: The Aesthetics of Salt in Lasana Sekou. Philipsburg, St. Martin: House of Nehesi, 176 pp.

Recent developments in literary criticism (especially at the intersection of world literature, environmental humanities and postcolonial studies) have stressed the importance of commodity frontiers as a framework to understand colonial and neocolonial realities, and their literary manifestations. An example is World Literature and Ecology: The Aesthetics of Commodity Frontiers, 1890-1950 (Niblett 2020), which explores the literary registrations of sugar, cacao, coal, and oil frontiers in Trinidad, Brazil, and Britain. Sara Florian's monograph, Caribbean Counterpoint: The Aesthetics of Salt in Lasana Sekou, might be said to partly cover similar grounds. This book explores the writings of Lasana Sekou, "poet, short story writer, essayist, author of dramatic monologues and historical profiles, and journalist from the Caribbean island of St. Martin" (2). St. Martin, divided between France and the Netherlands, is one of the few territories in the region to remain under the control of European powers. It is also noticeable because its history has been uniquely intertwined with the salt trade: salt was the main product of the island during the Slavery period, and its extraction left significant traces on the island's ecosystem. Both aspects, as Florian argues through-

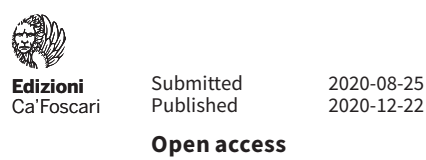

(C) 2020 | ()( Creative Commons Attribution 4.0 International Public License 
out the book, are constitutive of Sekou's work, whose nationalism, anticolonialism, anti-imperialism and internationalist political solidarities, as well as aesthetics, poetics and literary craft, are systematically interwoven with images of salt and saltiness. Florian's book uses salt as a point of departure to introduce Sekou's aesthetics and poetic vision, and then moves on to explore a great variety of issues within Sekou's oeuvre, while simultaneously paying attention to the wider cultural world of the Caribbean and to the history and ecology of St. Martin.

The book's title is useful to understand Florian's methodology. As she points out:

The title of this work on Lasana M. Sekou, Caribbean Counterpoint, hints - in the use of a piece of musical vocabulary - at his poetic voice as both independent from and interdependent with other Caribbean voices. [...] Poets of the Caribbean exhibit their own literary contours and colours, but they sing together, expressing different aspects of their society and creating exquisite, complex poetic ensemble in their production as a whole. (129-30)

This vision is embodied in the structure of the book, whose three central chapters (on salt, on marronage and on "West Indian" aesthetics, respectively) differ quite significantly in their focus: the former two are almost entirely dedicated to Sekou's work (albeit with several references to and comparisons with other Caribbean authors like such as Braithwaite, Walcott, Lamming and Glissant), while in the latter Sekou is much less prominent, and Florian focuses rather on other Caribbean voices. This shift, and other minor ones within each chapter, are rather abrupt, and not always fully justified to the readers as they move from one section to the other. As a matter of fact, the concept of "Caribbean Counterpoint", which helps to understand why Florian alternates between in-depth analyses of Sekou's work and long digressions on wider literary debates, is only fully introduced, rather belatedly, in the conclusion. Nevertheless, when the book ends, a clear design emerges out of its seemingly disjointed chapters, rewarding the reader's effort.

The book opens with an introductory chapter on the poet and his "I-land" (as he calls it in a poem). This brief chapter provides useful coordinates to set the scene of Sekou's poetry, including details on the (colonial) history of St. Martin: most notably, the marine salt trade and the island's diverse linguistic heritage, which play a crucial role in Sekou's work and in Florian's analysis. After these premises, Florian, in arguably the most fascinating chapter of the book, systematically maps the salt imagery in Sekou's work. As she argues, salt (at times in combination with sugar) presents itself as a versatile set of symbols and images that connects the various facets of Se- 
kou's poetic imagination. Salt, for instance, allows Sekou to find the images and the words to talk about slavery and colonial violence in St. Martin: a recurring image in his poems is that of the Great Salt Pond of St. Martin (as in the poem "The Great Salt Pond Speaks", from the 2005 collection The Salt Reaper), which has collected the blood and sweat of the slaves extracting the salt for the colonial masters and is therefore a literal repository of colonial history. The connection between salt as trade good and (salty) bodily fluids is a crucial one in Florian's analysis, as it allows her to connect slave labour and colonial history with rather different elements in Sekou's work: for instance desires and eroticism, or a sense of fertility that is also connected with the spread of revolutionary ideas. She also points out that salt, for Sekou, is metaphorically connected with the power to cauterize, preserve, season and heal, and that its connotation ranges from the physical to the spiritual and the political. Florian's attempt to pinpoint an "aesthetics of salt" - as the subtitle of her work recites - is at its best when it highlights the overlapping between these (at times widely diverging) associations. Moreover, Florian correctly points out that salt, while uniquely prominent in Sekou's work, also connects him to his fellow Caribbean writers. Florian, throughout the chapter, is always putting Sekou in dialogue with other Caribbean writers and thinkers.

After having established the aesthetics of salt as the founding element of Sekou's poetry, Florian moves on to discuss other aspects of his work. The following chapter is dedicated to marronage - the historical practice, widespread in the Caribbean and throughout America, of escaping plantations and forming communities at the fringes of slave societies. Florian explores how the recovery of the maroon figure is crucial for Sekou's politics and nationalism, as a symbol of struggle for freedom. Most crucially, she argues:

If we extend the concept [of the maroon] to encompass all human beings who resist abuse or the negative aspects of colonial experience, then we can argue that maroons still exist today. They are indeed present in Sekou's poetry and in his narratives. (56)

The maroon, therefore, is not only ubiquitous in Sekou's poetry, but takes different forms and resonates with a variety of different contexts, variously engaged in struggles for liberation - an internationalism that is facilitated by the multiplicity of colonial societies across the Caribbean and America where marronage took place. As she points out, "Sekou is continually portraying the maroon spirit as adaptable and unconquerable, as quite alive in the present day, ready to fight current forms of oppression and to remember" (84). Florian frames Sekou's engagement with marronage as a diverse set of images and references that allow him to engage with a variety of locales 
and situations, similarly to how he uses salt imagery. Both the aesthetics of salt and marronage, moreover, have a role within the project of spiritual and political awakening that is at the heart of Sekou's writing. As the chapter progresses, the internationalism inherent to the figure of the maroon gives Florian her cue to discuss other ways in which Sekou practices a form of transnational poetry: the coexistence of multiple languages within his work - St. Martin English, Caribbean and African languages, European languages, as well as Chinese - and his incorporation of various musical and dance traditions as part of his own aesthetics. Sekou's poetry, therefore, emerges as profoundly glocal - rooted in St. Martin's history, but engaged, with its imagery and style, with the wider world.

The last chapter further explores Sekou's engagements and influences by discussing the evolution of a "West Indian" aesthetics, connected with the development of a "West Indian" identity. The chapter is rather ambitious, discussing crucial topic such as orality, the use of creoles and the role of the Caribbean artist. As mentioned earlier, Sekou himself takes the back seat in this chapter, which is meant to provide the reader with a wider literary and cultural context for the in-depth analysis of Sekou's work presented in the previous two chapters. While, as discussed above, this results in a sudden shift in the book's focus which is not handled flawlessly, Florian's ability to locate Sekou within wider critical and literary debate is ultimately admirable and makes for a better, more satisfying portrait of his poetic universe. To this aim, it should be noted that the book also includes an interview with Sekou, which complements very well the critical reflections carried out within Caribbean Counterpoint.

Overall, the book is a rich source of insight on a leading Caribbean author, being both exhaustive and framed within a coherent, suggestive interpretative framework. I would argue that one specific passage of the conclusion beautifully encapsulates Florian's reading of Sekou's work, weaving together the overarching concerns of the book - salt, marronage, language and politics:

Sekou's voice, arising out of the swamp of St. Martin, his salt marsh, his pond, or climbing and descending the heights with the maroons does not always speak in creole, but he often uses the creole languages of the Caribbean, forms of speech, including his St. Martin nation tongue to define his roots and characterize his writing. His voice is polysemic and polyglottic at the same time. His poet-warrior voice is strong, resembling the battle given by the maroons, and it is salty too, salty as unholy slavery. (130) 


\section{Bibliography}

Florian, S. (2019). Caribbean Counterpoint: The Aesthetics of Salt in Lasana Sekou. Philipsburg, St. Martin: House of Nehesi.

Niblett, M. (2020). World Literature and Ecology: The Aesthetics of Commodity Frontiers, 1890-1950. Basingstoke: Palgrave Macmillan. 



\section{Listen, the Landscape: The Nature Narratives in Jacob Ross' Short Story Collection Tell No-One About This}

Michelle Ramos-Rodriguez

University of Puerto Rico - English Department, College of Humanities

Review of Ross, J. (2017). Tell No-One About This. England: Peepal Tree, 351 pp.

"Short stories are like a shot of vodka or gin - concentrated and full of impact", explains Britain-based Grenadian author Jacob Ross in an interview with Sofia Aatkar, the perfect description for his forty-two years of short stories encompassed in Tell No-One About This (Peepal Tree Press, 2017). The book is divided into four sections: Dark, Dust, Ocean, and Flight, each subtitle representing an aspect of the setting or characters on which his writing centers. To exemplify this, in Dark, the story "A Game of Marbles" describes a boy using his slingshot and marbles during the night to provide for his family. Dust presents "And There Were no Fireflies", the story of a young girl experiencing the changes of her body while admiring a different type of beauty she begins to recognize through her aunt's figure, for

what remained of the dying day settled on the woman's skin like dust. (Ross 2017, 172)

As a transition from Dust to Oceans, "A Way to Catch the Dust" tells the story of an elderly man who describes the sea as a taker and giver of life to his young apprentice whom he is teaching how to predict the weather by comprehending the language of nature. Lastly,

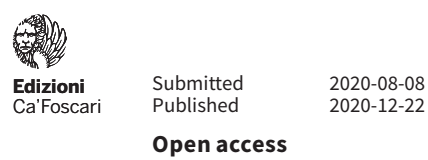

(C) 2020 | ()( Creative Commons Attribution 4.0 International Public License 
in Flight, "A Better Man" portrays a migrant woman demonstrating her strength after finding a remnant of her past in the strange and unknown London. Each section highlights human interaction in the form of love, compassion, violence, conflict, and heartbreak. This is clear in the presence of themes such as mother/child relationships, gender roles, education, grief, racism, politics, tourism and class struggle which play important parts in his framing of the Caribbean, particularly his home island Grenada.

Furthermore, Ross represents Caribbean people still struggling with the remnants of colonization. Their exploitation is closely tied to the natural landscape, for it, too, suffered from the colonizer's quest for power. Colonialism stripped the land of its natural resources through deforestation and the plantation system in order to satisfy the needs of those at the other side of the world. In Ross' short stories, nature is a character that observes and interacts with the human environment because he recognizes the importance of all the life found in the Caribbean landscape.

In one of the most compelling stories in the collection, "De Laughin Tree", a grandmother fights to retain her land instead of selling and letting it be developed into a tourism area to resemble the reality of many Caribbean islands. The biggest appeal of the land is its position - a clear view of where the sun and the sea meet that opens the eyes of our child narrator into understanding:

Like it had a voice inside o me dat was sleeping, an is only dat sight dat wake it up. It tell me dat nutting can't belong to nobody. (Ross, 2017, 118)

The grandmother understands this principle and plants the indomitable, 'unrootable' tree - the laughin tree - as a defence. While the laughin tree grows in this fertile land, the rest of the community is trapped in the tourism economy and displaced to a land that offers nothing but sickness. As the story progresses and the tree's presence is defended, the narrator's statement feels truer: nature is alive and deserves to co-exist in our landscapes without having to be threatened by capitalist economies that rely on distortion and bounded beauties. The symbiotic relationship in this story between human and nature is what can ensure the survival of the Caribbean ecosystem.

Likewise, in "Roses for Mister Thorne", another elderly woman, Miss Anni, grows roses at the request of the political figure Missa Thorne. As she finds herself uncertain about the future, she dedicates her days to nurture her flowers regardless of the physical damage to her body, paradoxically through the object reflected in his name:

How in growing this gift for him, there was also cruelty: the destruction of the stunned and malformed, the burning and uproot- 
ing. She might make him aware that she paid for her cruelty with blood. Becuz this rosebush wore a fortress of thorns that did not spare her hands. (Ross 2017, 139)

She considers the limits of growing and cutting flowers that give aroma and beauty before being replaced when they wither. Miss Anni explains that the roots are the essential part; they are "the part that grow" (Ross 2017, 144). The roses become a metaphor for the future of Grenada and the Caribbean. While there are claims of progressive political strategies to guarantee a fruitful future, their superficial and attractive ideals do not enfranchise the entire community. The roots represent the people, and all the pain is worth it, if it means that the roots are planted in fertile ground; it is the only way they will truly thrive.

Differently, the sea sets the atmosphere in "Listen, the Sea". Amos, the main character, acknowledges how the people on the island abide by its rules:

You listen to the sea like how a person' voice and unnerstand their mood. You watch the water move and know what that mean. (Ross 2017, 270)

He finds himself intrigued by a white U.S. tourist, Nancy, who challenges it each time she jumps into the waters without hesitation, yet he understands that her success will not last:

Whatever skill or trick she uses to defy the tide will not save her. Those killing waters will swallow her and spit her out in some dirty shore in South America. (Ross 2017, 252)

As he recognizes her recklessness, Amos ignores how he too puts his life at the mercy of the sea. His mother's house rests on the edge of a cliff since the waters have slowly taken chunks of the hill, and from the sea-facing wall he can see the stones below. When he wakes up from the terror of loneliness,

the frenzy of the waters below threaten to rise up and suck him down. (Ross 2017, 259)

The possibility of falling or being pulled into the sea is not enough to move him; he remains at the edge carelessly. In this story, Amos and Nancy are a reflection of each other. They each suffer from unhealed traumas and are looking for ways to cope in the Grenadian landscape. They are actively and passively seeking the sea in hopes of having the waters choose when to set them free.

The presence of nature in some of Ross' stories recognizes the inescapable connection Caribbean people have to their landscape. His 
nature becomes a place of reflection, a place of personal and collective battles and revolutions, a generative place to grow a new and better future for Caribbean people. In "Bird", a little girl finds a Dreamflyer or Soul-carrier, the bird her recently lost friend had described as the animal version of the wind. She reflects

if people found this bird with her, they would kill it becuz on Kara Isle, anything they did not like or understand they destroyed... in all the sea-stories she'd ever heard them tell, at the end of every one, something always died. (Ross 2017, 348)

This child understands the assault nature continues to endure in contemporary times, a residue of colonization, and decides to ensure this bird's safe flight. Ross' storytelling leaves his characters and readers questioning what they must do in their quest for liberation, without selling, undermining or killing the natural presence that surrounds them. 


\section{Carla Tempestoso Il teatro di Caryl Phillips. Transnazionalità, identità e appartenenze}

Giuseppe Capalbo

Università di Verona, Italia

Review of Tempestoso, C. (2020). II teatro di Caryl Phillips. Transnazionalità, identità e appartenenze. Roma: Aracne, 180 pp.

In a creative piece of writing entitled "Rude Am I in My Speech", Caryl Phillips stated: "My father is no Othello" (Phillips 2011b, 190). In so doing, the difficult-to-be-labelled author drew an eerie parallelism between his father and a Shakespearean character: in the first place, it is grounded on what Frantz Fanon described as "epidermal racial schema" (Fanon [1952] 2008, 92) because both his father and Othello share the same skin colour, which defines them beforehand' as "extravagant and wheeling stranger" (Oth., 1.1.135); in the second place, it connects Phillips with drama, the first genre he undertook at the dawn of his career.

According to Bénédicte Ledent, his early plays can be viewed as 'threshold texts' for his later writings (Ledent 2015, 85): and yet, Phillips's scholarship mainly focuses on either his novels or non-fiction works. Carla Tempestoso's Il teatro di Caryl Phillips seeks to fill this void by analysing, in the light of the Postcolonial Studies, the three stage plays performed and published between 1981 and 1984: Strange Fruit (1981), Where There is Darkness (1982), and The Shelter (1984). The volume consists of an introduction, three chapters and an afterword by Bruna Mancini.

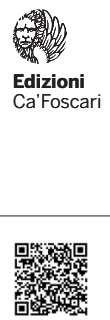


In the introduction, Tempestoso, following Ledent's insight, outlines the commonalities of Phillips's plays and novels: as many secondgeneration writers, his oeuvre is moulded upon the themes of belonging, displacement, homelessness (12). Mindful of Phillips's biography, she briefly relates them to his 'assimilation fatigue' (Phillips 2011a, 15): he was born in St. Kitts in 1958, brought up in England and, in 1978, he moved to the USA, where he now teaches Literatures in English at Yale University. Tempestoso describes these journeys as both physical and metaphorical: Phillips did not just move from one place to the other but, in the process, he had to go through the prejudices of the "European tribe" as well (13). Consequently, he often felt - just as Hanif Kureishi - "pretty out of place" (Kureishi 1986, 17); by writing, he was able to free himself from a fixed concept of 'belonging' and give birth to an 'itinerant identity' (14). The adjective 'itinerant' calls into question the idea of movement, which is at the core of this monograph because, through the lens of the Postcolonial Studies, Tempestoso analyses how Phillips's "diasporic wanderers" are affected by - and affect in return - Eurocentric spaces and places.

Chapter 1 - Multiple Identities in Transnational Spaces - consists of a theoretical discussion on the very concept of 'identity': according to Tempestoso, as migrations have redefined humanity as a whole, Postcolonial Studies and its 'progeny' (e.g. Diaspora Studies, Migration Studies, Transnational Studies) should take an intersectional approach to the analysis of the Twenty-first-century migrant experience (22). In an attempt to do so and highlight what she otherwise calls 'in-between spaces' (31), Tempestoso interweaves postcolonial voices, such as those of Bill Ashcroft, Homi Bhabha, and Stuart Hall. She specifically deals with the topographical dimension of their writings: for instance, by taking her cue from Bhabha's The Location of Culture, Tempestoso states that the so-called 'Third Space' - "which makes the structure of meaning and reference an ambivalent process" (Bhabha [1994] 2004, 54) - aims at re-enhancing one's own agency, thus reassessing our understanding of race as a Derridean pharmakon (Bassi 2016, 75). In the case of Caryl Phillips, the Third Space - complex, fluid, interstitial - is represented by the Atlantic Ocean, being a site which carries no connotations of either colour or race (Mancini 2014, 3): thus, he turns upside down the common idea of belonging as land-based, in favour of the water-based counterpart. Tempestoso is aware of the side effects of such a choice: by looking at both Phillips's life and writing, she points out how the feeling of physical, psychological, and cultural displacement has massively influenced his Dasein, his being-in-the-world (44).

Following these theoretical underpinnings, Chapter 2 - Identities and Belonging in Caryl Phillips's Theatrical World - is centred on the genesis of Phillips's early writing: from his experience as stagehand in Edinburgh to the creative process itself. Beyond its unquestiona- 
ble therapeutic effect, the act of writing turns out to be a way of negotiating his identity and becoming visible among the white British writers (80). Furthermore, thanks to her research period at Yale University (USA), where she met Phillips, Tempestoso offers invaluable information regarding genre classification: for example, she makes reference to the existence of another stage play - Rough Crossings (2007) - which the author himself refused to consider so, as it is a 'commissioned adaptation' of Simon Schama's novel (71).

The Shakespearean parallelism, which opened the review, is extensively explored in this chapter, through Phillips's collection of essays The European Tribe (1987) - specifically, "In the Ghetto" relates how the tragedy of Othello affected him while in Venice (De Chiara 1996, 179) - and the novel The Nature of Blood (1997), which "interweaves the fictional story of Othello and the true history of Venetian Jews" (Bassi 2011, 243). Moreover, Tempestoso highlights the importance of both the paratextual apparatus and the intertextual references, be they direct or indirect, to Othello, The Merchant of Venice, and The Tempest: for instance, in Strange Fruit, a non-white man defines himself as an "old black ram" (Oth., 1.1.89); in The Shelter, the black man and the white woman who inhabit a deserted island remind us of Caliban and Miranda in The Tempest.

Chapter 3 - Literary Displacements on Stage - is devoted to the analytical analysis of each and every stage play. With regard to Strange Fruit, Tempestoso at first puts the stress on the title: it refers back to a song of the same name which, as Phillips himself remarks, "made reference to racially motivated violence in the American South" (Philips 2011a, 99). In her view, besides the racial matrix, to mention the so-called 'strange fruits' is to hint at the exoticisation and alienation of Phillips's non-white characters in the white Western society (102). She then pinpoints the characters' naming procedure: the quality of "singular referring terms" (Casagranda 2013, 12) is lost because Phillips chose common names (e.g. "Mother"); however, according to Tempestoso, this allows the reader to better identify with the dramatis personae, as if they were archetypes (103).

One of the major themes, which Tempestoso is keen on analysing, is the communication between second-generation and third-generation migrants, which creates a powerful connection with the following play, namely Where There is Darkness. Whereas she previously focussed on either paratextual elements or naming procedures, here Tempestoso offers a geocritical analysis of the setting: on the one hand, there is the conservatory, which represents the unheimlich; on the other hand, the house as the place of security (121).

The aforementioned dialectic replicates the margin-centre relationship and implies a meditation upon the post-migration stress and psychological disorders a person can develop. This issue led Tempestoso to The Shelter, the last of Phillips's plays: by using Frantz Fanon's 
theory of the white gaze and Simone de Beauvoir's The Second Sex (1949), she focuses her attention on the spatial dynamics interracial relationships are exposed to.

Bruna Mancini's afterword, Spaces of Memory, Identity, and Rebirth in Caryl Phillips's Theatre, offers a literary-geography-oriented insight into the world of Caryl Phillips. She defines him as a cartographer because, in his entire oeuvre, he re-writes spaces and places, thus redefining power relations (151). By leveraging her experience as the Italian translator of The Shelter, Mancini identifies the stage as an inter-ethnic laboratory, a Third Space, where Phillips's characters can acknowledge their past, master their wounds, and reshape the white space they inhabit (152).

Overall, Carla Tempestoso's Il teatro di Caryl Phillips breaks new ground and adds significantly to the current scholarship on Phillips. Through the lens of the Postcolonial Studies, Tempestoso is able to characterise his three plays as a long continuum, rich in similarities, which redeems the "queer migrant" - i.e. the non-European, non-white, and non-Christian migrant (Chambers 2020, 18) - from any subaltern position.

Nevertheless, what the monograph lacks, and it could be the starting point for future research, is a textual analysis of Phillips's stage plays in the light of the Performance Studies: along with the drama text, the representation of the dramatic fiction could be an inviting field to be investigated, by looking at both its performances - recorded, ongoing, or upcoming- and at the dynamic relationship among sourcer (e.g. author), producers (e.g. directors, designers, technicians), performers, and spectators (Schechner 2020, 60).

\section{Bibliography}

Bassi, S. (2011). "Barefoot to Palestine: The Failed Meetings of Shylock and Othello”. Bassi, S.; Tosi, L. (eds), Visions of Venice in Shakespeare. Farnham, Surrey-Burlington (VT): Ashgate, 231-349.

Bassi, S. (2016). "I pericoli della razza: riflessioni italiane a partire da Paul Gilroy”. From the European South, 1, 75-82.

Bhabha, H.K. [1994] (2004). The Location of Culture. London; New York: Routledge.

Casagranda, M. (2013). Procedure di naming nel paesaggio linguistico canadese. Trento: Tangram Edizioni Scientifiche.

Chambers, I. (2020). "Broken Archives in a Migrating Modernity". Socioscapes. International Journal of Societies, Politics and Cultures, 1(1), 17-28.

De Chiara, M. (1996). “Otello è ancora a Venezia: Caryl Phillips narra la tragedia di un colore”. Di Michele, L. (ed), Aspetti di Othello. Napoli: Liguori, 175-81.

Fanon, F. [1952] (2008). Black Skin, White Masks. English translation by Richard Philcox. New York: Grove Press. 
Kureishi, H. (1986). My Beautiful Laundrette and The Rainbow Sign. London: Faber and Faber.

Ledent, B. (2015). "Caryl Phillips's Drama: Liminal Fiction under Construction?". Journal of Postcolonial Writing, 51(1), 84-94.

Mancini, C.B. (2014). "Across the Atlantic. Caryl Phillips e la questione dell'appartenenza". Phillips, C., The Shelter/Il rifugio. Translated and annotated with introduction by C. Bruna Mancini. Napoli: Liguori, 1-18.

Phillips, C. (2011a). Colour Me English: Selected Essays. London: Harvill Secker.

Phillips, C. (2011b). "Rude Am I in My Speech". Bassi, S.; Oboe, A. (eds), Experiences of Freedom in Postcolonial Literatures and Cultures. London; New York: Routledge, 187-91.

Schechner, R. (2020). Performance Studies: An Introduction. 4th ed. London; New York: Routledge.

Sanders, N. (ed.) (2012). William Shakespeare: Othello. Cambridge: Cambridge University Press.

Tempestoso, C. (2020). Il teatro di Caryl Phillips. Transnazionalità, identità e appartenenze. Roma: Aracne. 



\section{Inua Ellams Three Sisters}

Ilaria Oddenino

Università degli Studi di Torino, Italia

Recensione di Ellams, I. (2019). Three Sisters. London: Oberon Books, 112 pp.

Il 19 febbraio 2020 si è conclusa al National Theatre di Southbank, Londra, la rappresentazione di Three Sisters, adattamento del capolavoro di Čechov firmato dal poeta e drammaturgo londinese di origina nigeriana Inua Ellams. Per Ellams è stato il primo ritorno a teatro dopo il successo travolgente di Barbershop Chronicles (2017), lo spettacolo ambientato nei saloni di barbiere di Johannesburg, Harare, Kampala, Lagos, Accra e Londra che lo ha consacrato come una delle personalità più interessanti della nuova scena culturale britannica. A differenza di Barbershop Chronicless, Three Sisters è un lavoro di riscrittura che si inserisce idealmente nel solco della produzione poetica dell'autore; nel 2015, infatti, Ellams aveva dato alle stampe \#Afterhours, una raccolta in cui instaurava un dialogo immaginario con poeti inglesi e irlandesi del passato attraverso una serie di 'response poems' che portavano lo stesso titolo dell'originale, seguito da 'after' e dal nome dell'autore scelto. Più che vere e proprie risposte, le poesie di \#Afterhours erano narrazioni inedite e personali ispirate ai temi, alle voci e al ritmo dei testi di partenza, testi che rappresentavano quindi una sorta di matrice porosa entro la quale espandersi fino a trasformarsi in qualcosa di interamente altro. Quello di Ellams è un Three Sisters 'after Cechov', come recita il sottotitolo dell'opera; è dunque una riscrittura che se da un lato si mostra per molti versi rispettosa dell'originale di cui riveste lo stampo, dall'altro sradica le sorelle dalle campagne russe di fine '800 e le traspor-

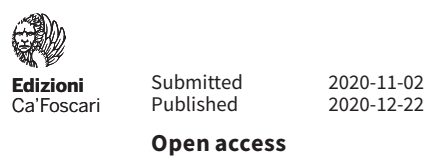

(C) 2020 | ()( Creative Commons Attribution 4.0 International Public License 
ta nel sud-est della Nigeria tra il 1967 e il 1970, intrecciando le loro vicende personali con il dramma collettivo della guerra del Biafra. ${ }^{1}$ In questo senso, Three Sisters non rappresenta un classico esempio di postcolonial rewriting, in cui rispondere a un testo significa scardinarlo e ribaltarne il paradigma, le dinamiche di potere e le proiezioni imperiali, ma ha più a che vedere con un'idea di ibridità e commistione come cifra artistica, un'abitudine al dialogo tra epoche, luoghi, voci e storie più o meno lontane. Detto questo, una volta proiettato nella Nigeria di fine anni '60, il Three Sisters di Inua Ellams assume inevitabilmente i contorni di una lucida critica del passato coloniale e neocoloniale del paese africano e della narrazione 'canonica' dello stesso, caratteristica che segna una dipartita significativa dal dramma russo di origine.

Il sipario si apre sulla grande casa della famiglia Onuzo a Owerri, Igboland; è un martedì di maggio del 1967, pochi mesi prima dello scoppio del conflitto. La simmetria tra le due versioni appare subito evidente: la Ol'ga cechoviana, maggiore delle sorelle, è qui Lolo, nubile, devota ai suoi alunni e al lavoro di insegnante prima e di direttrice scolastica poi; Maša è Nne Chukwu, intrappolata in un infelice matrimonio combinato con Onyinyechukwu, che lavora nella stessa scuola di Lolo e di cui quest'ultima, scopriremo, è (stata) innamorata; Irina è Udu, la più giovane, spensierata e vivace delle tre, simbolo di un esistere ancora in potenza che presto vedremo soccombere all'immobilismo che le assegnerà, inesorabile, il destino. Le sorelle Onuzo sono parte di una colta famiglia Igbo che undici anni prima aveva lasciato Lagos per seguire il padre, che aveva preso il comando di una brigata militare di stanza a Owerri. La scelta di trasferirsi nella regione rappresentava per lui un'esplicita volontà di allontanarsi da quella che definiva «erosione culturale coloniale» ${ }^{2}$ e ripristinare un più autentico contatto tra la sua famiglia e le tradizioni Igbo radicate nel territorio del sud-est. Come Mosca per le sorelle Prozorov, Lagos diventa fin da subito una sorta di terra promessa verso cui fantasticare un ritorno, un altrove ideale su cui proiettare aspirazioni e sogni di felicità lontano dalla prigione asfissiante del presente. Il valore simbolico di Lagos è ben riassunto da un commento del personaggio di Ikemba/Veršinin, nuovo comandante della guarnigione locale arrivato da poco proprio da Lagos, con cui Nne Chukwu intreccia una relazione. Alla fine del secondo atto, durante un temporale, lei gli dice che se fosse a Lagos non si accorgerebbe neanche

1 Il Biafra, territorio a maggioranza Igbo, aveva fondato una repubblica indipendente dal governo centrale, ma il tentativo di secessione ebbe vita breve e dal gennaio 1970 la regione, con una popolazione decimata da tre anni di guerra e malnutrizione, fu reintegrata nella federazione nigeriana.

2 Atto I. Tutte le traduzioni del testo sono dell'Autrice. 
che sta piovendo; Ikemba le risponde che se fosse a Lagos non si accorgerebbe neanche di Lagos, perché il desiderio, unico vero motore delle nostre esistenze, sarebbe ormai spento. Lagos/Mosca, quindi, come simbolo di una felicità mai veramente raggiungibile: non siamo fatti per conoscerla, ma solo per desiderarla.

A Lagos le sorelle non faranno mai ritorno, e le promesse di felicità o soddisfazione che sembrano, in momenti fugaci, illuminare le loro vite si risolvono tutte in un amaro fallimento. Lolo accetta un ruolo da preside nell'istituto in cui lavora solo a condizione di poter riscrivere il programma scolastico («Mi rifiuto di usare quei libri inglesi che dicono che Mungo Park ha scoperto il fiume Niger! Com'è possibile, se i nostri antenati si bagnavano in quelle acque quando i suoi non erano nemmeno nati?». Atto II); ma con la fine del conflitto il sogno di un Biafra indipendente andrà in fumo, portando con sé le idee riformiste di lei: la sovranità del governo federale verrà ripristinata e le tre regioni Hausa, Yoruba e Igbo verranno divise in dodici stati, all'interno dei quali la Nigeria istituirà 'Unity Schools' per «mostrare che possiamo essere tutti dei veri nigeriani» (Atto IV); e in queste scuole si dovrà mantenere il vecchio programma. Nne Chukwu e Ikemba si innamorano e in quell'amore lei sembra finalmente trovare un senso, ma lui ripartirà per Lagos con le figlie e la moglie inferma lasciandola lì, con un marito dall'ottimismo ottuso e ostinato che finge di non essersi accorto di nulla. Udu è sul punto di tornare a Lagos davvero quando accetta di sposare Nmeri (Tuzenbach nella versione originale), ma alla fine del quarto atto il futuro sposo verrà ucciso con un colpo di pistola da Igwe (Solenyj), anch'egli innamorato di lei. Tutto è destinato a rimanere fermo.

Mentre il tempo di Čechov è un tempo soggettivo, scandito dai languori dell'anima e da un astratto filosofare sui grandi temi della vita, nel Three Sisters di Ellams entra prepotentemente sulla scena il tempo collettivo di un dramma a cui nessuno può sottrarsi. Il peso ineluttabile della storia si affaccia dapprima nelle conversazioni tra le sorelle e i diversi personaggi (tra cui il fratello Dimgba, l'odiata moglie Abosede - qui un'esuberante e spietata donna Yoruba, lo 'zio' medico Eze, ma soprattutto i militari) che orbitano intorno alla casa degli Onuzo, mentre la realtà del conflitto si fa sempre più vicina e pressante. Si discute, tra le altre cose, del concetto di decolonizzazione, ${ }^{3}$

3 NMERI: Hanno inventato la Nigeria, messo forzatamente insieme duecentocinquanta lingue e gruppi etnici, creato questa pentola a pressione che ora è sul punto di esplodere.

IKEMBA: Nmeri, la colonizzazione è finita.

NMERI: Solo nove anni fa!

IKEMBA: Sì, e da allora la responsabilità è nostra!

NMERI: Pensi davvero che nove anni siano abbastanza per rimediare a centosessanta anni di distruzione culturale, e ai duecento prima ancora in cui i nostri padri e le nos- 
delle responsabilità britanniche nella guerra civile ${ }^{4}$ e degli interessi economici della (ex) potenza coloniale nel territorio, ${ }^{5}$ discorsi che aiutano a capire quanto i tentacoli del colonialismo si allunghino ben oltre l'ufficiale indipendenza, avvenuta il primo ottobre del 1960; l'autore si serve spesso di queste conversazioni - talvolta caratterizzate da un taglio apertamente didascalico - per offrire agli spettatori quante più informazioni possibile e restituire la complessità che caratterizza il quadro della storia nigeriana di quegli anni. È proprio la storia, infatti, la protagonista più dirompente e forse per certi versi più ingombrante di questa rilettura, e se la sua potenza serve da un lato a far sbiadire contingenze e problemi altrimenti importanti (la dipendenza da gioco d'azzardo del fratello e i debiti che ne derivano, ad esempio, che pur costeranno alle sorelle la casa di famiglia, oppure l'alcolismo dello zio Eze), dall'altro arricchisce i personaggi di una multidimensionalità inedita: date le circostanze, la ricerca di senso e prospettiva delle sorelle, qui impegnate in prima linea nelle operazioni di supporto e soccorso, non può che farsi sociale e politica, oltre che personale. Così quando alla fine di tutto le tre si interrogano sul perché di tanto dolore sappiamo che stanno sì pensando alle loro esistenze individuali, ma anche a quelle di un popolo intero. La domanda rimane, prevedibilmente, senza risposta. Nelle ultime righe, quando Udo si chiede se mai riusciranno a comprendere il senso di una tale sofferenza, Lolo ci congeda con queste parole:

«Solo i nostri antenati conoscono la risposta, e finché saremo in vita non ce la daranno. Come loro, anche noi verremo dimenticate;

tre madri sono stati venduti come schiavi? [...] Se mi pugnali nella schiena e poi tiri fuori il coltello di venti centimetri, quello non è progresso. Il progresso inizia quando la ferita guarisce. I britannici non hanno neanche estratto il coltello, e prima di guarire ci vorranno generazioni. (Atto III)

4 UDO: C'è gente disperata che arrostisce ratti e lucertole là fuori.

NNE CHUKWU: E gli inglesi fanno finta di niente. Il loro primo ministro potrebbe almeno far pressione su Gowon e sulla Nigeria affinché revochino il blocco del cibo. IKEMBA: E invece lui continua a fornire armi.

NNE CHUKWU: Manda la Croce Rossa a nutrire i nostri bambini, e al tempo stesso aiuta quelli che li fanno morire di fame.

5 NMERI: L'Inghilterra qui in Biafra ha infrastrutture per un valore di duecentocinquanta milioni di sterline. Giacimenti petroliferi, raffinerie, impianti di trivellazione, di tutto.

UDO: Quindi dovrebbero supportare noi, non la Nigeria.

NMERI: Ma hanno gli stessi interessi anche in Nigeria, e il problema è che sono convinti che la Nigeria vincerà.

ONYINYECHUKWU: Perché non facciamo saltare in aria le infrastrutture britanniche? NMERI: Perché sono la nostra sola merce di scambio, l'unico motivo per cui non ci hanno distrutti. Gran Bretagna, Shell, BP, hanno tutti investimenti qui. Se non li avessero armerebbero la Nigeria abbastanza da radere al suolo il Biafra, l'intera regione. Sarebbe un genocidio.

IKEMBA: Che vinciamo noi o la Nigeria, L'Inghilterra continuerà comunque a prendere petrolio da entrambi. (Atto III) 
nessuno si ricorderà di queste tre sorelle che hanno sopportato così tanto dolore, sono sopravvissute, ma non hanno mai conosciuto la felicità. Quando moriremo scopriremo il perché, ma vorrei tanto saperlo adesso». 

Necrologi | Obituaries | Nécrologies 



\section{In memory of Jacques Coursil, 1938-2020}

Paola Irene Galli Mastrodonato

Università degli Studi della Tuscia, Italia

If postcolonial studies still have some sort of sense beyond being just an empty label flapping in the wind of our career-prone academic departments, certainly Jacques Coursil filled that void with an overwhelming and precious presence. I had met him as a young woman of sixteen during my studies at the prestigious United Nations International School in New York City, where he landed suddenly as a professor of French literature in the late sixties. A handsome, tall, athletic black man in his early thirties, smoking a pipe at times, deep basso voice, long graceful hands that played his favourite jazz instrument - the trumpet - and a ground breaking view of what literature - and language - should be and are. His lessons on Stendhal's Le rouge et le noir already contained the imperative that what is inside the text is linked to what is outside the text, a notion that brought him to consider both Saussure and Benveniste - at the heart of two of his scholarly titles ${ }^{1}$ - as well as the passionate political manifestos of his fellow Martinicans Fanon, Césaire, and Glissant - a personal friend.

Born in the Paris banlieu from immigrant parents, he started early his path towards liberation from the heavy burden of the slave African past by joining the négritude movement of Senghor as an adjunct in his government and then moved to the Big Apple in the wake of Malcolm X's assassination, where he started jazz experimenting with Sunny Murray and Frank Wright producing a few years later - back in Europe - his first album Black Suite. After a thirty-five years span in which he left his trumpet on the nail for two PhDs in Linguistics and in Mathematics and a teaching career in literature, linguistics, and philosophy at the University of Caen in Normandy (where I met

1 La fonction muette du langage (2000) and Valeurs pures, le programme systemique de F. de Saussure (2015). 
him last in 1976), and at the University of the West Indies in Martinique, at Cornell University and University of California Irvine, he joined his former UNIS student John Zorn in 2005 and released his comeback recording Minimal Brass. Coursil recently conceived new voice, sound and text experiences with his album Clameurs where he recites an outstanding rethinking of Fanon's 1952 classic Peau noire masques blancs, and the long monologue in music Trail of Tears where he "investigates the forced migration of tens of thousands of Indigenous North Americans throughout the nineteenth century, situating this atrocity alongside the transatlantic slave trade, two foundational processes of racial capitalism", ${ }^{2}$ reaching with his art a final statement: "Je ne veux plus être l'esclave de l'esclavage".

In a paper he presented at a meeting in Siena in 2010 dedicated to Caribbean literatures, ${ }^{3}$ Coursil defined what he thought was the 'dead end' of definitions pertaining to Black Studies and African postcolonial awareness since a 'grammar' that is still based on the duality Prospero-Caliban cannot account for "l'accident historique d'avoir été encagé, transbordé et mis à la chaîne", and for this same reason "toute chaîne possède deux bouts aux points desquels se trouvent des sujets; chacun dit sa version fragmentaire et imaginaire d'une même histoire réelle", forever uniting us whites to our 'mirrored' counterparts which European colonialism has ravished centuries ago from our common Mother Africa.

Jacques died on June 26 at 82 years of age in Aachen, Germany, and he is survived by his wife Irene and two children.

2 Scott, C. (2020). "A Tribute to Jacques Coursil". Music \& Literature, July 21, 2020. https://www.musicandliterature.org/features/2020/7/21/a-tribute-tojacques-coursil.

3 “NÉGRITUDE: La Grammaire de Caliban". Francofonia, 61, 2011, 13-26. I have quoted Coursil's critical insight of 'assigning to a place, its subject' in my recent essay "Challenging Past and Present: Translating David Fennario’s Bilingual Theatre”. Di Vito, S. (a cura di), Lingue nella contemporaneità: Analisi linguistica, didattica e traduzione tra nuovi media e nuove sfide del multilinguismo. Roma: Aracne editrice, 2018, 167-81. 
Bio-bibliografie | Bio-bibliographies | Bio-bibliographies 



\title{
Bio-bibliographies
}

\begin{abstract}
ADAMI Esterino|esterino.adami@unito.it Esterino Adami is an associate professor of English language and translation at the University of Turin, Italy. His research areas include diasporic discourse, stylistics and narratology. He has written on the notion of naming in literary and non-literary texts, identity in Indian English fiction and the metaphorical rendition of specialised registers. He has authored Railway Discourse. Linguistic and Stylistic Representations of the Train in the Anglophone World (2018) and co-edited Other Worlds and the Narrative Construction of Otherness (2017, with F. Bellino and A. Mengozzi) and Within and Across: Language and Construction of Shifting Identities in Post-Colonial Contexts (2012, with A. Martelli).
\end{abstract}

ALOUANI Sara|sara.alouani@gmail.com Graduated in 2014 at the Department of Foreign Languages and Literatures of the Università of Verona. Her thesis, Rebellious Arab Women, Between Exile and Tradition: the Voice of Fawzia Zouari analysed in particular the works of the Franco Tunisian writer and journalist whom she met in Paris for an interview included in the dissertation. She, then, obtained a Master's degree in Contemporary Literature and Culture at the Birkbeck University of London, with the dissertation Exploring Islamic Radicalisation in Twenty-first Century British and American Literature. Since 2018 she collaborates as a publicist with the local newspaper II Corriere del Trentino. She is currently continuing her research on French-language and post-colonial literature, specialising in the analysis of Fawzia Zouari's works and emerging North-African Francophones writers.

BERARDI Alessia|854878@stud.unive.it Alessia Berardi graduated from Ca' Foscari University of Venice in European, American and Postcolonial Languages and Literatures - Master Européen en Études Françaises et Francophones with a score of 110/110 cum laude. During her university experience, she attended seminaries in Sorbonne Nouvelle (Paris, France, 2018), Université Laval (Québec City, Canada, 2018), and Université d'Orléans (Orléans, France, 2015). Her main interest is 20th century engaged literature, with a special focus on identity and gender issues.

BORASO Silvia | silvia.boraso@unive.it Silvia Boraso graduated in 2017 from the University of East Piedmont. Her thesis Writing Orality. Representations of Speech in Five Postcolonial Novels is a comparative study that analyses, from both a linguistic and hermeneutic perspective, the ways in which orality is reproduced within the boundaries of narrative fiction. She's currently a PhD student at Ca' Foscari University of Venice and is working on a research project focused on the representation of natural landscape in 19th-century Haitian novels. List of works published in /l Tolomeo: "Ahmadou Kourouma et la transposition de la parole malinké. Analyse de la représen- 
tation langagière et du projet de légitimation linguistique dans Allah n'est pas obligé" (2017); "The Sieve of Translation. Reflections on the French Version of The Butcher Boy by Patrick McCabe” (2018); Review of Intercultuerel Francophonies's monographic issue, Abdelkhébir Khatibi: le penser-écrire d'un intellectuel perspectiviste by Atmane Bissani (2019); Review of Yasmina Khadra's last novel, Khalil (2019).

BRANCAGLION Cristina | cristina.brancaglion@unimi.it Cristina Brancaglion is associate professor at the Department of Foreign Languages and Literatures of the University of Milan, where she teaches French language and linguistics. Her research interests lie in the area of linguistic variation in France and French-speaking countries, studied through lexicographic and literary sources or through documents taken from the press and social media. Her most recent works aim to deepen, from a historical sociolinguistics point of view, the corrective movement that spread in Montréal in the 20 th century with the development of diction schools. Cristina Brancaglion is the editorial assistant of the journal Ponti / Ponts. Langues, littératures civilizations et des Pays francophones, and coordinates the section "Etudes linguistiques". She is also responsible of some mobility agreements with France and Canada, and is the contact person for the double master's degree with the Université d'Avignon et des Pays de Vaucluse.

CAPALBO Giuseppe | giuseppe.capalbo@univr.it Giuseppe Capalbo is a PhD student in Literatures in English at the University of Verona. He earned a Master's degree cum laude in Modern Languages and Literatures in 2019 (thesis title: Queering the Body: Hanif Kureishi, Andrew McMillan, Will Self). In November 2018, he presented a paper entitled "Psychic Wounds, Pain, and Bodily Issues between Italy and the USA: The Case of Louise DeSalvo" at the conference The Cultural Language(s) of Pain: European Literary Perspectives (University of London, UK). In September 2019, he attended-as speaker and chair - the International Conference on Gender Studies Gender (Non) Conformity (University of Oxford, UK). His areas of interest include Disability, Gender and Postcolonial Studies.

CAZZATO Luigi Carmine | luigicarmine.cazzato@uniba.it Luigi Carmine Cazzato is Full Professor at the University of Bari Aldo Moro (Department of Education, Psychology and Communication Studies), where he currently teaches Cultures in English and Decoloniality. He co-leads the international research group "Un/Walling the Mediterranean" (https://smuraremediterraneo.wordpress.com/). From 2016 up to 2019, he was AISCLI vice-chair (Associazione Italiana per lo Studio delle Culture e delle Letterature in inglese: http://www.aiscli.it/). Moreover, he is in the board of the PhD School "Science of Human Relationships" and is the Director of the Master of Arts of Journalism at the University of Bari. Heis the author of several essays on the re-reading of the cultural relations between England and Italy, and on the South at large, from a postcolonial and decolonial perspective. His last monograph is Sguardo inglese e Mediterraneo italiano. Alle radici del meridionismo (Milano, 2017). His last essay "In spazi estremi, estremi disegni: l'isola di Doctor Wells fra memoria e amnesia coloniale. Un approccio decoloniale”. Brazzelli, N. (a cura di), Spazi e narrazioni nella letteratura in lingua inglese. Milano, 2020.

CERMINARA Adriana | adriana.cerminara@edu.unito.it Adriana Cerminara has recently graduated in Modern Languages for International Communication (MA) from the University of Turin with a thesis entitled Literature and Environment: the Case of Alissa York. Being deeply committed to the environmental cause and being interested in investigating the impact of human beings on the planet through literature, she 
found Ecocriticism to be the best way to explore these issues. She is willing to continue her studies with a PhD in this field.

CHEMLA Yves | yves.chemla@u-paris.fr Yves Chemla is a literary critic. PhD La Sorbonne-Paris-4 (Question of the Other in the contemporary Haitian novel, 1999), he teaches academic methodologies at University Paris Descartes / University of Paris. Visiting professor at the University of Parma in 2018, to teach contemporary Haitian literature. Member of the Francophone Manuscripts team of the Institute of Modern Texts and Manuscripts (CNRS-ENS). Recent publications: La Question de l'autre dans le roman haïtien contemporain (Cayenne, 2003); Le Roman haïtien : intertextualité, parenté, affinité (Lecce, 2007); Haïti, une traversée littéraire (Port-au-Prince; Paris, 2010); Littérature haïtienne, 1980-2015 (Port-au-Prince, 2015); with L.-F. Hoffmann, Euvres complètes de Jacques Roumain (Paris, 2018).

COSTANTINI Alessandro|costalex@unive.it Associate professor at Ca' Foscari University of Venice. His research and teaching work focuses on Francophone literatures (mainly Haitian \& Caribbean), French Algeria literature, History of French colonization; literary diglossia, Francophone linguistics. Editor of the journal /l Tolomeo (Le Nuove Letterature). Member of the Editorial Board of Interfrancophonies. Main latest publications: Fantasmi narrativi e sovversione linguistica nel romanzo haitiano moderno e contemporaneo (Milano, 2002); codirected with Yves Chemla, Interculturel Francophonies, num. 12, Le roman haïtien: intertextualité, parentés, affinités (2007); (dir.) Intefrancophonies, 7, "Nouvelles formes de l'engagement dans les littératures francophones" (2016); "De quelques catéchismes créoles anciens: oublis, pertes, disparitions, réapparitions, découvertes". Annali di Ca' Foscari. Serie occidentale, 49, 2015, 75-114; "De la littérature dite sabir. Regards coloniaux franco-algériens sur l'Autre". Annali di Ca' Foscari. Serie occidentale, 52, 2018, 141-74; "Fables et contes en sabir : avatars, réussites, échecs d'un genre paradoxal”. QUADERNI DI SEMANTICA, n.s. 6, 2020, 381-99.

D'AUTILIA Antonella | antonelladautilia@yahoo.it Antonella D'Autilia is PhD student in Human Relations Sciences at Department of Education, Psychology and Communication Studies (University of Bari Aldo Moro). She has published "Fantasmagorie politiche. Per una decostruzione delle strategie retoriche e iconografiche del populismo digitale”. Im@go Journal. Rivista di Studi Sociali sull'Immaginario, 14, 69-86.

DE CAPITANI Lucio | lucio.decapitani@unive.it Lucio De Capitani holds a PhD in Modern Languages, Cultures and Societies and Language Sciences (English Literature) from the Ca' Foscari University of Venice. His research interests include colonial, postcolonial and world literatures (especially Indian writing in English), the connections between anthropology and literary studies, travel writing, activist writing and literary journalism. He has published papers on Amitav Ghosh, Anita Desai and Robert Louis Stevenson. From 2019 to 2020 he took part, as a Ca' Foscari Research Fellow, to the FAMI Impact Veneto Project, leading workshops on migration and autobiographical writing in a high school setting. He currently teaches English Literature and English Language modules at Ca' Foscari, the IUAV University of Venice and the University of Bologna as an adjunct professor.

DOWLING Gregory | dowling@unive.it Gregory Dowling is Associate Professor of North-American Language and Literature at Ca' Foscari University of Venice. His academic research has been focused on British and American literature, with a special interest in the Romantic poets and in 20th-and 21st-century poetry. He is a member 
of the Academic Committee for the Lord Byron Museum in Ravenna, which is to be inaugurated in 2020. He has published six novels, the most recent of which (Ascension, 2015; The Four Horsemen, 2017) are set in 18th-century Venice.

FAZZINI Marco|mfazzini@unive.it Marco Fazzini is a lecturer at Ca' Foscari University of Venice. He has studied at the universities of Ca' Foscari and KwaZulu-Natal (Durban, South Africa). He has published articles and books on post-colonial literatures and has translated some of the major English-language contemporary poets: Philip Larkin, Norman MacCaig, Douglas Dunn, Edwin Morgan, Geoffrey Hill, Charles Tomlinson, Douglas Livingstone, Kenneth White. His latest books are a study on poetry and songwriting, Canto un mondo libero (2012), a book on poetry and poets, At the Back of My Ear (2019), and two collections of interviews: Conversations with Scottish Poets (2015) and The Saying of It (2017). His major poetry collections are: Nel vortice (1999); Riding the Storm (2015), 21 Poesie/Poemas/Poems (2017), Il canto dell'isola (2019) and Poesie scelte (2020).

FIANCO Fabiana | fabiana.fianco@gmail.com Fabiana Fianco graduated in Modern Languages and Literatures at University of Trento in 2016 with a dissertation called De la Négritude à la Créolité, which analyses the evolution of the francophone racial perspective through the most relevant literary theories conceived between the 1930s and the 1990s. In 2018, she completed a double degree programme with the University of Lausanne and Ca' Foscari University of Venice, where she obtained her Master's degree in European, American and Postcolonial Language and Literature. Her thesis explored the themes of sexuality and corporeality in the work of African and Haitian female novelists in contemporary times (1970-2013). She currently teaches French language in high school.

GALLI MASTRODONATO Paola Irene | paolai.galli@unitus.it Paola Irene Galli Mastrodonato has taught for nearly forty years English Language and Literature, Angloamerican Literature and Literature of Anglophone Countries in Italian State Universities, lately at Tuscia University-Viterbo. She holds a PhD in Comparative Literature from McGill University, and her publishing record includes 13 books (essays, translations, critical editions, collective volumes) and over 80 scholarly articles, ranging from the Eighteenth Century Novel during the Revolutionary Period, to Canadian playwright David Fennario, the Postcolonial analysis of Emilio Salgari's adventure novels, and Women's Studies.

GENNARI Martina | martinagennari94@gmail.com Martina Gennari graduated at the University of Macerata in Western and Eastern Languages and Cultures in April 2017 and received a master's degree in Languages, Cultures and Literary Translation in April 2020. Both her theses focus on French-Italian translation. In her first thesis, The translation of youth speak. "Les Boloss des Belles Lettres", Between Classical and Innovated Language, she analyses the varieties of spoken French, especially the Français Contemporain des Cités, in the work Les Boloss des Belles Lettres (2014), by Michel Pimpant and Quentin Leclerc. Her second thesis, The Meeting of Languages and Cultures in the Text. a Proposed Translation, Analysis and Commentary of the Novel "La vérité sort de la bouche du cheval" by Meryem Alaoui, explores the complex world of beur literature, linked to the question of identity, arguing whether this is the right collocation of Meryem Alaoui's work, according to the themes and the language employed in the text.

GIROTTO Alice | alice.girotto@unive.it Alice Girotto holds a PhD in Modern languages, cultures and societies from Ca' Foscari University of Venice with a thesis en- 
titled Forms of Representation of Power in Angola: a Journey from Literature to Visual Arts (and Back). Her main research interests concern literary and artistic expressions of Portuguese-speaking African countries within the field of Lusophone postcolonialism. She participated in several international conferences, among which the XLV Conference of the African Literature Association (Columbus, Ohio, 2019). She currently holds a position as lecturer of Portuguese and Brazilian literature at Università Ca' Foscari Venezia and of Portuguese language and translation at the University of Trieste.

GUARDUCCI Maria Paola | mariapaola.guarducci@uniroma3.it Maria Paola Guarducci (PhD in Comparative Literature) is Associate professor of English Literature at Roma Tre University. She has published on African novels in English, Victorian and contemporary British literature, South African women's poetry. She is author of Dopo l'interregno. Il romanzo sudafricano e la transizione (2008) and of articles published in Italian and English on Jane Austen, W.M. Thackeray, Joseph Conrad, Samuel Beckett, Sam Selvon, George Lamming, Monica Ali, J.M. Coetzee, women's poetry etc.

GYSSELS Kathleen | kathleen.gyssels@uantwerpen.be Kathleen Gyssels is Professor of Francophone Postcolonial Literature and Culture at Antwerp University, and member of the Institute of Jewish Studies. She is also on the board of the Society for Caribbean Studies and of several international peer reviewed journals in Caribbean literatures and postcolonial studies. Her publications are principally concerned with African American, Caribbean, Jewish and African Diasporas, Francophone authors. She studies postcolonial authors from a broad, comparative perspective. Her current research has extended her reach to include conflictual issues, such as the Memory Laws and the Memory Wars in the French Republic and postcolonial countries. Her $\mathrm{PhD}$ was on the novels of André and Si-mone Schwarz-Bart (Filles de Solitude, Essai sur l'identité antillaise dans les (auto-)biographies de Simone et André Schwarz-Bart, online with UQAC and Limag; 1996). Marrane et maronne. La co-écriture réversible d'André et de Simone Schwarz-Bart (2014); Passes et impasses dans le comparatisme caribéen postcolonial. Cinq traverses (Paris, 2010). Other essays include: Black-Label ou les déboires de L.G. Damas (2016); special issues on Damas with Dalhousie French Studies, Essays in French Literature and Culture, and René Maran for Etudes Caribéennes.

HAMON Yannick | yannick.hamon@unive.it Yannick Hamon is a researcher at the Ca' Foscari University of Venice. In 2013 he became PhD in Translation, Interpretation and Interculturality at the Department of Interpretation and Translation (DIT) of the University of Bologna, where he taught for ten years. There he also completed a postdoc contract on collaborative and distant translation. His research focuses on the use of technologies for teaching/learning languages in the specific field of writing skills and translation. He is also interested in the analysis of political discourse. Since 2018, he has been teaching French language courses in the DSLCC department, editing and translating for the journal /l Tolomeo.

LAMROUS Lila | lila.lamrous@uca.fr Lila Lamrous is an Assistant Professor of French and francophone literature at the University of Clermont-Auvergne. Her main areas of interest are poetics of contemporary francophone literature: the uses of languages, hybridity of genres and identities. She has published several articles about exile writing and migrations. Lately she has been taking an interest in francophone female literature and particularly women's agency.

LUPPI Fabio | fabio.luppi@uniroma3.it Research Fellow at Roma Tre University for Lingua e Traduzione Inglese. Phd in Comparative Literature (Roma Tre, 2008). Short-term fellowship at the Folger Shakespeare Library (2014). Author of the books 
Cerimonie e Artifici nel Teatro di W.B. Yeats (Roma, 2011); Fathers and Sons at the Abbey Theatre (1904-1938) (BrownWalker Press 2018), and Tradurre il teatro. Due case studies dall'inglese (Roma, 2019). Main fields of interest: Irish studies, Post-Colonial Studies, and Translation Studies.

MARINARO Isabella | ismarinaro@tiscali.it Isabella Marinaro is a third year PhD student in Textual Studies (English studies) at Sapienza University of Rome. She is an English teacher in Italian upper secondary schools and she also taught Spanish language and literature in Italian schools. Her main field of interest is the pedagogy of literature in Italian licei with special focus on the possible actions to enrich the English literary canon in Italian syllabi with new Anglophone authors worldwide by putting diverse texts in dialogue with one another with the help of stylistics. She is also interested in Political Discourse Analysis.

MICHIELETTO Anna | leilaflower@yahoo.it After following her studies in French and English languages and literatures with a particular focus on post-colonial studies, Anna Michieletto obtained her MA at Ca' Foscari University of Venice cum laude in 2006. Meanwhile she spent one year studying at Université Paris-Sorbonne (Paris IV) thanks to an Erasmus scholarship granted by the EU. She has taught English and French language and literature at secondary school in Italy and Italian language for foreigners at "Laboratorio Internazionale della Comunicazione" in Gemona del Friuli. She worked as a teacher of French language in Spain (EU project "Comenius") and she taught Italian at "Hacettepe University" in Ankara (Turkey). She has collaborated with /l Tolomeo. She obtained another MA in Anthropology at Università Ca' Foscari cum laude in 2012 and is very interested in travelling and studying other cultures.

MILANESIO Lia| lia.milanesio@hotmail.it Lia Milanesio graduated in 2020 with a master thesis titled Les récits animaliers de René Maran. Une analyse at Ca' Foscari University of Venice within the double degree programme "Master européen en études françaises et francophones" with the Humboldt University of Berlin (Germany). She attended her bachelor's degree at the University of Trento (Italy), where she studied Foreign Languages and Literatures - French and German - and spent a semester at Aix-Marseille University (France). Her study curriculum focused on Francophone literature and history, African cultures and languages, and French literature.

NEWBOLD David|newbold@unive.it David Newbold is associate professor of English Language and Linguistics at Ca' Foscari University of Venice. He has a background in language teaching, a long-standing interest in language testing and assessment, and a more recent interest in the development of World Englishes and English as a lingua franca, and non-native writing in English. He has published numerous materials for learners of English, and contributed to the development of a number of tests. In 2019 he was made an honorary member of Trinity College London for his contribution to language testing research.

ODDENINO Ilaria | ilaria.oddenino@unito.it Ilaria Oddenino holds a PhD in English and Postcolonial Literature from the University of Turin. She works as a lecturer at SSML Vittoria and at the University of Turin, and as a freelance translator.

PAGONE Sara | sara.pagone@yahoo.it Sara Pagone pursued a master's degree in Foreign Languages and Literatures at University of Trieste in 2019. She specialised in French and Francophone Literature. Her studies focus on the twentieth-century 
French Literature and the contemporary Moroccan Literature, particularly the French writer Paul Morand and the Moroccan author Fouad Laroui. She recently broadened her research interests to the Francophone Literature of the Indian Ocean, specifically through the works of the French comparatist Daniel-Henri Pageaux. She currently teaches French language and culture in high school.

PERRY, Amanda T. | aperry@champlaincollege.qc.ca Amanda T. Perry works on the literature and history of the Anglophone, Francophone, and Spanish-speaking Caribbean. She holds a PhD in Comparative Literature from New York University, and her publications have appeared in The Global South, Small Axe, Journal of Early Modern Cultural Studies, and several edited collections. Her book project seeks to reframe the Cuban Revolution as a Caribbean event. Dr. Perry currently resides in Montreal, where she teaches Caribbean literature at Concordia University and literatures in English at Champlain College-Saint Lambert.

PILIA Nicola | Nicola.pilia01@gmail.com Nicola Pilia is an Italian teacher of English Language who lives and works in Brescia. He graduated in Modern Languages and Literatures in April 2020 from University of Turin with a thesis titled Postcolonial Ecologies: an Ecocritical Reading of the Works by Amitav Ghosh. With his dissertation, he has investigated upon the relevance of environmental questions in the postcolonial contexts presented by Ghosh especially in The Hungry Tide and The Great Derangement. His work, supervised by Professor Carmen Concilio, was received with honours and later recommended for publication by the Degree Commission.

RAMOS-RODRÍGUEZ Michelle | michelle.ramos1@upr.edu Michelle Ramos-Rodríguez holds a MA in English with a focus on Caribbean literature and a BA in Anthropology with a minor in Literature, both from the University of Puerto Rico. She has collaborated in published interviews with various Caribbean authors including Richard Georges, Ann-Margaret Lim, and Lisa Allen-Agostini. Her academic work focuses on topics related to family, violence, trauma and nature in Caribbean literature and film.

SCARSELLA Alessandro| alescarsella@unive.it Alessandro Scarsella is professor at Ca' Foscari University of Venice. He is the author of monographs and studies on comparative literature, on the history and theory of literature, on translation studies and on hermeneutics of the text. His main areas of research are bibliophilia, the history of books, illustration and comics/graphic novels. In Venice he is the director of the Laboratory for Literary Study of Comics. He is director of Charta and the member of the scientific committee of many refereed journals such as Ermeneutica Letteraria, IF, Archivio d'Annunzio and has published the anthology Read the Italian Constitution (2008). He has written numerous voices for the Princeton Encyclopedia of Italian Literary Studies (Routledge, 2006). He has collaborated with the Einaudi Dictionary of American Literature (2011) and published the international volume Venise (Paris, 2016) and the essay novel Venetian Neuronarratology (Venice).

SCHIAVONE Cristina | cristina.schiavone@gmail.com Cristina Schiavone is Lecturer in French Language and Translation, teaching the undergraduate and graduate courses in Linguistics and Translation at the Department of Human Studies, University of Macerata (Italy), and Francophone Literatures at Alma Mater Studiorum University of Bologna (Italy); responsible for the binational degree in Lingue moderne per la comunicazione e la cooperazione internazionale/Études interculturelles francoitaliennes;coordinator of the bilateral agreement between University of Macerata 
(Italy) and Cheikh Anta Diop University, Dakar (Senegal). Research Fields: Sociolinguistic variation, Francophony, Translation, Difference and Identity, Specialised Lexicon, Language rights, Subsaharian Studies.

TERRENATO Francesca | terre@libero.it Francesca Terrenato, PhD, is Associate Professor of Dutch Language and Literature at Sapienza University of Rome. Her research interests include early modern cultural transfer and translation, gender issues in (early) modern and contemporary literary works and Afrikaans literature (especially poetry). She has published books and articles on Afrikaans women poets and migrant women authors in the Netherlands and in Flanders.

VIGNOLI Alessia | a.vignoli@uw.edu.pl Alessia Vignoli is a PhD candidate in French and Francophone Studies at the University of Warsaw (Poland). Her main research topic is the Caribbean literature of French expression, especially Haitian post-earthquake literature. She has received a research grant (2018-2021) from the Polish National Science Centre (NCN). Her PhD dissertation focuses on the writing of natural disasters in Haiti and in the French Caribbean (Martinique, Guadeloupe). Last published works: "L'universalisation de la catastrophe haïtienne dans Tout bouge autour de moi de Dany Laferrière". Dalhousie French Studies, 116, 2020; "Surmonter les traumatismes, réagir aux préjugés : Les voyages de Merry Sisal de Gisèle Pineau", in Couti, J.; Gyssels, K. (éds), “'Mines de rien': l'Antillaise et l'Afropéenne face aux tropologies, entre mythes et réalités au fil du temps". Essays in French Literature and culture, 56, 2019.

XAUSA Chiara | chiara.xausa2@unibo.it Chiara Xausa obtained a MA in Modern Philology from the University of Padua (2016), and a MA in Women's and Gender Studies from Alma Mater Studiorum University of Bologna and the University of Utrecht (2018), and she is currently a PhD candidate in Intercultural Studies at Alma Mater Studiorum University of Bologna, and a visiting PhD researcher at Bath Spa University (Research Centre for the Environmental Humanities). Her broader interests lie in the intersections between gender studies, postcolonial studies and posthuman studies. Her current research, titled Women Writing Intersectional Climate Justice from the Margins: an Alternative to the Apocalyptic Discourse of Contemporary Cli-fi, examines the work of a range of contemporary women of colour and native authors from the anglophone world, including C. Morgan Babst, Cherie Dimaline, Nnedi Okorafor, Jesmyn Ward and Alexis Wright, and is concerned with how these authors respond to the mainstream apocalyptic discourse of climate change.

ZOPPELLARI Anna | zoppelan@units.it Anna Zoppellari is Associate Professor of French Literature at the University of Trieste. Her main areas of interest are contemporary Francophone literatures and the relationships between literature and visual arts. She published several text and articles on French and Maghrebi authors. 

Rivista annuale Annual journal Revue annuelle Università Ca' Foscari Venezia

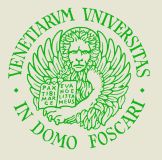

Università Ca'Foscari Venezia 